\title{
L'utilisation des services de centre de jour par les personnes âgées qui présentent des incapacités
}

\author{
par \\ Jacinthe Savard \\ Département d'administration de la santé \\ Faculté de Médecine
}

\begin{abstract}
Thèse présentée à la Faculté des études supérieures en vue de l'obtention du grade de Docteur en Santé publique

option Organisation des soins de santé
\end{abstract}

Octobre 2008

(C) Jacinthe Savard, 2008 



\section{Université de Montréal \\ Faculté des études supérieures}

Cette thèse intitulée :

L'utilisation des services de centre de jour par les personnes âgées qui présentent des incapacités

présentée par :

Jacinthe Savard

a été évaluée par un jury composé des personnes suivantes :

Régis Blais, président-rapporteur

Nicole Leduc, directeur de recherche

Paule Lebel, co-directeur de recherche

Danièle Roberge, membre du jury

Nicole Dubuc, examinateur externe

Annie Rochette, représentant du doyen de la FES 



\section{Résumé}

Contexte : Les centres de jour offrent des interventions de groupe à des personnes âgées qui présentent des incapacités, dans le but de favoriser leur maintien à domicile. Des études récentes tendent à démontrer qu'une utilisation régulière du service serait nécessaire pour induire des effets bénéfiques.

Objectifs : Cette recherche visait à documenter l'utilisation des centres de jour par des personnes âgées qui présentent des incapacités. Elle comportait trois principaux objectifs :

1) Caractériser les utilisateurs de centre de jour et ce qui les distingue des nonutilisateurs et analyser les déterminants de l'utilisation du centre de jour.

2) Explorer l'adéquation entre les activités offertes par les centres de jour et les caractéristiques d'autonomie et de santé des utilisateurs.

3) Définir les facteurs associés à la régularité de la participation.

Méthodes : Cette recherche s'appuie sur une analyse secondaire de données recueillies auprès de 723 personnes âgées qui ont participé au projet de démonstration SIPA (Services intégrés pour personnes âgées) implanté dans deux CLSC de la région de Montréal. L'utilisation du centre de jour a été documentée pendant une période de six mois, auprès des cinq centres de jour existant sur ce même territoire. Des informations sur le fonctionnement des centres de jour ont été obtenues par des entrevues et des rencontres de groupe auprès de coordonnateurs de ces centres. Les données ont été analysées à l'aide de statistiques descriptives, d'analyses en regroupement et d'analyses de régression logistique et multiple.

Résultats : Les résultats sont présentés dans trois articles, soit un pour chacun des objectifs. Article 1 : La proportion d'utilisateurs de centre de jour est de $18,8 \%\left(\mathrm{IC}_{0,95}\right.$ : 16,0 à $21,7 \%$ ). Les facteurs suivants augmentent la probabilité d'utiliser le centre de jour: être plus jeune (RC: 1,12; $\mathrm{IC}_{0,95}: 1,06$ à 1,19); ne pas avoir une éducation universitaire ( $\mathrm{RC}: 1,92 ; \mathrm{IC}_{0,95}: 1,04$ à 3,57); recevoir l'aide du CLSC pour les activités de vie quotidienne plus d'une fois par semaine (RC : 1,73 et 2,48 pour aide de deux à cinq fois par 
semaine et aide de six à sept fois par semaine respectivement; $\mathrm{IC}_{0,95}: 1,06$ à 2,80 et 1,22 à 5,06); faire partie du bassin de desserte d'un centre de jour dont les coûts sont moins élevés ( $\mathrm{RC}: 1,054 ; \mathrm{IC}_{0,95}: 1,001$ à 1,108 pour chaque augmentation de $1 \$$ ); et pour les hommes seulement, avoir déjà subi un accident vasculaire cérébral et présenter davantage d'incapacités fonctionnelles (interaction entre le sexe et la présence d'un AVC: RC: 0,$298 ; \mathrm{IC}_{0,95}: 0,108$ à 0,825 ; interaction entre le sexe et les capacités fonctionnelles mesurées à l'OARS: RC : 1,096; $\mathrm{IC}_{0,95}: 1,019$ à 1,178). De plus, on observe une plus grande probabilité d'utiliser le centre de jour chez les personnes qui cohabitent avec une personne de soutien. Toutefois, cette relation ne s'observe que chez les personnes nées au Canada (interaction entre la cohabitation avec la personne de soutien et le pays de naissance : $\mathrm{RC}: 0,417 ; \mathrm{IC}_{0,95}: 0,185$ à 0,938$)$. Article 2 : Des analyses en regroupement ont permis de distinguer quatre profils de participants ayant des caractéristiques similaires : 1) les personnes fragilisées par un âge avancé et un grand nombre de problèmes de santé; 2) les participants plus jeunes et plus autonomes que la moyenne, qui semblent des utilisateurs précoces; 3) les personnes qui présentent des incapacités d'origine cognitive; et 4) celles qui présentent des incapacités d'origine motrice. Les activités de groupe des centres de jour ont été regroupées en huit catégories: exercices physiques; groupe spécifique pour un diagnostic ou un problème fonctionnel commun; activités fonctionnelles; stimulation cognitive; activités musicales ou de réminiscence; sports et jeux physiques; intégration sociale; prévention et promotion de la santé. Les activités les plus fréquentes sont les exercices physiques et les activités d'intégration sociale auxquelles ont participé plus de $90 \%$ des utilisateurs de centre de jour, et ce en moyenne à respectivement $78 \%( \pm 23 \%)$ et $72 \%( \pm 24 \%)$ de leurs présences au centre de jour. Les autres catégories d'activités rejoignent de $45 \%$ à $77 \%$ des participants, et ce en moyenne à $35 \%( \pm 15 \%)$ à $46 \%( \pm 33 \%)$ de leurs présences. La participation aux diverses catégories d'activités a été étudiée pour chaque profil d'utilisateurs et comparée aux activités recommandées pour divers types de clientèle. On observe une concordance partielle entre les activités offertes et les besoins des utilisateurs. Cette concordance apparaît plus grande pour les participants qui présentent des problèmes de santé physique ou des incapacités d'origine motrice et plus faible pour ceux qui présentent des symptômes dépressifs ou des atteintes cognitives. 
$\underline{\text { Article } 3}$ : Les participants au centre de jour y sont inscrits en moyenne à raison de 1,56 $( \pm 0,74)$ jours par semaine mais sont réellement présents à $68,1 \%$ des jours attendus. Les facteurs suivants sont associés à une participation plus régulière au centre de jour en termes de taux de présences réelles / présences attendues: ne pas avoir travaillé dans le domaine de la santé (b : ,209; $\mathrm{IC}_{0,95}:, 037$ à ,382); recevoir de l'aide du CLSC les jours de fréquentation du centre de jour (b : ,124; $\mathrm{IC}_{0,95}$ : ,019 à ,230); être inscrit pour la journée plutôt que la demi-journée (b : ,209: $\mathrm{IC}_{0,95}:, 018$ à ,399); lors de ses présences au centre de jour, avoir une moins grande proportion d'activités de prévention et promotion de la santé (b : ,223; $\mathrm{IC}_{0,95}:, 044$ à ,402); et enfin, avoir un aidant qui présente un fardeau moins élevé pour les personnes avec une atteinte cognitive et un fardeau plus élevé pour les personnes sans atteinte cognitive (interaction entre la présence d'atteinte cognitive et le fardeau de l'aidant : b : -,008; $\mathrm{IC}_{0,95}$ : -,014 à -,044).

Conclusion : Conformément à leur mission, les centres de jour rejoignent une bonne proportion des personnes âgées qui présentent des incapacités. Cette étude fait ressortir les caractéristiques des personnes les plus susceptibles d'y participer. Elle suggère la nécessité de revoir la planification des activités pour assurer une offre de services qui tienne davantage compte des besoins des participants, en particulier de ceux qui présentent des atteintes cognitives et des symptômes de dépression. Elle démontre aussi que l'intensité d'exposition au service semble faible, ce qui soulève la question des seuils d'exposition nécessaires pour induire des effets favorables sur le maintien à domicile et sur la qualité de vie de la clientèle cible.

Mots-clés : centre de jour, personnes âgées, soutien à domicile, activités thérapeutiques, utilisation des services 


\section{Abstract}

Background: Adult Day Centers (ADC) offer group-based interventions to older, community-dwelling individuals with functional limitations, with the aim of helping them remain in the community for as long as possible. Recent studies seem to indicate that regular use is needed for positive effects on participants and their caregivers.

Objectives: The purpose of this study was to document the use of ADCs by seniors with functional limitations. There were three main objectives:

1) To identify the characteristics of ADC users versus non-users and to identify the determinants of use.

2) To explore how well ADC program activities matched the different needs of participants with various health and functional characteristics profile.

3) To examine the factors related to regularity of ADC attendance.

Methods: This study was based on a secondary analysis of data collected from 723 seniors participating in the Services intégrés pour personnes âgées (SIPA) demonstration project implemented in two CLSCs in Montreal. ADC use data were obtained for a six month period, from the five ADCs serving the same catchment area. To better understand the ADC program characteristics, individual interviews and focus groups were carried out with ADC coordinators. Data were analysed with descriptive statistics, cluster analysis, and logistic and multiple regression analysis.

Results: The results are presented in three articles, one for each main objective. Article 1: The proportion of $\mathrm{ADC}$ users was $18.8 \%\left(\mathrm{CI}_{0.95:} 16.0-21.7 \%\right)$. The following factors increased the probability of $\mathrm{ADC}$ use: being younger (OR: 1.12; $\mathrm{CI}_{0.95}: 1.06-1.19$ ); not having a university degree (OR: 1.92; $\mathrm{CI}_{0.95}$ : 1.04 - 3.57); receiving help from the CLSC for daily living activities more than once a week (OR: 1.73 and 2.48 for help two to five times per week and six to seven times per week respectively; $\mathrm{CI}_{0.95:} 1.06-2.80$ and $1.22-5.06$ ); being in the catchment population of an $\mathrm{ADC}$ with a lower daily fee (OR: $1.054 ; \mathrm{CI}_{0.95}$ : 
$1.001-1.108$ for each $1 \$$ fee increase); and for men only, having had a stroke or presenting with more functional incapacities (interaction between gender and presence of a stroke: OR: $0.298 ; \mathrm{CI}_{0.95:} 0.108-0.825$; interaction between gender and functional capacity as measured by OARS: OR: 1.096; $\mathrm{CI}_{0.95:} 1.019$ - 1.178). In addition, the probability of ADC use was higher among individuals who live with the person identified as their caregiver. However, this association was found only among persons born in Canada (interaction between whether they lived with caregiver and country of birth: OR: 0.417; $\mathrm{CI}_{0.95:} 0.185$ 0.938). Article 2: Four different participant profile groups were identified using cluster analysis: 1) frail seniors of advanced age who had a large number of health problems; 2 ) individuals who were younger and more independent than the average participants who appeared early users of ADC; 3 ) individuals with functional impairment related to cognitive problems; and 4) those with movement disorders which affected their independence. Classification of program activities by a panel of experts distinguished eight categories of activities: physical exercise; groups for those with common diagnoses or functional problems; functional activities; cognitive stimulation; music or reminiscence activities; sports and physical games; social skills and interaction; disease prevention and health promotion activities. The most frequent activities were physical exercise, and social skills and interaction activities in which more of $90 \%$ of ADC users participated, respectively at a mean of $78 \%( \pm 23 \%)$ and $72 \%$ ( $\pm 24 \%)$ of their attendances at the ADC program. Other categories of activities were provided to 45 to $77 \%$ of participants, and at a mean of $35 \%$ $( \pm 15 \%)$ to $46 \%( \pm 33 \%)$ of their $\mathrm{ADC}$ attendances. Participation in these activities categories was examined for each profile group, and compared to the recommended activities for such client group. Partial correspondence was found between program activities and participants' needs. Concordance seemed higher for individuals with physical health problems or mobility impairments and weaker for those presenting with depressive symptoms or cognitive impairments. Article 3: ADC participants were registered with their program for a mean of $1.56( \pm 0.74)$ days per week, but were actually present $68.1 \%$ of the scheduled days. More regular attendance (higher proportion of scheduled days attended) was associated with: a previous profession of ADC participant or spouse in a sector other than health care (b: .209; $\mathrm{CI}_{0.95} .037$ - .382); the individual receiving formal help for 
viii

activities of daily living on days of expected ADC participation (b: .124; $\mathrm{CI}_{0.95}$ : .019 .230); enrolment for the whole day rather than half a day (b: $209: \mathrm{CI}_{0.95} .018$ - .399); lower participation rates in prevention and health promotion activities (b: $.223 ; \mathrm{CI}_{0.95}$ $.044-.402)$; and among participants with cognitive impairment, lower caregiver burden, and among persons without cognitive impairment, higher caregiver burden (interaction between cognitive impairment and caregiver burden: b: -.008; $\left.\mathrm{CI}_{0.95:}-.014--.044\right)$.

Conclusion: In keeping with their mission, adult day centres attract a large portion of seniors with disabilities. This study has identified the characteristics of the clientele who are most likely to participate in centre activities. Results also suggest a need to review the planning of activity program components to ensure that the services offered best respond to client needs, particularly clients presenting with depressive symptoms or cognitive impairments. It has also demonstrated that the intensity of exposure to this service tends to be low overall, which raises the question of the level of exposure needed to have a positive influence on the quality of life and ability to remain in the community among this population.

Keywords: Adult Day Care, Adult Day Services, elderly, frail seniors, community longterm care, therapeutic activities, service use. 


\section{Table des matières}

Page

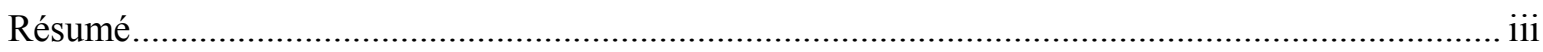

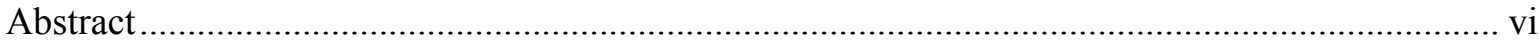

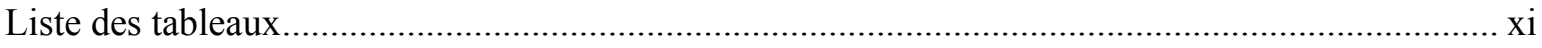

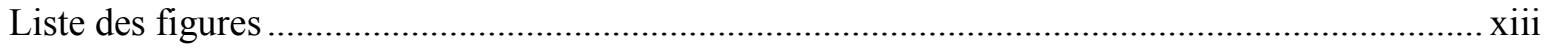

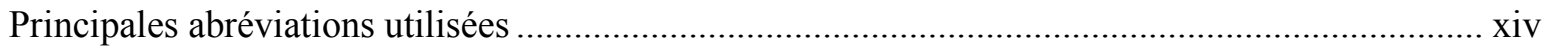

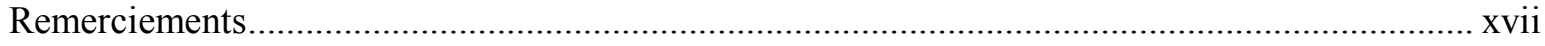

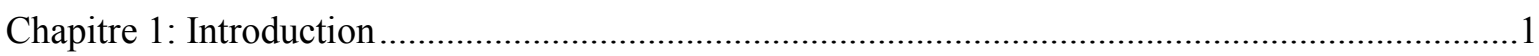

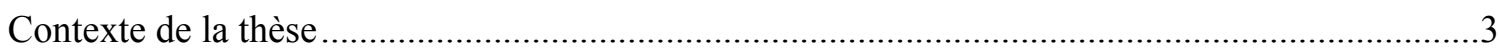

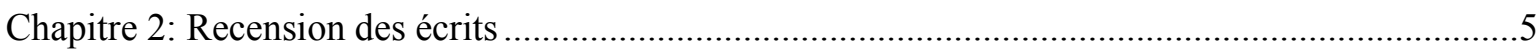

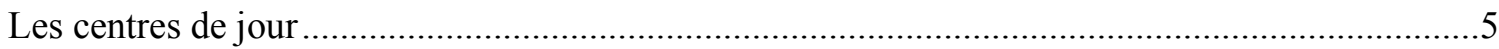

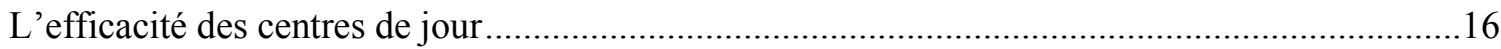

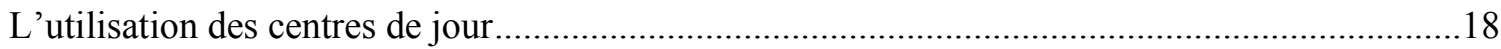

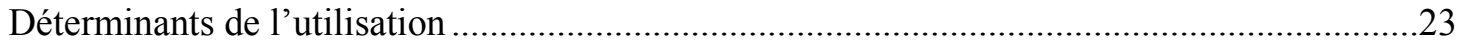

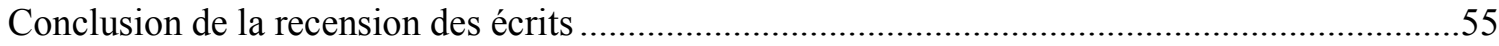

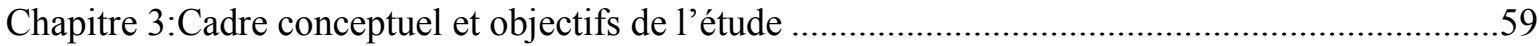

Cadres théoriques utilisés dans les études récentes sur l'utilisation des centres de jour ...............59

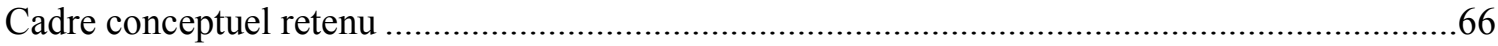

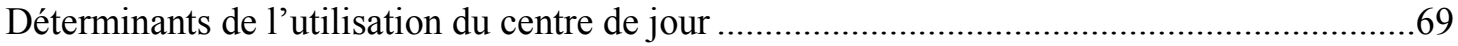

Limites relatives à la disponibilité des données .......................................................................73

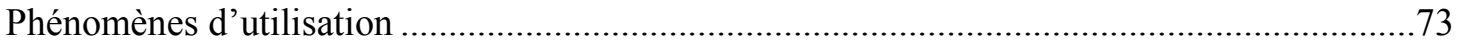

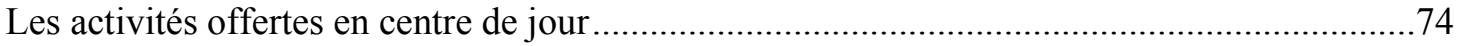

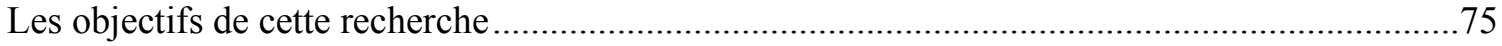

Adaptation du cadre théorique global aux objectifs de recherche relatifs aux déterminants

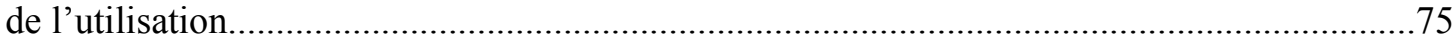

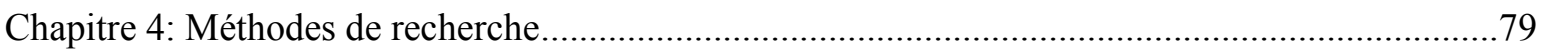

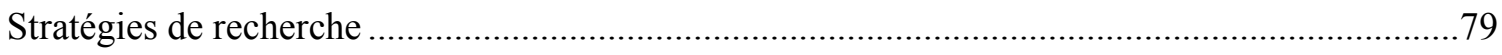

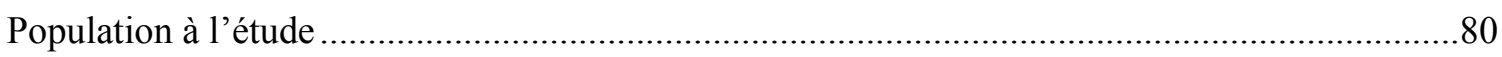

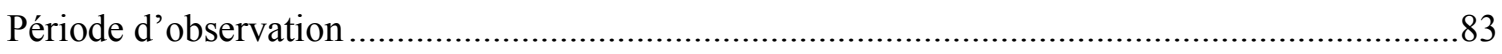

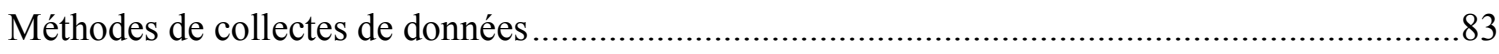

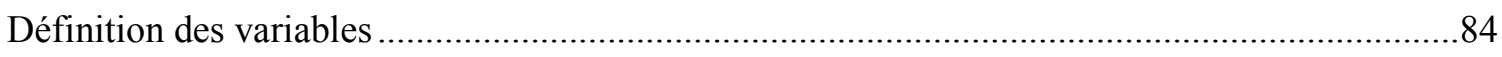

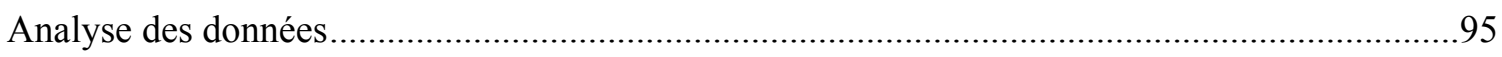

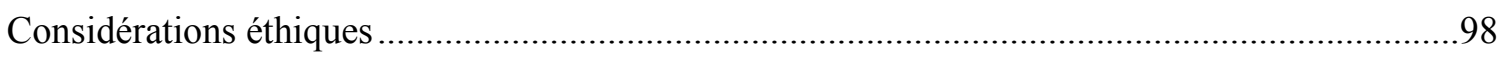




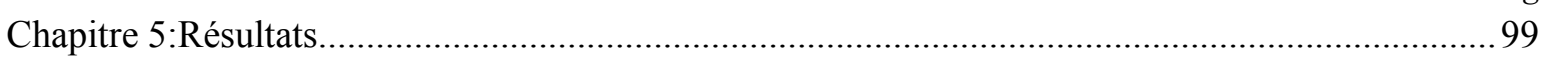

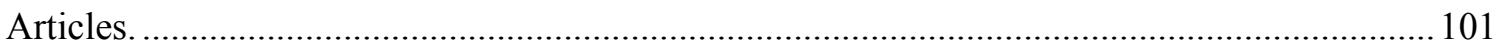

Article 1: L'utilisation des services de centre de jour par les personnes âgées qui présentent des incapacités.

Article 2: Les activités de groupe des centres de jour pour personnes âgées correspondent-elles aux besoins des différents profils de participants?

Article 3: Determinants of Adult Day Center attendance among older adults with

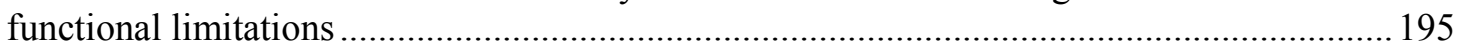

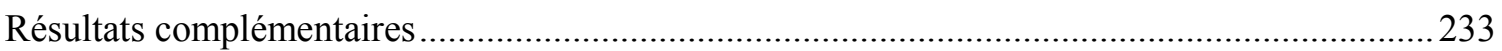

Déterminants du contact avec le centre de jour: interaction entre le sexe et les facteurs de

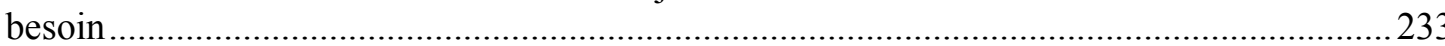

Déterminants du contact avec le centre de jour: Les facteurs de besoin des personnes de

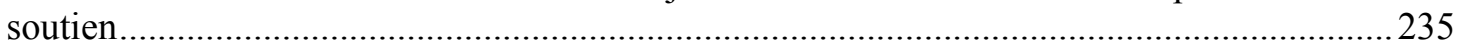

Régularité de participation pour divers profils de participants ...........................................240

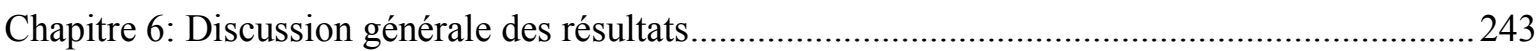

Éléments mis en lumière dans chacun des trois volets de l'étude..............................................244

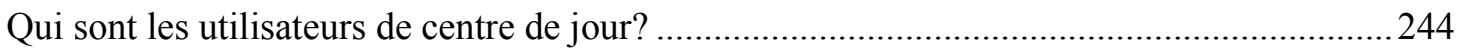

À quels types d'activités participent les personnes âgées lors de leur présence en centre

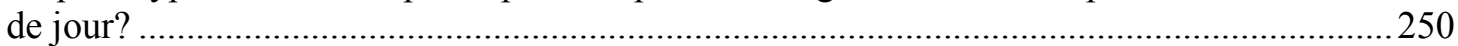

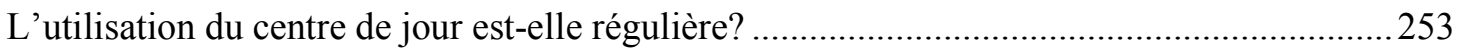

Parmi les utilisateurs de centres de jour, quels sont ceux qui le font avec le plus de

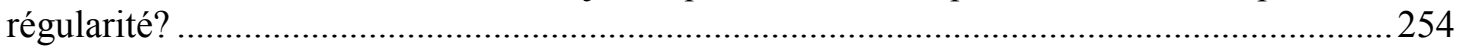

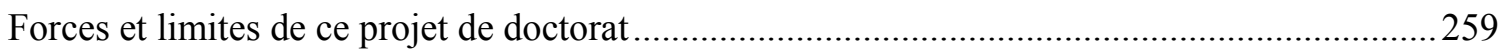

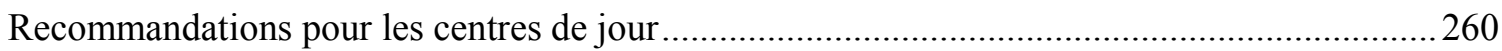

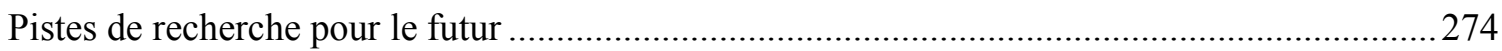

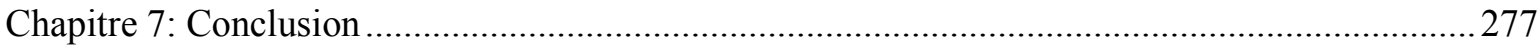

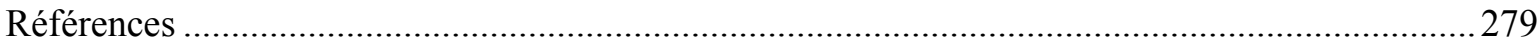

Annexe I : Article rédigé pour répondre aux exigences de l'examen de synthèse : Caregiver satisfaction with support services: Influence of different types of services ....................................i-1

Annexe II : Variables étudiées dans les principales recherches présentées à la recension des

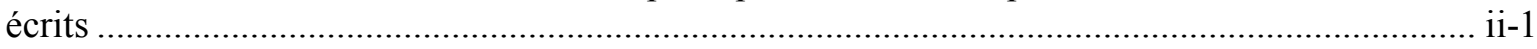

Annexe III : Guide d'entrevue : Entrevue individuelle avec les coordonnateurs des cinq centres

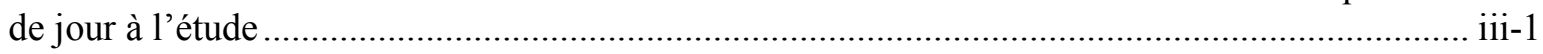

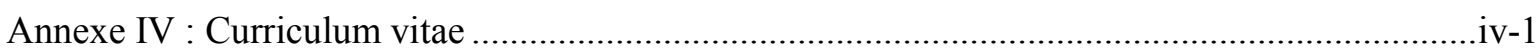




\section{Liste des tableaux}

\section{Recension des écrits}

Tableau I : Classifications des centres de jour. 6

Tableau II : Activités en centre de jour : adapté du Cadre de référence de l'Association des centres d'accueil du Québec (1991).....

Tableau III : Caractéristiques des études recensées pour identifier les déterminants de l'utilisation des centres de jour

Tableau IIIa: études des déterminants de l'utilisation des centres de jour pour une clientèle générale

Tableau IIIb: études des déterminants de l'utilisation des centres de jour pour une clientèle atteinte de démence............................................. 30

Tableau IIIc : études des déterminants de l'utilisation de services de répit et autres services connexes .............................................................. 31

Tableau IIIc: études des déterminants de l'utilisation de services de répit et autres services connexes (suite)

Tableau IIId : études descriptives sur les raisons qui motivent ou limitent l'utilisation de ces services ........................................................... 33

Tableau IIIe : études des déterminants de l'utilisation des services à domicile..... 34

\section{Méthodes de recherche}

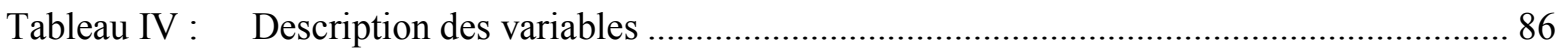

Tableau V： Qualité des instruments de mesure ........................................................................... 94

\section{Résultats}

\section{Article 1:}

Tableau 1: $\quad$ Facteurs prédisposants ou caractéristiques sociales des personnes âgées

Tableau 2: $\quad$ Facteurs de besoin ou état de santé des personnes âgées ........................................ 136

Tableau 3: $\quad$ Facteurs de capacité de nature organisationnelle ................................................... 137

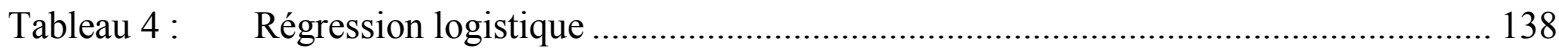

Tableau 5: Ratios de cote ajustés pour pays de naissance et lien avec la personne de soutien

Tableau 6: Ratios de cote ajustés selon l'âge, le sexe, le nombre de problèmes de santé physique, la présence d'AVC et les capacités fonctionnelles 
Article 2:

Tableau 1: Caractéristiques des participants 180

Tableau 2: Différences entre les caractéristiques des membres des groupes formés par les analyses en regroupement

Tableau 3: Activités en centre de jour, selon leurs buts

Tableau 4: Activités recommandées en centre de jour pour les divers sous-programmes (ACAQ, 1991) et rangs de priorité établis par le groupe d'experts

Tableau 5: Activités au centre de jour pour l'ensemble des participants, puis pour les quatre profils de participants.

Tableau 6: Correspondance entre l'exposition aux diverses activités pour les membres du groupe 1 (personnes fragiles) et les activités recommandées

Tableau 7: Correspondance entre l'exposition aux diverses activités pour les membres du groupe 3 (incapacités d'origine cognitive) et les activités recommandées...

Tableau 8: Correspondance entre l'exposition aux diverses activités pour les membres du groupe 4 (incapacités d'origine motrice) et les activités recommandées.

Article 3:

Table 1: Classification of ADC group activities 222

Table 2: Characteristics of the sample: Predisposing characteristics.

Table 3: $\quad$ Characteristics of the sample: Need factors 224

Table 4: $\quad$ Aspects of service delivery 225

Table 5: Number of ADC attendances during a period of 26 weeks, presented by frequency of registration. 226

Table 6: Multiple linear regression of level of ADC attendance on predisposing and enabling factors, and aspects of service delivery

\section{Résultats complémentaires:}

Tableau VI: Régression linéaire simple entre le contact avec le centre de jour et des variables de la personne de soutien.....................................................................2235

Tableau VII : $\quad$ Répartition des absences lors des jours de centre de jour prévus ............................239

Tableau VIII : Régularité de participation pour chacun des quatre groupes formés par les

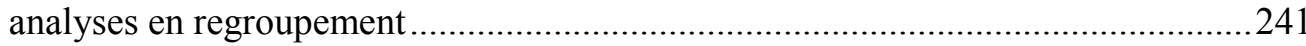

Tableau IX : Régularité de participation des personnes qui ont des atteintes cognitives ..............242

\section{Discussion:}

Tableau X: $\quad$ Recommandations pour les centres de jour 


\section{Liste des figures}

\section{Recension des écrits}

Figure 1: Cheminement d'une référence au centre de jour

Figure 2: Déterminants de 1'utilisation du centre de jour ou des services de répit recensés dans les écrits

\section{Cadre conceptuel et objectifs de l'étude}

Figure 3: Facteurs qui influencent l'utilisation du centre de jour

Méthodes de recherche

Figure 4: Participants inclus à chacune des étapes de cette étude sur les centres de jour

\section{Résultats}

\section{Article 1:}

Figure 1: Facteurs qui influencent l'utilisation du centre de jour

Figure 2: Relation entre l'utilisation du centre de jour et les capacités fonctionnelles.

\section{Article 2:}

Figure 1: Deux modèles d'horaire d'activités en CJ et lien avec le registre des activités

\section{Article 3:}

Figure 1: Factors influencing regularity of ADC attendance

\section{Résultats complémentaires:}

Figure 5: Interaction entre sexe et présence d'un AVC

Figure 6: Interaction entre sexe et capacités fonctionnelles.

Figure 7 : Interaction : âge, sexe et problèmes de santé.

Figure 8: Contact avec le centre de jour et capacités fonctionnelles de la personne de soutien .. 236

Figure 9: Contact avec le centre de jour et durée de l'aide par la personne de soutien 236

Figure 10: Contact avec le centre de jour et fardeau de la personne de soutien 237

Figure 11: Participation au centre de jour : présences réelles par semaine 238

Figure 12: Régularité de la participation en centre de jour, en pourcentage des journées prévues 
xiv

\section{Principales abréviations utilisées}

$\pm: \quad$ Écart-type

ACAQ : Association des centres d'accueil du Québec

ADC : $\quad$ Adult Day Center

ADRLSSS : Agence de développement des réseaux locaux de services de santé et de services sociaux *

ADDTC : Alzheimer Disease Diagnostic and Treatment Center Program

ANOVA : Analyse de variance

ASSS Agence de la santé et des services sociaux

AVC : $\quad$ Accident vasculaire cérébral

AVD : $\quad$ Activité de vie domestique

AVQ : $\quad$ Activité de vie quotidienne

BRDRS : $\quad$ Blessed-Roth Dementia Rating Scale

CJ : $\quad$ Centre de jour

CLSC : Centre local de services communautaires

CSSS : $\quad$ Centre de santé et de services sociaux

EDG : Échelle de dépression gériatrique

EPESE Established populations for epidemiological studies of the elderly

ETC: Équivalent temps complet

HMO : $\quad$ Health Maintenance Organization

HTA : $\quad$ Haute tension artérielle

$\mathrm{IC}_{0.95}: \quad$ Intervalle de confiance à $95 \%$

IRSC : Instituts de recherche en santé du Canada 
JEDI : Jonction et diffusion informatique (Base de données sur les clients, les présences et les activités en centre de jour)

MMSE : $\quad$ Mini-Mental State Evaluation

OARS: $\quad$ Older American Resources and Services

PA : $\quad$ Personne(s) âgée(s)

PS : $\quad$ Personne(s) de soutien

RAMQ : $\quad$ Régie de l'assurance-maladie du Québec

RC : $\quad$ Ratio de cote

SCO : $\quad$ Syndrome cérébral organique

SIPA : $\quad$ Services intégrés pour les personnes âgées

SMAF : $\quad$ Système de mesure de l'autonomie fonctionnelle

SPMSQ Short Portable Mental Status Questionnaire

* Ce sigle et ce nom sont utilisés dans l'article 1. Cette entité se nomme maintenant Agence de la santé et des services sociaux, terme utilisé dans les autres sections de la thèse. 
À François, à mes parents et à mes collègues de l'École des sciences de la réadaptation de l'Université d'Ottawa qui $n$ 'ont jamais cessé de m'encourager tout au long de ce travail 


\section{Remerciements}

Un travail de longue haleine comme celui-ci a été possible grâce à la collaboration de nombreuses personnes et organismes.

Je veux d'abord remercier la Société Alzheimer du Canada, le Groupe de recherche SOLIDAGE (financé par les IRSC), la Fondation canadienne d'ergothérapie, la Faculté de médecine et la Faculté des études supérieures de l’Université de Montréal, pour le soutien financier reçu tout au cours de mes études doctorales et de la rédaction de la présente thèse.

Cette étude utilise des données du projet SIPA, financé par le Fonds d'adaptation des services de santé de Santé Canada, la Fondation canadienne de recherche sur les services de santé et le Ministère de la santé et des services sociaux du Québec. Ma gratitude va aussi aux trois principaux chercheurs de l'équipe SIPA, François Béland, Howard Bergman et Paule Lebel, pour m'avoir permis d'utiliser ces données, de même qu'à Luc Dallaire et John Fletcher, coordonnateurs de recherche du projet SIPA, pour leur aide précieuse relative à la gestion et à la transmission des données.

Je ne saurais trop dire ma vive reconnaissance à Anne-Marie Boivin, Christian-Paul Gaudet, Mélanie Léonard, Manon Pouliot et Tina Tenenbaum, coordonnateurs des centres de jour à l'étude pour avoir partagé des informations sur le fonctionnement de leur centre de jour, ainsi qu'à Jacinthe Auger, Lucie Bernier, Christian-Paul Gaudet, Françoise Hébert, Jacinthe Labbé, Thérèse Lafontaine, Odette Descarries et Tina Tenenbaum qui ont participé aux différentes rencontres qui m'ont permis d'établir une classification des activités en centre de jour et de valider les premiers résultats obtenus auprès des intervenants du milieu.

Mes remerciements les plus chaleureux vont à mes deux directrices, Paule Lebel et Nicole Leduc, pour le temps qu'elles m'ont consacré, leurs judicieux conseils, leur soutien indéfectible et leurs encouragements constants. Je suis privilégiée d'avoir travaillé avec elles. 



\section{Chapitre 1 : Introduction}

Comme la plupart des pays industrialisés, le Québec est engagé dans un processus de vieillissement de sa population. Selon le scénario le plus probable, la proportion de personnes âgées passera de $13 \%$ en 2001 à $29,7 \%$ en 2051. De plus, on observe un vieillissement de la population âgée. En effet, l'accroissement des 85 ans et plus sera beaucoup plus important que celui des 65-74 ans. Le nombre des 85 ans et plus pourrait passer de 95000 à 584000 durant cette période et leur proportion dans la population totale pourrait grimper de $1,3 \%$ à $7,5 \%$ (Gauthier, 2004).

Confronté à ce changement démographique et aux besoins particuliers des personnes âgées, à l'instar de la plupart des pays industrialisés, le Québec a développé une gamme variée de services gérontologiques et gériatriques qui visent le maintien de l'autonomie et de la qualité de vie de cette population. Parmi ces services, on retrouve le centre de jour, un service offert aux personnes âgées qui demeurent à domicile et présentent des incapacités. Les interventions en centre de jour sont offertes principalement au moyen d'activités de groupe et comprennent des activités de surveillance de l'état de santé, des activités d'enseignement et de promotion de la santé, des activités de réadaptation, des interventions psychosociales et de loisirs, ainsi que du répit et du soutien aux familles (Dabelko et Zimmerman, 2008; Gutman, Milstein, Killam, Lewis et Hollander, 1993a; 1993b; Regroupement des centres d'hébergement et de soins de longue durée de la région de Montréal, 2005). Par ces interventions, le centre de jour vise à favoriser le maintien dans la communauté des personnes âgées qui présentent des incapacités.

Les centres de jour pour personnes âgées ont commencé à s'implanter au Québec au début des années 1970 (Tourigny, Côté, Laberge, Paradis et Joubert, 1993) et sont maintenant présents dans presque tous les territoires de Centres locaux de services communautaires (CLSC). Cependant, même lorsque ce type de services est accessible, des études auprès de centres de jour (ou d'un ensemble de services destinés aux personnes âgées en perte d'autonomie, incluant des centres de jour) ont démontré un faible taux d'utilisation par la clientèle âgée (Baumgarten, Lebel, Laprise, Leclerc et Quinn, 2002; Montgomery et Borgotta, 1989) et par la clientèle atteinte de démence (Douglass et Visconti, 1998; Lawton, Brody et Saperstein, 1989). Or, selon Zarit, Stephens, Townsend et 
Greene (1998), une utilisation régulière serait nécessaire pour induire des effets bénéfiques. Dans un tel contexte, il importe de se questionner sur les déterminants de l'utilisation des centres de jour.

Les quelques études qui ont porté sur les facteurs qui influencent l'utilisation des services de centres de jour sont encore à ce jour non concluantes. Les méthodes sont variées, de même que les variables étudiées. Très peu de travaux ont inclus le type d'activités offertes parmi les variables étudiées. Ainsi, il est difficile de tirer des conclusions sur les déterminants de l'utilisation des centres de jour. De plus, ces études sont en majorité américaines et nous ne savons pas si la dynamique d'utilisation des services est similaire au Québec. En effet, il existe une variété de modèles de centres de jour aux États-Unis : modèle social, modèle médical, centres spécifiques pour les personnes atteintes de démence (Gaugler et Zarit, 2001; Weissert, Elston, Bolda, Cready, Zelman, Sloane et coll., 1989). Des modèles mixtes, combinant des caractéristiques des modèles médical et social, ont aussi été identifiés (Cox et Reifler, 1994; Dabelko et Zimmerman, 2008; National Institute on Adult Day Care, 1990). Selon la description des services offerts dans les centres de jour québécois (Regroupement des centres d'hébergement et de soins de longue durée de la région de Montréal, 2005), il semble que les centres de jour au Québec sont mixtes et génériques, c'est-à-dire qu'ils offrent à la fois des services habituellement considérés comme des services de santé (suivi infirmier de l'état de santé, activités de réadaptation) et des services de nature plus sociale (activités de socialisation pour des personnes isolées en raison d'une perte d'autonomie importante, répit aux personnes de soutien). Ils acceptent une clientèle variée et adaptent leurs activités aux besoins diversifiés de cette clientèle. De plus, aux États-Unis la participation à ces services, offerts dans un marché compétitif, est parfois limitée par la capacité financière des individus. Au Québec, elle est plutôt limitée par la capacité d'accueil des centres qui ont souvent des listes d'attente, bien que les coûts reliés au transport et aux repas (généralement inférieurs aux coûts des centres de jour aux États-Unis) puissent aussi représenter des barrières à l'utilisation. Par conséquent, les déterminants de l'utilisation des centres de jour, dans le contexte québécois, restent encore à définir. Enfin, il faut préciser que différents aspects du 
phénomène de l'utilisation, à savoir le contact, le volume ou l'intensité d'utilisation et la durée, auront des prédicteurs différents (Andersen et Newman, 1973).

Le but de la présente recherche est de documenter l'utilisation des centres de jour dans un contexte québécois, par des personnes âgées qui vivent avec des incapacités. Elle comporte trois objectifs généraux:

1) Caractériser les utilisateurs de centre de jour et ce qui les distingue des nonutilisateurs et analyser les déterminants de l'utilisation du centre de jour.

2) Identifier les activités qui sont offertes au groupe d'utilisateurs de centre de jour, ainsi que l'adéquation entre ces activités et les caractéristiques de santé des utilisateurs.

3) Définir, pour le groupe d'utilisateurs, les facteurs qui influencent la régularité d'utilisation du centre de jour.

Cette recherche apporte un éclairage utile pour la communauté scientifique bien sûr, mais aussi pour les personnes âgées et leur famille ainsi que pour les planificateurs de soins et de services. La compréhension des facteurs qui influencent l'utilisation des services de centres de jour permettra de mieux cibler les interventions pour les personnes les plus susceptibles d'en bénéficier et de revoir l'offre de services de façon à mieux l'adapter aux besoins de la clientèle cible.

\section{Contexte de la thèse}

La recherche de ce projet d'études doctorales s'appuie sur une analyse secondaire d'informations provenant du projet de démonstration de Services intégrés pour personnes âgées (SIPA), un projet qui consistait en l'expérimentation d'un modèle de services intégrés pour les personnes âgées qui ont des incapacités, expérimenté de juin 1999 à mars 2001, grâce à un financement de \$ 7,6 millions du Fonds d'adaptation des services de santé de Santé Canada et du Ministère de la Santé et des Services sociaux du Québec. Dans le cadre de ce projet, 1230 personnes âgées de la région de Montréal ont été réparties de façon aléatoire en deux groupes, l'un recevant les services intégrés, l'autre recevant les services 
réguliers. Parallèlement à l'expérimentation, une équipe de recherche a complété une cueillette exhaustive de données comportant 3 questionnaires auprès des personnes âgées (entre juin 1999 et septembre 2002), un questionnaire auprès des personnes de soutien (à l'été 2000), ainsi qu'un relevé de leur utilisation des services de santé, d'après les bases de données des organismes et des établissements de santé (tels la Régie d'assurance maladie du Québec, l'Agence de la santé et des services sociaux de Montréal, les hôpitaux et les centres de jour) (Béland, Bergman et Lebel, 2001; Béland, Bergman, Lebel, Clarfield et coll., 2006; Béland, Bergman, Lebel, Dallaire et coll., 2006).

Même si ce projet utilise des données provenant du projet SIPA, il s'agit d'études entièrement distinctes. En effet, l'utilisation du centre de jour n'est d'intérêt dans le projet SIPA que pour estimer les coûts de santé des participants à l'étude. Ainsi, cette étude sur les déterminants de l'utilisation des centres de jour vise des objectifs complètement différents. Il est donc clair que les données provenant du projet SIPA ont été explorées et analysées de façon différente et novatrice.

De plus, une collecte originale de données auprès de coordonnateurs de centre de jour a permis de mieux comprendre l'ensemble de données relatives à la participation au centre de jour recueillies par l'équipe du projet SIPA. La section qui porte sur les méthodes de collecte de données expliquera de façon plus détaillée, la provenance de chacun des types de données utilisées et la portée de la collecte de données originales.

Les résultats de cette recherche doctorale sont présentés dans trois articles inclus dans la présente thèse. Une étude de la satisfaction des personnes de soutien des participants au projet SIPA a aussi été réalisée pour répondre aux exigences de l'examen de synthèse du programme d'études doctorales en santé publique (Savard, Leduc, Lebel, Béland et Bergman, 2006 - joint à l'annexe I). 


\section{Chapitre 2 : Recension des écrits}

Nous décrirons d'abord globalement les centres de jour, leurs objectifs, ainsi que leur mode de fonctionnement. Nous présenterons ensuite les principales conclusions des études qui ont porté sur leur efficacité. Nous verrons que ces conclusions sont influencées par les taux d'utilisation du service. Nous décrirons ensuite les études qui ont porté sur l'utilisation des centres de jour ou des services similaires pour en dégager ce qui a été observé sur les déterminants de l'utilisation de ces services.

\section{Les centres de jour}

Les centres de jour peuvent être définis de façon générale comme un endroit qui offre des services de jour à l'extérieur de la maison, dans un milieu protégé, pour des personnes âgées qui présentent une variété de types d'incapacités. Ces services incluent généralement la surveillance de l'état de santé, des activités thérapeutiques incluant des soins de santé, des services de réadaptation et des services sociaux, de la socialisation, le repas du midi et des services de transport (Dabelko et Zimmerman, 2008; Gaugler et Zarit, 2001).

Toutefois, les services offerts par les divers centres de jour varient énormément d'un centre à un autre. Ainsi, aux États-Unis, on a tenté de classifier les divers modèles de centres de jour. Le tableau I présente un résumé des différentes classifications recensées. Les classifications les plus connues sont sans doute celles de Weissert et collaborateurs (1976; 1989) qui distinguent deux, puis trois groupes de centres de jour : modèle médical et modèle social, puis des centres spécifiques pour les clientèles particulières (Gutman et coll., 1993b; Gaugler et Zarit, 2001). Avec la grande variété de centres de jour qui sont maintenant disponibles, de même que l'évolution des besoins des clients et en particulier de la clientèle atteinte de démence, les distinctions entre ces différents modèles ne semblent pas très nettes (Leitsch, Zarit, Townsend et Greene, 2001; National Institute on Adult Day Care, 1990). Plusieurs centres se décrivent comme des modèles mixtes (Cox et Reifler, 1994; Dabelko et Zimmerman, 2008). 
Tableau I : Classifications des centres de jour

\begin{tabular}{|c|c|c|c|}
\hline & $\begin{array}{l}\text { Classification intuitive selon le type de soins (O'Brien, 1982; } \\
\text { Szekais, 1985; Tate, 1988) }\end{array}$ & $\begin{array}{l}\text { Classification empirique selon l'intensité des diverses } \\
\text { catégories de services (Conrad et coll., 1993) }\end{array}$ & $\begin{array}{l}\text { Classifications empiriques de } \\
\text { Weissert et coll. (1976 et 1989) }\end{array}$ \\
\hline $\begin{array}{l}\text { vocation } \\
\text { médicale }\end{array}$ & $\begin{array}{l}\text { Hôpital de jour (O'Brien, 1982; Szekais, 1985), centre de soins de } \\
\text { rétablissement en milieu communautaire (Szekais, 1985) et } \\
\text { modèle de restauration (Tate, 1988) : ils s'adressent aux } \\
\text { personnes qui nécessitent des soins médicaux et de réadaptation } \\
\text { importants, habituellement après un congé de l'hôpital. Les } \\
\text { services sont habituellement pour une durée limitée (O'Brien, } \\
\text { 1982; Szekais, 1985). } \\
\text { Centre de traitement de jour: Offre des services de } \\
\text { rétablissement (réadaptation et services sociaux) à une clientèle } \\
\text { ayant un handicap mental ou souffrant de maladies chroniques. } \\
\text { La durée peut être illimitée (O'Brien, 1982) }\end{array}$ & $\begin{array}{l}\text { Centre de type réadaptation qui offre beaucoup de } \\
\text { services cliniques et de services de réadaptation et de } \\
\text { réentraînement aux activités quotidiennes et peu } \\
\text { d'activités récréatives. } \\
\text { Centre à intensité clinique élevée avec composante } \\
\text { sociale: offre beaucoup de services cliniques et de } \\
\text { services de réadaptation et de réentraînement aux } \\
\text { activités quotidiennes, mais aussi des activités sociales et } \\
\text { récréatives et du soutien aux familles. }\end{array}$ & $\begin{array}{l}\text { Modèle médical : (Weissert, 1976) } \\
\text { Auspice 1: centres affiliés aux } \\
\text { établissements de longue durée et de } \\
\text { réadaptation (Weissert, 1989), qui } \\
\text { reçoivent une clientèle avec plus } \\
\text { d'incapacités, mais moins de problèmes } \\
\text { mentaux que celle des autres modèles. } \\
\text { lls offrent en général plus de services } \\
\text { thérapeutiques et de santé. }\end{array}$ \\
\hline $\begin{array}{l}\quad \downarrow \\
\text { vocation } \\
\text { sociale }\end{array}$ & $\begin{array}{l}\text { Programme de préservation de la santé (O'Brien, 1982; Szekais, } \\
\text { 1985) ou modèle de maintien (Tate, 1988) : services infirmiers, } \\
\text { surveillance de l'état de santé, promotion de la santé, services } \\
\text { limités de réadaptation et activités sociales pour des individus à } \\
\text { risques qui nécessitent des soins de longue durée. Pour Szekais } \\
\text { (1985), la socialisation et les loisirs sont importants dans ce } \\
\text { programme. } \\
\text { Centre de soins de jour (O'Brien, 1982) ou modèle psychosocial: } \\
\text { (Tate, 1988) : programme d'activités sociales en milieu protégé } \\
\text { pour des personnes dont la santé est délicate ou qui ont des } \\
\text { incapacités et qui sont isolées. } \\
\text { Service de relève : offre du répit aux familles qui donnent des } \\
\text { soins à une personne nécessitant de la supervision continuelle } \\
\text { sur } 24 \text { heures (Szekais, 1985) }\end{array}$ & $\begin{array}{l}\text { Centre à but général : offre peu de services cliniques et } \\
\text { de services sociaux, très peu de services de réadaptation } \\
\text { et un nombre modéré d'activités sociales et récréatives. } \\
\text { Semble approprié pour les personnes qui ont des } \\
\text { incapacités très légères. } \\
\text { Centre à faible intensité d'activités: offre peu de services } \\
\text { dirigés, ce sont possiblement des centres } \\
\text { communautaires pour des clients autonomes. }\end{array}$ & $\begin{array}{l}\text { Modèle social : (Weissert, 1976) } \\
\text { Auspice } 2 \text { : centres affiliés aux centres } \\
\text { communautaires ou de services sociaux } \\
\text { surtout, ainsi que centres non affiliés } \\
\text { (Weissert, 1989), qui rejoignent une } \\
\text { clientèle avec moins d'incapacités et } \\
\text { offrent généralement plus d'activités de } \\
\text { nature sociale. }\end{array}$ \\
\hline $\begin{array}{l}\text { vocation } \\
\text { spéciale }\end{array}$ & $\begin{array}{l}\text { Centre psychiatrique de jour (O’Brien, 1982) ou Services } \\
\text { psychosociaux (Szekais, 1985): pour une clientèle qui souffre de } \\
\text { maladie mentale aiguë ou chronique. }\end{array}$ & $\begin{array}{l}\text { Centre pour personne avec démence : offre moins de } \\
\text { services cliniques et de services de réadaptation, mais } \\
\text { plusieurs types de loisirs thérapeutiques et beaucoup de } \\
\text { soutien aux familles. }\end{array}$ & $\begin{array}{l}\text { Centres à vocation spéciale (Weissert, } \\
\text { 1989) : ils s'adressent à une clientèle } \\
\text { précise : anciens combattants, non- } \\
\text { voyants, personnes atteintes de } \\
\text { démence, etc. }\end{array}$ \\
\hline
\end{tabular}


Au Canada, Gutman et collaborateurs (1993a; 1993b) décrivent des centres de jour de la Colombie-Britannique qui se répartissent sur un continuum de services de nature médicale à sociale, tandis qu'en Alberta, on semble distinguer plus clairement des hôpitaux de jour et des centres de jour de nature sociale (Ross-Kerr, Warren, Schalm, Smith et Godkin, 2003; Warren, Ross-Kerr, Smith et Schalm, 2003). Au Québec, les centres de jour semblent correspondre au niveau de services du modèle mixte.

Le ministère de la Santé et des Services sociaux du Québec (1986) définit le centre de jour comme « une ressource qui offre, à une clientèle inscrite et qui présente une perte d'autonomie pouvant entraver son maintien à domicile, des programmes et des activités de jour de nature préventive, thérapeutique ou de réadaptation» (p. 10). Les centres de jour québécois se distinguent nettement des hôpitaux de jour par la nature et le niveau d'intensité des interventions qui sont offertes. L'hôpital de jour utilise toutes les ressources hospitalières disponibles et nécessaires pour l'évaluation, le diagnostic et le traitement. La clientèle requiert des soins médicaux, infirmiers et de réadaptation qu'elle ne pourrait recevoir ailleurs sans être hospitalisée. L'intervention en hôpital de jour est habituellement de durée limitée (Gratton, 1995; Ministère de la Santé et des Services sociaux, 1986). Bien que le centre de jour compte des professionnels de la santé et de la réadaptation parmi son personnel, il ne comprend généralement pas de personnel médical. La nature des activités est davantage préventive avec un accent sur le maintien et la consolidation des capacités. Les durées d'intervention des centres de jour peuvent être longues, et se poursuivre tant que la fréquentation du centre de jour continue de contribuer au maintien à domicile de la personne âgée (Agence de la santé et des services sociaux de Montréal, 2005). Selon ce même document, le but du centre de jour est de :

« favoriser le maintien à domicile de la clientèle âgée et donc de retarder, sinon éviter, le recours à un milieu d'hébergement institutionnel, et ce, dans le contexte d'une qualité de vie optimale. Pour ce faire, le centre de jour poursuit essentiellement trois objectifs :

- le maintien ou l'amélioration de l'état de santé biopsychosociale;

- le maintien et la consolidation des capacités;

- le soutien aux proches aidants.

Il vise également à soutenir l'interaction entre l'usager et son réseau social. Il doit faciliter l'intégration de la clientèle aux activités de la communauté, notamment des organismes communautaires, lorsque celle-ci est en mesure de le faire. $\gg($ p.21) 
Le comité d'orientation des centres de jour du Regroupement des centres d'hébergement et de soins de longue durée de la région de Montréal précise la façon d'opérationnaliser ces objectifs. Le maintien et la consolidation de l'autonomie de la personne âgée s'actualisent par des activités de prévention et des activités thérapeutiques. Les activités de prévention incluent la surveillance de l'état de santé et, lorsqu'elle survient, l'identification rapide de la détérioration de la santé, ce qui permet une intervention précoce par une référence à une ressource appropriée. Elles comprennent aussi des activités qui visent le maintien ou l'amélioration de la qualité de vie, de l'estime de soi et des liens sociaux qui favorisent la vie dans la communauté. Les activités thérapeutiques incluent des interventions pour stimuler le maintien ou la consolidation des capacités motrices, sensorielles, cognitives et psychologiques et favoriser l'utilisation des capacités résiduelles ainsi que l'apprentissage de moyens de compensation pour les pertes de capacités qui demeurent (Regroupement des centres d'hébergement et de soins de longue durée Région de Montréal, 2001; 2005).

Le soutien aux proches aidants apparaît essentiel pour favoriser le maintien à domicile des personnes âgées en perte d'autonomie, puisqu'au Canada comme dans plusieurs pays, ces personnes reçoivent la majorité de leur soutien de la part de membres de leur famille ou d'autres proches non rémunérés. (Garant et Bolduc, 1990 et Connidis, 1989, cités par Ducharme, Lebel et Bergman, 2001; Canadian Study of Health and Aging Working Group, 1994). Plusieurs études ont démontré que ces tâches peuvent entraîner un sentiment de fardeau, ainsi que des risques pour la santé tant physique que psychologique de ces personnes de soutien (Centers for Disease Control and Prevention, 2007; Chenoweth et Spencer, 1986; Ducharme, Levesque, Zarit, Lachance et Giroux, 2007; Pinquart et Sörensen, 2003, Pushkar-Gold, Feldman-Reis, Markiewicz et Andres, 1995; Schulz et Beach, 1999). La majorité des centres de jour considèrent le soutien aux familles comme un rôle important du centre de jour, qui se concrétise de plusieurs façons. Le centre de jour peut offrir un répit aux personnes de soutien. En effet, dans le cas de personnes âgées qui demandent une assistance ou une supervision continue, la participation de la personne âgée au centre de jour permet à la personne de soutien de se libérer de ces tâches pendant 
quelques heures par semaine. Par la stimulation des capacités résiduelles de la personne âgée, elle peut aussi faciliter certaines tâches d'assistance quotidienne accomplies par la personne de soutien. De plus, certains centres de jour offrent des groupes d'éducation et de soutien pour les familles ou encore permettent la participation des personnes de soutien à certaines fêtes ou activités spéciales incluses à leur programmation, de façon à diminuer leur isolement (Curran, 1996; Gitlin, Reever, Dennis, Mathieu et Hauck, 2006; Reilly, Venables, Hughes, Challis et Abendstern, 2006). Les orientations de l'Agence de la santé et des services sociaux de Montréal (2005) précisent que «c'est particulièrement au niveau des services de répit que le centre de jour est appelé à intervenir auprès des proches aidants » (p. 22), d'autres activités d'éducation et de soutien aux familles pouvant être mises en place dans les CLSC.

Le Regroupement des centres d'hébergement et de soins de longue durée de la région de Montréal $(2001,2005)$ et l'Agence de la santé et des services sociaux de Montréal (2005) demeurent très généraux quant aux activités à mettre en place dans les centres de jour. Le document le plus détaillé retrouvé à ce sujet est Le centre de jour: Un cadre de référence de l'Association des centres d'accueil du Québec en 1991, qui regroupe les activités des centres de jour en six sous-programmes portant sur les aspects suivants :

- incapacités fonctionnelles d'origine motrice;

- incapacités fonctionnelles d'origine sensorielle;

- incapacités fonctionnelles d'origine cognitive;

- facteurs socio-environnementaux;

- santé physique;

- santé mentale.

Le cadre de référence propose, pour chacun des sous-programmes, une liste d'activités de groupe et d'activités individuelles qui peuvent être réalisées par les centres de jour (voir tableau II). Ces derniers adaptent, bien sûr, ces suggestions en fonction des besoins spécifiques de leur clientèle et des ressources dont ils disposent. 
Tableau II : Activités en centre de jour : adapté du Cadre de référence de l'Association des centres d'accueil du Québec (1991)

\begin{tabular}{|c|c|c|c|c|c|c|}
\hline $\begin{array}{l}\text { Sous- } \\
\text { programmes } \\
\text { Activités }\end{array}$ & $\begin{array}{c}\text { Incapacité d'origine } \\
\text { motrice }\end{array}$ & $\begin{array}{l}\text { Incapacité d'origine } \\
\text { sensorielle }\end{array}$ & $\begin{array}{c}\text { Incapacité d'origine } \\
\text { cognitive }\end{array}$ & $\begin{array}{l}\text { Facteurs socio- } \\
\text { environnementaux }\end{array}$ & Santé physique & Santé mentale \\
\hline Évaluation & $\begin{array}{l}\text { - } \text { bilan articulaire et } \\
\text { musculaire } \\
\text { - limitations ou } \\
\text { déformation } \\
\text { - compréhension des } \\
\text { consignes } \\
\text { - vitesse de réaction } \\
\text { - équilibre } \\
\text { - dextérité manuelle } \\
\text { - endurance et } \\
\text { tolérance à l'effort } \\
\text { - capacités } \\
\text { fonctionnelles }\end{array}$ & $\begin{array}{l}\text { Vue } \\
\text { - évaluation } \\
\text { ophtalmologique } \\
\text { - évaluation fonctionnelle: } \\
\text { AVQ, orientation et } \\
\text { mobilité } \\
\text { Ouïe } \\
\text { - dépistage } \\
\text { - évaluation audiologique } \\
\text { - évaluation du handicap } \\
\text { fonctionnel de } \\
\text { communication }\end{array}$ & $\begin{array}{l}\text { - fonctions cognitives } \\
\text { - capacités fonctionnelles } \\
\text { - sécurité à domicile } \\
\text { - réseau de soutien }\end{array}$ & $\begin{array}{l}\text { - } \begin{array}{l}\text { identification de la } \\
\text { problématique }\end{array} \\
\end{array}$ & $\begin{array}{l}\text { - } \text { profil de santé } \\
\text { - bilan fonctionnel } \\
\text { - dépistage des clients à } \\
\text { risque }\end{array}$ & $\begin{array}{ll}\text { - } & \text { histoire de la } \\
\text { problématique } \\
\text { - } \text { identification de la } \\
\text { problématique actuelle }\end{array}$ \\
\hline $\begin{array}{l}\text { Classe } \\
\text { d'exercices }\end{array}$ & $\begin{array}{l}\text { - régulière } \\
\text { - groupe d'ambulation } \\
\text { - groupe de préhension } \\
\end{array}$ & - pour les yeux & - régulière & & - régulière & \\
\hline $\begin{array}{l}\text { Groupe de } \\
\text { tâches }\end{array}$ & $\begin{array}{l}\text { - } \text { menuiserie } \\
\text { - } \text { horticulture } \\
\text { - artisanat } \\
\text { - cuisine } \\
\text { - etc. } \\
\end{array}$ & & $\begin{array}{l}\text { - cuisine } \\
\text { - artisanat adapté } \\
\text { - tâches domestiques } \\
\text { (ex. : préparer les } \\
\text { serviettes de table) } \\
\end{array}$ & & & \\
\hline Groupe d'AVQ & $\begin{array}{l}\text { enseignement et } \\
\text { entraînement AVQ } \\
\text { - enseignement sur } \\
\text { aides techniques } \\
\end{array}$ & & $\begin{array}{l}\text { Intégrer dans la routine } \\
\text { au CJ } \\
\text { - se vêtir et se dévêtir } \\
\text { - utiliser la toilette } \\
\end{array}$ & & & \\
\hline $\begin{array}{l}\text { Groupe } \\
\text { information } \\
\text { santé ou groupe } \\
\text { d'activités } \\
\text { spécifiques } \\
\text { pour la } \\
\text { problématique }\end{array}$ & $\begin{array}{l}\text { Pour tous } \\
\text { - tension artérielle } \\
\text { - médicaments } \\
\text { - hygiène des pieds } \\
\text { - alimentation } \\
\text { Groupe spécifique pour } \\
\text { certaines pathologies } \\
\text { - hémiplégie } \\
\text { - sclérose en plaques } \\
\text { - arthrite } \\
\text { - Parkinson }\end{array}$ & $\begin{array}{l}\text { Stratégies de } \\
\text { communication } \\
\text { - discrimination par le } \\
\text { toucher, les odeurs } \\
\text { - stratégie d'écoute, } \\
\text { lecture labiale, etc. } \\
\text { - exercices de mémoire } \\
\text { et de concentration }\end{array}$ & 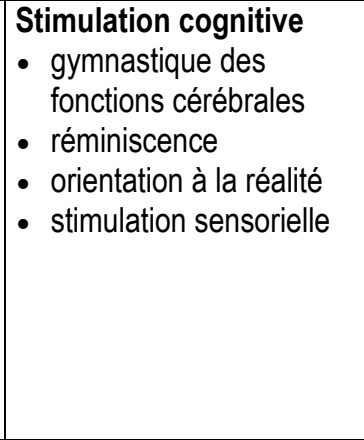 & & $\begin{array}{l}\text { Pour tous } \\
\text { - nutrition } \\
\text { - médication } \\
\text { - sécurité } \\
\text { - vie saine } \\
\text { - information sur } \\
\text { certaines pathologies } \\
\text { Groupe spécifique pour } \\
\text { certaines pathologies } \\
\text { - diabète }\end{array}$ & $\begin{array}{l}\text { Groupe d'information } \\
\text { et de prévention en } \\
\text { santé mentale } \\
\text { - relaxation } \\
\text { - médication }\end{array}$ \\
\hline
\end{tabular}




\begin{tabular}{|c|c|c|c|c|c|c|}
\hline $\begin{array}{l}\begin{array}{l}\text { Sous- } \\
\text { programmes }\end{array} \\
\text { Activités }\end{array}$ & $\begin{array}{c}\text { Incapacité d'origine } \\
\text { motrice }\end{array}$ & $\begin{array}{l}\text { Incapacité d'origine } \\
\text { sensorielle }\end{array}$ & $\begin{array}{c}\text { Incapacité d'origine } \\
\text { cognitive }\end{array}$ & $\begin{array}{l}\text { Facteurs socio- } \\
\text { environnementaux }\end{array}$ & Santé physique & Santé mentale \\
\hline $\begin{array}{l}\text { Groupe } \\
\text { psychosocial }\end{array}$ & $\begin{array}{l}\text { - } \text { remotivation } \\
\text { - } \text { deuil } \\
\text { - } \text { estime de soi } \\
\text { hanre avec un } \\
\text { houticap (groupe de } \\
\text { - information sur } \\
\text { services } \\
\text { communautaires }\end{array}$ & $\begin{array}{l}\text { - vivre avec un handicap } \\
\text { visuel (groupe } \\
\text { d'adaptation et de } \\
\text { soutien) } \\
\text { - vivre avec un handicap } \\
\text { auditif(groupe } \\
\text { d'adaptation et de } \\
\text { soutien) } \\
\text { - information sur } \\
\text { ressources disponibles }\end{array}$ & $\begin{array}{l}\text { - groupe de valorisation } \\
\text { et de socialisation (peut } \\
\text { inclure jeux, } \\
\text { zoothérapie, musique, } \\
\text { etc.) }\end{array}$ & $\begin{array}{l}\text { - groupe d'échange sur } \\
\text { des sujets communs } \\
\text { de préoccupation } \\
\text { (vieillissement, stress, } \\
\text { testament) } \\
\text { - socialisation (jeux, } \\
\text { chants, excursions) } \\
\text { - groupe de préparation } \\
\text { à l'hébergement } \\
\text { - groupe de réinsertion } \\
\text { sociale }\end{array}$ & $\begin{array}{l}\text { - } \text { adaptation au } \\
\text { vieillissement } \\
\text { - relaxation } \\
\text { - information sur } \\
\text { services } \\
\text { communautaires }\end{array}$ & $\begin{array}{l}\text { - } \text { prévention des } \\
\text { situations potentielles } \\
\text { de détresse } \\
\text { - } \text { revalorisation du rôle } \\
\text { social et estime de soi } \\
\text { - groupe d'entraide à la } \\
\text { suite d'un deuil } \\
\text { - groupe de suivi relié à } \\
\text { la consommation } \\
\text { abusive d'alcool ou de } \\
\text { médicaments }\end{array}$ \\
\hline $\begin{array}{l}\text { Interventions } \\
\text { individuelles }\end{array}$ & $\begin{array}{l}\text { - } \text { ergothérapeute } \\
\text { - physiothérapeute } \\
\text { - infirmière } \\
\text { - intervenant } \\
\text { psychosocial }\end{array}$ & $\begin{array}{l}\text { - } \text { audiologiste } \\
\text { - ergothérapeute }\end{array}$ & $\begin{array}{l}\text { - } \text { infirmière } \\
\text { - } \text { ergothérapeute } \\
\text { - intervenant psycho- } \\
\text { social }\end{array}$ & $\begin{array}{l}\text { - intervenant psycho- } \\
\text { social }\end{array}$ & $\begin{array}{l}\text { - infirmière (suivi de TA, } \\
\text { de poids et de diète, } \\
\text { de médication, soins } \\
\text { infirmiers sous } \\
\text { prescription médicale) } \\
\text { - ergothérapeute et } \\
\text { physiothérapeute }\end{array}$ & $\begin{array}{l}\text { - } \text { intervenant psycho- } \\
\text { social } \\
\text { - infirmière }\end{array}$ \\
\hline $\begin{array}{l}\text { Activités } \\
\text { indirectes } \\
\text { générales }\end{array}$ & $\begin{array}{l}\text { - } \text { rencontre avec aidant } \\
\text { (programme } \\
\text { d'exercice à domicile) } \\
\text { - contact avec } \\
\text { ressources du milieu }\end{array}$ & $\begin{array}{l}\text { - } \text { rencontre avec aidant : } \\
\text { suivi des stratégies de } \\
\text { communication } \\
\text { - contact avec ressources } \\
\text { du milieu }\end{array}$ & $\begin{array}{l}\text { - } \text { rencontre avec aidant : } \\
\text { enseignement et } \\
\text { soutien } \\
\text { - } \text { contact avec } \\
\text { ressources du milieu }\end{array}$ & $\begin{array}{ll}\text { - } & \text { rencontre avec aidant } \\
\text { - } & \text { contact avec } \\
& \text { ressources du milieu }\end{array}$ & $\begin{array}{l}\text { - rencontre avec } \\
\text { aidant : enseignement } \\
\text { (insuline, dosette) } \\
\text { - } \text { contact avec } \\
\text { ressources du milieu }\end{array}$ & $\begin{array}{ll}\text { - } & \text { rencontre avec } \\
& \text { aidant (groupe de } \\
& \text { soutien) } \\
\text { - } & \text { contact avec } \\
& \text { ressources du milieu }\end{array}$ \\
\hline $\begin{array}{l}\text { Activités } \\
\text { indirectes } \\
\text { spécifiques }\end{array}$ & $\begin{array}{l}\text { démarches pour } \\
\text { transport adapté, } \\
\text { vignettes de } \\
\text { stationnement, } \\
\text { demande de fauteuil } \\
\text { roulant, finition } \\
\text { d'orthèse, recherche } \\
\text { ou fabrication d'aide } \\
\text { technique }\end{array}$ & $\begin{array}{l}\text { - démarches pour } \\
\text { subvention pour } \\
\text { appareils spécialisés, } \\
\text { transport adapté, etc. } \\
\text { - référence en } \\
\text { réadaptation spécialisé } \\
\text { - aménagement des } \\
\text { locaux (éclairage, } \\
\text { réverbération du son, } \\
\text { système d'amplification } \\
\text { de groupe, etc.) }\end{array}$ & $\begin{array}{l}\text { - } \text { visite préinscription à } \\
\text { - domicile recommandée } \\
\text { - } \text { accueil avec les } \\
\text { personnes significatives } \\
\text { - } \text { identification d'un } \\
\text { intervenant } \\
\text { responsable } \\
\text { - } \text { aménagement des } \\
\text { locaux (diminuer stimuli } \\
\text { non utiles) }\end{array}$ & & $\begin{array}{l}\text { - démarches pour } \\
\text { transport adapté, } \\
\text { vignettes de } \\
\text { stationnement, achat } \\
\text { d'équipement }\end{array}$ & $\begin{array}{l}\text { - référence à des } \\
\text { ressources } \\
\text { spécialisées en } \\
\text { psychiatrie ou en } \\
\text { désintoxication } \\
\text { - suivis avec les } \\
\text { ressources } \\
\text { intermédiaires, les } \\
\text { centres de crises, etc. }\end{array}$ \\
\hline
\end{tabular}


À ce jour, aucune étude n'a été effectuée pour vérifier dans quelle mesure ces activités sont réellement offertes dans les centres de jour. À notre connaissance, ce cadre de référence n'a pas non plus été mis à jour depuis 1991.

Au Québec, la majeure partie du budget des centres de jour provient du ministère de la Santé et des Services sociaux, toutefois une participation financière de l'usager est exigée pour couvrir une partie des coûts de transport et de repas, lorsque ceux-ci sont offerts à l'usager. Le Regroupement des centres d'hébergement et de soins de longue durée de la région de Montréal (2005) recommande d'uniformiser la contribution financière des utilisateurs de centre de jour de la région à $2 \$$ pour le transport aller-retour et $5 \$$ pour le repas du midi. Ce regroupement n'ayant qu'un rôle consultatif, les centres de jour sont libres de suivre ou non cette recommandation.

Les critères d'admissibilité des divers centres de jour du Québec varient, entre autres, quant aux services offerts à la clientèle atteinte de troubles cognitifs et à celle qui présente de l'incontinence (Tourigny et coll., 1993). Puisque ces clients ont aussi grandement besoin de services s'ils souhaitent demeurer à domicile, les orientations gouvernementales actuelles préconisent de ne pas les exclure des centres de jour, sans nécessairement accorder les ressources nécessaires à leur intégration. Ceci donne lieu à des directives qui laissent place à interprétation, comme celle de l'Agence de la santé et des services sociaux de Montréal (2005) :

«Certaines clientèles ne peuvent ou ne doivent pas être admises en centre de jour compte tenu des objectifs poursuivis par ce programme. C'est le cas de la clientèle :

- dont les besoins sont strictement d'ordre social ou récréatif et qui peuvent fréquenter les ressources de la communauté (...);

- qui, malgré les interventions qui ont été effectuées à ce niveau, présente un comportement perturbateur tel qu'il compromet l'atteinte des objectifs du groupe et sa sécurité;

- qui nécessite un encadrement selon un ratio $1 / 1$ de façon continue.

Aucun autre motif ne devrait justifier le refus d'admettre un usager en centre de jour s'il rencontre les critères d'admissibilité. Par exemple, l'incontinence et le besoin d'assistance pour les transferts à la toilette ne devraient pas constituer des motifs d'exclusion d'un usager au centre de jour. Cependant, dans un contexte où les ressources sont limitées, certains centres de jour 
pourraient faire le choix de ne pas admettre la clientèle nécessitant l'assistance de deux personnes pour le transfert à la toilette ou celle requérant de l'aide à l'alimentation. Il apparait toutefois souhaitable que ces décisions soient prises en procédant à une analyse cas par cas qui tiendrait compte d'un ensemble de facteurs, dont des impacts pour l'usager et sa famille de ne pas fréquenter le centre de jour » (p. 66).

De même, selon l'Agence de la santé et des services sociaux de Montréal (2005), la fréquence de participation au centre de jour peut varier d'une à cinq visites par semaine, mais en raison de ressources limitées «l'intensité des services ou la fréquence des visites, doivent faire l'objet d'une mise en priorité » (p.22). Les données statistiques présentées dans ce document pour 2001-2002 et 2002-2003 montrent que $60 \%$ des participants sont inscrits à raison d'une journée par semaine et $30 \%$ à raison de deux jours par semaine. Ceci semble similaire à ce qui est observé ailleurs au Canada où des taux de participation moyens allant de 1,5 à 1,8 jour par semaine sont rapportés (Gottlieb et Johnson, 2000; Gutman et coll., 1993b; Neufeuld et Strang, 1992; Ross-Kerr et coll., 2003), mais inférieur à ce qui est observé aux États-Unis où on rapporte des participations moyennes d'un peu plus de trois jours par semaine (Conrad, Hanrahan et Hughes, 1990; Weissert et coll., 1989; Zarit et coll., 1998).

Dans le contexte québécois, le premier contact avec le centre de jour est rarement initié par la personne âgée ou sa personne de soutien. En effet, pour assurer la coordination des interventions qui visent le maintien à domicile des personnes âgées vulnérables, l'Agence souhaite que toutes les demandes de participation en centre de jour soient d'abord évaluées par le CLSC, à moins qu'elles ne proviennent d'un centre hospitalier, auquel cas la demande peut être faite simultanément au centre de jour et au CLSC. Ainsi, à Montréal en 2002-2003, 88,7\% des participants en centre de jour y avaient été dirigés par leur CLSC (Agence de la santé et des services sociaux de Montréal, 2005).

Le premier contact avec un professionnel de la santé peut donc avoir été initié par la personne âgée qui demande de l'aide au CLSC pour réaliser diverses activités de la vie quotidienne ou par un proche qui demande du soutien. Il peut aussi provenir d'une détérioration de l'état de santé qui a entrainé une hospitalisation. Le plan de congé du 
centre hospitalier peut inclure une référence au centre de jour. La figure 1 présente de façon graphique les étapes du cheminement d'une référence en centre de jour, en partant de l'identification du besoin, en passant par la référence et l'admission, jusqu'au congé.

Les motifs de congé les plus fréquents sont l'hébergement, l'hospitalisation, le décès, le refus de participer et la détérioration trop grande de l'état de santé qui empêche de fréquenter le centre de jour. Plus rarement, on observera des congés pour atteinte des objectifs ou amélioration de l'état de santé, pour des problèmes de transport ou des raisons financières, ou encore pour un déménagement (Cox, Reifler et Yates, 1998; Dabelko, 2004; Mace et Rabins, 1984; Neufeuld et Strang, 1992; Tourigny et coll., 1993; Wallace, 1987). Les problèmes de comportement sont aussi une raison qui amène à cesser l'utilisation des centres de jour chez les personnes atteintes de troubles cognitifs (Lawton, Brody et Saperstein, 1991; Mace et Rabins, 1984; Zarit, Stephens, Townsend, Greene et Leitsch, 1999b). 
Figure 1 : Cheminement d'une référence au centre de jour

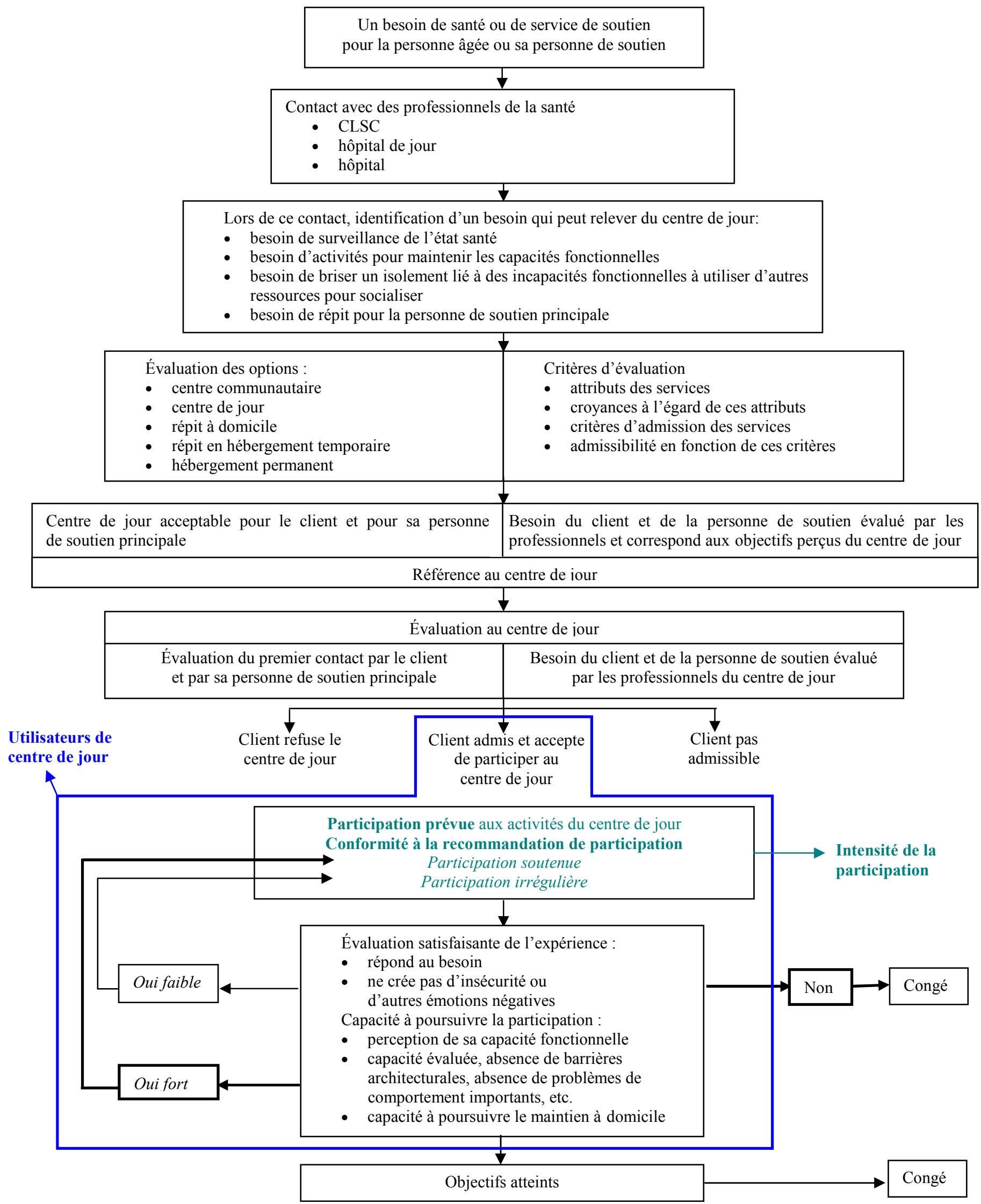




\section{L'efficacité des centres de jour}

À ce jour, les études sur l'efficacité des centres de jour pour réduire ou retarder l'institutionnalisation de la clientèle ou pour réduire le fardeau des personnes de soutien ont démontré des résultats variables (voir Zarit, Gaugler et Jarrott, 1999a; Gaugler et Zarit, 2001). Plusieurs études ont observé de faibles taux d'utilisation des services, sans toutefois considérer cette variable dans l'analyse des résultats. En général, ces études n'ont pu démontrer d'effets à la participation à un centre de jour. Les études qui ont contrôlé les taux d'utilisation sont plus encourageantes. Ces études sont en majorité des études quasiexpérimentales avec groupe témoin non-équivalent, sauf l'étude de Baumgarten et collaborateurs qui consiste en un essai randomisé et celle de Wilson et collaborateurs qui est une étude de cohorte. Bien que les données probantes provenant d'études de qualité soient encore insuffisantes (Mason et coll., 2007), les quelques études recensées ci-dessous suggèrent tout de même des effets positifs à la fréquentation du centre de jour.

L'étude de Baumgarten et collaborateurs (2002), menée auprès d'une clientèle générale (c.-à.-d. une clientèle qui comprend des personnes avec ou sans démence), a démontré une réduction du fardeau des personnes de soutien des participants qui avaient un minimum de 13 visites au centre de jour en 13 semaines, alors que pour ceux qui avaient moins de 13 visites, le fardeau des personnes de soutien semble légèrement augmenté.

Dans l'étude de Zank et Schacke (2002), auprès d'une clientèle générale comportant tout de même $71 \%$ de participants atteints de démence, les participants étaient inscrits à un centre de jour à raison d'un minimum de deux fois par semaine. Les membres du groupe d'utilisateurs et du groupe témoin présentent des caractéristiques sociodémographiques et de santé similaires au début de l'étude. Les analyses incluent deux mesures pour chaque variable : la valeur obtenue par la personne au début de l'étude et le niveau de changement pendant neuf mois. Les auteurs rapportent une stabilisation du bien-être psychologique et une légère amélioration du fonctionnement cognitif des participants au centre de jour, comparativement à une détérioration pour le groupe témoin. 
Dans les études suivantes les participants sont tous des personnes atteintes de démence. L'étude de Schacke et Zank (2006) utilisent une méthode identique à celle de Zank et Schacke (2002). Les auteurs ont observé une réduction significative du stress des personnes de soutien des participants comparativement à un groupe témoin constitué de non-utilisateurs de centre de jour.

Dans l'étude de Zarit et collaborateurs (1998), les participants étaient aussi inscrits à un centre de jour à raison d'un minimum de deux fois par semaine. Pour diminuer les biais possibles reliés à la répartition non aléatoire des groupes, la valeur obtenue par la personne au début de l'étude pour les différentes mesures de résultats, de même que les variables sociodémographiques pour lesquelles les utilisateurs et les non-utilisateurs différaient au début de l'étude ont été incluses comme variable de contrôle dans les analyses multivariées. Cette étude a trouvé des niveaux de stress significativement inférieurs chez les personnes de soutien d'utilisateurs de centre de jour comparativement à ceux des non-utilisateurs. Des analyses subséquentes de cette même étude ont démontré une diminution du temps consacré à la gestion des comportements dérangeants et une diminution du nombre de comportements problématiques rapportés par les personnes de soutien des utilisateurs de centre de jour (Gaugler, Jarrot, Zarit, Stephens, Townsend et Greene, 2003).

Dans une étude suédoise, en excluant du groupe expérimental les participants qui n'avaient utilisé le centre de jour qu'une ou deux fois, on a observé, sur une période d'un an, des taux d'institutionnalisation nettement inférieurs parmi le groupe expérimental (24\%) par rapport au groupe témoin constitué de personnes sur la liste d'attente du centre de jour (44 \%) (Wimo, Mattsson, Adolfsson, Eriksson et Nelvig, 1993). Les membres du groupe expérimental et du groupe témoin présentaient des caractéristiques sociodémographiques et de santé similaires au début de l'étude.

Gitlin et collaborateurs (2006) ont démontré qu'un soutien régulier apporté à l'aidant du participant au centre de jour dans le but de développer des habiletés de résolution de problèmes chez l'aidant, augmentait l'utilisation du centre de jour. Lorsque comparé à un groupe ayant reçu les interventions courantes du centre de jour, le groupe 
ayant reçu ce soutien particulier a démontré un meilleur bien-être de l'aidant et une diminution de l'institutionnalisation. Afin de contrôler les différences préexistantes possibles entre les membres des deux groupes, le bien-être de l'aidant au début de l'étude, de même que les variables sociodémographiques pour lesquelles les utilisateurs et les nonutilisateurs différaient au début de l'étude ont été incluses comme variables de contrôle dans les analyses multivariées.

Dans une étude de cohorte, Wilson, McCann, Li, Aggarwal, Gilley et Evans (2007) ont démontré que l'utilisation du centre de jour préalable au placement en institution a un effet protecteur sur le déclin cognitif de personnes atteintes de démence lors du placement et que cet effet est positivement corrélé à l'intensité de l'utilisation du centre de jour avant le placement.

Ainsi, il apparaît important de contrôler le niveau d'exposition au centre de jour lorsqu'on cherche à évaluer les effets de ce service sur les participants et leurs personnes de soutien. Plus encore, pour maximiser les effets du centre de jour, de niveaux minimaux de participation doivent faire partie des buts explicites des centres de jour (Baumgarten et coll., 2002). Les facteurs qui influencent la participation au centre de jour doivent être bien compris afin d'agir en fonction de ce but.

\section{L'utilisation des centres de jour}

Lors de la mise en place des centres de jour au Québec au début des années 1970, il était prévu que ces programmes toucheraient près de $5 \%$ de la population de leur territoire âgée de plus de 65 ans. En 1992, ils en rejoignaient effectivement $3 \%$ (Tourigny et coll., 1993). Selon les données statistiques fournies par les centres de jour, 3869 personnes étaient inscrites dans l'un des 32 centres de jour de l'Île de Montréal au 31 mars 2001. Considérant les départs et les nouvelles inscriptions, un total de 5932 personnes avaient participé aux activités d'un centre de jour à un moment ou un autre au cours de l'année 2001-2002. De ce nombre, 446 avaient moins de 65 ans (Agence de la santé et des services sociaux de Montréal, 2005). Les 5486 participants de plus de 65 ans représentent $2 \%$ des 
276695 personnes de ce groupe d'âge dans l'île de Montréal selon le recensement de 2001 (Statistique Canada, 2007).

Le pourcentage des personnes en perte d'autonomie qui reçoivent les services d'un centre de jour au Québec ou à Montréal n'est toutefois pas connu. Cette donnée serait pertinente, étant donné leur mandat orienté vers cette clientèle. Dans les écrits scientifiques, les données à ce sujet sont aussi peu nombreuses. Dans une étude auprès de participants inscrits à quatre Health Maintenance Organization (HMO), Lynch, Harrington et Newcomer (1999) ont trouvé qu'entre 1,9 et 11,7 \% des personnes chez qui ils ont identifié une incapacité pour au moins une activité de vie quotidienne ou deux activités de vie domestique ont utilisé un centre de jour. La grande variation semblait provenir de la disponibilité différente des centres de jour à l'intérieur de la gamme de services couverts par chacun des HMO à l'étude. Dans l'étude de Kosloski et Montgomery (1994) auprès de personnes âgées ayant au moins deux limitations d'activités quotidiennes, $6 \%$ des participants utilisaient un centre de jour. Dans une enquête populationnelle de 11 comtés du Kentucky et de l'Indiana, Barber, Paton et Wishnia (1993) ont estimé que 5439 familles comptaient au moins une personne âgée de plus de 60 ans qui présentait des incapacités et nécessitait au moins cinq heures de soins par jour de la part d'un membre de la famille. Les données sur le nombre d'utilisateurs de centre de jour dans la région étudiée faisaient état de 149 participants, soit environ 2,7\% du bassin de population qui présente théoriquement des besoins pour ce type de service. De l'étude manitobaine de Strain et Blandford (2002) auprès de 293 personnes âgées qui présentaient au moins une incapacité dans au moins une activité de vie quotidienne ou domestique, $6 \%$ avaient utilisé un centre de jour dans la dernière année.

L'utilisation d'un service peut être vue comme un indicateur de l'adaptation du service à la population qu'il est censé servir (concept de «goodness of fit» dans Dabelko, 2004). La recherche sur ce sujet démontre que le centre de jour n'est pas adapté à toute la population qui répond aux critères d'éligibilité. Selon Caserta, Lund, Wright et Redburn (1987), les personnes de soutien de personnes atteintes de démence mentionnent qu'elles ont besoin de services tels que le répit et les centres de jour, pourtant seulement $57 \%$ de 
ceux qui connaissent l'existence de ces services les utilisent. De plus, seule la moitié des utilisateurs le font de façon régulière. Même lorsque ce type de services est accessible gratuitement ou à faible coût, des études ont démontré un faible taux d'utilisation par la clientèle âgée (Baumgarten et coll., 2002; Montgomery et Borgotta, 1989) et par la clientèle atteinte de démence (Douglass et Visconti, 1998; Lawton et coll., 1989). Par exemple, dans l'étude de Baumgarten et collaborateurs (2002), seulement $34 \%$ des sujets inscrits au centre de jour ont eu au moins une présence par semaine, et 8,3\% (9 personnes) n'ont aucune présence en 13 semaines.

De forts taux d'abandon sont aussi rapportés dans les trois premiers mois d'utilisation : entre $20 \%$ et $42 \%$ dans des études auprès de la clientèle atteinte de démence (Gottlieb et Johnson, 2000; Schacke et Zank, 2006; Wells, Jorm, Jordan et Lefroy, 1990; Zarit et coll., 1999b) et entre 40 et $50 \%$ dans des études auprès de la clientèle générale (Dabelko, 2004; Neufeld et Strang, 1992). Il semble qu'après les deux ou trois premiers mois de participation, les taux d'abandon diminuent grandement (Dabelko, 2004). Les personnes qui se sont bien adaptées au centre de jour peuvent y participer pendant plusieurs années; les statistiques des centres de jour de la région de Montréal pour 2001-2002 font état de $41 \%$ d'utilisateurs ayant des durées de séjours de plus de 24 mois (Agence de la santé et des services sociaux de Montréal, 2005)

Les faibles taux d'utilisation des centres de jour surprennent étant donné les taux élevés de satisfaction chez les utilisateurs (Henry et Capitman, 1995; Jarrott, Zarit, ParrisStephens, Townsend et Greene, 1999; Weissert, Elston, Bolda, Zelman, Mutran et Magnum, 1990) et le besoin de répit fréquemment exprimé par les personnes de soutien de la clientèle atteinte de démence (Brodaty, Thomson, Thomson et Fine, 2005; Caserta et coll., 1987; Fortinsky et Hathaway, 1990; Montoro-Rodriguez, Kosloski et Montgomery, 2003; Philp et coll., 1995; Theis, Moss et Pearson, 1994).

Quelques études, qui portent majoritairement sur la clientèle atteinte de démence, se sont penchées sur les raisons mentionnées par les personnes de soutien pour utiliser les services de centre de jour ou de répit. Selon Monahan (1993), les personnes de soutien qui 
utilisent les centres de jour mentionnent : les bienfaits pour le parent atteint de démence $(84,6 \%)$, la possibilité de socialisation pour le parent $(80,3 \%)$, le répit pour la personne de soutien $(76,9 \%)$, pour pouvoir travailler $(50,0 \%)$, pour éviter un placement $(50,0 \%)$. Par ailleurs, la raison la plus importante pour la majorité des personnes de soutien est d'obtenir du répit. Selon Cotrell (1996), les personnes de soutien qui sont des enfants corésidants indiquent une préférence pour le centre de jour plutôt que d'autres formes de répit, pour les bienfaits de la socialisation qu'il procure à leur parent atteint de démence. De plus, le centre de jour représente un coût moins élevé que d'autres formes de supervision. Les conjoints perçoivent le répit de deux façons, soit un abandon du conjoint malade et un pas vers l'institutionnalisation, ou encore, une occasion de socialisation pour le conjoint. Ceux qui le perçoivent de la seconde façon ont tendance à utiliser davantage ce service. La perception du bienfait pour la personne âgée est aussi une raison d'utilisation rapportée par Beisecker, Wright, Chrisman et Ashworth (1996), peu importe la relation de la personne de soutien avec la personne âgée. Enfin, soulignons que selon Gottleib et Johnson (2000), lorsque cette option leur est présentée, une majorité de personnes de soutien indique que l'utilisation du centre de jour est aussi une façon de vérifier comment la personne âgée s'adapte à des soins prodigués par d'autres et d'obtenir du soutien pour la planification des soins de longue durée.

D'autres auteurs se sont penchés sur les raisons rapportées pour ne pas utiliser les services. Les personnes âgées inscrites à un centre de jour mais qui omettent de s'y présenter mentionnent le trop grand effort exigé pour la participation aux activités, l'incapacité de se préparer si tôt le matin, les troubles de l'ouïe ou de la vue qui nuisent à la participation en groupe, l'incapacité de sortir du domicile en raison d'obstacles physiques au domicile (marches), les déplacements trop longs, les frais de déplacement et de repas (Gutman et coll., 1993a), ainsi que le manque d'individualisation du programme de récréation qui ne correspond pas à leurs intérêts ou du programme d'exercices qui ne correspond pas au but recherché (Neufeuld et Strang, 1992).

Tant pour la clientèle atteinte de démence (Casserta et coll., 1987) que pour la clientèle générale (Strain et Blandford, 2002), la principale raison rapportée par les 
personnes de soutien pour ne pas utiliser ces services est le manque de besoin immédiat. La raison qui occupe la seconde place pour ne pas utiliser le centre de jour dans l'étude de Strain et Blandford est le manque de connaissance à propos du service. Il est utile de noter que si environ $10 \%$ de l'échantillon ne connaissaient pas l'existence des divers types d'aide à domicile (entretien ménager, soins personnels, soins infirmiers, livraison de repas), $35 \%$ des personnes de soutien ne savaient pas que le centre de jour était un service disponible, alors que de 40 à $50 \%$ ne connaissaient pas les autres types de services de répit.

Les autres raisons rapportées par les personnes de soutien pour ne pas utiliser les centres de jour et les services de répit incluent: la difficulté de laisser le parent avec un étranger (Beisecker et coll., 1996; Caserta et coll., 1987; Cotrell, 1996), le sentiment que c'est un devoir de s'occuper du proche (Cohen-Mansfield, Besansky, Watson et Bernhard, 1994: raison rapportée par les femmes seulement), le peu d'habitude d'utilisation des services formels (Montgomery et Borgotta, 1989), la difficulté à préparer le parent (Lawton et coll., 1991), la perception que le besoin du parent est différent de celui des autres clients du programme (Cohen-Mansfield et coll., 1994) ou que le programme ne répond pas aux besoins du proche (Beisecker et coll., 1996), la présence de problèmes de comportement importants, le refus de ce type d'activités par la personne âgée (Beisecker et coll., 1996; Cohen-Mansfield et coll., 1994; Cotrell, 1996) et la détérioration de l'état de santé (Cotrell, 1996). Certaines caractéristiques organisationnelles comme le manque de transport, le coût, les critères d'exclusion de certains centres (incontinence, problèmes de comportement, risques de fugues, etc.), limitent aussi la participation aux centres de jour (Beisecker et coll., 1996; Caserta et coll., 1987; Lawton et coll., 1991; Wallace, 1987). Le manque de personnel adéquatement formé pour s'occuper de personnes atteintes de démence, le ratio personnel/patient inadéquat, un environnement inadéquat (malpropreté, espace insuffisant, etc.) sont aussi cités comme des barrières à l'utilisation du centre de jour (Beisecker et coll., 1996).

Les personnes de soutien semblent utiliser le répit tardivement dans le processus de maladie de la personne dont elles prennent soin. Les niveaux de stress et de fardeau des aidants sont habituellement déjà élevés lorsqu'ils choisissent de recourir à des services 
(Cox, 1997; Cox et coll., 1998; Gaugler et Zarit, 2001; Gottlieb et Johnson, 2000; Zarit et coll., 1999b). Pour certains, la situation de soins à domicile a déjà commencé à se désintégrer et les démarches pour le placement en institution sont déjà entreprises lors de l'admission au centre de jour. Ces personnes sont généralement de brefs utilisateurs de centre de jour (Gottlieb et Johnson, 2000). Si un des objectifs du centre de jour est de retarder le placement en institution, les politiques de financement et d'accès à ce service doivent reconnaître le besoin d'y avoir recours tôt dans le processus (Gaugler, Kane, Kane et Newcomer, 2005). Pour favoriser une utilisation précoce du centre de jour, il faut aussi agir sur les perceptions des personnes de soutien, diminuer le sentiment d'échec associé au recours à l'aide extérieure et la peur que l'utilisation de services formels leur fasse perdre le contrôle qu'elles ont sur les décisions relatives à la personne dont elles prennent soin, de même que les rassurer sur le bien-être de la personne âgée lorsqu'elle participe au programme (Gottlieb et Johnson 2000).

Cependant, adoucir la transition vers le placement peut aussi être un objectif du centre de jour, un but recherché par certaines personnes de soutien. Les centres de jour peuvent mettre en place des interventions à cet effet et la planification efficace du placement est un résultat mesurable (Gottlieb et Johnson, 2000). Par exemple, l'utilisation du centre de jour préalablement au placement peut avoir un effet protecteur sur le déclin cognitif de personnes atteintes de démence lors du placement (Wilson et coll., 2007). L'évaluation de l'efficacité des centres de jour devrait toutefois tenir compte de ces deux types de besoins : les personnes qui cherchent une transition vers le placement et celles qui cherchent à améliorer leur capacité de poursuivre le maintien à domicile.

\section{Déterminants de l'utilisation}

Puisque le centre de jour ne semble pas convenir à l'ensemble de la population âgée qui présente des incapacités, il est pertinent de connaitre les caractéristiques des clients qui, par leur taux d'utilisation plus élevé, semblent mieux adaptés à ce service. 
L'état des connaissances sur les déterminants de l'utilisation des centres de jour sera présenté en fonction de quatre types de facteurs les plus fréquemment retrouvés dans les écrits recensés. Les trois premiers s'inspirent du modèle d'utilisation des services de santé d'Andersen (Andersen et Newman, 1973; Andersen, 1995). Ces trois premiers groupes de facteurs incluent :

- les facteurs prédisposants, des facteurs indépendants de l'état de santé immédiat qui, de façon générale, influencent indirectement l'utilisation de services de santé;

- les facteurs de capacité ou facteurs facilitants (en anglais «enabling factors»), qui peuvent être des ressources personnelles ou communautaires qui facilitent l'accès aux services;

- les facteurs de besoin qui sont reliés aux symptômes ressentis ou évalués. Ils ont une influence directe sur l'utilisation des services de santé.

Dans le contexte des services de soutien à domicile pour ces trois groupes de facteurs, on tiendra compte des caractéristiques des personnes de soutien principales en plus de celles des personnes âgées. Outre le fait que ces personnes ont un rôle important dans la connaissance qu'ont les personnes âgées des services formels et dans l'utilisation qu'elles font de ces services (Bass et Noelker, 1987; Cotrell et Engel, 1998; Strain et Blandford, 2002), certaines études de l'utilisation du centre de jour pour les personnes atteintes de démence considèrent la personne de soutien comme le principal utilisateur du répit offert par le centre de jour.

S'inspirant de Kosloski, Montgomery et Youngbauer (2001) et de MontoroRodriquez et collaborateurs (2003), le quatrième groupe de facteurs sera constitué des caractéristiques organisationnelles des services étudiés. Les caractéristiques organisationnelles des services influencent l'expérience du service, une fois que le besoin a été identifié. Pour des services de nature discrétionnaire, où la substitution de services est possible, ces facteurs peuvent avoir une influence directe sur la décision d'utiliser ou non un service particulier et de poursuivre son utilisation. 
On observe dans les écrits une certaine variabilité dans la compréhension du modèle d'Andersen par les divers auteurs des études d'utilisation recensées. Par exemple, certains placent le revenu familial ou le réseau de soutien social dans les facteurs prédisposants, alors que d'autres les placent dans les facteurs de capacité personnels et communautaires. Pour faciliter la lecture, des choix ont été effectués pour présenter chaque type de variable sous un seul type de facteur, peu importe comment ils sont présentés par les chercheurs dont les travaux sont rapportés. À ce sujet, la figure 2 présente le type de variables inclus sous chacun de ces quatre groupes de facteurs dans notre recension des déterminants de l'utilisation du centre de jour. Cette figure présente d'abord les facteurs prédisposants, dont l'influence sur l'utilisation est plus distale et termine par les caractéristiques des services, dont l'influence sur l'utilisation est plus immédiate ou proximale.

Quelques études ont porté sur les déterminants de l'utilisation des centres de jour. Puisqu'elles sont peu nombreuses, l'identification des variables susceptibles d'influencer cette utilisation inclura aussi une recension des recherches portant sur des services connexes. Les études sur les services de répit incluent souvent une proportion de sujets qui reçoivent leur répit par l'intermédiaire d'un centre de jour et seront donc considérées. Les études sur l'utilisation des services à domicile pourraient paraître intéressantes parce que ces services visent le même but que le centre de jour, soit le maintien à domicile des personnes âgées. De plus, plusieurs d'entre elles ont pris en considération des facteurs reliés aux personnes de soutien. Toutefois, les caractéristiques des utilisateurs de centre de jour et celles des utilisateurs de soins à domicile diffèrent sur plusieurs points. Selon Dabelko et Balaswamy (2000), les utilisateurs de centre de jour sont généralement plus jeunes et présentent davantage de troubles cognitifs que les utilisateurs de soins à domicile. Bien que les capacités fonctionnelles des deux groupes soient similaires selon les échelles d'AVQ et AVD, les utilisateurs de soins à domicile sont hospitalisés plus souvent et utilisent plus d'équipement médical ou d'aides techniques. Ces résultats sont cohérents avec le fait qu'il faut certaines capacités de déplacement pour utiliser le centre de jour. Ainsi, les résultats de ces études ne seront présentés que lorsqu'ils permettront d'apporter un éclairage sur certains facteurs peu étudiés dans les études sur les centres de jour ou les services de répit. 
Figure 2 : Déterminants de l'utilisation du centre de jour ou des services de répit recensés dans les écrits

\begin{tabular}{|c|c|c|c|}
\hline \multicolumn{4}{|c|}{ Influence sur l'utilisation } \\
\hline $\begin{array}{l}\text { Facteurs prédisposants } \\
\text { reliés à la personne âgée : } \\
\text { - âge } \\
\text { - sexe } \\
\text { - éducation } \\
\text { - profession } \\
\text { - réseau de soutien } \\
\text { reliés à la personne de soutien : } \\
\text { - âge } \\
\text { - } \text { sexe } \\
\text { - éducation } \\
\text { réseau de soutien } \\
\text { autant pour la personne âgée } \\
\text { que la personne de soutien : } \\
\text { - appartenance culturelle } \\
\text { (origine ethnique et religion) } \\
\text { type de lien entre PA et PS } \\
\text { (conjoint, enfant, amis ou } \\
\text { autre; cohabitation) } \\
\text { - croyances et attitudes envers la } \\
\text { santé, les services de santé et } \\
\text { les responsabilités de soins au } \\
\text { sein de la famille }\end{array}$ & $\begin{array}{l}\text { Facteurs de capacité } \\
\text { ressources personnelles et } \\
\text { communautaires de la famille : } \\
\text { - } \quad \text { connaissance des services } \\
\text { - } \text { revenu familial } \\
\text { - } \quad \text { source de financement pour les } \\
\text { services } \\
\text { - } \quad \text { existence des services dans le } \\
\text { milieu de vie }\end{array}$ & $\begin{array}{l}\text { Facteurs de besoin } \\
\text { de la personne agée : } \\
\text { - santé physique } \\
\text { - santé mentale } \\
\text { - troubles cognitifs } \\
\text { - incapacités fonctionnelles } \\
\text { de la personne de soutien : } \\
\text { - santé physique, troubles } \\
\text { cognitifs ou incapacités } \\
\text { - fonctionnelles } \\
\text { - santé mentale } \\
\text { - fardeau } \\
\text { - aspects positifs ou négatifs du } \\
\text { - durée de l'aide } \\
\text { - responsabilités concurrentes } \\
\text { (famille, emploi) }\end{array}$ & $\begin{array}{l}\text { Caractéristiques des services } \\
\text { du centre de jour : } \\
\text { - facilité d'accès et disponibilité } \\
\text { au moment opportun } \\
\text { - durée et fréquence du service } \\
\text { - fiabilité du service } \\
\text { - coût du service } \\
\text { - transport } \\
\text { - type d'activités offertes } \\
\text { d'autres services : } \\
\text { - présence d'autres ressources } \\
\text { formelles qui facilitent la } \\
\text { participation au centre de jour }\end{array}$ \\
\hline
\end{tabular}


Enfin, il faut rappeler que différents aspects du phénomène de l'utilisation, à savoir la prise de contact, l'intensité et la durée, auront des prédicteurs différents (Andersen et Newman, 1973). Bass et Noelker (1987) en fournissent l'illustration dans une étude de l'utilisation des services de soutien à domicile. Ils démontrent des différences entre les caractéristiques qui déterminent les probabilités de contact avec les soins à domicile et celles qui sont associées à un plus fort volume de services.

Les résultats contradictoires de diverses études sur l'utilisation des centres de jour pourraient aussi s'expliquer, au moins en partie, par des définitions différentes de l'utilisation. Il importe donc de préciser les aspects du phénomène de l'utilisation qui sont étudiés. Dans la présente recension des écrits, on retrouve des études sur chacun des phénomènes d'utilisation des centres de jour : le contact et l'intensité de la participation, ainsi que la durée d'utilisation. Chacun de ces phénomènes d'utilisation peut encore être défini de plusieurs façons :

- Contact : contact à un moment ou un autre du processus de vieillissement à domicile, contact à un moment précis (statut d'utilisateur ou de non-utilisateur à ce moment) ou au cours d'une période de temps donnée.

- Intensité de la participation: volume de services utilisés pendant l'ensemble de l'épisode d'utilisation du centre de jour, volume de services utilisés pendant une période donnée, ou encore, volume d'utilisation conforme à la recommandation de participation pour une période donnée. Dans toutes les études recensées, le volume d'utilisation est présenté en nombre de jours de présence au centre de jour. Il serait toutefois possible de mesurer le volume d'utilisation autrement, par exemple en calculant le nombre d'heures passées au centre de jour.

- Durée d'utilisation : mesurée de façon continue en nombre de mois d'utilisation ou de façon dichotomique par le fait de poursuivre ou non la fréquentation du centre de jour après un certain nombre de mois.

Les caractéristiques des études recensées, incluant le phénomène d'utilisation étudié, sont présentées au tableau III, selon cinq catégories : 
a) études sur l'utilisation des centres de jour auprès d'une clientèle générale (incluant une analyse corrélationnelle des déterminants de l'utilisation des centres de jour);

b) études sur l'utilisation des centres de jour auprès d'une clientèle atteinte de démence (incluant une analyse corrélationnelle des déterminants de l'utilisation des centres de jour);

c) études sur les déterminants de l'utilisation de services connexes (ex. : répit, divers services incluant les centres de jour, services de loisirs);

d) études descriptives sur les raisons d'utilisation ou de non-utilisation des centres de jour ou de services connexes;

e) études sur l'utilisation des services à domicile.

Pour chacune des recherches corrélationnelles portant sur les déterminants de l'utilisation des centres de jour et des services connexes (catégories a à c de la liste cidessus), la liste des variables étudiées est présentée à l'annexe II. 
Tableau III : Caractéristiques des études recensées pour identifier les déterminants de l'utilisation des centres de jour

Tableau IIIa : études des déterminants de l'utilisation des centres de jour pour une clientèle générale

\begin{tabular}{|c|c|c|c|c|c|c|c|}
\hline Auteurs & Chappell & Wallace & Kosloski et coll. & Henry et Capitman & Lynch et coll. & Baumgarten et coll. & Dabelko \\
\hline Année & 1983 & 1987 & 1994 & 1995 & 1999 & 2002 & $2004 ; 2005$ \\
\hline Pays & Canada & États-Unis & États-Unis & États-Unis & États-Unis & Canada & États-Unis \\
\hline Clientèle & $\begin{array}{l}\text { Personnes âgées ( } 50 \\
\text { ans +) }\end{array}$ & $\begin{array}{l}\text { Personnes âgées (50 } \\
\text { ans +) }\end{array}$ & $\begin{array}{l}\text { Personnes âgées } \\
\text { avec au moins } 2 \\
\text { limitations d'AVQ }\end{array}$ & $\begin{array}{l}\text { Personnes âgées } \\
\text { atteintes de démence } \\
(68 \%) \text { ou non }(32 \%)\end{array}$ & $\begin{array}{l}\text { Clientèle âgée avec } \\
\text { au moins une } \\
\text { incapacité AVQ ou } 2 \\
\text { incapacités AVD }\end{array}$ & $\begin{array}{l}\text { Personnes âgées (60 } \\
\text { ans +) }\end{array}$ & $\begin{array}{l}\text { Personnes âgées (50 } \\
\text { ans +) }\end{array}$ \\
\hline $\begin{array}{l}\text { Type d'étude } \\
\text { (étude } \\
\text { principale) }\end{array}$ & $\begin{array}{l}\text { Étude prospective de } \\
\text { l'état de santé des } \\
\text { participants au CJ. }\end{array}$ & $\begin{array}{l}\text { Corrélationnelle: } \\
\text { Analyse rétrospective } \\
\text { des dossiers du CJ }\end{array}$ & $\begin{array}{l}\text { Corrélationnelle : } \\
\text { Caractéristiques à T1, } \\
\text { utilisation dans les } 6 \\
\text { mois précédant }\end{array}$ & Corrélationnelle & $\begin{array}{l}\text { Corrélationnelle : } \\
\text { Caractéristiques à T1, } \\
\text { utilisation dans } \\
\text { l'année qui suit }\end{array}$ & Essai randomisé & $\begin{array}{l}\text { Corrélationnelle: } \\
\text { Analyse rétrospective } \\
\text { des dossiers. }\end{array}$ \\
\hline $\begin{array}{l}\text { But principal } \\
\text { de l'étude }\end{array}$ & $\begin{array}{l}\text { Vérifier l'effet du } \\
\text { centre de jour sur } \\
\text { divers indicateurs de } \\
\text { santé de la PA. }\end{array}$ & \multirow[t]{2}{*}{$\begin{array}{l}\text { Déterminer les } \\
\text { caractéristiques des } \\
\text { participants qui sont } \\
\text { reliées à leur } \\
\text { utilisation du CJ. }\end{array}$} & \multirow{2}{*}{$\begin{array}{l}\text { Identifier les } \\
\text { déterminants du } \\
\text { contact avec divers } \\
\text { types de services, } \\
\text { dont le CJ. En } \\
\text { particulier, vérifier la } \\
\text { présence d'interac- } \\
\text { tion entre le type de } \\
\text { lien PA-PS et les } \\
\text { facteurs de besoin. }\end{array}$} & \multirow{2}{*}{$\begin{array}{l}\text { Déterminer l'influence } \\
\text { de l'approche } \\
\text { thérapeutique choisie } \\
\text { par le CJ sur } \\
\text { l'utilisation du service } \\
\text { et la satisfaction des } \\
\text { PS envers le service. }\end{array}$} & \multirow{2}{*}{$\begin{array}{l}\text { 1) Identifier les } \\
\text { déterminants du } \\
\text { contact avec le CJ. } \\
\text { 2) Identifier les } \\
\text { déterminants de } \\
\text { l'intensité d'utilisation } \\
\text { du CJ. }\end{array}$} & $\begin{array}{l}\text { Vérifier l'effet du } \\
\text { centre de jour sur } \\
\text { divers indicateurs de } \\
\text { santé de la PA et de } \\
\text { sa PS. }\end{array}$ & \multirow{2}{*}{$\begin{array}{l}\text { 1) Déterminer les } \\
\text { facteurs qui influencent } \\
\text { la durée de séjour en } \\
\text { centre de jour (Dabelko, } \\
\text { 2004). } \\
\text { 2) Comparer les } \\
\text { utilisateurs qui } \\
\text { abandonnent } \\
\text { rapidement à ceux qui } \\
\text { pousuivent la partici- } \\
\text { pation (Dabelko, 2005). }\end{array}$} \\
\hline $\begin{array}{l}\text { But de l'étude } \\
\text { de l'utilisation } \\
\text { (si différent } \\
\text { du but } \\
\text { principal) }\end{array}$ & $\begin{array}{l}\text { Comparer les } \\
\text { caractéristiques des } \\
\text { utilisateurs de CJ à } \\
\text { d'autres groupes de } \\
\text { PA du Manitoba. }\end{array}$ & & & & & $\begin{array}{l}\text { À l'intérieur du groupe } \\
\text { expérimental, } \\
\text { comparer les } \\
\text { caractéristiques des } \\
\text { forts et des faibles } \\
\text { utilisateurs. }\end{array}$ & \\
\hline $\begin{array}{l}\text { Sujets pour } \\
\text { l'étude de } \\
\text { l'utilisation }\end{array}$ & $100 \mathrm{PA}$ & $177 \mathrm{PA}$ & $\begin{array}{l}503 \text { dyades } \\
\text { PA -PS }\end{array}$ & $\begin{array}{l}312 \text { dyades } \\
\text { (PA - PS) }\end{array}$ & $\begin{array}{l}\text { 1) } 1868 \mathrm{PA} \\
\text { 2) } 89 \mathrm{PA}\end{array}$ & 103 PA et 84 PS & $\begin{array}{l}\text { 1) } 280 \text { PA nouvellement } \\
\text { inscrites au CJ } \\
\text { 2) } 143 \text { PA qui ont cessé } \\
\text { la participation dans les } \\
15 \text { semaines de l'étude }\end{array}$ \\
\hline $\begin{array}{l}\text { Phénomène } \\
\text { d'utilisation } \\
\text { et définition } \\
\text { de la variable } \\
\text { d'utilisation }\end{array}$ & $\begin{array}{l}\text { Contact: } \\
\text { 1) Utilisateurs du CJ } \\
\text { versus utilisateurs de } \\
\text { soins à domicile. } \\
\text { 2) Utilisateurs du CJ } \\
\text { versus population de } \\
\text { personnes âgées en } \\
\text { général. }\end{array}$ & $\begin{array}{l}\text { Hybride d'intensité } \\
\text { et de durée : } \\
\text { Nombre de jours de } \\
\text { participation entre } \\
\text { l'admission et le } \\
\text { congé. }\end{array}$ & $\begin{array}{l}\text { Contact: } \\
\text { Utilisation ou non du } \\
\text { CJ dans les } 6 \text { mois } \\
\text { précédant l'entrevue }\end{array}$ & $\begin{array}{l}\text { Intensité : } \\
\text { nombre moyen de } \\
\text { jours utilisés par } \\
\text { semaine pendant } 6 \\
\text { mois. } \\
\text { Durée : } \\
\text { Congé pendant les } 6 \\
\text { mois de l'étude, } \\
\text { excluant décès. }\end{array}$ & $\begin{array}{l}\text { Contact: } \\
\text { Utilisation ou non du } \\
\text { CJ dans les } 12 \\
\text { derniers mois } \\
\text { Intensité : } \\
\text { Pour les utilisateurs, } \\
\text { nombre de jours } \\
\text { d'utilisation en } 12 \\
\text { mois }\end{array}$ & $\begin{array}{l}\text { Intensité : } \\
\text { Forts utilisateurs : au } \\
\text { moins } 13 \\
\text { participations en } 13 \\
\text { semaines; faibles } \\
\text { utilisateurs : moins de } \\
13 \text { participations en } \\
13 \text { semaines. }\end{array}$ & $\begin{array}{l}\text { Durée : } \\
\text { 1) nombre de mois } \\
\text { d'utilisation entre } \\
\text { l'inscription et la fin de } \\
\text { l'étude (max. } 15 \text { sem.). } \\
\text { 2) Maintien de } \\
\text { l'utilisation après } 4 \\
\text { semaines versus congé } \\
\text { après } 4 \text { semaines. }\end{array}$ \\
\hline
\end{tabular}


Tableau IIIb : études des déterminants de l'utilisation des centres de jour pour une clientèle atteinte de démence

\begin{tabular}{|c|c|c|c|c|c|c|c|}
\hline Auteurs & Wells et coll. & Gottlieb et Johnson & Beiseker et coll. & Zarit et coll. & Douglass et Visconti & $\begin{array}{l}\text { Montoro-Rodriguez } \\
\text { et coll. }\end{array}$ & Mävall et Thorslund \\
\hline Année & 1990 & $\begin{array}{l}\text { 2000, cite étude de } \\
1995\end{array}$ & 1996 & 1999 & 1998 & 2003 & 2006 \\
\hline Pays & Australie & Canada & États-Unis & États-Unis & États-Unis & États-Unis & Suède \\
\hline Clientèle & $\begin{array}{l}\text { Clientèle atteinte de } \\
\text { démence }\end{array}$ & $\begin{array}{l}\text { Clientèle atteinte de } \\
\text { démence }\end{array}$ & $\begin{array}{l}\text { Clientèle atteinte de } \\
\text { démence de type } \\
\text { Alzheimer }\end{array}$ & $\begin{array}{l}\text { Clientèle atteinte de } \\
\text { démence }\end{array}$ & $\begin{array}{l}\text { Clientèle atteinte de } \\
\text { démence de type } \\
\text { Alzheimer }\end{array}$ & $\begin{array}{l}\text { Clientèle atteinte de } \\
\text { démence }\end{array}$ & $\begin{array}{l}\text { Clientèle atteinte de } \\
\text { démence }\end{array}$ \\
\hline $\begin{array}{l}\text { Type d'étude } \\
\text { (étude } \\
\text { principale) }\end{array}$ & $\begin{array}{l}\text { Étude quasi } \\
\text { expérimentale }\end{array}$ & $\begin{array}{l}\text { Étude prospective de } \\
16 \text { programmes de } \\
\text { jour pour personnes } \\
\text { atteintes de démence }\end{array}$ & $\begin{array}{l}\text { Analyse descriptive, } \\
\text { avec sujets } \\
\text { volontaires }\end{array}$ & $\begin{array}{l}\text { Étude quasi } \\
\text { expérimentale avec } \\
\text { groupe témoin }\end{array}$ & $\begin{array}{l}\text { Corrélationnelle: } \\
\text { Analyse rétrospective } \\
\text { des dossiers du } \\
\text { ADDTC }\end{array}$ & Étude corrélationnelle & $\begin{array}{l}\text { Étude prospective de } \\
\text { l'état de santé PS }\end{array}$ \\
\hline $\begin{array}{l}\text { But principal } \\
\text { de l'étude }\end{array}$ & $\begin{array}{l}\text { Mesurer les effets du } \\
\text { CJ sur divers } \\
\text { indicateurs de santé } \\
\text { des PS. }\end{array}$ & \begin{tabular}{|l|} 
Vérifier l'effet du \\
centre de jour sur \\
divers indicateurs de \\
santé de la PS
\end{tabular} & \multirow{2}{*}{$\begin{array}{l}\text { Connaître les } \\
\text { bénéfices perçus de } \\
\text { l'utilisation des CJ et } \\
\text { les barrières à leur } \\
\text { utilisation. } \\
\text { Identifier certaines } \\
\text { caractéristiques qui } \\
\text { distinguent les } \\
\text { utilisateurs des non- } \\
\text { utilisateurs. }\end{array}$} & $\begin{array}{l}\text { Mesurer les effets du } \\
\text { CJ sur divers } \\
\text { indicateurs de santé } \\
\text { des PS. }\end{array}$ & \multirow{2}{*}{$\begin{array}{l}\text { Déterminer les } \\
\text { facteurs qui } \\
\text { influencent l'utilisation } \\
\text { du centre de jour, } \\
\text { parmi la clientèle } \\
\text { suivie par l'Alzheimer } \\
\text { Disease Diagnostic } \\
\text { and Treatment Center } \\
\text { Program (ADDTC). }\end{array}$} & \multirow[t]{2}{*}{$\begin{array}{l}\text { Identifier les } \\
\text { déterminants de } \\
\text { l'intensité d'utilisation } \\
\text { du CJ. }\end{array}$} & $\begin{array}{l}\text { Comparer les } \\
\text { indicateurs de stress et } \\
\text { de santé des PS qui } \\
\text { utilisent le CJ, selon le } \\
\text { statut de cohabitation } \\
\text { avec PA. }\end{array}$ \\
\hline $\begin{array}{l}\text { But de } \\
\text { l'étude de } \\
\text { l'utilisation } \\
\text { (si différent } \\
\text { du but } \\
\text { principal) }\end{array}$ & $\begin{array}{l}\text { Comparer certains } \\
\text { indicateurs des PS qui } \\
\text { ont cessé l'utilisation } \\
\text { du CJ à l'intérieur de } 3 \\
\text { mois à ceux qui } \\
\text { l'utilisent encore à } 3 \\
\text { mois. }\end{array}$ & $\begin{array}{l}\text { Identifier certaines } \\
\text { caractéristiques des } \\
\text { PS reliées à l'intensité } \\
\text { de l'utilisation }\end{array}$ & & $\begin{array}{l}\text { Pour le groupe } \\
\text { recevant l'intervention, } \\
\text { comparer les } \\
\text { caractéristiques des } \\
\text { brefs utilisateurs à } \\
\text { celles des utilisateurs } \\
\text { de plus longue durée. }\end{array}$ & & & $\begin{array}{l}\text { Comparer certains } \\
\text { indicateurs des PS qui } \\
\text { ont cessé l'utilisation } \\
\text { du CJ à l'intérieur de } 4 \\
\text { mois à ceux qui } \\
\text { l'utilisent encore à } 4 \\
\text { mois. }\end{array}$ \\
\hline $\begin{array}{l}\text { Sujets pour } \\
\text { l'étude de } \\
\text { l'utilisation }\end{array}$ & $52 \mathrm{PS}$ & non précisé & 104 PS & $245 \mathrm{PS}$ & $737 \mathrm{PA}$ & 1158 PS & $51 \mathrm{PS}$ \\
\hline $\begin{array}{l}\text { Phénomène } \\
\text { d'utilisation } \\
\text { et définition } \\
\text { de la variable } \\
\text { d'utilisation }\end{array}$ & $\begin{array}{l}\text { Durée : } \\
\text { Maintien de l'utilisation } \\
\text { versus congé à } \\
\text { l'intérieur de } 3 \text { mois. }\end{array}$ & $\begin{array}{l}\text { Intensité : } \\
\text { Nombre de jours } \\
\text { d'utilisation pendant } \\
15 \text { semaines. }\end{array}$ & $\begin{array}{l}\text { Contact : } \\
\text { Utilisent ou ont déjà } \\
\text { utilisé CJ versus ceux } \\
\text { qui ne l'ont jamais } \\
\text { utilisé. }\end{array}$ & $\begin{array}{l}\text { Durée : } \\
\text { Maintien de } \\
\text { l'utilisation pendant } \\
\text { un an ou plus versus } \\
\text { congé à l'intérieur des } \\
3 \text { mois qui suivent } \\
\text { l'inscription. } \\
\end{array}$ & $\begin{array}{l}\text { Contact: } \\
\text { Utilise le CJ dans les } \\
3 \text { mois précédant } \\
\text { l'entrevue de suivi qui } \\
\text { a lieu un an après } \\
\text { l'admission au } \\
\text { ADDTC. } \\
\end{array}$ & $\begin{array}{l}\text { Intensité : } \\
\text { Nombre de jours de } \\
\text { services utilisés dans } \\
\text { le dernier mois. }\end{array}$ & $\begin{array}{l}\text { Durée : } \\
\text { Maintien de l'utilisation } \\
\text { versus congé à } \\
\text { l'intérieur de } 4 \text { mois. }\end{array}$ \\
\hline
\end{tabular}

Note :

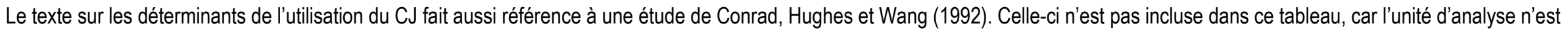

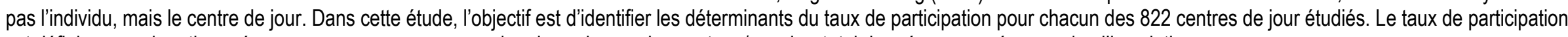
est défini comme le ratio : présences en moyenne par semaine dans chacun des centres / nombre total de présences prévues selon l'inscription. 
Tableau IIIc : études des déterminants de l'utilisation de services de répit et autres services connexes

\begin{tabular}{|c|c|c|c|c|c|}
\hline Auteurs & Casserta et coll. & Montgomery et coll. & Lawton et coll. & Biegel et coll. & Kosloski et Montgomery. \\
\hline Année & 1987 & 1988-1989 & 1989,1991 & 1993 & 1993 \\
\hline Pays & États-Unis & États-Unis & États-Unis & États-Unis & États-Unis \\
\hline Clientèle & $\begin{array}{l}\text { Clientèle atteinte de } \\
\text { démence }\end{array}$ & Personnes âgées & $\begin{array}{l}\text { Clientèle atteinte de } \\
\text { démence de type Alzheimer }\end{array}$ & $\begin{array}{l}\text { Clientèle atteinte de démence } \\
\text { de type Alzheimer }\end{array}$ & $\begin{array}{l}\text { Clientèle atteinte de } \\
\text { démence }\end{array}$ \\
\hline Services & Services de répit 1 & $\begin{array}{l}15 \text { services de santé ou de } \\
\text { soutien }\end{array}$ & Services de répit 2 & $\begin{array}{l}\text { Services de soutien à domicile } \\
\text { et hors domicile }{ }^{3}\end{array}$ & Services de répit 4 \\
\hline $\begin{array}{l}\text { Type d'étude } \\
\text { (étude principale) }\end{array}$ & $\begin{array}{l}\text { Corrélationnelle: } \\
\text { Caractéristiques et utilisation } \\
\text { à T1. }\end{array}$ & $\begin{array}{l}\text { Corrélationnelle : Groupe } \\
\text { formé à T1, utilisation } \\
\text { mesurée à T1 et un an plus } \\
\text { tard. }\end{array}$ & Essai randomisé & $\begin{array}{l}\text { Corrélationnelle : } \\
\text { Caractéristiques à T1, } \\
\text { utilisation pendant les } 6 \text { mois } \\
\text { précédents }\end{array}$ & $\begin{array}{l}\text { Corrélationnelle: } \\
\text { Caractéristiques à T1et } \\
\text { utilisation dans les } 6 \text { mois } \\
\text { précédents. }\end{array}$ \\
\hline $\begin{array}{l}\text { But principal de } \\
\text { l'étude }\end{array}$ & \multirow{2}{*}{$\begin{array}{l}\text { Connaître la proportion de } \\
\text { PS qui connaissent et } \\
\text { utilisent les services de répit } \\
\text { disponibles dans leur région, } \\
\text { ainsi que les déterminants de } \\
\text { l'utilisation de ces services. }\end{array}$} & \multirow{2}{*}{$\begin{array}{l}\text { Vérifier si l'expérience des } \\
\text { PS de personnes atteintes de } \\
\text { troubles cognitifs diffère de } \\
\text { celle des PS de personnes } \\
\text { non atteintes, en particulier } \\
\text { quant à l'utilisation des } \\
\text { services de soutien formels. }\end{array}$} & $\begin{array}{l}\text { Déterminer les effets de } \\
\text { services de répit sur divers } \\
\text { indicateurs de santé des PS. }\end{array}$ & $\begin{array}{l}\text { Identifier les déterminants de } \\
\text { l'utilisation de divers types de } \\
\text { services de soutien }\end{array}$ & \multirow{2}{*}{$\begin{array}{l}\text { Identifier les déterminants de } \\
\text { l'intensité d'utilisation des } \\
\text { services de répit, } \\
\text { en particulier déterminer } \\
\text { l'influence des attitudes } \\
\text { envers les services offerts } \\
\text { sur l'utilisation. }\end{array}$} \\
\hline $\begin{array}{l}\text { But de l'étude de } \\
\text { l'utilisation (si } \\
\text { différent du but } \\
\text { principal) }\end{array}$ & & & $\begin{array}{l}\text { À l'intérieur du groupe } \\
\text { expérimental, comparer les } \\
\text { caractéristiques des } \\
\text { différents utilisateurs. }\end{array}$ & & \\
\hline $\begin{array}{l}\text { Sujets pour l'étude } \\
\text { de l'utilisation }\end{array}$ & 597 PS & $\begin{array}{l}351 \text { dyades } \\
\text { PA-PS } \\
\end{array}$ & $\begin{array}{l}177 \text { dyades } \\
\text { PA-PS } \\
\end{array}$ & 171 PS & 114 PS \\
\hline $\begin{array}{l}\text { Phénomène } \\
\text { d'utilisation et } \\
\text { définition de la } \\
\text { d'utilisation }\end{array}$ & $\begin{array}{l}\text { Contact : } \\
\text { Utilisation de services, versus } \\
\text { PS qui ne sentent pas encore } \\
\text { le besoin de services mais } \\
\text { pensent utiliser plus tard ou } \\
\text { non-utilisateurs pour d'autres } \\
\text { raisons. }\end{array}$ & $\begin{array}{l}\text { Intensité : } \\
\text { Nombre de jours d'utilisation } \\
\text { de } 15 \text { services différents } \\
\text { pendant un mois. }\end{array}$ & $\begin{array}{l}\text { Intensité : } \\
\text { Nombre d'heures d'utilisation } \\
\text { de chacun des services } \\
\text { pendant une période d'un } \\
\text { an : répit à domicile, en } \\
\text { centre d'hébergement, en } \\
\text { centre de jour. }\end{array}$ & $\begin{array}{l}\text { Contact : } \\
4 \text { groupes d'utilisateurs: } \\
\text { Serv. à domicile seulement } \\
\text { Serv. hors domicile seulement } \\
\text { Serv. à domicile et hors } \\
\text { domicile } \\
\text { Aucun service }\end{array}$ & $\begin{array}{l}\text { Intensité : } \\
\text { Quantité de répit utilisé dans } \\
\text { les } 6 \text { mois précédant l'étude. }\end{array}$ \\
\hline
\end{tabular}

Notes:

1. Inclut le répit à domicile, le centre de jour ou des soins à domicile, parce que pour les répondants, les définitions de ces services étaient interchangeables.

2. Inclut le répit à domicile, l'hébergement temporaire et le répit en centre de jour fournis par une des agences à l'étude (les utilisateurs et les non-utilisateurs pouvaient recevoir du répit d'autres sources).

3. Les services hors domicile incluent des services de transport, de centre de jour, de repas de groupe pour personnes âgées.

4. Inclut le répit à domicile et le répit en centre de jour fournis par une des agences à l'étude (les utilisateurs et les non-utilisateurs pouvaient aussi recevoir du répit d'autres sources). 
Tableau IIIc : études des déterminants de l'utilisation de services de répit et autres services connexes (suite)

\begin{tabular}{|c|c|c|c|c|c|c|}
\hline Auteurs & Cox & Cotrell \& Engel & Pedlar et coll. & Kosloski et coll. & Kosloski et coll. & Li \\
\hline Année & 1997 & 1998 & 1999 & 1999 & 2001 & 2004 \\
\hline Pays & États-Unis & États-Unis & États-Unis & États-Unis & États-Unis & États-Unis \\
\hline Clientèle & $\begin{array}{l}\text { Clientèle atteinte de } \\
\text { démence }\end{array}$ & $\begin{array}{l}\text { Clientèle atteinte de } \\
\text { démence }\end{array}$ & $\begin{array}{l}\text { Hommes âgés atteints de } \\
\text { démence et leurs } \\
\text { épouses }\end{array}$ & $\begin{array}{l}\text { Clientèle atteinte de } \\
\text { démence }\end{array}$ & $\begin{array}{l}\text { Clientèle atteinte de } \\
\text { démence }\end{array}$ & $\begin{array}{l}\text { PS de personnes âgées } \\
(60 \text { ans }+) \text { avec au moins } \\
\text { une limitation d'activité }\end{array}$ \\
\hline Services & Services de répit 1 & Services de répit 2 & $\begin{array}{l}\text { Divers services de } \\
\text { soutien, dont les services } \\
\text { pour PS (répit, éducation) }\end{array}$ & $\begin{array}{l}\text { Divers services de } \\
\text { soutien }\end{array}$ & Services de répit 3 & $\begin{array}{l}8 \text { types de services de } \\
\text { soutien à domicile }\end{array}$ \\
\hline $\begin{array}{l}\text { Type d'étude } \\
\text { (étude principale) }\end{array}$ & $\begin{array}{l}\text { Corrélationnelle : } \\
\text { Caractéristiques à } \\
\text { l'admission au prog., } \\
\text { utilisation } 6 \text { mois plus tard }\end{array}$ & $\begin{array}{l}\text { Corrélationnelle : } \\
\text { Caractéristiques et } \\
\text { utilisation à T1 }\end{array}$ & $\begin{array}{l}\text { Corrélationnelle : } \\
\text { Caractéristiques à T1, } \\
\text { utilisation dans les } 4 \\
\text { semaines précédentes }\end{array}$ & $\begin{array}{l}\text { Corrélationnelle : } \\
\text { Caractéristiques et } \\
\text { utilisation à T1 }\end{array}$ & $\begin{array}{l}\text { Corrélationnelle : } \\
\text { Caractéristiques et } \\
\text { utilisation à T1 }\end{array}$ & $\begin{array}{l}\text { Corrélationnelle: } \\
\text { Caractéristiques et } \\
\text { utilisation à T1. }\end{array}$ \\
\hline $\begin{array}{l}\text { But principal de } \\
\text { l'étude }\end{array}$ & $\begin{array}{l}\text { Comparer les } \\
\text { caractéristiques des } \\
\text { utilisateurs de répit, } 6 \\
\text { mois après l'inscription, à } \\
\text { celles des non-utilisateurs } \\
\text { et de ceux qui ont cessé } \\
\text { l'utilisation avant } 6 \text { mois }\end{array}$ & $\begin{array}{l}\text { Vérifier si les membres } \\
\text { du réseau informel et les } \\
\text { professionnels de la } \\
\text { santé agissent comme } \\
\text { médiateurs pour faciliter } \\
\text { l'utilisation de } 3 \text { types de } \\
\text { services de répit. }\end{array}$ & $\begin{array}{l}\text { Identifier les } \\
\text { déterminants de } \\
\text { l'utilisation des services } \\
\text { pour PS, en particulier } \\
\text { déterminer l'influence des } \\
\text { attitudes envers l'aide } \\
\text { formelle sur l'utilisation. }\end{array}$ & $\begin{array}{l}\text { Vérifier s'il y a interaction } \\
\text { entre l'ethnicité et les } \\
\text { facteurs de besoin dans } \\
\text { les déterminants de } \\
\text { l'utilisation des services }\end{array}$ & $\begin{array}{l}\text { Identifier les } \\
\text { déterminants de } \\
\text { l'utilisation de répit. Pour } \\
\text { les non-utilisateurs, } \\
\text { vérifier les différences } \\
\text { entre ceux qui ont pris } \\
\text { des informations sur les } \\
\text { services et les autres. }\end{array}$ & $\begin{array}{l}\text { Identifier les } \\
\text { déterminants de } \\
\text { l'utilisation des services } \\
\text { d'aide, en particulier } \\
\text { déterminer l'influence des } \\
\text { réseaux sociaux sur } \\
\text { l'utilisation. }\end{array}$ \\
\hline $\begin{array}{l}\text { Sujets pour l'étude } \\
\text { de l'utilisation }\end{array}$ & $228 \mathrm{PS}$ & 100 PS & 82 PS (épouses) & 2947 PS & $458 \mathrm{PS}$ & 292 PS (épouses et filles) \\
\hline $\begin{array}{l}\text { Phénomène } \\
\text { d'utilisation et } \\
\text { définition de la } \\
\text { d'utilisation }\end{array}$ & $\begin{array}{l}\text { Contact et durée : } \\
\text { Utilisateurs de services de } \\
\text { répit } 6 \text { mois après } \\
\text { l'inscription, utilisateurs } \\
\text { ayant cessé l'utilisation et } \\
\text { non-utilisateurs. }\end{array}$ & $\begin{array}{l}\text { Contact : } \\
\text { Utilisent ou non : } \\
\text { - centre de jour } \\
\text { - répit à domicile } \\
\text { - hébergement } \\
\text { temporaire }\end{array}$ & $\begin{array}{l}\text { Contact : } \\
\text { Utilisent ou non : } \\
\text { - aide ménagère } \\
\text { - service pour PS } \\
\text { - soins personnels } \\
\text { - transport }\end{array}$ & $\begin{array}{l}\text { Contact : } \\
\text { Utilisent ou non : } \\
\text { - centre de jour } \\
\text { - répit à domicile, } \\
\text { - repas } \\
\text { - soins santé à domicile }\end{array}$ & $\begin{array}{l}\text { Contact: } \\
\text { Utilisation ou non des } \\
\text { services de répit offerts } \\
\text { par les agences étudiées. }\end{array}$ & $\begin{array}{l}\text { Contact: } \\
\text { Utilisation ou non d'au } \\
\text { moins un des services au } \\
\text { moment de l'entrevue. }\end{array}$ \\
\hline
\end{tabular}

Notes :

1. II semble que cela inclut uniquement le répit à domicile, l'hébergement temporaire et le répit en centre de jour fournis par une des agences du programme.

2. Inclut le répit à domicile, l'hébergement temporaire et le répit en centre de jour, obtenu de toute source.

3. Inclut le répit à domicile et le répit en centre de jour fournis par une des agences à l'étude (les utilisateurs et les non-utilisateurs pouvaient recevoir du répit d'autres sources). 
Tableau IIId : études descriptives sur les raisons qui motivent ou limitent l'utilisation de ces services

\begin{tabular}{|c|c|c|c|c|c|c|}
\hline Auteurs & Tourigny et coll., & Monahan & $\begin{array}{l}\text { Cohen-Mansfield et } \\
\text { coll. }\end{array}$ & Cotrell & Strain et Blandford & Brodaty \\
\hline Année & 1993 & 1993 & 1994 & 1996 & 2002 & 2005 \\
\hline Pays & Canada & États-Unis & États-Unis & États-Unis & Canada & Australie \\
\hline Clientèle & Personnes âgées & $\begin{array}{l}\text { Clientèle atteinte de } \\
\text { démence }\end{array}$ & Personnes âgées & $\begin{array}{l}\text { Clientèle atteinte de } \\
\text { démence }\end{array}$ & Personnes âgées & $\begin{array}{l}\text { Clientèle atteinte de } \\
\text { démence ou de pertes } \\
\text { de mémoire }\end{array}$ \\
\hline Services & Centre de jour & $\begin{array}{l}\text { Service de répit en } \\
\text { centre de jour }\end{array}$ & Centre de jour & Services de répit 1 & $\begin{array}{l}\text { Divers services analysés } \\
\text { séparément, dont le } \\
\text { centre de jour }\end{array}$ & $\begin{array}{l}7 \text { types de services de } \\
\text { soutien à domicile, dont } \\
\text { le répit }\end{array}$ \\
\hline $\begin{array}{l}\text { But principal de } \\
\text { l'étude }\end{array}$ & $\begin{array}{l}\text { Évaluer le programme } \\
\text { québécois de centre de } \\
\text { jour quant aux: } \\
\text { Similitudes et différences } \\
\text { entre les divers centres } \\
\text { Forces et faiblesses du } \\
\text { fonctionnement } \\
\text { Conditions favorables à la } \\
\text { poursuite efficace du } \\
\text { programme }\end{array}$ & $\begin{array}{l}\text { Décrire les } \\
\text { caractéristiques des } \\
\text { participants (PA et PS) } \\
\text { et les raisons pour } \\
\text { lesquelles les PS } \\
\text { souhaitent la } \\
\text { participation de la PA au } \\
\text { CJ. }\end{array}$ & $\begin{array}{l}\text { Connaitre les sources } \\
\text { d'information sur le CJ } \\
\text { et les raisons invoquées } \\
\text { pour ne pas utiliser le } \\
\text { CJ. }\end{array}$ & $\begin{array}{l}\text { Raisons invoquées pour } \\
\text { ne pas recourir à des } \\
\text { services de répit, ou } \\
\text { encore pour débuter } \\
\text { et/ou cesser d'utiliser de } \\
\text { ces services. }\end{array}$ & $\begin{array}{l}\text { Raisons invoquées pour } \\
\text { ne pas recourir aux } \\
\text { services et } \\
\text { caractéristiques des } \\
\text { répondants associées à } \\
\text { divers types de raisons. }\end{array}$ & $\begin{array}{l}\text { Développer une } \\
\text { typologie des personnes } \\
\text { de soutien qui n'utilisent } \\
\text { pas les services. }\end{array}$ \\
\hline Sujets & $\begin{array}{l}\text { Échantillon orienté de } 22 \\
\text { centres de jour. }\end{array}$ & $\begin{array}{l}42 \text { PA (tous les } \\
\text { participants au } \\
\text { programme) et } 62 \% \text { de } \\
\text { leurs PS. }\end{array}$ & $\begin{array}{l}\text { 111 PS qui avaient pris } \\
\text { des informations auprès } \\
\text { de } 4 \text { CJ et n'ont pas } \\
\text { utilisé. }\end{array}$ & 100 PS volontaires & $\begin{array}{l}293 \text { dyades PA-PS de } \\
\text { l'étude manitobaine sur } \\
\text { la santé et le } \\
\text { vieillissement. }\end{array}$ & $\begin{array}{l}109 \text { PS dont la PA a } \\
\text { une démence, tiré d'un } \\
\text { échantillon } \\
\text { populationnel de PS } \\
\text { prennent soin de PA } \\
\text { ayant des problèmes } \\
\text { variés. }\end{array}$ \\
\hline
\end{tabular}

Notes:

1. Inclut le répit à domicile, l'hébergement temporaire et le répit en centre de jour. 
Tableau IIIe : études des déterminants de l'utilisation des services à domicile

\begin{tabular}{|c|c|c|c|c|c|c|c|}
\hline Auteurs & Grene & Bass \& Noelker & Tennstedt et coll. & Miller \& McFall & Bass et coll. & Crets & $\begin{array}{l}\text { Dabelko et } \\
\text { Balaswamy }\end{array}$ \\
\hline Année & 1983 & 1987 & 1989 & 1991 & 1992 & 1996 & 2000 \\
\hline Pays & États-Unis & États-Unis & États-Unis & États-Unis & États-Unis & Belgique & États-Unis \\
\hline Clientèle & $\begin{array}{l}\text { PA (60 ans ou +) } \\
\text { inscrites au service } \\
\text { de gestion de cas } \\
\text { (CSS) de la ville de } \\
\text { Tuscon en Arizona. }\end{array}$ & $\begin{array}{l}\text { PA (60 ans ou +) de la } \\
\text { région de Cleaveland, } \\
\text { qui nécessitent de } \\
\text { l'aide pour au moins un } \\
\text { soin personnel et } \\
\text { cohabitent avec sa PS. }\end{array}$ & $\begin{array}{l}\text { PA (70 ans ou +) du } \\
\text { Massachusetts, } \\
\text { identifiées comme } \\
\text { fragiles selon le HRCA } \\
\text { Vulnerability Index. }\end{array}$ & $\begin{array}{l}\text { Personnes inscrites à } \\
\text { Medicare qui ont au } \\
\text { moins une limitation } \\
\text { dans une activité de vie } \\
\text { quotidienne et qui ont } \\
\text { identifié une PS. }\end{array}$ & $\begin{array}{l}\text { PA admises dans un } \\
\text { service de soins à } \\
\text { domicile, qui ont utilisé } \\
\text { les services pendant au } \\
\text { moins } 30 \text { jours et ont } \\
\text { identifié une PS. }\end{array}$ & $\begin{array}{l}\text { PA (75 ans et }+ \text { ) qui } \\
\text { vivent à domicile, } \\
\text { dans la ville de } \\
\text { Antwerp. }\end{array}$ & $\begin{array}{l}\text { Personnes âgées de } \\
70 \text { ans et + }\end{array}$ \\
\hline $\begin{array}{l}\text { Type d'étude } \\
\text { (étude } \\
\text { principale) }\end{array}$ & $\begin{array}{l}\text { Corrélationnelle : } \\
\text { caractéristiques des } \\
\text { PA à T1 et utilisation } \\
\text { pendant les mois } \\
\text { précédents. }\end{array}$ & $\begin{array}{l}\text { Corrélationnelle: } \\
\text { caractéristiques des PA } \\
\text { et des PS à T1, } \\
\text { utilisation pendant les } 6 \\
\text { mois précédents. }\end{array}$ & $\begin{array}{l}\text { Corrélationnelle : Type } \\
\text { de PS à T1 et } \\
\text { utilisation au même } \\
\text { moment. }\end{array}$ & $\begin{array}{l}\text { Étude longitudinale: } \\
\text { Caractéristiques à T1 } \\
\text { et à T2 (deux ans plus } \\
\text { tard), utilisation dans la } \\
\text { semaine précédente. }\end{array}$ & $\begin{array}{l}\text { Corrélationnelle : } \\
\text { caractéristiques des PA } \\
\text { et des PS à l'inscription, } \\
\text { utilisation dans les } 2 \\
\text { mois suivants. }\end{array}$ & $\begin{array}{l}\text { Corrélationnelle: } \\
\text { caractéristiques des } \\
\text { PA à T1 et utilisation } \\
\text { au même moment. }\end{array}$ & $\begin{array}{l}\text { Corrélationnelle: } \\
\text { caractéristiques des } \\
\text { PA à T1 et utilisation } \\
\text { dans les } 12 \text { mois } \\
\text { précédant l'étude. }\end{array}$ \\
\hline $\begin{array}{l}\text { But principal } \\
\text { de l'étude }\end{array}$ & \multirow{2}{*}{$\begin{array}{l}\text { Étudier la relation } \\
\text { entre le soutien fourni } \\
\text { par le CSS et le } \\
\text { soutien fourni par la } \\
\text { famille et les amis, } \\
\text { ainsi que les } \\
\text { déterminants des } 2 \\
\text { types de soutien. }\end{array}$} & $\begin{array}{l}\text { Évaluer comment les } \\
\text { PS qui résident avec la } \\
\text { PA sont affectées par } \\
\text { cette situation. }\end{array}$ & $\begin{array}{l}\text { Évaluer l'importance et } \\
\text { le rôle des PS } \\
\text { secondaire dans les } \\
\text { soins aux PA. }\end{array}$ & \multirow{2}{*}{$\begin{array}{l}\text { Évaluer les } \\
\text { changements dans } \\
\text { l'utilisation des services } \\
\text { à domicile, identifier les } \\
\text { déterminants de } \\
\text { l'utilisation à T2. }\end{array}$} & \multirow{2}{*}{$\begin{array}{l}\text { Identifier les } \\
\text { déterminants de } \\
\text { l'utilisation des services } \\
\text { à domicile } \\
\text { Données proviennent } \\
\text { de l'entrevue initiale } \\
\text { avec PA et PS. }\end{array}$} & \multirow[t]{2}{*}{$\begin{array}{l}\text { Identifier les } \\
\text { déterminants de } \\
\text { l'utilisation des } \\
\text { services à domicile. }\end{array}$} & \multirow{2}{*}{$\begin{array}{l}\text { Comparer les } \\
\text { utilisateurs de } \\
\text { services à domicile } \\
\text { aux utilisateurs de } \\
\text { centre de jour }\end{array}$} \\
\hline $\begin{array}{l}\text { But: étude de } \\
\text { l'utilisation } \\
\text { (si différent } \\
\text { du but } \\
\text { principal) }\end{array}$ & & $\begin{array}{l}\text { Identifier les } \\
\text { déterminants de } \\
\text { l'utilisation des services } \\
\text { à domicile. }\end{array}$ & $\begin{array}{l}\text { Déterminer si la } \\
\text { présence d'une PS } \\
\text { secondaire influence } \\
\text { l'utilisation des services } \\
\text { formels. }\end{array}$ & & & & \\
\hline Sujets & $124 \mathrm{PA}$ & $\begin{array}{l}\text { Données proviennent } \\
\text { de } 586 \text { PS, concernant } \\
\text { services utilisés pour la } \\
\text { PA. }\end{array}$ & 635 PA et 429 PS & $\begin{array}{l}604 \text { PA (parmi les } 904 \\
\text { initiales) toujours à } \\
\text { domicile à T2. }\end{array}$ & $\begin{array}{l}97 \text { PA qui reçoivent des } \\
\text { services de santé et } \\
246 \text { PA qui reçoivent } \\
\text { services de nature } \\
\text { sociale ou aide pour les } \\
\text { soins personnels. } \\
\end{array}$ & 393 PA & $\begin{array}{l}62 \text { utilisateurs de CJ } \\
\text { et } 91 \text { utilisateurs de } \\
\text { soins de santé à } \\
\text { domicile }\end{array}$ \\
\hline $\begin{array}{l}\text { Phénomène } \\
\text { d'utilisation } \\
\text { et définition } \\
\text { de la variable } \\
\text { d'utilisation }\end{array}$ & $\begin{array}{l}\text { Contact: } \\
\text { Soutien reçu ou non } \\
\text { pour } 12 \text { types de } \\
\text { soins personnels ou } \\
\text { de tâches } \\
\text { domestiques. }\end{array}$ & $\begin{array}{l}\text { Contact et intensité: } \\
\text { Avoir reçu ou non des } \\
\text { services à domicile. } \\
\text { Nombre d'heures de } \\
\text { services à domicile } \\
\text { pour } 14 \text { tâches. }\end{array}$ & $\begin{array}{l}\text { Intensité: } \\
\text { Nombre d'heures par } \\
\text { semaine d'aide } \\
\text { formelle. }\end{array}$ & $\begin{array}{l}\text { Contact: } \\
\text { Utilisation ou non } \\
\text { d'aide formelle pour } \\
\text { certaines activités de } \\
\text { soins personnels ou } \\
\text { tâches domestiques. }\end{array}$ & $\begin{array}{l}\text { Intensité: } \\
\text { Nombre d'heures de } \\
\text { services à domicile } \\
\text { utilisées. }\end{array}$ & $\begin{array}{l}\text { Contact: } \\
\text { Utilisation ou non de } \\
3 \text { types d'aide } \\
\text { (informelle, formelle, } \\
\text { commerciale). }\end{array}$ & $\begin{array}{l}\text { Contact : } \\
\text { Utilisation ou non de : } \\
\text { - centre de jour } \\
\text { - soins de santé à } \\
\text { domicile. }\end{array}$ \\
\hline
\end{tabular}




\section{Facteurs prédisposants}

Ces facteurs incluent les caractéristiques démographiques comme l'âge et le sexe, qui représentent des facteurs biologiques influençant la probabilité d'avoir besoin des services de santé, ainsi que des mesures de la structure sociale comme le niveau d'éducation, la profession, l'appartenance culturelle, qui donnent une indication de la probabilité de vivre dans un environnement sain et qui influencent la capacité de faire face aux problèmes qui surviennent. Ils incluent aussi les croyances sur la santé et la perception de l'utilité des services (Andersen, 1995).

\section{Personne âgée}

L'influence de l'âge est variable. Pour la clientèle générale, l'âge semble négativement corrélé à l'utilisation du centre de jour (contact: Dabelko et Balaswamy, 2000; intensité : Henry et Capitman, 1995; durée: Dabelko, 2004), sauf dans l'étude de Baumgarten et collaborateurs (2002) où l'on rapporte une association positive entre l'intensité de l'utilisation du centre de jour et l'âge. Pour la clientèle atteinte de démence, l'âge semble corrélé positivement à l'utilisation des services de répit (contact : Casserta et coll., 1987; intensité : Lawton et coll., 1991), mais les études qui s'intéressaient spécifiquement au centre de jour n'ont trouvé aucune relation entre ces deux variables (Douglass et Visconti, 1998; Zarit et coll., 1999b).

Les différences d'utilisation selon le sexe sont aussi variables. Les hommes semblent participer plus intensivement au centre de jour dans deux études auprès d'une clientèle générale (Wallace, 1987; Baumgarten et coll., 2002), alors que les femmes démontrent une participation de plus longue durée dans l'étude de Zarit et collaborateurs (1999b) auprès d'une clientèle atteinte de démence. Au moins trois études n'ont trouvé aucune association significative entre le sexe du participant et l'utilisation du centre de jour (Chappell, 1983; Dabelko, 2004; Douglass et Visconti, 1998)

Le niveau d'éducation semble associé négativement à l'utilisation du centre de jour (contact: Chappell, 1983; durée: Dabelko, 2005). Deux autres études n'ont toutefois 
démontré aucune association significative (Baumgarten et coll., 2002; Douglass et Visconti, 1998).

La profession peut être vue comme une mesure de la structure sociale (Andersen, 1995) qui peut influencer le besoin de services, de même que la connaissance des services disponibles et leur utilisation. Deux études du contact avec le centre de jour ont étudié cette variable. Selon Chappell (1983), les utilisateurs de centre de jour sont moins nombreux à avoir œuvré dans des postes professionnels ou de gestion que l'ensemble des utilisateurs de services à domicile, alors que dans l'étude de Douglass et Visconti (1998), aucune association significative n'a été démontrée.

Le réseau de soutien peut influencer l'utilisation des services de deux façons. Il peut augmenter la connaissance des services disponibles (Cotrell et Engel, 1998; Miller et McFall, 1991), favoriser le rappel des rendez-vous et stimuler l'utilisation des services. Il est aussi possible que les personnes âgées qui ont un plus grand réseau de soutien informel utilisent moins de services formels, car leurs besoins peuvent être davantage comblés par leur réseau informel. Cette dernière hypothèse (diminution de l'utilisation) ne semble vérifiée que dans le cas de l'utilisation de services à domicile (Bass, Looman et Ehrlich, 1992; Crets, 1996; Greene, 1983). Pour ce qui est de l'utilisation des centres de jour, bien que le fait de vivre seul ne semble pas associé à l'utilisation du centre de jour (Baumgarten et coll., 2002; Douglass et Visconti, 1998; Lynch et coll., 1999; Wallace, 1987), d'autres indicateurs de la présence d'un réseau ont été corrélés à une plus forte intensité d'utilisation du centre de jour, soit : la présence d'une personne de soutien (Baumgarten et coll., 2002), le fait d'avoir des enfants (Wallace, 1987) et d'avoir un conjoint (Wallace, 1987). Ce serait vrai en particulier pour les hommes mariés (Wallace, 1987). Une seule étude démontre une association négative entre la présence d'un conjoint et l'intensité d'utilisation du centre de jour (Lynch et coll., 1999). De plus, Dabelko (2004) observe une durée d'utilisation plus grande pour les personnes mariées. 
Personne de soutien

L'âge de la personne de soutien est associé positivement à l'intensité de la participation au centre de jour dans l'étude de Baumgarten et collaborateurs (2002). Par contre, il est associé négativement à la durée d'utilisation dans deux études (Henry et Capitman, 1995; Zarit et coll., 1999b). Dans l'étude de Lawton et collaborateurs (1991), l'âge de la personne de soutien est associé positivement à l'utilisation du répit en hébergement temporaire, mais pas aux autres formes de répit. Les études dont la variable dépendante était un ensemble indifférencié de services de soutien n'ont pas démontré d'association entre l'âge de la personne de soutien et l'utilisation de ces services (Casserta et coll., 1987; Cotrell et Engel, 1998; Kosloski et coll., 2001; Li, 2004; Pedlar et Biegel, 1999).

Le sexe de la personne de soutien ne semble pas associé à l'utilisation du centre de jour ou des services de répit. Dans l'ensemble des études recensées, seuls Baumgarten et collaborateurs (2002) ont observé une association significative. Dans leur étude, l'intensité de la participation au centre de jour semble plus grande lorsque la personne de soutien est une femme.

L'éducation de la personne de soutien est négativement corrélée à l'intensité d'utilisation du centre de jour dans l'étude d'Henry et Capitman (1995) et positivement à la durée d'utilisation dans l'étude de Zarit et collaborateurs (1999b). Deux autres études n'ont toutefois démontré aucune association significative (Baumgarten et coll., 2002; MontoroRodriguez et coll., 2003).

Le réseau de soutien de la personne de soutien peut aussi influencer l'utilisation des services formels de plusieurs façons. La présence d'un tel réseau peut diminuer le besoin d'aide de la part des services formels. D'ailleurs, dans l'étude de Miller et McFall (1991), la perte d'une personne de soutien secondaire augmente de 1,6 fois les chances d'utiliser des services à domicile. Les personnes de soutien qui n'utilisaient pas les services de répit (Cox, 1997; Kosloski et coll., 2001) et en particulier, celles qui ne les utilisaient pas parce qu'elles n'en sentaient pas encore le besoin (Casserta et coll., 1987) étaient légèrement plus 
satisfaites envers leur réseau de soutien informel (Cox, 1997; Casserta et coll., 1987) ou étaient plus nombreuses à rapporter la présence d'un autre aidant pouvant prendre la relève en cas de besoin (Kosloski et coll., 2001). Cette association négative entre le réseau de soutien de l'aidant et l'utilisation de services formels ne semble pas observée dans le cas du centre de jour.

D'autres chercheurs ont observé que le réseau de soutien peut favoriser la connaissance des services et des attitudes plus positives envers leur utilisation. Au moins deux études rapportent une corrélation positive entre la présence de personnes de soutien secondaires et l'utilisation des services à domicile (Bass et Noelker, 1987; Tennstedt, McKinlay et Sullivan, 1989). L'influence des soutiens secondaires peut différer selon qu'il s'agit d'amis ou de membres de la famille. Les proches aidants qui rapportent recevoir le soutien d'amis plutôt que d'autres membres de la famille pour les soins apportés à la personne âgée utilisaient davantage de services formels ( $\mathrm{Li}, 2004)$, suggérant que les amis aideraient davantage à établir des ponts entre les proches aidants et le réseau formel d'aide. Pour l'utilisation du centre de jour par une clientèle atteinte de troubles cognitifs, l'influence d'un membre du réseau informel ou d'un professionnel sur la personne de soutien semble importante dans la décision d'utiliser ou non le centre de jour et d'autres services de répit (Beiseker et coll., 1996; Cotrell et Engel, 1998). Selon Cotrell et Engel (1998), l'influence d'un membre du réseau informel paraît d'ailleurs plus importante pour l'utilisation du centre de jour que pour les autres formes de répit. Enfin, la satisfaction envers le réseau de soutien informel a aussi été associée à l'utilisation de services de soutien à l'extérieur du domicile, alors que les personnes de soutien qui rapportent une plus faible satisfaction envers leur réseau informel se retrouvent davantage chez les nonutilisateurs et chez les utilisateurs de services à domicile seulement (Biegel, Bass, Schulz et Morycz, 1993).

Soulignons que le nombre de personnes vivant sous le toit de la personne de soutien n'a pas été associé à l'utilisation du centre de jour ou des services de répit dans les études recensées (Dabelko 2004; 2005). Cette variable semble inclure une trop grande variété de situations différentes (par exemple : conjoint ou adolescents en âge d'apporter du soutien, 
ou encore, enfants en bas âge ou autres personnes dépendantes) pour pouvoir servir de proxy pour mesurer un soutien à l'aidant.

\section{Personne âgée et personne de soutien}

L'appartenance culturelle peut influencer les croyances sur la santé et sur l'acceptabilité de solliciter de l'aide pour les soins d'une personne âgée, et en particulier de recourir à des services d'aide formelle pour faciliter le maintien à domicile. Elle peut aussi influencer le niveau socioéconomique et la capacité de payer les services le cas échéant. Peu de recherches ont exploré cette variable sauf pour ce qui est de la différence entre Noirs, Hispaniques et Blancs aux États-Unis. En ce qui concerne l'utilisation du centre de jour, l'intensité d'utilisation du centre de jour serait plus grande chez les personnes d'origine afro-américaine (Henry et Capitman, 1995; Montoro-Rodriguez et coll., 2003) ou hispaniques (Montoro-Rodriguez et coll., 2003). Toutefois, les durées d'utilisation seraient plus courtes pour la clientèle noire (Henry et Capitman, 1995). Cette association n'est pas observée dans l'étude de Zarit et collaborateurs (1999b), où au contraire, les Blancs étaient proportionnellement plus nombreux dans le groupe des brefs utilisateurs. Toutefois, cette association n'est plus significative dans les analyses multivariées qui contrôlaient les variables suivantes : sexe de la personne âgée, lien avec la personne de soutien, éducation de la personne de soutien, nombre d'incapacités et nombre de problèmes de comportement de la personne âgée, fardeau et bien-être psychologique de la personne de soutien. Les résultats des études de Dabelko (2004; 2005) sont contradictoires selon la variable dépendante considérée (durée d'utilisation en mois ou mesure dichotomique de la durée: congé ou non à l'intérieur de 4 semaines de l'inscription). Dans le contexte canadien, Chappell (1983) a observé qu'une moins grande proportion de personnes nées à l'extérieur du Canada utilisait un centre de jour.

Le type de lien entre la personne âgée et sa personne de soutien : Il semble que la préférence pour certains types de services varie selon que la personne de soutien soit le conjoint, un enfant qui cohabite avec la personne âgée, ou une autre personne qui ne cohabite pas avec la personne de soutien (Cotrell et Engel, 1998). Cela pourrait expliquer 
que plusieurs études sur des services de répit non différenciés n'aient pas démontré d'association significative entre cette variable et l'utilisation des services (Biegel et coll., 1993; Cox, 1997; Kosloski et coll., 2001; Lawton et coll., 1991). Certains auteurs rapportent tout de même que les personnes de soutien qui sont le conjoint de la personne âgée utilisent moins de services de répit. Pour les conjoints surtout, la présence de soutiens secondaires semble avoir un rôle important dans l'utilisation du répit (Cotrell et Engel, 1998; Li, 2004). Des conjoints ont rapporté qu'ils n'auraient jamais utilisé les services de répit si leurs enfants ne les avaient pas encouragés à le faire (Cotrell et Engel, 1998). Dans l'étude de Caserta et collaborateurs (1987), les conjoints sont plus nombreux dans le groupe de personnes de soutien qui se disent pas encore prêtes à utiliser les services de répit, mais le feraient si le besoin devenait plus important. Selon Kosloski et Montgomery (1994), les facteurs de besoin contribuaient davantage à prédire l'utilisation du centre de jour lorsque la personne de soutien est le conjoint, ce qui semble cohérent avec l'observation précédente de Casserta et collaborateurs.

L'influence du type de lien sur l'utilisation du centre de jour est aussi variable. Dans une étude après d'une clientèle générale, l'intensité d'utilisation plus élevée est associée au fait que la personne de soutien soit le conjoint (Baumgarten et coll., 2002), alors dans une étude auprès d'une clientèle atteinte de démence, Gottlieb et Johnson (2000) observent le contraire. Par ailleurs, la durée d'utilisation semble plus courte lorsque la personne de soutien est le conjoint, tant dans l'étude d'Henry et Capitman (1995) qui contient $68 \%$ de clients atteints de démence que dans l'étude de Zarit et collaborateurs (1999b) auprès d'une clientèle atteinte de démence. Ceci pourrait s'expliquer par la différence dans le but de la fréquentation du centre de jour pour les divers groupes de clients, le répit étant le but le plus souvent recherché pour la clientèle atteinte de troubles cognitifs. Selon Cotrell (1996), les enfants corésidants indiquent une préférence pour le centre de jour plutôt que d'autres formes de répit, tandis que les conjoints qui ont besoin de répit semblent préférer le répit à domicile. Cette préférence pourrait être associée à une plus faible utilisation des centres de jour par les conjoints de personnes atteintes de troubles cognitifs. Selon Gottlieb et Johnson (2000), les enfants acceptent peut-être mieux l'idée d'un programme de groupe, ou encore, 
ils ont davantage besoin de répit que les conjoints, étant donné les responsabilités familiales et de travail concomitantes à leur rôle d'aidant.

Les croyances et attitude envers l'utilisation des services : Outre les études sur les raisons de l'omission de se présenter discutées précédemment, nous n'avons recensé aucune recherche sur les attitudes envers l'utilisation du centre de jour par les personnes âgées elles-mêmes. Les études des croyances et les attitudes des personnes de soutien envers le rôle d'aidant et envers l'utilisation des services sont plus nombreuses. La confiance envers les services de la communauté pour prendre soin d'un conjoint a été associée positivement à l'utilisation de services destinés aux proches aidants (Pedlar et Biegel, 1999). Selon Kosloski et collaborateurs (2001), les personnes qui ont une plus grande perception que c'est leur devoir d'aider leur conjoint ou leur parent vieillissant sont moins nombreuses à utiliser les services de répit. Celles qui perçoivent une plus grande utilité aux services de répit (Kosloski et Montgomery, 1993) et aux centres de jour (Montoro-Rodriguez et coll., 2003) sont plus nombreuses à utiliser ces services. L'attitude envers l'utilisation du centre de jour et de l'aide formelle peut être influencée positivement par la participation à un groupe d'éducation pour les personnes de soutien (Robinson et Yates, 1994). Par ailleurs, selon Beiseker et collaborateurs (1996), les utilisateurs sont plus nombreux que les non-utilisateurs à percevoir dans leur fréquentation du centre de jour des bénéfices pour la personne âgée, en plus des bénéfices pour la personne de soutien.

L'utilisation antérieure de services similaires indique une attitude positive à leur égard et la maîtrise des barrières à l'utilisation, tant en ce qui a trait à la connaissance qu'à l'accessibilité au service (Miller et McFall, 1991). Ainsi, l'utilisation du centre de jour pour un épisode antérieur de soins pourrait faciliter l'utilisation lors de l'apparition d'un nouveau besoin. Toutefois, l'effet pourrait être inverse si l'épisode antérieur s'est soldé par la nonparticipation parce que la personne n'a pas aimé la première expérience. Les résultats des recherches effectuées à ce jour sont incomplets à ce sujet. Douglass et Visconti (1998) ont observé que l'utilisation du centre de jour antérieure à l'évaluation initiale est le plus important prédicteur de son utilisation lors de l'entrevue de suivi un an plus tard. Henry et Capitman (1995) ont aussi observé que les personnes qui utilisaient le centre de jour depuis 
plus longtemps avaient moins de probabilité de cesser l'utilisation à l'intérieur des six prochains mois. Il s'agit ici d'une utilisation continue et non d'une utilisation pour un épisode antérieur.

L'utilisation de services de répit autres que le centre de jour peut donner une indication des croyances positives des personnes de soutien quant aux services d'aide formelle et pourrait être associée à une plus grande utilisation du centre de jour. Par contre, l'utilisation de ces services pourrait aussi diminuer le besoin du répit offert par le centre de jour (substitution de services). Dans deux études qui ont examiné cette relation, l'utilisation antérieure de soutien formel n'a pas été associée à l'utilisation des nouveaux services de répit proposé par leur projet (Cox, 1997; Lawton et coll., 1991), alors que Kosloski et Montgomery (1993) ont observé une association positive entre l'utilisation d'aide à domicile et l'utilisation des services de répit, infirmant l'hypothèse de substitution de service. Cette hypothèse est aussi infirmée par Beiseker et collaborateurs (1996) qui observent que les utilisateurs de centre de jour ont aussi recours en moyenne à plus d'autres services de répit et de soutien à domicile que les non-utilisateurs.

\section{Facteurs de capacité personnels ou communautaires}

Ce sont encore une fois des facteurs indépendants de l'état de santé immédiat qui, de façon générale, facilitent l'accès aux services. Ils incluent la connaissance des services, les ressources financières ou l'accès à une assurance-santé, de même que la disponibilité des services dans la communauté où vit la personne. Plusieurs auteurs considèrent les ressources à la disposition de la famille, sans distinguer les ressources des personnes âgées et de celles des personnes de soutien.

Il va de soi que la connaissance du service est préalable à son utilisation. La méconnaissance au sujet de la disponibilité du service est rapportée comme raison de nonutilisation du répit (Beisecker et coll., 1996; Strain et Blandford, 2002) et l'influence de cette variable sur le contact avec un service de répit ou un centre de jour est décrite dans deux études corrélationnelles (Cotrell et Engel, 1998; Montoro-Rodriguez et coll., 2003). 
Cette connaissance peut être influencée par plusieurs facteurs. Dans l'étude de Strain et Blandford (2002), les personnes de soutien de sexe masculin et celles qui vivent à l'extérieur des grands centres urbains étaient plus nombreuses à ne pas connaître le service de centre de jour. De plus, comme discuté plus haut, la présence d'un réseau de soutien favorise la connaissance des services d'aide.

De façon étonnante, le revenu familial n'est pas associé à l'utilisation du centre de jour (Beiseker et coll., 1996; Dabelko, 2004; Henry et Capitman, 1995; Kosloski et Montgomery, 1994; Lynch et coll.; 1999; Zarit et coll., 1999b), même dans le contexte américain où les coûts de ces services sont parfois élevés. On retrouve une exception dans l'étude de Montoro-Rodriguez et collaborateurs (2003) où le revenu de la personne de soutien est associé à une plus grande intensité d'utilisation du centre de jour. Il faut noter que cette variable n'était pas significative avant l'ajout dans le modèle statistique d'un ensemble de variables reliées à la connaissance du service, à son attrait et à la facilité d'utilisation. De plus, leur analyse subséquente incluant des variables d'interaction démontre que ce facteur est plus important pour les personnes d'origine afro-américaine.

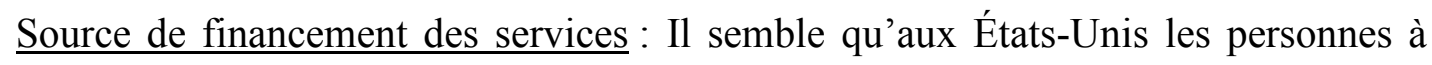
faibles revenus soient admissibles à différents programmes qui subventionnent, au moins en partie, les coûts des services. Nous avons recensé trois études mesurant l'effet de cette variable sur la durée de l'utilisation du centre de jour. Le fait de pouvoir bénéficier de financement extérieur pour défrayer les coûts du centre de jour est associé à une plus longue utilisation du centre de jour dans l'étude de Wallace (1987). Dans les études de Dabelko, un financement privé (la personne défraie le coût) est associé à une plus grande probabilité de cesser l'utilisation à l'intérieur des quatre premières semaines suggérant que le manque de ressources financières peut limiter l'accès au service (Dabelko 2005). Toutefois, lorsque l'analyse porte sur la durée de séjour (variable continue), le financement public est associé à une plus courte utilisation (Dabelko, 2004). Une explication possible est que le critère d'accès au financement public retarde l'utilisation du centre de jour jusqu'au moment où les incapacités sont déjà très grandes. Le placement en institution ne 
peut alors être retardé aussi longtemps que pour les personnes qui utilisent le centre de jour à un stade plus précoce.

Enfin, la disponibilité du service dans sa zone de résidence (Zarit et coll., 1998) ou dans l'ensemble des services offerts par une Health Maintenance Organization (Lynch et coll., 1999) influence bien sûr l'utilisation du centre de jour. Si on suggère que les centres de jour sont moins présents en milieu rural qu'en milieu urbain (Strain et Blandford, 2002), l'étude de Montoro-Rodriguez et collaborateurs (2003), la seule à tester cette variable parmi les études recensées, n'a pu démontrer d'association entre l'utilisation du centre de jour et la densité de population de la zone de résidence.

\section{Facteurs de besoin}

Ces facteurs influencent directement l'utilisation des services. Dans le cas des centres de jour, ils incluent les problèmes de santé physique ou de santé mentale, ainsi que la présence de troubles cognitifs et d'incapacités fonctionnelles. Pour les personnes de soutien, il faudra aussi considérer les facteurs reliés à ce rôle comme la durée de l'aide et le fardeau ressenti, ainsi que les responsabilités concommitantes qui pourraient augmenter le besoin de répit comme le fait d'occuper un emploi .

\section{Personne âgée}

La participation au centre de jour pourrait être limitée par un état de santé trop fragile, indiqué par un nombre élevé de maladies, un recours fréquent à l'hospitalisation ou par un nombre élevé de jours d'hospitalisation. Si plusieurs indicateurs de problèmes de santé physique ont été associés avec une plus grande utilisation des soins à domicile (Bass et Noelker, 1987; Bass et coll., 1992), les résultats ne sont pas aussi consistants pour ce qui est de l'utilisation des centres de jour. Plusieurs auteurs n'ont démontré aucune relation significative (Dabelko, 2004; 2005; Douglas et Visconti, 1998; Henry et Capitman, 1995; Wallace, 1987). Une mauvaise santé a été associée à une plus faible probabilité d'utiliser le centre de jour (Lynch et coll., 1999), mais pas à l'intensité d'utilisation pour le groupe d'utilisateurs. La présence de diabète a été associée à une plus grande intensité d'utilisation, 
alors que la présence de troubles urinaires a été associée à une plus faible intensité d'utilisation (Lynch et coll., 1999). Un plus grand risque nutritionnel a été associé à l'utilisation du centre de jour sur une plus courte période (Dabelko, 2004). Le fait d'avoir été hospitalisé dans les 2,12 ou 36 mois précédant l'étude n'a pas été associé à l'utilisation du centre de jour (Dabelko, 2004; 2005; Henry et Capitman, 1995; Lynch et coll., 1999).

La santé mentale : En ce qui a trait à l'intensité d'utilisation, selon Baumgarten et collaborateurs (2002), les plus grands utilisateurs de centres de jour présenteraient des scores de dépression légèrement supérieurs à ceux des faibles utilisateurs, mais des scores d'anxiété légèrement inférieurs à ces derniers. Pour ce qui est de la durée d'utilisation, dans les études de Dabelko, la présence de dépression et d'anxiété est associée à une plus grande probabilité de cesser l'utilisation à l'intérieur des 4 premières semaines suggérant que ces problèmes peuvent nuire à l'adaptation au centre de jour (Dabelko, 2005). Toutefois, lorsque l'analyse porte sur la durée de séjour (variable continue), la présence de dépression et d'anxiété est associée à une plus longue utilisation (Dabelko, 2004), suggérant que ceux qui arrivent à s'y adapter maintiennent l'utilisation. Ces variables ne sont pas étudiées dans les travaux sur l'utilisation des centres de jour par la clientèle atteinte de démence.

Les troubles cognitifs: L'utilisation de services de répit (contact: Cox, 1997; Cotrell et Engel, 1998) et de centre de jour (contact: Dabelko et Balaswamy, 2000; intensité : Baumgarten et coll., 2002; contact et intensité : Lynch et coll., 1999) serait positivement associée à la présence d'atteinte cognitive chez la personne âgée. Il faut souligner aussi deux études qui n'ont pas observé d'association significative (contact: Kosloski et Montgomery, 1994; intensité : Wallace, 1987).

Dans une étude sur l'utilisation de 15 services de santé ou de soutien, les personnes avec ou sans troubles cognitifs utilisent le même nombre de jours de services, mais les personnes avec troubles cognitifs utilisent plus certains types de services. Entre autres, les personnes atteintes de démence de type Alzheimer utilisent davantage le centre de jour (intensité : Montgomery, Kosloski et Borgotta, 1988-1989). Toutefois, à l'intérieur de la clientèle atteinte de troubles cognitifs, les personnes ayant plus de problèmes de 
comportement, utilisent moins le centre de jour (intensité : Lawton et coll., 1991; durée : Zarit et coll., 1999b) et davantage le répit à domicile (intensité : Lawton et coll., 1991).

Les incapacités fonctionnelles : Dans les études auprès d'une clientèle générale, les observations sont contradictoires. En ce qui concerne le contact avec le centre de jour, Chappell (1983) rapporte que les utilisateurs de centre de jour présentent plus d'incapacités fonctionnelles que les utilisateurs de soins à domicile alors que Dabelko et Balaswamy (2000) n'observent pas de différence entre les deux groupes. En ce qui a trait à l'intensité d'utilisation, dans au moins deux études, on note que les plus forts utilisateurs de centre de jour ont aussi plus d'atteintes fonctionnelles (Baumgarten et coll., 2002; Lynch et coll., 1999). Cette variable ne semble pas prédire la durée d'utilisation (Dabelko, 2004; 2005; Henry et Capitman, 1995).

Dans les études auprès de personnes démentes, on observe généralement que les personnes qui présentent plus d'incapacités physiques ou d'atteintes fonctionnelles utilisent davantage les services de répit en général (Brodaty et coll. 2005; Casserta et coll. 1987; Cotrell et Engel, 1998; Kosloski et coll., 1993; 2001). En ce qui concerne le centre de jour toutefois, Zarit et collaborateurs (1999b) rapportent que les personnes qui ont plus d'atteintes fonctionnelles sont plus nombreuses dans le groupe ayant une faible durée d'utilisation. Pour ce qui est du contact avec le centre de jour, Biegel et collaborateurs (1993) constatent que les personnes qui ont moins d'incapacités utilisent davantage le répit hors du domicile comme le centre de jour alors que celles qui présentent plus d'incapacités sont plus nombreuses chez les utilisateurs de répit à domicile et chez les non-utilisateurs.

\section{Personne de soutien}

La personne de soutien qui est elle-même affectée par un problème de santé, que ce soit un problème de santé physique, un trouble cognitif ou une diminution de sa capacité fonctionnelle sera davantage limitée dans sa capacité d'aider la personne âgée. Il semble donc logique que cette personne de soutien ait un plus grand besoin de services que celle qui est en excellente santé. Par contre, ses problèmes de santé peuvent limiter sa capacité à rechercher de l'aide pour la personne âgée qu'elle soutient (Boucher, Renvall et Jackson, 
1996). Toutefois, lorsqu'un premier contact avec des services de soutien a eu lieu, les intervenants peuvent leur recommander davantage de services jugeant que leur niveau de besoin est supérieur. À ce jour, les études sur l'utilisation des centres de jour (Douglass et Visconti, 1998; Kosloski et Montgomery, 1994) ou des services de répit (Kosloski et coll., 2001; Lawton et coll., 1989; Li, 2004; Pedlar et Biegel, 1999) qui ont considéré la santé physique de la personne de soutien n'ont pas démontré de relation significative.

Le bien-être psychologique des personnes de soutien est davantage étudié. Les indicateurs de bien-être psychologique ont démontré des effets variables. On a observé que les personnes de soutien plus anxieuses et présentant plus de symptômes dépressifs étaient plus nombreuses à cesser l'utilisation des services de répit en moins de 6 mois (Cox, 1997) et plus nombreuses à cesser l'utilisation du centre de jour dans les trois premiers mois en raison de l'hébergement du proche (Wells et coll., 1990). Dans l'étude de Mävall et Thorslund (2006), un indicateur de dépression plus élevée chez la personne de soutien est aussi relié à une plus courte utilisation du centre de jour, mais seulement pour les personnes de soutien qui ne cohabitent pas avec la personne aidée. Par contre, dans d'autres études, un plus haut niveau de dépression de la personne de soutien est associé à une utilisation plus intense des services de répit (Lawton et coll., 1991) et à l'utilisation du centre de jour sur une plus longue période (Zarit et coll., 1999b). On retrouve aussi deux études où aucune association significative n'est démontrée (Douglass et Visconti, 1998; Montoro-Rodriguez et coll., 2003).

Le fardeau de la personne de soutien pourrait influencer l'utilisation du centre de jour de deux façons. Un plus grand fardeau pourrait indiquer un plus grand besoin de répit et augmenter l'utilisation du centre de jour. Toutefois, la personne de soutien plus fatiguée n'a peut-être pas l'énergie pour stimuler la personne à se rendre au centre de jour, ce qui pourrait se traduire par une plus faible utilisation pour certains usagers. Les recherches à ce jour ont démontré que le fardeau est généralement positivement corrélé à l'utilisation de services de répit (Casserta et coll., 1987; Cox, 1997, Lawton et coll., 1991) et à une utilisation plus intense du centre de jour (Baumgarten et coll., 2002) pour une clientèle générale. Dans les études limitées à l'utilisation du centre de jour par la clientèle atteinte de 
démence, la relation semble à l'inverse de celle observée par Baumgarten et collaborateurs Selon Biegel et collaborateurs (1993), les utilisateurs de centre de jour ont un moins grand fardeau émotionnel (sentiments d'impuissance, de dépression, de culpabilité, de craintes pour le futur et d'isolement reliés au rôle d'aidant) par rapport aux utilisateurs de répit à domicile et aux non-utilisateurs. De même, Zarit et collaborateurs (1999b) ont trouvé qu'un plus fort sentiment de captivité relié au rôle de personne de soutien est associé à une plus courte utilisation du centre de jour. Dans l'étude de Mävall et Thorslund (2006) auprès d'une clientèle atteinte de démence, trois indicateurs de fardeau (sentiment de captivité relié au rôle, inquiétudes et sentiment de surcharge) sont aussi reliés à une plus courte utilisation du centre de jour, mais seulement pour les personnes de soutien qui ne cohabitent pas avec la personne aidée. Une explication possible serait que quelques heures de centre de jour par semaine n'apporteraient pas une diminution suffisante des inquiétudes reliées à la supervision nécessaire à la personne atteinte de démence. Les personnes de soutien qui ne cohabitent pas avec la personne âgée se tourneraient plus rapidement vers des ressources qui peuvent apporter une supervision 24/h sur 24 .

Les aspects positifs ou négatifs du rôle de personne de soutien ont aussi été étudiés. Les personnes de soutien qui ont choisi d'exercer ce rôle sont associées à une utilisation plus intense du centre de jour (Henry et Capitman, 1975). Les personnes de soutien pour lesquelles ce rôle apporte peu de bénéfices sont plus nombreuses à cesser d'utiliser les services de répit à l'intérieur de 6 mois (Cox, 1997), possiblement en raison de l'hébergement de leur proche.

Durée de l'aide: Dans une étude, les personnes de soutien qui exercent ce rôle depuis moins d'un an étaient plus nombreuses à cesser l'utilisation du centre de jour dans une période de 6 mois (Henry et Capitman, 1995). Trois autres études n'ont démontré aucune association avec l'utilisation du centre de jour (Zarit et coll., 1999b) ou des services de répit (Biegel et coll., 1993; Pedlar et Biegel, 1999).

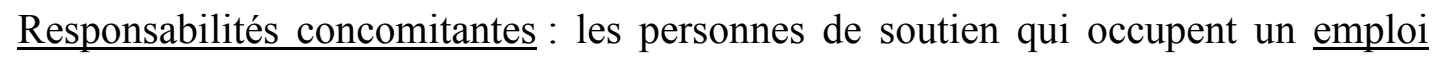
pourraient avoir davantage besoin du centre de jour ou d'autres services de répit, surtout si 
leur proche âgé ne peut être laissé seul durant la journée. Selon Cotrell et Engel (1998), les personnes de soutien qui occupent un emploi ont utilisé davantage de services de répit. Toutefois, cinq autres études qui ont considéré ce facteur n'ont pas démontré d'association significative avec l'utilisation du centre de jour (Henry et Capitman, 1995) ou des services de répit (Biegel et coll., 1993; Cox, 1997; Kosloski et coll., 2001; Li, 2004).

\section{Caractéristiques des services}

Les caractéristiques des services peuvent faciliter l'accès à un service particulier, une fois le besoin d'aide identifié ou favoriser la poursuite de l'utilisation après le contact initial. Pour le centre de jour, ils incluent la facilité d'accès et la disponibilité du service au moment opportun, le coût du service pour l'utilisateur, la durée et la fiabilité du service, le transport, le type d'activités offertes, ainsi que la présence d'autres services qui peuvent faciliter l'utilisation du centre de jour.

La facilité d'accès et la disponibilité du service au temps opportun seront surtout fonction des critères d'admissibilité et des listes d'attente. Pour ce qui est des critères d'admissibilité, plusieurs auteurs mentionnent un accès au centre de jour plus limité pour les personnes qui présentent des problèmes de comportement ou d'incontinence (Beisecker et coll., 1996; Lawton et coll., 1991; Mace et Rabins, 1984; Tourigny et coll., 1993; Wallace, 1987). Aucune des études recensées ne discute de l'impact des listes d'attente, bien qu'en toute logique, leur présence limitera l'accès aux services. Ceci s'explique possiblement par le fait que la plupart des études sont américaines, et qu'aux États-Unis, les centres de jour ont rarement des listes d'attente. Au contraire, ils semblent avoir de la difficulté à remplir toutes les places disponibles (Cox et coll., 1998; Kosloski et coll., 2001; Weissert et coll., 1990). Enfin, l'obligation d'utiliser le service de façon régulière plutôt que selon les besoins a aussi été rapportée comme raison de non-utilisation des services de répit (Lawton et coll., 1991).

La durée et la fréquence des activités: Pour une clientèle générale, une plus longue durée d'utilisation du centre de jour a été associée au fait d'être inscrit à raison de trois 
jours par semaine. Une explication avancée est que la participation à raison d'une ou deux journées par semaine ne serait pas suffisante pour incorporer le centre de jour dans les habitudes de la personne âgée, alors qu'une participation initiale de plus de trois jours pourrait provoquer un changement trop brusque de routine, à la fois pour le participant et sa personne de soutien (Wallace, 1987). Cette association n'est pas retrouvée dans les études de Dabelko (2004; 2005) auprès d'une clientèle générale, ni dans celle de Mävall et Thorslund (2006) auprès d'une clientèle atteinte de démence. Nous n'avons pas recensé d'analyse de l'utilisation du centre de jour en fonction de la durée des services offerts chaque jour. Toutefois, certaines personnes mentionnent comme raison de non-utilisation le fait que les heures d'ouverture ne conviennent pas aux besoins (Beiseker et coll., 1996; Lawton et coll. 1991). La possibilité d'obtenir une quantité suffisante de répit par le centre de jour a aussi été associée à son utilisation par la clientèle atteinte de démence (MontoroRodriguez et coll., 2003).

La fiabilité du service : Dans l'étude de Kosloski et coll. (2001), les personnes de soutien qui accordaient une plus grande importance à la régularité et à la fiabilité des heures de répit étaient plus nombreuses à utiliser les services de répit dispensés par les agences étudiées, incluant le centre de jour ou du répit à domicile.

Le coût du centre de jour : Dans l'étude de Dabelko (2005), un coût moyen plus élevé (coût réel pour le client tenant compte des subventions possibles) est observé chez les personnes qui quittent le centre de jour dans les quatre premières semaines de fréquentation, suggérant que le coût peut être un frein à l'utilisation. Henry et Capitman (1995) observent au contraire qu'un coût plus élevé pour les services du centre de jour comme tel serait associé à une utilisation plus intense, toutefois, un coût plus élevé pour les services auxiliaires et une insatisfaction par rapport aux coûts amèneraient une utilisation moins intense. Les coûts moyens présentés dans cette étude semblent beaucoup plus élevés que ceux observés dans l'étude de Dabelko, mais les informations fournies ne sont pas suffisamment détaillées pour saisir l'origine de ces différences et les interprétations possibles. S'il s'agit du coût total du service et non du coût pour le client, il est possible qu'un service plus coûteux soit aussi un service de plus grande qualité. Dans le contexte 
québécois de gratuité des soins de santé, seuls les coûts des services auxiliaires (ex. : repas, transport) seront pertinents. Il est tout de même possible que, pour certains, ces frais soient une barrière à l'utilisation (Gutman et coll., 1993a). Certains centres de jour peuvent avoir une politique de paiement flexible qui permet aux plus démunis de participer aux activités sans acquitter de frais de repas ou de transport.

Les difficultés de transport ont été rapportées comme barrières à l'utilisation du centre de jour (Beisecker et coll., 1996; Lawton et coll., 1991). L'effet positif de cette variable sur l'utilisation du centre de jour est démontré dans les deux études recensées à ce sujet (intensité : Montoro-Rodriguez et coll., 2003, durée : Dabelko, 2005).

Le type d'activités offertes peut aussi influencer l'utilisation du centre de jour. Henry et Capitman (1995) se sont attardés à l'influence du type d'activités offertes aux personnes âgées sur l'intensité et la durée de participation. Ils ont trouvé que les participants à des programmes qui mettent l'accent sur le maintien de la santé utilisent moins intensément le centre de jour et sont plus susceptibles de cesser la participation que ceux qui participent à des programmes qui mettent l'accent sur le répit ou sur les activités de stimulation et de croissance. Gitlin et collaborateurs (2006) ont analysé l'effet d'une intervention auprès des aidants (un suivi d'en moyenne une heure par mois) pour aborder leur préoccupation. Ce suivi a permis une amélioration du taux d'utilisation du centre de jour par la personne aidée, surtout par une diminution de l'abandon précoce de la participation. Conrad, Hughes et Wang (1992) ont étudié l'effet des activités offertes par divers centres de jour sur les taux de participation moyens de ces centres. Leur unité d'analyse est donc le centre de jour et non l'individu. Les centres qui offraient à la fois des services thérapeutiques (ergothérapie, physiothérapie, orthophonie), de l'entraînement aux soins personnels et des activités récréatives thérapeutiques (art, musique) et moins d'éducation aux familles obtenaient de plus forts taux de participation.

Dabelko $(2004 ; 2005)$ a inclus dans ses études plusieurs variables reliées à l'environnement des services: taille du centre de jour, ratio personnel/personnes âgées, diversité du personnel, espace disponible, localisation du programme dans une institution 
de soins de longue durée, centre qui accepte ou non la clientèle ayant des troubles cognitifs. Aucune de ces variables n'a démontré d'association avec l'utilisation du centre de jour.

Puisque, dans le domaine du soutien à domicile, diverses agences ou organisations travaillent de concert pour offrir aux personnes âgées l'ensemble des services que leur condition nécessite (Griffin, 1993; Agence de la santé et des services sociaux de Montréal, 2005), la présence d'autres services de soutien à domicile et les caractéristiques de ces $\underline{\text { services, }}$ peuvent aussi faciliter l'utilisation des centres de jour. L'influence de ces services a été très peu étudiée. On retrouve un exemple dans l'étude de Douglass et Visconti (1998) auprès de la clientèle de l'Alzheimer's Disease Diagnostic and Treatment Center Program (ADDTC), où le fait d'être référé au centre de jour par le personnel du ADDTC est, avec l'utilisation du centre de jour avant l'inscription au programme ADDTC, le seul prédicteur significatif du fait d'avoir recours au centre de jour un an plus tard.

Certains auteurs ont émis des recommandations pour améliorer l'organisation des services offerts en centre de jour de façon à en augmenter l'utilisation. Parmi ces éléments d'organisation dont l'effet sur l'utilisation du centre de jour mériterait d'être étudié, mentionnons entre autres : une gestion de cas plus intensive des participants à risque de se désister (Dabelko, 2004), un suivi individualisé pour une meilleure intégration au groupe des personnes qui ont des problèmes de santé mentale (Dabelko, 2005), la possibilité d'offrir non seulement le transport « de porte à porte (door to door) », mais un service « indoor to door», soit une aide à l'intérieur du domicile pour aider la personne âgée à se préparer pour le centre de jour (Dabelko, 2005).

\section{Interaction entre divers facteurs}

Quelques rares études ont recherché la présence d'interactions entre les divers facteurs qui expliquent l'utilisation du centre de jour ou d'autres services de soutien. Celles qui l'ont fait ont obtenu des résultats intéressants. Les interactions les plus souvent démontrées incluent des interactions entre : 1) plusieurs facteurs et le type de lien qui unit 
la personne de soutien et la personne âgée 2) divers facteurs et l'appartenance culturelle et 3) divers facteurs et la présence de troubles cognitifs.

\section{Type de lien entre la personne de soutien et la personne âgée}

Dans l'étude de Kosloski et Montgomery (1994), lorsque la personne de soutien est le conjoint, les personnes qui se perçoivent en moins bonne santé que d'autres personnes de leur âge et les personnes atteintes de démence de type Alzheimer sont plus nombreuses à utiliser le centre de jour. Celles qui ont le sentiment d'être plus dépendantes des autres sont moins nombreuses à utiliser le centre de jour. Ces relations ne sont pas observées lorsque la personne de soutien est un descendant.

Henry et Capitman (1995) ont trouvé que pour les personnes dont l'aidant est le conjoint, le fait que centre de jour favorise la stimulation et la croissance, plutôt que le répit ou le maintien de la santé, est associé à une plus grande intensité d'utilisation. Lorsque le type de relation avec la personne aidante n'était pas considéré, ils avaient observé une utilisation plus faible pour les programmes qui mettent l'accent sur la santé, mais n'avaient pas vu de différence avec les programmes orientés vers le répit.

L'influence des soutiens secondaires pourrait aussi différer selon que la personne de soutien principale est le conjoint de la personne âgée ou un descendant. Dans une étude auprès de conjointes et de filles, Li (2004) a observé que les conjointes utiliseraient davantage de services formels lorsqu'il y a présence de personnes de soutien secondaires. Ceux qui ont une fille comme personne de soutien principale utilisent généralement un bon nombre de services formels lorsque cette personne de soutien est la seule responsable des soins ou lorsqu'elle est aidée dans ce rôle par des amis. Par contre, lorsque cette fille reçoit de l'aide d'autres membres de la famille, ils utiliseraient moins de services formels.

La cohabitation entre la personne âgée et la personne de soutien est une autre façon de conceptualiser le type de lien qui existe entre eux. Dans l'étude de Mävall et Thorslund (2006) auprès d'une clientèle atteinte de démence, les trois indicateurs de fardeau (sentiment de captivité relié au rôle, inquiétudes et sentiment de surcharge) et un indicateur 
du bien-être psychologique de la personne de soutien sont reliés à une plus courte utilisation du centre de jour, mais seulement pour les personnes de soutien qui ne cohabitent pas avec la personne aidée.

\section{Appartenance culturelle}

Deux études (Kosloski, Montgomery et Karner, 1999; Montoro-Rodriguez et coll., 2003) ont démontré que les facteurs qui influencent le plus l'utilisation du centre de jour diffèrent selon l'appartenance culturelle des participants et de leur personne de soutien. Par exemple, dans leur échantillon de personnes de soutien de personne atteinte de démence, Montoro-Rodriguez et collaborateurs (2003) ont trouvé que le revenu de la personne de soutien serait un facteur plus important pour prédire l'intensité d'utilisation du centre de jour par les personnes d'origine afro-américaine, alors que la possibilité d'obtenir une quantité de répit suffisante serait plus importante pour les personnes d'origine caucasienne. Kosloski et collaborateurs (1999) ont observé que les personnes d'origine afro-américaine sont plus nombreuses à utiliser le centre de jour lorsque la personne de soutien est un enfant alors que cette variable n'a pas d'influence chez les personnes d'origine caucasienne et chez les hispanophones. L'âge de la personne âgée était relié à une plus grande probabilité d'utiliser le centre de jour chez les hispaniques. Le fait que la personne de soutien occupe un emploi était relié au contact avec le centre de jour pour les personnes d'origine caucasienne et celles d'origine afro-américaine, mais pas pour les hispaniques.

\section{Présence de troubles cognitifs}

Dans une étude des déterminants de l'utilisation des soins à domicile, Bass et collaborateurs (1992) ont observé des interactions significatives entre la présence de troubles cognitifs et de nombreuses autres variables: le fait que la personne âgée vive seule, la présence de dépression chez la personne âgée, la présence de soutiens secondaires en plus de la personne de soutien principale, le fardeau de la personne de soutien principale. La présence d'interaction entre divers facteurs et la présence de troubles cognitifs n'a pas été testée dans les études de l'utilisation du centre de jour que nous avons recensées. Toutefois, certains résultats divergents des recherches auprès d'une clientèle générale par 
rapport aux recherches auprès de la clientèle atteinte de démence pourraient s'expliquer par la présence d'interactions similaires.

\section{Conclusion de la recension des écrits}

Il ressort de cette recension des écrits que l'utilisation des centres de jour est un phénomène complexe, et ce, pour plusieurs raisons. Premièrement, il existe une grande variété de centres de jour qui offrent une gamme très diversifiée de services. Il ne s'agit donc pas d'un service homogène. La raison prioritaire d'utilisation peut être aussi bien la surveillance de l'état de santé pour une personne fragile, la volonté de participer à des activités physiques pour une personne ayant fait un AVC, le désir de socialiser pour une personne dépressive ou le répit pour les proches aidants d'une personne qui présente de grandes incapacités physiques ou une démence. Il est donc logique de penser que les déterminants de l'utilisation pourraient varier pour ces divers motifs d'utilisation. De plus, l'utilisation peut être définie de plusieurs façons : contact (probabilité d'utiliser ou non), intensité de l'utilisation, durée de l'utilisation. Les facteurs expliquant le contact avec un service peuvent être très différents de ceux expliquant la poursuite de l'utilisation de ce service (Andersen et Newman, 1973). Certains déterminants sont toutefois ressortis plus fréquemment ou avec une plus grande constance dans l'ensemble des facteurs étudiés. En voici un résumé.

Parmi les facteurs prédisposants, on observe que les personnes plus âgées parmi l'ensemble des aînés semblent de moins forts utilisateurs de centre de jour, peu importe le phénomème d'utilisation étudié, sauf pour la clientèle atteinte de démence où l'âge semble avoir moins d'importance. De plus, la présence d'un réseau de soutien pour la personne âgée tout comme pour la personne de soutien semble favoriser l'utilisation du centre de jour. Lorsque la personne de soutien est le conjoint, la présence de soutiens secondaires semble plus importante pour faciliter la recherche d'aide formelle. Les personnes qui vivent en couple ont pris soin l'une de l'autre depuis de nombreuses années. Il semble que lorsque l'état de santé de l'une d'elles se dégrade, l'autre ait besoin de sentir que les autres 
membres de sa famille ou de son entourage lui permettent de ne plus s'occuper seule de cette tâche.

Parmi les facteurs de capacité personnels ou communautaires, il apparaît que le revenu n'a pas un très grand impact sur l'utilisation du centre de jour, parce que dans la majorité des cas où ce service n'est pas gratuit, il peut être subventionné en fonction de la capacité de payer des individus. Par ailleurs, l'accès à ce type de soutien financier apparait important pour l'accès au centre de jour et son utilisation continue par les clients moins aisés.

Parmi les facteurs de besoin de la personne âgée, il semble que la présence de troubles cognitifs soit plus souvent associée positivement à l'utilisation du centre de jour, peu importe le phénomème d'utilisation étudié. Toutefois, à l'intérieur de la clientèle atteinte de troubles cognitifs, les personnes qui ont plus de problèmes de comportement utilisent le centre de jour moins intensivement et pour de moins longues périodes. Plusieurs études n'ont pas trouvé de lien entre l'utilisation du centre de jour et les capacités fonctionnelles. Toutefois, celles qui ont observé une différence significative ont trouvé le plus souvent une association positive avec le contact et l'intensité d'utilisation pour la clientèle générale et une association négative pour le contact et la durée d'utilisation dans les études auprès de la clientèle atteinte de démence. Enfin, il faut noter que l'influence des problèmes de santé mentale (tels que dépression, stress ou anxiété) sont étudiés presque exclusivement dans les recherches auprès de la clientèle générale. Comme ces études sont moins nombreuses que celles auprès de la clientèle atteinte de démence, cette variable demeure peu étudiée.

Parmi les facteurs de besoin de la personne de soutien, il semble que la santé physique de cette dernière ne soit pas associée à l'utilisation du centre de jour. Les variables mesurant le bien-être psychologique ou le sentiment de fardeau de la personne de soutien semblent plus souvent indiquer que, lorsque la situation d'aide est trop difficile, l'utilisation du centre de jour est plus faible. Ces facteurs ont été mis en lumière dans les études auprès d'une clientèle atteinte de démence et sont généralement associés à une 
utilisation brève du centre de jour, probablement en raison d'un recours plus rapide à l'hébergement en institution.

Les caractéristiques organisationnelles des services ont été peu étudiées. La seule variable pour laquelle il semble possible de tirer une conclusion est l'organisation du transport par le centre de jour, qui est associé positivement à l'utilisation du centre de jour. Cependant, certains auteurs ont émis des recommandations pour améliorer l'organisation des services offerts en centre de jour de façon à en augmenter l'utilisation. 



\section{Chapitre 3 : Cadre conceptuel et objectifs de l'étude}

\section{Cadres théoriques utilisés dans les études récentes sur l'utilisation des centres de jour}

Parmi les études effectuées au cours des dix dernières années et ayant permis de dégager les déterminants de l'utilisation du centre de jour à la section précédente, certaines ne présentaient aucun cadre théorique de l'utilisation. C'est le cas notamment des études pour lesquelles l'identification des déterminants de l'utilisation n'était pas l'objectif principal, mais le résultat d'analyses secondaires (Baumgarten et coll., 2002; Gottlieb et Johnson, 2000; Mävall et Thorslund, 2006) et d'études qui portaient sur l'identification des raisons de non-utilisation (Strain et Blandford, 2002). Parmi celles qui ont utilisé un cadre conceptuel de l'utilisation, on retrouve principalement quatre approches théoriques :

1) Trois études (Douglass et Visconti, 1998; Kosloski et coll., 1999; Lynch et coll., 1999) ont utilisé le modèle d'utilisation des services de santé élaboré par Andersen en 1968 et repris par Andersen et plusieurs collègues (pour un résumé de l'évolution du modèle, voir Andersen, 1995).

2) Deux études (Kosloski et coll., 2001; Montoro-Rodriguez et coll., 2003) ont utilisé les facteurs du modèle d'utilisation d'Andersen, complété par un groupe de facteurs tirés du modèle axé sur la pratique (Practice-oriented Model) décrit par Yeatts, Crow et Folts (1992).

3) Dabelko (2004 et 2005) utilise un modèle social écologique (Social Ecological Model) inspiré des travaux de Bloom (1996) et de Germain et Bloom (1999).

4) Zarit et collaborateurs (1999b) fondent leur étude sur le modèle de processus du stress chez les aidants (Stress Process Model of Caregiving: Pearlin, Mullan, Semple et Skaff, 1990).

Voici un bref survol de ce que chacune de ces approches peut apporter à l'étude des déterminants de l'utilisation des centres de jour. 
Le modèle d'utilisation des services de santé d'Andersen (Andersen et Newman, 1973; Aday et Andersen, 1974; Andersen, 1995) permet une conceptualisation globale intéressante de trois types de facteurs qui peuvent expliquer l'utilisation des services de santé :

- les facteurs prédisposants, des facteurs indépendants de l'état de santé immédiat qui, de façon générale, influencent indirectement l'utilisation de services de santé. (ex. : facteurs démographiques, structure sociale, croyances et attitudes envers la santé et l'utilisation des services, etc.);

- les facteurs de capacité ou facteurs facilitants (en anglais " enabling factors »), des facteurs personnels ou communautaires qui facilitent l'accès aux services chez des personnes prédisposées à y recourir (ex. : revenus, présence d'assurance-maladie, disponibilité des services dans la communauté, etc.);

- les facteurs de besoin de services, des facteurs reliés aux symptômes ressentis ou évalués, qui ont une influence directe sur l'utilisation des services de santé (Andersen, 1995).

Ce modèle a été largement utilisé dans des études sur l'utilisation des services de soutien à domicile (Bass et Noelker, 1987; Bass et coll., 1992; Crets, 1996; Forbes et Janzen, 2004; Jackson et Mittlemark, 1997; Kadushin, 2004; Miller et McFall, 1991; Starret, Rodgers et Walters, 1989) et dans plusieurs études sur l'utilisation des services de répit ou de centres de jour (Biegel et coll., 1993; Cox, 1997; Douglas et Visconti, 1998; Kosloski et coll., 1994; 1999; Lynch et coll., 1999). Ces chercheurs ont eu recours à la typologie du modèle d'Andersen, en l'adaptant à leur situation particulière. Le plus souvent, ces auteurs ont inclus les caractéristiques des personnes âgées et celles de leurs personnes de soutien dans les divers facteurs étudiés (Bass et Noelker, 1987; Bass et coll., 1992; Biegel et coll., 1993; Douglas et Visconti, 1998; Kosloski et coll., 1994; 1999; Miller et McFall, 1991). En effet, il a été démontré que les personnes de soutien jouent un rôle important dans la connaissance qu'ont les personnes âgées des services formels et dans l'utilisation qu'elles font de ces services (Bass et Noelker, 1987; Cotrell et Engel, 1998; Strain et Blandford, 2002). Selon Strain et Blandford (2002), les caractéristiques des 
personnes de soutien seraient plus importantes que les caractéristiques des personnes âgées pour prédire la connaissance de la disponibilité de plusieurs types de services de soutien à domicile, connaissance préalable à l'utilisation. De plus, les centres de jour, comme plusieurs autres services gérontologiques, considèrent aussi les besoins de la personne de soutien lorsqu'ils évaluent le besoin de service d'une personne âgée.

Le modèle d'Andersen a été critiqué par plusieurs chercheurs parce qu'en général, il ne permet d'expliquer qu'une faible proportion de la variation dans l'utilisation des services de santé des personnes âgées (Wolinsky et Johnson, 1991, Kosloski et coll., 1993; 1994). Dans les études recensées pour cette recherche et qui fournissent cette donnée, il explique entre 18 à $47 \%$ de la variance dans l'utilisation des services (Bass et Noelker, 1987; Bass et coll., 1992; Lynch et coll., 1999; Starrett et coll., 1989). Il faut noter que les indicateurs retenus pour mesurer les trois types de facteurs du modèle varient d'une étude à l'autre et la plupart du temps, ne couvrent pas l'ensemble des éléments compris dans le modèle. Par exemple, dans la majorité des études qui se disent fondées sur le modèle d'Andersen, les croyances et attitudes envers la santé n'ont pas été prises en compte (Kosloski et Montgomery, 1993). De plus, seuls un petit nombre de chercheurs l'ont réellement utilisé comme un modèle interactif plutôt qu'un modèle additif (Kosloski et Montgomery 1994; Calsyn et Winter, 2000). Une seconde critique énoncée par rapport à ce modèle est qu'il inclut surtout des facteurs qui relèvent des caractéristiques des individus, mais ne tient pas compte des facteurs organisationnels qui influencent la façon dont les services sont livrés. Ainsi, à l'exception des croyances envers la santé et l'utilisation des services, les fournisseurs de soins peuvent difficilement influencer les autres facteurs. Ce modèle offrira donc peu de pistes pour les gestionnaires de services qui veulent améliorer l'utilisation ou l'accès à leurs services (Yeats et coll., 1992; Kosloski et coll., 2001; Montoro-Rodriquez et coll., 2003). En effet, si le modèle inclut certaines caractéristiques relevant de l'organisation du système de santé à un niveau global (présence d'assurance-maladie, disponibilité de services dans la communauté, etc.) (Andersen et Newman 1973; Andersen 1995), il accorde peu d'attention aux caractéristiques organisationnelles propres à chaque fournisseur de services (ex. : structure du personnel). Ces caractéristiques influenceront 
vraisemblablement l'expérience de l'individu qui cherche à accéder à un service particulier ou qui doit décider de poursuivre ou non l'utilisation d'un service donné. Enfin, notons que ce modèle a été élaboré pour expliquer l'utilisation de services médicaux. Les facteurs qui expliquent l'utilisation du centre de jour, un service à la fois de nature sociale et de santé, pourraient différer.

Yeats et collaborateurs (1992) proposent un modèle d'utilisation axé sur la pratique (Practice-oriented model). Ce modèle cherche à rendre compte des facteurs qui pourraient stimuler l'amélioration de l'utilisation de services sociaux par divers groupes minoritaires. Il inclut trois types de facteurs sur lesquels les fournisseurs de services peuvent agir :

- les facteurs reliés aux connaissances qui comprennent la connaissance de l'existence des services, la connaissance des procédures pour accéder aux services, ainsi que la connaissance des symptômes qui justifient une consultation, ce qui permet l'identification du besoin de service par l'individu;

- les facteurs reliés à l'accès qui comportent au moins trois types de barrières qui peuvent nuire à l'utilisation des services. La première concerne la capacité de se rendre à l'endroit où les services sont offerts. Dans le cas des personnes âgées, il faut souvent considérer une capacité de déplacement restreinte. Ainsi, le fait de fournir des services de transport est un facteur pouvant faciliter l'accès aux services. La seconde barrière concerne l'aspect abordable des services. Ce facteur implique une mise en relation du coût du service par rapport aux revenus de l'individu. La troisième barrière relève de la disponibilité du service de façon à en permettre un accès au temps opportun et en quantité suffisante pour répondre aux besoins;

- les facteurs reliés à l'intention d'utiliser un service. On conçoit que même si un besoin de service est reconnu et qu'un service est accessible pour répondre à ce besoin, l'utilisation n'aura lieu que si les caractéristiques du service disponible sont suffisamment attrayantes et conformes aux valeurs de l'individu pour induire le comportement d'utilisation. Dans les services de nature sociale, les éléments inclus dans ce facteur peuvent varier selon le type de services offerts. Ils peuvent comprendre la qualité de la nourriture servie, les activités sociales adaptées aux goûts 
des participants, la concordance culturelle entre les fournisseurs de services et les clients, de même que les attitudes envers le fait de recevoir des services de nature sociale (qui peuvent inclure, par exemple, un sentiment d'humiliation ou la peur du ridicule).

Bien qu'il présente des concepts intéressants pour décrire certains aspects de l'utilisation des centres de jour, ce modèle semble incomplet en ce qu'il ne comporte aucune mesure objective des besoins pouvant justifier l'utilisation du centre de jour. Dans le contexte québécois où l'accès au centre de jour n'est possible que si un besoin est reconnu par un professionnel de la santé, il semble important d'inclure des facteurs de besoin dans le cadre conceptuel de la présente étude. Enfin, notons que ce modèle axé sur la pratique a été peu testé de façon empirique. Si l'étude de Kosloski et collaborateurs (2001) s'inspirait de certains facteurs tirés de ce modèle, nous n'avons recensé qu'une seule étude (Montoro-Rodriguez et coll., 2003), qui utilisait formellement ce modèle, en complément au modèle d'Andersen, pour analyser l'utilisation des centres de jour et des services de répit.

Le modèle social écologique utilisé par Dabelko (2004 et 2005) suppose que les comportements sociaux sont fonction de l'interaction entre l'individu et son environnement. Des tâches d'adaptation sont effectuées pour atteindre une adéquation entre la personne et son environnement. Dans ce contexte, l'individu utilisera des services de santé et des services sociaux s'ils contribuent à l'aider à répondre aux besoins d'adaptation entre ses sous-systèmes individuels (biologique, affectif, cognitif et comportemental) et les sous-systèmes de son environnement (culturel, sociétal et physique). Dans ses deux études, Dabelko cherche donc à déterminer les facteurs individuels et environnementaux qui influencent la durée d'utilisation du centre de jour. Les facteurs individuels incluent : les facteurs biologiques (âge et le genre), affectifs (présence de dépression ou d'anxiété); cognitifs (diagnostic de démence et problèmes de comportement reliés aux troubles cognitifs) ainsi que des facteurs de santé (hospitalisation antérieure, hébergement temporaire en institution; capacités fonctionnelles démontées par des scores d'AVQ et 
d'AVD; risque nutritionnel; incontinence et présence de diagnostics multiples). Les facteurs environnementaux comprennent les facteurs culturels (groupe ethnique; langue; religion), les facteurs sociaux relatifs aux ressources financières (revenu; source de financement pour les services; coût à assumer personnellement pour les services), les facteurs sociaux relatifs aux ressources sociales (état civil; niveau d'éducation; nombre de personnes dans la maison; lien avec l'aidant principal; l'intensité des services de centre de jour auxquels la personne a accès en nombre de jours par semaine; l'accès à un service de transport), les facteurs physiques relatifs au centre de jour fréquenté (capacité du centre de jour/nombre de clients servis; dimension du centre de jour/nombre de pieds carrés; ratio client/personnel; diversité culturelle du personnel adaptée à celle des clients; programme qui accepte ou refuse les clients avec des troubles cognitifs).

À notre connaissance, Dabelko est la seule auteure à avoir utilisé ce modèle pour cerner les déterminants de l'utilisation du centre de jour. Les indicateurs retenus par cette auteure pour chacun des groupes de variables sont discutables. On peut se demander, par exemple, pourquoi la diversité culturelle du centre de jour est placée sous les facteurs de l'environnement physique, plutôt que de l'environnement social. Enfin, bien qu'elle explique ce modèle comme un modèle interactif, l'expérimentation qui s'ensuit ne tient aucunement compte de possibles interactions, ni d'un ordre de priorité des variables. Ainsi, la contribution possible de ce modèle à l'étude de l'utilisation des centres de jour demeure incertaine. La majorité des variables étudiées pourraient être incluses dans un cadre conceptuel combinant le modèle d'utilisation d'Andersen et le modèle axé sur la pratique de Yeats et collaborateurs.

Zarit et collaborateurs (1999b) fondent leur étude de l'utilisation du centre de jour par des personnes atteintes de démence sur le modèle de processus du stress chez les personnes de soutien (Stress Process Model of Caregiving : Pearlin et coll., 1990). Selon ce modèle, une personne de soutien est en constant processus de stress et d'adaptation, au cours duquel la décision d'utiliser des services représente un effort pour s'adapter aux facteurs primaires de stress de la situation d'aide. Cette décision est influencée par plusieurs facteurs, dont : 
- le contexte de la situation d'aide qui inclut: les caractéristiques sociales de la personne âgée et de sa personne de soutien; le type de relation (relation maritale ou enfant - parent); la qualité de la relation avant l'apparition de la maladie; et le type de ressources auxquelles la personne de soutien a accès. Ce sont des facteurs indépendants de l'état de santé de la personne âgée mais qui influencent la réaction de la personne de soutien à la situation d'aide;

- les facteurs primaires de stress comprennent des indicateurs objectifs du niveau d'aide dont la personne âgée a besoin (incapacités fonctionnelles, troubles cognitifs, problèmes de comportement), ainsi que des indicateurs subjectifs de l'effort consacré à cette aide par la personne de soutien (fardeau ressenti);

- les facteurs secondaires de stress sont composés d'autres éléments stressants de la vie de la personne de soutien, non reliés directement à la situation d'aide, comme les conflits de responsabilités entre travail et responsabilités familiales, les problèmes économiques, etc.;

- l'évaluation que la personne de soutien fait des facteurs de stress influence son bienêtre psychologique. Ces facteurs influencent directement son besoin d'adaptation, entre autres, par le recours à des services d'aide formelle pour une prise en charge partielle (ex. : centre de jour, répit, etc.) ou totale (ex. : placement en institution) de la situation.

Selon Zarit et collaborateurs (1999b), lorsque les facteurs de stress primaires et la détresse psychologique de la personne de soutien sont élevés, l'utilisation du centre de jour sera de plus courte durée, car le service n'apportera pas le répit suffisant pour éviter le placement en institution.

Ce modèle apporte un éclairage intéressant sur l'aspect subjectif des besoins des personnes de soutien envers un service de répit comme le centre de jour. Toutefois, s'il s'applique bien à la situation de l'utilisation des centres de jour par les personnes atteintes de démence, il ne peut expliquer l'ensemble de l'utilisation des centres de jour par une clientèle générale, puisque dans ce dernier cas, le centre de jour répond à un ensemble varié de besoins qui, parfois, n'ont rien à voir avec le répit de la personne de soutien. 
L'étude de ces modèles nous amène à concevoir un modèle d'utilisation des services de centre de jour qui permettra à la fois d'analyser cette utilisation et de suggérer des façons de l'améliorer.

\section{Cadre conceptuel retenu}

Le cadre retenu sera adapté aux caractéristiques particulières des centres de jour du Québec, ainsi qu'à notre situation particulière de recherche. Il s'inspire de la typologie de facteurs décrite par Andersen (1995). Il est complété par des facteurs reliés aux caractéristiques des services, s'inspirant des travaux de Yeats et collaborateurs (1992), de Kosloski et collaborateurs (2001) et de Montoro-Rodriguez et collaborateurs (2003). Les caractéristiques propres aux services que la personne reçoit ont un impact certain sur leur capacité à répondre aux besoins identifiés. Pour des services de nature discrétionnaire, où la substitution de services est possible, ces facteurs auront aussi une influence directe sur la décision d'utiliser ou non un service particulier et de poursuivre son utilisation.

Ainsi, nous retenons premièrement deux grandes catégories de facteurs de la typologie d'Andersen : les facteurs prédisposants et les facteurs de besoin. Les facteurs de capacité personnels ou communautaires ne sont pas retenus pour cette étude pour plusieurs raisons. En premier lieu, les centres de jour sont accessibles sur tout le territoire sur lequel l'étude s'est déroulée. Ensuite, dans le contexte québécois, les services des centres de jour sont des services subventionnés pour tous les utilisateurs, sans égard à leur revenu. Enfin, les données sur le revenu présentaient un fort taux de données manquantes. Ceci est souvent le cas dans les études populationnelles, et même lorsque ces données ont été incluses dans les études d'utilisation, elles n'avaient que très rarement un effet sur l'utilisation parce que les services sont souvent subventionnés ou remboursés par des assurances (Kosloski et coll., 1999).

Deuxièmement, à l'instar de toutes les recherches récentes sur l'utilisation des centres de jour, les caractéristiques des personnes de soutien sont considérées. Comme le propose le modèle de processus du stress chez les personnes de soutien (Pearlin et coll., 
1990; Zarit et coll., 1999b), c'est surtout l'évaluation que la personne de soutien fait de la situation qui influera sur son niveau de besoin. Cette évaluation peut se mesurer par le fardeau ressenti ou par le bien-être psychologique de la personne de soutien. Cette évaluation est influencée par les caractéristiques des personnes de soutien (âge, sexe, état de santé, rôle d'aidant choisi ou imposé, durée de l'aide, etc.), ainsi que par plusieurs caractéristiques des personnes âgées (âge, sexe, type de lien avec la personne de soutien, qualité de la relation antérieure à la maladie, niveaux de besoin amenés par la maladie).

Troisièmement, pour nous adapter au contexte des centres de jour du Québec, parmi les caractéristiques des services, nous inclurons à la fois des caractéristiques de centres de jour et des caractéristiques des CLSC qui orientent les personnes âgées vers le centre de jour et qui parfois offrent même un soutien à cette participation par exemple, en aidant les personnes âgées à se préparer pour cette activité.

Le cadre conceptuel retenu est représenté graphiquement à la figure 3. Dans ce modèle, les facteurs prédisposants sont des facteurs qui sont présents avant que le besoin de services ne se fasse sentir. Ils influencent la propension à utiliser les services, mais aussi le niveau de besoin pour les services offerts en centre de jour. Les facteurs de besoin sont directement reliés aux besoins que le centre de jour tente de combler. Les caractéristiques des services sont des facteurs qui peuvent interagir avec les facteurs de besoin pour modifier les taux d'utilisation du centre de jour. En effet, pour expliquer le contact avec le centre de jour, on peut penser par exemple qu'à besoin égal, une moins grande proportion de sujets utiliseront le centre de jour d'un territoire si ce dernier présente une liste d'attente plus longue. Toutefois, si les centres de jour ont établi des critères de priorisation, l'effet de la liste d'attente pourrait être différent chez les personnes avec un niveau de besoin plus faible que chez celle avec un niveau de besoin plus élevé. Pour ce qui est de l'intensité de la participation, on peut penser qu'un certain type d'activités pourrait favoriser une participation plus grande au centre de jour pour des sous-groupes de clients (ex. : hommes vs femmes) auxquels ces activités sont mieux adaptées. 
Figure 3 : Facteurs qui influencent l'utilisation du centre de jour

\section{Facteurs prédisposants des personnes âgées \\ * Variables sociodémographiques \\ * Réseau social de la PA \\ * Utilisation antérieure du CJ \\ * Connaissance du français ou de l'anglais \\ * Attitudes envers activités de groupe}

Facteurs prédisposants des personnes de soutien

* Variables sociodémographiques

* Réseau social de la PS

* Utilisation de services de répit (autre que $\mathrm{CJ}$ )

Facteurs prédisposants des dyades personne âgée / personne de soutien

* Appartenance culturelle

* Lien avec la personne âgée

* Qualité de la relation entre PA et PS

* Attitudes envers la santé, les services formels, les responsabilités de soins dans la famille

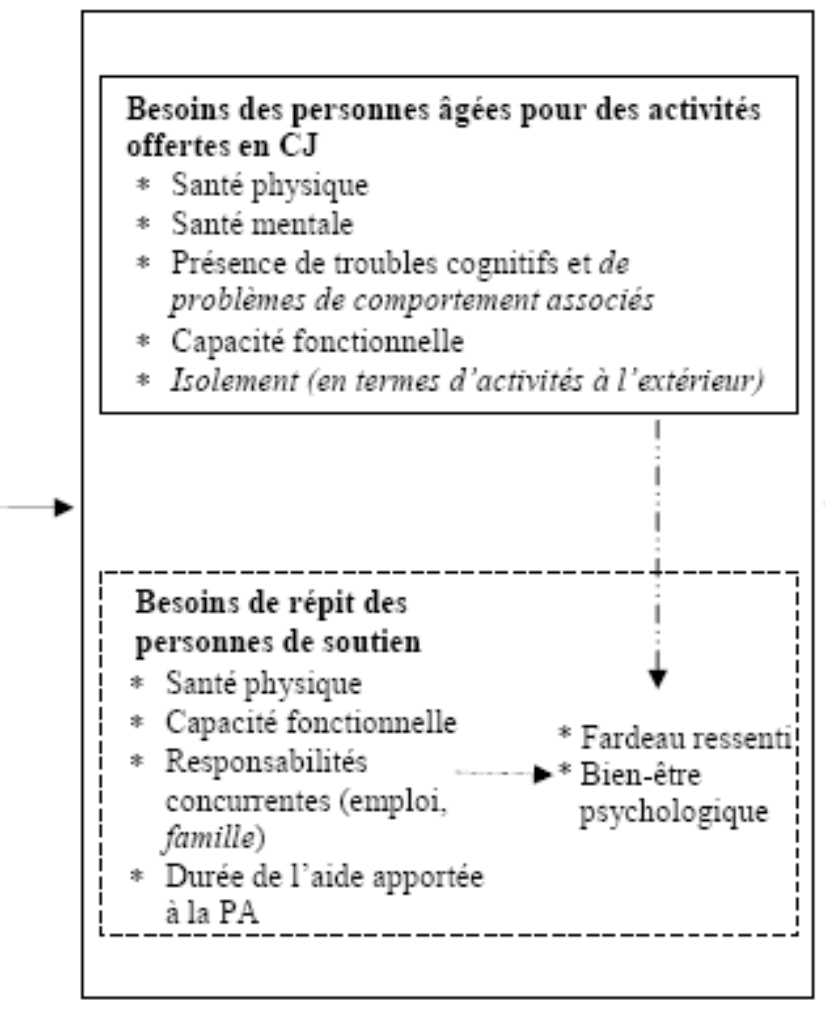

\section{Caractéristiques des services}

Centre de jour

* Admissibilité au CJ du territoire

Liste d'attente au CJ du territoire

* Téléphones de rappel

Coût du CJ du territoire

* Politique de transport et de paiement du CJ

Type d'activités offertes : groupes spécifiques

Durée et fréquence des activités offertes

CLSC

* Processus de référence an CJ

* Caractéristiques de l'intervenant pivot ou gestionnaire de cas

Type de services : SIPA ou régulier

* Intensité de l'aide reçue du CLSC pour AVQ-AVD

* Aide du CLSC pour AVQ le même jour que CJ

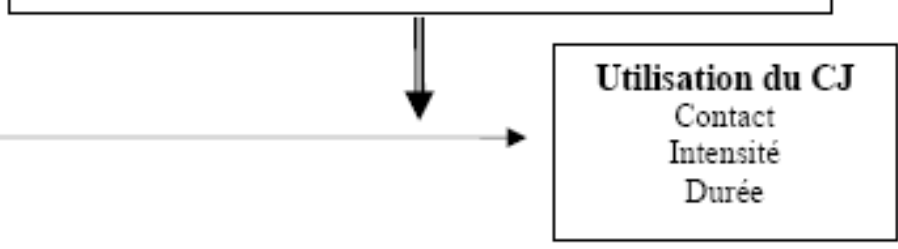

\section{Légende}

En caractères noirs : facteurs qui peuvent influencer autant la probabilité d'un contact que l'utilisation continue du service

En caractères bleus : facteurs qui influenceront surtout la probabilité d'un contact

En caractères aqua : facteurs qui influenceront surtout l'intensité d'utilisation du service et sa durée

En italiques : facteurs pour lesquels les données ne sont pas disponibles 


\section{Déterminants de l'utilisation du centre de jour}

Les facteurs qui pourraient être associés à l'utilisation du centre de jour ont été ciblés à partir de la recension des écrits sur l'utilisation des centres de jour et de services connexes, ainsi que de notre expérience personnelle en centre de jour. C'est surtout parmi les caractéristiques des services, qui nous sont apparues les moins étudiées à ce jour, que les facteurs inclus dans notre modèle seront plus innovateurs.

Les facteurs prédisposants de la personne âgée incluent l'âge, le sexe, l'éducation et le type de profession antérieure, ainsi que la présence d'un réseau de soutien pour la personne âgée. De plus, dans le contexte des services de longue durée que sont les centres de jour, services où les congés sont peu fréquents, le fait d'avoir un épisode antérieur d'utilisation du centre de jour peut être le signe que le centre de jour ne répondait pas aux goûts et aux besoins de la personne âgée, la prédisposant négativement à utiliser le service à nouveau. C'est pourquoi nous l'incluons dans les facteurs prédisposants. Dans un contexte québécois, la capacité de communiquer en français ou en anglais prédisposera aussi à poursuivre une activité de groupe. Enfin, les attitudes envers les activités de groupe influenceront aussi la prédisposition à utiliser les services de centre de jour.

Les facteurs prédisposants de la personne de soutien comprennent l'âge, le sexe, le lien avec la personne âgée, l'éducation, le type de profession antérieure ou actuelle, la présence et la qualité du réseau de la personne de soutien. Plusieurs chercheurs ont observé une réticence des personnes de soutien à utiliser des services de soutien formels pour les aider dans leur rôle ou à laisser le parent âgé en perte d'autonomie avec un étranger (Caserta et coll., 1987; Cotrell, 1996; Paquet, 1996). Dans ce contexte, l'utilisation d'autres services de répit par la personne de soutien peut être considérée comme un facteur prédisposant à l'utilisation du centre de jour, puisque ce sera une indication que la personne de soutien a moins de réticences à laisser des étrangers prendre soin de la personne âgée. 
D’autres facteurs prédisposants peuvent être considérés soit pour la personne âgée, soit pour sa personne de soutien, soit simultanément pour les deux. Il s'agit de: l'appartenance culturelle (pays d'origine, ethnie, langue parlée à la maison et religion), pour laquelle la donnée de la personne âgée est fortement corrélée à celle de la personne de soutien; du type de lien entre la personne âgée et sa personne de soutien (conjoint, enfant, amis ou autre; cohabitation); la qualité de la relation entre ces deux personnes antérieurement à la maladie, ainsi que des croyances et attitudes envers la santé, les services formels de soutien à domicile et les responsabilités de soins au sein de la famille.

Les facteurs de besoin de la personne âgée incluent des indicateurs de la santé physique et de la santé mentale, la présence de troubles cognitifs et de problèmes de comportement qui y sont associés, le degré de capacité fonctionnelle de la personne âgée, ainsi que ses hospitalisations pendant la période d'observation. De plus, le fait d'avoir peu d'autres activités à l'extérieur du domicile peut aussi être considéré comme un facteur de besoin puisqu'un des buts du centre de jour est de réduire l'isolement créé par les incapacités fonctionnelles des personnes âgées.

Les facteurs de besoin de la personne de soutien incluent des indicateurs de sa santé physique, de sa capacité fonctionnelle, ses responsabilités concurrentes comme un emploi ou des responsabilités familiales (ex. : jeunes enfants), la durée de l'aide apportée à la personne âgée, ainsi que le bien-être psychologique et le fardeau ressenti par la personne de soutien.

Les caractéristiques des services du centre de jour comprennent les critères d'admissibilité au centre de jour du territoire, la durée d'attente moyenne pour l'admission, les téléphones de rappel, les politiques de transport, le coût pour le transport et les repas, la flexibilité de la politique de paiement, le ratio de personnel du centre, la durée et le type d'activités offertes, ainsi que la fréquence de participation recommandée.

Nous pouvons supposer que l'admission d'une personne en centre de jour pourrait être retardée par la présence de liste d'attente au centre de son territoire. De plus, les 
professionnels qui font les recommandations de services peuvent être moins enclins à recommander un service pour lequel ils savent que l'attente est longue. Cela ferait qu'à besoin égal, certaines personnes pourraient ne pas avoir accès à ce service.

Certains centres font des appels la veille ou le matin même de la présence prévue au centre de jour, de façon à remémorer le rendez-vous au participant. On peut penser que de tels appels peuvent stimuler l'intensité de la participation, de même que le maintien de la participation, donc augmenter la probabilité de contact avec le centre de jour au cours d'une certaine période de temps.

Dans le contexte québécois, la majorité des centres de jour fournissent le transport aux usagers. Toutefois, des politiques différentes (utilisation de taxis ou de minibus adaptés ou utilisation du transport adapté de la Société de transport de Montréal, réservation du transport par les usagers ou par le personnel du centre) pourraient faciliter ou limiter l'utilisation du centre de jour.

La structure de personnel, tant au plan du ratio de clients / membre de personnel, qu'au plan du type de formation des intervenants peut influencer l'utilisation du centre de jour. Un plus faible ratio de clients / membre de personnel peut permettre un suivi plus personnalisé qui pourrait être de nature à favoriser la participation au centre de jour. De même, la formation du personnel peut être plus ou moins adéquate pour répondre aux besoins d'un participant donné, en fonction des besoins particuliers de ce participant.

La durée des visites, la fréquence de participation recommandée et le type d'activités offertes peuvent aussi influencer le degré de satisfaction des participants. Par exemple, certains types d'activités pourraient plaire davantage à certains sous-groupes (ex. : hommes vs femmes, personnes qui présentent ou non des troubles cognitifs, etc.).

Enfin, les caractéristiques des services reçus des CLSC peuvent influencer l'utilisation du centre de jour. Par exemple, l'intervenant pivot ou le gestionnaire de cas attitré à la personne âgée, celui qui peut ou non la diriger vers le centre de jour, a un rôle 
important. Plusieurs caractéristiques relatives à cet individu (âge, sexe, appartenance culturelle, type de formation, nombre d'années d'expérience dans la profession et dans les soins aux personnes âgées, expériences antérieures positives ou négatives avec le centre de jour du territoire) peuvent influencer sa façon d'interpréter les données sur l'état de santé de la personne âgée et le bien-être ou la détresse de sa personne de soutien pour y déceler un besoin pour les activités en centre de jour. De même, la gestion de cas plus intensive fournie par le projet SIPA (en cours au moment de cette étude) apporte, aux personnes âgées qui en bénéficient, un suivi qui pourrait être de nature à favoriser l'identification plus rapide des besoins et une meilleure adhésion aux recommandations de participation au centre de jour. Une fréquence plus élevée d'aide de la part du CLSC pour les AVQ et AVD peut être un soutien important au maintien à domicile, qui favorisera une plus grande durée d'utilisation du centre de jour en évitant un recours rapide à l'hébergement. Enfin, pour les personnes qui ont besoin d'aide pour l'hygiène et l'habillement, la présence d'aide du CLSC pour compléter ces activités les jours de présence en centre de jour pourrait faciliter la participation aux activités du centre de jour. Pour les personnes seules, sans cette aide, la participation ne serait probablement pas possible. Pour les personnes qui demeurent avec une personne de soutien, sans cette aide, la participation au centre de jour peut accentuer le fardeau de la personne de soutien en exigeant que la routine de soins soit accomplie plus rapidement les jours de présence en centre de jour ${ }^{1}$.

1 Communication personnelle : Discussion à la Table de concertation des centres de jour de Montréal-centre, le 1 er février 2002. 


\section{Limites relatives à la disponibilité des données}

Malheureusement, certaines variables identifiées comme déterminants possibles de l'utilisation du centre de jour dans le cadre conceptuel retenu ne pourront être étudiées dans la présente recherche. En effet, à l'époque où cette étude a été amorcée, on constatait un manque d'homogénéité dans les données consignées par les centres de jour concernant l'état de santé des usagers. Ainsi, l'analyse secondaire de données recueillies dans le cadre d'une étude plus large sur les services intégrés aux personnes âgées a permis d'obtenir des données valides tant sur les caractéristiques des individus que sur leurs présences en centre de jour, mais a toutefois limité les types de variables qui ont pu être prises en compte dans les analyses effectuées pour cette recherche. Ainsi, il n'y avait pas de données disponibles sur les intervenants qui dirigent les participants vers le centre de jour, ni sur les attitudes des participants envers les activités de groupe, les services de centre de jour ou les services de soutien à domicile. Il n'y avait aucune donnée sur les problèmes de comportement. Les données sur les activités sociales des participants à l'étude portaient sur le nombre de sorties par mois. Cette donnée n'a pas pu être utilisée comme mesure de l'isolement social des participants préalable à l'utilisation du centre de jour, parce que la réponse pouvait inclure les sorties pour fréquenter le centre de jour. De même, les données sur l'utilisation du répit par les personnes de soutien pouvaient inclure le centre de jour. Enfin, dans l'ensemble de l'échantillon (participants et non-participants au centre de jour), il y avait beaucoup de données manquantes dans les questionnaires aux personnes de soutien. Ainsi, les données sur la qualité de la relation entre les personnes âgées et leur personne de soutien n’ont pu être utilisées.

\section{Phénomènes d'utilisation}

Dans la présente étude, nous nous penchons sur deux phénomènes d'utilisation des centres de jour :

1) Le contact ou le fait d'utiliser le centre de jour au cours d'une certaine période de temps : Comme la figure 1 le démontre, un premier contact avec le centre de jour 
survient lorsqu'un intervenant recommande à une personne âgée ou à sa personne de soutien les services offerts par le centre de jour et que la personne âgée accepte de s'y présenter pour une évaluation. Le recours au centre de jour se poursuit si la personne âgée (ou sa personne de soutien) juge que la fréquentation d'un centre de jour est une intervention valable pour répondre au besoin identifié. Cette étude cherche à cerner les caractéristiques qui distinguent, parmi un groupe de personnes âgées qui présentent des incapacités, celles qui ont eu au moins un contact avec le centre de jour au cours d'une période donnée.

2) L'intensité de la participation : Dans le contexte québécois où l'accès au centre de jour est souvent limité par les listes d'attente, l'intensité de la participation peut relever à la fois du personnel du centre de jour qui décidera de la fréquence de participation à offrir et de la personne âgée elle-même pour ce qui est du degré de conformité avec la recommandation de fréquentation ou de la régularité de la participation. Dans cette étude, nous nous attarderons à la régularité de la participation, une mesure de l'adhésion au centre de jour, définie comme le taux participations réelles / participations prévues. Ce taux peut être calculé à partir des journées de fréquentation prévues pour chaque personne, en excluant les journées où le centre de jour était fermé, ainsi que celles où la personne était hospitalisée ou placée en hébergement temporaire, et donc dans l'impossibilité de fréquenter le centre de jour. Cette mesure permettra de distinguer les caractéristiques des personnes qui ont une participation soutenue de celles qui ont une participation irrégulière au centre de jour.

\section{Les activités offertes en centre de jour}

L'influence des caractéristiques des services sur l'utilisation du centre de jour a été peu étudiée. Parmi ces caractéristiques, l'influence du type d'activités offertes en centre de jour l'est encore moins. Ce n'est pas étonnant, puisqu'un ensemble très diversifié d'activités est offert dans différents centres de jour et qu'il n'y a pas de classification reconnue permettant d'opérationnaliser cette variable dans des études quantitatives. Nous 
nous sommes donc attardés à décrire les activités qui sont offertes par les centres de jour, ainsi qu'à établir une classification qui permettra d'opérationnaliser cette variable afin de pouvoir l'utiliser dans notre étude de la régularité de la participation au centre de jour.

\section{Les objectifs de cette recherche}

Le but de la présente recherche est d'approfondir les connaissances des facteurs qui influencent l'utilisation des centres de jour par les personnes âgées qui présentent des incapacités. Elle comporte trois groupes d'objectifs dont les résultats sont présentés dans les trois articles inclus dans la présente thèse :

Objectifs de l'article 1: Estimer la proportion d'utilisateurs de centre de jour parmi une clientèle âgée qui présente des incapacités, caractériser ces utilisateurs et ce qui les distingue des non-utilisateurs et analyser les déterminants du contact avec le centre de jour.

Objectifs de l'article 2 : Identifier des groupes d'utilisateurs de centre de jour ayant des caractéristiques semblables, identifier les activités qui sont offertes par les centres de jour, classifier ces activités et déterminer l'adéquation entre les activités auxquelles participent les divers groupes d'utilisateurs et leurs caractéristiques de santé.

Objectifs de l'article 3: Pour le groupe d'utilisateurs, décrire la régularité de la participation au centre de jour et définir les facteurs associés à la régulatité de la participation.

\section{Adaptation du cadre théorique global aux objectifs de recherche relatifs aux déterminants de l'utilisation}

Les divers facteurs présentés dans le cadre théorique sont susceptibles d'influencer de manière différente l'utilisation du centre de jour selon que l'on s'intéresse au contact avec le centre de jour ou à la régularité de la participation. Par conséquent, des modèles 
légèrement différents ont été utilisés pour analyser ces deux phénomènes d'utilisation du centre de jour.

Ainsi, pour le premier article qui porte sur le contact avec le centre de jour au moment de l'étude et ce qui distingue les utilisateurs des non-utilisateurs de centre de jour, le modèle adapté est présenté à la figure 1 de l'article 1 (voir page 133). Lors de cette analyse, les différentes caractéristiques de la personne de soutien n'ont pas été considérées étant donné le nombre de questionnaires aux personnes de soutien qui étaient manquants ou incomplets. La prise en compte de ces variables aurait entraîné une réduction importante de la taille de l'échantillon de l'étude. Les facteurs prédisposants considérés sont l'âge, le sexe, l'appartenance culturelle, l'éducation de la personne âgée, le type de profession antérieure de la personne âgée ou de son conjoint, la capacité de la personne âgée à communiquer en français ou en anglais, la présence d'un épisode antérieur d'utilisation du centre de jour, ainsi que comme indicateur du réseau de soutien, la cohabitation ou non avec une personne de soutien. Les facteurs de besoin comprennent des indicateurs de la santé physique et de la santé mentale, la présence de troubles cognitifs, le degré de capacité fonctionnelle de la personne âgée. Les caractéristiques des services du centre de jour incluent l'admissibilité au centre de jour du territoire (critères d'exclusion), la durée d'attente moyenne pour l'admission au centre de jour, le coût demandé par le centre de jour, la présence de téléphones de rappel, ainsi que la structure de personnel. Les caractéristiques des services des CLSC incluent le type de services: services intégrés (SIPA) ou services réguliers et la fréquence de l'aide reçue du CLSC pour les AVQ et les AVD.

Pour le troisième article qui vise à définir les facteurs associés à la régularité de la participation au centre de jour, le modèle adapté est présenté à la figure 1 de l'article 3 (voir page 221). Pour cette analyse, bien que l'échantillon soit plus petit puisqu'il ne comprend que les sujets ayant au moins une présence au centre de jour, la proportion de questionnaires aux personnes de soutien manquants ou incomplets est plus faible. Ainsi, nous avons choisi d'inclure les principales caractéristiques de la personne de soutien dans 
l'analyse, tout en faisant preuve de parcimonie pour éviter un trop grand nombre de variables par rapport à la taille de l'échantillon. Ainsi, les facteurs prédisposants considérés sont l'âge, le sexe, l'appartenance culturelle, l'éducation de la personne âgée, le type de profession antérieure de la personne âgée ou de son conjoint, la capacité de la personne âgée à communiquer en français ou en anglais, la présence d'un épisode antérieur d'utilisation du centre de jour, ainsi qu'une variable combinant le type de lien avec la personne de soutien et la cohabitation ou non avec cette personne comme indicateur du réseau de soutien. Les facteurs de besoin comprennent des indicateurs de la santé physique et de la santé mentale, la présence de troubles cognitifs, le degré de capacité fonctionnelle de la personne âgée. Ils comprennent aussi l'âge, le sexe et la capacité fonctionnelle de la personne de soutien, la durée de l'aide, ainsi que le fardeau subjectif de la personne de soutien. Les caractéristiques des services reçus du centre de jour incluent la durée d'une présence au centre de jour, la fréquence de participation recommandée, ainsi que le type d'activités auxquelles la personne participe. Les caractéristiques des services reçus des CLSC incluent le type de services: services intégrés (SIPA) ou services réguliers, la fréquence de l'aide reçue du CLSC pour les AVQ et les AVD, ainsi que la présence d'aide du CLSC lors des jours de participation au centre de jour. 



\section{Chapitre 4 : Méthodes de recherche}

\section{Stratégies de recherche}

Il s'agit d'une étude synthétique comparative fondée sur l'analyse statistique d'un ensemble de relations interdépendantes dans le but de tester un modèle théorique. (Contandriopoulos, Champagne, Potvin, Denis et Boyle, 1990). La recherche s'appuie sur des informations provenant de deux bases de données existantes, ainsi que sur une collecte originale de données. Les deux bases de données existantes sont :

1) Les données tant sociodémographiques que reliées à la santé physique et psychologique des personnes âgées et des personnes de soutien, recueillies dans le cadre du projet SIPA : Services intégrés pour personnes âgées.

2) Les données statistiques recueillies de façon routinière par quatre des cinq centres de jour qui desservent ce même territoire, à l'aide d'un logiciel commun : Jonction et diffusion informatique (JEDI) ${ }^{2}$. Ce logiciel fut conçu spécialement pour recueillir des données sur les clients, les activités et les présences aux centres de jour.

Ces informations ont été complétées par une cueillette originale de données dans les dossiers du cinquième centre de jour (qui n'utilise pas le logiciel JEDI), cueillette de données planifiée en collaboration avec l'équipe du projet SIPA. De plus, des entrevues avec les coordonnateurs de centres de jour ont permis de préciser les caractéristiques des activités offertes, ainsi que les ressources, les procédures et les critères d'admission propres à chacun des centres de jour.

${ }^{2}$ Le logiciel JEDI (pour Jonction et diffusion informatiques, la compagnie qui a créé ce logiciel), est un système de bases de données et de rapports périodiques créé sur la plateforme Access de Microsoft et conçu spécialement pour recueillir des données sur les clients, les activités et les présences en centre de jour. 
Enfin, un groupe d'experts, composé de membres du Groupe d'intérêt des responsables de centres de jour de Montréal, a été rencontré dans le but de procéder à une classification des activités en centre de jour, à partir des activités recensées dans les registres d'activités des divers centres de jour. Cette classification était préalable à l'étude de l'adéquation entre les activités des centres de jour et les caractéristiques de santé des utilisateurs.

\section{Population à l'étude}

Les personnes admises au projet SIPA ont été recrutées par le biais des services de soutien à domicile de trois Centres locaux de services communautaires (CLSC) de la région de Montréal. Ces personnes devaient vivre à domicile au début de l'étude et présenter des incapacités fonctionnelles indiquées par un résultat de -10 ou moins au Système de mesures de l'autonomie fonctionnelle (SMAF) (Desrosiers, Bravo, Hébert et Dubuc, 1995; Hébert, Carrier et Bilodeau, 1988). Elles devaient aussi accepter de participer à une étude randomisée sur les services intégrés pour personnes âgées et pouvoir s'exprimer en français ou en anglais avec les membres du projet SIPA, ou avoir un proche en mesure de le faire (Béland et coll., 2001; 2006a; 2006b).

Il s'agit donc d'une partie de la population âgée habituellement identifiée comme présentant des limitations d'activités de modérées à graves. Dans une étude populationnelle réalisée par Béland, Haldemann, Martin, Bourque, Ouellette et Lavoie (1998), on observe que de 12 à $16 \%$ de la population âgée de 65 ans et plus présentent des incapacités correspondant à un résultat de -10 ou moins au SMAF. Ces données sont cohérentes avec une étude de Développement Ressources humaines Canada (2003) qui trouvait des taux d'incapacités graves allant de 11 à $19 \%$ selon les provinces, chez la population âgée de 65 ans et plus du Canada.

Dans le cadre du projet SIPA, 1230 personnes âgées vulnérables de la région de Montréal ont été recrutées. Parmi celles-ci, des sous-groupes différents ont été inclus dans chacun des volets de la présente étude. La sélection des personnes incluses et exclues dans 
chacun de ces volets est décrite dans le texte qui suit, ainsi que de façon graphique à la figure 4 .

Pour le premier article qui définit les caractéristiques des utilisateurs de centre de jour et ce qui les distingue des non-utilisateurs, l'échantillon de départ est composé de 812 personnes âgées de 65 ans ou plus, toujours suivies par l'équipe de recherche SIPA à la fin de la première phase du projet, en mai 2000. De ce nombre, 44 personnes hébergées de façon permanente au moment du questionnaire, 2 personnes hospitalisées pour toute la durée de la période d'observation, ainsi que 43 sujets qui présentaient des données manquantes pour des variables importantes à l'étude ont été exclus des analyses. L'échantillon final comprend donc 723 sujets. Certaines différences entre les sujets inclus et exclus sont observées dans la proportion de sujets pour lesquels le questionnaire est rempli par un substitut. Les sujets exclus sont plus nombreux à présenter de trop grandes incapacités fonctionnelles pour répondre aux questions par eux-mêmes ou à ne pas avoir une connaissance suffisante du français ou de l'anglais. Pour plus d'information à ce sujet, voir l'article 1.

De ces 723 personnes, 136 ont participé aux activités d'un centre de jour pendant la période étudiée. Toutefois, les données sur les activités auxquelles ces personnes ont participé étaient manquantes pour quatre d'entre elles. Ainsi, pour la seconde partie de cette étude qui vise à décrire les activités auxquelles ces personnes ont participé lors de leurs présences au centre de jour et à découvrir les liens entre les activités offertes et les caractéristiques des participants au centre de jour, l'échantillon final est de 132 participants. 
Figure 4 : Participants inclus à chacune des étapes de cette étude sur les centres de jour

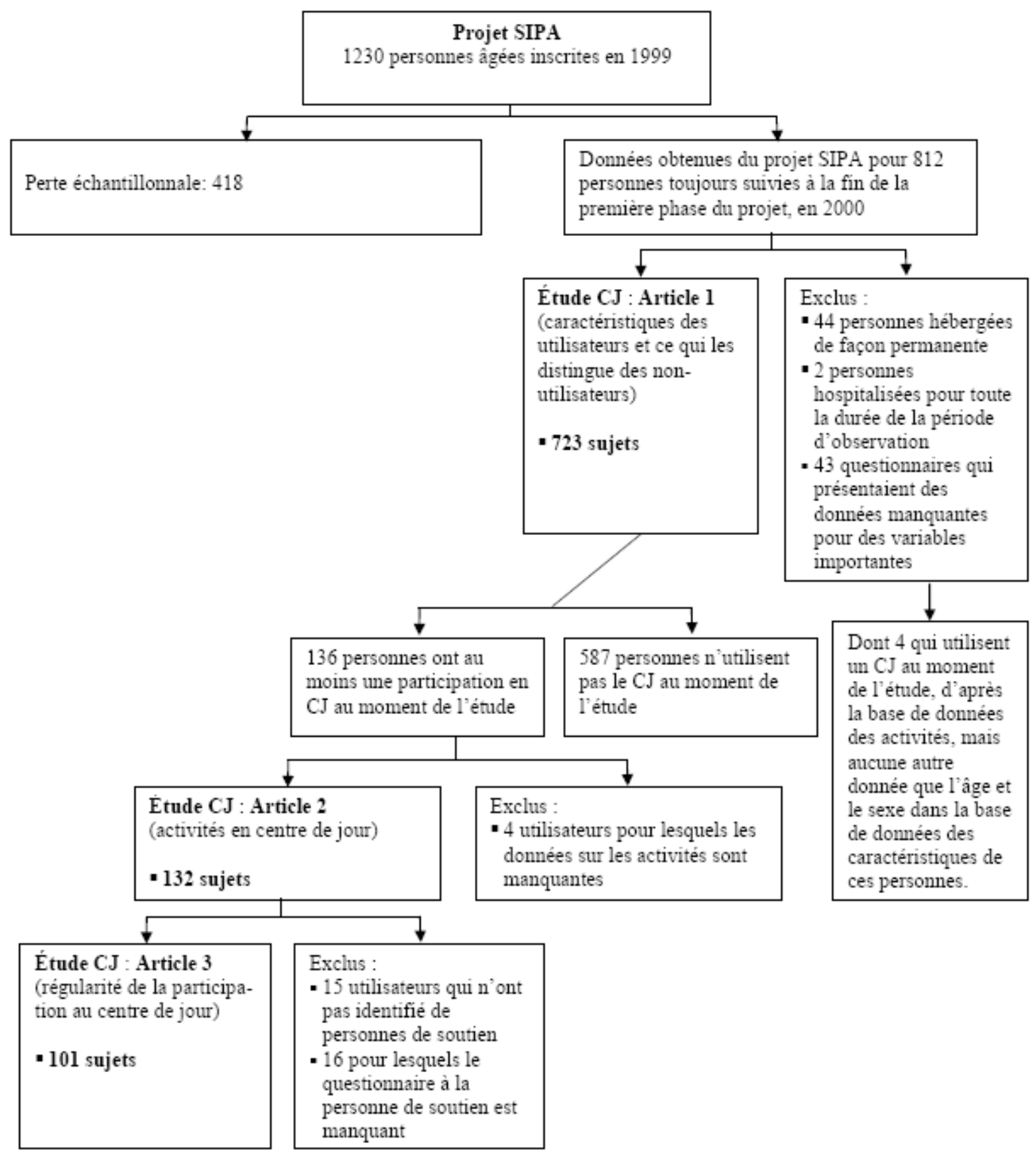


Puisque nous avons choisi d'inclure les caractéristiques des personnes de soutien pour le troisième article, nous avons aussi dû exclure des analyses les 15 participants qui n'avaient pas identifié de personne de soutien et les 16 pour lesquels le questionnaire à la personne de soutien manquait. Ainsi, pour cette partie de l'étude qui vise à identifier les déterminants de la régularité d'utilisation du centre de jour, l'échantillon final est de 101 participants.

Pour ces deux dernières parties, la seule différence statistiquement significative entre les caractéristiques démographiques et de santé des sujets inclus et exclus concerne le lien avec la personne de soutien : pour les sujets exclus, la personne de soutien est plus rarement le conjoint. Pour plus d'information à ce sujet, voir les articles 2 et 3 .

\section{Période d'observation}

Les observations se centrent autour du questionnaire T1 du projet SIPA, qui s'est échelonné du 29 mai au 10 novembre 2000. C'est la période pour laquelle nous disposons du maximum d'information sur les déterminants possibles de l'utilisation (le questionnaire aux personnes de soutien n'est disponible qu'à T1). Pour chaque individu, l'utilisation du centre de jour a été observée pendant une période de six mois, soit de trois mois avant à trois mois après la date du questionnaire. Cette période a été retenue en considérant deux facteurs importants. Puisque les caractéristiques de santé des personnes âgées sont susceptibles de changer rapidement, une courte période d'observation devait être privilégiée. Elle devait toutefois être suffisamment longue pour englober un échantillon représentatif des participations au centre de jour, malgré les absences qui peuvent être fréquentes pour certaines personnes.

\section{Méthodes de collectes de données}

Les caractéristiques des sujets et de leur personne de soutien ont été recueillies à la fin de la phase 1 du projet SIPA (mai à novembre 2000) par l'équipe de recherche SIPA, à l'aide des questionnaires remplis lors d'entrevues réalisées auprès des personnes âgées et de 
leurs personnes de soutien. Pour les personnes âgées incapables de répondre elles-mêmes au questionnaire, un substitut a été utilisé.

Un relevé de l'utilisation des services de santé par les personnes âgées et leurs personnes de soutien (lorsque ces dernières y ont consenti) a été réalisé à partir des bases de données des organismes et des établissements de santé (RAMQ, Agence de la santé et des services sociaux, hôpitaux, CLSC, centres de jour, etc.). Pour les centres de jour, les données d'utilisation proviennent des données statistiques recueillies de façon routinière par quatre des cinq centres de jour qui desservent ce même territoire, à l'aide du logiciel JEDI, conçu spécialement pour recueillir des données administratives sur les clients (ex. : adresse, téléphone, jours de fréquentation prévus), les activités et les présences aux centres de jour. Les mêmes informations ont été recueillies à partir du registre des présences et de l'horaire des activités consigné au dossier du client, pour le cinquième centre qui n'utilisait pas ce logiciel. Nous avons planifié cette cueillette dans les dossiers cliniques du cinquième centre de jour en collaboration avec l'équipe du projet SIPA, afin qu'elle réponde à la fois aux besoins de notre étude et à ceux du projet SIPA.

De plus, nous avons effectué des entrevues avec les coordonnateurs des cinq centres de jour à l'étude pour préciser diverses caractéristiques organisationnelles de ces centres : objectifs, critères d'admission et d'exclusion, liste d'attente, structure du personnel, capacité, caractéristiques des activités offertes, date des fermetures du centre de jour, coût, procédures pour le repas et les transports, etc. Le guide d'entrevue est joint à l'annexe III.

\section{Définition des variables}

Différentes variables sont mesurées pour chacun des trois groupes d'objectifs de cette étude. Elles sont présentées au tableau IV. Elles sont aussi décrites en détail à la section méthodologique de chacun des trois articles qui décrivent les résultats de cette thèse. Le tableau IV présente chacun des concepts évalués, les variables retenues pour représenter le concept, les indicateurs ou les échelles de mesure utilisés, ainsi que les objectifs de l'étude pour lesquels ils ont été utilisés. 
Lorsque des variables continues ont aussi été traitées de façon dichotomique, le score seuil pour identifier un problème selon la documentation scientifique (ex : Échelle de dépression gériatrique, Short Portable Mental Status Questionnaire, etc.), ou en l'absence d'un tel score seuil, la valeur médiane observée dans l'échantillon pour la variable en question (ex : âge, capacités fonctionnelles, etc.), ont servi à diviser les participants en deux groupes.

Certaines variables indépendantes, décrites au tableau IV, ont été mesurées à l'aide d'outils de mesure standardisés. Les qualités métrologiques de ces échelles de mesure sont présentées au tableau V. 
Tableau IV : Description des variables

\begin{tabular}{|c|c|c|c|c|c|}
\hline \multirow[t]{2}{*}{ Concepts } & \multirow[t]{2}{*}{ Variables et indicateurs } & \multirow[t]{2}{*}{ Source } & \multicolumn{3}{|c|}{ Utilisation } \\
\hline & & & Objectif 1 & Objectif 2 & Objectif 3 \\
\hline \multicolumn{6}{|l|}{ VARIABLES DEPENDANTES } \\
\hline Contact avec le centre de jour & $\begin{array}{l}\text { Présences au centre de jour : Les participants qui ontau } \\
\text { moins une présence au CJ pendant la période } \\
\text { d'observation sont définis comme utilisateurs }\end{array}$ & $\begin{array}{l}\text { Registre présences: } \\
\text { JEDI } \\
\text { dossiers du } 5^{\mathrm{e}} \mathrm{CJ}\end{array}$ & $\mathrm{X}$ & & \\
\hline Activités du centre de jour & $\begin{array}{l}\text { Activités dont le participant a bénéficié lors de ses } \\
\text { présences au centre de jour. }\end{array}$ & $\begin{array}{l}\text { Registre activités: } \\
\text { JEDI } \\
\text { dossiers du } 5 \text { e CJ }\end{array}$ & & $x$ & $\begin{array}{l}\text { En variable } \\
\text { indépendante, } \\
\text { voir plus bas }\end{array}$ \\
\hline Intensité de la participation & $\begin{array}{l}\text { Conformité à la recommandation de participation, } \\
\text { calculée en pourcentage : participation réelle / nombre de } \\
\text { participations prévues pendant la période d'observation. } \\
\text { Les participations prévues sont fonction de la fréquence } \\
\text { d'inscription, mais excluent les journées où le centre de } \\
\text { jour était fermé, ainsi que celles où la personne était } \\
\text { hospitalisée ou placée en hébergement temporaire, et } \\
\text { donc dans l'impossibilité de fréquenter le centre de jour. }\end{array}$ & $\begin{array}{l}\text { Participation réelle et fréquence } \\
\text { d'inscription : } \\
\text { - registres de présences } \\
\text { Dates de fermeture de CJ : } \\
\text { - entrevues aux coordonnateurs } \\
\text { Hospitalisations : } \\
\text { - Données MedEcho } \\
\text { obtenues par SIPA } \\
\text { Hébergements : } \\
\text { - Données de l'Agence } \\
\text { régionale obtenues par SIPA }\end{array}$ & & & $\mathrm{x}$ \\
\hline
\end{tabular}




\begin{tabular}{|c|c|c|c|c|c|}
\hline \multirow[t]{2}{*}{ Concepts } & \multirow[t]{2}{*}{ Variables et indicateurs } & \multirow{2}{*}{ Source } & \multicolumn{3}{|c|}{ Utilisation } \\
\hline & & & Objectif 1 & Objectif 2 & Objectif 3 \\
\hline \multicolumn{6}{|c|}{ VARIABLES INDEPENDANTES } \\
\hline \multicolumn{6}{|c|}{ Facteurs prédisposants de la personne âgée } \\
\hline Variables démographiques & $\begin{array}{l}\text { Âge (en années) : calculé avec date du questionnaire } \\
\text { moins date de naissance } \\
\text { Âge dichotomisé : } 85 \text { ans et plus vs plus jeunes } \\
\text { Sexe : masculin ou féminin }\end{array}$ & Questionnaire SIPA à la PA & $x$ & $\begin{array}{l}\mathrm{D} \\
\mathrm{X} \\
\mathrm{X}\end{array}$ & $\begin{array}{l}x \\
x\end{array}$ \\
\hline Structure sociale & $\begin{array}{l}\text { État civil : } \\
\text { - célibataire (jamais marié) } \\
\text { - marié ou conjoint de fait } \\
\text { - veuf } \\
\text { - séparé ou divorcé } \\
\text { - religieux } \\
\text { Éducation : scolarité sur une échelle à } 6 \text { niveaux : } \\
1: \text { aucune } \\
2: \text { primaire non complété } \\
3: \text { primaire complété } \\
4: \text { secondaire } \\
5: \text { école technique ou école de métier } \\
6: \text { universitaire } \\
\text { Proportion avec éducation universitaire } \\
\text { Éducation } \\
\text { - aucune ou primaire } \\
\text { - secondaire ou école technique ou école de métier } \\
\text { - universitaire } \\
\text { Profession antérieure : travail rémunéré que la personne } \\
\text { âgée ou son conjoint (le cas échéant) ont exercé le plus } \\
\text { longtemps au cours de leur vie, sur une échelle } \\
\text { dichotomique: } \\
1: \text { au moins un membre du couple a travaillé dans le } \\
\text { domaine de la santé ou des services sociaux } \\
0: \text { aucun membre du couple n'avait un travail dans le } \\
\text { domaine de la santé ou des services sociaux } \\
\text { Proportion des répondants qui estime que leur revenu } \\
\text { répond difficilement à leurs besoins }\end{array}$ & Questionnaire SIPA à la PA & $\mathrm{D}$ & $\mathrm{D}$ & $\mathrm{D}$ \\
\hline
\end{tabular}

Légende:

$X$ : utilisé dans toutes les analyses (descriptives, bivariées et multivariées)

$D:$ utilisé de manière descriptive et dans des analyses bivariées seulement

Les numéros indiqués après le nom d'un outil d'évaluation renvoient à la description de cet outil dans le tableau V. 


\begin{tabular}{|c|c|c|c|c|c|}
\hline \multirow[t]{2}{*}{ Concepts } & \multirow[t]{2}{*}{ Variables et indicateurs } & \multirow[t]{2}{*}{ Source } & \multicolumn{3}{|c|}{ Utilisation } \\
\hline & & & Objectif 1 & Objectif 2 & Objectif 3 \\
\hline Structure sociale (suite) & $\begin{array}{l}\text { Appartenance culturelle : } \\
\text { Pays de naissance = Canada ou autre }\end{array}$ & & $x$ & $x$ & $x$ \\
\hline & Religion = Catholique, juive ou autre & & $\mathrm{D}$ & & D \\
\hline & $\begin{array}{l}\text { Langue d'usage: } \\
\text { - français à la maison et avec professionnels } \\
\text { - anglais à la maison et avec professionnels } \\
\text { - anglais ou français avec les professionnels } \\
\text { (pas à la maison) } \\
\text { - autre langue à la maison, langue avec les } \\
\text { professionnels est manquante } \\
\text { - autre langue même avec les professionnels }\end{array}$ & Questionnaire SIPA à la PA & $\mathrm{D}$ & $\mathrm{D}$ & $\mathrm{D}$ \\
\hline $\begin{array}{l}\text { Réseau de soutien social de la } \\
\text { personne âgée }\end{array}$ & $\begin{array}{l}\text { Lien PA-PS - aucune PS } \\
\text { - conjoint(e) } \\
\text { - autre co-résident (enfant) } \\
\text { - autre, non co-résident (enfant) } \\
\text { Lien PA-PS dichotomisé : cohabite ou non } \\
\text { Lien PA-PS dichotomisé : conjoint ou non }\end{array}$ & $\begin{array}{l}\text { Questionnaire SIPA à la PA: } \\
\text { question sur le type de lien } \\
\text { (Conjoint, enfant, autre) et sur la } \\
\text { cohabitation avec la personne. }\end{array}$ & $x$ & D & $\begin{array}{c}\text { D } \\
\text { Les personnes qu } \\
\text { n'ont aucune PS } \\
\text { sont exclues de } \\
\text { cette analyse } \\
\text { X } \\
\text { X }\end{array}$ \\
\hline Utilisation antérieure du CJ & $\begin{array}{l}\text { Utilisation antérieure du CJ pour un autre épisode de } \\
\text { soins survenu entre } 1995 \text { et T1: } \\
\text { - contact (une seule visite enregistrée pour cet autre } \\
\text { épisode) } \\
\text { - participation (plus d'une visite lors de cet autre épisode) }\end{array}$ & $\begin{array}{l}\text { Registre présences: } \\
\text { JEDI } \\
\text { dossiers du } 5 \mathrm{e} \text { CJ }\end{array}$ & & & $x$ \\
\hline $\begin{array}{l}\text { Capacité à suivre une activité } \\
\text { de groupe dans le contexte } \\
\text { canadien }\end{array}$ & $\begin{array}{l}\text { Connaissance de l'anglais ou du français : à la question } \\
\text { sur la langue d'usage décrite ci-dessus, les deux } \\
\text { dernières catégories sont considérées comme n'ayant } \\
\text { pas une connaissance suffisante de l'une ou l'autre des } \\
\text { deux langues officielles }\end{array}$ & Questionnaire SIPA à la PA & $x$ & & $x$ \\
\hline
\end{tabular}




\begin{tabular}{|c|c|c|c|c|c|}
\hline \multirow[t]{2}{*}{ Concepts } & \multirow[t]{2}{*}{ Variables et indicateurs } & \multirow[t]{2}{*}{ Source } & \multicolumn{3}{|c|}{ Utilisation } \\
\hline & & & Objectif 1 & Objectif 2 & Objectif 3 \\
\hline \multicolumn{6}{|c|}{ Facteurs de besoin de la personne âgée } \\
\hline \multirow[t]{6}{*}{ Santé physique } & $\begin{array}{l}14 \text { problèmes de santé physique parmi une liste de } \\
\text { problèmes tirée de l'étude EPESE (Coroni-Huntley et } \\
\text { coll., 1986) : } \\
\text { Déjà eu cataractes } \\
\text { Mal jointures ou os (rhumatismes) } \\
\text { Problèmes de vessie, reins ou prostate } \\
\text { Problèmes de circulation } \\
\text { Maladie de cœur } \\
\text { Haute tension } \\
\text { Accident vasculaire cérébral (AVC) } \\
\text { Problèmes respiratoires } \\
\text { Déjà eu problèmes d'estomac } \\
\text { Diabète } \\
\text { Déjà eu tumeur ou cancer } \\
\text { Fracture hanche ou fémur dans l'année } \\
\text { Déjà eu glaucome } \\
\text { Maladie de Parkinson }\end{array}$ & Questionnaire SIPA à la PA & D & & D \\
\hline & Présence d'un AVC analysé séparément & & $\mathrm{X}$ & & $\mathrm{X}$ \\
\hline & $\begin{array}{l}\text { Nombre de problèmes sur 13, excluant AVC de la liste } \\
\text { Nombre de problèmes sur 13: de la liste ci-dessus, } \\
\text { glaucome et cataractes sont regroupés en un prob. }\end{array}$ & & $\mathrm{X}$ & D & $\mathrm{X}$ \\
\hline & $\begin{array}{l}\text { Valeur dichotomisée : } 5 \text { ou plus vs } 4 \text { ou moins } \\
\text { Deux types de problèmes de santé physique : } \\
\text {-présence d'un problème qui entraîne des incapacités } \\
\text { locomotrices (AVC, Parkinson et fracture de hanche ou } \\
\text { de jambe récente) }\end{array}$ & & & $\begin{array}{l}x \\
x\end{array}$ & \\
\hline & $\begin{array}{l}\text {-présence d'au moins un autre problème parmi : maladie } \\
\text { de cœur; problèmes respiratoires; diabète; tumeur; } \\
\text { problèmes de vessie, de reins ou de prostate; problèmes } \\
\text { d'estomac. }\end{array}$ & & & $\mathrm{X}$ & \\
\hline & $\begin{array}{l}\text { Hospitalisation : } \\
\text { Nombre de jours d'hospitalisation pendant la période } \\
\text { d'observation }\end{array}$ & $\begin{array}{l}\text { Hospitalisation : } \\
\text { - Données MedEcho } \\
\text { obtenues par SIPA }\end{array}$ & $X$ & $X$ & $\mathrm{x}$ \\
\hline
\end{tabular}

Légende:

$X$ : utilisé dans toutes les analyses (descriptives, bivariées et multivariées)

$\mathrm{D}$ : utilisé de manière descriptive et dans des analyses bivariées seulement

Les numéros indiqués après le nom d'un outil d'évaluation renvoient à la description de cet outil dans le tableau V. 


\begin{tabular}{|c|c|c|c|c|c|}
\hline \multirow[t]{2}{*}{ Concepts } & \multirow[t]{2}{*}{ Variables et indicateurs } & \multirow[t]{2}{*}{ Source } & \multicolumn{3}{|c|}{ Utilisation } \\
\hline & & & Objectif 1 & Objectif 2 & Objectif 3 \\
\hline Santé mentale & $\begin{array}{l}\text { Question de l'étude EPESE sur la présence de } \\
\text { problèmes émotifs (incluant nervosité, dépression, } \\
\text { anxiété, insomnie). } \\
\text { Présence de symptômes dépressifs tels qu'indiqués à } \\
\text { l'Échelle de dépression gériatrique, version courte } \\
\text { (EDG/GDS-15) (Sheikh et Yesavage, 1986)1 } \\
\text { - variable continue : résultat à l'EDG/15 } \\
\text { variables dichotomiques : } \\
\text { - dépression probable (10 ou plus à l'ÉDG) } \\
\text { - dépression possible (6 ou plus à l'ÉDG) }\end{array}$ & $\begin{array}{l}\text { Questionnaire SIPA à la PA } \\
\text { Questionnaire SIPA à la PA }\end{array}$ & $\begin{array}{l}D \\
D\end{array}$ & $\begin{array}{l}\text { données } \\
\text { manquantes } \\
\text { cotées comme } \\
\text { absence de } \\
\text { symptômes } \\
\text { X }\end{array}$ & $x$ \\
\hline Troubles cognitifs & $\begin{array}{l}\text { Présence de troubles cognitifs : } \\
\text { Pour les sujets qui répondent au questionnaire par eux- } \\
\text { mêmes : selon le résultat au Short Portable Mental } \\
\text { Status Questionnaire (SPMSQ) (Pfeiffer, 1975) }{ }^{2} \\
\text { Lorsqu'un substitut répond au questionnaire : la raison } \\
\text { est la détérioration cognitive ou une réponse positive à la } \\
\text { question de l'étude EPESE sur la présence de problèmes } \\
\text { de mémoire ou de maladie d'Alzheimer, confirmée par un } \\
\text { médecin }\end{array}$ & Questionnaire SIPA à la PA & $x$ & $x$ & $x$ \\
\hline Capacité fonctionnelle & $\begin{array}{l}\text { Activités de la vie quotidienne originalement mesurées } \\
\text { par l'IIndice de Barthel (Granger et coll., 1979) }{ }^{3} \text { et } \\
\text { activités de la vie domestique mesurées par } 7 \text { items } \\
\text { d'AVD du questionnaire Older American Resources and } \\
\text { Services (OARS) (Fillenbaum, 1988). } \\
\text { Pour créer un score unique incluant AVQ et AVD, les } \\
\text { résultats de l'Indice de Barthel ont été transformés pour } \\
\text { obtenir l'équivalent des } 7 \text { items d'AVQ du questionnaire } \\
\text { OARS et additionnés aux résultats d'AVD du } \\
\text { questionnaire OARS. C'est ce résultat (OARS / 28) qui } \\
\text { est utilisé dans les analyses. } \\
\text { Des scores partiels (AVQ, AVD, incontinence) sont aussi } \\
\text { présentés dans certains tableaux descriptifs. } \\
\text { Variable dichotomique: } 19 \text { ou + vs } 18 \text { ou - (I28) }\end{array}$ & Questionnaire SIPA à la PA & D & $x$ & $\mathrm{D}$ \\
\hline
\end{tabular}




\begin{tabular}{|c|c|c|c|c|c|}
\hline \multirow[t]{2}{*}{ Concepts } & \multirow[t]{2}{*}{ Variables et indicateurs } & \multirow[t]{2}{*}{ Source } & \multicolumn{3}{|c|}{ Utilisation } \\
\hline & & & Objectif 1 & Objectif 2 & Objectif 3 \\
\hline Capacité fonctionnelle (suite) & $\begin{array}{l}\text { Problèmes sensoriels: } \\
\text { - aucun problème sensoriel } \\
\text { - au moins un prob. avec un peu de difficulté } \\
\text { - au moins un prob. avec beaucoup de difficulté } \\
\text { Variable dichotomique isolant «beaucoup de difficulté } \\
\text { dans au moins une sphère sensorielle » } \\
\text { Difficulté de communication } \\
\text { - aucune } \\
\text { - un peu de difficulté } \\
\text { - beaucoup de difficulté } \\
\text { Variable dichotomique isolant « beaucoup de difficulté » }\end{array}$ & $\begin{array}{c}\text { Questionnaire SIPA à la PA : } \\
4 \text { questions demandant à la } \\
\text { personne si elle a pas, peu ou } \\
\text { beaucoup de difficulté à } \\
\text { reconnaître les personnes, à lire, à } \\
\text { entendre ou à se faire comprendre } \\
\text { lorsqu'elle parle, même en utilisant } \\
\text { les aides techniques appropriées } \\
\text { (lunettes, appareil auditif) s'il y a } \\
\text { lieu }\end{array}$ & 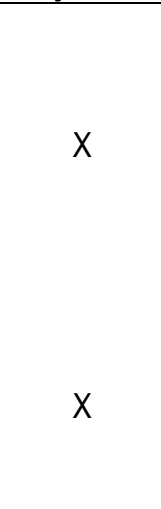 & $\begin{array}{l}x \\
x\end{array}$ & $\begin{array}{l}\mathrm{D} \\
\mathrm{X} \\
\mathrm{D} \\
\mathrm{X}\end{array}$ \\
\hline \multicolumn{6}{|c|}{ Facteurs de besoin de la personne de soutien } \\
\hline Variables démographiques & $\begin{array}{l}\text { Âge : calculé avec date du questionnaire moins date de } \\
\text { naissance (en années) } \\
\text { Sexe : Masculin ou féminin }\end{array}$ & Questionnaire SIPA à la PS & & & $\begin{array}{l}X \\
X\end{array}$ \\
\hline Capacité fonctionnelle & $\begin{array}{l}\text { Activités de la vie quotidienne originalement mesurées } \\
\text { par l'Indice de Barthel (Granger et coll., } 1979)^{3} \text { et } \\
\text { activités de la vie domestique mesurées par } 7 \text { items } \\
\text { d'AVD du Older American Resources and Services } \\
\text { (OARS) questionnaire (Fillenbaum, 1988). }{ }^{4} \\
\text { Pour créer un score unique incluant AVQ et AVD, les } \\
\text { résultats de l'Indice de Barthel ont été transformés pour } \\
\text { obtenir l'équivalent des } 7 \text { items d'AVQ du questionnaire } \\
\text { OARS et additionnés aux résultats d'AVD du } \\
\text { questionnaire OARS. C'est ce résultat au OARS sur } 28 \\
\text { qui est utilisé dans les analyses. } \\
\text { Des scores partiels (AVQ, AVD, incontinence) sont aussi } \\
\text { présentés dans certains tableaux descriptifs. }\end{array}$ & Questionnaire SIPA à la PS & & & D \\
\hline Durée de l'aide & $\begin{array}{l}\text { Calculée en années et mois à partir de la réponse à la } \\
\text { question : Quand êtes-vous devenu sa personne de } \\
\text { soutien principale sur une base continue? }\end{array}$ & Questionnaire SIPA à la PS & & & $\mathrm{x}$ \\
\hline
\end{tabular}

Légende:

$X$ : utilisé dans toutes les analyses (descriptives, bivariées et multivariées)

$\mathrm{D}$ : utilisé de manière descriptive et dans des analyses bivariées seulement

Les numéros indiqués après le nom d'un outil d'évaluation renvoient à la description de cet outil dans le tableau V. 


\begin{tabular}{|c|c|c|c|c|c|}
\hline \multirow[t]{2}{*}{ Concepts } & \multirow{2}{*}{ Variables et indicateurs } & \multirow[t]{2}{*}{ Source } & \multicolumn{3}{|c|}{ Utilisation } \\
\hline & & & Objectif 1 & Objectif 2 & Objectif 3 \\
\hline Fardeau ressenti & $\begin{array}{l}\text { Mesuré par l'Inventaire du fardeau (Burden Interview) } \\
\text { (Zarit et coll., 1985) }{ }^{5} \\
\text { Aussi estimé par l'utilisation ou non d'hébergement } \\
\text { - présence ou non d'épisodes d'hébergement temporaire } \\
\text { - hébergement dans les trois mois suivant le } \\
\text { questionnaire }\end{array}$ & $\begin{array}{l}\text { Questionnaire SIPA à la PS } \\
\text { Hébergement: } \\
\text { - Données de l'Agence } \\
\text { régionale obtenues par SIPA }\end{array}$ & $\mathrm{X}$ & $\mathrm{X}$ & $\mathrm{x}$ \\
\hline \multicolumn{6}{|c|}{ Caractéristique du centre de jour } \\
\hline Disponibilité du service & $\begin{array}{l}\text { Admissibilité au CJ du territoire : } \\
\text { CJ du territoire accepte ou non } \\
\text { - les personnes qui présentent de l'incontinence } \\
\text { fécale } \\
\text { - les personnes qui ont besoin d'être transférées à } \\
\text { l'aide de deux personnes } \\
\text { - les personnes qui ont besoin d'aide pour manger } \\
\text { Liste d'attente : durée moyenne, en mois, de l'attente } \\
\text { pour être admis au CJ de son territoire à l'été } 2000\end{array}$ & $\begin{array}{l}\text { Entrevues aux coordonnateurs des } \\
\text { cinq centres de jour }\end{array}$ & D & & \\
\hline Coût & $\begin{array}{l}\text { Tarif exigé par jour pour le repas et le transport par le CJ } \\
\text { du territoire }\end{array}$ & $\begin{array}{l}\text { Entrevues aux coordonnateurs des } \\
\text { cinq centres de jour }\end{array}$ & $x$ & & \\
\hline Téléphones de rappel & $\begin{array}{l}\text { Fait partie du bassin d'un CJ qui : } \\
\text { - n'appelle pas les participants } \\
\text { - appelle les cas particuliers } \\
\text { - appelle tous les participants } \\
\end{array}$ & $\begin{array}{l}\text { Entrevues aux coordonnateurs des } \\
\text { cinq centres de jour }\end{array}$ & D & & \\
\hline Structure du personnel & $\begin{array}{l}\text { Ratio personnes âgées inscrites par jour / ETC } \\
\text { Ratio personnes âgées présentes par jour / ETC } \\
\% \text { de personnel de soins } \\
\% \text { de personnel de réadaptation } \\
\% \text { d'intervenants en loisirs } \\
\% \text { d'intervenants psychosociaux } \\
\% \text { de personnel administratif et de soutien }\end{array}$ & $\begin{array}{l}\text { Entrevues aux coordonnateurs des } \\
\text { cinq centres de jour }\end{array}$ & D & & \\
\hline Durée des activités offertes & $\begin{array}{l}\text { Durée d'une visite type au CJ, en heures } \\
\text { Durée d'une visite type : } \\
\text { - demi-journée ( } 3,5 \text { heures ou moins) } \\
\text { - journée complète (plus de } 3,5 \text { heures) }\end{array}$ & $\begin{array}{l}\text { Entrevues aux coordonnateurs des } \\
\text { CJ : durées habituelles de chacun } \\
\text { des programmes proposés par les } \\
\text { CJ, mis en lien avec les registres } \\
\text { de présences pour la durée d'une } \\
\text { visite type pour chaque personne }\end{array}$ & & & $\begin{array}{l}\mathrm{D} \\
\mathrm{X}\end{array}$ \\
\hline
\end{tabular}




\begin{tabular}{|c|c|c|c|c|c|}
\hline \multirow[t]{2}{*}{ Concepts } & \multirow[t]{2}{*}{ Variables et indicateurs } & \multirow{2}{*}{ Source } & \multicolumn{3}{|c|}{ Utilisation } \\
\hline & & & Objectif 1 & Objectif 2 & Objectif 3 \\
\hline Fréquence de l'inscription & $\begin{array}{l}\text { Nombre de journées par semaine pour lesquelles des } \\
\text { activités en CJ sont prévues pour la personne }\end{array}$ & $\begin{array}{l}\text { Registres de présences : } \\
\text { JEDI } \\
\text { dossiers du } 5 \mathrm{e} \mathrm{CJ}\end{array}$ & & $\mathrm{X}$ & $\mathrm{X}$ \\
\hline Activités du centre de jour & $\begin{array}{l}\text { Variable dichotomique indiquant si l'individu a participé } \\
\text { ou non à chacun des types d'activités } \\
\text { Pour les personnes qui y ont participé, l'intensité de la } \\
\text { participation à chaque type d'activité est calculée par le } \\
\text { pourcentage suivant : présences au CJ au cours } \\
\text { desquelles la personne a participé à ce type d'activitél } \\
\text { présences totales au CJ. } \\
\text { A. Exercices physiques } \\
\text { B. Groupes spécifiques pour un diagnostic } \\
\text { physique ou un problème fonctionnel particulier } \\
\text { C. Activités fonctionnelles } \\
\text { D. Stimulation cognitive } \\
\text { E. Activités musicales ou de réminiscence } \\
\text { F. Sports et jeux physiques } \\
\text { G. Intégration sociale } \\
\text { H. Prévention et promotion de la santé }\end{array}$ & $\begin{array}{l}\text { Registres des activités : } \\
\text { JEDI } \\
\text { dossiers du } 5^{\mathrm{e}} \mathrm{CJ}\end{array}$ & & D & D \\
\hline \multicolumn{6}{|c|}{ Caractéristiques des services du CLSC } \\
\hline Intensité de la gestion de cas & $\begin{array}{l}\text { Participant SIPA reçoit l'intervention SIPA (groupe } \\
\text { expérimental) ou l'intervention habituelle (groupe } \\
\text { contrôle) }\end{array}$ & Fichier de recrutement SIPA & $\mathrm{x}$ & $\mathrm{x}$ & $\mathrm{X}$ \\
\hline Intensité des services & $\begin{array}{l}\text { Fréquence de l'aide du CLSC pour les activités } \\
\text { quotidiennes et domestiques (nombre de jours par } \\
\text { semaines) }\end{array}$ & $\begin{array}{l}\text { Fichier SIPA des données du } \\
\text { CLSC avec les dates et codes de } \\
\text { services reçus du CLSC dans les } \\
\text { catégories AVQ et AVD }\end{array}$ & $\mathrm{X}$ & $\mathrm{X}$ & $\mathrm{X}$ \\
\hline $\begin{array}{l}\text { Aide pour } A V Q \text { les jours de } \\
\text { fréquentation du CJ }\end{array}$ & $\begin{array}{l}\text { Reçoit ou non une aide régulière du CLSC pour se } \\
\text { préparer au CJ: Cette aide est considérée présente si } \\
\text { elle est observée sur une base régulière pour au moins } \\
\text { une des journées où la personne est inscrite au centre de } \\
\text { jour }\end{array}$ & $\begin{array}{l}\text { Fichier SIPA des données du } \\
\text { CLSC avec les dates et codes de } \\
\text { services reçus du CLSC dans les } \\
\text { catégories AVQ et AVD, mis en } \\
\text { lien avec les données des registres } \\
\text { de présences en CJ }\end{array}$ & & & $\mathrm{X}$ \\
\hline
\end{tabular}

Légende:

$X$ : utilisé dans toutes les analyses (descriptives, bivariées et multivariées)

$D:$ utilisé de manière descriptive et dans des analyses bivariées seulement

Les numéros indiqués après le nom d'un outil d'évaluation renvoient à la description de cet outil dans le tableau V. 


\section{Tableau V: Qualité des instruments de mesure ${ }^{1}$}

1. Échelle de dépression gériatrique, version courte (Sheikh et Yesavage, 1986) : L'échelle de dépression gériatrique originale contient 30 énoncés qui évaluent la présence de symptômes dépressifs. Cette échelle a démontré une bonne consistance interne (alpha de Chronbach $=0.94$ ) et une bonne fidélité test-retest (0.85, à une semaine d'intervalle) (Yesavage, Brink, Rose, Lum, Huang, Adey et Leirer, 1983). La version française (Bourque, Blanchard et Vézina, 1990) démontre aussi de bonnes qualités métrologiques. La forme courte, qui comporte 15 énoncés, est fortement corrélée ( $r=0.84)$ à l'évaluation originale (Sheikh et Yesavage, 1986). Les résultats possibles pour la forme courte vont de 0 à 15 . Un résultat plus élevé indique plus de symptômes dépressifs.

2. Short Portable Mental Status Questionnaire - SPMSQ (Pfeiffer, 1975): Ce questionnaire comporte 10 questions évaluant principalement la mémoire et l'orientation. La fidélité de cette échelle est démontrée par un alpha de Cronbach de 0.89 pour la consistance interne (Foreman, 1987), une fidélité test-retest à quatre semaines d'intervalle de 0.82 (Pfeiffer, 1975) et une fidélité interjuges entre 0.62 et 0.87 (Fillenbaum et Smyer, 1981). Pfeiffer (1975) suggère qu'un résultat de trois erreurs ou plus indiquerait possiblement un déficit cognitif pour une personne qui a une éducation de niveau secondaire. II suggère de permettre une erreur de plus aux personnes qui n'ont pas fréquenté l'école secondaire et une erreur de moins à ceux qui ont fréquenté le collège ou l'université. II recommande aussi d'accorder une erreur de plus aux AfroAméricains. Nous avons adapté cette correction pour l'origine ethnique au contexte multiethnique de Montréal, en accordant une erreur de plus aux sujets qui ne sont pas nés au Canada. En utilisant les critères suggérés par Pfeiffer, Foreman (1987) a obtenu un coefficient de prévision d'un test positif de 0.89.

3. Indice de Barthel : La version utilisée est celle décrite par Granger, Albrecht et Hamilton (1979), qui comporte 18 questions évaluant la capacité à réaliser diverses activités de la vie quotidienne comme se lever d'une chaise, se déplacer à l'intérieur et à l'extérieur de la maison, utiliser la toilette, se laver, s'habiller, etc. Les résultats possibles vont de 0 à 100 , un résultat plus élevé indiquant une plus grande autonomie. Cette échelle a démontré une bonne fidélité test-retest (0.89) et une bonne fidélité interjuges (au-dessus de 0.95) (Granger et coll., 1979).

4. Older American Resources and Services - OARS (Fillenbaum, 1988) : Cette échelle comporte 7 énoncés qui évaluent la capacité à réaliser diverses activités de la vie quotidienne (s'habiller, manger, contrôler ses sphincters, etc.) et 7 énoncés qui évaluent la capacité à réaliser diverses activités de la vie domestique comme utiliser le téléphone, utiliser les moyens de transport, magasiner ou faire l'épicerie, préparer les repas, etc. Les résultats possibles vont de 0 à 14, un résultat plus élevé indiquant une plus grande autonomie. L'ensemble du questionnaire OARS a démontré une bonne fidélité test-retest $(0.90,3$ à 8 semaines d'intervalle) et la section des soins personnels (énoncés de vie quotidienne et de vie domestique) a démontré une fidélité interjuges de 0.86 (Fillenbaum, 1988).

5. Inventaire du Fardeau (Burden Interview) (Zarit, Orr et Zarit, 1985) : Cet instrument comporte 22 énoncés qui évaluent le fardeau subjectif ressenti par les personnes de soutien. Hébert, Bravo et Girouard (1993) rapportent une bonne consistance interne (alpha de Cronbach de 0.85 à 0.91 ) et une bonne fidélité testretest $(0.71$ à 0.90$)$ pour les versions anglaise et française de cet instrument de mesure. Les résultats possibles vont de 0 à 88 , un résultat plus élevé indiquant un plus grand fardeau ressenti.

Notes:

1. Les numéros réfèrent aux numéros de renvois utilisés dans le tableau IV. 


\section{Analyse des données}

Les méthodes d'analyses de données sont décrites en détail à la section méthodologique de chacun des trois articles inclus à la section des résultats de cette thèse. Elles sont résumées brièvement ci-dessous.

Article 1:

Pour la première étape qui vise à connaître les taux d'utilisation du centre de jour par la clientèle cible, des statistiques descriptives (moyenne, fréquence avec intervalle de confiance) ont été calculées.

Pour décrire qui sont ces utilisateurs, des statistiques descriptives (moyenne, fréquence) ont été calculées. Les différences entre les utilisateurs et les non-utilisateurs ont été étudiées de façon bivariée à l'aide de tests de Student (test de t) et de tests du chi carré.

Pour mieux comprendre les effets de ces variables sur le fait d'avoir utilisé ou non le centre de jour pendant la période d'observation, des analyses multivariées (régressions logistiques multiples) ont ensuite été effectuées.

\section{Article 2 :}

Pour vérifier si les individus ayant un ensemble de caractéristiques similaires reçoivent des services similaires des centres de jour, des analyses en regroupement (cluster analysis) ont été d'abord été effectuées pour regrouper les clients les plus semblables en fonction de diverses variables sociodémographiques et de santé. Des analyses de variance (ANOVA) ont confirmé les différences significatives dans les caractéristiques des membres de chacun des groupes créés par ces analyses. 
En second lieu, les diverses activités de groupe offertes dans les cinq centres de jour à l'étude ont été regroupées en huit grandes catégories d'activités, dans un processus à trois étapes :

1) Validation des données: Les registres d'activités des cinq centres de jour contenaient 107 noms différents d'activités. Les rencontres avec les coordonnateurs de cinq centres de jour ont permis de comprendre ce qu'était chacune de ses activités : but de l'activité, clientèle visée, exemples de sous-activités. Par exemple, dans un centre, l'activité «Sports » comportait des jeux de quilles, du volleyball adapté, du curling adapté, etc.

2) Regroupement des activités similaires portant des noms différents : Les registres d'activités des cinq centres de jour participants contenaient 107 noms différents d'activités. Les rencontres avec les coordonnateurs de cinq centres de jour ont permis de regrouper les activités similaires qui portaient des noms différents dans les registres statistiques (par exemple, les activités «mémo », «méli-mélo », « intello», «cognitif» sont toutes des activités de stimulation cognitive). Ces rencontres ont permis de réduire la liste d'activités de 107 à 40 activités différentes.

3) Regroupement des activités selon leurs buts : Ces 40 activités ont à nouveau été regroupées en huit grandes catégories, en fonction de leurs buts. Ce regroupement a été effectué par consultation d'un groupe d'experts constitué de sept responsables de centre de jour de la région de Montréal, au moyen d'une méthode adaptée de la technique du groupe nominal (Delbecq, Van De Ven et Gustavson, 1975).

Par la suite, pour identifier les types d'activités dont ont bénéficié les participants à cette étude, des statistiques descriptives ont été calculées : 1) pour l'ensemble des utilisateurs de centre de jour, le nombre et le pourcentage d'individus ayant participé à chacune des catégories d'activités et 2) pour les individus ayant participé aux activités d'une catégorie donnée, la moyenne de l'intensité d'exposition à cette catégorie d'activités. À cette fin, le nombre de jours auxquels chaque individu a participé à chacune des huit grandes catégories d'activités a été dénombré. Pour tenir compte de la variation de participation des individus, le nombre de participations à chacune des catégories d'activités 
a ensuite été calculé en pourcentage des jours de présence au centre de jour. Cette variable donne une indication de l'intensité de l'exposition à chacune des activités (elle ne tient cependant pas compte de la durée de chacune des activités au cours d'une journée).

Des ANOVA ont permis de déterminer les différences significatives entre les divers profils de participants créés par les analyses en regroupement, pour la participation et l'intensité d'exposition à chaque type d'activité des individus ayant participé au moins une fois aux activités de chacune des catégories.

Enfin, les résultats de ces analyses ont été comparés aux types d'activités à privilégier pour les clientèles de chacun des six sous-programmes du cadre de référence des centres de jour (ACAQ, 1991), selon ce document ainsi que selon le groupe d'experts décrit ci-dessus.

Article 3 :

Pour connaître les déterminants de la régularité de la participation (mesurée par le pourcentage de présences réelles sur le nombre de présences attendues en fonction de la fréquence d'inscription), des analyses des relations bivariées entre les variables indépendantes et la variable dépendante ont d'abord été effectuées. Pour mieux comprendre les effets de ces variables sur la participation au centre de jour, des analyses de régressions linéaires multiples ont été réalisées. Les variables indépendantes incluaient les caractéristiques des utilisateurs et de leur personne de soutien, ainsi que certaines caractéristiques des services reçus, dont le type d'activités décrit en fonction des huit grandes catégories d'activités définies dans l'article 2.

Toutes les analyses statistiques ont été effectuées à l'aide du logiciel SPSS pour Windows, allant de la version 12.0 pour le premier article, à la version 14.0 pour le dernier article. 


\section{Considérations éthiques}

Ce projet repose sur une analyse secondaire de données. Le projet SIPA a reçu toutes les approbations éthiques nécessaires. Les sujets du projet SIPA ont consenti à répondre aux questionnaires sur leur état de santé et à ce que les chercheurs recueillent des informations sur leur utilisation des services de santé. La cueillette supplémentaire de données dans les dossiers des centres de jour est effectuée par la même équipe de recherche. De plus, l'utilisation des données du projet SIPA aux fins de la présente recherche a obtenu une approbation distincte du comité d'éthique de l'Hôpital Général Juif - Sir Mortimer B. Davis. 


\section{Chapitre 5 : Résultats}

Les résultats de chacun des objectifs de cette étude sont présentés dans les trois articles inclus à cette section.

L'article 1 rapporte les résultats obtenus en lien avec le premier objectif de l'étude qui vise à connaître la proportion d'utilisateurs de centre de jour ainsi que les déterminants de l'utilisation au sein d'un groupe de 723 personnes âgées de 65 ans et plus de la région de Montréal qui participaient au projet SIPA. Cet article est publié dans le numéro d'automne 2007 de la Revue canadienne du vieillissement.

Au moment de soumettre cet article, la réflexion sur le cadre conceptuel présenté aux pages 66 à 68 de la présente thèse n'était pas terminée. Ainsi, on notera que ce qui est décrit dans cet article comme des facteurs de capacité de nature organisationnelle est plutôt présenté simplement comme des caractéristiques des services dans la présentation du cadre conceptuel aux pages 66 à 68 .

L'article 2 présente les résultats obtenus en lien avec le second objectif qui cherche à vérifier l'adéquation entre les activités offertes par les centres de jour et les caractéristiques des utilisateurs, pour 132 participants des cinq centres de jour. Une classification des activités a été créée par un groupe d'experts. La participation à ces activités a été étudiée pour divers groupes d'utilisateurs créés par des analyses en regroupement. La version révisée de cet article a été soumise à la Revue canadienne du vieillissement le 2 octobre 2008.

L'article 3 rapporte les résultats du troisième objectif de recherche qui vise à définir les déterminants de la régularité de la participation au centre de jour, parmi un groupe de 101 personnes âgées de 65 ans et plus pour lesquels des données sur la personne de soutien et sur la fréquentation du centre de jour sont disponibles. La régularité de participation est calculée en pourcentage des présences réelles sur le nombre de participations attendues, compte tenu de la fréquence de l'inscription et des jours où le centre de jour est fermé, ainsi que des jours où la personne est hébergée ou hospitalisée et donc dans l'impossibilité de 
fréquenter le centre de jour. Cet article à été soumis le 3 octobre 2008 au Journal of Aging and Health.

\section{Rôle joué par chaque auteur dans la rédaction de ces articles.}

Jacinthe Savard a développé les idées et la conceptualisation des questions de recherche, elle a réalisé les analyses de données et assuré la rédaction des trois articles.

Nicole Leduc et Paule Lebel, codirectrices de recherche de l'étudiante, l'ont guidée pendant toute la durée de son projet de doctorat. À ce titre, elles ont participé à développer les idées et à conceptualiser les questions de recherche, elles ont guidé l'étudiante particulièrement pour la réalisation des analyses et ont assuré plusieurs révisions des trois articles.

François Béland, Howard Bergman et Paule Lebel ont conceptualisé et dirigé le projet SIPA duquel sont tirées la majorité des données utilisées pour cette recherche. À ce titre, ils ont été actifs dans le choix des outils de mesure et des méthodes de collectes de données utilisés pour cette recherche.

François Béland et Howard Bergman aussi participé à la rédaction des trois articles en révisant les versions préliminaires et en apportant des commentaires judicieux qui ont permis de clarifier la présentation et la discussion des résultats de chacun de ces articles. 
ARTICLE 1

\title{
L'utilisation des services de centre de jour par les personnes âgées qui présentent des incapacités.
}

\author{
Jacinthe Savard ${ }^{1,2}$ \\ Nicole Leduc ${ }^{1,3}$ \\ Paule Lebel ${ }^{1,4}$ \\ François Béland ${ }^{1,3}$ \\ Howard Bergman ${ }^{1,5}$
}

${ }^{1}$ Groupe de recherche SOLIDAGE, Faculté de Médecine, Université de Montréal et Université McGill

${ }^{2}$ Clinique universitaire interprofessionnelle de réadaptation, Université d'Ottawa

${ }^{3}$ Groupe de recherche interdisciplinaire en santé (GRIS), Faculté de Médecine, Université de Montréal

${ }^{4}$ Centre d'expertise sur la santé des personnes âgées et des aidants (CESPA), Institut universitaire de gériatrie de Montréal

${ }^{5}$ Division of Geriatric Medicine, Université McGill et Jewish General Hospital, Montréal

Soumis : 12/06/06, accepté pour publication : 27/03/07

Publié : Canadian Journal on Aging / La Revue canadienne du vieillissement, 26(3), 255-274 (2007) 


\begin{abstract}
This study consists of a secondary analysis of data collected during the SIPA demonstration project. Its purpose is to identify the proportion of Adult Day Care (ADC) users as well as the determinants of use in this group of persons 65 years old and over receiving home care services in Montreal. Results show that $18.8 \%$ of the sample have at least one ADC presence during a 6 months study period. The following factors increase the probability of ADC use: being younger; not having a university degree; being born outside of Canada or for persons born in Canada, living with a caregiver; receiving help from the CLSC for daily life activities more than once a week; being in the catchment population of an ADC which fees are lower; and for men only, having had a stroke or presenting more functional incapacities.
\end{abstract}

\title{
RÉSUMÉ
}

Cette étude consiste en une analyse secondaire de données recueillies dans le cadre du projet SIPA. Elle vise à identifier la proportion d'utilisateurs de centres de jour (CJ) ainsi que les déterminants de l'utilisation au sein de ce groupe de personnes âgées de 65 ans et plus de la région de Montréal qui reçoivent des services de maintien à domicile. Les résultats montrent que $18,8 \%$ de l'échantillon ont au moins une présence en CJ pendant la période d'observation de six mois. Les facteurs suivants augmentent la probabilité d'utiliser le $\mathrm{CJ}$ : être plus jeune; ne pas avoir une éducation universitaire; être né à l'extérieur du Canada ou, pour les personnes nées au Canada, cohabiter avec une personne de soutien; recevoir l'aide du CLSC pour les activités de vie quotidienne plus d'une fois par semaine; faire partie du bassin de desserte d'un CJ dont les coûts sont moins élevés; et pour les hommes seulement, avoir déjà subi un accident vasculaire cérébral et présenter davantage d'incapacités fonctionnelles.

Mots clés : vieillissement, Centre de jour, personnes âgées, maintien à domicile Keywords: aging, Adult Day Care, frail seniors, community long-term care 


\section{Remerciements}

Pour mener à bien cette étude, la première auteure a reçu une bourse de recherche doctorale de la Societé Alzheimer du Canada et une du groupe de recherche SOLIDAGE. Cette étude utilise des données du projet SIPA, financé par le Fonds d'adaptation des services de santé de Santé Canada, la Fondation canadienne de recherche sur les services de santé et le Ministère de la Santé et des Services sociaux du Québec. Les auteurs remercient Luc Dallaire et John Fletcher, coordonnateurs de recherche du projet SIPA, pour leur aide précieuse relative à la gestion et à la transmission des données. De plus, ils remercient Anne-Marie Boivin, Christian-Paul Gaudet, Mélanie Léonard, Manon Pouliot et Tina Tenenbaum, coordonnateurs de centres de jour pour avoir partagé des informations sur le fonctionnement de leur centre de jour, ainsi que Jacinthe Auger, Christian-Paul Gaudet et Tina Tenenbaum pour leurs commentaires sur les résultats de l'étude. 


\section{Introduction}

Confrontés au vieillissement de la population et aux besoins particuliers des personnes âgées, la plupart des pays industrialisés ont développé une gamme variée de services gérontologiques et gériatriques qui visent le maintien de l'autonomie et de la qualité de vie de cette population. Parmi ces services, on retrouve le centre de jour (CJ), un service offert aux personnes âgées qui demeurent à domicile et présentent des incapacités. Les interventions en CJ sont offertes principalement au moyen d'activités de groupe et comprennent des activités de surveillance de l'état de santé, des activités d'enseignement et de prévention, des activités de réadaptation visant essentiellement le maintien des capacités, des interventions psychosociales et de loisirs, ainsi que du répit et du soutien aux familles (Gutman, Milstein, Killam, Lewis et Hollander, 1993b; Regroupement des centres d'hébergement et de soins de longue durée Région de Montréal, 2001). Par ces interventions, le CJ vise à favoriser le maintien dans la communauté des personnes âgées qui présentent des incapacités.

Les études sur l'efficacité des CJ pour réduire ou retarder l'institutionnalisation de la clientèle ou pour réduire le fardeau des personnes de soutien ont démontré des résultats variables. Dans plusieurs études, même lorsque ce service est accessible, les taux d'utilisation par la clientèle âgée sont faibles (Baumgarten, Lebel, Laprise, Leclerc et Quinn, 2002; Douglass et Visconti, 1998; Lawton, Brody et Saperstein, 1989; Montgomery et Borgotta, 1989). Toutefois, lorsque les chercheurs ont contrôlé les taux d'utilisation, plusieurs ont pu démontrer des effets positifs à la fréquentation du CJ (Baumgarten et coll., 2002; Wimo, Mattsson, Adolfsson, Eriksson et Nelvig, 1993; Zarit, Stephens, Townsend, Greene et Leitsch, 1999). Dans un tel contexte, il importe de se questionner sur les caractéristiques des utilisateurs de CJ afin de mieux cibler les personnes les plus susceptibles d'en bénéficier ou encore de revoir l'offre de service de façon à mieux l'adapter aux besoins de la clientèle cible.

Les quelques recherches qui ont porté sur les facteurs qui influencent l'utilisation des CJ ont utilisé des méthodologies variées. Plus particulièrement, la définition de 
l'utilisation et du groupe de comparaison varie d'une étude à l'autre. Ainsi, nous avons identifié deux études qui ont comparé les utilisateurs de CJ aux utilisateurs de services à domicile qui ne participent pas au CJ. Dans une étude canadienne des années 1980, les utilisateurs de $\mathrm{CJ}$ sont généralement moins instruits et sont moins nombreux à avoir œuvré dans des postes professionnels ou de gestion que les utilisateurs de services à domicile. Ils sont aussi plus nombreux à être nés au Canada et ils présentent plus d'incapacités fonctionnelles (Chappell, 1983). Dans une étude américaine récente, les utilisateurs de CJ sont généralement plus jeunes et présentent davantage de troubles cognitifs que les personnes qui utilisent uniquement les soins à domicile. Bien que les capacités fonctionnelles des deux groupes soient similaires selon les échelles d'activités de la vie quotidienne (AVQ) et d'activités de la vie domestique (AVD), les utilisateurs de soins à domicile sont hospitalisés plus souvent et utilisent plus d'équipement médical ou d'aides techniques (Dabelko et Balaswamy, 2000). Ces résultats sont cohérents avec le fait que les personnes qui fréquentent le CJ doivent posséder certaines capacités de déplacement. Même lorsqu'un transport adapté est offert par le CJ, il est plus difficile de déplacer des personnes qui utilisent d'avantage d'équipement médical ou celles qui se sentent fragiles en raison d'une hospitalisation récente.

D'autres études, centrées sur des groupes d'utilisateurs de CJ, ont identifié certains facteurs qui distinguent les forts utilisateurs des plus faibles utilisateurs. Selon ces études, les facteurs associés à une plus forte utilisation étaient: des scores de dépression légèrement supérieurs; des scores d'anxiété légèrement inférieurs; des atteintes cognitives plus grandes (Baumgarten et coll., 2002); moins de problèmes de comportement (Lawton, Brody et Saperstein, 1991; Zarit et coll., 1999); la présence d'une personne de soutien; un plus grand fardeau ressenti par cette personne (Baumgarten et coll., 2002); le fait d'avoir des enfants; et le fait d'être inscrit à raison de 3 jours par semaine (Wallace, 1987).

Des études sur l'utilisation de services de répit par les aidants familiaux ont aussi démontré que les aidants de personnes atteintes de troubles cognitifs ont utilisé davantage le CJ que les autres groupes de sujets (Montgomery, Kosloski et Borgotta, 1988-1989) et que les aidants qui disent avoir été influencés par un membre de leur réseau informel ou par 
un professionnel utiliseraient davantage le CJ que ceux qui ne rapportent pas l'influence d'une tierce personne sur leur utilisation de services (Cotrell et Engel, 1998). De plus, dans une étude qualitative de Cotrell (1996) auprès d'une clientèle atteinte de démence, les personnes de soutien qui sont des enfants corésidents indiquent une préférence pour le CJ plutôt que d'autres formes de répit pour les bienfaits résultant de la socialisation qu'il procure à leur parent, tandis que les conjoints qui ont besoin de répit semblent préférer le répit à domicile qui leur permet de quitter la maison ou de vaquer librement à certaines occupations domestiques.

Il faut noter qu'à l'exception des travaux de Baumgarten et collaborateurs (2002) et de Chappell (1983), ces résultats proviennent d'études américaines. Les autres études canadiennes recensées au sujet des CJ (Gutman, Milstein, Killam, Lewis et Hollander, 1993a; 1993b; Tourigny, Côté, Laberge, Paradis et Joubert, 1993) n'abordent pas les facteurs qui influencent l'utilisation. Nous ne savons pas si la dynamique d'utilisation des services observée aux États-Unis est similaire à celle du Québec et du Canada. En effet, il existe une variété de modèles de CJ aux États-Unis : modèle social, modèle médical, centres spécifiques pour les personnes atteintes de démence (Gaugler et Zarit, 2001; Weissert et coll., 1989). Au Québec et ailleurs au Canada, les CJ sont plutôt génériques, c'est-à-dire qu'ils acceptent une clientèle variée et adaptent leurs activités aux besoins diversifiés de cette clientèle. On constate donc que les CJ offrent à la fois des services habituellement considérés comme des services de santé (suivi infirmier de l'état de santé, activités de réadaptation) et des services de nature plus sociale (activités de socialisation pour des personnes isolées en raison d'une perte d'autonomie importante, répit aux personnes de soutien). La majorité des activités sont offertes sous forme d'activités de groupe et la plupart des personnes âgées qui y participent reçoivent plus d'un type de services. De plus, aux États-Unis la participation à ces services, offerts dans un marché compétitif, est parfois limitée par la capacité financière des individus. Au Québec, elle est plutôt limitée par la capacité d'accueil des centres qui ont souvent des listes d'attente, bien que les coûts reliés au transport et aux repas (généralement inférieurs aux coûts des CJ aux États-Unis) puissent aussi représenter des barrières à l'utilisation. Par conséquent, les 
facteurs qui influencent l'utilisation des CJ dans le contexte québécois et canadien, restent encore à définir.

Les objectifs de la présente étude sont d'identifier la proportion d'utilisateurs de CJ parmi une clientèle âgée vulnérable de la région de Montréal, de caractériser ces utilisateurs et d'analyser les déterminants de l'utilisation du CJ. Les résultats fourniront des pistes de réflexion pour les intervenants qui dirigent les personnes âgées vers ce type de service.

Le modèle élaboré par Andersen dès 1968 pour expliquer l'utilisation de services médicaux et repris ensuite par Andersen et plusieurs collègues (voir Andersen, 1995) permet une conceptualisation globale intéressante de trois types de facteurs qui peuvent expliquer l'utilisation des services de santé: les facteurs prédisposants; les facteurs de capacité (enabling factors); et les facteurs de besoin. Toutefois, la façon dont ces facteurs influencent l'utilisation des services médicaux et l'utilisation du CJ peut différer grandement, ne serait-ce que parce que dans le contexte québécois, la demande pour fréquenter le CJ est plus rarement initiée par la personne elle-même, mais provient plus souvent d'une recommandation d'un professionnel de la santé. Dans cette étude, la typologie de facteurs décrite par Anderson et ses collègues sera adaptée aux caractéristiques particulières des $\mathrm{CJ}$, pour identifier les variables à inclure dans l'analyse des déterminants de l'utilisation des CJ (voir figure 1). Selon ce modèle adapté, les facteurs prédisposants sont des facteurs qui sont présents avant que le besoin de services ne se fasse sentir. Ils influencent la propension à utiliser les services, mais aussi l'état de santé de la personne âgée, donc le niveau de besoin pour les services offerts en CJ. Les facteurs de besoin sont reliés à l'état de santé de la personne âgée et aux besoins que le CJ tente de combler. On remarque que l'état de santé peut aussi influencer certaines caractéristiques sociales, comme le fait de vivre avec une personne de soutien. Par ailleurs, les facteurs de capacité inclus dans cette étude sont reliés aux caractéristiques organisationnelles des services qui peuvent faciliter ou limiter l'accès aux services. Dans le cas de l'utilisation du CJ, nous émettons l'hypothèse qu'elles interagiront avec les facteurs prédisposants et les facteurs de besoin pour modifier les taux d'utilisation. En effet, on peut penser par exemple qu'à besoin égal, une moins grande proportion de sujets utiliseront le CJ si ce dernier présente 
une liste d'attente plus longue. Toutefois, si les CJ ont établi des critères de priorité, l'effet de la liste d'attente pourrait être différent chez les personnes dont le niveau de besoin est plus faible que chez celles dont le niveau de besoin est plus élevé. De même, il est possible que des services plus intensifs de soutien à domicile puisse apporter un suivi qui serait de nature à favoriser l'identification plus rapide des besoins et une meilleure adhésion aux recommandations et donc, à besoin égal, augmenter l'utilisation du CJ.

\section{Insérer la figure 1 près d'ici}

\section{Méthodologie}

Cette étude s'appuie sur des informations provenant de deux bases de données existantes :

1) Les données tant sociodémographiques que reliées à la santé physique et psychologique des personnes âgées recueillies dans le cadre du projet de démonstration Services intégrés pour personnes âgées (SIPA) ${ }^{1}$. Le projet SIPA s'est déroulé de juin 1999 à mars 2001, auprès de 1230 personnes âgées qui présentent des incapacités et résident sur deux territoires de Centre local de services communautaires (CLSC) de la région de Montréal. Environ la moitié de ces personnes ont reçu les services intégrés évalués dans le cadre du projet SIPA, alors que l'autre moitié continuait de recevoir les services de maintien à domicile régulier de leur CLSC. Une équipe de recherche a complété une cueillette exhaustive de données comportant trois vagues de questionnaires auprès des personnes âgées, un questionnaire auprès des personnes de soutien, ainsi qu'un relevé de leur utilisation des services de santé pendant toute la durée de l'expérimentation, d'après les bases de données des organismes et des établissements de santé (Régie de l'assurance maladie du Québec, Agence de développement des réseaux locaux de services de santé et de services sociaux, hôpitaux, CJ, etc.). Pour les personnes âgées incapables de répondre elles-mêmes aux questionnaires, un substitut a été utilisé.

2) Les données statistiques recueillies de façon routinière par quatre des cinq CJ qui desservent ces deux mêmes territoires, à l'aide d'un logiciel commun : le JEDI ${ }^{2}$. Ce 
logiciel fut conçu spécialement pour recueillir des données sur les clients, les activités et les présences aux CJ. Au moment de l'étude, il est utilisé par 23 des 32 centres de jour de l'Île de Montréal.

Ces informations ont été complétées par une cueillette de données dans les dossiers du cinquième $\mathrm{CJ}$. De plus, des entrevues avec les coordonnateurs des cinq CJ ont permis de préciser les caractéristiques des activités offertes, les procédures pour le repas et les transports et les critères d'admission spécifiques à chacun des centres.

\section{Population à l'étude}

Les personnes admises au projet de démonstration SIPA devaient vivre à domicile au début de l'étude, recevoir des services de maintien à domicile de leur CLSC et présenter des incapacités fonctionnelles, indiquées par un résultat de -10 ou moins au Système de mesure de l'autonomie fonctionnelle (SMAF) (Hébert, Carrier et Bilodeau, 1988). Elles devaient aussi accepter de participer à une étude randomisée sur les services intégrés pour personnes âgées et pouvoir s'exprimer en français ou en anglais avec les membres du projet SIPA, ou avoir un proche en mesure de le faire (Béland, Bergman et Lebel, 2001). Il s'agit donc d'une partie de la population âgée habituellement identifiée comme présentant des limitations d'activités de modérées à graves. Dans des études de population menées au Canada, on a observé que de $11 \%$ à $19 \%$ des personnes âgées de plus de 65 ans présentent de telles incapacités (Béland, Haldemann, et coll., 1998; Développement des ressources humaines Canada, 2003).

Pour cette étude sur l'utilisation des CJ, l'échantillon de départ est composé de 812 personnes âgées de 65 ans ou plus, toujours suivies par l'équipe de recherche SIPA à la fin de la première phase du projet, en mai 2000. De ce nombre, 44 personnes hébergées de façon permanente et 2 personnes hospitalisées pour toute la durée de la période d'observation étaient dans l'incapacité de fréquenter un CJ et ont été exclues de l'étude. De plus, 43 personnes qui présentaient des données manquantes pour des variables importantes à l'étude ont été exclues des analyses. L'échantillon final comprend donc 723 sujets. 
On observe certaines différences entre les 43 sujets exclus en raison de données manquantes et les 723 sujets inclus : Les questionnaires exclus sont plus souvent remplis par des substituts (54\% contre $23 \%$ pour les questionnaires inclus, $p<0,001)$. Les sujets exclus sont plus nombreux à ne pas avoir une connaissance suffisante du français ou de l'anglais pour répondre par eux-mêmes au questionnaire $(18,6 \% \mathrm{vs} 7,3 \%, \mathrm{p}=0,008)$ et ils sont moins autonomes que les sujets inclus dans l'étude, mais cette différence est grandement due au plus grand nombre de sujets qui sont très dépendants $(0$ à 2 à 1 'échelle du Older Americans Resources and Services [OARS]) (12,5\% vs 1,2\%). Lorsque ces sujets ne sont pas considérés, la différence entre les deux groupes en terme d'autonomie fonctionnelle n'est plus significative.

\section{Caractéristiques des centres de jour}

Cinq CJ desservent le territoire sur lequel cette étude a été menée. Le nombre de personnes âgées inscrites chaque jour varie de 25 à 45 personnes par jour, selon les centres. Le nombre moyen de présences réelles par jour varie de 18,1 à 28,7. La fréquentation minimale pour une même personne varie d'une demi-journée à 1 journée par semaine et la fréquentation maximale de 2 à 3 journées par semaine, selon les centres. La durée de chaque visite varie de 4,5 à 6 heures par jour, pour les personnes inscrites pour la journée et de 2 à 3,5 heures pour celles inscrites à la demi-journée, dans les centres qui offrent ce type de fréquentation.

Bien que chacun des centres décrive ses critères d'admission de façon légèrement différente, ce sont tous des centres qui visent le maintien à domicile des personnes âgées qui présentent des incapacités. Deux centres acceptent les personnes de 60 ans et plus, deux autres à partir de 65 ans, alors qu'un autre exige un profil gériatrique sans mentionner un âge minimum. Tous les centres mentionnent que les personnes doivent être en perte d'autonomie. Un centre ajoute que cette perte d'autonomie fonctionnelle empêche de participer à d'autres activités offertes dans la communauté, tandis qu'un autre spécifie que les personnes admises ont besoin d'un environnement thérapeutique structuré en raison d'une perte d'autonomie temporaire ou permanente. Trois des cinq CJ de l'étude ont des 
critères d'admission spécifiques liés à la langue ou à la religion (anglophones, francophones, juifs). Ces trois centres desservent le même territoire et acceptent les personnes allophones selon leur préférence pour un milieu anglophone ou francophone, de sorte que le service de CJ est disponible pour la population âgée en perte d'autonomie de toute langue ou de toute religion sur ce territoire.

En ce qui concerne les critères d'exclusion, aucun des centres n'accepte les personnes qui présentent des comportements qui pourraient perturber le groupe ou constituer un danger pour les autres participants. Aucun n'accepte les personnes qui auraient besoin d'être transférées au levier pendant la journée puisqu'aucun ne possède ce type d'équipement. De plus, trois des cinq centres n'acceptent pas les personnes qui auraient besoin d'être transférées à l'aide de deux personnes pendant la journée, parce qu'ils n'ont pas le personnel nécessaire pour le faire. Les transferts à l'aide d'une personne sont possibles dans tous les centres. Tous les centres acceptent les personnes qui présentent de l'incontinence urinaire si elles portent les protections appropriées (p. ex : couches, serviettes hygiéniques). Un seul refuse celles qui présentent de l'incontinence fécale. Deux centres n'acceptent pas les personnes qui ont besoin d'aide pour se nourrir (c.-à.-d pour porter les aliments à leur bouche). Tous acceptent les personnes qui ont besoin d'aide pour préparer l'assiette (c.-à.-d. couper la viande, beurrer le pain et ouvrir les contenants s'il y a lieu).

Le coût de chaque participation varie de 5 \$̀ 17 \$ par jour, pour couvrir les frais de transport et de repas. Tous les centres sont prêts à faire des ajustements à leur tarif pour accommoder les personnes qui éprouvent des difficultés financières. Certaines personnes peuvent payer moins cher parce qu'elles n'utilisent pas le transport ou ne prennent pas le repas du CJ. La durée moyenne d'attente pour être admis au CJ à l'époque de l'étude variait d'environ un mois à 1 an selon les centres. Deux centres font des appels à tous les participants la veille ou le matin même, pour leur rappeler le jour de leur participation. Un centre effectue de tels appels seulement pour les cas particuliers comme les individus qui ont des problèmes de mémoire et qui habitent seuls. Les deux autres centres n'effectuent pas de relance téléphonique. 
Les CJ de cette étude peuvent compter sur un personnel variant de 4,7 à 7,9 équivalents à temps complet (ETC). Le ratio de personnes âgées inscrites par jour par membre de personnel varie de 3,8 à 6,6. Le ratio de personnes âgées présentes en moyenne par jour par membre de personnel varie de 3,1 à 4,2. Le personnel se répartit dans les catégories suivantes : personnel de soins $(13,2 \%$ à $35,2 \%)$, personnel de réadaptation $(14,8 \%$ à $41,2 \%)$, intervenants en loisirs (14,7\% à 37,9\%), intervenants psychosociaux $(0 \%$ à $15,4 \%)$ et personnel administratif et de soutien $(19,3 \%$ à $39,2 \%)$.

\section{Période d'observation}

Les données relatives aux caractéristiques des personnes âgées ont été recueillies entre mai et novembre 2000. Pour chaque sujet, l'utilisation du CJ a été observée pendant une période de six mois, dont le centre est la date du questionnaire à la personne âgée. Comme l'état de santé des personnes en perte d'autonomie peut changer rapidement, il a semblé important d'utiliser la période d'observation de l'utilisation des CJ la plus près possible du moment où les données sur l'état de santé ont été recueillies.

\section{Variables à l'étude}

La variable dépendante est la participation au CJ pendant la période d'observation. Les sujets qui ont au moins une présence au CJ pendant la période sont définis comme utilisateurs de CJ, alors que ceux qui n'ont aucune présence sont définis comme nonutilisateurs, qu'ils aient ou non un dossier ouvert dans un CJ pendant la période.

Les variables indépendantes considérées dans cette étude incluent les facteurs présdisposants, reliés aux caractéristiques sociales de la personne âgée; les facteurs de besoin, reliés aux caractéristiques de son état de santé, ainsi que les facteurs de capacités de nature organisationnelle reliés aux caractéristiques organisationnelles du CJ de son territoire et au type de services qu'elle reçoit de son CLSC (voir figure 1). 
Facteurs prédisposants ou caractéristiques sociales de la personne âgée

Les variables sociodémographiques considérées sont l'âge, le sexe, le niveau d'éducation, le fait pour la personne âgée ou son conjoint d'avoir exercé une profession du domaine de la santé, l'appartenance culturelle mesurée par le pays de naissance, la religion et la langue d'usage. La langue d'usage et le niveau d'éducation sont présentés sur des échelles à cinq et six niveaux respectivement pour les analyses descriptives.

Des variables dichotomiques ont aussi été créées, soit le fait d'avoir ou non une éducation universitaire et la connaissance ou non d'une des deux langues officielles. Ce sont ces variables dichotomiques qui seront utilisées dans les analyses multivariées. Enfin, une question demandant au sujet d'estimer si son revenu actuel lui permet de satisfaire ses besoins sera utilisée dans les analyses descriptives, mais ne pourra être utilisée dans les analyses multivariées en raison du grand nombre de réponses manquantes.

En ce qui concerne le réseau social de la personne âgée, la présence ou non d'une personne de soutien et le type de lien avec celle-ci ont été regroupés pour former une variable à quatre niveaux : une personne de soutien qui est le conjoint de la personne âgée; une personne de soutien autre que le conjoint qui réside avec la personne âgée; une personne de soutien qui ne réside pas avec la personne âgée; ou pas de personne de soutien. L'état civil a été enregistré pour des fins descriptives seulement en raison de sa forte corrélation avec la variable précédente concernant le lien avec la personne de soutien.

\section{Facteurs de besoin ou état de santé de la personne âgée}

Pour évaluer la santé physique des personnes âgées, le questionnaire demandait aux répondants d'indiquer s'ils présentaient l'une ou l'autre des 16 conditions suivantes tirées de l'étude Established Populations for Epidemiologic Studies of the Elderly (EPESE) (Coroni-Huntley, Brook, Ostfeld, Taylor et Wallace, 1986). Ces conditions incluent la

présence de : haute tension artérielle; maladie de cœur; problèmes de circulation; accident vasculaire cérébral (AVC); diabète; problèmes respiratoires; rhumatisme ou arthrite (douleur aux jointures ou aux os); tumeur ou cancer; problèmes émotionnels; maladie de Parkinson; problèmes de mémoire ou maladie d'Alzheimer; problèmes de vessie, de reins 
ou de prostate; cataractes; glaucome; problèmes d'estomac (gastrite, ulcères, hernie hiatale); fracture de hanche ou de jambe récente (dans la dernière année). Pour les analyses multivariées, la présence d'AVC, de problèmes émotionnels et de problèmes de mémoire ou de maladie d'Alzheimer a été exclue de cette liste afin de pouvoir tester l'influence de ces problèmes de façon séparée. Pour ces analyses, le total des réponses positives aux 13 questions restantes donne le nombre de problèmes de santé. Pour compléter ces informations sur la santé physique des participants, le nombre de jours d'hospitalisation du sujet pendant la période d'observation a été enregistré.

La santé psychologique de la personne âgée est mesurée par une question tirée de l'étude EPESE qui demande si la personne souffre de problèmes émotionnels (nervosité, dépression, anxiété ou insomnie). De plus, pour les personnes qui répondaient elles-mêmes au questionnaire, la santé psychologique a aussi été mesurée par l'Échelle de dépression gériatrique, version courte (EDG) (Sheikh et Yesavage, 1986). Cette donnée n'étant disponible que pour certains sujets, elle ne sera utilisée que dans les analyses descriptives. L'échelle de dépression gériatrique originale (30 énoncés) a démontré une bonne consistance interne (alpha de Chronbach $=0,94)$ et une bonne fidélité test-retest $(0,85$ à une semaine d'intervalle) (Yesavage et al., 1983). La forme courte, qui comporte 15 énoncés, est fortement corrélée $(r=0,84)$ à l'évaluation originale (Sheikh et Yesavage, 1986). Les résultats possibles pour la forme courte vont de 0 à 15 . Un résultat plus élevé indique plus de symptômes dépressifs.

La présence de troubles cognitifs est mesurée par le Short Portable Mental Status Questionnaire (SPMSQ) (Pfeiffer, 1975). Ce questionnaire comporte 10 questions évaluant principalement la mémoire et l'orientation. La fidélité de cette échelle est démontrée par un alpha de Cronbach de 0,89 pour la consistance interne (Foreman, 1987), une fidélité testretest à quatre semaines d'intervalle de 0,82 (Pfeiffer, 1975) et une fidélité interjuges entre 0,62 et 0,87 (Fillenbaum et Smyer, 1981). Pfeiffer (1975) suggère qu'un résultat de 3 erreurs ou plus indique possiblement un déficit cognitif pour une personne qui a une éducation de niveau secondaire. Il suggère de permettre une erreur de plus aux personnes qui n'ont pas fréquenté l'école secondaire et une erreur de moins à ceux qui ont fréquenté le 
collège ou l'université. Il recommande aussi d'accorder une erreur de plus aux AfricainsAméricains. Cette correction pour l'origine ethnique a été adaptée au contexte multiethnique de Montréal, en accordant une erreur de plus aux sujets qui ne sont pas nés au Canada. En utilisant les critères suggérés par Pfeiffer, Foreman (1987) a obtenu un coefficient de prévision d'un test positif de 0,89 .

Pour les personnes qui n'avaient pas la capacité de répondre au SPMSQ (difficulté de communication reliées à la connaissance de la langue ou à un problème de santé), la présence de troubles cognitifs est enregistrée lorsque le questionnaire indique qu'un substitut est utilisé parce que la personne souffre de troubles cognitifs ou lorsqu'à la question tirée de l'étude EPESE sur les problèmes de mémoire, le substitut indique que la personne souffre de la maladie d'Alzheimer ou de problèmes de mémoire attestés par un médecin.

La capacité fonctionnelle est évaluée par l'échelle des soins personnels du Older American Resources and Services (OARS) (Fillenbaum, 1988). Cette échelle comporte sept énoncés qui évaluent la capacité à réaliser diverses activités de la vie quotidienne (s'habiller, manger, contrôler ses sphincters, etc.) et sept énoncés qui évaluent la capacité à réaliser diverses activités de la vie domestique (utiliser le téléphone, utiliser les moyens de transport, magasiner ou faire l'épicerie, préparer les repas, etc.). Les résultats possibles vont de 0 à 28 , un résultat plus élevé indiquant une plus grande autonomie. L'ensemble du questionnaire OARS a démontré une bonne fidélité test-retest $(0,90$ pour trois à huit semaines d'intervalle) et la section des soins personnels a démontré une fidélité interjuges de 0,86 (Fillenbaum, 1988). Pour compléter cette information, la présence de troubles sensoriels a aussi été évaluée en demandant aux répondants s'ils avaient aucune difficulté, un peu de difficulté, ou beaucoup de difficulté à reconnaitre des personnes, à lire ou à entendre, même en utilisant les aides techniques appropriées (lunettes, appareil auditif) le cas échéant. Une variable à trois niveaux a été créée : aucune difficulté pour aucune de ces trois activités; un peu de difficulté pour au moins une des trois activités; et beaucoup de difficulté pour au moins une des trois activités. La présence de troubles de la parole a été 
évaluée en demandant aux sujets s'ils avaient aucune, un peu, ou beaucoup de difficulté à se faire comprendre lorsqu'ils parlent.

Le fait d'être hébergé dans les trois mois suivants le questionnaire peut indiquer une détérioration de la capacité fonctionnelle ou une diminution de la capacité du réseau social à compenser les incapacités. Cette information a été recueillie à partir des banques de données sur l'hébergement de l'Agence de développement des réseaux locaux de services de santé et de services sociaux (ADRLSSSS) de Montréal, agence responsable de la coordination des demandes de placement pour l'ensemble de l'île de Montréal.

\section{Facteurs de capacité de nature organisationnelle}

Deux caractéristiques relevant des services reçus du CLSC ont été considérées. La première est la fréquence de l'aide reçue du CLSC pour les activités de vie quotidienne ou domestique. La seconde est le type de service reçu du CLSC, le service régulier ou le service intégré expérimenté dans le cadre du projet SIPA. En effet, on peut penser que le service intégré, par une gestion de cas plus personnalisée, aurait pu influencer la participation au CJ.

En ce qui concerne les caractéristiques organisationnelles des CJ, les variables suivantes ont été considérées. Le fait que le CJ accepte ou non les personnes qui présentent de l'incontinence fécale, celles qui ont besoin d'être transférées à l'aide de deux personnes, celles qui ont besoin d'aide pour manger, sont observés de façon dichotomique. La

présence d'appels aux participants pour leur rappeler leur journée de participation est observée sur une échelle à trois niveaux : pas d'appels, appels pour les cas particuliers, appels à tous les participants. La durée de la liste d'attente, le coût du CJ et la disponibilité du personnel sont observés sur des échelles continues. La quantité de personnel disponible dans chacun des CJ est mesurée en considérant le ratio du nombre de participants au CJ sur le nombre d'employés en équivalents à temps complet (ETC). Le type de personnel disponible est calculé en pourcentage du total d'ETC, pour chacune des catégories suivantes : personnel de soins (infirmières, assistantes-infirmières, préposés); personnel de réadaptation (ergothérapeute, physiothérapeute, thérapeute en réadaptation physique et 
moniteur en réadaptation); intervenants en loisirs (techniciens en loisirs, éducateurs spécialisés, musicothérapeutes); intervenants psychosociaux (travailleurs sociaux, baccalauréat en psychologie, etc.) et personnel administratif et de soutien (coordonnateur, secrétaire, chauffeur, aide en alimentation).

\section{Analyse des données}

Pour identifier la proportion d'utilisateurs de CJ et décrire qui sont ces utilisateurs, des statistiques descriptives (moyenne, fréquence, intervalles de confiance) ont été calculées. Les différences entre les utilisateurs et les non-utilisateurs ont été étudiées de façon bivariée à l'aide de tests de Student (test de t) et de tests du chi-carré.

Pour mieux comprendre les effets de ces variables sur l'utilisation du CJ, des analyses multivariées (régressions logistiques multiples) ont ensuite été effectuées, en respectant l'ordre du modèle conceptuel (figure 1) pour l'introduction des variables dans ces analyses. Ainsi, toutes les variables relatives aux caractéristiques sociales et de santé ont été d'abord entrées dans les analyses et les variables significatives à $\mathrm{p}<0,10$ après cette première étape d'analyse ont été conservées. De plus, comme le suggèrent Hosmer et Lemeshow (2000), certaines variables jugées importantes cliniquement et démontrant possiblement des interactions avec d'autres variables ont aussi été conservées à ce stade, bien qu'elles n'étaient pas statistiquement significatives dans ces premières analyses. La présence d'interactions parmi ces variables a ensuite été évaluée en entrant chacune des interactions possibles une à une dans les analyses de régression. Les interactions qui individuellement paraissaient significatives à $\mathrm{p}<0,20$ ont alors été entrées simultanément dans le modèle. Un modèle préliminaire contenant les caractéristiques sociales et de santé ainsi que leurs interactions est ainsi créé, en ne conservant que les termes significatifs à $p<0,10$. Les variables organisationnelles ont ensuite été introduites dans le modèle et la procédure a été répétée pour tester l'influence de ces dernières et de leurs interactions. Enfin, les variables qui n'atteignaient pas le seuil de signification de $p<0,05$ ont été retirées du modèle. La qualité d'ajustement de ce modèle a été évaluée à l'aide du test de Hosmer et Lemeshow. 
En préalable à ces analyses, les associations entre les variables indépendantes ont été étudiées de façon à éviter les problèmes de multicolinéarité, et pour chacune des variables continues, la linéarité de la relation avec l'utilisation du CJ a été évaluée. Les analyses ont été effectuées à l'aide du logiciel SPSS pour Windows, version 12.0

Les résultats de l'étude ont été discutés avec les coordonnateurs de trois des CJ inclus dans l'étude et les points importants de ces échanges sont présentés dans la discussion de cet article.

\section{Résultats}

\section{Proportion d'utilisateurs de centre de jour}

Parmi les 723 sujets de l'étude, 136 ont au moins une présence dans un CJ pendant la période d'observation de 6 mois, soit 18,8 \% ( $\mathrm{IC}_{95}: 16,0 \%$ à 21,7\%) de l'échantillon.

\section{Description de l'échantillon}

Les principales caractéristiques des utilisateurs et des non-utilisateurs de CJ, ainsi que de l'échantillon total sont présentées aux tableaux 1, 2 et 3. La moyenne d'âge de l'échantillon est de 83,3 ans $( \pm 7,2)$. Comme on peut s'y attendre pour ce groupe d'âge, les participants sont en majorité des femmes (73,3\%) et des veuves ou veufs (53,3\%). L'étude ayant été menée dans deux quartiers très multiethniques de la ville de Montréal, on observe que 44,5\% des sujets sont nés dans un pays autre que le Canada. Ces immigrants vivent au Canada depuis plusieurs années (moyenne de 38,5 ans $\pm 19,3$ ) et seuls $4,7 \%$ sont au Canada depuis 10 ans ou moins. Dans l'ensemble de l'échantillon, 58,4\% des participants utilisent le français à la maison, 20,5\% utilisent l'anglais et $21,1 \%$ utilisent une autre langue seulement. Seulement $5 \%$ à $6 \%$ des sujets ne semblent pas avoir une connaissance suffisante du français ou de l'anglais pour utiliser ces langues avec les professionnels de la santé. Près de $89 \%$ des sujets ont identifié une personne de soutien principale. On observe que $30 \%$ des sujets sont mariés et que le conjoint est la personne de soutien pour $78 \%$ des

personnes mariées ou $23,4 \%$ de l'échantillon total. Les enfants (filles et fils) sont la 
personne de soutien principale pour $43,4 \%$ des sujets, et un peu plus des deux tiers d'entre eux n'habitent pas avec le parent dont ils prennent soin.

\section{Insérer le tableau 1 près d'ici}

Les sujets de cette étude présentent en moyenne 4,4 $( \pm 2,3)$ problèmes de santé parmi la liste présentée des 16 conditions. On note que $22 \%$ des sujets ont déjà subi un AVC. De plus, $33 \%$ présentent des problèmes émotionnels, alors que 10,6\% de ceux qui ont répondu à l'ÉDG obtiennent un résultat de plus de 10 à cette échelle, ce qui révèle la présence probable de dépression. Près de $27 \%$ de l'échantillon présentent des problèmes cognitifs. Les capacités fonctionnelles des sujets sont très variées, les résultats allant de 0 à 28 à l'échelle des soins personnels OARS. L'incontinence urinaire ou fécale affecte 42,6\% des sujets et les problèmes sensoriels importants touchent près du tiers de l'échantillon.

\section{Insérer le tableau 2 près d'ici}

\section{Différences entre utilisateurs et non-utilisateurs de centre de jour}

Les utilisateurs de CJ de notre échantillon sont en moyenne plus jeunes que les nonutilisateurs (tableau 1). Les utilisateurs de CJ sont proportionnellement moins nombreux parmi les sujets qui détiennent une formation universitaire. Ils sont proportionnellement plus nombreux chez les personnes qui ne sont pas nées au Canada. On observe aussi que $41 \%$ des utilisateurs disent que leur revenu leur permet difficilement de répondre à leurs besoins, comparativement à seulement $32 \%$ des non-utilisateurs.

La fréquence des problèmes de santé physique est similaire dans le groupe des utilisateurs et des non-utilisateurs, sauf en ce qui concerne la présence d'un AVC. En effet, $30 \%$ des utilisateurs de $\mathrm{CJ}$ ont déjà fait un $\mathrm{AVC}$, contre seulement $20 \%$ des nonutilisateurs (tableau 2). En ce qui concerne la santé psychologique des sujets, la présence de problèmes émotionnels est similaire dans les deux groupes. Toutefois, parmi les sujets qui ont répondu à l'ÉDG, les utilisateurs sont près de deux fois plus nombreux à présenter des symptômes indiquant la présence probable de dépression. 
Les capacités fonctionnelles des utilisateurs de CJ, mesurées à l'échelle OARS, sont légèrement inférieures à celles des non-utilisateurs. Une analyse graphique révèle que la relation entre l'utilisation du CJ et les capacités fonctionnelles n'est pas parfaitement linéaire (figure 2). En effet, de façon générale, les personnes qui présentent davantage d'incapacités utilisent le CJ dans une plus grande proportion. Toutefois, aucune des personnes dépendantes pour presque toutes les activités (résultat de 0 à 2 à l'échelle OARS) n'utilise le CJ. Il faut cependant noter que ces personnes ne représentent que 1,2\% de l'échantillon. Enfin, une analyse des résultats à chacune des questions de l'OARS révèle que les utilisateurs de CJ sont moins autonomes $(\mathrm{p}<0,05)$ pour les activités suivantes : prendre un bain, préparer les repas et gérer leur argent. Ils sont aussi plus nombreux à utiliser une orthèse ou une prothèse.

\section{Insérer la figure 2 près d'ici}

Pour ce qui est des facteurs de capacités de nature organisationnelle, on observe que les utilisateurs de CJ sont proportionnellement moins nombreux à ne recevoir aucune aide du CLSC pour leurs activités de vie quotidienne ou domestique et plus nombreux à recevoir de l'aide plus d'une fois par semaine (tableau 3). Ils sont aussi proportionnellement plus nombreux à faire partie du bassin de recrutement d'un CJ qui n'accepte pas les personnes ayant besoin d'être transférées à l'aide de 2 personnes pendant la journée et d'un CJ qui offre une plus forte proportion de personnel de réadaptation.

Insérer le tableau 3 près d'ici

\section{Facteurs qui influencent l'utilisation du centre de jour}

Pour mieux comprendre les facteurs qui influencent l'utilisation des CJ, des analyses de régression logistique ont été effectuées. Les variables relatives aux caractéristiques sociales et de santé ont d'abord été introduites dans les analyses et les variables significatives à $\mathrm{p}<0,10$ après cette première étape ont été conservées, soit : 1'âge, l'éducation, le pays de naissance, la connaissance du français ou de l'anglais et les capacités fonctionnelles. Certaines variables jugées importantes cliniquement en raison de 
la possibilité d'interactions avec d'autres variables ont aussi été conservées à ce stade : le sexe, la cohabitation avec la personne de soutien, le nombre de problèmes de santé, la présence d'un AVC, de problèmes émotifs et de troubles cognitifs. Un modèle préliminaire contenant les caractéristiques sociales et de santé ainsi que leurs interactions est ainsi créé. Les variables organisationnelles suivantes ont ensuite été introduites dans le modèle : le fait d'avoir reçu les services intégrés ou les services réguliers de CLSC, ainsi que la fréquence de l'aide reçue du CLSC pour les AVQ. Les variables reliées aux caractéristiques des CJ (critères d'exclusion, liste d'attente, coût, appels, ratio de personnel) sont fortement corrélées entre elles. Par exemple, les deux CJ qui ont les plus courtes listes d'attente sont aussi ceux qui ont les plus faibles coûts et font aussi partie des CJ qui ont le plus grand ratio de personnes âgées par membre de personnel. L'influence de ces variables a donc dû être testée dans des modèles distincts pour éviter les problèmes de multicolinéarité. Le modèle utilisant le coût présente les meilleures qualités d'ajustement. Aucune des interactions entre les caractéristiques organisationnelles et les caractéristiques sociales ou de santé n'est apparue significative. Le modèle ainsi créé est présenté au tableau 4. Le tableau de contingence du test de Hosmer et Lemeshow indique qu'il s'ajuste bien aux données. De légers écarts (supérieurs à 2 mais inférieurs à 5) dans le nombre de participations observées par rapport à ce que le modèle théorique prédit sont observés pour les $6^{\mathrm{e}}$ et $8^{\mathrm{e}}$ déciles seulement. Étant donné la présence d'interactions, les ratios de cotes ajustés du tableau 4 pour l'âge, le sexe, le pays de naissance, la cohabitation avec la personne de soutien, le nombre de problèmes de santé, la présence d'un AVC et les capacités fonctionnelles ne peuvent être interprétés directement. Les tableaux 5 et 6 présentent des ratios de cotes valables pour divers niveaux de ces variables.

\section{Insérer le tableau 4 près d'ici}

En s'attardant d'abord aux variables qui ne présentent pas d'interactions significatives avec les autres variables du modèle, on observe que, toutes autres choses étant égales, les personnes qui ont une éducation universitaire ont $1,9(1 / 0,515)$ fois moins de chances de fréquenter le $\mathrm{CJ}$ que les personnes qui ont une éducation primaire, secondaire ou collégiale. De plus, les personnes qui reçoivent de l'aide du CLSC pour leurs activités 
quotidiennes ou domestiques de deux à cinq fois par semaine ou six à sept fois par semaine ont respectivement 1,7 et 2,5 fois plus de chances de fréquenter le $\mathrm{CJ}$ que les personnes qui reçoivent une telle aide une fois par semaine ou moins. Enfin, les personnes qui font partie du bassin de desserte d'un CJ offrant un tarif plus élevé voient leurs chances de fréquenter le CJ diminuer de 0,054 fois $(1 / 0,949)$ pour chaque 1 \$ d'augmentation de tarif.

Lorsqu'on s'attarde à l'interaction entre le lieu de naissance et la cohabitation avec la personne de soutien (tableau 5), on observe qu'une personne née au Canada qui cohabite avec une personne de soutien a environ deux fois plus de chances de fréquenter le CJ qu'une personne née au Canada qui ne cohabite pas avec une personne de soutien. Par ailleurs, les personnes nées à l'extérieur du pays ont aussi environ 2 fois plus de chances de participer au CJ que cette dernière, peu importe qu'elles cohabitent ou pas avec une personne de soutien.

\section{Insérer le tableau 5 près d'ici}

Pour interpréter les résultats touchant l'âge, le sexe, le nombre de problèmes de santé, la présence d'un AVC et les capacités fonctionnelles ainsi que leurs interactions, le tableau 6 présente les ratios de cotes pour divers groupes de sujets, comparés au groupe le moins susceptible de fréquenter le $\mathrm{CJ}$, soit celui des hommes plus jeunes qui présentent plusieurs problèmes de santé tout en demeurant très autonomes. Ces résultats indiquent que, si de façon générale les personnes plus âgées sont proportionnellement moins nombreuses à fréquenter le CJ, lorsqu'elles présentent un nombre plus élevé de problèmes de santé, c'est l'inverse qui s'observe. Par exemple, une personne de 70 ans, sans aucun des 13 problèmes de santé étudiés, a 10,3 fois plus de chances de fréquenter le CJ qu'une personne de 90 ans dans la même situation, alors qu'à 8 problèmes de santé, la personne de 90 ans, a 1,1 fois plus de chance de fréquenter le CJ que celle de 70 ans. De plus, pour un même groupe d'âge, les hommes sont moins nombreux à participer au CJ s'ils ont davantage de problèmes de santé. Par exemple, un homme de 90 ans sans aucun des 13 problèmes de santé étudiés, a 2,1 fois plus de chances de fréquenter le CJ qu'un homme de 90 ans qui présente 8 problèmes de santé. On observe l'inverse pour les femmes de 80 ans et plus. 
Ainsi, une femme de 90 ans sans aucun des 13 problèmes de santé étudiés, a 3,9 fois moins de chances de fréquenter le CJ qu'une femme de 90 ans qui présente 8 problèmes de santé.

Les hommes sont plus nombreux à fréquenter le CJ lorsqu'ils présentent davantage d'incapacités fonctionnelles. Ainsi, pour tous les groupes d'âge et tous les niveaux de problèmes de santé, un homme qui obtient seulement 7 à l'échelle de capacités fonctionnelles OARS a 5,1 fois plus de chances de fréquenter le CJ que celui qui est complètement autonome (28 à l'OARS). Toutes choses étant égales par ailleurs, les hommes qui ont fait un AVC ont 3,1 fois plus de chances de fréquenter le CJ que ceux qui n'ont jamais eu d'AVC. Cependant, ces deux relations ne s'observent pas chez les femmes. Ces dernières tendent plutôt à utiliser davantage le CJ lorsqu'elles sont plus autonomes et n'ont pas fait d'AVC, mais ces différences sont peu importantes.

\section{Insérer le tableau 6 près d'ici}

Dans le tableau 6, on note aussi que le groupe le moins susceptible de fréquenter le CJ est celui des hommes plus jeunes qui n'ont pas fait d'AVC mais présentent plusieurs problèmes de santé tout en demeurant très autonomes. Le groupe le plus susceptible de le fréquenter est celui des hommes les plus jeunes, qui présentent un faible nombre de problèmes de santé, mais qui ont fait un AVC et présentent plusieurs incapacités fonctionnelles. Tous les groupes de femmes se situent entre ces deux extrêmes. Les femmes les plus susceptibles d'y participer sont les plus jeunes qui ont peu de problèmes de santé, suivies de celles (plus jeunes ou plus vieilles) qui ont plusieurs problèmes de santé, alors que les femmes les moins susceptibles d'y participer sont les plus âgées, qui ont peu de problèmes de santé.

\section{Discussion}

Le premier objectif de cette étude était d'identifier la proportion d'utilisateurs de CJ parmi une cohorte de personne âgée vulnérable de la région de Montréal qui participaient à une étude plus large sur les services intégrés aux personnes âgées. Une proportion de $18,8 \%$ de l'échantillon a utilisé le CJ pendant la période d'observation. Selon les 
coordonnateurs de $\mathrm{CJ}$ rencontrés pour discuter des résultats, il est réaliste de croire que ce résultat reflète la proportion d'usagers des CLSC du territoire concerné qui participe à un moment donné aux activités des CJ. Il peut être difficile de généraliser ce résultat, étant donné le caractère multiethnique du territoire sur lequel le projet s'est déroulé. Toutefois, dans l'hypothèse où l'utilisation de ce service serait limitée par sa disponibilité, alors il serait possible de penser qu'on obtiendrait un résultat similaire pour l'ensemble des territoires de CLSC de la région de Montréal, puisque la disponibilité, l'organisation et les objectifs des CLSC et des CJ sont similaires pour toute la région. La situation est différente dans les CJ des régions rurales de la province puisque ceux-ci doivent souvent adapter leur programmation en fonction de possibilités de transport sur un vaste territoire.

Le second objectif de cette étude était de déterminer les caractéristiques qui distinguent les utilisateurs de CJ des non-utilisateurs, parmi une clientèle en perte d'autonomie. Les résultats permettent d'identifier plusieurs de ces caractéristiques.

Ainsi, à l'instar de Dabelko et Balaswamy (2000), on note que les utilisateurs de CJ sont plus jeunes que l'ensemble des sujets de notre étude. Une partie de l'explication peut résider dans le fait que les CJ rejoignent peu la clientèle très âgée. En effet, sur les 34 sujets de 95 ans ou plus que comptaient cette étude, seuls 2 étaient des participants de CJ, et ils étaient âgés respectivement de 95 et 97 ans. Aucun des 8 centenaires ne fréquentait le CJ. Les responsables de CJ rencontrés ont observé que les personnes très âgées se sentent particulièrement fragiles et tolèrent moins bien le transport et les activités en groupe. Elles préfèrent donc recevoir des services à la maison.

Tout comme dans l'étude de Chappell (1983), les personnes plus instruites de cet échantillon utilisent moins le CJ. Toutefois, dans la présente recherche, des différences sont observées seulement lorsqu'on isole ceux et celles qui ont une éducation universitaire. Par ailleurs, selon Coulton et Frost (1982), les personnes plus instruites utiliseraient davantage les services récréatifs. Ainsi, les diplômés universitaires ont peut-être davantage accès à des ressources personnelles comme la physiothérapie en privé, ou communautaires comme des groupes de loisirs et des centres d'exercices et donc ressentent moins le besoin de 
fréquenter le CJ. Ou encore, du fait qu'ils sont peu nombreux dans le groupe des 65 ans et plus, ils se sentent moins à l'aise au CJ. Peut-être également que le type d'activités offertes en $\mathrm{CJ}$, conçues pour plaire à la majorité de la clientèle ne leur convient pas. Selon les coordonnateurs de CJ rencontrés, il est aussi possible que les professionnels à la retraite (p. ex. : avocats, médecins) ne soient pas à l'aise de participer à un groupe au coté d'anciens clients. Cette observation mériterait d'être testée dans des recherches ultérieures.

Cette étude démontre aussi que lorsque les services sont accessibles dans les quartiers multiethniques, les personnes immigrantes les utilisent autant que les personnes nées au Canada. Chappell (1983), qui avait obtenu un résultat contraire, avait remis en question l'accessibilité des services dans les secteurs plus multiethniques du Manitoba.

Comme on pouvait s'y attendre, chez les personnes nées au Canada, la cohabitation avec une personne de soutien augmente les chances d'utiliser le CJ. Le besoin de répit de la personne de soutien, de même que la stimulation que cette dernière peut fournir à la personne âgée pour lui faire voir les bienfaits que le CJ peut lui apporter, peuvent expliquer ce résultat. Un résultat plus surprenant est que chez les personnes qui sont nées à l'extérieur du Canada, cette relation n'est pas observée. Pourquoi ces personnes fréquentent-elles autant le CJ lorsqu'elles ne cohabitent pas avec une personne de soutien que lorsqu'elles cohabitent? Peut-être que les personnes originaires du Canada qui vivent seules le font par choix ou par souci d'indépendance, alors que les personnes d'une autre appartenance culturelle qui vivent seules le font par obligation, parce qu'elles sont isolées de leur famille et donc, recherchent davantage la participation à un groupe. Peut-être aussi que les intervenants qui dirigent les personnes âgées vers les services ajoutent le déracinement à la liste des critères qu'ils considèrent et les dirigent davantage vers les CJ. Peut-être aussi que des personnes nées dans des pays offrant peu de services publics se sentent-elles privilégiées de l'offre de services disponibles ici et acceptent-elles plus rapidement les services qui leur sont proposés par les intervenants. Ce ne sont là que quelques hypothèses qui peuvent expliquer ce résultat. Des études qualitatives permettraient de fournir des réponses plus pertinentes à cette question. 
Alors que les études antérieures n'avaient pas démontré de lien clair entre le sexe et l'utilisation du CJ, cette étude démontre que si les hommes et les femmes utilisent le CJ dans des proportions semblables, ils ne l'utilisent pas de la même façon. C'est lorsqu'ils ont subi un AVC et lorsqu'ils présentent plusieurs incapacités fonctionnelles que les hommes utilisent davantage le CJ. Pour les femmes, l'utilisation ne varie pas en fonction de ces variables. On peut penser que les hommes utilisent davantage le CJ pour y faire des exercices dans le but de maintenir ou d'améliorer leurs capacités fonctionnelles, ou encore comme un répit pour leur personne de soutien. Les femmes utilisent le CJ pour ces mêmes raisons ou encore pour briser l'isolement, puisqu'elles sont plus nombreuses à vivre seules. Cependant, ces résultats sont observés même lorsque la cohabitation avec une personne de soutien est contrôlée. Ainsi, il faut peut-être regarder de plus près le type de lien avec la personne de soutien. On remarque alors que pour les $68 \%$ d'hommes qui cohabitent avec une personne de soutien, la conjointe est la personne de soutien dans $85 \%$ des cas. Pour les $31 \%$ des femmes qui cohabitent avec une personne de soutien, le conjoint est présent dans $35 \%$ des situations, alors que dans $65 \%$ des cas, il s'agit d'un autre proche. On peut penser que lorsque l'homme présente peu d'incapacités, son épouse lui apporte facilement le soutien dont il a besoin. Habituée à partager le quotidien de son conjoint, elle ne sent peutêtre pas de besoin de répit lorsque que celui-ci peut être laissé sans surveillance et que les soins à lui apporter ne l'empêchent pas d'accomplir ses autres tâches. Par contre, lorsque les incapacités sont élevées, le répit s'imposera pour permettre le maintien à domicile du conjoint. À l'inverse, on peut imaginer la situation de la femme veuve qui emménage avec une tierce personne parce qu'elle ne peut plus vivre seule malgré un niveau d'incapacités peu élevé. Le simple fait de devoir s'habituer à vivre avec cette personne âgée quotidiennement peut demander un effort d'adaptation qui créera un fardeau et un besoin de répit pour la personne de soutien. Ou encore, si cette tierce personne occupe un emploi, la personne âgée peut se sentir isolée même lorsqu'elle cohabite avec sa personne de soutien et ressentir le besoin du CJ pour briser cet isolement. Encore ici, ce ne sont que quelques hypothèses qui pourraient être vérifiées dans des études qualitatives. 
Contrairement à celle de Dabelko et Balaswamy (2000), cette étude n'a pas démontré de différence significative dans le nombre de jours d'hospitalisation des participants au CJ. Par contre, les utilisateurs de CJ sont légèrement moins autonomes que les non-utilisateurs. De plus, comme on pouvait s'y attendre, les personnes qui présentent des incapacités très importantes (0 à 2 à l'OARS) ne fréquentent pas le CJ. Ces incapacités très importantes rendent probablement impossibles les déplacements hors du domicile. Pour les mêmes raisons, on aurait pu penser que les personnes ayant des incapacités importantes (3 à 6 et 7 à 10 à l'OARS) seraient aussi moins représentées chez les utilisateurs de CJ. Ce n'est pas le cas. Hormis les personnes ayant un résultat de 0 à 2 à l'OARS, les seuls groupes qui ont des taux de participation inférieurs à la moyenne sont les groupes les plus autonomes (18 et plus à l'OARS). Ainsi, les CJ remplissent leur mission de desservir les personnes en perte d'autonomie.

Contrairement à d'autres études (Baumgarten et coll., 2002; Dabelko et Balaswamy, 2000; Montgomery et coll., 1988-1989), celle-ci n’a pas révélé de relation entre la présence de troubles cognitifs et l'utilisation du CJ. Bien que les CJ reçoivent une grande proportion de personnes avec des troubles cognitifs afin d'offrir du répit à leur personne de soutien, il est possible de penser qu'un nombre aussi important est desservi à domicile par d'autres ressources, étant donné qu'il n'est pas toujours facile de faire accepter la participation au CJ à cette clientèle. De plus, lorsque la personne manifeste des troubles de comportement qui nuisent au fonctionnement du groupe, elle sera exclue du CJ. Par ailleurs, il est possible que le nombre de personnes atteintes de troubles cognitifs ait été sous évalué dans la présente étude. En effet, il se peut que le SPMSQ n'identifie pas bien toutes les formes de troubles cognitifs. Par exemple, contrairement au Mini examen de l'état mental (Folstein, Folstein et McHugh, 1975), il n'y a aucune question sur les praxies ou la planification motrice dans le SPMSQ.

En ce qui concerne la présence de symptômes de dépression, les résultats de cette étude sont cohérents avec ceux de Baumgarten et coll. (2002). Les utilisateurs de CJ présentent des scores de dépression légèrement supérieurs à ceux des non-utilisateurs. Toutefois, en raison d'un nombre important de réponses manquantes (questionnaires 
remplis par des substituts), l'importance de cette variable n'a pu être étudiée dans les analyses de régression. La présence de troubles émotionnels, qui peut inclure en plus de la dépression, de l'anxiété et des troubles du sommeil, peut influencer l'utilisation du CJ de façon différente selon le problème en question. Cette variable trop générale n'a pas permis de distinguer les utilisateurs des non-utilisateurs.

Deux facteurs organisationnels ont démontré une association avec l'utilisation du CJ. Premièrement, on remarque que la probabilité de fréquenter le CJ diminue dans les bassins des CJ qui facturent un montant plus élevé aux participants. Cette influence peut sembler peu importante puisque les chances de fréquenter le CJ diminuent de 0,054 fois pour chaque $1 \$$ d'augmentation de tarif. Cependant, lorsqu'on considère qu'un des CJ de cette étude facture $17 \$$ par présence à ses participants et que ceci est nettement au-dessus des coûts demandés par les autres CJ de l'étude ( 5 à $8,50 \$)$, on réalise que pour ce centre, la probabilité de fréquenter le $\mathrm{CJ}$ diminue de $68 \%$ par rapport à un centre qui facture le coût médian de $7 \$^{3}$.

Deuxièmement, on observe qu'à capacités fonctionnelles égales, une aide plus fréquente du CLSC pour les activités quotidiennes et domestiques est associée à une plus grande probabilité de fréquenter le CJ. Ceci peut s'expliquer de plusieurs façons. Ce peut être relié à la propension à accepter l'aide des services formels, tant de la part du CLSC que du CJ. Ce peut être une indication que la personne qui est plus encadrée par le CLSC sera plus stimulée par le personnel du CLSC à participer aux activités du CJ. À l'inverse, ce peut être la participation au CJ qui induit une plus grande aide de la part du CLSC. En effet, les intervenants des CJ peuvent s'apercevoir que la condition d'un client se détériore et faire plus rapidement une demande d'aide supplémentaire au CLSC. La personne ellemême pourrait attendre plus longtemps avant de faire une telle demande, malgré une détérioration de ses capacités. De plus, afin d'offrir un meilleur répit à leur personne de soutien, plusieurs participants sont préparés (aide à l'hygiène et à l'habillement) par le personnel du CLSC lors de leurs journées de fréquentation du CJ. Cette réalité peut faire augmenter la fréquence de l'aide reçue par les participants des CJ. 
Enfin, contrairement à ce que nous aurions pu croire, le fait de recevoir les services intégrés expérimentés dans le cadre du projet SIPA, plutôt que les services réguliers du CLSC n'a pas influencé l'utilisation du CJ. Les services des CJ sont probablement déjà bien connus par les intervenants réguliers des CLSC qui y dirigent des clients régulièrement. Les clients dirigés en CJ par les gestionnaires de cas du projet SIPA ou par les intervenants des services réguliers des CLSC, devaient se plier aux mêmes règles de gestion de la liste d'attente, le cas échéant. Car, si le budget additionnel des gestionnaires de cas SIPA permettait l'ajout d'heures de services à domicile, il n'était pas possible de faire l'achat de jours additionnels en CJ dans le contexte actuel de la gestion des CJ concernés.

\section{Forces et limitations de l'étude}

Bien que largement répandus comme service de soutien à domicile, les CJ ont été peu étudiés au Canada. Cette étude remédie en partie à cette lacune en décrivant les caractéristiques des utilisateurs de CJ dans le contexte québécois. À notre connaissance, cette recherche sur les déterminants de l'utilisation du CJ est l'une des premières à vérifier la présence d'interaction entre les diverses variables à l'étude, ce qui a permis avec un échantillon de taille moyenne, de faire ressortir des différences importantes dans l'utilisation du CJ entre les hommes et les femmes. Ces différences n'étaient pas ressorties dans les études précédentes.

Les éléments mis en lumière dans cette étude peuvent permettre de mieux cibler les interventions des CJ pour les personnes les plus susceptibles d'en bénéficier. Ainsi, en prenant connaissance de ces résultats, les intervenants qui dirigent les personnes âgées vers les CJ pourront réaliser l'importance de discuter de cette possibilité avec les hommes qui ont fait un $\mathrm{AVC}$ et qui présentent des incapacités importantes, clientèle qui répond positivement à l'offre de fréquentation du CJ. Les responsables de CJ, quant à eux, pourront se questionner sur les raisons qui font que certaines clientèles sont moins représentées au CJ, comme les personnes avec une éducation universitaire. Est-ce 
réellement parce que leurs besoins sont moins grands ou est-ce parce que leur programmation d'activités n'est pas adaptée aux besoins de ces clientèles?

Cette étude comporte aussi certaines limites. En ce qui concerne sa validité externe, il faut noter qu'il s'agit d'une analyse secondaire de données recueillies pour une étude plus large sur les services intégrés aux personnes âgées. L'échantillon n'est donc pas un échantillon représentatif de l'ensemble de la population âgée qui présente des incapacités. La population à l'étude était déjà connue des services de maintien à domicile. Combien y at-il de personnes âgées qui présentent des incapacités aussi importantes que celles de cet échantillon mais n'ont pas recours aux services des CLSC ? Ces personnes sont peut-être plus isolées et plus démunies en termes de ressources d'aide. Certaines d'entre elles pourraient sans doute bénéficier des services offerts par les CJ. Toutefois, les façons de les rejoindre sont limitées. De plus, les caractéristiques des utilisateurs de cette étude ne reflètent pas les caractéristiques de l'ensemble des utilisateurs de CJ pour personnes âgées, puisque certains $\mathrm{CJ}$ admettent des personnes de moins de 65 ans qui présentent un profil d'incapacités gériatriques. Ces personnes n'étaient pas admissibles à la présente étude. Il faut aussi noter aussi que la population du territoire sur lequel le projet s'est déroulé est plus multiethnique que celle de l'ensemble de l'île de Montréal (43,5 à 48 \% d'immigrants contre $28 \%$ pour Montréal) et, sur un des deux territoires de CLSC, plus instruite (seulement $15 \%$ d'adultes n'ayant pas terminé des études secondaires contre 25 à $27 \%$ pour l'autre territoire et l'ensemble de l'île de Montréal) (ADRLSSSS de Montréal, 2004). Ceci peut avoir influencé certains résultats, en particulier ceux qui concernent l'influence de l'éducation et du pays de naissance sur l'utilisation des CJ. Enfin, en réalisant une analyse secondaire de données déjà recueillies, il a été impossible de distinguer parmi les non-utilisateurs, les personnes qui ont déjà été dirigées au CJ et qui ont choisi de ne pas y participer, de celles qui n'ont jamais été dirigées vers ce type de services.

En ce qui concerne la validité interne de l'étude, il faut noter que différentes caractéristiques de la personne de soutien, comme le fardeau de cette personne (Baumgarten et coll., 2002) ou l'influence de son réseau formel et informel (Cotrell et Engel, 1998) peuvent aussi influencer l'utilisation du CJ par la personne âgée. Ces 
caractéristiques n'ont pas été considérées dans la présente étude, étant donné le nombre de questionnaires aux personnes de soutien qui étaient manquants ou incomplets. La prise en compte de ces variables aurait entraîné une réduction importante de la taille de l'échantillon de l'étude. Enfin, le fait que cette étude ne portait que sur cinq centres de jours a réduit la possibilité d'étudier l'influence des caractéristiques organisationnelles des CJ et des interactions entre ces dernières et les caractéristiques sociales ou de santé, puisque les caractéristiques de ces cinq CJ étaient fortement corrélées entre elles.

\section{Conclusion}

Nous croyons que les résultats obtenus peuvent être utiles aux gestionnaires de CJ et aux planificateurs de services pour les personnes âgées. Ces résultats indiquent d'abord que le coût des services crée une contrainte qui peut réduire l'accès au CJ. Ils montrent aussi que les hommes et les femmes n'utilisent pas le service de la même façon et ont probablement des attentes différentes envers ce type de service. Ces résultats fournissent des pistes à explorer pour mieux comprendre ces différences afin d'éventuellement mieux adapter les services aux besoins distincts de ces clientèles. Ils incitent aussi à approfondir les raisons qui font que les diplômés universitaires utilisent moins ce type de services. Étant donné que la proportion d'universitaires parmi la population âgée devrait augmenter dans les années à venir, il serait important de répondre à cette question pour s'assurer que les besoins de ce groupe seront pris en compte. Enfin, pour mieux comprendre les déterminants de la participation au CJ, il y aurait aussi lieu d'explorer les différences entre les groupes qui sont moins dirigés vers ces services et ceux qui, une fois dirigés vers un CJ, refusent d'y participer ou cessent plus rapidement leur participation. Les résultats présentés dans cette étude fournissent certaines pistes pour aborder ces questions dans des recherches ultérieures auprès des sources de référence des CJ. 


\section{Notes}

1 Le projet SIPA consiste en l'expérimentation d'un modèle de services intégrés pour les personnes âgées qui présentent des incapacités. Le modèle a été développé par un groupe de chercheurs l'Université McGill et de l'Université de Montréal (Béland, Bergman et Lebel, 2001; Béland, Bergman, Lebel, Clarfield, et coll., 2006; Béland, Bergman, Lebel, Dallaire, et coll., 2006). Ce modèle prévoit qu'une organisation unique exerce la responsabilité clinique des services peu importe l'endroit où ils sont prodigués. Il a été implanté dans deux CLSC de la région de Montréal comme programme distinct des services à domicile régulier et a été offert à un nombre limité de clients à titre de projet de démonstration. Les personnes du groupe expérimental ont été suivies par un gestionnaire de cas SIPA, assisté d'une équipe multidisciplinaire. Elles ont reçu de la part de l'équipe SIPA des services de soutien à domicile similaires à ce qu'elles recevaient du CLSC avant l'implantation du projet. De plus, le gestionnaire de cas avait la capacité de mobiliser rapidement des ressources additionnelles en cas de besoin (accès à des places réservées dans des résidences pour personnes âgées et à un budget pour permettre une augmentation temporaire des services à domicile), afin d'éviter une utilisation inappropriée des ressources hospitalières. En cas d'hospitalisation, il assurait un suivi auprès de l'équipe hospitalière. Les personnes qui recevaient les services intégrés SIPA avaient aussi accès à un service téléphonique 24 heures par jour, 7 jours par semaine, pour une réponse rapide à leur besoin. Chaque gestionnaire de cas SIPA n'avait pas plus de 40 clients sous sa responsabilité afin d'assurer une réponse personnalisée à chacun. Ce modèle de services intégrés a pu être expérimenté grâce à un financement de \$ 7,6 millions du Fonds d'adaptation des services de santé de Santé Canada et du Ministère de la Santé et des Services sociaux du Québec.

2 JEDI pour Jonction et diffusion informatiques, le nom de la compagnie qui a créé ce logiciel.

${ }^{3}$ Ce résultat provient de $\mathrm{B}=-0,052, \mathrm{e}^{10 \mathrm{~B}}=0,5951 / 0,595=1,682$ 


\section{Figure 1 : Facteurs qui influencent l'utilisation du centre de jour}

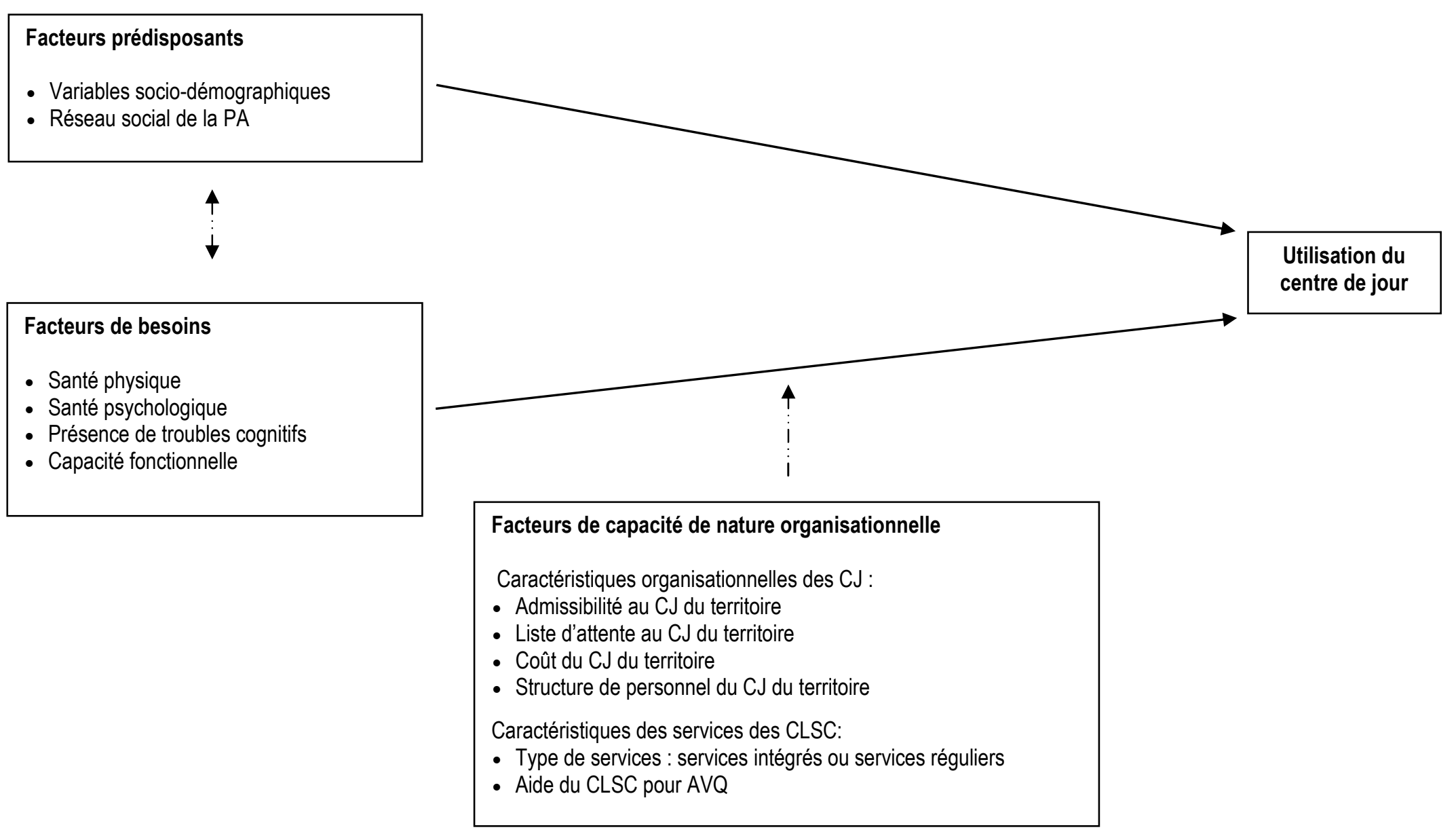


Figure 2 : Relation entre l'utilisation du centre de jour et les capacités fonctionnelles

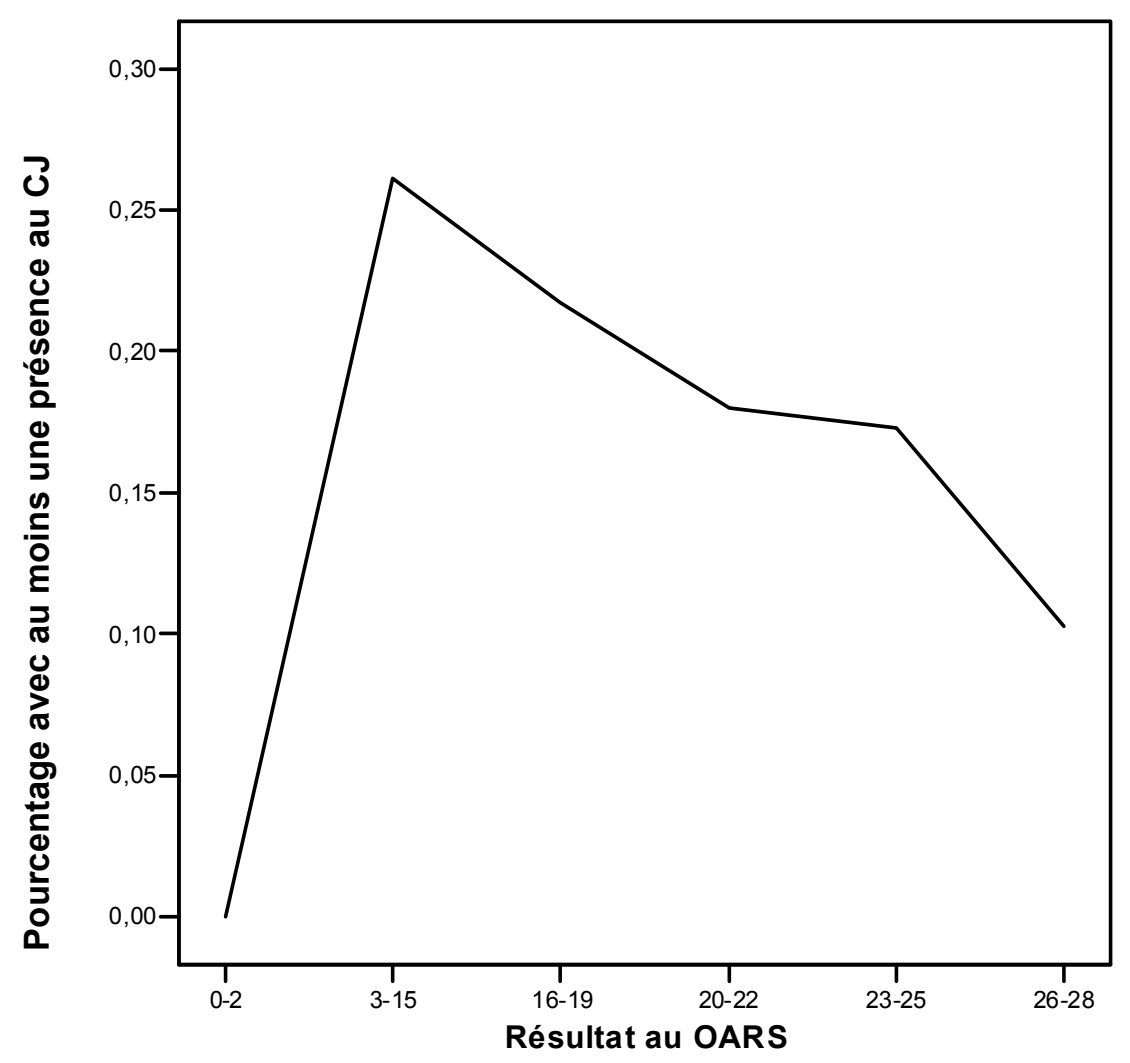

Note : sur le graphique ci-dessus, chacune des catégories de résultats à l'OARS représente environ $20 \%$ de l'échantillon, sauf la catégorie $0-2$ qui ne comprend que 1,2\% des sujets de l'étude. 
Tableau 1 : Facteurs prédisposants ou caractéristiques sociales des personnes âgées

\begin{tabular}{|c|c|c|c|c|c|c|c|c|c|}
\hline & \multicolumn{2}{|c|}{ Utilisateurs } & \multicolumn{2}{|c|}{ Non-utilisateurs } & \multirow[b]{2}{*}{ test } & \multirow[b]{2}{*}{ sig. } & \multicolumn{2}{|c|}{ Echantillon total } \\
\hline & & Moy. ou \% & $\mathbf{n}$ & Moy. ou \% & $\mathbf{n}$ & & & Moy. ou \% & $\mathrm{n}$ \\
\hline \multicolumn{10}{|c|}{ Variables sociodémographiques } \\
\hline \multicolumn{2}{|l|}{ Âge } & $80,68( \pm 6,83)$ & 136 & $83,92( \pm 7,08)$ & 587 & $\mathrm{t}$ &, $000 *$ & $83,31( \pm 7,15)$ & 723 \\
\hline \multicolumn{2}{|c|}{ Sexe (proportion de femmes) } & $73,5 \%$ & 136 & $73,3 \%$ & 587 & $\mathrm{t}$ & 948 & $73,3 \%$ & 723 \\
\hline État civil & $\begin{array}{l}\text { - Célibataire (Jamais marié) } \\
\text { - Marié ou conjoint de fait } \\
\text { - veuf } \\
\text { - séparé ou divorcé } \\
\text { - religieux }\end{array}$ & $\begin{array}{r}14,0 \% \\
33,8 \% \\
49,3 \% \\
2,9 \% \\
0,0 \%\end{array}$ & 136 & $\begin{array}{r}11,6 \% \\
29,1 \% \\
54,1 \% \\
5,0 \% \\
0,2 \%\end{array}$ & 585 & $\chi^{2}$ &, 549 & $\begin{array}{r}12,1 \% \\
30,0 \% \\
53,3 \% \\
4,6 \% \\
0,1 \%\end{array}$ & 721 \\
\hline Éducation & $\begin{array}{l}\text { - aucune } \\
\text { - primaire non complétée } \\
\text { - primaire complétée } \\
\text { - secondaire } \\
\text { - école techn. ou de métiers } \\
\text { - universitaire }\end{array}$ & $\begin{array}{r}3,0 \% \\
15,9 \% \\
15,9 \% \\
42,4 \% \\
11,4 \% \\
11,4 \%\end{array}$ & 132 & $\begin{array}{r}2,9 \% \\
13,9 \% \\
14,0 \% \\
39,5 \% \\
9,2 \% \\
20,5 \%\end{array}$ & 577 & $\chi^{2}$ & ,300 & $\begin{array}{r}3,0 \% \\
14,2 \% \\
14,4 \% \\
40,1 \% \\
9,6 \% \\
18,8 \%\end{array}$ & 709 \\
\hline \multicolumn{2}{|c|}{ Proportion avec éducation universitaire } & $11,4 \%$ & & $20,5 \%$ & & $\mathrm{t}$ &, 004 * & & \\
\hline \multicolumn{2}{|c|}{ Profession antérieure associée à la santé } & $6,6 \%$ & 136 & $8,9 \%$ & 587 & $t$ & ,398 & $8,4 \%$ & 723 \\
\hline \multicolumn{2}{|c|}{$\begin{aligned} \text { Pays de naissance } & \text { - Canada } \\
& \text { - autre }\end{aligned}$} & $\begin{array}{l}45,6 \% \\
54,4 \%\end{array}$ & 136 & $\begin{array}{l}57,8 \% \\
42,2 \%\end{array}$ & 587 & $t$ &, $010 *$ & $\begin{array}{l}55,5 \% \\
44,5 \%\end{array}$ & 723 \\
\hline Religion & $\begin{array}{l}\text { - catholique } \\
\text { - juive } \\
\text { - autre (incluant aucune) }\end{array}$ & $\begin{array}{l}59,6 \% \\
19,9 \% \\
20,6 \%\end{array}$ & 136 & $\begin{array}{l}61,0 \% \\
22,4 \% \\
16,6 \%\end{array}$ & 585 & $\chi^{2}$ &, 500 & $\begin{array}{l}60,7 \% \\
21,9 \% \\
17,3 \%\end{array}$ & 721 \\
\hline \multicolumn{10}{|c|}{ Langue } \\
\hline \multicolumn{2}{|c|}{$\begin{array}{l}\text { - français à la maison et avec } \\
\text { professionnels } 1\end{array}$} & $56,3 \%$ & 135 & $\begin{array}{l}58,9 \% \\
20,6 \%\end{array}$ & 587 & $\chi^{2}$ & 866 & $\begin{array}{l}58,4 \% \\
20.5 \%\end{array}$ & 722 \\
\hline \multicolumn{2}{|c|}{$\begin{array}{l}\text { anglais à la maison et avec } \\
\text { professionnels } 1\end{array}$} & $20,0,10$ & & & & & & & \\
\hline \multicolumn{2}{|c|}{$\begin{array}{l}\text { - anglais ou français avec les } \\
\text { professionnels (pas à la maison) }\end{array}$} & $17,8 \%$ & & $16,0 \%$ & & & & $16,3 \%$ & \\
\hline \multirow{2}{*}{\multicolumn{2}{|c|}{$\begin{array}{l}\text { - autre langue à la maison, langue avec } \\
\text { les professionnels est manquante } \\
\text { - autre langue (ni anglais ni français) } \\
\text { même avec les professionnels }\end{array}$}} & $2,2 \%$ & & $1,2 \%$ & & & & $1,4 \%$ & \\
\hline & & $3,7 \%$ & & $3,2 \%$ & & & & $3,3 \%$ & \\
\hline \multicolumn{2}{|c|}{$\begin{array}{l}\text { Proportion qui estime que leur revenu } \\
\text { répond difficilement à leurs besoins }\end{array}$} & $40,4 \%$ & 132 & $31,7 \%$ & 565 & $t$ &, $036 *$ & $33,6 \%$ & 697 \\
\hline \multicolumn{10}{|c|}{ Réseau social } \\
\hline \multicolumn{2}{|c|}{$\begin{array}{l}\text { Lien PA-PS - aucune PS } \\
\text { - Conjoint(e) } \\
\text { - Autre co-résident (enfant) } \\
\text { - Autre, non co-résident (enfant) }\end{array}$} & $\begin{array}{l}11,0 \% \\
27,2 \% \\
23,5 \%(16,9 \%) \\
38,2 \%(26,5 \%)\end{array}$ & 136 & $\begin{array}{l}11,2 \% \\
22,5 \% \\
16,4 \%(11,2 \%) \\
49,9 \%(32,2 \%)\end{array}$ & 587 & $\chi^{2}$ & 060 & $\begin{array}{l}11,2 \% \\
23,4 \% \\
17,7 \%(12,3 \%) \\
47,7 \%(31,1 \%)\end{array}$ & 723 \\
\hline
\end{tabular}

1 Ces personnes utilisent le français ou l'anglais à la maison parfois en plus d'une autre langue 
Tableau 2 : Facteurs de besoin ou état de santé des personnes âgées

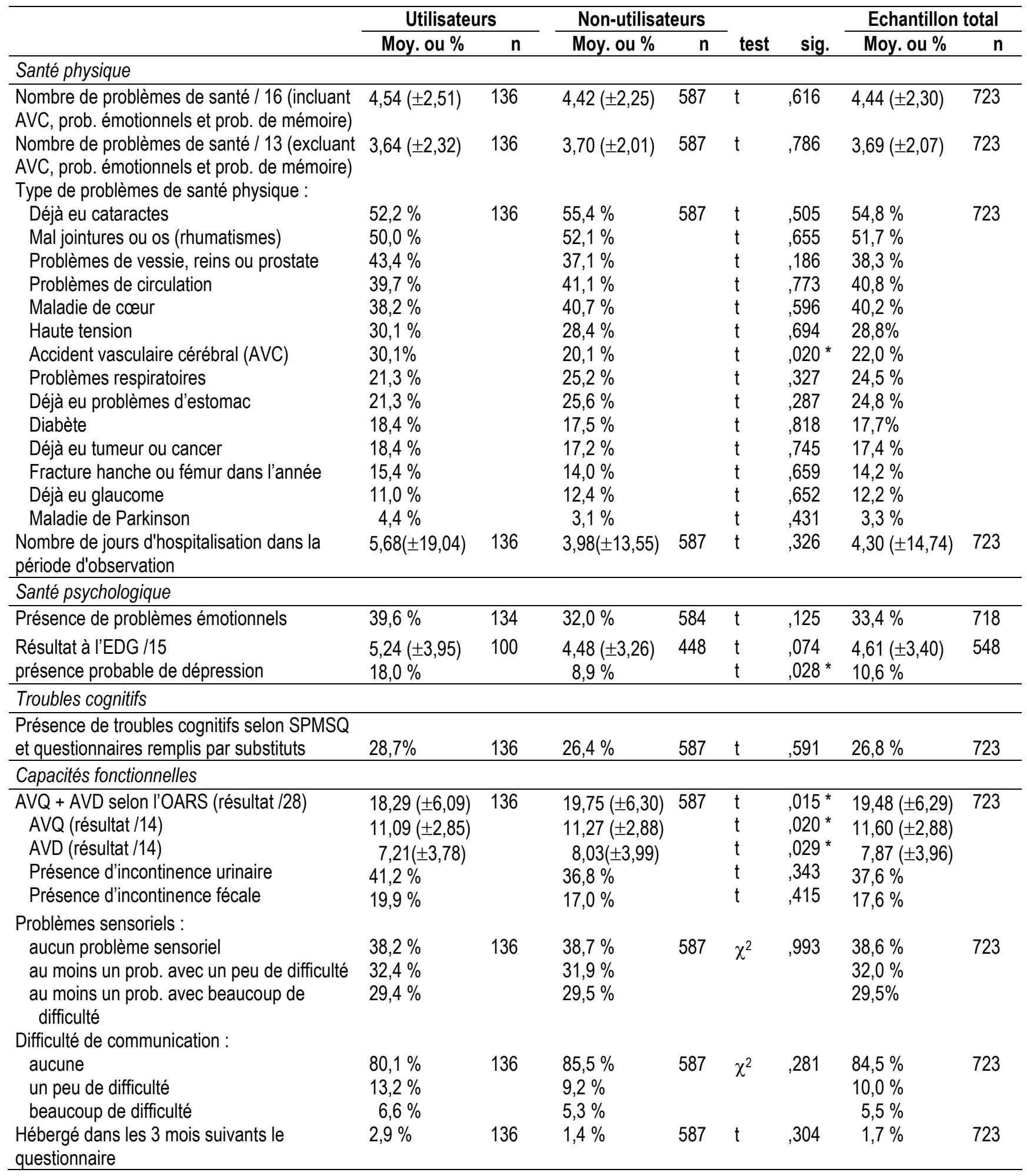


Tableau 3 : Facteurs de capacité de nature organisationnelle

\begin{tabular}{|c|c|c|c|c|c|c|c|c|}
\hline & \multicolumn{2}{|c|}{ Utilisateurs } & \multicolumn{2}{|c|}{ Non-Utilisateurs } & \multirow[b]{2}{*}{ test } & \multirow[b]{2}{*}{ sig. } & \multicolumn{2}{|c|}{ Echantillon total } \\
\hline & Moy. ou \% & $\mathrm{n}$ & Moy. ou \% & $\mathrm{n}$ & & & Moy. ou \% & $\mathrm{n}$ \\
\hline \multicolumn{9}{|l|}{ Services reçus du CLSC } \\
\hline \multicolumn{9}{|l|}{ Fréquence approx. de l'aide du CLSC : } \\
\hline $0:$ aucune intervention recensée & $36,8 \%$ & 136 & $50,3 \%$ & 587 & $\chi^{2}$ &, $011^{*}$ & $47,7 \%$ & 723 \\
\hline 1: moins d'une fois / sem. & $7,4 \%$ & & $8,2 \%$ & & & & $8,0 \%$ & \\
\hline $2: 1$ fois $/ \mathrm{sem}$ & $13,2 \%$ & & $14,3 \%$ & & & & $14,1 \%$ & \\
\hline $3: 2$ à 5 fois / sem. & $30,1 \%$ & & $20,4 \%$ & & & & $22,3 \%$ & \\
\hline $4: 6$ ou 7 fois $/$ sem. & $12,5 \%$ & & $6,8 \%$ & & & & $7,9 \%$ & \\
\hline Services intégrés (SIPA) & $53,7 \%$ & 136 & $52,5 \%$ & 587 & $t$ & 800 & $52,7 \%$ & 723 \\
\hline Services réguliers & $46,3 \%$ & & $47,5 \%$ & & & & $47,3 \%$ & \\
\hline \multicolumn{9}{|l|}{ Caractéristiques des CJ } \\
\hline \multicolumn{9}{|l|}{$\begin{array}{l}\text { Critères d'exclusion : proportion qui fait partie } \\
\text { du bassin d'un CJ qui n'accepte pas les } \\
\text { personnes: }\end{array}$} \\
\hline $\begin{array}{l}\text { qui doivent être transférées à } 2 \text { pers. } \\
\text { avec incontinence fécale }\end{array}$ & $\begin{array}{r}65 \% \\
8 \%\end{array}$ & 136 & $\begin{array}{r}48 \% \\
7 \%\end{array}$ & 587 & $\begin{array}{l}t \\
t\end{array}$ & $\begin{array}{l}.000 * \\
815\end{array}$ & $\begin{array}{r}51,2 \% \\
7,6 \%\end{array}$ & 723 \\
\hline qui ont besoin d'aide pour s'alimenter & $21 \%$ & & $15 \%$ & & $\mathrm{t}$ &, 119 & $16,5 \%$ & \\
\hline Liste attente du CJ en mois & $3,20( \pm 2,71)$ & 136 & $3,14( \pm 2,27)$ & 587 & $t$ & ,816 & $3,15( \pm 2,36)$ & 723 \\
\hline Coût du CJ & $\begin{array}{l}7,87 \$ \\
( \pm 4,18)\end{array}$ & 136 & $\begin{array}{l}8,57 \$ \\
( \pm 4,49)\end{array}$ & 587 & $\mathrm{t}$ & 086 & $\begin{array}{l}8,44 \$ \\
( \pm 4,44)\end{array}$ & 723 \\
\hline $\begin{array}{l}\text { Fait partie du bassin d'un CJ qui : } \\
\text { n'appelle pas les participants }\end{array}$ & & 136 & & 587 & & & & \\
\hline $\begin{array}{l}\text { nappelle pas les participants } \\
\text { appelle les cas particuliers }\end{array}$ & $\begin{array}{l}21,3 \% \\
18,4 \%\end{array}$ & 130 & $\begin{array}{l}15,3 \% \\
29,8 \%\end{array}$ & 581 & $\chi^{2}$ & , $01 /^{*}$ & $\begin{array}{l}16,5 \% \\
27,7 \%\end{array}$ & 123 \\
\hline appelle tous les participants & $60,3 \%$ & & $54,9 \%$ & & & & $55,9 \%$ & \\
\hline Ratio personnes âgées inscrites par jour/ETC & $5,68( \pm 1,07)$ & 136 & $5,56( \pm 1,08)$ & 587 & $\mathrm{t}$ & ,213 & $5,58( \pm 1,08)$ & 723 \\
\hline Ratio personnes âgées présentes par jour/ETC & $3,72( \pm 0,48)$ & & $3,64( \pm 0,45)$ & & $\mathrm{t}$ & 091 & $3,65( \pm 0,46)$ & \\
\hline$\%$ de personnel de soins & $20,7 \%( \pm 8,6)$ & 136 & $21,7 \%( \pm 8,0)$ & 587 & $t$ & 212 & $21,5 \%( \pm 8,1)$ & 723 \\
\hline$\%$ de personnel de réadaptation & $26,9 \%( \pm 12,6)$ & & $24,3 \%( \pm 11,9)$ & & $\mathrm{t}$ &, 031 * & $24,8 \%( \pm 12,0)$ & \\
\hline$\%$ d'intervenants en loisirs & $18,5 \%( \pm 6,9)$ & & $19,2 \%( \pm 6,9)$ & & $\mathrm{t}$ & ,281 & $19,0 \%( \pm 6,9)$ & \\
\hline$\%$ d'intervenants psychosociaux & $7,3 \%( \pm 4,8)$ & & $7,9 \%( \pm 5,6)$ & & $\mathrm{t}$ & ,181 & $7,8 \%( \pm 5,5)$ & \\
\hline$\%$ de personnel administratif et de soutien & $26,7 \%( \pm 5,4)$ & & $27,0 \%( \pm 6,0)$ & & $\mathrm{t}$ &, 575 & $27,0 \%( \pm 5,9)$ & \\
\hline
\end{tabular}


Tableau 4 : Régression logistique

\begin{tabular}{|c|c|c|c|c|}
\hline & $\begin{array}{c}\text { Coefficient } \\
\text { B }\end{array}$ & $\begin{array}{c}\text { Ratio de } \\
\text { cote ajusté }\end{array}$ & $\begin{array}{l}\text { IC } 95,0 \% \text { pour le } \\
\text { ratio de cote ajusté }\end{array}$ & Signif. \\
\hline \multicolumn{5}{|c|}{ Facteurs prédisposants (Caractéristiques sociales) } \\
\hline Âge & $-0,117$ & 0,890 & $(0,840-0,943)$ &, $000^{*}$ \\
\hline \multicolumn{5}{|l|}{ Sexe } \\
\hline Masculin & & 1,000 & & \\
\hline Féminin & $-1,941$ & 0,144 & $(0,026-0,782)$ &, 025 * \\
\hline \multicolumn{5}{|l|}{ Éducation } \\
\hline Primaire, secondaire ou collégial & & 1,000 & & \\
\hline Universitaire & $-0,664$ & 0,515 & $(0,277-0,957)$ &, 036 * \\
\hline \multicolumn{5}{|l|}{ Pays de naissance } \\
\hline Canada & & 1,000 & & \\
\hline Autre & 0,881 & 2,414 & $(1,356-4,297)$ &, 003 * \\
\hline \multicolumn{5}{|l|}{ Lien avec PS } \\
\hline $\begin{array}{l}\text { Pas de PS ou PS non co-résidant } \\
\text { PS corésidante }\end{array}$ & 0,687 & $\begin{array}{l}1,000 \\
1,989\end{array}$ & $(1,083-3,651)$ &, 027 * \\
\hline \multicolumn{5}{|l|}{ Facteurs de besoin (État de santé) } \\
\hline Nombre de problèmes de santé & $-1,454$ & 0,234 & $(0,072-0,754)$ & $015^{*}$ \\
\hline $\begin{array}{l}\text { Présence d'un AVC } \\
\text { Non }\end{array}$ & 1,127 & & & \\
\hline Oui & & 3,087 & $(1,323-7,199)$ &, 009 * \\
\hline Capacités fonctionnelles (OARS /28) & $-0,077$ & 0,926 & $(0,867-0,988)$ &, $021 *$ \\
\hline \multicolumn{5}{|c|}{ Interactions - caractéristiques sociales et état de santé } \\
\hline Interaction âge et problèmes de santé & 0,015 & 1,015 & $(1,001-1,030)$ &, $034^{*}$ \\
\hline Interaction sexe et problèmes de santé & 0,261 & 1,298 & $(1,013-1,663)$ & 039 * \\
\hline Interaction sexe et AVC & $-1,210$ & 0,298 & $(0,108-0,825)$ &, 020 * \\
\hline Interaction sexe et capacités fonct. & 0,091 & 1,096 & $(1,019-1,178)$ &, 014 * \\
\hline Interaction entre lien et pays & $-0,875$ & 0,417 & $(0,185-0,938)$ &, 034 * \\
\hline \multicolumn{5}{|c|}{ Facteurs de capacité de nature organisationnelle } \\
\hline \multicolumn{5}{|l|}{ Fréq. de l'aide du CLSC pour AVQ/AVD } \\
\hline Aucune ou une fois /sem ou moins & & 1,000 & & \\
\hline 2 à 5 fois / sem & 0,545 & 1,725 & $(1,064-2,797)$ &, 027 * \\
\hline 6 à 7 fois/ sem & 0,910 & 2,484 & $(1,219-5,058)$ &, 012 * \\
\hline Coût du CJ du territoire & $-0,052$ & 949 & $(0,902-0,999)$ &, 044 * \\
\hline
\end{tabular}


Tableau 5: Ratios de cote ajustés pour pays de naissance et lien avec la personne de soutien, les divers groupes étant comparés aux personnes nées au Canada et ne cohabitant pas avec une personne de soutien

\begin{tabular}{lll}
\hline \multirow{2}{*}{ Pays } & \multicolumn{2}{c}{ Lien avec PS } \\
& Ne co-habite pas & Co-habite \\
\hline Canada & 1,000 & 1,989 \\
Autre & 2,413 & 2,000 \\
\hline
\end{tabular}


Tableau 6: Ratios de cote ajustés selon l'âge, le sexe, le nombre de problèmes de santé physique, la présence d'AVC et les capacités fonctionnelles, les divers groupes étant comparés aux hommes sans AVC, avec 8 autres problèmes de santé et entièrement autonomes

\begin{tabular}{|c|c|c|c|c|c|c|c|c|c|}
\hline \multirow{2}{*}{ Hommes } & \multicolumn{4}{|c|}{ Capacités fonctionnelles (OARS) } & \multirow[t]{2}{*}{ Femmes } & \multicolumn{4}{|c|}{ Capacités fonctionnelles (OARS) } \\
\hline & 7 & 14 & 21 & 28 & & 7 & 14 & 21 & 28 \\
\hline 70 ans & & & & & 70 ans & & & & \\
\hline sans $A V C$, aucun autre problème de santé & 119,473 & 69,536 & 40,471 & 23,555 & sans AVC, aucun autre problème de santé & 32,511 & 35,850 & 39,532 & 43,591 \\
\hline sans AVC, 8 autres problèmes de santé & 5,072 & 2,952 & 1,718 & 1,000 & sans AVC, 8 autres problèmes de santé & 11,147 & 12,291 & 13,553 & 14,945 \\
\hline avec AVC, aucun autre problème de santé & 368,762 & 214,627 & 124,917 & 72,704 & avec AVC, aucun autre problème de santé & 29,920 & 32,992 & 36,380 & 40,116 \\
\hline avec AVC, 8 autres problèmes de santé & 15,655 & 9,112 & 5,303 & 3,087 & avec AVC, 8 autres problèmes de santé & 10,258 & 11,311 & 12,473 & 13,754 \\
\hline 80 ans & & & & & 80 ans & & & & \\
\hline sans AVC, aucun autre problème de santé & 37,234 & 21,671 & 12,613 & 7,341 & sans AVC, aucun autre problème de santé & 10,132 & 11,173 & 12,320 & 13,585 \\
\hline sans AVC, et 8 autres problèmes de santé & 5,303 & 3,086 & 1,796 & 1,045 & sans AVC, 8 autres problèmes de santé & 11,654 & 12,850 & 14,170 & 15,625 \\
\hline avec AVC, aucun autre problème de santé & 114,926 & 66,889 & 38,931 & 22,659 & avec AVC, aucun autre problème de santé & 9,325 & 10,282 & 11,338 & 12,502 \\
\hline avec AVC, 8 autres problèmes de santé & 16,368 & 9,526 & 5,544 & 3,227 & avec AVC, 8 autres problèmes de santé & 10,725 & 11,826 & 13,040 & 14,380 \\
\hline 90 ans & & & & & 90 ans & & & & \\
\hline sans AVC, aucun autre problème de santé & 11,604 & 6,754 & 3,931 & 2,288 & sans AVC, aucun autre problème de santé & 3,158 & 3,482 & 3,840 & 4,234 \\
\hline sans AVC, 8 autres problèmes de santé & 5,544 & 3,227 & 1,878 & 1,093 & sans AVC, 8 autres problèmes de santé & 12,184 & 13,435 & 14,815 & 16,336 \\
\hline avec AVC, aucun autre problème de santé & 35,817 & 20,846 & 12,133 & 7,062 & avec AVC, aucun autre problème de santé & 2,906 & 3,204 & 3,534 & 3,896 \\
\hline avec AVC, 8 autres problèmes de santé & 17,112 & 9,960 & 5,797 & 3,374 & avec AVC, 8 autres problèmes de santé & 11,213 & 12,364 & 13,634 & 15,034 \\
\hline
\end{tabular}




\section{Références}

ADRLSSS de Montréal. (2004). Portrait de la population: Centre de santé Côte-desNeiges, Métro et Parc Extension. En ligne [http://www.santemontreal.qc.ca/pdf /PDF_CSSS/pop_05.pdf]. Consulté le 28 juillet 2007.

ADRLSSS de Montréal. (2004). Portrait de la population : Centre de santé Nord de l'île et Saint-Laurent. En ligne [http://www.santemontreal.qc.ca/pdf/PDF_CSSS/pop_06 .pdf]. Consulté le 28 juillet 2007.

Andersen, R. M. (1995). Revisiting the behavioral model and access to medical care: Does it matter? Journal of Health and Social Behavior, 36(March), 1-10.

Baumgarten, M., Lebel, P., Laprise, H., Leclerc, C., \& Quinn, C. (2002). Adult day care for the frail elderly: Outcomes, satisfaction, and cost. Journal of Aging and Health, 14(2), 237-259.

Béland, F., Bergman, H., \& Lebel, P. (2001). SIPA : Un Système de services intégrés pour personnes âgées en perte d'autonomie. Évaluation de la phase I, juin 1999 à mai 2000. Montréal : Groupe de recherche Université de Montréal et Université McGill sur les services intégrés aux personnes âgées.

Béland, F., Bergman, H., Lebel, P., Clarfield, A. M., Tousignant, P., Contandriopoulos, A. P., et al. (2006). A system of integrated care for older persons with disabilities in Canada: Results from a randomized controlled trial. Journal of Gerontology Series A: Biological Sciences and Medical Sciences, 61(4), 367-373.

Béland, F., Bergman, H., Lebel, P., Dallaire, L., Fletcher, J., Contandriopoulos, A. P., et al. (2006). Des services intégrés pour les personnes âgées fragiles (SIPA): Expérimentation d'un modèle pour le Canada. Revue canadienne du vieillissement, $25(1), 5-42$.

Béland, F., Haldemann, V., Martin, J.-C., Bourque, P., Ouellette, P., \& Lavoie, J.-P. (1998). Vieillir dans la communauté : Santé et autonomie (No. R98-05). Montréal : Groupe de recherche interdisciplinaire en santé, Université de Montréal.

Chappell, N. L. (1983). Who benefits from adult day care: Changes in functional ability and mental functioning during attendance. Revue canadienne du vieillissement, 2(1), 926. 
Cornoni-Huntley, J., Brook, D. B., Ostfeld, A., Taylor, J. O., \& Wallace, R. B. (1986). Established Populations for Epidemiological Studies of the Elderly, Resources Data Book. National Institutes of Health: NIH no. 86-2443.

Cotrell, V. (1996). Respite use by dementia caregivers: Preferences and reasons for initial use. Journal of Gerontological Social Work, 26(3/4), 35-55.

Cotrell, V., \& Engel, R. J. (1998). The role of secondary supports in mediating formal services to dementia caregivers. Journal of Gerontological Social Work, 30(3/4), $117-132$.

Coulton, C., \& Frost, A. K. (1982). Use of social and health services by the elderly. Journal of Health and Social Behavior, 23(4), 330-339.

Dabelko, H. I., \& Balaswamy, S. (2000). Use of adult day services and home health care services by older adults: A comparative analysis. Home Health Care Services Quarterly, 18(3), 65-79.

Développement des ressources humaines Canada. (2003). L'incapacité au Canada: Un profil en 2001. En ligne [http://www.dsc.gc.ca/fr/pip/bcph/documents/EPLA /EPLA.pdf]. Consulté le 28 juillet 2005.

Douglass, C., \& Visconti, C. (1998). Factors influencing the use of adult day care by individuals with Alzheimer's Disease: A multivariate examination of the California Alzheimer's Disease Diagnostic and Treatment Center Program. Home Health Care Services Quarterly, 17(2), 53-69.

Fillenbaum, G. G. (1988). Multidimensional Functional Assessment of Older Adults: The Duke Older American Resources and Services Procedures. Hillsdale, NJ: Lawrence Erlbaum Associates.

Fillenbaum, G. G., \& Smyer, M. A. (1981). The development, validity and reliability of the OARS multidimentional functional assessment questionnaire. Journal of Gerontology, 36, 428-434.

Folstein, M. F., Folstein, S. E., \& McHugh, P. R. (1975). Mini-Mental State: A practical method for grading the cognitive state of patients for the clinician. Journal of Psychiatric Research, 12(3), 189-198. 
Foreman, M. D. (1987). Reliability and validity of mental status questionnaires in elderly hospitalized patients. Nursing Research, 36(4), 216-220.

Gaugler, J. E., \& Zarit, S. H. (2001). The effectiveness of adult day services for disabled older people. Journal of Aging and Social Policy, 12(2), 23-47.

Gutman, G. M., Milstein, S., Killam, J., Lewis, D., \& Hollander, M. J. (1993a). Les centres de soins de jour pour adultes en Colombie-Britannique : Caractéristiques des clients, raisons de l'aiguillage et raisons de l'omission de se présenter. Rapports sur la santé, 5(3), 321-333.

Gutman, G. M., Milstein, S., Killam, J., Lewis, D., \& Hollander, M. J. (1993b). Les centres de soins de jour pour adultes en Colombie-Britannique : Modèles, caractéristiques et services. Rapports sur la santé, 5(2), 189-207.

Hébert, R., Carrier, R., \& Bilodeau, A. (1988). Le Système de mesure de l'autonomie fonctionnelle (SMAF). La revue de gériatrie, 13(4), 161-167.

Hosmer, D. W., \& Lemeshow, S. (2000). Applied Logistic Regression (2 ${ }^{\text {nd }}$ ed.). New-York: John Wiley and Sons, inc.

Lawton, M. P., Brody, E. M., \& Saperstein, A. R. (1989). A controlled study of respite services for caregivers of Alzheimer's patients. The Gerontologist, 29(1), 8-16.

Lawton, M. P., Brody, E. M., \& Saperstein, A. R. (1991). Respite for caregivers of Alzheimer's patients: Research and practice. New-York: Springer Publishing Company.

Montgomery, R. J. V., \& Borgotta, E. F. (1989). The effects of alternative support strategies on family caregiving. The Gerontologist, 29(4), 457-464.

Montgomery, R. J. V., Kosloski, K., \& Borgotta, E. F. (1988-1989). The influence of cognitive impairment on service use and caregiver response. The Journal of Applied Social Sciences, 13(1), 142-169.

Pfeiffer, E. (1975). A Short Portable Mental Status Questionnaire for the assessment of organic brain deficit in elderly patients. Journal of the American Geriatrics Society, 23(10), 433-441. 
Regroupement des centres d'hébergement et de soins de longue durée, Région de Montréal. (2001). Les centres de jour aujourd'hui... et demain! Document III: Synthèse des consultations et propositions d'orientations. Montréal : Regroupement des centres d'hébergement et de soins de longue durée, Région de Montréal.

Sheikh, J. I., \& Yesavage, J. A. (1986). Geriatric Depression Scale (GDS): Recent evidence and development of a shorter version. Clinical Gerontologist, 5, 165-173.

Tourigny, A., Côté, L., Laberge, A., Paradis, M., \& Joubert, P. (1993). Évaluation du programme québécois des centres de jour. Québec: Centre hospitalier de l'Université Laval: Unité de recherche en santé communautaire, Centre de santé publique de Québec.

Wallace, J. (1987). Factors affecting attendance in adult day care centers. Journal of Gerontological Social Work, 11(3/4), 155-165.

Weissert, W. G., Elston, J. M., Bolda, E. J., Cready, C. M., Zelman, W. N., Sloane, P. D., et al. (1989). Models of adult day care: Findings from a national survey. The Gerontologist, 29(5), 640-649.

Wimo, A., Mattsson, B., Adolfsson, R., Eriksson, T., \& Nelvig, A. (1993). Dementia day care and its effects on symptoms and institutionalization: A controlled Swedish study. Scandinavian Journal of Primary Health Care, 11, 117-123.

Yesavage, J. A., Brink, T. L., Rose, T. L., Lum, O., Huang, V., Adey, M., \& Leirer, V. O. (1983). Development and validation of a geriatric depression screening scale: A premiminary report. Journal of Psychiatric Research, 17(1), 37-49.

Zarit, S. H., Stephens, M. A. P., Townsend, A., Greene, R., \& Leitsch, S. A. (1999). Patterns of adult day service use by family caregivers: A comparison of brief versus sustained use. Family Relations: Interdisciplinary Journal of Applied Family Studies, 48(4), 355-361. 


\begin{abstract}
ARTICLE 2
Les activités de groupe des centres de jour pour personnes âgées correspondent-elles aux besoins des différents profils de participants?
\end{abstract}

\author{
Jacinthe Savard ${ }^{1,2}$ \\ Paule Lebel ${ }^{1,3}$ \\ Nicole Leduc ${ }^{1,4}$ \\ François Béland ${ }^{1,4}$ \\ Howard Bergman 1,4,5
}

${ }^{1}$ Groupe de recherche SOLIDAGE, Faculté de Médecine, Université de Montréal et Université McGill

${ }^{2}$ Clinique universitaire interprofessionnelle de réadaptation, Université d'Ottawa

${ }^{3}$ Centre d'expertise sur la santé des personnes âgées et des aidants (CESPA), Institut universitaire de gériatrie de Montréal

${ }^{4}$ Groupe de recherche interdisciplinaire en santé (GRIS), Faculté de Médecine, Université de Montréal

${ }^{5}$ Division of Geriatric Medicine, Université McGill et Jewish General Hospital, Montréal

Soumis à la Revue canadienne du vieillissement : 16/12/07, accepté pour publication : 05/02/09

Sous-presse : Canadian Journal on Aging / La Revue canadienne du vieillissement, 28(1) 


\section{RESUME}

Cette étude exploratoire cherche à vérifier l'adéquation entre les activités offertes par les centres de jour et les caractéristiques d'autonomie et de santé des utilisateurs, pour 132 participants de 5 centres de jour de Montréal. Des analyses en regroupement ont permis de distinguer quatre profils de participants. Une classification des activités en centre de jour a été établie à l'aide d'un groupe d'experts. La participation à ces activités a été étudiée pour chaque profil et comparée aux activités recommandées par notre groupe d'experts ainsi que dans les écrits. Les résultats démontrent une concordance partielle entre les activités offertes et les besoins des participants. Cette concordance apparaît plus grande pour les personnes qui présentent des problèmes de santé physique ou des incapacités d'origine motrice et plus faible pour celles qui présentent des symptômes dépressifs ou des atteintes cognitives. Ces résultats suggèrent la nécessité de revoir la planification des activités pour assurer une offre de services qui tienne davantage compte des besoins des participants, et ainsi, optimiser les bénéfices de la participation à un centre de jour.

\section{ABSTRACT}

This study was carried out to explore the fit between health and functional characteristics of Day Program participants and the types of program activities they were offered in Day Programs. The subjects were 132 participants from 5 Day Programs in Montreal. Four different participant profile groups were identified using cluster analysis. A classification of program activities was created by a panel of experts. Participation in these activities was studied for each profile group, and compared to recommended activities for such client groups outlined through examination of the literature and consultation with our panel of experts. Results showed only partial correspondence between program activities and participants' needs. This correspondence seemed higher for individuals with physical health problems or mobility impairments and weaker for those presenting depressive symptoms or cognitive impairments. These results suggest a need to review activity program components and determine ways to better match these with client needs to optimise the benefits of Day Program participation.

Mots-clés : Centre de jour, personnes âgées, soutien à domicile, activités thérapeutiques Keywords : Adult Day Care, Adult Day Services, frail seniors, community long-term care, therapeutic activities 


\section{Remerciements}

Pour mener à bien cette étude, la première auteure a reçu une bourse de recherche doctorale de la Société Alzheimer du Canada et une du groupe de recherche SOLIDAGE (financé par les IRSC). Cette étude utilise des données du projet SIPA, financé par le Fonds d'adaptation des services de santé de Santé Canada, la Fondation canadienne de recherche sur les services de santé et le Ministère de la Santé et des Services sociaux du Québec. Les auteurs remercient Luc Dallaire et John Fletcher, coordonnateurs de recherche du projet SIPA, pour leur aide précieuse relative à la gestion et à la transmission des données. De plus, ils remercient Anne-Marie Boivin, Christian-Paul Gaudet, Mélanie Léonard, Manon Pouliot et Tina Tenenbaum, coordonnateurs des centres de jour à l'étude pour avoir partagé des informations sur le fonctionnement de leur centre de jour, ainsi que Jacinthe Auger, Lucie Bernier, Françoise Hébert, Jacinthe Labbé, Thérèse Lafontaine, Odette Descarries et Tina Tenenbaum qui ont participé au groupe d'experts sur les activités en centres de jour. 


\section{Introduction}

Parmi l'ensemble des services gérontologiques et gériatriques mis en place pour répondre aux besoins de la population vieillissante, on retrouve le centre de jour $(\mathrm{CJ})$, un service offert aux personnes âgées qui présentent des incapacités et qui demeurent à domicile. Les interventions en CJ incluent des activités de surveillance de l'état de santé, des activités d'enseignement et de prévention, des activités de réadaptation visant essentiellement le maintien des capacités, des interventions psychosociales et de loisirs, ainsi que du répit et du soutien aux familles (Gutman, Milstein, Killam, Lewis et Hollander, 1993b; Regroupement des centres d'hébergement et de soins de longue durée de la région de Montréal, 2005). Elles sont offertes principalement sous forme d'activités de groupe, bien que certaines interventions individuelles sont aussi réalisées.

Les interventions du centre de jour sont une composante importante des services de soutien à domicile, qui contribuent à améliorer le bien-être psychologique et la qualité de vie des personnes âgées, ainsi qu'à diminuer le stress et le sentiment de fardeau de leur personne de soutien (Baumgarten, Lebel, Laprise, Leclerc et Quinn, 2002; Schacke et Zank, 2006; Strain, Chappell et Blandford, 1987; Zank et Schacke, 2002; Zarit, Stephens, Townsend et Greene, 1998). De plus, lorsque le maintien à domicile n'est plus possible, l'utilisation du centre de jour préalable au placement en institution aurait un effet protecteur sur le déclin cognitif de personnes atteintes de démence lors du placement (Wilson, McCann, Li, Aggarwal, Gilley et Evans, 2007).

Plusieurs études ont décrit les caractéristiques des utilisateurs de CJ (Cefalu, Ettinger et Espeland, 1996; Cohen-Mansfield, Lipson, Brenneman et Pawlson, 2001; Gutman, Milstein, Killam, Lewis et Hollander, 1993a), ainsi que les types d'activités offertes dans divers modèles de CJ (Conrad et Guttman, 1991; Conrad, Hughes, Hanrahan et Wang, 1993; Gutman et coll., 1993b; Jarrott, Zarit, Berg et Johansson, 1998). Par exemple, aux États-Unis, Conrad et Guttman (1991) ont comparé les centres qui reçoivent $30 \%$ ou plus de clients atteints de démence à ceux qui en reçoivent moins de $30 \%$. Ils ont observé que les centres qui en accueillent plus de $30 \%$ offrent en général moins de services 
cliniques comme les soins dentaires et les examens auditifs, et plus de services tels l'assistance aux soins personnels et aux finances; le répit et le soutien aux familles; les activités récréatives thérapeutiques (thérapie par l'art, musicothérapie); les activités de divertissement comme les fêtes, les sorties, les groupes sociaux; les activités de loisirs ou passe-temps comme les jeux et l'artisanat. Jarrott et collaborateurs (1998) ont pour leur part comparé les activités offertes dans les centres de jour suédois et américains qui offrent des services à la clientèle atteinte de démence. Ils ont trouvé que la clientèle des CJ est similaire dans les deux pays, mais que les centres américains offrent une plus grande variété de services, comme les services de podiatrie et les groupes de soutien aux aidants qui ne sont pas offerts dans les centres suédois. Toutefois, ces études n’ont pas cherché à savoir si les activités offertes à chacun des participants variaient en fonction des caractéristiques individuelles des clients.

Des guides publiés aux États-Unis (Goldston, 1989; Lindeman, Corby, Downing et Sanborn, 1991; National Institute on Adult Day Care, 1990; Nissenboim et Vroman, 1998) décrivent comment mettre en place un CJ. Sauf les guides destinés spécifiquement aux CJ pour la clientèle atteinte de démence (Lindeman et coll., 1991; Nissenboim et Vroman, 1998), ces guides présentent les types d'activités à offrir, sans préciser la clientèle visée spécifiquement par chaque type d'activités. Au Canada, le seul guide recensé, le cadre de référence pour les centres de jour proposé par l'Association des centres d'accueil du Québec (ACAQ, 1991) regroupe la clientèle des CJ en six sous-programmes :

- incapacités fonctionnelles d'origine motrice;

- incapacités fonctionnelles d'origine sensorielle;

- incapacités fonctionnelles d'origine cognitive;

- facteurs socio-environnementaux;

- santé physique;

- santé mentale.

Pour chacun des sous-programmes, ce cadre de référence propose une liste d'activités de groupe et d'activités individuelles qui peuvent être offertes par les CJ. Ces derniers peuvent adapter ces suggestions en fonction des besoins particuliers de leur 
clientèle et des ressources dont ils disposent. Aucune étude n'a été effectuée pour vérifier dans quelle mesure ces activités sont réellement offertes dans les CJ. Ce cadre de référence n'a pas non plus été mis à jour depuis 1991.

L'objectif général de la présente étude est d'évaluer l'adéquation entre les activités offertes par les CJ et les caractéristiques de santé et d'autonomie des utilisateurs. Elle comporte trois objectifs spécifiques : 1) identifier, à l'aide d'analyses en regroupement, les profils de participants des $\mathrm{CJ}$; 2) Étudier et catégoriser les activités qui leur sont offertes; 3) comparer les activités offertes aux membres de chacun des profils préalablement identifiés à celles suggérées par le cadre de référence de l'ACAQ (1991) et aux recommandations d'un groupe d'experts. Il s'agit d'une étude exploratoire qui cherche à combler une lacune dans les études actuelles. En effet, bien que les CJ offrent majoritairement des activités de groupe, il arrive qu'ils offrent des programmations différentes pour les divers jours de la semaine ou encore, dans les centres suffisamment importants, deux ou trois activités simultanées pour divers groupes de clients, permettant de personnaliser l'offre de services. Une combinaison d'activités mieux adaptées aux besoins de chaque participant pourrait avoir une influence sur la capacité du CJ d'atteindre ses objectifs.

\section{Méthode}

L'étude s'appuie principalement sur une analyse secondaire de données recueillies de juin 1999 à mars 2001dans le cadre du projet de démonstration SIPA, qui consistait en l'expérimentation d'un modèle de services intégrés pour les personnes âgées qui présentent des incapacités (Béland et coll., 2006a; 2006b). Le projet SIPA a été mené auprès de 1230 personnes âgées qui résidaient sur deux territoires de CLSC de la région de Montréal. Environ la moitié de ces personnes ont reçu les services intégrés évalués dans le cadre du projet SIPA, alors que l'autre moitié continuait de recevoir les services de soutien à domicile réguliers de leur CLSC. 


\section{Population à l'étude}

Les personnes admises au projet SIPA devaient être âgées de 65 ans ou plus, vivre à domicile au début de l'étude, accepter de participer à une étude randomisée sur les services intégrés pour personnes âgées et pouvoir s'exprimer en français ou en anglais avec les membres du projet SIPA, ou avoir un proche en mesure de le faire. Elles devaient aussi présenter des incapacités fonctionnelles, indiquées par un résultat de -10 ou moins au Système de mesure de l'autonomie fonctionnelle - SMAF (Hébert, Carrier et Bilodeau, 1988; Desrosiers, Bravo, Hébert et Dubuc, 1995).

Parmi les personnes toujours à domicile et suivies par l'équipe de recherche SIPA à la fin de la première phase du projet en mai 2000, 140 avaient participé aux activités d'un centre de jour pendant la période d'observation. Huit de ces participants ont dû être exclus des analyses en raison d'un trop grand nombre de données manquantes. L'échantillon final comprend donc 132 personnes âgées. La seule différence statistiquement significative entre les caractéristiques démographiques et de santé des sujets inclus et exclus concerne le lien avec la personne de soutien : pour les sujets exclus, la personne de soutien n'est jamais le conjoint, alors qu'elle l'est pour $28 \%$ des sujets inclus.

\section{Caractéristiques des centres de jour}

Les caractéristiques des cinq CJ qui offrent des services sur le territoire des deux CLSC de l'étude ont été décrites plus en détail dans un article précédent (Savard, Leduc, Lebel, Béland et Bergman, 2007) et sont résumées ci-après.

Le nombre de personnes âgées inscrites varie de 25 à 45 personnes par jour, selon les centres. Le nombre moyen de présences réelles par jour varie de 18,1 à 28,7. Chaque personne peut être inscrite de $1 / 2$ à 3 journées par semaine, selon ses besoins et les politiques de chacun des centres. Exceptionnellement, une personne a pu être inscrite à raison de 4 journées par semaine. La durée de chaque visite varie de 4,5 à 6 heures par jour, pour les personnes inscrites pour la journée; et de 2 à 3,5 heures pour celles inscrites à la demijournée, dans les centres qui offrent ce type de fréquentation. Le coût de chaque 
participation varie de 5 à 17 \$ par jour, pour couvrir les frais de transport et de repas. Ces CJ peuvent compter sur un personnel variant de 4,7 à 7,9 équivalents à temps complet (ETC), qui inclut, en plus du personnel administratif et de soutien (coordonnateur, secrétaire, chauffeur), des membres des professions suivantes: infirmières (5 centres), ergothérapeutes (5 centres), physiothérapeutes ou thérapeutes en réadaptation physique (4 centres), intervenants en loisirs (4 centres) ou éducateurs spécialisés (2 centres), intervenants psychosociaux ( 4 centres), préposés aux bénéficiaires (4 centres).

Bien que chacun des centres décrive ses critères d'admission de façon légèrement différente, ce sont tous des centres qui visent le soutien à domicile des personnes âgées qui présentent des incapacités. Deux centres acceptent les personnes de 60 ans et plus, deux autres à partir de 65 ans, alors qu'un autre exige un profil gériatrique sans mentionner un âge minimum.

Aucun des centres à l'étude ne se définit comme un centre pour personnes atteintes de démence. Toutefois, quatre des cinq centres regroupaient les personnes atteintes des troubles cognitifs lors de certaines journées particulières. Le cinquième formait, cinq jours par semaine, un groupe spécifique pour les personnes qui ont des atteintes cognitives importantes. Lors de ces journées ou dans ce groupe, on pouvait mener les mêmes types d'activités que lors des groupes réguliers, mais en les adaptant aux capacités cognitives de la clientèle.

\section{Collecte de données}

Les caractéristiques des participants ont été recueillies par l'équipe de recherche SIPA à l'aide de questionnaires complétés par entrevue. Pour les personnes âgées incapables de répondre elles-mêmes au questionnaire, un substitut a été utilisé. L'équipe de recherche SIPA a aussi réalisé un relevé de l'utilisation des services de santé des participants à partir des bases de données des organismes et des établissements de santé (RAMQ, Agence de la santé et des services sociaux, hôpitaux, CLSC, centres de jour, etc.). Pour les centres de jour, ce relevé incluait les données d'utilisation recueillies à l'aide du 
logiciel $\mathrm{JEDI}^{1}$ par quatre des cinq centres de jour et des informations similaires recueillies dans les dossiers des clients, pour le cinquième centre qui n'utilisait pas ce logiciel.

\section{Période d'observation}

Les données relatives aux caractéristiques des personnes âgées ont été recueillies entre mai et novembre 2000. Les données obtenues des CJ sur les présences et les activités réalisées couvraient les années 2000 et 2001. Pour chaque individu, l'information a été relevée pour une période de six mois, allant de trois mois avant à trois mois après la date du questionnaire. Cette période a été retenue en considérant deux facteurs importants. Puisque cette étude s'intéresse aux liens entre les caractéristiques des individus et les activités auxquelles ils ont participé et parce que les caractéristiques de santé des personnes âgées sont susceptibles de changer rapidement, une courte période d'observation devait être privilégiée. Elle devait toutefois être suffisamment longue pour englober un échantillon représentatif des activités offertes à chaque participant, malgré les absences qui peuvent être fréquentes pour certains d'entre eux.

\section{Variables à l'étude}

\section{Caractéristiques des participants}

Les participants sont décrits à l'aide de variables sociodémographiques, d'indicateurs de santé et de variables d'utilisation des services de santé. Neuf de ces variables (âge, sexe et sept indicateurs de santé), traitées sous une forme dichotomique, ont servi à l'identification des profils de participants lors des analyses en regroupement. Les autres variables ont été utilisées pour la description de l'échantillon et pour la validation nomologique des profils créés.

Les variables sociodémographiques retenues sont l'âge, le sexe, l'état civil, l'éducation, le pays de naissance, la langue d'usage et la cohabitation avec une personne de soutien. Elles ont été choisies parce que des études antérieures ont démontré des différences dans l'utilisation des CJ en fonction de ces variables (Baumgarten et coll., 2002; Henry et 
Capitman, 1995; Savard et coll., 2007; Zarit, Stephens, Townsend, Greene et Leitsch, 1999).

Les indicateurs de l'état de santé considérés sont ceux qui permettront de faire des liens avec les divers sous-programmes décrits dans le cadre de référence (ACAQ, 1991), sauf pour le sous-programme relié aux facteurs socio-environnementaux, pour lequel il n'y a pas de données correspondantes fiables. Ainsi, les variables suivantes ont été retenues : la capacité fonctionnelle, la présence de troubles sensoriels, la présence de troubles cognitifs, le nombre et le type de problèmes de santé physique, ainsi que la présence de symptômes de dépression.

Les variables d'utilisation des services de santé qui ont été saisies sont les suivantes : le fait de recevoir les services intégrés du projet SIPA ou les services réguliers du CLSC (groupe contrôle), la fréquence de la participation au centre de jour, la fréquence de l'aide du CLSC pour ses activités de vie quotidienne ou domestique pendant la période d'observation, la présence et le nombre de jours d'hospitalisation ainsi que la présence d'hébergement temporaire pendant la période d'observation.

Les neuf variables ayant servi à l'identification des profils de participants lors des analyses en regroupement ont été opérationnalisées de la façon suivante :

Les variables sociodémographiques considérées sont l'âge et le sexe. Deux groupes d'âge ont été créés : 85 ans et plus vs 84 ans ou moins. Des analyses antérieures nous indiquaient que les personnes de 85 ans et plus étaient moins nombreuses à utiliser le CJ. Ce critère semble donc intéressant pour distinguer les divers groupes de participants.

La capacité fonctionnelle est évaluée par la Section des soins personnels du Older American Resources and Services (OARS) (Fillenbaum, 1988). Cette échelle comporte sept énoncés qui évaluent la capacité d'accomplir ses soins personnels (s'habiller, manger, contrôler ses sphincters, etc.) et sept énoncés qui évaluent la capacité d'accomplir diverses tâches de la vie domestique (utiliser le téléphone, magasiner ou faire l'épicerie, préparer les repas, etc.). Les résultats possibles vont de 0 à 28 , un résultat plus élevé indiquant une plus 
grande autonomie. Deux groupes ont été créés : les personnes plus autonomes que la moyenne (19 et plus à l'échelle OARS) vs les moins autonomes (18 et moins à l'échelle OARS). Des analyses antérieures nous indiquaient que les personnes âgées ayant un résultat de 19 ou plus à l'échelle OARS, étaient moins nombreuses à utiliser le CJ.

La présence de troubles sensoriels a été évaluée en demandant aux répondants s'ils avaient aucune, un peu ou beaucoup de difficulté à reconnaître des personnes, à lire ou à entendre, même en utilisant les aides techniques appropriées (lunettes, appareil auditif) le cas échéant. Pour les analyses, une variable dichotomique a été créée : aucune ou peu de difficulté pour chacune de ces trois activités ou beaucoup de difficulté pour au moins une des trois activités.

La présence de troubles cognitifs est mesurée par le Short Portable Mental Status Questionnaire (SPMSQ) (Pfeiffer, 1975). Ce questionnaire comporte 10 questions évaluant principalement la mémoire et l'orientation. Pfeiffer (1975) suggère qu'un résultat de trois erreurs ou plus indique un déficit cognitif possible pour une personne qui a une éducation de niveau secondaire. Il suggère de permettre une erreur de plus aux personnes qui n'ont pas fréquenté l'école secondaire et une erreur de moins à ceux qui ont fréquenté le collège ou l'université. Il recommande aussi d'accorder une erreur de plus aux Afro-Américains. Cette correction pour l'origine ethnique a été adaptée au contexte multiethnique de Montréal, en accordant une erreur de plus aux personnes qui ne sont pas nées au Canada. En utilisant les critères suggérés par Pfeiffer, Foreman (1987) a obtenu un coefficient de prévision d'un test positif de 0,89 .

Pour les personnes qui n'avaient pas la capacité de répondre au SPMSQ (difficulté de communication reliée à la connaissance de la langue ou à un problème de santé), la présence de troubles cognitifs est notée lorsque le questionnaire indique qu'un substitut est utilisé parce que la personne souffre de troubles cognitifs ou lorsqu'à la question sur les problèmes de mémoire tirée de l'étude Established Populations for Epidemiological Studies of the Elderly (EPESE) (Cornoni-Huntley, Brook, Ostfeld, Taylor et Wallace, 
1986), le substitut indique que la personne souffre de la maladie d'Alzheimer ou de problèmes de mémoire attestés par un médecin.

Pour évaluer la santé physique des personnes âgées, le questionnaire demandait aux répondants d'indiquer s'ils présentaient l'une ou l'autre des 16 conditions tirées de l'étude EPESE (Cornoni-Huntley et coll., 1986), et dans l'affirmative, si ce problème était confirmé par un médecin. Ces conditions incluent la présence de : haute tension; maladie de cœur; problèmes de circulation; accident vasculaire cérébral; diabète; problèmes respiratoires; rhumatisme ou arthrite (douleur aux articulations ou aux os); tumeur ou cancer; problèmes émotionnels; maladie de Parkinson; problèmes de mémoire ou maladie d'Alzheimer; problèmes de vessie, de reins ou de prostate; cataractes; glaucome; problèmes d'estomac (gastrite, ulcères, hernie hiatale); fracture de hanche ou de jambe récente (dans la dernière année). Pour créer un indicateur de l'état de santé physique, deux de ces conditions, soit la présence de problèmes émotionnels et la présence de problèmes de mémoire ou de maladie d'Alzheimer, ont été exclues. La présence de cataractes et de glaucome ont été regroupées pour indiquer la présence de problèmes aux yeux. Le nombre de problèmes de santé attesté par un médecin parmi ces 13 conditions a été retenu comme premier indicateur de la santé physique. Pour les analyses, deux groupes sont créés : les personnes qui ont davantage de problèmes que la médiane ( 5 ou plus) vs les autres ( 4 ou moins). Cette distinction semble cliniquement significative pour les responsables de CJ. Deux autres indicateurs de santé physique ont été retenus : la présence d'un problème qui entraîne des incapacités locomotrices (AVC, Parkinson et Fracture de hanche ou de jambe récente) et la présence d'au moins un autre problème parmi les suivants : maladie de cœur; problèmes respiratoires; diabète; tumeur; problèmes de vessie, de reins ou de prostate; problèmes d'estomac. La présence de problèmes articulaires ou rhumatismes n'est pas considérée dans les problèmes qui entraînent des incapacités locomotrices parce que les personnes âgées peuvent présenter ces problèmes sans limitation de leurs capacités locomotrices.

La santé psychologique a été mesurée par l'Échelle de dépression gériatrique, version courte (EDG) (Bourque, Blanchard et Vézina, 1990; Sheikh et Yesavage, 1986). 
Les résultats possibles pour la forme courte vont de 0 à 15 . Un résultat de 6 ou plus indique la présence possible de dépression. Cette donnée n'est disponible que pour les personnes qui répondaient elles-mêmes au questionnaire, soit 97 des 132 sujets. Lorsque le résultat à l'ÉDG n'est pas présent, la personne est considérée comme n'ayant pas de symptômes dépressifs. La question de l'EPESE portant sur les troubles émotifs, qui demande si la personne souffre de problèmes émotionnels (nervosité, dépression, anxiété ou insomnie) et qui était disponible pour tous les sujets, n'a pas été retenue. En effet, cette question est trop générale et ne peut représenter une estimation adéquate de la présence de signes dépressifs.

\section{Activités au centre de jour}

Les activités auxquelles chaque individu participait à chacune de ses présences en centre de jour ont été colligées. Pour quatre des cinq CJ, cela s'est fait à partir du registre quotidien des activités du logiciel JEDI. Ce logiciel permet à chaque CJ de créer sa propre liste d'activités, puis d'attribuer à chaque client la liste des activités auxquelles il est censé participer à chacune de ses visites. Le logiciel génère ensuite, pour chaque jour, la liste des clients de la journée avec les activités associées à chacun. À la fin de chacune des journées, les intervenants suppriment les participants absents, les activités annulées, et pour chaque client présent, ils éliminent les activités auxquelles il n’a pas effectivement participé et ajoutent les activités non prévues auxquelles il a participé, puis sauvegardent ces informations dans le registre quotidien d'activités.

Les mêmes informations ont été recueillies à partir du registre des présences et de l'horaire des activités consignés au dossier des clients, pour le centre qui n'utilisait pas ce logiciel. Dans ce cas, les présences, les absences et les activités spéciales comme les fêtes ou les sorties sont inscrites chaque jour sur le registre de présences de chaque participant. Toutefois, l'horaire des activités prévues pour chacun est révisé seulement périodiquement et la non-participation occasionnelle à une activité (par exemple si une personne manque le groupe d'exercices pour rencontrer individuellement l'infirmière) n'est pas enregistrée.

Il faut noter que seules les activités de groupe sont considérées dans cette étude, parce que les cinq CJ ne colligeaient pas tous quotidiennement les activités individuelles 
réalisées auprès des personnes âgées. De même, les activités d'accueil et les périodes de repos n'ont pas été prises en compte. Bien que seulement certains centres inscrivaient ces activités à leurs registres statistiques, presque tous les offraient. Il aurait été donc inadéquat de calculer un nombre précis de présences à ces activités seulement pour les centres qui les enregistraient.

Les variables pour l'étude et la catégorisation des activités sont les 107 activités de groupe recensées dans les registres d'activités.

Pour comparer les activités offertes à celles recommandées, les variables utilisées sont reliées aux huit catégories d'activités établies à l'étape de la catégorisation des activités. Le fait d'avoir bénéficié ou non de chacune de ces catégories d'activités a été enregistré. De plus, le nombre de jours auxquels chaque individu a participé à chacune des catégories d'activités a été dénombré. Si le registre des activités contenait deux activités de la même catégorie le même jour, une seule participation à cette catégorie était enregistrée pour cette journée. Pour tenir compte de la variation de participation des individus (certains n'avaient qu'une ou deux présences au CJ pendant la période de 26 semaines et d'autres en comptaient jusqu'à 95), le nombre de participations à chacune des catégories d'activités a ensuite été calculé en pourcentage des jours de présence au CJ. Cette variable donne une indication de l'intensité de l'exposition à chacune des catégories d'activités (elle ne tient cependant pas compte de la durée de chacune des activités au cours d'une journée). Enfin, le fait d'avoir participé au CJ à l'intérieur d'un groupe spécifique pour les personnes qui ont des atteintes cognitives a aussi été relevé.

\section{Analyse des données}

\section{Profils de participants}

Pour décrire les participants, des statistiques descriptives (moyenne, fréquence) ont été d'abord calculées pour l'ensemble des participants. Puis, des analyses en regroupement ont été effectuées pour créer des profils de participants ayant un ensemble de caractéristiques similaires, en considérant les variables suivantes : âge, sexe, capacités 
fonctionnelles, nombre de problèmes de santé, présence de problèmes sensoriels importants, de troubles cognitifs, de problèmes de santé qui entraînent des incapacités locomotrices, d'autres problèmes de santé et de symptômes dépressifs. Comme suggéré par Everitt, Landau et Leese (2001), les résultats de plusieurs méthodes d'analyses en regroupement ont été comparés, soit deux méthodes hiérarchiques (Ward, distance moyenne) et une méthode d'optimisation (méthode des nuées dynamiques ou K-means). Les résultats des méthodes hiérarchiques ont été utilisés pour déterminer les nombres de groupes possibles, nombres qui furent entrés comme paramètre dans les analyses avec la méthode des nuées dynamiques.

Deux séries d'ANOVA ont ensuite été effectuées pour identifier les différences significatives dans les caractéristiques des membres de chacun des groupes créés par ces analyses. La première série portait sur les variables déjà incluses dans les analyses en regroupement, pour confirmer que les différences identifiées étaient statistiquement significatives pour toutes ces variables. La seconde série portait sur les variables sociodémographiques et sur les variables d'utilisation des services non incluses dans les analyses en regroupement, pour tester la validité nomologique des profils créés.

Si les profils créés représentent vraiment des groupes de participants ayant des caractéristiques de santé et d'autonomie semblables, les profils devraient différer quant à la cohabitation avec une personne de soutien, de même que pour la majorité des variables d'utilisation des services de santé. Toutefois, le pourcentage d'individus recevant les services intégrés du projet SIPA ne devrait pas varier selon les profils, puisque la sélection des individus pour le groupe expérimental du projet SIPA était aléatoire. Les profils pourraient aussi différer quant au niveau d'éducation puisque la présence de certaines conditions de santé est corrélée à l'éducation, ainsi que pour la proportion d'immigrants, si les immigrants diffèrent dans les types de problèmes qui les amènent à demander des services de soutien à domicile. 
Étude et catégorisation des activités

La catégorisation des activités a nécessité plusieurs étapes : 1) validation des données; 2) regroupement des activités similaires portant des noms différents; 3) regroupement des activités selon leurs buts.

Pour valider les données et préciser les caractéristiques des activités enregistrées, nous avons rencontré les coordonnateurs des cinq centres à l'étude en 2004, leur demandant des précisions sur les activités de l'année 2000. Bien que des biais de rappel soient toujours possibles, ceux-ci sont minimisés par le fait que les objectifs des CJ et les programmes d'activités avaient peu changé pendant cette période. Ces rencontres ont permis de constater que la majorité des centres inscrivaient aux registres statistiques les activités spécifiques auxquelles les participants assistaient, tels qu'artisanat, exercices, échanges (figure 1, modèle 1). Toutefois, pour certains groupes de participants, deux centres inscrivaient plutôt le nom d'un groupe de participants prédéterminés, alors que plusieurs activités pouvaient se dérouler à l'intérieur de ce groupe (figure 1, modèle 2). Les rencontres ont aussi permis de réaliser que des activités similaires portaient des noms différents dans les registres statistiques des divers centres (par exemple, les activités « mémo », « méli-mélo », « intello », « cognitif » étaient toutes des activités de stimulation cognitive).

Les activités similaires portant des noms différents ont donc été regroupées. Pour les inscriptions effectuées selon le modèle 2 , les activités réalisées ont été calculées en fonction des informations recueillies auprès du responsable du centre sur l'horaire type de ces groupes. Par exemple, pour un groupe répit qui comportait à chaque présence des exercices physiques et de la stimulation cognitive, ainsi qu'en alternance des activités d'artisanatbricolage ou des jeux physiques, nous avons inscrit des présences aux exercices physiques et à la stimulation cognitive pour chaque présence à ce groupe, ainsi qu'une présence aux activités d'artisanat pour $50 \%$ des présences à ce groupe et une présence aux jeux physiques pour $50 \%$ des présences à ce groupe. Cette étape a permis de réduire la liste d'activités de 107 à 40 activités différentes. 
Les 40 activités résultant de ce premier processus de réduction ont ensuite été regroupées en fonction de leurs buts, à l'aide d'un groupe d'experts. L'ensemble des responsables de CJ de la région de Montréal a été invité à participer à ce groupe. Sept de ces personnes, parmi les plus engagés dans les activités de la table de concertation des CJ de la région et ayant en moyenne 17,8 années d'expérience en CJ ont accepté l'invitation. Ils ont participé à une rencontre au cours de laquelle des catégories d'activités ont été créées au moyen d'une méthode adaptée de la technique du groupe nominal (Delbecq, Van De Ven et Gustavson, 1975) qui comportait trois étapes.

Premièrement, le groupe d'experts a été invité à générer une liste de buts pour les interventions en centre de jour. Deuxièmement, chaque expert a été appelé à choisir, dans la liste générée, un maximum de huit buts qu'il considérait comme les buts principaux des CJ. Seuls les buts qui ont reçu plus de $50 \%$ des votes ont été conservés. Après ce vote, sept buts ont été retenus. Troisièmement, le groupe d'experts a été appelé à voter sur les buts de chacune des 40 activités recensées dans les CJ à l'étude, à partir de la liste de sept buts générés précédemment. Même s'il est possible qu'une activité permette d'atteindre plusieurs buts, chaque participant devait forcer le choix d'un seul but par activité. Lors de l'analyse des résultats de ce vote, lorsqu'il y avait dispersion des votes, un maximum de deux buts a été conservé pour chaque activité. Cela a mené à une classification comprenant huit grandes catégories d'activités.

\section{Adéquation des activités pour chaque profil de participants}

Avant de se prononcer sur l'adéquation des activités pour chaque profil de participants, il a fallu compiler les types d'activités dont ont bénéficié les membres de chaque profil, puis déterminer les activités recommandées pour chacun.

Pour chaque profil de participants, des statistiques descriptives ont été calculées pour 1) le pourcentage d'individus ayant participé à chacune des catégories d'activités et 2) pour les individus ayant participé aux activités d'une catégorie donnée, la moyenne de l'intensité d'exposition à cette catégorie d'activité. Puis, des ANOVA ont permis de 
déterminer les différences significatives entre les divers profils de participants, pour la participation et l'intensité d'exposition à chaque type d'activité.

Les activités recommandées pour chaque profil de participants ont été déterminées de deux manières : d'abord à partir des recommandations d'activités pour les divers sousprogrammes incluses dans le cadre de référence des centres de jour (ACAQ, 1991), puis à partir des activités recommandées par le groupe d'experts consultés. Pour chaque sousprogramme décrit dans le cadre de référence, les membres du groupe d'experts devaient indiquer, par ordre de priorité, les cinq activités qu'il serait le plus pertinent d'offrir. Soulignons qu'au moment de se prononcer sur ces activités, le groupe d'experts n'avait pas accès à la liste des activités recommandées par le cadre de référence, alors qu'il venait d'étudier la liste des 40 activités offertes par les CJ de cette étude. Lors de l'analyse des résultats de ce vote, les activités ont été d'abord associées à la catégorie d'activités à laquelle ils appartenaient, selon les résultats obtenus à l'étape précédente. L'importance de chacune des catégories d'activités pour chaque sous-programme a été déterminée à l'aide d'un système de points, accordant 5 points chaque fois qu'une catégorie apparaissait en première position, 4 points pour chaque deuxième position, etc.

Les analyses statistiques ont été effectuées à l'aide du logiciel SPSS pour Windows, version 13.0.

\section{Résultats}

\section{Description de l'échantillon}

Les principales caractéristiques des participants sont présentées au tableau 1. La moyenne d'âge de l'échantillon est de 80,3 ans $( \pm 6,7)$. Les participants sont en majorité des femmes $(72,7 \%)$ et des veuves ou veufs (49,2\%). L'étude ayant été menée dans deux quartiers à caractère multiethnique de la ville de Montréal, on observe que 56,1\% des participants sont nés dans un pays autre que le Canada. Ces immigrants vivent au Canada depuis plusieurs années (moyenne de 35,1 ans \pm 18,8) et seuls 5,4 \% sont au Canada depuis moins de dix ans. Près de $89 \%$ des participants ont identifié une personne de soutien 
principale. On observe que $35 \%$ des participants sont mariés et que le conjoint est la personne de soutien pour $80 \%$ des personnes mariées ou $28 \%$ de l'échantillon total. Les enfants sont la personne de soutien principale pour $43,2 \%$ des participants, et environ $60 \%$ d'entre eux n'habitent pas avec le parent dont ils prennent soin.

\section{Insérer le tableau 1 près d'ici}

Les participants de cette étude présentent en moyenne 3,9 $( \pm 2,3)$ problèmes de santé physique parmi les 13 conditions incluses dans cette variable. Les capacités fonctionnelles des participants sont très variées, les résultats allant de 3 à 28 à l'échelle OARS. Les troubles cognitifs sont présents chez $28,0 \%$ des participants et près du tiers de l'échantillon présente au moins un problème sensoriel important. De plus, 43,3\% de ceux qui ont répondu à l'ÉDG présentent des symptômes de dépression.

Ces personnes sont inscrites à leur centre de jour à raison de $1,5( \pm 0,7)$ fois par semaine en moyenne, toutefois les présences réelles sont en moyenne d'un peu moins d'une fois par semaine ( $23,2 \pm 18,6$ présences en 26 semaines d'observation). Près des deux tiers des participants reçoivent de l'aide à domicile de leur CLSC pour les activités quotidiennes ou domestiques, et ce, en moyenne à raison de 2,0 $( \pm 2,3)$ fois par semaine.

\section{Les profils de participants}

Les analyses en regroupement ont permis de classer les clients qui présentent des caractéristiques similaires en quatre groupes (tableau 2). Les résultats obtenus avec la méthode des nuées dynamiques (K-means), qui fait partie des méthodes dites d'optimisation des regroupements, sont présentés. Selon Everitt et collaborateurs (2001), ce type de méthode permet d'augmenter l'homogénéité à l'intérieur des groupes et ainsi d'accroître la distance entre les groupes. C'est aussi ce que nos données ont démontré. Les méthodes hiérarchiques (Ward, distance moyenne) ont créé des groupes moins contrastés. 
Les groupes 1 et 2 sont composés surtout de personnes plus autonomes que la moyenne, alors que les groupes 3 et 4 comprennent majoritairement des personnes moins autonomes que la moyenne. Le groupe 1 est composé principalement de femmes qui présentent peu d'incapacités, sont relativement intactes au plan cognitif, mais semblent présenter une plus grande fragilité en raison d'un âge avancé, d'un grand nombre de problèmes de santé et de symptômes dépressifs. Pour y référer ultérieurement à l'aide d'une caractéristique principale, nous retiendrons la fragilité. Le groupe 2 est composé principalement de femmes un peu plus jeunes que la moyenne. En général, les problèmes de santé des membres de ce groupe sont peu nombreux. Par exemple, cinq personnes n'ont que des troubles cognitifs, quatre n'ont que des problèmes de vision et un autre problème de santé (ex.: haute tension artérielle ou rhumatisme), deux n'ont que des symptômes dépressifs et trois n'ont aucun problème de santé parmi la liste de 13 problèmes présentés. Seulement cinq membres de ce groupe ont un nombre de problèmes de santé plus élevé que la moyenne et ces cinq personnes sont plus autonomes que la moyenne (OARS de 25 à 28). Ce sont des personnes qui semblent fréquenter le centre de jour plus tôt dans le processus d'apparition des incapacités. Bien que ce terme ne reflète pas nécessairement la réalité de chacun des individus du groupe, nous les qualifierons d'utilisateurs précoces. Le groupe 3 est composé surtout de femmes plus âgées, peu autonomes et qui présentent, pour la plupart, des troubles cognitifs. La caractéristique distinctive de ce groupe serait les incapacités d'origine cognitive. Le groupe 4 est composé surtout d'hommes plus jeunes et moins autonomes que l'ensemble de l'échantillon, qui présentent principalement des problèmes de santé qui entraînent des incapacités locomotrices, soit les AVC, la maladie de Parkinson ou les fractures de hanche ou de jambe récentes. La caractéristique distinctive de ce groupe serait les incapacités d'origine motrice. Les ANOVA confirment les différences entre les caractéristiques des membres de chacun des groupes pour chacune des variables ayant servi à créer la classification. Elles confirment aussi des différences significatives pour un ensemble de variables n'ayant pas été utilisées dans la création des regroupements : pays de naissance, cohabitation avec une personne de soutien, aide reçue du CLSC et nombre de jours d'hospitalisation. 


\section{Les catégories d'activités en centre de jour}

Selon le groupe d'experts, les activités en centre de jour visent sept principaux buts : 1) maintien des capacités physiques; 2) maintien de l'autonomie fonctionnelle; 3) maintien des fonctions cognitives; 4) amélioration de l'affect; 5) amélioration de la qualité du réseau de soutien (par le soutien direct aux proches aidants); 6) prévention et promotion de la santé; 7) intégration sociale (incluant maintien ou réapprentissage des habiletés sociales et participation à la communauté).

Le regroupement des activités en fonction de leurs buts, en retenant un maximum de deux buts par activité, a mené à la création de huit catégories d'activités. Ces catégories, ainsi que les buts identifiés pour chacune, sont présentées au tableau 3. On note qu'aucune des activités recensées dans cette étude ne vise le soutien direct aux proches aidants puisqu'un seul centre offrait des groupes de soutien ou d'éducation pour les aidants, mais il n'enregistrait pas les présences des aidants dans le registre des activités du participant au CJ. Enfin, la dernière colonne du tableau 3 présente les activités associées à chacune des catégories d'activités, ainsi que le nombre de centres de jour qui offraient ces activités.

\section{Insérer le tableau 3 près d'ici}

\section{Recommandations d'activités}

Les activités recommandées pour la clientèle de chacun des six sous-programmes selon le cadre de référence (ACAQ, 1991) sont présentées au tableau 4. Pour chaque catégorie d'activités, le rang indique le degré de priorité à lui accorder dans chacun des sous-programmes selon le groupe d'experts.

Insérer le tableau 4 près d'ici 


\section{Participation aux diverses catégories d'activités}

Le tableau 5 présente le nombre d'individus qui ont bénéficié de chacune des catégories d'activités puis, pour ceux qui ont bénéficié des activités de la catégorie, la moyenne de l'intensité d'exposition.

\section{Insérer le tableau 5 près d'ici}

Près de $90 \%$ des individus ont participé à des groupes d'intégration sociale et à des groupes d'exercices physiques. Ces activités sont les plus fréquentes puisqu'en moyenne, les personnes qui ont participé à ces activités l'ont fait lors de 72 à $78 \%$ de leurs présences au CJ. Suivent les sports et jeux physiques, les activités fonctionnelles, les activités de prévention et de promotion de la santé, ainsi que les activités de stimulation cognitive qui rejoignent de 65 à $77 \%$ des individus lors de 35 à $46 \%$ de leurs présences. Enfin, les activités qui rejoignent le moins grand nombre de participants sont les groupes spécifiques pour un problème physique commun et les activités musicales et de réminiscence, auxquelles ont participé respectivement $50 \%$ et $45 \%$ des individus, lors de 36 à $43 \%$ de leurs présences.

On peut aussi noter que $26 \%$ des individus ont participé aux activités du CJ lors des journées ou des groupes destinés aux personnes qui ont des troubles cognitifs. En moyenne, les participants ont bénéficié d'activités appartenant à 5,6 $( \pm 1,7)$ catégories différentes, parmi les huit catégories étudiées.

\section{Différences entre les activités dont ont bénéficié les membres des divers profils}

Les membres du groupe 1 sont parmi les plus nombreux à avoir bénéficié de groupes spécifiques pour un problème physique commun et d'activités fonctionnelles. Ils sont moins nombreux dans les journées destinées aux personnes atteintes de troubles cognitifs. Ceux du groupe 2 sont parmi les plus nombreux à avoir bénéficié de groupes d'exercices généraux et d'activités fonctionnelles. Ils sont aussi peu nombreux dans les journées destinées aux personnes atteintes de troubles cognitifs. Ceux du groupe 3 sont parmi les plus nombreux à avoir bénéficié de groupes d'exercices généraux et les moins 
nombreux pour les groupes pour un problème physique commun et parmi les moins nombreux à participer aux activités fonctionnelles. Ils sont par contre plus nombreux dans les journées destinées aux personnes atteintes de troubles cognitifs. Ceux du groupe 4 sont parmi les moins nombreux à avoir bénéficié de groupes d'exercices généraux et parmi les plus nombreux dans les groupes spécifiques pour un problème physique commun. Comme ceux du groupe 3, ils sont parmi les moins nombreux à participer aux activités fonctionnelles et parmi les plus nombreux dans les journées destinées aux personnes atteintes de troubles cognitifs.

En ce qui concerne l'intensité de l'exposition aux activités, on note des différences significatives pour quatre catégories d'activités: les exercices physiques, les groupes spécifiques, la stimulation cognitive ainsi que les sports et les jeux physiques. Elles vont toutes dans le sens d'une plus grande régularité de participation pour les membres des groupes 3 et 4 . Ces derniers ont aussi participé à un moins grand nombre de catégories d'activités que ceux des profils 1 et 2 .

\section{Accord entre l'exposition aux divers types d'activités et les activités recommandées}

Les membres du groupe 1 présentent plusieurs problèmes de santé physique. Ils sont aussi nombreux à présenter des symptômes dépressifs et des incapacités d'origine sensorielle, tout en étant majoritairement plus autonomes que la moyenne. Les activités auxquelles ils ont participé ont donc été comparées aux activités recommandées pour les trois sous-programmes suivants: incapacités fonctionnelles d'origine sensorielle, santé physique et santé mentale (tableau 6). On observe que les activités recommandées de façon prioritaire pour ces sous-programmes sont aussi des activités dont ont bénéficié la majorité des membres de ce groupe, à l'exception des activités de réminiscence qui peuvent être bénéfiques pour les personnes atteintes de dépression et auxquelles seulement la moitié du groupe a participé. On note aussi que les groupes spécifiques auxquels ils ont participé sont reliés à des problèmes physiques. On ne relève aucun groupe spécifique pour les problématiques sensorielles comme recommandé dans le cadre de référence. 
Les membres du groupe 2 se sont regroupés par le fait qu'ils sont majoritairement plus jeunes que l'ensemble des participants à l'étude et qu'ils présentent peu de problèmes de santé. Ces caractéristiques ne permettent pas de les associer à un sous-programme particulier des CJ. Les besoins de chacun de ces individus sont associés à des sousprogrammes différents. La comparaison avec les activités recommandées n'est donc pas possible pour ce groupe.

Les membres du groupe 3 présentent, pour la plupart, des atteintes cognitives. Les activités auxquelles ils ont participé ont donc été comparées aux activités recommandées pour le sous-programme relié aux incapacités fonctionnelles d'origine cognitive (tableau 7). On observe ici que les activités dont ont bénéficié la majorité des membres de ce groupe sont les exercices physiques, les activités d'intégration sociale ainsi que les sports et les jeux physiques. Malgré que les activités de stimulation cognitive et de réminiscence soient jugées prioritaires pour cette clientèle par le groupe d'experts consultés, un faible pourcentage des membres de ce groupe a participé à ce type d'activités. On note par ailleurs que ceux qui ont participé à de la stimulation cognitive l'ont fait avec une certaine régularité (67\% de leurs présences).

\section{Insérer le tableau 7 près d'ici}

Les membres du groupe 4 présentent presque tous des problèmes de santé qui entraînent des incapacités locomotrices. Les activités auxquelles ils ont participé ont donc été comparées aux activités recommandées pour le sous-programme relié aux incapacités fonctionnelles d'origine motrice (tableau 8). On observe que les membres de ce groupe ont participé en grande majorité aux activités d'intégration sociale, aux exercices physiques et aux groupes spécifiques pour un problème physique commun. Ces activités sont aussi celles qui sont recommandées de façon prioritaire pour ce groupe. On note aussi que tous les membres de ce groupe ont bénéficié d'exercices physiques, que ce soit dans des groupes d'exercices physiques généraux ou dans des groupes spécifiques pour un problème physique commun. 


\section{Discussion}

Bien que largement présents dans la gamme des services de soutien à domicile, les CJ ont été peu étudiés au Canada. Les études canadiennes recensées à ce jour portent essentiellement sur leur structure (Gutman et coll., 1993a, 1993b; Ross-Kerr, Warren et Godkin, 1995; Strang, Greschuk, Mcllveen, Gadacz et Neufeld, 1992; Tourigny, Côté, Laberge, Paradis et Joubert, 1993) ou sur leurs résultats (Baumgarten et coll., 2002; Strain et coll., 1987), avec très peu d'attention apportée au processus d'intervention, les auteurs se limitant à décrire de façon succincte les activités offertes.

Dans cette étude exploratoire, nous avons cherché à vérifier l'adéquation entre les activités offertes dans les CJ et les caractéristiques de santé et d'autonomie des utilisateurs, afin de combler une lacune dans les études actuelles. Ce type de recherche est important et probablement préalable aux études sur l'efficacité des centres de jour, car une combinaison d'activités mieux adaptées aux besoins de chaque participant pourrait avoir une influence sur la capacité des CJ à atteindre leurs objectifs reliés au maintien des capacités physiques, cognitives, affectives et d'intégration sociale, au répit offert aux familles et ultimement, au maintien dans la communauté. Comme peu de chercheurs se sont intéressés à ce sujet jusqu'à maintenant, nous n'avons pas trouvé une classification reconnue des activités offertes en CJ. Les activités sont décrites et regroupées de façon différente dans chaque étude, en fonction des préférences des auteurs plutôt qu'en raison de fondements théoriques. Nous avons fait appel à un groupe d'experts pour créer la classification utilisée dans la présente étude. Malgré certaines différences, cette classification rejoint globalement celle proposée dans le manuel d'activités de Norman et Horton (1996), ce qui lui apporte une certaine validité apparente. Ainsi, cette étude présente une méthode originale et structurée qui pourrait servir de référence pour des études similaires auprès d'un plus grand nombre de centres de jour.

L'analyse en regroupement a permis d'identifier quatre profils de participants et d'examiner les activités dont ont bénéficié les membres de chacun de ces profils. L'importance de considérer de tels groupes vient de la nécessité de prendre en compte les multiples caractéristiques des participants dans la programmation des activités. Par 
exemple, les personnes qui présentent des atteintes cognitives peuvent avoir des besoins différents selon leur niveau de capacités fonctionnelles. Les différences statistiquement significatives observées dans l'utilisation des services de santé et dans les types d'activités dont ont bénéficié les membres de chacun des groupes confèrent une certaine validité apparente à ces regroupements. Il y aurait certes lieu de tester leur robustesse en vérifiant s'ils peuvent être reproduits dans d'autres études. Ils permettent tout de même d'apporter un éclairage intéressant sur les liens entre les activités en CJ et les caractéristiques des utilisateurs.

Parmi les éléments mis en lumière par les différentes analyses de cette étude, on notera tout d'abord que les quatre profils de participants identifiés par les analyses en regroupement ne correspondent que partiellement aux six sous-programmes décrits dans le cadre de référence (ACAQ, 1991). D'autres observations nous amènent aussi à remettre en question l'existence réelle de ces sous-programmes dans les CJ. Par exemple, dans les recommandations de notre groupe d'experts, les activités de deux sous-programmes (incapacités fonctionnelles d'origine motrice et santé physique) semblaient relativement similaires. Ensuite, les données empiriques ne démontrent aucune activité particulière pour les personnes qui présentent des difficultés sensorielles. On note aussi que les divers sousprogrammes de ce cadre de référence distinguent les individus davantage en fonction de l'origine de leurs difficultés qu'en fonction de leur niveau fonctionnel. Cependant, lorsqu'on planifie des activités de groupe, il semble important de tenir compte du niveau fonctionnel pour planifier des activités qui présentent le niveau de stimulation approprié aux capacités des participants.

On constate que les membres du groupe 2, qualifié d'utilisateurs précoces, semblent fréquenter le centre de jour en raison d'un problème qui diffère pour chaque personne, mais avant que les incapacités reliées à cette condition ne deviennent importantes $(87 \%$ sont dans la moitié la plus autonome de l'échantillon). On peut se demander si cette clientèle a réellement besoin de fréquenter un $\mathrm{CJ}$ ou si elle pourrait fréquenter des ressources plus légères (groupes communautaires et associatifs) pour laisser les places en CJ aux personnes qui présentent des besoins plus importants. Cependant, surtout pour les personnes qui 
présentent une atteinte cognitive, plusieurs chercheurs recommandent que l'utilisation du CJ débute à un stade plus précoce de la maladie afin de vraiment contribuer au soutien à domicile. Lorsque le répit aux proches arrive tardivement dans le processus de maladie, les niveaux de stress et de fardeau sont déjà élevés et les démarches d'hébergement sont souvent en cours et ne peuvent être retardées (Gaugler, Kane, Kane et Newcomer, 2005; Gaugler et Zarit, 2001; Gottlieb et Johnson, 2000; Zarit et coll., 1999).

En ce qui concerne les activités offertes, on constate que les exercices physiques et les activités d'intégration sociale sont les activités dont ont bénéficié le plus grand nombre d'individus. Ces activités correspondent aux besoins d'un grand nombre de personnes âgées qui fréquentent les CJ. En effet, les activités d'intégration sociale sont recommandées pour tous les sous-programmes, tant dans le cadre de référence de l'ACAQ (1991) que par le groupe d'experts consultés, bien qu'avec un rang de priorité moindre pour les sousprogrammes de santé physique et d'incapacités d'origine cognitive. Selon ces mêmes sources, les exercices physiques sont aussi indiqués pour un grand nombre de sousprogrammes, malgré que le degré de priorité de ce type d'activités ne soit élevé que pour deux des sous-programmes (incapacités d'origine motrice et santé physique). De plus, ces deux types d'activités sont facilement réalisables en groupe et probablement facilement acceptées par les participants des CJ.

Les exercices physiques et les jeux physiques ne faisaient pas parties des activités prioritaires pour la clientèle atteinte de démence selon le groupe d'experts consultés pour cette recherche. Nos données démontrent que, dans la réalité, les responsables de CJ les offrent régulièrement à cette clientèle. Les guides d'activités pour les personnes atteintes de démence leur donnent raison. Selon ces guides, les activités physiques qui demandent peu d'apprentissage sont pertinentes pour favoriser la participation. De plus, elles permettent de stimuler le maintien des capacités physiques et de prévenir l'incapacité excessive; d'amener une saine fatigue qui diminue l'agitation et l'errance; de faire vivre des succès; et d'avoir du plaisir et de célébrer la vie (Lindeman et coll., 1991; Nissenboim et Vroman, 1998). 
Dans les cinq CJ à l'étude, les groupes spécifiques sont tous des groupes d'exercices physiques pour un diagnostic ou un problème physique commun. Les groupes spécifiques pour la santé mentale et les incapacités d'origine sensorielle ne semblent pas être offerts. S'ils existent, ils ne sont pas traités comme tels dans les données statistiques des CJ et ils n'ont pas été identifiés lors de nos rencontres avec les coordonnateurs des cinq CJ à l'étude visant à préciser les caractéristiques des activités recensées dans chacun des centres. Certains groupes d'intégration sociale (ex. : groupe de valorisation) visaient certainement la prévention et la promotion de la santé mentale, mais n'étaient pas propres à un groupe d'individus ayant démontré des besoins particuliers.

Lorsque l'on s'intéresse aux différences entre les hommes et les femmes, on observe que parmi les quatre profils de participants identifiés, les hommes se retrouvent principalement dans un groupe de personnes moins autonomes, qui présentent un plus grand nombre de problèmes entrainant des incapacités locomotrices. Les femmes se regroupent dans trois groupes distincts, suggérant pour les femmes une plus grande variété de raisons de fréquenter un CJ. Ceci est cohérent avec les résultats d'une première étude qui indiquaient que les hommes participent davantage au CJ lorsqu'ils ont fait un AVC et lorsqu'ils sont moins autonomes (Savard et coll., 2007).

Comme on pouvait s'y attendre, les membres du groupe 3 (incapacités d'origine cognitive) sont proportionnellement plus nombreux que ceux des autres groupes à avoir participé aux journées destinées aux personnes atteintes de troubles cognitifs. Ce qui étonne, c'est que $41 \%$ des membres du groupe 4 (incapacités d'origine motrice) ont participé à ces journées alors que seulement $28 \%$ de ces personnes présentent des troubles cognitifs. Lorsqu'on analyse les données pour l'ensemble des personnes qui présentent une atteinte cognitive, on observe un résultat similaire : la participation aux journées destinées aux personnes atteintes de troubles cognitifs est constatée pour $57 \%$ des personnes qui ont une atteinte cognitive et $14 \%$ des personnes sans ce type d'atteinte. Les membres du groupe 4 sont majoritairement moins autonomes que la moyenne de l'échantillon. Est-il possible que les lourdes pertes d'autonomie soient faussement associées à des pertes cognitives? Il est aussi possible que notre étude sous-estime le nombre de personnes 
atteintes de troubles cognitifs. En effet, il se peut que le SPMSQ ne dépiste pas bien toutes les formes de troubles cognitifs. Par exemple, contrairement au Mini examen de l'état mental (Folstein, Folstein et McHugh, 1975), il n'y a aucune question sur les praxies ou la planification motrice dans le SPMSQ.

Les données démontrent que les activités de stimulation cognitive sont offertes autant aux membres du groupe 3 (incapacités d'origine cognitive) qu'aux membres des autres groupes. Un résultat similaire est observé lorsqu'on compare l'ensemble des personnes qui présentent des atteintes cognitives à celles qui n'en ont pas. Ceci contraste avec les recommandations du cadre de référence de l'ACAQ et du groupe d'experts qui ne recommandent ces activités que pour les personnes qui présentent des atteintes cognitives. Il est possible que des activités de stimulation cognitive de niveau avancé soient intéressantes pour des personnes sans atteinte cognitive et qu'elles puissent aussi être effectuées sous forme de jeux qui favorisent l'intégration sociale. Malheureusement, les données dont nous disposions pour cette étude ne permettaient pas de différencier entre divers types de stimulation cognitive. De plus, bien que les études à ce sujet soient encore insuffisantes (Pope, Shue et Beck, 2003), il existe certaines données voulant que la stimulation cognitive puisse aider à prévenir l'apparition de déficits cognitifs (Wilson, Mendes de Leon, Barnes, Schneider, Bienias et coll., 2002).

Par ailleurs, on peut remettre en question le fait que seules $76 \%$ des personnes qui ont des atteintes cognitives et seules $57 \%$ des personnes du groupe 3 (qui inclut $74 \%$ de personnes avec des atteintes cognitives) aient participé à des activités de stimulation cognitive. En effet, ce type d'activités est présenté comme prioritaire pour cette clientèle par le groupe d'experts consultés et la recherche a aussi démontré l'utilité de la stimulation cognitive pour cette clientèle (Knapp et coll., 2006; Spector, Orrell, Davies et Woods, 2000; Spector et coll., 2003). Pourquoi cet écart? Il y a sans doute la difficulté à animer des activités de stimulation cognitive en groupe avec des personnes de niveaux cognitifs différents ou avec des personnes qui ont des atteintes très importantes. Les intervenants en CJ doivent aussi tenir compte de l'ensemble des besoins et des goûts des clients, comme des besoins prioritaires reliés à leur état de santé physique ou le manque d'intérêt à 
participer à la stimulation cognitive. Il peut aussi exister une certaine perception chez les intervenants en $\mathrm{CJ}$ voulant que la stimulation cognitive ne donne pas de résultats avec les personnes atteintes de troubles cognitifs. En effet, l'orientation à la réalité et la stimulation cognitive ont fait l'objet de critiques en raison de l'application trop mécanique de ces techniques dans le passé (Spector et al., 2000).

On observe aussi que peu de personnes ont bénéficié des activités de réminiscence. Compte tenu des effets bénéfiques de ce type d'activités pour des personnes dépressives (Anderson, 2002; Bohlmeijer, Smit et Cuijpers, 2003; Frazer, Christensen et Griffiths, 2005) et pour des personnes qui présentent des troubles cognitifs (Woods, Spector, Jones, Orrell et Davies, 2005), on aurait pu s'attendre à ce qu'un grand nombre de membres du groupe 1 (dont $80 \%$ présentent des symptômes dépressifs) et du groupe 3 (dont $74 \%$ présentent des atteintes cognitives) aient participé à des activités de réminiscence. Est-ce parce que ce type d'activités est mal connu des intervenants en centre de jour? Est-ce en raison de la difficulté à regrouper les participants qui ont des besoins homogènes pour ce type d'activités? Est-il possible que de telles activités aient été offertes à l'intérieur d'activités identifiées comme activités sociales, et qu'elles n'aient pas été répertoriées comme activités de réminiscence? Ce sont là des questions importantes auxquelles les données de cette étude ne permettent pas de répondre. On peut tout de même recommander que les centres de jour s'assurent d'avoir suffisamment d'intervenants formés à l'intervention psychosociale pour animer ce type d'activités de groupe.

La socialisation apportée par le centre de jour (Webb, 1989), de même que les exercices (Frazer et coll. 2005) offerts à la majorité des participants des CJ, sont certes bénéfiques pour les personnes qui présentent des symptômes dépressifs. Toutefois, d'autres approches ayant démontré des résultats probants (réminiscence, thérapie cognitive behaviorale -- voir Frazer et coll., 2005) pourraient certainement être intégrées aux services offerts à cette clientèle. Compte tenu de la grande proportion de participants en CJ qui présentent des symptômes dépressifs, il serait approprié que les responsables de CJ apportent une attention particulière aux services offerts à cette population. 
Avant de terminer, un mot sur la fréquence de la participation aux diverses activités. Sauf pour les exercices physiques et les activités d'intégration sociale, les sujets de cette étude participent aux autres catégories d'activités en moyenne à moins de $50 \%$ de leurs présences en CJ. Considérant que leur présence réelle au CJ est d'un peu moins d'un jour par semaine en moyenne, on peut se demander si l'intensité d'exposition aux diverses activités proposées par les CJ est suffisante pour engendrer les effets bénéfiques escomptés de chacune d'elles. Toutefois, ces données moyennes sont insuffisantes pour porter un jugement. Il y aurait surtout lieu de s'assurer d'une intensité d'exposition suffisante aux catégories d'activités prioritaires pour chaque profil de participants.

Nos données font ressortir très peu de différences dans l'intensité d'exposition à diverses activités en fonction des caractéristiques des participants, à l'exception de leur degré d'autonomie. Les membres des profils 3 et 4 (moins autonomes), lorsqu'ils participent aux exercices physiques, à la stimulation cognitive, aux sports et aux jeux physiques, ainsi qu'à des groupes spécifiques, le font avec plus de régularité. Puisque les exercices physiques, les sports et jeux physiques et les groupes spécifiques sont bénéfiques pour les incapacités d'origine motrice et que la stimulation cognitive de même que les sports et jeux physiques sont recommandés pour les incapacités d'origine cognitive, ces résultats sont encourageants. Toutefois, on aurait pu s'attendre à trouver d'autres différences pour mieux refléter les besoins variés des divers profils de participants.

\section{Limites de l'étude}

Les statistiques colligées par les CJ comportent certaines imprécisions. Par exemple, certaines données sont enregistrées sous le nom d'un groupe qui pouvait contenir plusieurs types d'activités (par exemple, groupe répit) et nous avons fait des inférences sur les présences à chaque type d'activités à partir du pourcentage approximatif d'activités de chaque type à l'intérieur du groupe. Puisqu'il est possible que la répartition réelle des activités ait varié légèrement par rapport à celle décrite, ou que certaines activités effectuées occasionnellement n'aient pas été mentionnées dans la description du groupe, ces données sont moins précises que celles où les présences sont enregistrées pour chaque 
type d'activités. Ces variations ne semblent cependant pas majeures et ne devraient pas affecter les conclusions de l'étude.

À l'époque où cette étude a été menée, on constatait un manque d'homogénéité dans les données consignées par les CJ concernant l'état de santé des usagers. Ainsi, l'analyse secondaire de données recueillies dans le cadre d'une étude plus large sur les services intégrés aux personnes âgées a permis d'obtenir des données valides tant sur les caractéristiques des individus que sur leurs présences en CJ. Toutefois, l'échantillon n'est pas pleinement représentatif de l'ensemble de la population qui fréquente les CJ québécois.

Premièrement, ce n'est pas un échantillon aléatoire des personnes fréquentant les cinq centres étudiés. Toutefois, selon les responsables de ces cinq centres, les caractéristiques des participants à cette étude seraient assez semblables à celles de la clientèle de leur $\mathrm{CJ}$, à l'exception de la proportion de personnes qui ont des atteintes cognitives, qui semble légèrement plus élevée dans la clientèle de leur CJ (entre 30 et $40 \%$ ) que chez les participants à cette étude (28\%).

Deuxièmement, cet échantillon n'inclut que des personnes de 65 ans et plus. Ces dernières ont été recrutées majoritairement parmi les clients des services à domicile des CLSC. Bien que certains CJ montréalais acceptent d'autres types de clients, ceux de plus de 65 ans forment plus de $90 \%$ de leur clientèle et plus de $85 \%$ y sont dirigés par les services de soutien à domicile de leur CLSC (Regroupement des centres d'hébergement et de soins de longue durée de la région de Montréal, 2005).

Troisièmement, cet échantillon n'inclut que des personnes fréquentant l'un des cinq CJ d'un secteur bien délimité de l'Ile de Montréal. La population des deux territoires de CLSC sur lequel le projet s'est déroulé est plus multiethnique que celle de l'ensemble de l'île de Montréal (44 à $48 \%$ d'immigrants contre $28 \%$ pour Montréal) (Agence de la santé et des services sociaux de Montréal, 2004a; 2004b). Il s'agit donc d'un échantillon d'une grande métropole à composition multiethnique comme on en observe de plus en plus dans les différentes métropoles des pays occidentaux. Enfin, malgré cette limite, les résultats sont possiblement au moins en partie représentatifs des activités des CJ de la région de 
Montréal, les responsables des CJ de cette région ayant entrepris une réflexion commune sur les orientations des CJ (Regroupement des centres d'hébergement et de soins de longue durée de la région de Montréal, 2001; 2005). On peut cependant penser que des résultats différents auraient pu être obtenus dans les CJ des régions rurales de la province puisqu'en plus de desservir une population aux caractéristiques différentes, ceux-ci doivent souvent adapter leur programmation en fonction des possibilités de transport sur un vaste territoire. On notera toutefois l'importance d'offrir des services adéquats aux personnes âgées de la région métropolitaine, puisque $46 \%$ des personnes âgées du Québec vivent dans la région métropolitaine de Montréal (Institut de la statistique du Québec, 2005-2006; 2007).

\section{Conclusion}

Les résultats de cette étude seront utiles aux gestionnaires de CJ et aux planificateurs de services pour les personnes âgées. Ils démontrent une concordance partielle entre les activités offertes et les besoins des utilisateurs. Cette concordance apparaît plus grande pour les personnes qui présentent des problèmes de santé physique ou des incapacités d'origine motrice. Bien qu'ils mériteraient d'être confirmés par des études plus poussées, les éléments mis en lumière dans cette étude soulèvent des questions sur l'adéquation entre la programmation d'activités et les objectifs visés, en particulier pour les personnes atteintes de troubles cognitifs et celles qui présentent des symptômes dépressifs. Ils suggèrent la nécessité de revoir la planification des activités pour assurer une offre de services qui tienne davantage compte des besoins des participants et des interventions démontrées efficaces. Les résultats de cette étude identifient quatre profils de participants qui pourraient servir de point de départ à cette révision de la programmation des activités.

Dans le futur, des recherches plus approfondies sur les liens entre le type d'activités offertes en CJ et l'atteinte des objectifs des CJ seraient pertinentes. De plus, puisqu'une fréquentation régulière du CJ semble nécessaire pour induire des effets bénéfiques, des recherches sur les liens entre les activités offertes et la participation au CJ constituent une étape préalable essentielle. 
Enfin, cette étude fait ressortir l'importance d'adopter une classification des activités de CJ qui permettrait un recueil de données plus fiable et subséquemment une meilleure comparaison entre les CJ.

\section{Notes}

1 Le logiciel JEDI (pour Jonction et diffusion informatiques, la compagnie qui a créé ce logiciel), est un système de bases de données et de rapports périodiques créé sur la plateforme Access de Microsoft et conçu spécialement pour recueillir des données sur les clients, les activités et les présences en CJ. 
Figure 1 : Deux modèles d'horaire d'activités en CJ et lien avec le registre des activités

Modèle 1:

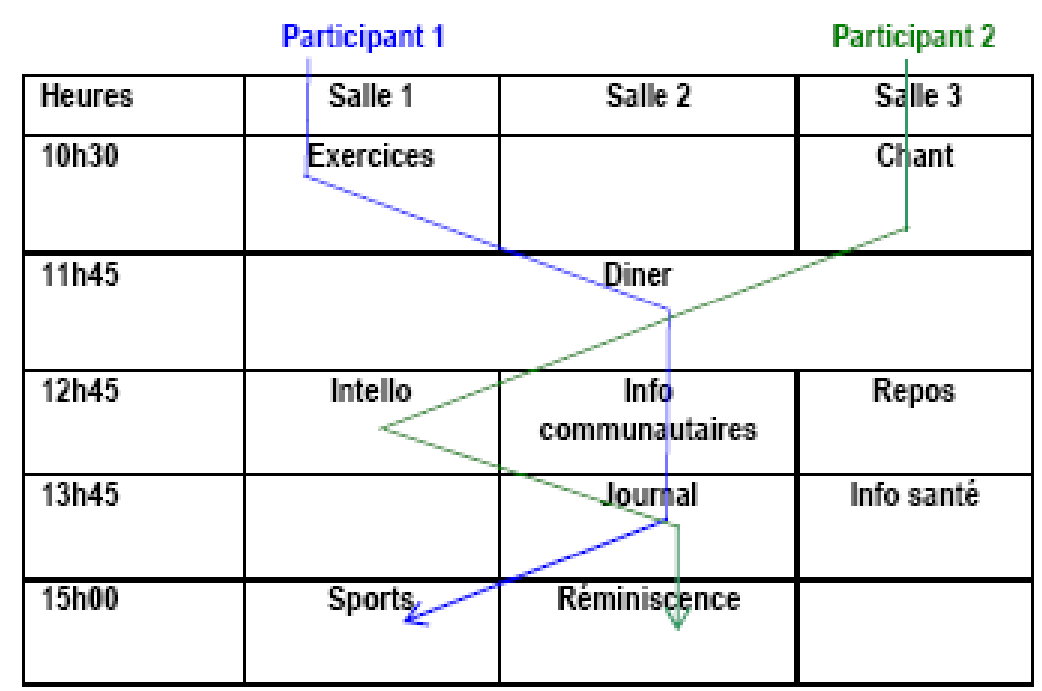

Registre d'activités :

Participant 1 : Activités physiques, Diner, Info communautaires, Joumal, Sports

Participant 2 : Chant, Diner, Intello, Journal, Réminiscence
Modèle 2:

Participant 1

\begin{tabular}{|c|c|c|c|}
\hline Heures & $\begin{array}{l}\text { Salle 1: } \\
\text { Socialisation }\end{array}$ & $\begin{array}{l}\text { Salle 2: } \\
\text { Maintien } \\
\text { Physique }\end{array}$ & $\begin{array}{l}\text { Salle } 3: \\
\text { Groupe répit }\end{array}$ \\
\hline $9 h 45$ & Accueil & Accueil & Accueil \\
\hline $10 h 30$ & $\begin{array}{c}\text { Info } \\
\text { communautaires }\end{array}$ & Exercices & Exercices \\
\hline $11 \mathrm{~h} 45$ & & Diner & \\
\hline $12 \mathrm{~h} 45$ & & Repos & \\
\hline $13 \mathrm{~h} 15$ & Jeux physiques & Info santé & $\begin{array}{l}\text { Stimulation } \\
\text { cognitive }\end{array}$ \\
\hline $14 h 15$ & $\begin{array}{cc}\text { Intello ou } \\
\text { jeux de tables }\end{array}$ & Jeux physiques & $\begin{array}{l}\text { Bricolage ou } \\
\text { jeux physiques }\end{array}$ \\
\hline
\end{tabular}

\section{Registre d'activités :}

Participant 1 : Socialisation

Participant 2 : Groupe répit 
Tableau 1 : Caractéristiques des participants

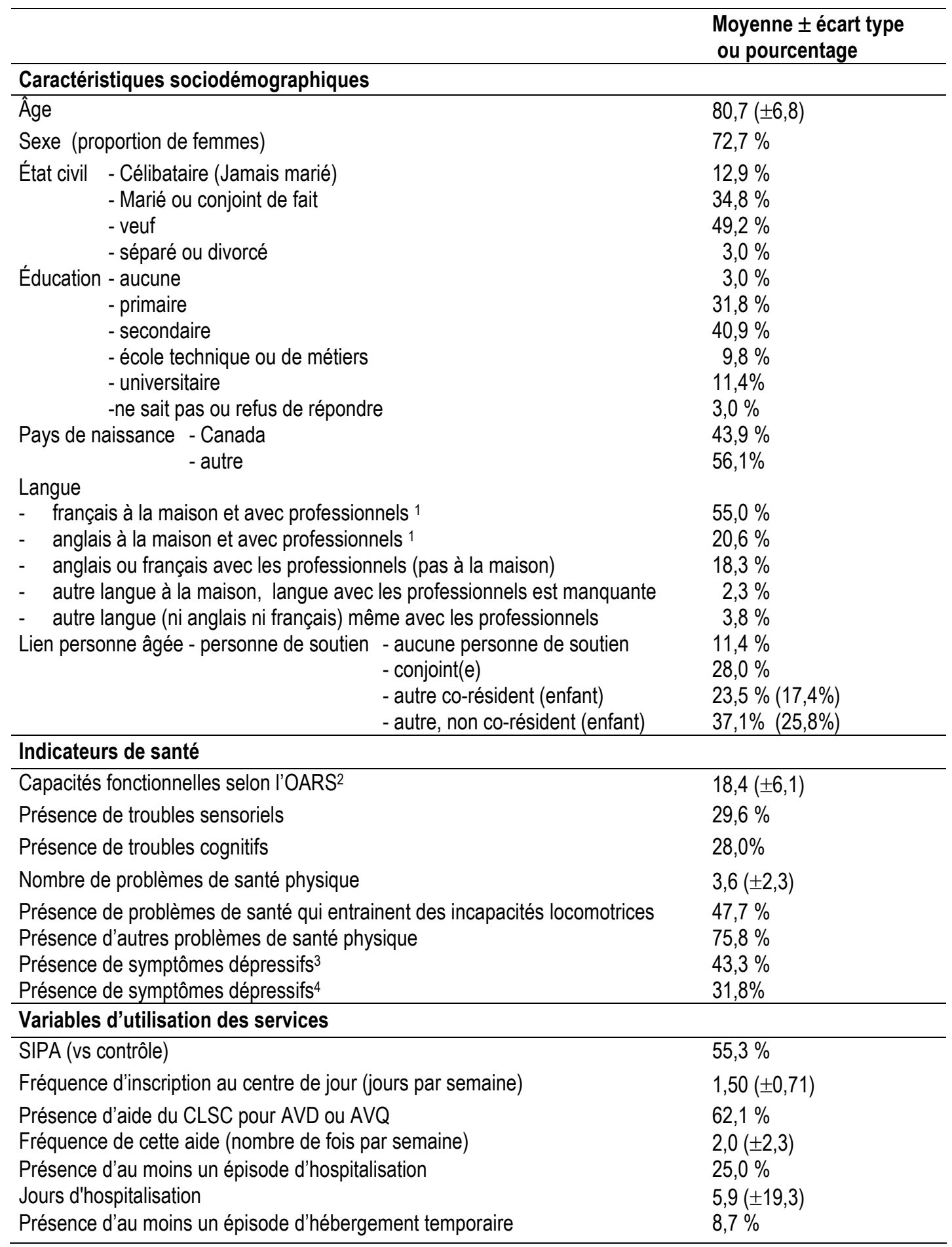

${ }^{1}$ Ces personnes utilisent le français ou l'anglais à la maison parfois en plus d'une autre langue

2 Les résultats possibles à l'OARS vont de 0 à 28 , un résultat plus élevé indique une plus grande autonomie

${ }^{3}$ n=97 car disponible seulement pour les personnes qui répondent elles-mêmes au questionnaire

${ }^{3} n=132$ lorsque les individus dont les résultats sont manquants sont considérés comme ne présentant pas de symptômes dépressifs 
Tableau 2: Différences entre les caractéristiques des membres des groupes formés par les analyses en regroupement

\begin{tabular}{|c|c|c|c|c|c|}
\hline Variables & $\begin{array}{c}\text { Groupe } 1 \\
\mathrm{n}=35 \\
\text { Moyenne et écart type } \\
\text { ou pourcentage }\end{array}$ & $\begin{array}{c}\text { Groupe 2 } \\
\mathrm{n}=45 \\
\text { Moyenne et écart type } \\
\text { ou pourcentage }\end{array}$ & $\begin{array}{c}\text { Groupe } 3 \\
\mathrm{~N}=23 \\
\text { Moyenne et écart type } \\
\text { ou pourcentage }\end{array}$ & $\begin{array}{c}\text { Groupe } 4 \\
\mathrm{~N}=29 \\
\text { Moyenne et écart type } \\
\text { ou pourcentage }\end{array}$ & ANOVA \\
\hline \multicolumn{6}{|c|}{ Variables utilisées pour créer les groupes } \\
\hline Age (\% de 85 et +$)$ & $49 \%$ & $22 \%$ & $65 \%$ & $7 \%$ &, $000^{*}$ \\
\hline Sexe (\% de femmes) & $83 \%$ & $91 \%$ & $87 \%$ & $21 \%$ &, $000^{*}$ \\
\hline $\begin{array}{l}\text { OARS ( } \% \text { plus autonomes que la } \\
\text { moyenne) }\end{array}$ & $71 \%$ & $87 \%$ & $0 \%$ & $24 \%$ &, $000^{*}$ \\
\hline Problèmes sensoriels & $51 \%$ & $16 \%$ & $57 \% 1$ & $3 \%$ &, $000^{*}$ \\
\hline Troubles cognitifs & $6 \%$ & $22 \%$ & $74 \%$ & $28 \%$ &, $000^{*}$ \\
\hline Prob. $\rightarrow$ incapacités locomotrices & $49 \%$ & $13 \%$ & $56 \%$ & $93 \%$ &, $000^{*}$ \\
\hline Prob. santé autre & $100 \%$ & $53 \%$ & $78 \%$ & $79 \%$ &, $000^{*}$ \\
\hline Nombre prob santé (\% de 5 ou +) & $83 \%$ & $11 \%$ & $48 \%$ & $24 \%$ &, $000^{*}$ \\
\hline Troubles dépressifs & $80 \%$ & $16 \%$ & $0 \%$ & $24 \%{ }^{2}$ &, $000^{*}$ \\
\hline \multicolumn{6}{|c|}{ Valeur des variables continues ou combinées } \\
\hline Âge & $82,3 \pm 6,8$ & $79,6 \pm 6,6$ & $84,5 \pm 5,2$ & $77,3 \pm 6,4$ &, $000^{*}$ \\
\hline AVQ + AVD selon l'OARS ${ }^{3}$ & $20,8 \pm 4,5$ & $21,8 \pm 4,9$ & $11,9 \pm 4,9$ & $15,2 \pm 4,7$ &, $000^{*}$ \\
\hline Nombre de prob de santé / 13 & $5,6 \pm 1,5$ & $2,4 \pm 1,7$ & $4,7 \pm 2,7$ & $3,3 \pm 1,56$ &, $000^{*}$ \\
\hline $\begin{array}{l}\text { Prob. } \rightarrow \text { incapacités locomotrices } \\
\text { - AVC } \\
\text { - Parkinson } \\
\text { - Fracture récente }\end{array}$ & $\begin{array}{c}29 \% \\
0 \% \\
20 \%\end{array}$ & $\begin{array}{l}9 \% \\
0 \% \\
4 \%\end{array}$ & $\begin{array}{c}35 \% \\
4 \% \\
22 \%\end{array}$ & $\begin{array}{l}59 \% \\
17 \% \\
24 \%\end{array}$ & $\begin{array}{l}, 000^{*} \\
, 002^{*} \\
, 075\end{array}$ \\
\hline \multicolumn{6}{|c|}{ Variables pour la validation nomologique des groupes } \\
\hline $\begin{array}{l}\text { Éducation } \\
\text { - aucune ou primaire } \\
\text { - secondaire ou technique } \\
\text { - universitaire }\end{array}$ & $\begin{array}{l}29 \% \\
60 \% \\
11 \%\end{array}$ & $\begin{array}{l}33 \% \\
53 \% \\
13 \%\end{array}$ & $\begin{array}{c}61 \% \\
30 \% \\
9 \%\end{array}$ & $\begin{array}{l}31 \% \\
59 \% \\
10 \%\end{array}$ &, 173 \\
\hline $\begin{array}{l}\text { Pays naissance autre que } \\
\text { Canada }\end{array}$ & $51 \%$ & $38 \%$ & $83 \%$ & $69 \%$ &, $001^{*}$ \\
\hline $\begin{array}{l}\text { Cohabite avec personne de } \\
\text { soutien }\end{array}$ & $34 \%$ & $38 \%$ & $57 \%$ & $90 \%$ &, $000^{*}$ \\
\hline Fréquence d'inscription au CJ & $1,46 \pm 0,64$ & $1,40 \pm 0,73$ & $1,55 \pm 0,72$ & $1,64 \pm 0,78$ & .539 \\
\hline Aide du CLSC pour AVD ou AVQ & $69 \%$ & $56 \%$ & $43 \%$ & $79 \%$ &, $036^{*}$ \\
\hline $\begin{array}{l}\text { Fréquence de l'aide } \\
\text { (nombre de fois par semaine) }\end{array}$ & $1,6 \pm 2,0$ & $1,6 \pm 2,1$ & $1,8 \pm 2,4$ & $3,2 \pm 2,5$ &, $013^{*}$ \\
\hline Avec épisode d'hospitalisation & $22,8 \%$ & $13,3 \%$ & $34,8 \%$ & $37,9 \%$ & ,068 \\
\hline Jours d'hospitalisation & $4,7 \pm 15,0$ & $0,4 \pm 1,2$ & $6,1 \pm 14,2$ & $15,6 \pm 34,1$ &, $009^{*}$ \\
\hline Avec épisode d'héb. temporaire & $2,9 \%$ & $8,9 \%$ & $17,4 \%$ & $6,9 \%$ & ,273 \\
\hline SIPA (vs Contrôle) & $68,8 \%$ & $53,3 \%$ & $47,8 \%$ & $48,3 \%$ & ,306 \\
\hline $\begin{array}{l}\text { Caractéristique distinctive } \\
\text { du groupe }\end{array}$ & Personnes fragiles & $\begin{array}{l}\text { Utilisateurs } \\
\text { précoces }\end{array}$ & $\begin{array}{c}\text { Incapacités } \\
\text { d'origine cognitive }\end{array}$ & $\begin{array}{c}\text { Incapacités } \\
\text { d'origine motrice }\end{array}$ & \\
\hline
\end{tabular}

1 dont $31 \%$ chez des personnes avec atteinte cognitive et $26 \%$ chez des personnes sans atteinte cognitive

2 tous pour des personnes qui ont une atteinte neurologique (AVC, Parkinson)

${ }^{3}$ les résultats possibles à l'OARS vont de 0 à 28 , un résultat plus élevé indique une plus grande autonomie 
Tableau 3 : Activités en centre de jour, selon leurs buts

\begin{tabular}{|c|c|c|}
\hline $\begin{array}{l}\text { Nom de la catégorie } \\
\text { (nombre de CJ qui l'offre) }\end{array}$ & $\begin{array}{l}\text { But de ces activités } \\
\text { (commentaires sur la classification) }\end{array}$ & $\begin{array}{l}\text { Activités } \\
\text { (nombre de CJ qui l'offre) }\end{array}$ \\
\hline A. Exercices physiques (5) & 1. Maintien des capacités physiques & $\begin{array}{l}\text { Exercices généraux (5) } \\
\text { Musique et mouvement (2) } \\
\text { Groupe de marche (2) } \\
\text { Exercices adaptés (assis) (1) } \\
\text { Exercices en piscine (1) }\end{array}$ \\
\hline $\begin{array}{l}\text { B. Groupes spécifiques pour } \\
\text { un problème physique } \\
\text { commun (4) }\end{array}$ & $\begin{array}{l}\text { 1. Maintien des capacités physiques } \\
\text { 2. Maintien de l'autonomie fonctionnelle }\end{array}$ & $\begin{array}{l}\text { Groupe Parkinson (3) } \\
\text { Groupe AVC (2) } \\
\text { Groupe diabète (1) } \\
\text { Groupe arthrite (1) } \\
\text { Groupe de dextérité manuelle (1) } \\
\text { Groupe de douleur (1) }\end{array}$ \\
\hline C. Activités fonctionnelles (5) & $\begin{array}{l}\text { 2. Maintien de l'autonomie fonctionnelle } \\
\text { 4. Amélioration de l'affect } \\
\text { (Ces activités ont en commun qu'elles } \\
\text { permettent de réaliser quelque chose). }\end{array}$ & $\begin{array}{l}\text { Arts plastiques ou artisanat (5) } \\
\text { Cuisine (4) } \\
\text { Horticulture (2) } \\
\text { Beauté (coiffure, maquillage, soins des ongles)(1) } \\
\text { Thérapie animale (1) }\end{array}$ \\
\hline D. Stimulation cognitive (5) & 3. Maintien des fonctions cognitives & $\begin{array}{l}\text { Stimulation cognitive }(5) \\
\text { Stimulation sensorielle (1) }\end{array}$ \\
\hline $\begin{array}{l}\text { E. Activités musicales ou de } \\
\text { réminiscence (5) }\end{array}$ & $\begin{array}{l}\text { 3. Maintien des fonctions cognitives } \\
\text { 4. Amélioration de l'affect } \\
\text { (Dans les écrits scientifiques, la réminiscence } \\
\text { est utilisée pour améliorer l'affect chez les } \\
\text { personnes dépressives (Frazer et coll., 2005) } \\
\text { et l'affect et les fonctions cognitives chez } \\
\text { celles avec tr. cognitifs (Woods et coll., 2005)) }\end{array}$ & $\begin{array}{l}\text { Musique ou musicothérapie (4 CJ, dont un CJ qui } \\
\text { a plusieurs groupes distincts : composition } \\
\text { musicale, exploration sonore, lames sonores, } \\
\text { écoute musicale). } \\
\text { Réminiscence (3) } \\
\text { Chant (2) }\end{array}$ \\
\hline F. Sports et jeux physiques (5) & $\begin{array}{l}\text { 1. Maintien des capacités physiques } \\
7 \text { : intégration sociale } \\
\text { (le groupe d'experts mentionne ces activités } \\
\text { comme moyen de maintenir les capacités } \\
\text { physiques pour le sous-programme } \\
\text { d'incapacités d'origine motrice et comme } \\
\text { moyen de favoriser l'intégration sociale pour } \\
\text { les sous-programmes des incapacités } \\
\text { d'origine cognitive ou sensorielle) }\end{array}$ & $\begin{array}{l}\text { Jeux physiques (5) (Jeux de sac de sables, } \\
\text { curling adapté, volleyball adapté, quilles } \\
\text { adaptées, etc.) }\end{array}$ \\
\hline G. Intégration sociale (5) & $\begin{array}{l}\text { 4. Amélioration de l'affect } \\
\text { 7. Intégration sociale } \\
\text { (La description des activités des groupes de } \\
\text { valorisation/croissance indiquent bien qu'ils } \\
\text { visent les } 2 \text { objectifs d'affect et intégration: } \\
\text { Discussion sur thèmes affectifs (ex: exprimer } \\
\text { des émotions en groupe, accepter les pertes } \\
\text { physiques ou psychologiques), acquérir des } \\
\text { stratégies de communication efficaces pour } \\
\text { faciliter l'intégration à un réseau social, etc.) }\end{array}$ & $\begin{array}{l}\text { Jeux de table (cartes, scrabble, Bingo, etc.) (5) } \\
\text { Sorties (5) } \\
\text { Fêtes, dîner anniversaire ou spectacles (4) } \\
\text { Infos communautaires ou psychosociales (4) } \\
\text { Échanges (Période formelle) (3) } \\
\text { Valorisation/croissance (appartenance, santé } \\
\text { mentale, bien-être) (3) } \\
\text { Actualités (2) } \\
\text { Journal (2) } \\
\text { Ordinateur - Internet (2) } \\
\text { Activités spirituelles (messe, pastorale, } \\
\text { préparation des fêtes juives) (2) } \\
\text { Valorisation par des tâches utiles au CJ ou au } \\
\text { CLSC auquel il est affilié (1) } \\
\text { Participation à la communauté (1) } \\
\text { Comité planification clients (1) } \\
\text { Poésie - écriture (1) }\end{array}$ \\
\hline $\begin{array}{l}\text { H. Prévention et promotion de } \\
\text { la santé (4) }\end{array}$ & 6. Prévention et promotion de la santé & $\begin{array}{l}\text { Info santé (4) } \\
\text { Relaxation dirigée (2) } \\
\text { Cuisine santé (1) } \\
\text { Prévention des chutes (1) }\end{array}$ \\
\hline
\end{tabular}


Tableau 4 : Activités recommandées en centre de jour pour les divers sous-programmes $(A C A Q, 1991)$ et rangs de priorité établis par le groupe d'experts

\begin{tabular}{|c|c|c|c|c|c|c|}
\hline \multirow[b]{2}{*}{ Activités } & \multicolumn{6}{|c|}{ Sous-programmes } \\
\hline & $\begin{array}{l}\text { Incapacités d'origine } \\
\text { motrice }\end{array}$ & $\begin{array}{l}\text { Incapacités d'origine } \\
\text { sensorielle }\end{array}$ & $\begin{array}{l}\text { Incapacités d'origine } \\
\text { cognitive }\end{array}$ & $\begin{array}{l}\text { Facteurs socio- } \\
\text { environnementaux }\end{array}$ & Santé physique & Santé mentale \\
\hline \multirow[t]{2}{*}{ Exercices physiques } & $\begin{array}{l}\text { Classes d'exercices: } \\
\text { régulière, groupe d'ambula- } \\
\text { tion, groupe de préhension }\end{array}$ & & Classe d'exercices régulière & & $\begin{array}{l}\text { Classe d'exercices } \\
\text { régulière }\end{array}$ & \\
\hline & Rang 1 & $\begin{array}{l}\text { Rang } 5 \text { : moyen de } \\
\text { valorisation pour cette } \\
\text { clientèle }\end{array}$ & Rang 6 & Rang 6 & Rang 3 & Rang 7 \\
\hline \multirow[t]{2}{*}{ Groupes spécifiques } & $\begin{array}{l}\text { Groupe d'activités pour: } \\
\text { hémiplégie, sclérose en } \\
\text { plaques, arthrite, Parkinson }\end{array}$ & $\begin{array}{l}\text { Groupe de soutien } \\
\text { Exercices pour les yeux } \\
\text { Stratégies de communication: } \\
\text { discrimination par le toucher, } \\
\text { les odeurs, stratégie d'écoute, } \\
\text { lecture labiale, exercices de } \\
\text { mémoire et de concentration }\end{array}$ & & & $\begin{array}{l}\text { Groupe spécifique pour } \\
\text { certaines pathologies : } \\
\text { diabète }\end{array}$ & $\begin{array}{l}\text { Groupe de prévention en } \\
\text { santé mentale }\end{array}$ \\
\hline & Rang 2 & Rang 2 & & & Rang 2 & Rang 4/5 \\
\hline Activités fonctionnelles & $\begin{array}{l}\text { Groupe de tâches: } \\
\text { menuiserie, horticulture, } \\
\text { artisanat, cuisine, } \\
\text { enseignement et } \\
\text { entraînement AVQ }\end{array}$ & Rang 3/4 & $\begin{array}{l}\text { Groupe de tâches : cuisine, } \\
\text { artisanat adapté, tâches } \\
\text { domestiques (ex. : préparer } \\
\text { les serviettes de table). } \\
\text { Intégrer AVQ dans la routine } \\
\text { au CJ: se vêtir et se dévêtir, } \\
\text { utiliser la toilette, etc. } \\
\text { Rang } 3\end{array}$ & Rang 2 & Rang 4/5 & Rang 2 \\
\hline Stimulation cognitive & & & $\begin{array}{l}\text { Gymnastique des fonctions } \\
\text { cérébrales : réminiscence, } \\
\text { orientation à la réalité, } \\
\text { stimulation sensorielle } \\
\text { Rang } 1\end{array}$ & & & \\
\hline $\begin{array}{l}\text { Activités musicales ou } \\
\text { de réminiscence }\end{array}$ & & Rang 6 & $\begin{array}{l}\text { Dans le cadre, inclus avec } \\
\text { stimulation cognitive et avec } \\
\text { groupe psychosocial } \\
\text { Rang } 2\end{array}$ & Rang 5 & & Rang 3 \\
\hline $\begin{array}{l}\text { Sports et jeux } \\
\text { physiques }\end{array}$ & $\begin{array}{l}\text { Mentionné au rang } 1 \\
\text { comme exercices physiques }\end{array}$ & $\begin{array}{l}\text { Mentionné au rang } 5 \text { comme } \\
\text { exercices physiques }\end{array}$ & $\begin{array}{l}\text { Mentionné au rang } 5 \\
\text { comme intégration sociale }\end{array}$ & & & \\
\hline
\end{tabular}




\begin{tabular}{|c|c|c|c|c|c|c|}
\hline \multirow[b]{2}{*}{ Activités } & \multicolumn{6}{|c|}{ Sous-programmes } \\
\hline & $\begin{array}{l}\text { Incapacités d'origine } \\
\text { motrice }\end{array}$ & $\begin{array}{l}\text { Incapacités d'origine } \\
\text { sensorielle }\end{array}$ & $\begin{array}{l}\text { Incapacités d'origine } \\
\text { cognitive }\end{array}$ & $\begin{array}{l}\text { Facteurs socio- } \\
\text { environnementaux }\end{array}$ & Santé physique & Santé mentale \\
\hline Intégration sociale & $\begin{array}{l}\text { Groupe psychosocial : } \\
\text { remotivation, deuil, estime } \\
\text { de soi, vivre avec un } \\
\text { handicap (groupe de } \\
\text { soutien), information sur } \\
\text { services communautaires }\end{array}$ & $\begin{array}{l}\text { Groupe psychosocial : vivre } \\
\text { avec un handicap visuel, vivre } \\
\text { avec un handicap auditif, } \\
\text { information sur ressources } \\
\text { disponibles }\end{array}$ & $\begin{array}{l}\text { Groupe de valorisation et de } \\
\text { socialisation (peut inclure } \\
\text { jeux, zoothérapie, musique, } \\
\text { etc.) }\end{array}$ & $\begin{array}{l}\text { Groupes psychosociaux: } \\
\text { échanges sur des } \\
\text { préoccupations courantes } \\
\text { (vieillissement, stress, } \\
\text { testament), socialisation } \\
\text { (jeux, chants, excursions), } \\
\text { groupe de préparation à } \\
\text { l'hébergement, groupe de } \\
\text { réinsertion sociale } \\
\text { Rang } 1\end{array}$ & $\begin{array}{l}\text { Groupe psychosocial: } \\
\text { adaptation au } \\
\text { vieillissement, relaxation, } \\
\text { information sur services } \\
\text { communautaires }\end{array}$ & $\begin{array}{l}\text { Groupe psychosocial: } \\
\text { prévention des situations } \\
\text { potentielles de détresse, } \\
\text { revalorisation du rôle social } \\
\text { et estime de soi, groupe } \\
\text { d'entraide à la suite d'un } \\
\text { deuil, groupe relié aux } \\
\text { problèmes de consommation } \\
\text { d'alcool ou de médicaments } \\
\text { Rang } 1\end{array}$ \\
\hline $\begin{array}{l}\text { Prévention et promotion } \\
\text { de la santé }\end{array}$ & $\begin{array}{l}\text { Groupe d'information } \\
\text { santé: tension artérielle, } \\
\text { médicaments, hygiène des } \\
\text { pieds, alimentation } \\
\text { Rang } 5\end{array}$ & & & & $\begin{array}{l}\text { Groupe d'information } \\
\text { santé : nutrition, } \\
\text { médication, sécurité, vie } \\
\text { saine, information sur } \\
\text { certaines pathologies } \\
\text { Rang } 1\end{array}$ & $\begin{array}{l}\text { Groupe d'information santé } \\
\text { relaxation, médication }\end{array}$ \\
\hline $\begin{array}{l}\text { Soutien au réseau } \\
\text { d'aide }\end{array}$ & $\begin{array}{l}\text { Avec aidant : suivi du } \\
\text { programme d'exercice à } \\
\text { domicile } \\
\text { Rang } 6\end{array}$ & $\begin{array}{l}\text { Avec aidant : suivi des } \\
\text { stratégies de communication }\end{array}$ & $\begin{array}{l}\text { Avec aidant : enseignement } \\
\text { et soutien }\end{array}$ & Rencontre avec aidant & $\begin{array}{l}\text { Avec aidant : enseigne- } \\
\text { ment (insuline, dosette) }\end{array}$ & $\begin{array}{l}\text { Avec aidant : enseignement } \\
\text { et soutien }\end{array}$ \\
\hline
\end{tabular}




\section{Tableau 5 : Activités au centre de jour pour l'ensemble des participants, puis pour les quatre profils de participants}

(Pourcentage d'individus qui ont bénéficié de chaque type d'activités, puis moyenne de l'intensité de l'exposition pour ceux qui en ont bénéficié)

\begin{tabular}{|c|c|c|c|c|c|c|}
\hline Activités au CJ & $\begin{array}{l}\text { Pour les } 132 \\
\text { participants }\end{array}$ & $\begin{array}{c}\text { Groupe } 1: \text { Personnes } \\
\text { fragiles }(n=35)\end{array}$ & $\begin{array}{c}\text { Groupe } 2 \text {; Utilisateurs } \\
\text { précoces }(n=45)\end{array}$ & $\begin{array}{c}\text { Groupe } 3: \text { Incapacités } \\
\text { d'origine cognitive }(n=23)\end{array}$ & $\begin{array}{l}\text { Groupe } 4 \text { : Incapacités } \\
\text { d'origine motrice }(n=29)\end{array}$ & ANOVA \\
\hline A. Exercices physiques & $\begin{array}{c}91 \% \\
78 \% \pm 23 \% 1\end{array}$ & $\begin{array}{c}89 \% \\
0,71 \pm 0,25\end{array}$ & $\begin{array}{c}96 \% \\
0,74 \pm 0,20\end{array}$ & $\begin{array}{c}100 \% \\
0,92 \pm 0,16\end{array}$ & $\begin{array}{c}79 \% \\
0,79 \pm 0,26\end{array}$ & $\begin{array}{l}, 037^{*} \\
, 005^{*}\end{array}$ \\
\hline B. Groupe spécifique & $\begin{array}{c}50 \% \\
43 \% \pm 29 \%\end{array}$ & $\begin{array}{c}69 \% \\
0,33 \pm 0,20\end{array}$ & $\begin{array}{c}49 \% \\
0,37 \pm 0,22\end{array}$ & $\begin{array}{c}23 \% \\
0,66 \pm 0,41\end{array}$ & $\begin{array}{c}52 \% \\
0,60 \pm 0,35\end{array}$ & $\begin{array}{l}, 006^{*} \\
, 004^{*}\end{array}$ \\
\hline C. Activités fonctionnelles & $\begin{array}{c}73 \% \\
35 \% \pm 15 \%\end{array}$ & $\begin{array}{c}86 \% \\
0,33 \pm 0,17\end{array}$ & $\begin{array}{c}80 \% \\
0,34 \pm 0,12\end{array}$ & $\begin{array}{c}65 \% \\
0,37 \pm 0,19\end{array}$ & $\begin{array}{c}55 \% \\
0,39 \pm 0,16\end{array}$ & $\begin{array}{l}, 023^{*} \\
, 658\end{array}$ \\
\hline D. Stimulation cognitive & $\begin{array}{c}65 \% \\
46 \% \pm 33 \%\end{array}$ & $\begin{array}{c}51 \% \\
0,37 \pm 0,28\end{array}$ & $\begin{array}{c}73 \% \\
0,35 \pm 0,26\end{array}$ & $\begin{array}{c}57 \% \\
0,67 \pm 0,40\end{array}$ & $\begin{array}{c}76 \% \\
0,58 \pm 0,36\end{array}$ & $\begin{array}{l}, 092 \\
, 003^{*}\end{array}$ \\
\hline $\begin{array}{l}\text { E. Activités musicales ou de } \\
\text { réminiscence }\end{array}$ & $\begin{array}{c}45 \% \\
36 \% \pm 26 \%\end{array}$ & $\begin{array}{c}49 \% \\
0,34 \pm 0,27\end{array}$ & $\begin{array}{c}51 \% \\
0,36 \pm 0,26\end{array}$ & $\begin{array}{c}39 \% \\
0,38 \pm 0,28\end{array}$ & $\begin{array}{c}38 \% \\
0,40 \pm 0,27\end{array}$ & $\begin{array}{l}, 633 \\
, 962\end{array}$ \\
\hline F. Sports et jeux physiques & $\begin{array}{c}77 \% \\
36 \% \pm 17 \%\end{array}$ & $\begin{array}{c}74 \% \\
0,32 \pm 0,16\end{array}$ & $\begin{array}{c}84 \% \\
0,33 \pm 0,15\end{array}$ & $\begin{array}{c}74 \% \\
0,44 \pm 0,16\end{array}$ & $\begin{array}{c}69 \% \\
0,42 \pm 0,19\end{array}$ & $\begin{array}{l}, 452 \\
, 031^{*}\end{array}$ \\
\hline G. Intégration sociale & $\begin{array}{c}92 \% \\
72 \% \pm 24 \%\end{array}$ & $\begin{array}{c}100 \% \\
0,67 \pm 0,25\end{array}$ & $\begin{array}{c}93 \% \\
0,71 \pm 0,22\end{array}$ & $\begin{array}{c}83 \% \\
0,74 \pm 0,27\end{array}$ & $\begin{array}{c}86 \% \\
0,76 \pm 0,25\end{array}$ & $\begin{array}{l}, 074 \\
, 494\end{array}$ \\
\hline Évènements spéciaux & $\begin{array}{c}58 \% \\
17 \% \pm 13 \%\end{array}$ & $\begin{array}{c}51 \% \\
0,21 \pm 0,14\end{array}$ & $\begin{array}{c}76 \% \\
0,16 \pm 0,12\end{array}$ & $\begin{array}{c}57 \% \\
0,18 \pm 0,17\end{array}$ & $\begin{array}{c}38 \% \\
0,13 \pm 0,10\end{array}$ & $\begin{array}{l}, 010^{*} \\
, 381\end{array}$ \\
\hline H. Prévention et promotion santé & $\begin{array}{c}67 \% \\
35 \% \pm 24 \%\end{array}$ & $\begin{array}{c}71 \% \\
0,33 \pm 0,22\end{array}$ & $\begin{array}{c}78 \% \\
0,37 \pm 0,23\end{array}$ & $\begin{array}{c}52 \% \\
0,32 \pm 0,31\end{array}$ & $\begin{array}{c}59 \% \\
0,37 \pm 0,26\end{array}$ & $\begin{array}{l}\text {,117 } \\
\text {,842 }\end{array}$ \\
\hline Journées pour troubles cognitifs & $26 \%$ & $9 \%$ & $16 \%$ & $52 \%$ & $41 \%$ &, $000^{*}$ \\
\hline Nombre de catégories d'activités & $5,6 \pm 1,7$ & 5,9 & 6,0 & 4,9 & 5,1 &, $019^{*}$ \\
\hline
\end{tabular}

${ }^{1}$ ce résultat signifie que, par exemple, ceux qui ont participé à des exercices physiques y ont participé en moyenne à $78 \%$ de leurs présences au CJ 
Tableau 6 : Correspondance entre l'exposition aux diverses activités pour les membres du groupe 1 (personnes fragiles ; n= 35) et les activités recommandées

\begin{tabular}{|c|c|c|c|c|c|}
\hline Activités & $\begin{array}{l}\text { Recommandation pour } \\
\text { Incapacités d'origine } \\
\text { sensorielles }\end{array}$ & $\begin{array}{l}\text { Recommandation pour } \\
\text { santé physique }\end{array}$ & $\begin{array}{l}\text { Recommandation pour } \\
\text { santé mentale }\end{array}$ & $\begin{array}{l}\text { Personnes du groupe } 1 \text { qui ont } \\
\text { bénéficié d'activités de cette } \\
\text { catégorie } \\
\text { (Pourcentage) }\end{array}$ & $\begin{array}{l}\text { Pour ceux qui ont bénéficié } \\
\text { des activités de la catégorie, } \\
\text { intensité relative d'exposition } \\
\text { (Moyenne et écart-type) }\end{array}$ \\
\hline G. Intégration sociale & Rang 1 & Rang 4/5 & Rang 1 & $100 \%$ & $0,67 \pm 0,25$ \\
\hline $\begin{array}{l}\text { H. Prévention et promotion } \\
\text { de la santé }\end{array}$ & Rang $3 / 4$ & Rang 1 & Rang $4 / 5$ & $71 \%$ & $0,33 \pm 0,22$ \\
\hline B. Groupes spécifiques & Rang 2 & Rang 2 & Rang $4 / 5$ & $69 \%$ & $0,33 \pm 0,20$ \\
\hline C. Activités fonctionnelles & Rang $3 / 4$ & Rang 4/5 & Rang 2 & $86 \%$ & $0,33 \pm 0,17$ \\
\hline $\begin{array}{l}\text { E. Activités musicales ou } \\
\text { de réminiscence }\end{array}$ & Rang 6 & & Rang 3 & $49 \%$ & $0,34 \pm 0,27$ \\
\hline A. Exercices physiques & & & & $89 \%$ & $0,71 \pm 0,25$ \\
\hline F. Sports et jeux physiques & Rang 5 & Rang 3 & Rang 7 & $74 \%$ & $0,32 \pm 0,16$ \\
\hline I. Soutien au réseau d'aide & Rang 7 & Rang 6 & Rang 5 & $N / D$ & $\mathrm{~N} / \mathrm{D}$ \\
\hline
\end{tabular}

$\mathrm{N} / \mathrm{D}$ : données non disponibles 
Tableau 7 : Correspondance entre l'exposition aux diverses activités pour les membres du groupe 3 (incapacités d'origine cognitive; $n=23)$ et les activités recommandées

\begin{tabular}{|c|c|c|c|}
\hline Activités & $\begin{array}{l}\text { Recommandation pour Incapacités } \\
\text { d'origine cognitive }\end{array}$ & $\begin{array}{c}\text { Personnes du groupe } 3 \text { qui ont } \\
\text { bénéficié d'activités de cette catégorie } \\
\text { (Pourcentage) }\end{array}$ & $\begin{array}{l}\text { Pour ceux qui ont bénéficié des activités de } \\
\text { la catégorie, intensité relative d'exposition } \\
\text { (Moyenne et écart-type) }\end{array}$ \\
\hline D. Stimulation cognitive & Rang 1 & $57 \%$ & $0,67 \pm 0,40$ \\
\hline E. Activités musicales ou de réminiscence & Rang 2 & $39 \%$ & $0,38 \pm 0,28$ \\
\hline C. Activités fonctionnelles & Rang 3 & $65 \%$ & $0,37 \pm 0,19$ \\
\hline I. Soutien au réseau d'aide & Rang 4 & $N / D$ & $N / D$ \\
\hline G. Intégration sociale & & $83 \%$ & $0,74 \pm 0,27$ \\
\hline F. Sports et jeux physiques & Rang 5 & $74 \%$ & $0,44 \pm 0,16$ \\
\hline A. Exercices physiques & Rang 6 & $100 \%$ & $0,92 \pm 0,16$ \\
\hline
\end{tabular}

N/D : données non disponibles 
Tableau 8: Correspondance entre l'exposition aux diverses activités pour les membres du groupe 4 (incapacités d'origine motrice, $n=29)$ et les activités recommandées

\begin{tabular}{|c|c|c|c|}
\hline Activités & $\begin{array}{l}\text { Recommandation pour Incapacités } \\
\text { d'origine motrice }\end{array}$ & $\begin{array}{l}\text { Personnes du groupe } 4 \text { qui ont } \\
\text { bénéficié d'activités de cette catégorie } \\
\text { (Pourcentage) }\end{array}$ & $\begin{array}{l}\text { Pour ceux qui ont bénéficié des activités de } \\
\text { la catégorie, intensité relative d'exposition } \\
\text { (Moyenne et écart-type) }\end{array}$ \\
\hline A. Exercices physiques & Rang 1 & $79 \%$ & $0,79 \pm 0,26$ \\
\hline B. Groupes spécifiques & Rang 2 & $69 \%$ & $0,42 \pm 0,19$ \\
\hline C. Activités fonctionnelles & Rang 4 & $55 \%$ & $0,39 \pm 0,16$ \\
\hline H. Prévention et promotion de la santé & Rang 5 & $59 \%$ & $0,37 \pm 0,26$ \\
\hline
\end{tabular}

N/D : données non disponibles 


\section{Références}

Agence de la santé et des services sociaux de Montréal. (2004a). Portrait de la population : Centre de santé Côte-des-Neiges, Métro et Parc Extension. En ligne [http://www.santemontreal.qc.ca/pdf/PDF_CSSS/pop_05.pdf]. Consulté le 28 juillet 2007.

Agence de la santé et des services sociaux de Montréal. (2004b). Portrait de la population : Centre de santé Nord de l'île et Saint-Laurent. En ligne [http://www.santemontreal.qc.ca/pdf/PDF_CSSS/pop_06.pdf]. Consulté le 28 juillet 2007.

Anderson, A. J. (2002). Treatment of Depression in Older Adults [Electronic Version]. International Journal of Psychosocial Rehabilitation, 6, 69-78. En ligne [http://psychosocial.com/current_2002/OLDER_Adults.html]. Consulté le 8 mai 2006.

Association des centres d'accueil du Québec (ACAQ) (1991). Le centre de jour : Un cadre de référence. Montréal: auteur.

Baumgarten, M., Lebel, P., Laprise, H., Leclerc, C., \& Quinn, C. (2002). Adult day care for the frail elderly: Outcomes, satisfaction, and cost. Journal of Aging and Health, 14(2), 237-259.

Béland, F., Bergman, H., Lebel, P., Clarfield, A. M., Tousignant, P., Contandriopoulos, A. P., et al. (2006a). A system of integrated care for older persons with disabilities in Canada: Results from a randomized controlled trial. Journal of Gerontology Series A: Biological Sciences and Medical Sciences, 61(4), 367-373.

Béland, F., Bergman, H., Lebel, P., Dallaire, L., Fletcher, J., Contandriopoulos, A. P., et al. (2006b). Des services intégrés pour les personnes âgées fragiles (SIPA): Expérimentation d'un modèle pour le Canada. Revue canadienne du vieillissement, 25(1), 5-42.

Bohlmeijer, E., Smit, F., \& Cuijpers, P. (2003). Effects of reminiscence and life review on late-life depression: A meta-analysis. International Journal of Geriatric Psychiatry, 18(12), 1088-1094. 
Bourque, P., Blanchard, L., \& Vézina, J. (1990). Étude psychométrique de l'Échelle de dépression gériatrique. Revue canadienne du vieillissement, 9(4), 348-355.

Cefalu, C. A., Ettinger, W. H., \& Espeland, M. (1996). A study of the characteristics of the dementia patients and caregivers in dementia-nonspecific adult day care programs. Journal of the American Geriatrics Society, 44(6), 654-659.

Cohen-Mansfield, J., Lipson, S., Brenneman, K. S., \& Pawlson, L. G. (2001). Health status of participants of adult day care centers. Journal of Health and Social Policy, 14(2), 71-89.

Conrad, K. J., \& Guttman, R. (1991). Characteristics of Alzheimer's versus nonAlzheimer's adult day care centers. Research on Aging, 13(1), 96-116.

Conrad, K. J., Hughes, S. L., Hanrahan, P., \& Wang, S. (1993). Classification of adult day care: A cluster analysis of services and activities. Journal of Gerontology, 48(3), S112-122.

Cornoni-Huntley, J., Brook, D. B., Ostfeld, A., Taylor, J. O., \& Wallace, R. B. (1986). Established Populations for Epidemiological Studies of the Elderly, Resources Data Book. National Institutes of Health: NIH no. 86-2443.

Delbecq, A. L., Van De Ven, A. H., \& Gustavson, D. H. (1975). Group Techniques for Program Planning, A Guide to Nominal Group and Delphi Processes. Glenview, Illinois: Scott, Foresman and Company.

Desrosiers, J., Bravo, G., Hébert, R., \& Dubuc, N. (1995). Reliability of the revised functional autonomy measurement system (SMAF) for epidemiological research. Age and Ageing, 24(5), 402-406.

Everitt, B. S., Landau, S., \& Leese, M. (2001). Cluster Analysis (4 ${ }^{\text {th }}$ ed.). London: Arnold.

Fillenbaum, G. G. (1988). Multidimensional functional assessment of older adults: The Duke Older American Resources and Services procedures. Hillsdale, NJ: Lawrence Erlbaum Associates.

Folstein, M. F., Folstein, S. E., \& McHugh, P. R. (1975). Mini-Mental State: A practical method for grading the cognitive state of patients for the clinician. Journal of Psychiatric Research, 12(3), 189-198.

Foreman, M. D. (1987). Reliability and validity of mental status questionnaires in elderly hospitalized patients. Nursing Research, 36(4), 216-220. 
Frazer, C. J., Christensen, H., \& Griffiths, K. M. (2005). Effectiveness of treatments for depression in older people. Medical Journal of Australia, 182(12), 627-632.

Gaugler, J.E., Kane, R.L., Kane, R.A., \& Newcomer, R. (2005). Early community-based service utilization and its effects on institutionalization in dementia caregiving. The Gerontologist, 45(2), 177-185.

Gaugler, J.E., \& Zarit, S.H. (2001). The effectiveness of adult day services for disabled older people. Journal of Aging and Social Policy, 12(2), 23-47.

Goldston, S. M. (1989). Adult day care: A basic guide. Owings Mills, Maryland: National Health Publishing (a division of Williams \& Wilkins).

Gottlieb, B.H., \& Johnson, J. (2000). Respite programs for caregivers of persons with dementia: A review with practice implications. Aging and Mental Health, 4(2), 119129.

Gutman, G. M., Milstein, S., Killam, J., Lewis, D., \& Hollander, M. J. (1993a). Les centres de soins de jour pour adultes en Colombie-Britannique: Caractéristiques des clients, raisons de l'aiguillage et raisons de l'omission de se présenter. Rapports sur la santé, 5(3), 321-333.

Gutman, G. M., Milstein, S., Killam, J., Lewis, D., \& Hollander, M. J. (1993b). Les centres de soins de jour pour adultes en Colombie-Britannique: Modèles, caractéristiques et services. Rapports sur la santé, 5(2), 189-207.

Hébert, R., Carrier, R., \& Bilodeau, A. (1988). Le système de mesure de l'autonomie fonctionnelle (SMAF). La revue de gériatrie, 13(4), 161-167.

Henry, M. E., \& Capitman, J. A. (1995). Finding satisfaction in adult day care: Analysis of a national demonstration of dementia care and respite services. Journal of Applied Gerontology, 14(3), 302-320.

Institut de la statistique du Québec. (31 janvier 2007). Données démographiques régionales: Estimation de la population des régions métropolitaines de recensement selon le groupe d'âge et le sexe, Québec, au ler juillet des années 1996 à 2006. En ligne [http://www.stat.gouv.qc.ca/donstat/societe/demographie/ dons_regnl/regional/index.htm]. Consulté le 3 juillet 2007. 
Institut de la statistique du Québec. (2005-2006). Données générales sur la population du Québec : Population par année d'âge et par sexe, Québec, le 1er juillet 2005-révisé et Population par année d'âge et par sexe, Québec le ler juillet 2006- provisoire. En ligne [http://www.stat.gouv.qc.ca/donstat/societe/demographie/struc_poplt/ index.htm]. Consulté le 3 juillet 2007.

Jarrott, S. E., Zarit, S. H., Berg, S., \& Johansson, L. (1998). Adult day care for dementia: A comparison of programs in Sweden and the United States. Journal of CrossCultural Gerontology, 13(2), 99-108.

Knapp, M., Thorgrimsen, L., Patel, A., Spector, A., Hallam, A., Woods, B., et al. (2006). Cognitive stimulation therapy for people with dementia: Cost-effectiveness analysis. British Journal of Psychiatry, 188, 574-580.

Lindeman, D. A., Corby, N. H., Downing, R., \& Sanborn, B. (1991). Alzheimer's Day Care: A Basic Guide. Washington, DC: Hemisphere Publishing Corp.

National Institute on Adult Day Care. (1990). Standards and Guidelines for Adult Day Care. Washington, DC: National Council on Aging.

Nissenboim, S., \& Vroman, C. (1998). The Positive Interactions Program of Activities for People with Alzheimer's Disease. Baltimore, MD: Health Professions Press.

Norman, J. L., \& Horton, E. R. (1996). Adult Day Care Therapeutic Activity Manual: A Continuous Quality Improvement Approach. Gaithersburg, MD: Aspen Publishers.

Pfeiffer, E. (1975). A Short Portable Mental Status Questionnaire for the assessment of organic brain deficit in elderly patients. Journal of the American Geriatrics Society, 23(10), 433-441.

Pope, S. K., Shue, V. M., \& Beck, C. (2003). Will a healthy lifestyle help prevent Alzheimer's disease. Annual Review of Public Health, 24, 111-132.

Regroupement des centres d'hébergement et de soins de longue durée de la région de Montréal. (2001). Les centres de jour aujourd'hui... et demain! Document III: Synthèse des consultations et propositions d'orientations. Montréal: Regroupement des centres d'hébergement et de soins de longue durée de la région de Montréal. 
Regroupement des centres d'hébergement et de soins de longue durée de la région de Montréal. (2005). Cadre de référence régional pour le programme Centre de jour. Montréal: Regroupement des centres d'hébergement et de soins de longue durée de la région de Montréal.

Ross-Kerr, J. C., Warren, S., \& Godkin, M. D. (1995). Evaluation of adult day programs in Alberta: Final report. Edmonton: Alberta Health, Long Term Care Branch.

Savard, J., Leduc, N., Lebel, P., Béland, F., \& Bergman, H. (2007). L'utilisation des services de centres de jour par les personnes âgées qui présentent des incapacités. Revue canadienne du vieillissement, 26(3), 255-274.

Schacke, C., \& Zank, S. R. (2006). Measuring the effectiveness of adult day care as a facility to support family caregivers of dementia patients. Journal of Applied Gerontology, 25(1), 65-81.

Sheikh, J. I., \& Yesavage, J. A. (1986). Geriatric Depression Scale (GDS): Recent evidence and development of a shorter version. Clinical Gerontologist, 5, 165-173.

Spector AE, Orrell M, Davies SP, Woods B. (2000). Reality orientation for dementia. Cochrane Database of Systematic Reviews 2000, Issue 3. Art. No.: CD001119. DOI: 10.1002/14651858. CD001119.pub2. Last update 30 August 2005. Récupéré le 23 novembre 2006 de http://www.mrw.interscience.wiley.com.proxy.bib. uottawa.ca/cochrane/clsysrev/ articles/CD001119/frame.html

Spector, A., Thorgrimsen, L., Woods, B., Royan, L., Davies, S., Butterworth, M., et al. (2003). Efficacy of an evidence-based cognitive stimulation therapy programme for people with dementia. British Journal of Psychiatry, 183, 248-254.

Strain, L. A., Chappell, N. L., \& Blandford, A. A. (1987). Changes in life satisfaction among participants of Adult Day Care and their informal caregivers. Journal of Gerontological Social Work, 11(3-4), 115-129.

Strang, V., Greschuk, J., McIlveen, K., Gadacz, R. R., \& Neufeld, A. (1992). Adult day care: An evaluation study. Edmonton: Victorian Order of Nurses, Edminton Brabch.

Tourigny, A., Côté, L., Laberge, A., Paradis, M., \& Joubert, P. (1993). Évaluation du programme québécois des centres de jour. Québec: Centre hospitalier de l'Université Laval: Unité de recherche en santé communautaire, Centre de santé publique de Québec. 
Webb, L.C. (Eds.) (1989). Planning and Managing Adult Day Care: Pathways to Success. Owing Mills, Maryland: National Health Publishing (a division of Williams \& Wilkins).

Wilson, R. S., McCann, J. J., Li, Y., Aggarwal, N. T., Gilley, D. W., \& Evans, D. A. (2007). Nursing home placement, day care use, and cognitive decline in Alzheimer's disease. American Journal of Psychiatry, 164(6), 910-915.

Wilson, R. S., Mendes de Leon, C. F., Barnes, L. L., Schneider, J. A., Bienias, J. L., et al. (2002). Participation in cognitively stimulating activities and risk of incident Alzheimer disease. JAMA, 287, 742-748.

Woods, B., Spector, A., Jones, C., Orrell, M., \& Davies, S. (2005). Reminiscence therapy for dementia. [update of Cochrane Database Syst Rev. 2000;(4):CD001120; PMID: 11034700]. The Cochrane Database of Systematic Reviews (2), CD001120.

Zank, S. \& Schacke, C. (2002). Evaluation of geriatric day care units: Effects on patients and caregivers. Journal of Gerontology, Serie B: Psychological Sciences and Social Sciences, 57(4), 348-357.

Zarit, S. H., Stephens, M. A., Townsend, A., \& Greene, R. (1998). Stress reduction for family caregivers: Effects of adult day care use. Journals of Gerontology, Serie B: Psychological Sciences and Social Sciences, 53, S267-277.

Zarit, S. H., Stephens, M. A. P., Townsend, A., Greene, R., \& Leitsch, S. A. (1999). Patterns of adult day service use by family caregivers: A comparison of brief versus sustained use. Family Relations: Interdisciplinary Journal of Applied Family Studies, 48(4), 355-361. 


\begin{abstract}
ARTICLE 3
Determinants of Adult Day Center attendance among older adults with functional limitations
\end{abstract}

\author{
Jacinthe Savard ${ }^{1,2}$ \\ Nicole Leduc ${ }^{1,3}$ \\ Paule Lebel ${ }^{1,4}$ \\ François Béland ${ }^{1,3}$ \\ Howard Bergman 1,3,5
}

${ }^{1}$ Groupe de recherche SOLIDAGE, Facultés de Médecine, Université de Montréal et Université McGill

${ }^{2}$ Clinique universitaire interprofessionnelle de réadaptation, Université d'Ottawa

${ }^{3}$ Groupe de recherche interdisciplinaire en santé (GRIS), Faculté de Médecine, Université de Montréal

${ }^{4}$ Centre d'expertise sur la santé des personnes âgées et des aidants (CESPA), Institut universitaire de gériatrie de Montréal

${ }^{5}$ Division of Geriatric Medicine, McGill University, and Jewish General Hospital, Montréal 


\section{AUTHOR'S NOTE}

The first author was supported during the present study through doctoral awards, first from the Alzheimer Society of Canada and later from the SOLIDAGE Research Group (funded by the Canadian Institute of Health Research - CIHR). The study used data collected during the System of Integrated Care for Older Persons (SIPA) project, funded by the Health Transition Funds of Health Canada, the Canadian Health Services Research Foundation, and the Ministry of Health and Social Services of the province of Quebec. The authors wish to thank Luc Dallaire and John Fletcher, research coordinators with the SIPA project, for their helpful cooperation during data analysis, and Mary Egan for her helpful comments on the manuscript. 


\begin{abstract}
Objective: To examine factors related to regularity of Adult Day Center (ADC) attendance among seniors with functional limitations. Methods: Using data collected as part of a larger study, we identified the proportion of scheduled days attended among 101 ADC users in Montréal and identified determinants of this attendance. Results: More regular attendance was associated with: previous profession of ADC participant or spouse in a sector other than health care, receiving formal help for ADL or IADL on days of expected ADC participation, participating for the whole day rather than half a day, lower participation in prevention and health promotion activities, and lower caregiver burden among persons with cognitive impairments and higher caregiver burden among persons without cognitive impairments. Discussion: To enhance ADC intervention effects, levels of participation should be maximized. Study findings suggest ways to promote more regular attendance.
\end{abstract}

Key words: Adult Day Center, Adult Day Services, frail seniors, community long-term care, utilization of services 


\section{Introduction}

Most industrialized countries have developed a range of geriatric services aimed at promoting autonomy and quality of life among vulnerable members of their aging populations. Among these services, are Adult Day Centers (ADC), programs offered to older, community-dwelling individuals with functional limitations. At these centers, interventions include health monitoring, health education, rehabilitation activities oriented towards the maintenance of functional capacities, psychosocial interventions and leisure activities, primarily provided through group activities, as well as respite and support to families (Dabelko \& Zimmerman, 2008; Gutman, Milstein, Killam, Lewis, \& Hollander, 1993; Regroupement des centres d'hébergement et de soins de longue durée de la région de Montréal, 2005). Studies focusing on the effectiveness of ADCs to reduce or delay the institutionalization of participants or to reduce caregiver burden have showed variable results. In several studies, even when this service is accessible, the participation rates are low (Baumgarten, Lebel, Laprise, Leclerc, \& Quinn, 2002; Douglass \& Visconti, 1998; Lawton, Brody, \& Saperstein, 1989; Montgomery \& Borgotta, 1989). However, a minimum amount of ADC exposure would seem to be necessary to ensure beneficial effects (Zarit, Stephens, Townsend, \& Greene, 1998). A number of studies that included only individuals with a specified minimum attendance showed a reduction of caregiver burden or stress (Baumgarten et al., 2002; Gaugler, Jarrot, Zarit, Stephens, Townsend \& Greene, 2003; Schacke et Zank, 2006; Zarit et al., 1998), and stabilization or improvement of participants cognitive status (Zank et Schacke, 2002). In such a context, it is important to question the determinants of regularity of ADC use to better target potential users or better adapt services to support attendance among present users.

The determinants of ADC use can be studied in a variety of ways. Distinguishing ADC users from non-users allows for the identification of persons most likely to accept this type of service. Determining the characteristics of those who present higher ADC use from those who frequently miss sessions or quit prematurely would allow the identification of factors important to regular participation, and help service providers enhance use among those who may benefit from this service. 
Studies of factors that distinguish ADC users from non-users include an American study of individuals of 65 years and older. Among this group, ADC users (with or without home care services) were generally younger and presented with more cognitive deficits than individuals who used home care without ADC. Although the functional capacities of the two groups, as reflected in ADL and IADL scores, were similar, those who used only home care were hospitalized more often and used more medical equipment or assistive devices (Dabelko \& Balaswamy, 2000). In our previous study of 723 individuals 65 years and older, the probability of ADC use was higher among those who were younger; did not have a university degree; received help for daily life activities from home care services more than once a week; or were in an area where ADC fees were lower. For men only, history of stroke and greater functional impairment were associated with ADC use (Savard, Leduc, Lebel, Béland, \& Bergman, 2007).

Intensity of ADC use has been defined in a number of ways in studies designed to identify factors associated with higher use: number of attendances over a 3-month period (Baumgarten et al., 2002; Lawton Brody, \& Saperstein,1991; Montgomery, Kosloski, \& Borgotta, 1988-1989) or a 12-month period (Lynch, Harrington, \& Newcomer, 1999), number of attendances between admission and discharge (Wallace, 1987), average number of attendances per week during 6 months (Henry \& Capitman,1995), maintenance of participation during at least 6 months (Henry \& Captiman, 1995) or at least a year (Zarit, Stephens, Townsend, Greene, \& Leitsch, 1999).

The following factors have been associated with higher intensity of ADC use: male gender (Wallace, 1987; Baumgarten et al., 2002), higher ADL needs (Baumgarten et al., 2002; Lynch et al., 1999), slightly higher depression scores and slightly lower anxiety scores (Baumgarten et al., 2002), presence of cognitive deficits (Baumgarten et al., 2002; Lynch et al., 1999; Montgomery et al., 1988-1989), fewer behavioral problems (Lawton et al., 1991; Zarit et al., 1999), the presence of a spouse caregiver, higher caregiver subjective burden (Baumgarten et al., 2002), being registered for 3 days a week, and for men only, having a spouse and children (Wallace, 1987). In addition, programs which focused on 
respite or on stimulation and growth activities were attended with greater regularity than those that focused on health activities (Henry \& Capitman, 1995).

With the exception of Baumgarten et al., these results are derived from American studies. The dynamics of ADC use observed in the United States may differ in Canada or Québec. In fact, there are a variety of models of ADC in the United States: social model, medical model, mixed models, specific Dementia Day Centers. In Québec and elsewhere in Canada, ADCs are rather generic, which means that they accept a varied clientele and adapt their activities to the diverse needs of this clientele. Most ADCs offer what are usually considered health services (nursing follow-up of health status, rehabilitation activities) as well as services of a more social nature (socialization activities for persons isolated due to a significant loss of autonomy, respite for caregivers). The majority of activities are offered in the form of group activities and most of the participants receive more than one type of service. In addition, in the United States, many of these services are offered privately; participation is sometimes limited by individual financial resources. In Québec, ADCs are subsidized by the Ministry of Health and Social Services. Here participation is limited by the intake capacity of the centers, although individuals may be charged for transportation and meals (fees generally inferior to the costs of the ADC in the United States) and these charges may restrict participation. Due to differences between the two contexts, a separate consideration of factors that influence the intensity of ADC use in a Canadian context is warranted.

Research on ADC use have generally relied on the behavioral model elaborated by Andersen in 1968 and subsequently revisited (Andersen, 1995) to investigate factors affecting utilization. This model allows an interesting global conceptualization of three types of factors that can explain the utilization of health services: predisposing characteristics, enabling factors and need factors. However, research stemming from this model has focused on individual characteristics of potential services users to explain services use (Kosloski, Montgomery \& Youngbauer, 2001). Yeatts and colleagues (Yeatts, Crow \& Folts, 1992) emphasize the importance of studying factors that can be altered by service providers to increase service use, including: knowledge of services and procedures 
to access the services, access to transportation, affordability and availability of services, as well as attractiveness of services offered. In addition, in the Québec context where ADC enrollment is rarely initiated by the person himself or herself, but more often originates with the recommendation of a CLSC (Centre local de services communautaires) ${ }^{1}$ health professional, it seemed relevant to include aspects of the CLSC services in the potential factors explaining ADC use. Thus, following Kosloski et al. (2001), the conceptual framework used in this study includes predisposing characteristics and need factors from the typology described by Andersen, as well as aspects of service delivery, inspired by the works of Yeatts et al. (1992). We also included factors specific to the Québec context (see figure 1). When planning services for frail older persons, the needs of the informal caregiver must also be considered (Bass \& Noelker, 1987; Douglass \& Visconti, 1998; Ducharme, Lebel, \& Bergman, 2001) and are included in this conceptual framework. In the presence of subsidized services, enabling factors such as revenue and individual insurance coverage lose their relevance, and were omitted from the model.

\section{Insert figure 1 around here}

Predisposing characteristics are factors that are present before the need for services occurs. They influence the tendency to use formal services, but also the health status of the individual, and therefore may be related to the need for services. Need factors are linked with the health status of the senior individual and the characteristics of his or her caregiver. Aspects of service delivery included in the model are factors which can facilitate or limit access to services. We hypothesized that these factors would interact with predisposing characteristics and need factors to modify regularity of attendance. For example, it is possible that given equal need, individuals participating in activities that are well-suited to their conditions would have more regular program attendance.

The objective of the present study was to identify factors associated with the regularity of ADC attendance. Due to limited availability of ADC spaces, planned frequency of weekly attendance is determined both by the participants' desires and the participants' need for attendance as evaluated by the ADC team. Thus, in this study we 
examined regularity of attendance in terms of actual days attended as a proportion of scheduled days, rather than as the absolute number of attendances over the study period.

\section{Methods}

This study used data collected as part of a larger study, the Services Intégrés pour Personnes Agées (System of Integrated Care for Older Persons; SIPA), a demonstration project undertaken to evaluate a model of integrated services for frail seniors (Béland et al., 2006a; 2006b). The project took place from June 1999 to March 2001 and recruited 1230 study participants through two CLSCs in Montréal. About half of these seniors were randomized to receive integrated care, while the others continued to receive standard care from their CLSC. Data collection for the SIPA project included three waves of questionnaires to the SIPA participants, one questionnaire to the participants' primary caregiver in year 2000, and monitoring of health services utilization throughout the SIPA project.

\section{Study Population}

Participants to the SIPA project met the following criteria: they were aged 65 or over, were living at home at the beginning of the study, were able to speak French or English or had a relative or close friend who could communicate with health care professionals and research personal in French or English. In addition, they presented with functional limitations as demonstrated by a score of -10 or less on the Functional Autonomy Measurement System (Hébert, Carrier, \& Bilodeau, 1988).

For this study of ADC attendance, data from 812 individuals still followed by the SIPA team in 2000 were examined. Of these individuals, 140 persons attended an ADC during a 6-month observation period. Thirty-nine of these individuals were excluded from the analyses ( 8 with numerous missing data, 15 who did not identify a caregiver, 16 whose caregiver did not complete the questionnaire). The final sample therefore included 101 ADC participants. Comparison between included and excluded individuals on the sociodemographic variables listed in Table 1 revealed only one statistically significant difference 
$(p<.05)$ : the caregiver is the spouse of $35 \%$ of the included individuals and of only $8 \%$ of the excluded individuals.

\section{Data collection}

Data related to the characteristics of the older individuals and their caregivers were obtained from questionnaires administered by interview between May and November 2000 as part of the SIPA project. For the 101 retained ADC participants, 29\% of the interviews were completed by a proxy, as the individual was unable to answer because of frailty, cognitive impairment, or language barrier. In another $17.8 \%$ of the sample, the participant was helped by a relative or a friend to answer the questionnaire. In addition, the health services utilization of participants was monitored by the SIPA research team through examination of the relevant agency databases (Régie de l'assurance-maladie du Québec, Agence de la santé et des services sociaux (ASSS) de Montréal, CLSCs, hospitals, ADCs, etc.). These databases included information on the use of home care services (dates and types of services), ADC attendance (dates and types of activities), hospitalization (dates and length of stay), and institutional placement (dates).

\section{Observation period}

Data on ADC attendance over 6 months, 3 months prior to and 3 months after the participant interview, were examined. This period was chosen to take two important factors into account. Since this study was considering the links between the characteristics of the individuals and their ADC attendance and because the health characteristics of older persons are likely to change quickly, a short observation period was required. It nevertheless had to be sufficiently long to capture a representative sample of attendance taking into consideration expected absences due to hospitalizations, the use of institutional respite or other such events.

For the 16 individuals who enrolled in an ADC during the observation period, a minimum of 8 weeks of observation was sought. For three individuals, this meant extending the observation period beyond three months after the interview date. 


\section{Measurements}

The dependent variable, the regularity of ADC attendance, was calculated as the actual attendances divided by the expected attendances given the frequency with which the individual was scheduled to attend the ADC, corrected for ADC closure, hospitalizations and days in overnight respite care.

The independent variables considered in this study are presented according to the categories of the conceptual model: predisposing factors, needs factors of the senior person and of his or her caregiver and aspects of services delivery pertaining to services received from the CLSC and from the ADC (Figure 1).

\section{Predisposing characteristics}

Sociodemographic information considered included age, gender, education, whether the elderly person or his or her spouse had worked in the health care sector, and cultural background as measured by the country of birth and language spoken at home and with health professionals. The level of education is analyzed on a 3-level scale. The language spoken is presented on a 5-level scale for descriptive purpose, but analyzed as dichotomous variable (knowledge of at least one of the two Canadian official languages). Religion was recorded for descriptive purpose only as it was highly correlated to country of birth and language spoken.

For social network information, living situation and the relationship with the caregiver were grouped together in a combined variable with three categories: spouse caregiver, other co-resident caregiver, and non co-resident caregiver.

The presence of a previous episode of participation in an ADC was also considered as predisposing factors. We hypothesed that this variable could indicate a lesser inclination to use an $\mathrm{ADC}$, since an earlier episode ended in resignation of participation, which is often the result of irregular participation. 
Need factors

Health and functional status of participants

To evaluate their health status, respondents were asked if they had any of the 16 conditions included in the EPESE study (Cornoni-Huntley, Brook, Ostfeld, Taylor, \& Wallace, 1986). Of these conditions, 14 related to their physical health, one to their mental health and one to memory problems specifically. The first physical health score used in the present study is the number of health problems among 13 of these conditions (listed in table 3 ). The $14^{\text {th }}$ condition, history of stroke, was analyzed separately. To complete the assessment of the physical health of the participants, the number of days of hospitalization during the observation period was recorded.

The mental health of the participant was measured using a question taken from the EPESE study that asks if the person suffers from emotional problems (nervousness, depression, anxiety or insomnia).

Cognitive impairment was assessed with the Short Portable Mental Status Questionnaire (SPMSQ) (Pfeiffer, 1975). This questionnaire includes 10 questions which mainly evaluate memory and orientation. Pfeiffer (1975) suggests that a score of 3 or more errors is indicative of possible cognitive impairment for persons with at least some high school education. He suggests allowing one additional error for persons with less than high school education and one less error for those with college or university education, as well as an additional error for African-Americans. Pfeiffer's recommendations were used to classify subjects as cognitively impaired or not, adapting the correction for ethnicity to the multiethnic context of the city of Montréal, allowing one additional error for subjects who were not born in Canada. While using the criteria suggested by Pfeiffer, Foreman (1987) has obtained a coefficient of prediction of a positive test of 0,89 .

For persons unable to communicate with the interviewer because of high level of impairment or lack of French or English knowledge, the presence of cognitive deficits was recorded if the questionnaire indicated that a proxy responded because of the participant's cognitive deficits, or on the EPESE question on memory, the proxy indicated that a 
physician had reported that the person suffers from Alzheimer disease or memory problems.

Functional capacity was measured using the section on personal care from the Older American Resources and Services (OARS) (Fillenbaum, 1988). This scale includes 7 items evaluating the capacity to complete various activities of daily living - ADL (to wash, get dressed, eat, etc.) and 7 items evaluating the capacity to complete various instrumental activities of daily living - IADL (to use the telephone, use means of transportation, prepare meals, etc.). Scores can range from 0 to 28 , a higher score indicating higher independence.

Presence of sensory problems was evaluated by asking respondents if they had no difficulty, some difficulty or a lot of difficulty recognizing people, reading, or hearing, even when using appropriate technical aids (glasses, hearing aid). Similarly, presence of communication problems was evaluated by asking respondents if they had no difficulty, some difficulty or a lot of difficulty being understood when they speak. For the multivariate analyses, dichotomous scores were created with sensory problems indicating a lot of difficulty in at least one of the three sensory activities, and communication problem indicating a lot of difficulty when speaking.

Placement in a long term care facility in the 3 months following the questionnaire can indicate deterioration in functional capacity or a decrease in the capacity of the social network to compensate the disabilities. This information was collected from the database on placement of the Montréal ASSS.

\section{Caregivers need}

Three types of variables were selected as indicative of caregivers need for respite. They included caregiver sociodemographic indicators such as age and gender, functional capacity, and variables related to the caregiving role such as length of time in caregiving and subjective burden.

Caregivers functional capacity was measured with the section on personal care from Older American Resources and Services (OARS) (Fillenbaum, 1988), as described above. 
Length of time in caregiving was recorded in years. Subjective burden of the caregiver was measured with the 22-item Burden Interview (Hébert, Bravo, \& Girouard, 1993; Zarit, Orr, \& Zarit, 1985).

Aspects of service delivery

Three characteristics pertaining to the services received from the CLSC were considered. The first was frequency of assistance received from the CLSC for ADL and IADL activities. The second considered whether assistance was received on the days of scheduled attendance to the ADC. In fact, the two CLSCs offered some individuals assistance preparing for ACD attendance (hygiene, dressing, etc.) to lessen strain on the caregiver. This assistance could favor ADC participation. Crosschecking data on the ADC attendance and data on the services received from the CLSC allowed us to determine if the participant had received assistance for ADL or IADL on the days of scheduled ADC attendance. Preparation assistance was considered present if it was observed on a regular basis for at least one of the ADC scheduled day each week. Finally, a variable reflecting type of service received from the CLSC was used to denote whether the participant was receiving standard service or integrated service within the SIPA project. This was done since integrated service, with more personalized case management, may have influenced ADC attendance.

Regarding the characteristics of the services received from ADC, the following variables were considered: which $\mathrm{ADC}$ was attended, the duration and the frequency of scheduled visits, and type of group activities offered. The ADC attended represented a variety of organizational characteristics that were identical for every participant attending the same center. The other three variables presented individual variations for participants of the same ADCs. The duration of scheduled visits was considered as a continuous and a dichotomous variable (half-day ( 3.5 hours or less) or entire day (more than 3.5 hours)). The frequency of registration was considered on a continuous scale (number of days per week). When the frequency changed during the observation period, the frequency was prorated. The classification of the group activities of the ADC established in a previous study (Savard, Lebel, Leduc, Béland, \& Bergman, 2008 submitted; see summary in Table 1) was 
used to determine first, on a dichotomous scale, whether an individual participated in this type of activity, and then to establish on a continuous scale, the intensity of participation in this type of activity (percentage of ADC attendances in which the person participated in this type of activity).

(Insert Table 1 around here)

\section{Participating Adult Day Centers}

Five ADCs served the study region. Their characteristics are described in more detail elsewhere (Savard et al., 2007) and are summarized below. The number of persons registered varied from 25 to 45 per day. The average number of attendances per day varied from 18.1 to 28.7 . This corresponds to an attendance rate of 64 to $85 \%$ (mean of $73 \%$ ) for the 4 ADCs that provided this information. Participants may be registered between $1 / 2$ to 3 days a week, according to evaluated needs and the policies of the centre. Exceptionally, one person was registered for 4 days a week. The duration of each attendance varied from 4.5 to 6 hours a day, for persons registered for the whole day; and from 2 to 3.5 hours for those registered for a half-day. The cost of each attendance varied from $\$ 5$ to $\$ 17$ a day (mean \$8), to cover transportation and meal expenses. All the centers mentioned that their fees could be adjusted to accommodate people who request this due to financial difficulties. Two centers phoned all participants prior to their scheduled attendance to remind them of their expected participation. One center carried out such phone calls only for special cases such as for persons with memory problems who live alone.

These ADCs employed 4.7 to 7.9 full-time equivalent (FTE) staff, for mean ratios of 5.6 registered participants per FTE, and 3.7 real ADC presences per FTE. Personnel generally included, in addition to administrative and support personnel (coordinator, secretary, driver), the following care staff: nurses, occupational therapists, physiotherapists or physical rehabilitation therapists (a special category of college-trained personnel available in Québec), recreation technicians, social workers, and support workers. 
All of the programs endeavored to support older adults with functional limitations remain in non institutional settings. Two centers accepted persons of 60 years and older, two accepted those at least 65 years, while the remaining center specified that they were a geriatric center but did not exclude clients based on a minimum age. Each center hosted persons with cognitive difficulties on particular days or in specific groups, which ran parallel to other groups and included activities adapted for individuals with cognitive deficits.

\section{Analysis}

Descriptive statistics were computed first for each of the variables, followed by bivariate analysis of the relationship between each independent variables and ADC attendance.

To better understand the influence of the different variables on ADC attendance, multiple regression analyses were performed. Before performing the regression analysis, continuous variables were tested for linearity of their relationship to ADC attendance. As suggested by Hosmer and Lemeshow (2000), variables which demonstrated a relationship with ADC attendance at the $\mathrm{p} \leq 0.25$ level in the bivariate analyses were considered for entry in the regression analyses, as it is possible that variables weakly associated with the outcome at the bivariate level may become important predictors when considered together. Variables that have demonstrated important associations with ADC attendance in previous studies were also entered into the regression analysis. They include age, gender, relationship with caregiver, functional capacity, presence of emotional problems and cognitive deficits, caregiver burden, and frequency of scheduled attendance.

Variables were tested in three steps in the regression analyses. According to the conceptual model, predisposing characteristics variables were first entered in the model, followed by needs factors, and in the final step aspects of service delivery. At each step, variables were retained if they reached the threshold of $p \leq 0.25$ in the presence of the variables already in the model, or if they were identified in previous studies as associated with ADC attendance. After each step, the possible interactions between each of the 
retained variables were introduced into the model. The interaction terms were kept in the model if they maintained a significance threshold of $p \leq 0.25$ in the presence of the other variables and if they did not cause colinearity problems $(\mathrm{VIF}<10)$. After these steps were completed, the variables that did not reach the significance threshold of $\mathrm{p} \leq 0.05$ were withdrawn from the model. The quality of the final model was evaluated using residual diagnostic procedures.

All analyses were conducted with SPSS software for Windows, version 14.0

\section{Results}

\section{Description of the sample}

Main characteristics of ADC participants and their caregivers are reported in Tables 2 and 3. Mean age for the sample was $80.9( \pm 6.9)$. As expected among this age group, participants were mostly women (70.3\%) and a high proportion was widowed (46.5\%). Reflecting the high multiethnic composition of the areas of the city where the study took place, $46.5 \%$ of the participants were born in Canada and 53.5\% elsewhere. The immigrants had lived in Canada for many years (average of $34.5 \pm 19.0$ years) and only $5.4 \%$ have been in Canada 10 years or less. Among the 21.0 to $23.0 \%$ who were not using one of the two Canadian official languages at home, only 4 to $6 \%$ did not seem to have a sufficient knowledge of French or English to use these languages with health professionals. There were $36.6 \%$ of the subjects who were married and the spouse was the caregiver for $94.8 \%$ of the married people or $34.7 \%$ of the total sample. Children (daughters and sons) were the main caregivers for $45.5 \%$ of the subjects, and two-thirds of these did not live with the care recipient.

\section{(Insert Table 2 around here)}

Participants had on average $3.4( \pm 2.4)$ health problems among the list of 13 conditions presented. In addition, 30.7\% had experienced a stroke, 38.6\% relate having emotional problems, and $32.7 \%$ presented with cognitive impairments. Finally, functional 
capacity scores indicated a variety of independence levels (range 3-28). The caregivers were primarily women $(66.3 \%)$ whose functional capacities were generally intact. Approximately $25 \%$ of the caregivers felt moderate or severe burden.

(Insert Table 3 around here)

With regards to services, we observed that just over half of the participants received assistance from the CLSC for ADL and IADL at least once per week (table 4). Almost 25\% regularly received assistance on ADC attendance days.

(Insert Table 4 around here)

\section{ADC attendance}

The absolute number of attendances varied, of course, according to the frequency of scheduled registation (Table 5). Participants in this study were scheduled to attend an average of $1.56( \pm 0.74)$ times a week (range from 1 time/two weeks to 4 times/week, median 1 time/week). They were registered to attend the ADC for an average of $24( \pm 5)$ weeks during the observation period of 26 weeks. Shorter periods of registration were attributable to the 16 individuals who joined the ADC after the beginning of the observation period and the 9 individuals who discontinued attendance during this time. Participants had an average of $24( \pm 19)$ attendances to the ADC during the observation period. The range was 1 to 95 attendances.

\section{(Insert Table 5 around here)}

On average, the participants attended the ADC for $68.1 \%$ (95\% confidence interval: 63 to $73 \%$ ) of the scheduled visits, with $50 \%$ of the subjects attending over $72.8 \%$ of the scheduled visits. The participants with the lowest proportion of scheduled attendance had only one attendance (representing 3\% of the days registered), while 6 individuals demonstrated $100 \%$ attendance. 


\section{Factors influencing ADC attendance}

In bivariate analysis of associations between the independent variables and ADC attendance, the following were associated with higher ADC attendance $(p<0.05)$ : at least some high school but no university education, participant or spouse had not worked in health care, help from the CLSC for ADL or IADL activities once a week or more, help from the CLSC on ADC scheduled days, higher number of days per week registered at the $\mathrm{ADC}$, lower proportion of prevention and health promotion activities during ADC visits.

In the first step of the regression analysis, predisposing characteristics significant at the $\mathrm{p}<0.25$ level in bivariate analysis (gender, education, profession) were entered in the model along with age and relationship with caregiver, variables deemed important, as they relate to ADC use in previous studies.

In the following step, need variables significant at the $\mathrm{p}<0.25$ level in bivariate analysis (older individuals number of health problems, presence of cognitive impairment, presence of important sensory problems and functional capacity, as well as caregivers functional capacity and subjective burden) were entered in the model, along with a history of stroke and presence of emotional problems, variables deemed important from previous studies.

In the last step, six service delivery factors significant at the $p<0.25$ level when tested individually were entered in the model. These included: help received from the CLSC for ADL or IADL activities, frequency of ADC registration, registration for half day or full day, proportion of the ADC visits that included cognitive stimulation, prevention and health promotion activities, and special events. Help received from the CLSC for ADL or IADL activities was tested in two forms separately (frequency of services and receipt of service on scheduled ADC attendance day) as these two variables are highly correlated $($ Spearman rho $=0.723, \mathrm{p}<.001)$.

After all these steps, the variables that did not reach the significance threshold of $\mathrm{p}<0.05$ were withdrawn one by one from the model to produce the final model (Table 6). 
Six variables were identified as predictors of ADC attendance: one predisposing characteristic, two need factors and three service delivery factors. Among the predisposing characteristics, results indicate attendance was lower among participants who had been or were married to health care workers. Among the need factors, presence of cognitive impairments was a predictor of higher attendance when caregiver burden was low, but an indicator of lower attendance when the burden was high. As well, caregiver burden was a predictor of higher attendance for individuals without cognitive impairments. Among service delivery factors, receiving help the day of expected ADC day and participating for the whole day rather than half day were identified as predictors of higher attendance. Finally, greater participation in prevention and health promotion activities was an indicator of lower attendance. The model explained $23.8 \%$ of the observed variance.

\section{(Insert Table 6 around here)}

Residual analysis showed residual variance to be smaller for higher values of predicted ADC attendance. This variation may be explained by a ceiling effect. As the dependant variable is a proportion, observed and expected ADC attendance cannot be higher than one, limiting the possibility of large positive residuals at this end of the scale. Five cases were found with a Cook's distance higher than 0.10 but smaller than 0.20 . Such potential outliers can influence the model. Among these cases, three had higher observed attendance than what is predicted by the model, and the remaining two had lower observed attendance. Examination of the data revealed that these cases had observed values for all independent variables that were possible values, so these cases were kept in the analysis.

\section{Discussion}

The objective of the present study was to identify factors associated with the regularity of $\mathrm{ADC}$ attendance. Among the various ways of measuring ADC use, this study focused on the more voluntary component of attendance. Attendance was defined as observed attendances compared to scheduled attendances excluding days of hospitalization, days in respite beds and holidays when the ADC is closed. 
The mean attendance rate of $68,1 \%$ for the sample is close to the $73 \%$ mean attendance rate for all clients of the participating ADCs (as reported by the ADC managers), thus increasing the confidence in the possible generalization of study results to other samples of similar populations. Attendance rates have important programming implications for programs with waiting lists. Programs with attendance rates similar to those seen in this study would be able to enroll 25 to $30 \%$ more participants over estimated capacity before daily attendance would exceed capacity.

When looking at these results, one must keep in mind that they describe a group composed of $78 \%$ of established users, that is, users registered with the ADC for at least three months prior to the beginning of the study period. A different result would be expected from a sample composed of people beginning to attend an ADC, as drop out rates are higher in the first three to six months of registration (Cox, 1997; Dabelko, 2004; Neufeld \& Strang, 1992; Schacke \& Zank 2006; Wells, Jorm, Jordan, \& Lefroy, 1990; Zarit et al., 1999).

As suggested by Baumgarten et al. (2002), to maximize intervention effects, high levels of participation should be incorporated as an explicit ADC program goal. Thus factors influencing attendance must be understood and acted upon. Among the determinants of attendance identified in the conceptual model, service delivery factors present a higher degree of potential mutability (Kosloski et al., 2001; Yeatts et al., 1992). These factors can be modified through programming and policy decisions. In general, predisposing and need factors which relate to participant or caregiver characteristics are considered less mutable, although some need factors such as caregiver burden could be influenced by provision of targeted services. Factors with lower degrees of mutability still must be understood in order to program services that are sensitive to the utilization behaviors of clients with various characteristics and needs.

Looking first at predisposing characteristics, a somewhat surprising result is that the only factor among these that attained statistical significance was that the participant or spouse had worked in the health care sector, and that this characteristic predicted lower 
attendance. A possible explanation may be that previous work in the health care sector is associated with university education (Odds ratio: 16.5, 95\% confidence interval: 3.1 - 89.2). In a previous study, university education was associated with a lower probability of ADC use (Savard et al., 2007). In this study, university educated individuals seemed to have slightly lower ADC attendance, but the difference was not statistically significant. However, this may be due to a small sample size and in particular to the small number of university educated participants. It can be hypothesized that as university educated persons are less common in the present cohort of individuals of 65 years and over, and as ADC activities (mainly group activities) are designed to the needs of the majority, activities may be less well adapted for persons with higher education. This potential explanatory variable requires further investigation, first to confirm if individuals who have higher education and/or who have worked in health care have different experiences of ADC participation, and then to examine possible service adaptations to better meet the needs of these individuals.

With regards to need factors, presence of cognitive impairments and higher caregiver burden were important predictors of attendance. This is consistent with findings from other studies (Baumgarten et al., 2002; Montgomery et al., 1988-1989). However, the present study went a step further in testing interaction and found that presence of cognitive impairments interacts with caregiver burden to predict ADC attendance. To our knowledge this is the first time this interaction has been formally identified. However, this result is consistent with reports from qualitative studies about caregivers feeling that preparing a relative with dementia for day care is exhausting and sometimes more work than keeping the person at home (Graham, 1989; Lawton et al., 1991), and that some persons with dementia are reluctant to attend the ADC (Beisecker, Wright, Chrisman, \& Ashworth, 1996; Cotrell, 1996), making it even more difficult for caregivers with an already high level of burden to take on the extra work of convincing the cognitively impaired person to attend an ADC. This may also explains why Wells et al. (1990) found that high levels of caregiver distress at initiation of services is predictive of discontinuation of Day Care attendance among individuals with dementia. 
Contrary to the previous finding from Wallace (1987), in this study, gender was not an indicator of attendance when other factors are controlled. Unfortunately, the data available for this study did not permit testing of some predictors identified in previous research including, scores on scales for depression, anxiety, or behavioral problems. We did examine the relationship between a positive response to a general question regarding depression, anxiety and sleeping difficulties, but found no relationships. This is not surprising as these different emotional problems may influence ADC attendance in diverging ways. Baumgarten et al. (2002) found that depression was associated with higher $\mathrm{ADC}$ attendance and anxiety with lower attendance.

Three service delivery factors included in the present study influenced ADC attendance. As expected, receiving home services for ADL or IADL activities from the CLSC on expected ADC days was associated with higher attendance. Although the available data did not allow differentiation between services received expressly to help the person prepare for the ADC and other kinds of help (for example, help received later during the day), knowing that the participating CLSCs offered services to prepare individuals for ADC attendance allows us to assume that much of the CLSC help recorded was of this type. This relationship may be the result of a confounding variable not included in the study. For example, it is possible that CLSCs offer this kind of help to persons demonstrating high motivation for ADC participation. However, because of scarcity of resources, this help is most probably offered only to persons living alone or to caregivers experiencing increased burden assisting individuals to prepare to attend the ADC. If this is the case, it may represent an efficient use of resources to prevent ADC irregular attendance or termination, and in this way to provide effective respite to the caregivers.

Participating for the whole day rather than half day was also a predictor of higher attendance. Considering the burden of preparing for ADC activities either for caregivers or for older individuals themselves, a full day of participation may be necessary for perceived benefits of participation to exceed this burden. 
Finally, a higher participation rate in prevention and health promotion activities was an indicator of lower attendance. This category includes three types of activities: didactic presentations on various health topics, guided relaxation, and healthy cooking. However, $70 \%$ of the 65 persons participating in health promotion activities were offered mainly didactic activities. Henry and Capitman (1995) found that programs which focused on respite or on stimulation and growth activities had stronger participation than those focusing on health activities. However, they included many individual activities such as health assessment, referrals to needed care, etc. in their definition of health activities, whereas the present study included only group activities. Participant may prefer to be more active, and this may explain the relationship between higher proportions of health promotion activities in their schedule and lower attendance. This assumption would need to be tested in future studies.

Contrary to the previous findings of Wallace (1987), in this study, the number of times a person was registered to attend an ADC each week was not indicative of attendance when other factors were controlled.

Service delivery factors represent organizational choices made by the various participating ADC and the other agencies of the Health and Social Service system that aim at helping older individuals remain in the community for as long as possible. These important variables provide useful information for quality improvement. Yet, they have rarely been included in studies on the determinants of ADC use. As stated by Henry and Capitman (1995), it seems that "details of ADC programming count". Findings from this study suggest that to maximize levels of participation, programs should consider providing help to prepare participants for ADC, provide health promotion activities with parsimony, and offer services of sufficient daily duration. A threshold of 4 hours per day, that is a participation including lunch, seemed to positively influence attendance. Because of the limited sample size and the limited number of ADCs represented in this study, findings would need to be confirmed in future studies.

Unfortunately, some service delivery factors included in previous studies could not be considered in the present study, limiting comparison with their results. Since 
transportation and meals were offered by all the participating ADCs, we could not consider the effects of the presence or absence of these factors. In addition, a number of potentially important variables were unavailable including reason for referrals, and number and type of individual services received. Finally, variation in a number of other variables was completely attributable to the specific ADC program. These included whether participants were provided with reminder phone calls, cost of the ADC, and structure of the ADC personnel. Thus, the influence of each of these precise variables could not be tested. Inclusion of the ADC attended in the regression analysis did not demonstrate any relationship with regularity of attendance.

\section{Strengths and limitations of the study}

Most previous studies of ADC were conducted during the 1980's (general participants) and the 1990's (participants with dementia). Today, ADCs are widely available as a service to support aging in place. These early studies have had substantial influence on present day provision of services. For example, transportation difficulties were identified in previous studies as an important barrier to ADC use, and many ADCs now provide this service. Current studies are however needed to determine how well present programs respond to current needs. This study on the determinants of regularity of ADC attendance in a Canadian context provides such information.

An important strength of this study on ADC attendance is the inclusion of service delivery factors representing organizational choices made by ADCs that have not been extensively studied to date. It sheds light on additional factors such as the collaboration with the home support agency to help a person prepare for ADC attendance. It is hoped that these results will stimulate new research on areas of high importance for ADC programming and policy. Nevertheless, results must be interpreted in the light of the study limitations.

First, there are limitations attributable to the use of secondary data analysis. At the time this study was conducted, there was a lack of homogeneity in the data recorded by the 
ADCs related to the health status of their participants and the characteristics of caregivers. Thus, using data uniformly collected as part of a larger study on integrated services for the frail seniors has been a strategy to obtain valid data on the characteristics of the participants and their caregivers, as well as data on their ADC presences. However, the sample is not completely representative of the entire ADC population in Canada or Québec, for two main reasons. For one, it is not a random sample of all the participants of the five participating ADCs. Nevertheless, according to the managers of the five participating ADCs, participant characteristics in this study are quite similar to those of their entire clientele. Second, this sample was taken from persons from two well-delimited area of the city of Montréal. Population from the two CLSC catchment areas from which the participants were recruited is more multiethnic than that of the whole city of Montréal (44 to $48 \%$ of immigrants versus $28 \%$ for the entire city) (ASSS de Montréal, 2004a, 2004b). It is therefore a sample from a large multiethnic urban center. Despite this limitation, results may be at least partially representative of the situation in Montréal, as objectives and organization of the CLSCs and ADCs are similar for the entire region. ADC use and attendance may be different in rural areas as, in addition to serving populations with different characteristics, rural ADCs often must adapt their programs according to transportation possibilities over a vast area. Working with a secondary analysis of data has also limited the number of centers included in the study, restricting our ability to study the influence of a number of organizational factors.

Second, an important limit in testing the influence of the type of activities offered by the ADC on attendance is the lack of a standardized classification for ADC group activities. Such a classification would permit more reliable comparison. Finally, to include caregiver factors in the analysis restricted the sample to only those persons for whom caregiver has been identified and reached. This has reduced the sample size to 101 participants, and persons with a spouse caregiver are slightly overrepresented in this sample as compare to the general ADC population. Having a spouse caregiver has been associated with higher ADC attendance in a previous study (Baumgarten et al., 2002). Our data also showed a higher mean attendance rate for participants with spouse versus non-spouse caregivers, and for included participants versus individuals excluded because of lack of 
caregiver data. However, in both cases, these differences did not reach statistical significance.

\section{Conclusion}

High levels of participation frequency are required to maximize ADC intervention effects. Findings from this study present important programming and policy implications that deserve consideration. First, although one goal of ADC is to provide respite to families to alleviate the burden of caregiving, it is a challenge to offer respite to participants with cognitive difficulties and high caregiver burden. These participants tend to have lower ADC attendance, and additional efforts may be needed to help them. Suggestions include that such individuals may be better served through provision of help to prepare ADC participants and services of sufficient duration to warrant preparation effort. Second, activity programming can influence attendance, and future research with more comparable data on activities is needed.

\section{Footnotes}

1 In Québec, the Centres locaux de services communautaires (CLSC) are part of the Ministry of Health and Social Services network and exist throughout the province, although they were recently merged with other institutions (hospitals and nursing homes) to form the Centres de santé et de services sociaux (CSSS). They are the main source of home care services for seniors. Services include home nursing, social work, rehabilitation, help with personal care and instrumental activities of daily living, home respite, referrals to community agencies, and evaluation of admissibility and referrals for admission to long term care institutions. These services are free of charge and access is on the basis of evaluated need. 
Figure 1: Factors influencing regularity of ADC attendance

\begin{tabular}{|l|l|l|}
\hline $\begin{array}{l}\text { Presdisposing characteristics } \\
\text { of the older individual }\end{array}$ & $\begin{array}{l}\text { Needs } \\
\text { Older individual } \\
\text { - Socio-demographic variables } \\
\text { - Social network } \\
\text { - Former ADC participation }\end{array} \quad \longrightarrow \quad \begin{array}{l}\text { - Mental health } \\
\text { - Cognitive limitation } \\
\text { - Functional capacity } \\
\text { Caregiver } \\
\text { - Age and sex of caregiver } \\
\text { - Functional capacity of caregiver } \\
\text { - Length of time as a caregiver } \\
\text { - Caregiver subjective burden }\end{array}$ \\
\hline
\end{tabular}

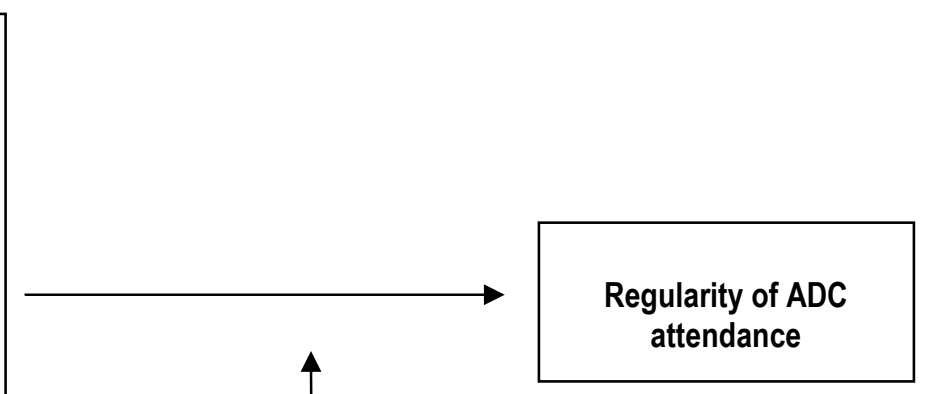

Aspects of service delivery

CLSC:

- Type of service received: SIPA or standard

- Frequency of assistance from CLSC

- ADL assistance from CLSC on days attending ADC

ADC:

- Organizational characteristics (operationalized with the identity of the ADC attended)

- Frequency of services offered

- Duration of each ADC participation

- Types of activities attended 


\section{Table 1: Classification of ADC group activities}

\begin{tabular}{ll}
\hline Name of the category & Examples of activities \\
\hline A. Physical exercises & $\begin{array}{l}\text { General exercises, Adapted exercises (sitting), Walking group, } \\
\text { Pool exercises }\end{array}$
\end{tabular}

B. Specific groups designed for a physical diagnosis or a particular functional problem

Groups for Parkinson's disease, Stroke, Diabetes, Arthritis, Pain

C. Functional activities

D. Cognitive stimulation

E. Musical or reminiscence activities

F. Sports and physical games

G. Social integration

$H$. Prevention and health promotion activities
Arts and crafts, Cooking, Horticulture, Beauty, Pet therapy

Cognitive stimulation, Sensory stimulation

Music therapy, Musical composition, Listening to music, Signing, Reminiscence

Sandbags game, Adapted curling, Adapted volleyball, Adapted bowling, etc.

Discussions (formal), Current events, Community information, Table games (cards, scrabble, Bingo, etc.), Outings, Anniversary dinners, special events and concerts, Computer- Internet, Client planning committee

Health information, healthy cooking, guided relaxation 
Table 2: Characteristics of the sample: Predisposing characteristics

\begin{tabular}{|c|c|}
\hline & Average or $\%$ \\
\hline \multicolumn{2}{|l|}{ Sociodemographic information } \\
\hline Age & $80.87( \pm 6.89)$ \\
\hline Gender (proportion of women) & $70.3 \%$ \\
\hline \multicolumn{2}{|l|}{ Education } \\
\hline - none or elementary school & $39.6 \%$ \\
\hline - high school, technical or trade school & $49.5 \%$ \\
\hline - university & $10.9 \%$ \\
\hline Participant or spouse worked in the health care field & $6.9 \%$ \\
\hline \multicolumn{2}{|l|}{ Country of birth } \\
\hline - Canada & $46.5 \%$ \\
\hline - other & $53.5 \%$ \\
\hline \multicolumn{2}{|l|}{ Religion } \\
\hline - Catholic & $58.4 \%$ \\
\hline - Jewish & $21.8 \%$ \\
\hline - other (including none) & $19.8 \%$ \\
\hline \multicolumn{2}{|l|}{ Language } \\
\hline - French at home and with professionals ${ }^{1}$ & $56.0 \%$ \\
\hline - English at home and with professionals ${ }^{1}$ & $21.0 \%$ \\
\hline - English or French with professionals (not at home) & $17.0 \%$ \\
\hline - other language at home, language with professionals missing & $2.0 \%$ \\
\hline - other language (neither English or French) even with professionals & $4.0 \%$ \\
\hline \multicolumn{2}{|l|}{ Social Network } \\
\hline \multicolumn{2}{|l|}{ Relationship with the caregiver } \\
\hline - spouse & $34.7 \%$ \\
\hline - other caregiver residing with participant (child) & $22.8 \%(15.8 \%)$ \\
\hline - other caregiver not residing with participant (child) & $42.6 \%(29.7 \%)$ \\
\hline \multicolumn{2}{|l|}{ Previous episode of $A D C$ participation } \\
\hline \multicolumn{2}{|l|}{ Previous episode of ADC participation } \\
\hline - none & $89.1 \%$ \\
\hline - contact (one visit) & $3.0 \%$ \\
\hline - participation (more than one visit) & $7.9 \%$ \\
\hline
\end{tabular}

1 These persons speak French or English at home, sometimes in addition to another language 


\section{Table 3: Characteristics of the sample: Need factors}

\begin{tabular}{|c|c|}
\hline & Average or $\%$ \\
\hline \multicolumn{2}{|l|}{ Physical Health } \\
\hline Number of health problems/ 13 & $3.40( \pm 2.35)$ \\
\hline \multicolumn{2}{|l|}{ Type of health problems } \\
\hline - cataracts & $47.5 \%$ \\
\hline - rheumatism or arthritis (joint or bone pain) & $46.5 \%$ \\
\hline - circulation problems & $39.6 \%$ \\
\hline - bladder, kidneys or prostate problems & $36.6 \%$ \\
\hline - heart disease & $34.7 \%$ \\
\hline - high blood pressure & $25.7 \%$ \\
\hline - stomach problems & $19.8 \%$ \\
\hline - diabetes & $19.8 \%$ \\
\hline - respiratory problems & $17.8 \%$ \\
\hline - tumor or cancer & $16.8 \%$ \\
\hline - recent hip or leg fracture (over the past year) & $16.8 \%$ \\
\hline - glaucoma & $11.9 \%$ \\
\hline - Parkinson's disease & $5.9 \%$ \\
\hline Stroke & $30.7 \%$ \\
\hline Days of hospitalization during the observation period & $6.82( \pm 20.70)$ \\
\hline \multicolumn{2}{|l|}{ Mental health } \\
\hline Emotional problems (yes) & $38.6 \%$ \\
\hline \multicolumn{2}{|l|}{ Cognitive impairment } \\
\hline Cognitive impairment & $32.7 \%$ \\
\hline \multicolumn{2}{|l|}{ Functional capacity } \\
\hline IADL + ADL (OARS score/28) & $17.65( \pm 6.24)$ \\
\hline - ADL (score /14) & $1083(+299)$ \\
\hline - IADL (score /14) & $6.82( \pm 3.76)$ \\
\hline - urinary incontinence 1 & $41.6 \%$ \\
\hline - fecal incontinence & $21.8 \%$ \\
\hline \multicolumn{2}{|l|}{ Presence of sensory problems } \\
\hline - no difficulties recognizing people, reading or hearing & $40.6 \%$ \\
\hline - some difficulties in at least one of the 3 activities & $31.7 \%$ \\
\hline - a lot of difficulties in at least one of the 3 activities & $27.7 \%$ \\
\hline \multicolumn{2}{|l|}{ Presence of communication problems } \\
\hline - none & $78.2 \%$ \\
\hline - a little bit of difficulty & $14.9 \%$ \\
\hline - a lot of difficulties & $6.9 \%$ \\
\hline Permanent placement in a long term care facility in the 3 months following the questionnaire & $3.0 \%$ \\
\hline \multicolumn{2}{|l|}{ Caregivers sociodemographic indicators } \\
\hline Age & $62.34( \pm 13.73)$ \\
\hline Gender (proportion of women) & $66.3 \%$ \\
\hline \multicolumn{2}{|l|}{ Caregivers functional capacities } \\
\hline ADL + ADL (OARS score/28) & $27.56( \pm 0.87)$ \\
\hline - ADL (score /14) & $13.89( \pm 0.34)$ \\
\hline - IADL (score /14) & $13.67( \pm 0.69)$ \\
\hline \multicolumn{2}{|l|}{ Variables associated with the caregiver role } \\
\hline Length of time of caregiving & $8.07( \pm 8.38 ;$ median 5.06) \\
\hline Subjective burden (result/88) & $29.01( \pm 16.66)$ \\
\hline - absent to mild-moderate $(0-40)$ & $75.2 \%$ \\
\hline - moderate-severe to severe (41-88) & $24.8 \%$ \\
\hline
\end{tabular}

${ }^{1}$ urinary or fecal incontinence, or both $=48.5 \%$ of the sample 


\section{Table 4: Aspects of service delivery}

\begin{tabular}{ll}
\hline Services received from the CLSC & Average or $\%$ \\
\hline Frequency of assistance received from the CLSC for ADL and IADL activities & \\
- no intervention recorded & $38.6 \%$ \\
- less than once per week & $7.9 \%$ \\
- once per week or more & $53.5 \%$ \\
& \\
Assistance received on the days of ADD attendance & $22.8 \%$ \\
Type of service received from the CLSC & \\
- integrated service within the SIPA project & $54.5 \%$ \\
- regular service & $45.5 \%$ \\
\hline Characteristics of ADC visits & \\
\hline Duration of scheduled visits (hours) & \\
Proportion of individuals with half-day scheduled visits (3,5 hours or less) & $4.98 \pm 0.72$ \\
Frequency of registration (days/week) & $5,9 \%$ \\
\hline & \\
Activities at the ADC & $1.56 \pm 0.74$ \\
& $\% \%$ individuals who participated \\
(for those with participation, \\
A. Physical Exercises & intensity of participation) \\
B. Specific group for a physical diagnosis or a particular functional problem & $89.1 \%(0.71 \pm 0,32)$ \\
C. Functional activities & $50.5 \%(0.23 \pm 0,31)$ \\
D. Cognitive stimulation & $69.3 \%(0.26 \pm 0,22)$ \\
E. Musical or reminiscence activities & $66.3 \%(0.31 \pm 0,35)$ \\
F. Sports and physical games & $44.6 \%(0.16 \pm 0,25)$ \\
G. Social integration & $77.2 \%(0.28 \pm 0,22)$ \\
Special events (celebrations, shows, outings) & $90.1 \%(0.66 \pm 0,32)$ \\
H. Prevention and health promotion activities & $54.5 \%(0.09 \pm 0,13)$ \\
\hline & $64.4 \%(0.24 \pm 0,26)$ \\
\hline
\end{tabular}


Table 5: Number of ADC attendances during a period of 26 weeks, presented by frequency of registration

The shaded boxes correspond to expected observation if actual attendance matched scheduled attendance.

\begin{tabular}{|c|c|c|c|c|c|c|c|c|c|}
\hline Frequency of registration & $\begin{array}{c}\text { Once per } 2 \\
\text { weeks }\end{array}$ & $\begin{array}{c}1 \text { time/ } \\
\text { week }\end{array}$ & $\begin{array}{c}\text { Between } \\
1 \& 2 \text { times / } \\
\text { week }^{*}\end{array}$ & $\begin{array}{c}2 \text { times/ } \\
\text { week }\end{array}$ & $\begin{array}{c}\text { between } 2 \\
\& 3 \text { times } / \text { week }^{*}\end{array}$ & $\begin{array}{l}3 \text { times/ } \\
\text { week }\end{array}$ & $\begin{array}{l}\text { between } 3 \\
\& 4 \text { times / } \\
\text { week }^{*}\end{array}$ & $\begin{array}{l}4 \text { times/ } \\
\text { week }\end{array}$ & Total \\
\hline \multicolumn{10}{|l|}{ Actual attendance } \\
\hline 1 to 13 & 1 & 26 & & 6 & & & & & 33 \\
\hline 14 to 26 & & 28 & 4 & 5 & & & & & 37 \\
\hline 27 to 39 & & & 2 & 7 & & 2 & & & 11 \\
\hline 40 to 52 & & & 1 & 6 & 1 & 2 & & & 10 \\
\hline 53 to 65 & & & & & 1 & 3 & & & 4 \\
\hline 66 to 78 & & & & & & 5 & & & 5 \\
\hline 79 to 91 & & & & & & & 0 & & 0 \\
\hline 92 to 104 & & & & & & & & 1 & 1 \\
\hline Total & 1 & 54 & 7 & 24 & 2 & 12 & 0 & 1 & 101 \\
\hline
\end{tabular}

* This represents individuals for whom the scheduled attendance changed during the observation period 
Table 6: Multiple linear regression of level of ADC attendance on predisposing and need factors, and aspects of service delivery

\begin{tabular}{llll}
\hline & Coefficient B & IC $95,0 \%$ for B & p value \\
\hline Predisposing characteristics & & & \\
\hline Participant or spouse worked in the field of health care & -.209 & $(-.382 ;-.037)$ & .018 \\
\hline Need factors & .281 & $(.080 ; .483)$ & .007 \\
\hline Cognitive impairment & .003 & $(.000 ; .006)$ & .042 \\
Caregiver burden & -.008 & $(-.014 ;-.002)$ & .009 \\
Interaction between burden and cognitive impairment & & & \\
\hline Aspects of service delivery & .124 & $(.019 ; .230)$ & .022 \\
\hline Assistance received from CLSC on the days of ADC attendance & .209 & $(.018 ; .399)$ & .032 \\
Duration of attendance $\geq 3,5 \mathrm{~h}$ & -.223 & $(-.402 ;-.044)$ & .015 \\
Participation in prevention and health promotion activities & & $\mathrm{R} 2: 0.238$ Ra2: 0.181 & \\
\hline Explained variance & & & \\
\hline
\end{tabular}




\section{References}

ASSS de Montréal. (2004a). Portrait de la population: Centre de santé Côte-des-Neiges, Métro et Parc Extension [Population description: Health sectors Côte-des-Neiges, Métro and Parc Extension] Retrieved July 28, 2007 from http://www.santemontreal.qc.ca/pdf/PDF_CSSS/pop_05.pdf

ASSS de Montréal. (2004b). Portrait de la population: Centre de santé Nord de l'Île et Saint-Laurent [Population description: Health sectors Nord de l'Île and SaintLaurent]. Retrieved July 28, 2007 from http://www.santemontreal.qc.ca/pdf /PDF_CSSS/pop_06.pdf

Andersen, R. M. (1995). Revisiting the behavioral model and access to medical care: Does it matter? Journal of Health and Social Behavior, 36, 1-10.

Bass, D. M., \& Noelker, L. S. (1987). The influence of family caregivers on elder's use of in-home services: An expanded conceptual framework. Journal of Health and Social Behavior, 28, 184-196.

Baumgarten, M., Lebel, P., Laprise, H., Leclerc, C., \& Quinn, C. (2002). Adult day care for the frail elderly: Outcomes, satisfaction, and cost. Journal of Aging and Health, $14(2), 237-259$.

Beisecker, A. E., Wright, L. J., Chrisman, S. K., \& Ashworth, J. (1996). Family caregiver perceptions of benefits and barriers to the use of adult day care for individuals with Alzheimer's disease. Research on Aging, 18(4), 430-450.

Béland, F., Bergman, H., Lebel, P., Clarfield, A. M., Tousignant, P., Contandriopoulos, A. P., et al. (2006a). A system of integrated care for older persons with disabilities in Canada: Results from a randomized controlled trial. . Journal of Gerontology Series A: Biological Sciences and Medical Sciences, 61(4), 367-373.

Béland, F., Bergman, H., Lebel, P., Dallaire, L., Fletcher, J., Contandriopoulos, A. P., et al. (2006b). Integrated services for frail elders (SIPA): A trial of a model for Canada. Canadian Journal on Aging, 25(1), 5-42.

Cornoni-Huntley, J., Brook, D. B., Ostfeld, A., Taylor, J. O., \& Wallace, R. B. (1986). Established Populations for Epidemiological Studies of the Elderly, Resources Data Book. National Institutes of Health: NIH no. 86-2443. 
Cotrell, V. (1996). Respite use by dementia caregivers: Preferences and reasons for initial use. Journal of Gerontological Social Work, 26(3/4), 35-55.

Cox, C. (1997). Findings from a statewide program of respite care: A comparison of service users, stoppers, and nonusers. The Gerontologist, 37(4), 511-517.

Dabelko, H. I. (2004). Individual and environmental factors that influence length of stay in adult health care programs. Journal of Gerontological Social Work, 43(1), 83-105.

Dabelko, H. I., \& Balaswamy, S. (2000). Use of adult day services and home health care services by older adults: A comparative analysis. Home Health Care Services Quarterly, 18(3), 65-79.

Dabelko, H. I., \& Zimmerman, J.A. (2008). Outcomes of adult day services for participants: A conceptual model. Journal of Applied Gerontology, 27(1), 78-92.

Douglass, C., \& Visconti, C. (1998). Factors influencing the use of adult day care by individuals with Alzheimer's Disease: A multivariate examination of the California Alzheimer's Disease Diagnostic and Treatment Center Program. Home Health Care Services Quarterly, 17(2), 53-69.

Ducharme, F., Lebel, P., \& Bergman, H. (2001). Vieillissement et soins, l'urgence d'offrir des services de santé intégrés aux familles du XXIe siècle [Aging and care: Urgency to offer integrated health services to families of 21 st century]. Ruptures, revue transdisciplinaire en santé, 8(2), 110-121.

Fillenbaum, G. G. (1988). Multidimensional functional assessment of older adults: The Duke Older American Resources and Services procedures. Hillsdale, NJ: Lawrence Erlbaum Associates.

Foreman, M. D. (1987). Reliability and validity of mental status questionnaires in elderly hospitalized patients. Nursing Research, 36(4), 216-220.

Gaugler, J. E., Jarrot, S. E., Zarit, S. H., Stephens, M. A. P., Townsend, A., \& Greene, R. (2003). Respite for dementia Caregivers: The effects of adult day service use on caregiving hours and care demands. International Psychogeriatrics, 15(1), 37-58.

Graham, R. W. (1989). Adult day care: How families of the dementia patient respond. Journal of Gerontological Nursing, 15(3), 27-31, 40-41. 
Gutman, G. M., Milstein, S., Killam, J., Lewis, D., \& Hollander, M. J. (1993). Adult Day Care centres in British Columbia: Models, characteristics and services. Health Reports, 5(2), 189-207.

Hébert, R., Bravo, G., \& Girouard, D. (1993). Fidélité de la traduction française de trois instruments d'évaluation des aidants naturels de malades déments [Reliability of the French translation of three evaluation instruments for family caregivers and dementia patients]. Canadian Journal on Aging, 12(3), 324-337.

Hébert, R., Carrier, R., \& Bilodeau, A. (1988). The Functional Autonomy Measurement System (SMAF): Description and validation of an instrument for the measurement of handicaps. Age and Ageing, 17, 293-302.

Henry, M. E., \& Capitman, J. A. (1995). Finding satisfaction in adult day care: Analysis of a national demonstration of dementia care and respite services. Journal of Applied Gerontology, 14(3), 302-320.

Hosmer, D. W., \& Lemeshow, S. (2000). Applied Logistic Regression (2 ed.). New-York: John Wiley \& Sons, inc.

Kosloski, K., Montgomery, R. J. V., \& Youngbauer, J. G. (2001). Utilization of respite services: A comparison of users, seekers, and nonseekers. Journal of Applied Gerontology, 20(1), 111-132.

Lawton, M. P., Brody, E. M., \& Saperstein, A. R. (1989). A controlled study of respite services for caregivers of Alzheimer's patients. The Gerontologist, 29(1), 8-16.

Lawton, M. P., Brody, E. M., \& Saperstein, A. R. (1991). Respite for caregivers of Alzheimer's patients: Research and practice. New-York: Springer Publishing Company.

Lynch, M., Harrington, C., \& Newcomer, R. (1999). Predictors of use of chronic services by impaired members in the Social Health Maintenance Organization demonstration. Journal of Applied Gerontology, 18(3), 283-304.

Montgomery, R. J. V., \& Borgotta, E. F. (1989). The effects of alternative support strategies on family caregiving. The Gerontologist, 29(4), 457-464. 
Montgomery, R. J. V., Kosloski, K., \& Borgotta, E. F. (1988-1989). The influence of cognitive impairment on service use and caregiver response. The Journal of Applied Social Sciences, 13(1), 142-169.

Neufeld, A., \& Strang, V. (1992). Issues in the evaluation of small-scale adult day care programs. International Journal of Nursing Studies, 29(3), 261-273.

Pfeiffer, E. (1975). A Short Portable Mental Status Questionnaire for the assessment of organic brain deficit in elderly patients. Journal of the American Geriatrics Society, 23(10), 433-441.

Regroupement des centres d'hébergement et de soins de longue durée de la région de Montréal. (2005). Cadre de référence régional pour le programme Centre de jour [A Regional Frame of Reference for the Adult Day Center Program]. Montréal: Regroupement des centres d'hébergement et de soins de longue durée de la région de Montréal.

Savard, J., Lebel, P., Leduc, N., Béland, F., \& Bergman, H. (2008, submitted). Les activités de groupe des centres de jour pour personnes âgées correspondent-elles aux besoins des différents profils de participants. [Are group activities of Adult Day Centers fit to the needs of different participants' profile]. Canadian Journal on Aging.

Savard, J., Leduc, N., Lebel, P., Béland, F., \& Bergman, H. (2007). L'utilisation des services de centres de jour par les personnes âgées qui présentent des incapacités [Utilization of Adult Day Care services by frail seniors]. Canadian Journal on Aging, 26(3), 255-274.

Schacke, C., \& Zank, S. R. (2006). Measuring the effectiveness of adult day care as a facility to support family caregivers of dementia patients. Journal of Applied Gerontology, 25(1), 65-80.

Wallace, J. (1987). Factors affecting attendance in adult day care centers. Journal of Gerontological Social Work, 11(3/4), 155-165.

Wells, Y., Jorm, A., Jordan, F., \& Lefroy, R. (1990). Effects on care-givers of special day care programmes for dementia sufferers. Australian and New Zealand Journal of Psychiatry, 24(1), 82-90. 
Yeatts, D. E., Crow, T., \& Folts, E. (1992). Service use among low-income minority elderly: Strategies for overcoming barriers. The Gerontologist, 32(1), 24-32.

Zank S., \& Schacke, C. (2002). Evaluation of geriatric day care units: Effects on patients and caregivers. Journal of Gerontology, Series B: Psychological Sciences and Social Sciences, 52(4), 348-357.

Zarit, S. H., Orr, N. K., \& Zarit, J. M. (1985). The Hidden Victims of Alzheimer's Disease. New York: New York University Press.

Zarit, S. H., Stephens, M. A., Townsend, A., \& Greene, R. (1998). Stress reduction for family caregivers: Effects of adult day care use. Journals of Gerontology, Series B: Psychological Sciences and Social Sciences, 53, S267-277.

Zarit, S. H., Stephens, M. A. P., Townsend, A., Greene, R., \& Leitsch, S. A. (1999). Patterns of adult day service use by family caregivers: A comparison of brief versus sustained use. Family Relations: Interdisciplinary Journal of Applied Family Studies, 48(4), 355-361. 


\section{Résultats complémentaires}

Déterminants du contact avec le centre de jour: interaction entre le sexe et les facteurs de besoin

Les résultats présentés au tableau 6 de l'article 1 démontrent que si les hommes et les femmes utilisent le centre de jour dans des proportions semblables, ils ne l'utilisent pas de la même façon. C'est lorsqu'ils ont subi un AVC et lorsqu'ils présentent plusieurs incapacités fonctionnelles que les hommes utilisent davantage le centre de jour. Pour les femmes, l'utilisation ne varie pas en fonction de ces variables. Les femmes semblent utiliser davantage le centre de jour lorsqu'elles sont plus autonomes. Mais ces différences sont peu importantes. De plus, les hommes et les femmes plus jeunes semblent utiliser davantage le centre de jour lorsqu'ils ou elles présentent moins de problèmes de santé, alors que c'est l'inverse pour les femmes plus âgées.

En complément de ce qui est présenté à l'article 1, il apparait intéressant de démontrer ces résultats de façon graphique avec les figures 5 à 7 ci-dessous.

Figure 5 : Interaction entre sexe et présence d'un AVC

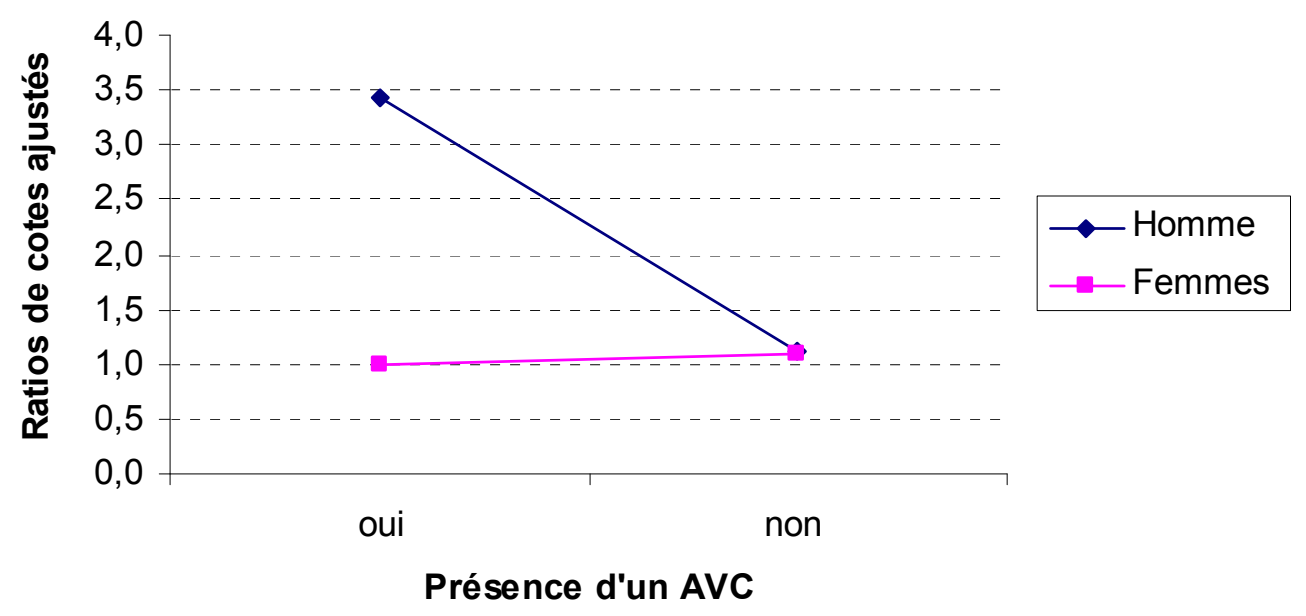


Figure 6 : Interaction entre sexe et capacités fonctionnelles

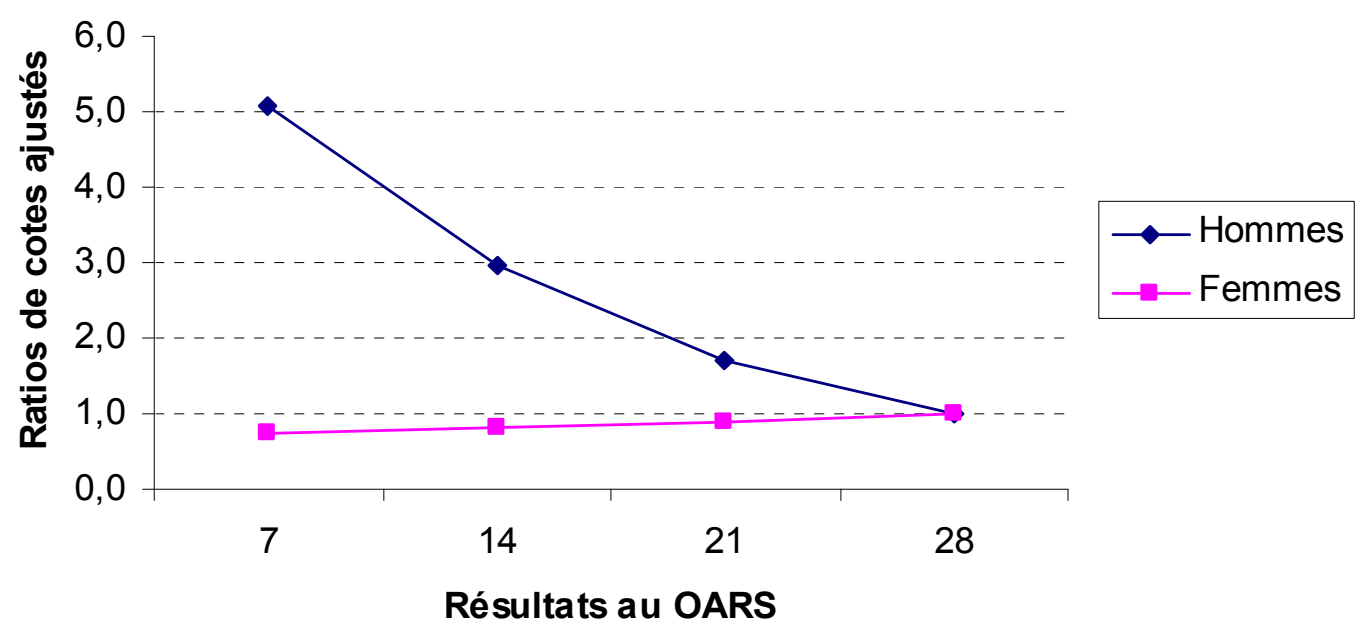

Figure 7 : Interaction : âge, sexe et problèmes de santé

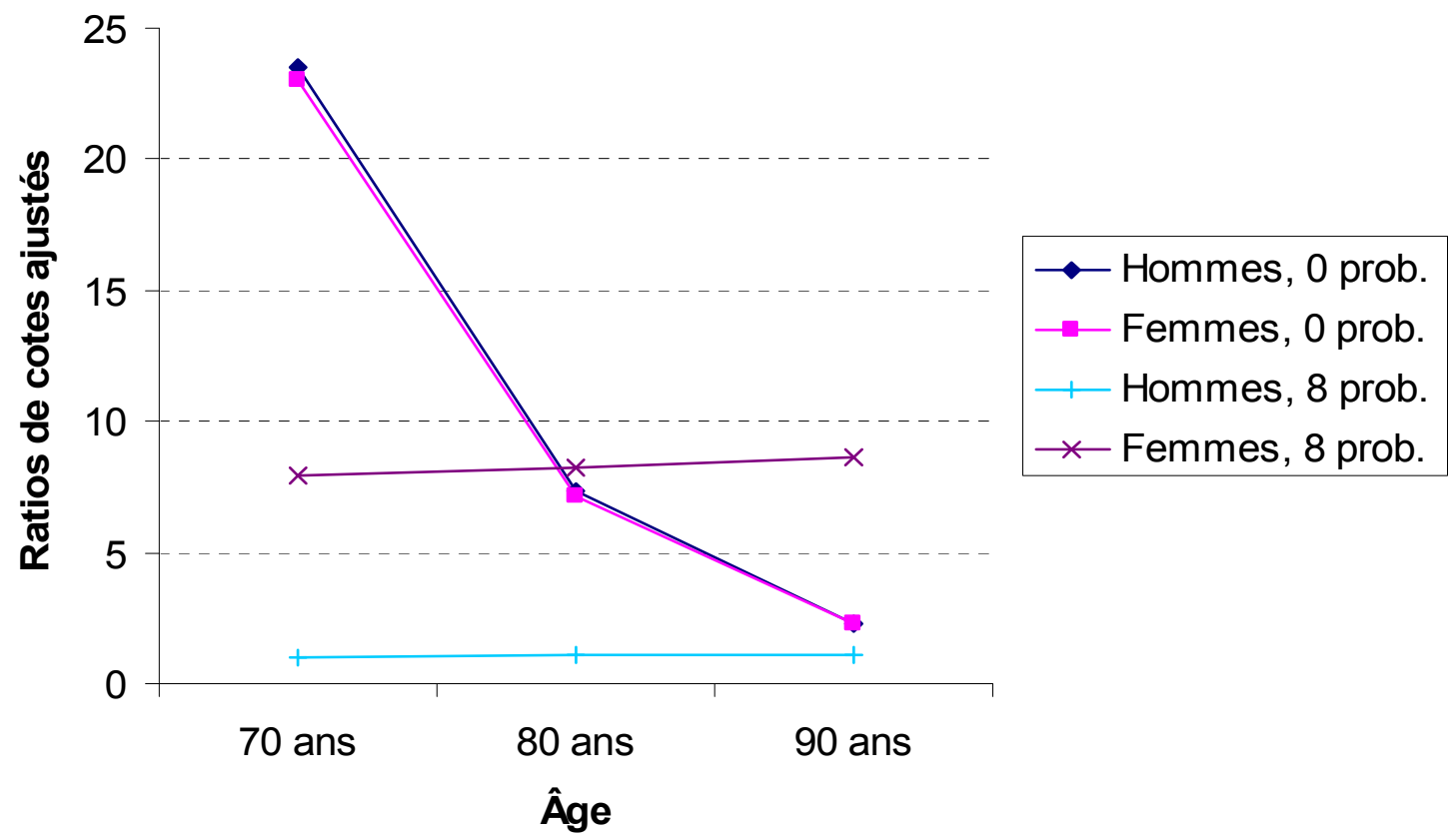


Déterminants du contact avec le centre de jour: Les facteurs de besoin des personnes de soutien

Dans l'article 1, l'influence des facteurs prédisposants et des facteurs de besoin des personnes de soutien sur le contact avec le centre de jour n'a pas été analysé pour ne pas diminuer la taille de l'échantillon utilisé pour les analyses multivariées. En effet, nous disposons de données complètes que pour 510 personnes de soutien. Les analyses bivariées entre les principales variables des personnes de soutien et le contact avec le centre de jour laissent toutefois supposer que certains facteurs de besoin des personnes de soutien, présentés au tableau VI ci-dessous, pourraient influencer le contact avec le centre de jour.

Tableau VI : Régression linéaire simple entre le contact avec le centre de jour et des variables de la personne de soutien

\begin{tabular}{lccccc}
\hline Variable & Forme & étendue & $\mathbf{n}$ & \multicolumn{2}{c}{ Relation bivariée } \\
\cline { 5 - 6 } & & & & coefficient B & signification \\
\hline Activités de la vie quotidienne et domestique & Continue & $(9$ à 28/28) & 524 &, 017 &, 048 \\
Durée de l'aide & Continue & $(0,25$ à 80 ans $)$ & 514 &,- 003 &, 049 \\
Fardeau ressenti & Continue & $(0$ à 85/88) & 510 &, 003 &, 002 \\
\hline
\end{tabular}

Ces relations, qui sont représentées graphiquement aux figures 8 à 10 ci-dessous, laissent entrevoir qu'une personne âgée serait plus susceptible de fréquenter un centre de jour si sa personne de soutien ne présente aucune incapacité fonctionnelle, est aidante depuis moins longtemps et présente un fardeau relativement élevé, sans être un fardeau très lourd. Elles mériteraient d'être explorées dans des analyses multivariées des études futures. 
Figure 8 : Contact avec le centre de jour et capacités fonctionnelles de la personne de soutien

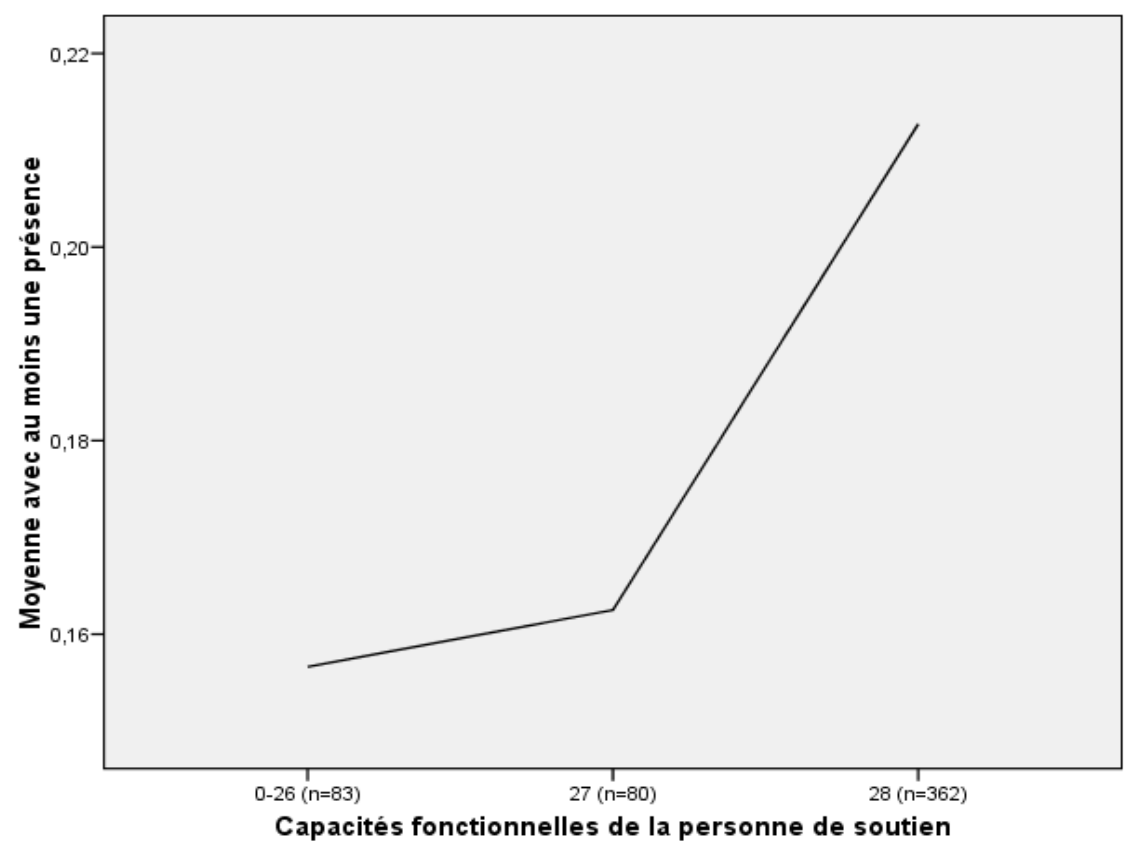

Figure 9 : Contact avec le centre de jour et durée de l'aide par la personne de soutien

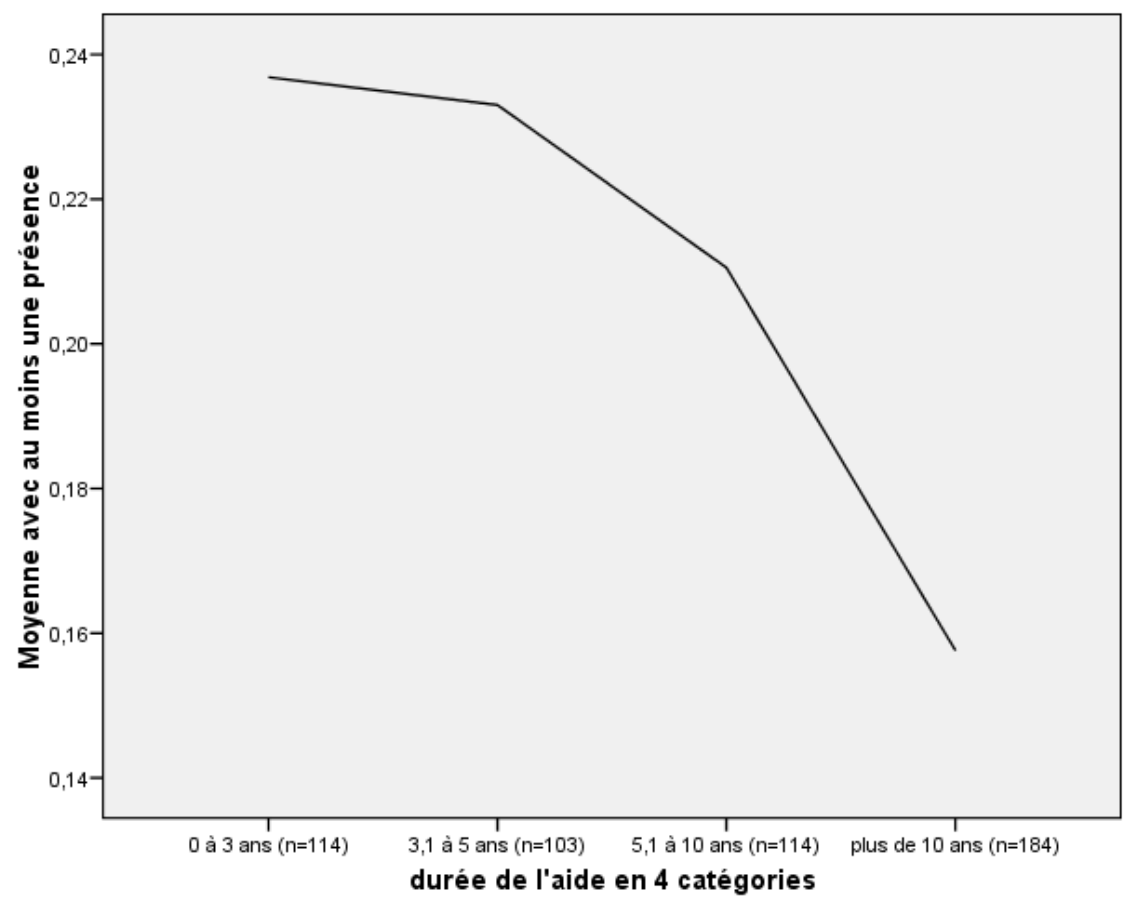


Figure 10 : Contact avec le centre de jour et fardeau de la personne de soutien

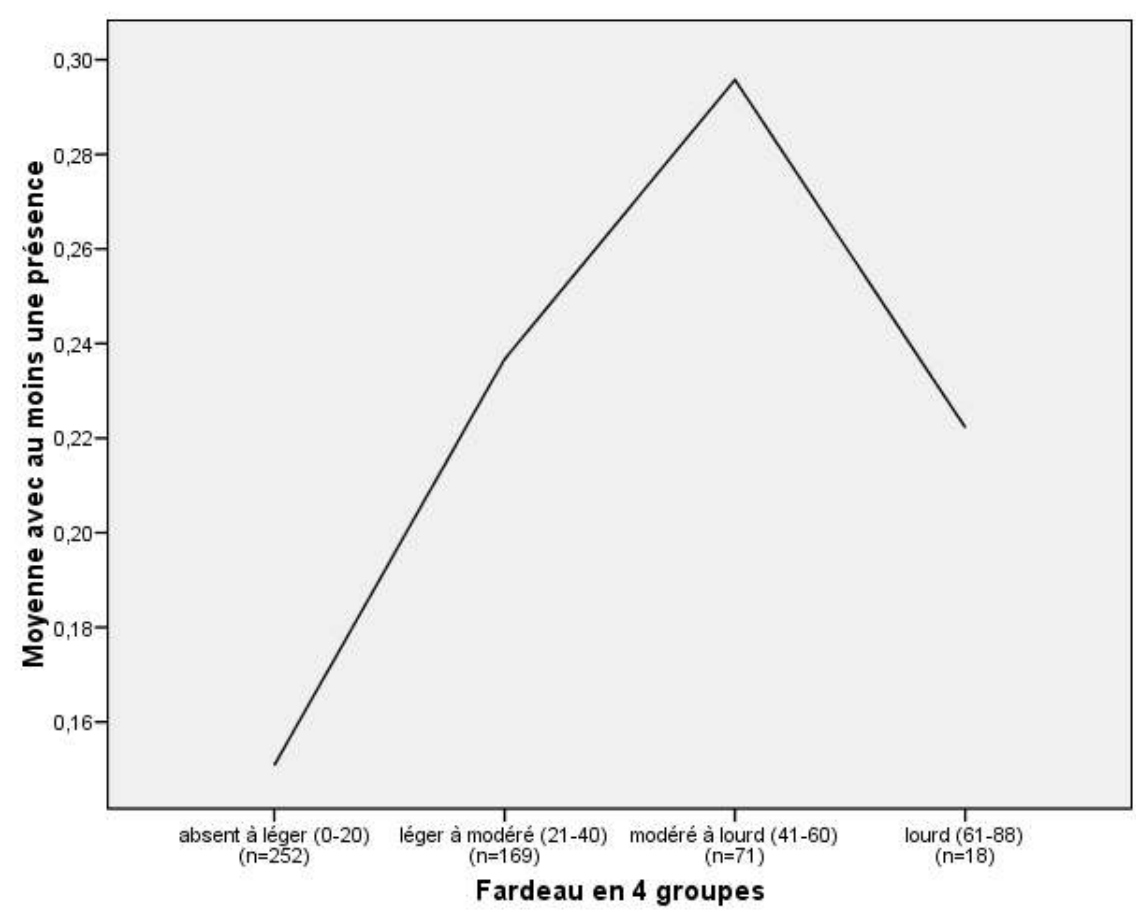


Présences réelles, régularité de la participation et types d'absence

En moyenne, les utilisateurs de centre de jour de notre échantillon étaient inscrits au centre de jour à raison de $1,56( \pm 0,74)$ jours par semaine et y ont effectivement été présents 1,0 fois par semaine en moyenne. Cependant, la moyenne du nombre de présences par semaine est rehaussée par les forts utilisateurs. La médiane est de 0,73 présence par semaine (figure 11).

Figure 11 : Participation au centre de jour : présences réelles par semaine

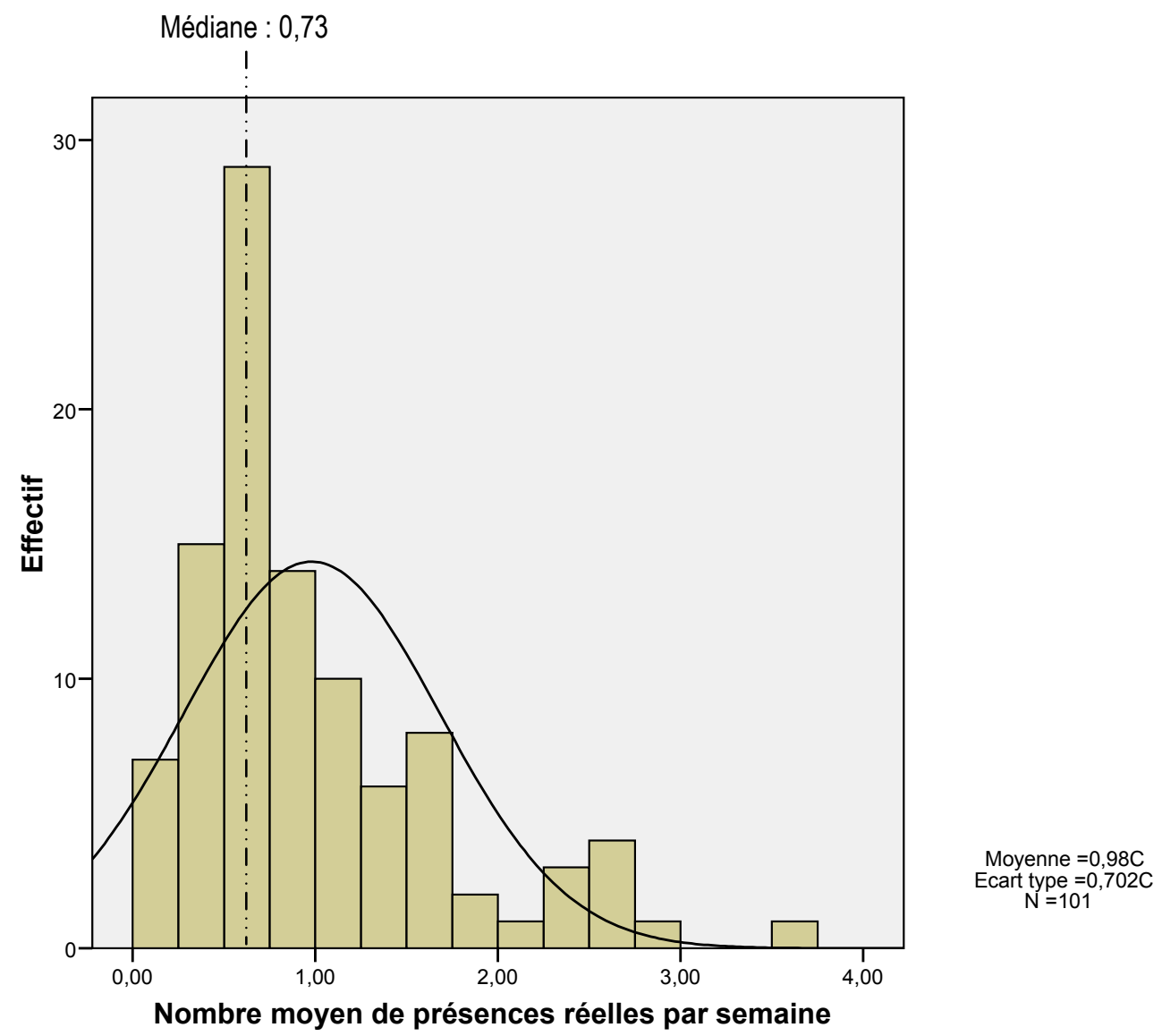


L'exposition au centre de jour a été en moyenne de $64,1 \%$ de ce qui était recommandé. Toutefois, une partie des absences sont indépendantes de la volonté de l'individu, par exemple, lorsque le centre de jour était fermé ou alors que la personne était hospitalisée ou placée en hébergement temporaire. Le tableau VII ci-dessous démontre la répartition des absences dues à ces raisons hors de la volonté de la personne et les absences pour d'autres raisons.

Tableau VII : Répartition des absences lors des jours de centre de jour prévus

\begin{tabular}{lcccc}
\hline & Minimum & Maximum & Moyenne & Écart-type \\
\hline nombre d'absences pendant la période d'observation de 26 semaines & 0 & 51 & 14,1 & 10,97 \\
absence parce que le centre de jour est fermé & 0 & 7 & 2,3 & 2,21 \\
absences pendant lesquelles la personne est hospitalisée & 0 & 43 & 1,6 & 5,64 \\
absences pendant lesquelles la personne est hébergée & 0 & 11 &, 5 & 1,87 \\
absences pour raisons autres & 0 & 40 & 9,7 & 8,92 \\
\hline
\end{tabular}

Lorsqu'on exclut ces journées où la personne est dans l'impossibilité de fréquenter le centre de jour, le taux de participation moyen est de $68,1 \%$ de ce qui serait attendu, alors que la médiane est de 72,8\%. Ainsi, une majorité d'utilisateurs ont une participation qu'on pourrait qualifier de régulière, alors qu'ils sont présents à près des trois quarts des moments prévus et possibles. On observe par contre que près de $25 \%$ des participants sont présents $50 \%$ ou moins des jours prévus et possibles (figure 12). 
Figure 12 : Régularité de la participation en centre de jour, en pourcentage des journées prévues

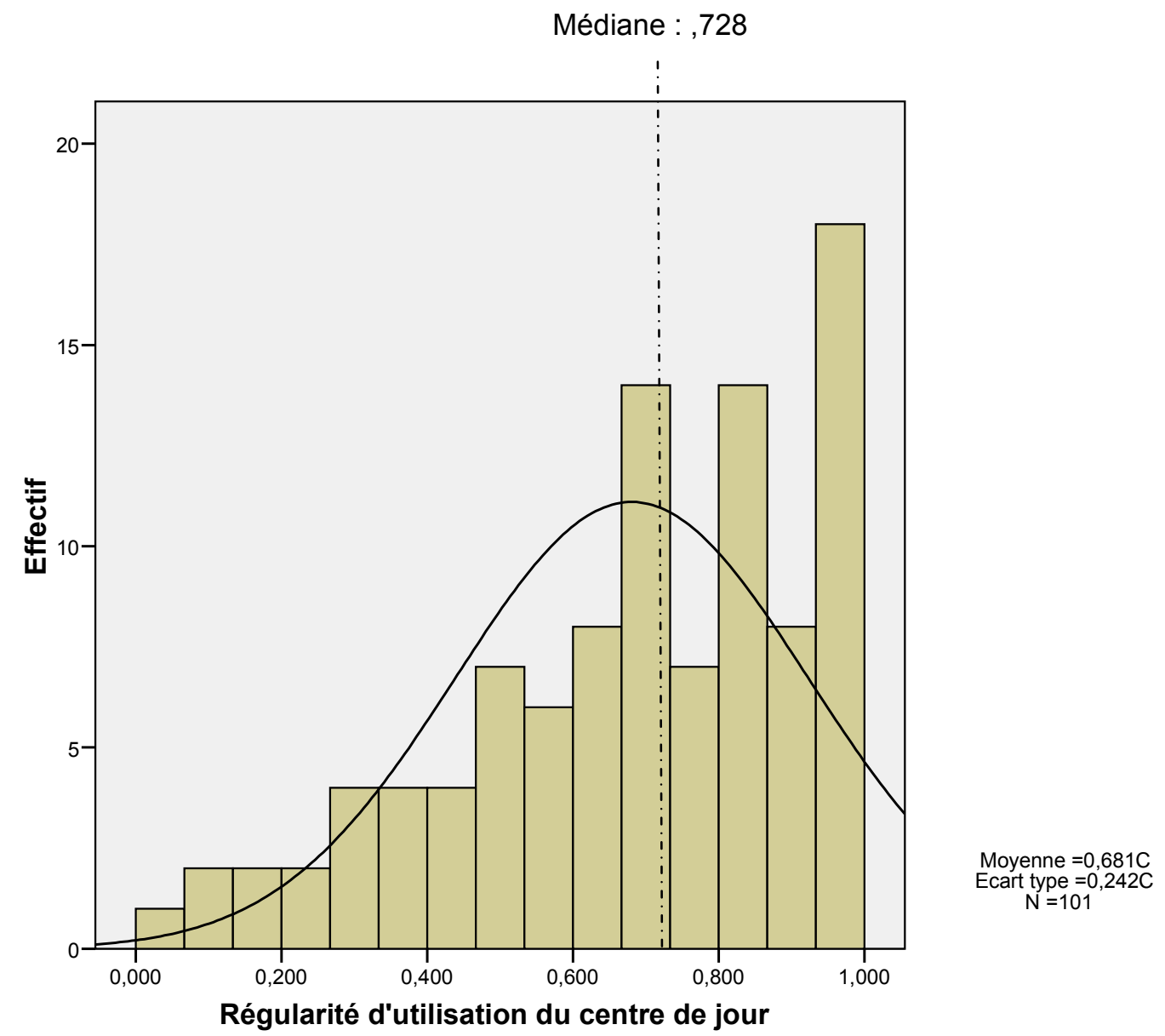

\section{Régularité de participation pour divers profils de participants}

Il est intéressant de noter les tendances observées dans la régularité de la participation en centre de jour pour divers profils de participants, même lorsque les variables de comparaison ne sont pas ressorties comme déterminants de la régularité de participation en centre de jour, car elles n'atteignent pas le seuil de signification statistique de $\mathrm{p}<, 05$. Pour comparer les résultats de divers profils de participants, rappelons que la régularité de participation moyenne pour l'ensemble de l'échantillon est de $68,1 \%( \pm 0,24)$. 
En ce qui concerne les caractéristiques des participants prises isolément, on observe que la participation des hommes semblent plus régulière que celle des femmes $(74,2 \%$ vs $65,5 \% ; \mathrm{p}=, 100)$. On note aussi que la moitié la moins autonome de l'échantillon semble participer plus régulièrement au centre de jour que la partie la plus autonome $(71,8 \%$ vs $63,2 \%, \mathrm{p}=, 076)$.

Lorsqu'on s'attarde à la régularité de participation des quatre profils de participants créés par les analyses en regroupement à l'article 2 , on observe que les différences dans les taux de régularité de participation de ces divers groupes ne sont pas statistiquement significatives, mais qu'ils démontrent une progression qu'il est pertinent de noter (voir tableau VIII) et qui est cohérente avec les observations du paragraphe précédent.

Tableau VIII : Régularité de participation pour chacun des quatre groupes formés par les analyses en regroupement

\begin{tabular}{lcccc}
\hline & $\mathbf{n}$ & Moyenne & Écart-type & Intervalle de confiance à 95\% \\
\hline 1 : Personnes fragiles & 21 &, 626 &, 226 & $(0,524-0,729)$ \\
2 : Utilisateurs précoces & 35 &, 642 &, 224 & $(0,565-0,719)$ \\
3 : Incapacités majoritairement d'origine cognitive & 19 &, 714 &, 244 & $(0,596-0,831)$ \\
4 : Incapacités majoritairement d'origine motrice & 26 &, 752 &, 268 & $(0,644-0,860)$ \\
\hline
\end{tabular}

Rappelons que les trois premiers groupes sont composés principalement de femmes, alors que le dernier groupe est composé surtout d'hommes. Les deux premiers groupes sont composés principalement de personnes plus autonomes que la moyenne et les deux derniers, de personnes moins autonomes que la moyenne.

Selon le cadre conceptuel retenu, les caractéristiques des services pourraient interagir avec les facteurs de besoin pour modifier les taux d'utilisation du centre de jour et, par exemple, un certain type d'activités pourrait favoriser une participation plus grande au centre de jour pour des sous-groupes de clients auxquels ces activités sont mieux adaptées. 
Lors d'analyses graphiques, on observe que les interactions suivantes semblent présentes en lien avec la régularité d'utilisation du centre de jour, même si aucune de ces interactions n'atteint le seuil de signification statistique de $\mathrm{p}<0,05$ lors des analyses à l'aide du modèle linéaire général contenant les deux variables principales et le terme d'interaction entre ces variables :

- les hommes semblent fréquenter plus régulièrement un centre de jour lorsqu'ils participent plus souvent à des groupes spécifiques pour un problème fonctionnel commun, alors que ce semble l'inverse pour les femmes $(p=, 103)$;

- les hommes semblent fréquenter moins régulièrement un centre de jour lorsqu'ils sont inscrits à un groupe spécifique pour personnes qui ont une atteinte cognitive, alors que ce semble l'inverse pour les femmes $(p=, 199)$;

- les personnes qui présentent des troubles cognitifs selon le SPMSQ et qui sont inscrites au centre de jour dans des groupes spécifiques pour ce type de personnes semblent participer plus régulièrement que les autres utilisateurs $(p=, 132)$.

Pour ces deux dernières observations, puisqu'il s'agit de variable dichotomique, il est possible de calculer les taux de participation moyen pour chacun des sous-groupes (tableau IX).

Tableau IX : Régularité de participation des personnes qui ont des atteintes cognitives

\begin{tabular}{lcccccc}
\hline & \multicolumn{2}{c}{$\begin{array}{c}\text { Groupe pour personnes qui } \\
\text { ont des troubles cognitifs }\end{array}$} & \multicolumn{2}{c}{ Groupes réguliers } & \multicolumn{2}{c}{$\begin{array}{c}\text { Terme } \\
\text { d'interaction }\end{array}$} \\
\hline & Moyenne & Ecart-type & Moyenne & Ecart-type & $\mathbf{F}$ & $\mathbf{p}$ \\
\hline Personnes avec atteinte cognitive &, 774 &, 197 &, 676 &, 275 & & \\
Personnes sans atteinte cognitive &, 614 &, 302 &, 664 &, 231 & 1,675 &, 199 \\
Hommes &, 700 &, 277 &, 778 &, 223 & & \\
Femmes &, 722 &, 235 &, 634 &, 235 & 2,313 &, 132 \\
\hline
\end{tabular}




\section{Chapitre 6 : Discussion générale des résultats}

Les centres de jour sont présents sur l'ensemble du territoire québécois comme une des composantes du réseau de services de soutien à domicile offerts par le système public de santé aux personnes âgées. Malgré cela, ils ont été peu étudiés à ce jour. Avec le vieillissement de la population et le besoin grandissant de services de soutien à domicile, il importe de mieux comprendre comment ils peuvent agir pour favoriser la qualité de vie et le maintien des personnes âgées dans leur milieu de vie. Puisque les centres de jours offrent un ensemble hétérogène de services à des clientèles variées et que ces clientèles ne semblent pas toujours utiliser le centre de jour avec des taux de participation qui permettent de générer des effets, la première étape pour améliorer cette compréhension a été d'étudier leur utilisation. Ce projet de doctorat visait donc à documenter l'utilisation des centres de jour dans un contexte québécois, par des personnes âgées qui présentent des incapacités d'origine variée (clientèle générale).

Plus précisément, deux aspects du phénomène d'utilisation ont été étudiés : le contact avec le centre de jour pendant une période donnée et la régularité de la participation définie comme le taux participations réelles / participations prévues. Dans un contexte où la fréquence d'inscription est déterminée par les intervenants du centre de jour en fonction du besoin évalué, cette régularité de participation peut être considérée comme le degré de conformité à la recommandation de participation.

De plus, une analyse des activités auxquelles ont participé les utilisateurs de centre de jour a été effectuée afin de déterminer s’il existe une concordance entre les activités et les caractéristiques de santé des utilisateurs. Elle a aussi permis d'inclure le type d'activités comme variable dans les analyses des déterminants de la régularité de participation. 
La première partie de cette discussion résumera les éléments mis en lumière dans chacun des trois articles de cette thèse en reprenant certaines des grandes questions soulevées par cette étude :

- Qui sont les utilisateurs de centre de jour? (article 1)

o Qu'est-ce qui les distingue des non-utilisateurs? (article 1)

o Parmi les utilisateurs, peut-on distinguer des profils d'utilisateurs ayant des caractéristiques semblables? (article 2)

- À quels types d'activités participent les personnes âgées lors de leur présence en centre de jour? Y a-t-il des liens entre ces activités et les caractéristiques des participants? (article 2)

- L'utilisation du centre de jour est-elle régulière? (article 3)

- Parmi les utilisateurs de centres de jour, quels sont ceux qui le font avec le plus de régularité? (article 3)

Chacune de ces questions de la discussion intégrera une synthèse de l'état des connaissances aux résultats de la présente étude.

La seconde partie précisera les forces et les limites de cette recherche.

Enfin, nous proposerons des recommandations pour améliorer l'utilisation des centres de jour et ses effets bénéfiques pour les participants, ainsi que des suggestions pour des recherches futures.

\section{Éléments mis en lumière dans chacun des trois volets de l'étude}

\section{Qui sont les utilisateurs de centre de jour?}

Parmi une cohorte de personnes âgées de la région de Montréal qui présentent des incapacités indiquées par un résultat de -10 ou moins au SMAF et qui participaient à l'étude SIPA, 18,8 \% ont utilisé un centre de jour pendant la période d'observation de six mois. Selon les coordonnateurs de centres de jour rencontrés pour cette étude, il est réaliste de croire que ce résultat reflète la proportion d'usagers des CLSC du territoire concerné qui 
participe aux activités des centres de jour. La proportion de personnes qui auront recours aux services d'un centre de jour à un moment ou un autre au cours de leur processus de vieillissement sera légèrement plus importante.

On retrouve peu d'information sur la proportion d'utilisateurs de centre de jour dans les écrits scientifiques, et lorsqu'on en retrouve, les données sont peu comparables entre elles et avec les résultats de la présente étude, car la définition des incapacités varie d'une étude à l'autre. Les études recensées font état de 1,9 à 11,7\% des personnes qui ont au moins une incapacité pour une activité de vie quotidienne ou domestique qui participaient à un centre de jour (Barber et coll., 1993; Kosloski et coll., 1994; Lynch et coll., 1999; Strain et Blandford, 2002). Sauf celle de Strain et Blanford, ces études sont américaines.

Le plus grand pourcentage obtenu dans notre étude ( $18,8 \%$ vs 1,9 à $11,7 \%)$ pourrait s'expliquer de plusieurs manières. La disponibilité restreinte de ce service dans certains territoires peut expliquer les plus faibles pourcentages observés. Par ailleurs, le critère d'inclusion dans notre étude requérait de plus grandes incapacités. En effet, un seuil d'incapacités de -10 au SMAF, indique un minimum de deux incapacités complètes ou de plusieurs incapacités partielles. Les personnes de notre étude devaient avoir accepté de participer au projet SIPA, une étude expérimentale. Les personnes qui acceptent de participer à ce type de recherche sont peut-être plus susceptibles de participer aussi à des activités recommandées par les intervenants du système de santé comme les activités en centre de jour. Il est aussi possible que les centres de jour québécois offrent leurs services à une plus grande quantité d'individus, en limitant l'intensité de la participation. En effet, dans la présente étude, les participants au centre de jour y étaient inscrits en moyenne à raison de 1,56 jour/semaine, alors que dans les études américaines sur le sujet, la fréquence moyenne d'inscription est de plus de trois jours par semaine. Enfin, il est possible que ces différences soient aussi en partie explicables par des différences dans les caractéristiques de notre échantillon (milieu urbain, majoritairement francophone et catholique quoique comportant une forte proportion d'individus d'origine pluriethnique). Parmi les études recensées, seule celle de Lynch et collaborateurs (1999) est limitée comme la nôtre à des 
sites urbains. La composition des milieux urbains américains diffère vraisemblablement des milieux urbains québécois.

\section{Qu'est-ce qui distingue les utilisateurs des non-utilisateurs?}

\section{Facteurs prédisposants}

Nos résultats démontrent que les utilisateurs de centre de jour sont plus jeunes que l'ensemble du groupe étudié. Une partie de l'explication peut résider dans le fait que les centres de jour rejoignent peu la clientèle très âgée (95 ans et plus), qui semble se sentir plus fragile, tolérer moins bien le transport et les activités en groupe, et préférer recevoir des services à la maison. Ces résultats sont cohérents avec ceux des études antérieures auprès de la clientèle générale qui démontraient une association négative entre l'âge et l'utilisation du centre de jour (Dabelko, 2004; Dabelko et Balaswamy, 2000; Henry et Capitman, 1995).

Dans la plupart des études antérieures, on a observé que les hommes et les femmes utilisent le centre de jour dans des proportions semblables (Chappell, 1983; Dabelko, 2004; Douglass et Visconti, 1998). La présente étude confirme ce résultat, mais démontre aussi qu'ils ne l'utilisent pas de la même façon. C'est lorsqu'ils ont subi un AVC et lorsqu'ils présentent plusieurs incapacités fonctionnelles que les hommes utilisent davantage le centre de jour. Pour les femmes, l'utilisation ne varie pas vraiment en fonction de ces variables. De plus, les hommes ainsi que les femmes plus jeunes semblent utiliser davantage le centre de jour lorsqu'ils ou elles présentent moins de problèmes de santé, alors que c'est l'inverse pour les femmes plus âgées.

Les personnes plus instruites de cet échantillon utilisent moins le centre de jour. Toutefois, des différences statistiquement significatives sont observées seulement lorsqu'on isole ceux et celles qui ont une éducation universitaire. Ce résultat est cohérent avec ceux de deux études antérieures (Chappell, 1983; Dabelko, 2005). Des résultats similaires avaient été observés pour le statut professionnel, où le fait d'avoir œuvré dans des postes au statut social plus élevé (postes professionnels ou de gestion) avait été associé négativement 
au contact avec le centre de jour (Chappell, 1983). Dans la présente étude, le statut professionnel est mesuré par rapport au contact avec le milieu de la santé (la personne âgée ou son conjoint ont travaillé dans le domaine de la santé). Nous cherchions à vérifier si les personnes ayant déjà travaillé dans le domaine de la santé, par une plus grande connaissance des services disponibles, auraient une plus grande probabilité de contact avec le centre de jour. Nos résultats n'ont pas démontré ce type d'association. Il faut noter ici que les personnes âgées qui ont une éducation universitaire, de même que celles avec un statut professionnel associé au domaine de la santé, sont très peu nombreuses dans notre échantillon. Ces résultats mériteraient donc d'être confirmés dans d'autres études.

Dans la présente étude, où les services de centre de jour sont accessibles dans les quartiers multiethniques, les personnes immigrantes les utilisent autant ou même un peu plus que les personnes nées au Canada. Chappell (1983), qui avait obtenu un résultat contraire, avait remis en question l'accessibilité des services dans les secteurs plus multiethniques du Manitoba.

Enfin, de façon cohérente avec les études antérieures qui ont démontré que la présence d'un réseau de soutien pour la personne âgée semblait favoriser l'utilisation du centre de jour (Baumgarten et coll., 2002; Dabelko, 2004; Wallace, 1987), nos résultats démontrent que les personnes âgées qui cohabitent avec une personne de soutien semblent plus nombreuses à utiliser le centre de jour (contact). Il faut noter cependant que dans notre échantillon multiethnique, ceci s'observe uniquement pour les personnes nées au Canada.

\section{Facteurs de besoin}

Les utilisateurs de centres de jour sont légèrement moins autonomes que les nonutilisateurs. Ainsi, les centres de jour remplissent leur mission de répondre aux besoins des personnes en perte d'autonomie. Ces résultats sont cohérents avec les résultats d'études antérieures auprès de la clientèle générale qui avaient observé le plus souvent que les personnes qui présentent plus d'incapacités utilisaient davantage le centre de jour (Baumgarten et coll., 2002; Chappell, 1983; Lynch et coll., 1999). Toutefois, dans notre étude, cette différence est due aux hommes, puisque pour les femmes, la probabilité de 
contact avec le centre de jour ne semble pas influencée par le statut fonctionnel. De plus, notons que nous n'avons observé aucune interaction entre le statut fonctionnel et le statut cognitif de la personne âgée. Cette interaction était suggérée par les résultats divergents des études auprès de la clientèle générale et celles auprès de la clientèle atteinte de démence. Ces dernières observaient plus souvent que les personnes démentes qui présentaient moins d'incapacités fonctionnelles utilisaient davantage le centre de jour (Biegel et coll., 1993; Zarit et coll., 1999b).

La présence d'atteintes cognitives ne semble pas influencer le fait d'utiliser ou non un centre de jour. Environ $30 \%$ des utilisateurs de centres de jour présentent des troubles cognitifs. On observe une proportion similaire de personnes atteintes de troubles cognitifs chez les non-utilisateurs. Dans le passé, la présence de troubles cognitifs a été plus souvent associée positivement avec l'utilisation du centre de jour (Baumgarten et coll., 2002; Dabelko et Balaswamy, 2000; Lynch et coll., 1999; Montgomery et coll., 1988-1989).

Les utilisateurs de centres de jour présentent des scores de dépression légèrement supérieurs à ceux des non-utilisateurs. Toutefois, en raison d'un nombre important de réponses manquantes (questionnaires remplis par des substituts), l'importance de cette variable n'a pu être étudiée dans les analyses de régression. La présence de troubles émotionnels, qui peut inclure des symptômes de dépression, de l'anxiété et des troubles du sommeil, n'a pas permis de distinguer les utilisateurs des non-utilisateurs. En effet, ces divers problèmes émotionnels peuvent influencer l'utilisation du centre de jour de façon différente selon le problème en question. Baumgarten et collaborateurs (2002) ont trouvé que la dépression était associée à une plus grande intensité d'utilisation du centre de jour, alors que l'anxiété était associée à une plus faible intensité d'utilisation.

\section{Caractéristiques des services}

Les utilisateurs de centre de jour sont plus nombreux dans les bassins de desserte des centres de jour dont les coûts sont moins élevés. Ces résultats corroborent ceux de Dabelko (2005), où un coût moyen plus élevé (coût réel pour le client tenant compte des subventions possibles) est observé chez les personnes qui quittent le centre de jour dans les 
quatre premières semaines de fréquentation, suggérant que le coût peut être un frein à l'utilisation.

Enfin, à capacités fonctionnelles égales, les utilisateurs de centre de jour sont plus nombreux chez ceux qui reçoivent une aide plus fréquente du CLSC pour les activités quotidiennes et domestiques. Il est toutefois difficile de dire si c'est l'aide du CLSC qui favorise la participation au centre de jour (les intervenants du CLSC peuvent renforcer l'importance du centre de jour auprès du client), ou s'il s'agit d'une variable individuelle, en l'occurrence la propension à accepter de l'aide formelle, qui agit à la fois pour augmenter la probabilité de participer au centre de jour et augmenter la probabilité d'utiliser l'aide du CLSC.

\section{Profils d'utilisateurs de centre de jour}

Des analyses en regroupement ont permis de classer les utilisateurs de centre de jour qui présentent des caractéristiques similaires en quatre groupes (voir tableau 5 de l'article 2). À notre connaissance, c'est la première fois que de telles analyses sont réalisées.

Le premier groupe rassemble des personnes qui semblent fragiles. Elles sont relativement autonomes et intactes au plan cognitif, mais semblent fragilisées en raison d'un âge avancé, d'un grand nombre de problèmes de santé et de symptômes dépressifs. Le deuxième groupe est composé d'individus que nous pouvons qualifier d'utilisateurs précoces. Ce sont principalement des femmes qui présentent peu de problèmes de santé et qui semblent fréquenter le centre de jour en raison d'un problème qui diffère pour chaque personne, mais avant que les incapacités reliées à cette condition ne deviennent importantes ( $87 \%$ sont dans la moitié la plus autonome de l'échantillon). Le troisième groupe regroupe majoritairement des femmes plus âgées, dont l'autonomie est compromise surtout en raison de troubles cognitifs. Enfin, le dernier groupe est composé surtout d'hommes dont l'autonomie semble compromise surtout en raison d'incapacités locomotrices.

Ces quatre groupes d'utilisateurs suggérent des besoins différents à combler par le centre de jour. Les personnes plus autonomes des deux premiers groupes semblent avoir 
besoin davantage des activités de prévention et de maintien des capacités. Une grande partie des gens du groupe de personnes fragiles présentent des symptômes dépressifs et bénéficieraient aussi d'activités thérapeutiques reliées à la santé mentale. Les personnes moins autonomes des deux derniers groupes semblent avoir davantage besoin d'activités thérapeutiques qui favorisent l'utilisation des capacités résiduelles, l'apprentissage de moyens de compensation et le répit pour les proches. Nous en rediscuterons un peu plus loin, dans nos recommandations pour les centres de jour.

Notons aussi que les femmes se retrouvent à peu près également dans les trois premiers groupes, alors que le quatrième groupe est composé majoritairement d'hommes qui présentent un plus grand nombre d'incapacités locomotrices. Ceci suggère une plus grande variété de raisons de fréquenter un centre de jour pour les femmes que pour les hommes. Ce résultat est cohérent avec le résultat du premier article de cette thèse qui indique que les hommes sont plus nombreux à fréquenter un centre de jour lorsqu'ils ont fait un AVC et lorsqu'ils sont moins autonomes.

\section{À quels types d'activités participent les personnes âgées lors de leur présence en centre de jour?}

Une classification des activités en centre de jour a été établie par un groupe d'experts. Les activités des centres de jour ont été regroupées en huit catégories : exercices physiques; groupe spécifique pour un diagnostic ou un problème fonctionnel commun (ex.: groupe AVC, groupe Parkinson, groupe de dextérité manuelle); activités fonctionnelles; stimulation cognitive; activités musicales ou de réminiscence; sports et jeux physiques; intégration sociale; prévention et promotion de la santé.

Cette étude s'est aussi intéressée à connaître la fréquence à laquelle les personnes âgées participaient aux divers types d'activités et s'il y avait des différences entre les activités auxquelles elles participaient en fonction de leurs caractéristiques individuelles.

Ce volet de l'étude est très novateur. Nous n'avons pas trouvé une classification reconnue des activités offertes en centre de jour. Dans les travaux antérieurs, les activités 
sont décrites et regroupées de façon différente dans chaque étude, en fonction des préférences des auteurs plutôt qu'en raison de fondements théoriques. De plus, à notre connaissance, c'est la première fois que l'on cherche à établir des liens entre les caractéristiques des individus et les activités auxquelles ils participent.

Les résultats démontrent qu'en moyenne, les participants ont bénéficié d'activités appartenant à 5,6 $( \pm 1,7)$ catégories différentes, parmi les huit catégories étudiées. Les exercices physiques et les activités d'intégration sociale sont les activités dont ont bénéficié le plus grand nombre d'individus (plus de $90 \%$ des participants) et ce, de façon régulière, c'est-à-dire à une grande proportion de leur présence en centre de jour. Ces activités correspondent aux besoins d'un grand nombre de personnes âgées qui fréquentent les centres de jour. De plus, ces deux types d'activités sont facilement réalisables en groupe et probablement facilement acceptées par les participants des centres de jour. Les autres catégories d'activités rejoignent de 45 à $77 \%$ des individus lors de 35 à $46 \%$ de leurs présences.

On observe que $26 \%$ des individus ont participé aux activités du centre de jour à l'intérieur de journées ou de groupes spécialement adaptés pour les personnes qui ont des troubles cognitifs. Dans les cinq centres de jour à l'étude, à l'exception des journées à l'intention des personnes atteintes de troubles cognitifs, les groupes spécifiques sont tous des groupes d'exercices physiques pour un diagnostic physique ou un problème fonctionnel commun. Les groupes spécifiques reliés à l'adaptation à des problèmes sensoriels ne semblent pas être offerts. Les activités utiles pour améliorer la santé mentale (comme les activités d'intégration sociale et les activités de réminiscence) semblent disponibles pour tous plutôt qu'offertes à des groupes spécifiques.

Les résultats ont démontré une concordance partielle entre les caractéristiques des individus et les catégories d'activités dont ils ont bénéficié.

En lien avec les résultats du premier article de cette thèse qui indiquaient que les hommes participent davantage au centre de jour lorsqu'ils ont fait un AVC et lorsqu'ils sont moins autonomes, on observe que les hommes ont participé davantage aux journées pour 
personnes atteintes de troubles cognitifs et plus régulièrement aux groupes spécifiques pour un problème fonctionnel commun.

Comme on pouvait s'y attendre, les membres du troisième groupe (incapacités d'origine cognitive) sont proportionnellement plus nombreux que ceux des autres groupes à avoir participé aux journées pour les personnes avec troubles cognitifs. De même, pour l'ensemble des participants, les personnes qui présentent des atteintes cognitives sont proportionnellement plus nombreuses que celles sans atteintes cognitives à avoir participé aux activités du centre de jour lors des journées dédiées à ces personnes (57\% vs $14 \%$ ). Ce qui étonne, c'est que $14 \%$ des personnes identifiées comme sans atteintes cognitives selon les résultats du SPMSQ sont inscrites aux journées ou groupes pour personnes qui présentent des troubles cognitifs. Est-ce parce que de lourdes pertes d'autonomie ont été faussement associées à des pertes cognitives ou parce que le SPMSQ sous-estime le nombre de personnes atteintes de troubles cognitifs?

Les données démontrent que les activités de stimulation cognitive sont offertes autant aux personnes qui présentent des atteintes cognitives qu'à celles qui n'en ont pas. Cela n'est pas nécessairement mauvais. Des activités de stimulation cognitive de divers niveaux (de base, modérée ou avancé) sont possiblement indiquées pour des personnes se situant à divers endroits sur le continuum de fonctionnement cognitif. Malheureusement, les données dont nous disposions pour cette étude ne nous permettaient pas de différencier entre divers types de stimulation cognitive. Toutefois, environ le quart des personnes qui ont des atteintes cognitives n'ont pas participé à des activités de stimulation cognitive, bien que ce type d'activités semble indiqué pour cette clientèle (Knapp et coll., 2006; Spector, Orrell, Davies et Woods, 2005; Spector et coll., 2003). Ce constat mériterait d'être davantage exploré.

On observe aussi que peu de personnes ont bénéficié des activités de réminiscence. Compte tenu des effets bénéfiques de ce type d'activités pour des personnes dépressives (Anderson, 2002; Bohlmeijer, Smit et Cuijpers, 2003; Frazer, Christensen et Griffiths, 2005 ) et pour des personnes qui présentent des troubles cognitifs (Woods, Spector, Jones, 
Orrell et Davies, 2005), on pourrait s'attendre à ce que ce type d'activité soit davantage exploité en centre de jour.

L'intensité d'exposition aux diverses activités proposées par les centres de jour mériterait aussi davantage d'attention. Sauf pour les exercices physiques et les activités d'intégration sociale, les sujets de cette étude participent aux autres catégories d'activités en moyenne à moins de $50 \%$ de leurs présences en centre de jour. Considérant que, pour plus de $50 \%$ des participants, les présences réelles au centre de jour sont de moins d'un jour par semaine, elles participent à ces diverses catégories d'activités environ toutes les deux semaines. On peut donc se demander si l'intensité d'exposition aux diverses activités proposées est suffisante pour engendrer les effets bénéfiques escomptés de chacune d'elles. Toutefois, ces données moyennes sont insuffisantes pour porter un jugement. Il y aurait surtout lieu de s'assurer d'une intensité d'exposition suffisante pour les profils de participants priorisés pour chacun des types d'activités.

\section{L'utilisation du centre de jour est-elle régulière?}

En moyenne, les utilisateurs de centre de jour de notre échantillon étaient inscrits au centre de jour à raison de 1,56 jour par semaine et y ont effectivement été présents 1,00 fois par semaine. Toutefois, la médiane n'est que de 0,73 fois par semaine. Ceci est similaire au résultat d'une autre étude québécoise, celle de Baumgarten et collaborateurs (2002) où la participation médiane est de 0,7 présence par semaine.

Lorsqu'on exclut les journées où la personne était dans l'impossibilité de fréquenter le centre de jour parce que le centre était fermé ou parce que la personne était hospitalisée ou placée en hébergement temporaire, une majorité de participants ont une participation qu'on pourrait qualifier de régulière, alors qu'ils sont présents à près des trois quarts des moments prévus. On observe par contre que $25 \%$ des participants sont présents à moins de $50 \%$ des jours prévus.

En l'absence de données probantes sur l'intensité optimale d'exposition aux activités et de données comparatives sur la régularité de participation, il est difficile de 
porter un jugement sur ces résultats. Zarit et collaborateurs (1998) suggéraient, d'après une consultation auprès de responsables de centres de jour, qu'une participation à raison de deux fois par semaine serait nécessaire pour induire des effets bénéfiques, en particulier pour la clientèle atteinte de démence. Plusieurs études expérimentales ont donc inclus uniquement des personnes inscrites à raison de deux fois par semaine dans l'évaluation des effets de la participation au centre de jour (Gaugler et coll., 2003; Schacke et Zank, 2006; Zank et Schacke, 2002; Zarit et coll., 1998). Toutefois, dans leur étude expérimentale, Beaumgarten et collaborateurs (2002) ont démontré une réduction du fardeau des personnes de soutien des participants qui avaient une participation minimale réelle d'une fois par semaine, alors qu'il avait augmenté pour ceux ayant une participation moindre. À la lumière de ces faibles indications, on peut s'inquiéter du fait que pour plus de $50 \%$ de l'échantillon, la participation réelle n'atteint pas une fois par semaine.

Il faut aussi rappeler que nos résultats concernent un groupe composé de $78 \%$ d'utilisateurs qui fréquentent le centre de jour depuis au moins trois mois au début de la période d'observation. Un résultat différent aurait pu être obtenu avec un échantillon constitué de nouveaux utilisateurs de centre de jour. Plusieurs études démontrent que les taux d'abandon sont élevés dans les premiers trois à six mois après l'inscription (Cox, 1997; Dabelko, 2004; Neufeld et Strang, 1992; Schacke et Zank 2006; Wells et coll., 1990; Zarit et coll., 1999b) et ces abandons peuvent être précédés d'une période d'absences au cours de laquelle l'inscription est maintenue.

\section{Parmi les utilisateurs de centres de jour, quels sont ceux qui le font avec le plus de régularité?}

Les résultats de la présente étude apportent une contribution originale à l'état des connaissances sur l'utilisation du centre de jour en identifiant des déterminants de la régularité de l'utilisation du centre de jour, une forme d'évaluation de l'intensité d'utilisation qui, à notre connaissance, n'avait pas encore été étudiée à ce jour. En effet, à ce jour, la plupart des études de l'intensité d'utilisation du centre de jour ne tenaient pas compte du fait que le volume d'utilisation possible n'est pas le même pour tous. Dans le 
contexte américain, où le nombre de places disponibles en centre de jour dépasse la demande, il n'était sans doute pas apparu important de tenir compte de l'intensité en termes de pourcentage de la participation prévue. Dans le contexte québécois où la fréquence d'inscription est limitée par la capacité des divers centres de jour, cela prend un sens particulier puisqu'il s'agit de la partie du volume d'utilisation sur laquelle la personne âgée peut exercer un contrôle.

\section{Facteurs prédisposants}

L'âge des participants n'a pas été associé à la régularité de la participation dans la présente étude, bien qu'il soit associé négativement à divers autres phénomènes d'utilisation dans les écrits sur les centres de jour (Dabelko, 2004; Dabelko et Balaswamy, 2000; Henry et Capitman, 1995).

Il semble que lorsque les participants ou leur conjoint ont travaillé dans le domaine de la santé, leur utilisation du centre de jour est moins régulière. Il est possible que ce résultat soit lié au fait que, dans cet échantillon, les travailleurs du domaine de la santé et leur conjoint sont plus susceptibles d'avoir une éducation universitaire $\left(\mathrm{RC}: 16,5, \mathrm{IC}_{0,95}\right.$ : 3,1 à 89,2 ). Les personnes qui ont fréquenté l'université semblent utiliser le centre de jour avec une moins grande régularité, mais ce résultat n'atteint pas le seuil de signification statistique de $\mathrm{p}<0,05$. Toutefois, puisque les personnes âgées qui ont une éducation universitaire, de même que celles avec un statut professionnel associé au domaine de la santé, sont très peu nombreuses dans notre échantillon, il est possible que ce résultat soit en partie attribuable au niveau d'éducation, même si le niveau d'éducation pris isolément n'a pas démontré une association statistiquement significative avec la régularité d'utilisation.

\section{Facteurs de besoin}

En ce qui concerne les facteurs de besoin des personnes âgées, le statut fonctionnel de la personne âgée ne semble pas influencer la régularité d'utilisation du centre de jour.

De façon cohérente avec les études démontrant une plus grande intensité d'utilisation par les personnes atteintes de troubles cognitifs (Baumgarten et coll., 2002; 
Lynch et coll., 1999; Montgomery et coll., 1988-1989), la présente étude a démontré une plus grande régularité de participation par ces personnes, mais uniquement lorsque le fardeau de la personne de soutien est moins élevé.

Lorsque l'on s'intéresse aux facteurs de besoin de la personne de soutien, les variables les plus étudiées concernent le bien-être psychologique ou le sentiment de fardeau de cette dernière. Les études antérieures à ce sujet indiquaient plus souvent que, lorsque la situation d'aide était trop difficile, l'utilisation du centre de jour était plus faible. Ces facteurs ont été mis en lumière dans les études auprès d'une clientèle atteinte de démence et ont généralement été associés à une utilisation brève du centre de jour, probablement en raison d'un recours plus rapide à l'hébergement en institution (Mävall et Thorslund, 2006; Zarit et coll., 1999b). Par contre, auprès d'une clientèle générale, Baumgarten et collaborateurs (2002) avait observé une utilisation plus intense du centre de jour lorsque le fardeau de la personne de soutien était plus élevé. La présente étude semble confirmer ces résultats antérieurs. Nos données démontrent en effet que les individus qui présentent des atteintes cognitives sont plus susceptibles d'utiliser le centre de jour avec une plus grande régularité lorsque le fardeau de leur personne de soutien est plus faible et, à l'inverse, ceux sans atteintes cognitives sont susceptibles d'être des utilisateurs plus réguliers si le fardeau de leur personne de soutien est élevé.

\section{Caractéristiques des services}

Les caractéristiques organisationnelles des services avaient été peu étudiées à ce jour. Nos résultats viennent corroborer certains constats déjà observés concernant le type d'activités offertes. Ils apportent un nouvel éclairage sur l'influence de la durée des activités et de la présence d'aide à domicile sur l'utilisation du centre de jour.

Dans la présente étude, les personnes qui ont une moins grande proportion d'activités de prévention et de promotion de la santé lors de leurs présences en centre de jour semblent être des utilisateurs plus réguliers du centre de jour. Ces résultats concordent avec ceux d'Henry et Capitman (1995), qui avaient trouvé que les participants à des programmes qui mettent l'accent sur le maintien de la santé utilisent moins intensément le 
centre de jour et sont plus susceptibles de cesser la participation que ceux qui participent à des programmes qui mettent l'accent sur le répit ou sur les activités de stimulation et de croissance. Notons que les définitions des activités axées sur la santé diffèrent dans ces deux études. L'étude d'Henry et Capitman incluait des activités individuelles de suivi de l'état de santé alors que notre étude porte uniquement sur les activités de groupe.

Nos résultats suggèrent qu'une durée minimale serait nécessaire pour compenser l'effort fait pour se préparer à aller au centre de jour puisque les individus qui sont inscrits pour la journée entière plutôt que la demi-journée se sont avérés des utilisateurs plus réguliers du centre de jour. L'influence de cette variable ne semble pas avoir été étudiée auparavant.

Dans les analyses bivariées, on a observé que les participants qui reçoivent de l'aide du CLSC pour les activités de vie quotidienne ou de vie domestique au moins une fois par semaine, de même que ceux qui reçoivent ce type d'aide les jours où ils sont inscrits au centre de jour sont des utilisateurs plus réguliers du centre de jour. En présence des autres variables dans les analyses de régression, la présence d'aide lors des journées de présences au centre de jour demeure significative. Bien que ce constat nécessite d'être confirmé par d'autres études, il est possible que sans cette aide, certains seraient incapables de se préparer pour se rendre au centre de jour. L'aide à domicile pour préparer la personne âgée pour le centre de jour est recommandée par Dabelko (2005), mais son influence sur l'utilisation du centre de jour n'avait pas encore été étudiée à ce jour.

\section{Interaction entre facteurs de besoin et caractéristiques des services}

Le cadre conceptuel retenu pour cette étude, suggère que les caractéristiques des services pourraient interagir avec les facteurs de besoin pour modifier les taux d'utilisation du centre de jour et que, par exemple, un certain type d'activités pourrait favoriser une participation plus grande au centre de jour pour des sous-groupes de clients auxquels ces activités sont mieux adaptées. Les résultats n'ont pas démontré d'interactions statistiquement significatives entre les activités et les facteurs de besoin. Certaines tendances ont toutefois été observées. Elles concernent premièrement la participation aux 
groupes pour un problème fonctionnel commun ainsi qu'aux groupes pour les personnes qui présentent des troubles cognitifs, par les hommes et les femmes et, en second lieu, la participation aux groupes pour les personnes qui présentent des troubles cognitifs par les personnes qui présentent ou non de telles atteintes. Il est probable que la taille de l'échantillon n'ait pas été suffisante pour confirmer des interactions qui pouvaient être présentes avec le degré de puissance statistique souhaité. Ces observations mériteraient donc d'être étudiées dans des études futures auprès d'un plus grand nombre d'utilisateurs de centre de jour.

Dans l'étude des déterminants du contact avec le centre de jour, nous avons noté que les hommes qui ont subi un AVC et présentent plusieurs incapacités fonctionnelles sont plus nombreux à utiliser le centre de jour. Une des hypothèses avancées pour expliquer cette observation était que les hommes voyaient peut-être davantage le centre de jour comme un endroit pour faire des exercices afin de maintenir ou d'améliorer leurs capacités alors que les femmes pouvaient l'utiliser davantage pour briser l'isolement. Le fait que les hommes semblent participer plus régulièrement au centre de jour lorsqu'ils sont inscrits à des groupes pour un problème fonctionnel commun (ex. : groupe AVC, groupe Parkinson, etc.) apporte un argument de plus à cette hypothèse.

L'observation à l'effet que les personnes qui ont des atteintes cognitives semblent participer plus régulièrement au centre de jour lorsqu'elles sont inscrites à des groupes qui leurs sont dédiés, alors que celles sans atteintes cognitives inscrites à de tels groupes semblent participer moins régulièrement, semble d'une logique évidente. Il y a lieu de se questionner sur les raisons qui font que des personnes sans atteintes cognitives apparentes au SPMSQ aient été inscrites à de tels groupes. Nous y reviendrons dans nos recommandations pour les centres de jour. 


\section{Forces et limites de ce projet de doctorat}

\section{Forces}

Bien que largement répandus comme services de soutien à domicile, les centres de jour ont été peu étudiés au Canada. Cette étude remédie en partie à cette lacune en décrivant les caractéristiques des utilisateurs de centres de jour, les services qu'ils reçoivent lors de leurs présences en centre de jour, ainsi que les déterminants de la régularité de la participation, et ce, dans un contexte québécois.

Par une analyse exhaustive des différentes possibilités d'interaction suggérées par le modèle conceptuel, cette recherche sur les déterminants de l'utilisation du centre de jour a pu, avec un échantillon de taille moyenne, faire ressortir des différences importantes dans le contact avec le centre de jour entre les hommes et les femmes, qui n'étaient pas ressorties dans les études précédentes. Elle a aussi pu faire ressortir des différences dans la régularité de participation des personnes qui ont des atteintes cognitives et celles qui n'en ont pas, en fonction du fardeau de la personne de soutien. Diverses observations notées par d'autres chercheurs menaient dans cette direction, mais à notre connaissance, cette interaction n'avait jamais été testée de façon formelle.

En incluant dans les variables à l'étude, plusieurs caractéristiques des services qui n'avaient pas été beaucoup étudiées à ce jour, cette étude a mis en lumière l'influence de certains facteurs qui représentent des choix organisationnels faits par les centres de jour, choix qui peuvent être modifiés pour mieux rejoindre la clientèle susceptible de bénéficier du centre de jour et mieux cibler les interventions pour améliorer les taux de participation.

De plus, parmi les multiples façons d'étudier l'intensité d'utilisation du centre de jour, cette étude a retenu la régularité de participation, définie d'une façon qui excluait une partie importante des absences indépendantes de la volonté de l'individu, par exemple, lorsque le centre de jour était fermé ou alors que la personne était hospitalisée ou placée en hébergement temporaire. Ainsi, cette régularité de participation représente, au moins en partie, la conformité à la recommandation de participation. 
Enfin, cette étude comportait un volet exploratoire, dans lequel nous avons cherché à identifier les liens entre les activités qui sont offertes par les centres de jour et les caractéristiques des utilisateurs. Ce volet tente de remédier à une lacune importante dans les études actuelles, lacune identifiée entre autres par Bea Van Beveren (1998) et par Dabelko et Zimmerman (2008), ainsi que par notre recension des écrits. En particulier, les études canadiennes recensées portaient essentiellement sur la structure des centres de jour (Gutman et al., 1993a, 1993b; Ross-Kerr, Warren et Godkin, 1995; Strang, Greschuk, McIlveen, Gadacz et Neufeld, 1992; Tourigny et coll., 1993) ou sur leurs résultats (Baumgarten et coll., 2002; Strain, Chappell et Blandford, 1987), avec très peu d'attention apportée au processus d'intervention. Selon Bea van Beveren (1998), une bonne connaissance descriptive des centres de jour est essentielle au design de bonnes études évaluatives.

Comme peu de chercheurs s'étaient intéressés auparavant à décrire les activités offertes en centre de jour, nous n'avons pas trouvé une classification reconnue des activités offertes. Nous avons fait appel à un groupe d'experts pour créer la classification utilisée dans la présente étude. Malgré certaines différences, cette classification rejoint globalement celle proposée dans le manuel d'activités de Norman et Horton (1996), ce qui lui apporte une certaine validité apparente. Ainsi, cette étude présente une méthode originale et structurée qui pourrait servir de référence pour poursuivre l'étude du processus d'intervention en centres de jour.

\section{Limites}

Comme toute recherche, cette étude comporte certaines limites. La première et la plus importante vient sans doute du recours à une analyse secondaire de données recueillies dans le cadre d'une étude plus large sur les services intégrés aux personnes âgées. À l'époque où cette étude a été menée, on constatait un manque d'homogénéité dans les données consignées par les centres de jour concernant l'état de santé des usagers. Ainsi, l'analyse secondaire de données a permis d'obtenir des données valides tant sur les 
caractéristiques des individus que sur leurs présences en centre de jour. Toutefois, cette stratégie impose certaines limites.

Premièrement, la disponibilité des données a influencé le type d'analyse possible et le choix d'un cadre conceptuel. Par exemple, cette étude de l'utilisation a permis de définir les caractéristiques des utilisateurs et des non-utilisateurs de centre de jour, mais ne pouvait pas analyser le processus de prise de décision du professionnel qui dirige une personne vers le centre de jour et de la personne âgée ou de son aidant qui accepte ou non cette recommandation..L'étude de cet aspect important de l'utilisation. aurait nécessité une collecte de données prospective pour comprendre ce qui se passe au moment de la décision.

Parmi les divers cadres conceptuels recensés pour étudier les déterminants de l'utilisation, le modèle d'utilisation d'Andersen demeure le modèle le plus utilisé et, dans sa version révisée (Andersen, 1995), un modèle assez complet. En plus des caractéristiques individuelles (facteurs prédisposants, facteurs facilitants et facteurs de besoin) décrites dans le modèle initial, ce modèle révisé fait mention de l'influence de l'environnement et de la rétroaction obtenue par l'utilisation des services, incluant la satisfaction envers les services, sur l'utilisation future, reconnaissant ainsi, à l'instar de Donabedian (1973), que l'utilisation est la résultante de la rencontre entre un individu et un professionnel de la santé (Model of the Medical Care Process). Toutefois, les données sur la satisfaction envers les services n'étaient pas disponibles et les composantes de l'environnement décrites par Andersen et ses collègues relèvent davantage de l'organisation du système de santé à un niveau global (présence d'assurance-maladie, disponibilité de services dans la communauté, etc.) que des caractéristiques organisationnelles propres à chaque fournisseur de services (ex. : taille, structure du personnel, accueil, etc.), caractéristiques qui influenceront vraisemblablement l'expérience de l'individu qui cherche à accéder à un service particulier ou qui doit décider de poursuivre ou non l'utilisation d'un service donné. Ainsi, nous avons choisi de compléter le modèle d'Anderson par des facteurs reliés aux caractéristiques des services, s'inspirant des travaux de Yeats et collaborateurs (1992), de Kosloski et collaborateurs (2001) et de Montoro-Rodriguez et collaborateurs (2003). Ces caractéristiques tels le coût des services, le type d'activités offertes, la structure du personnel peuvent avoir une 
influence sur la satisfaction envers les services et le désir d'y retourner. Nous avons aussi inclus dans ces caractéristiques des services, certaines caractéristiques des CLSC par lesquels se fait le tri et les recommandations d'utilisation du centre de jour. Idéalement, nous aurions inclus aussi des caractéristiques des intervenants des CLSC, comme la connaissance que ces intervenants ont des critères d'admission et des places disponibles dans les centres de jour situés sur leur territoire, etc. Cependant, certaines variables potentiellement importantes tant pour l'étude des caractéristiques individuelles que pour l'étude des caractéristiques des services n'étaient pas disponibles, incluant la raison de la référence au centre de jour, les attitudes préalables des personnes âgées envers ce type de services ou envers les activités de groupe en général, le nombre et le type de services individuels reçus, les données sur la satisfaction envers les services reçus, ainsi que les données sur les caractéristiques des intervenants des CLSC. Il a aussi été impossible de distinguer parmi les non-utilisateurs, les personnes qui ont déjà été dirigées au centre de jour et qui ont choisi de ne pas y participer, de celles qui n'ont jamais été dirigées vers ce type de service.

Deuxièmement, l'échantillon n'est pas pleinement représentatif de l'ensemble de la population qui fréquente les centres de jour québécois, pour au moins trois raisons.

Tout d'abord, ce n'est pas un échantillon aléatoire des personnes fréquentant les cinq centres étudiés. Toutefois, selon les responsables de ces cinq centres, les caractéristiques des participants à cette étude seraient assez semblables à celles de la clientèle de leur centre de jour, à l'exception de la proportion de personnes qui ont des atteintes cognitives, qui semble légèrement plus élevée dans la clientèle de leur centre de jour (entre 30 et $40 \%)$ que dans les participants à cette étude $(27 \%, 28 \%$ et $33 \%$ pour les objectifs 1,2 et 3 respectivement).

Ensuite, cet échantillon n'inclut que des personnes de 65 ans et plus. Ces dernières ont été recrutées majoritairement parmi les clients des services de soutien à domicile des CLSC. Bien que certains centres de jour montréalais acceptent d'autres types de clients, ceux de plus de 65 ans forment plus de $90 \%$ de leur clientèle et plus de $85 \%$ y sont dirigés 
par leur CLSC (Regroupement des centres d'hébergement et de soins de longue durée de la région de Montréal, 2005).

Enfin, cet échantillon ne porte que sur des personnes fréquentant les centres de jour d'une portion bien délimitée de l'Île de Montréal. La population des deux territoires de CLSC sur lequel le projet s'est déroulé est plus multiethnique que celle de l'ensemble de l'île de Montréal (44 à $48 \%$ d'immigrants contre $28 \%$ pour Montréal) (Agence de la santé et des services sociaux de Montréal, 2004a; 2004b). Il s'agit donc d'un échantillon d'une grande métropole à composition multiethnique comme on en observe de plus en plus dans les différentes métropoles des pays occidentaux. Enfin, malgré cette limite, les résultats sont possiblement au moins en partie représentatifs des activités des centres de jour de la région de Montréal, les responsables des centres de jour de cette région ayant entrepris une réflexion commune sur les orientations des centres de jour (Regroupement des centres d'hébergement et de soins de longue durée de la région de Montréal, 2001; 2005). On peut cependant penser que des résultats différents auraient pu être obtenus dans les centres de jour des régions rurales de la province puisqu'en plus d'offrir leurs services à une population aux caractéristiques différentes, ceux-ci doivent souvent adapter leur programmation en fonction de possibilités de transport sur un vaste territoire. On notera toutefois l'importance d'offrir des services adéquats aux personnes âgées de la région métropolitaine, puisque $46 \%$ des personnes âgées du Québec vivent dans la région métropolitaine de Montréal (Institut de la statistique du Québec, 2005-2006; 2007).

Troisièmement, si le nombre de sujets est élevé pour le premier objectif de l'étude qui cherche à distinguer les utilisateurs des non-utilisateurs de centre de jour, le nombre d'utilisateurs de centre de jour est relativement petit. Cela a pu limiter la capacité à identifier certains déterminants de la régularité de participation avec le niveau de puissance statistique souhaité.

Quatrièmement, cette étude ne portait que sur cinq centres de jour. Ces cinq centres ne présentent pas un portrait complet des caractéristiques organisationnelles retrouvées en centre de jour au Québec, même s'ils sont assez représentatifs de ce qui se fait en milieu urbain à Montréal. De plus, cela a réduit la possibilité d'étudier l'influence d'une variété de 
caractéristiques organisationnelles des centres de jour et des interactions entre ces dernières et les caractéristiques sociales ou de santé. En effet, comme chaque centre présente un ensemble de caractéristiques organisationnelles qui lui est propre, des analyses multiniveaux auraient été nécessaires pour isoler l'effet de caractéristiques organisationnelles précises. De plus, les sujets qui proviennent d'un même centre de jour ne sont pas des unités d'analyse complètement indépendantes. Ils risquent de présenter des caractéristiques semblables. Cela viole l'un des postulats des procédures telles que la régression logistique et la régression multiple utilisées dans cette étude, celui de l'indépendance des observations. Idéalement, il aurait fallu utiliser des procédures statistiques qui tiennent compte de cette auto-corrélation, telles que les modèles multi-niveaux ou encore les GEE (Generalized Estimating Equations). Les effectifs et le nombre de centres de jour à l'étude étaient insuffisants pour procéder à ce type d'analyse.

En plus des limites inhérentes à l'analyse secondaire de données, les données manquantes ont aussi imposé certaines limites à cette étude. Par exemple, pour l'analyse des déterminants du contact avec le centre de jour, les caractéristiques des personnes de soutien n'ont pas été considérées étant donné le nombre de questionnaires aux personnes de soutien qui étaient manquants ou incomplets.

Enfin, une limite importante pour tester l'influence du type d'activités sur la régularité de participation est le manque de classification standardisée des activités. Lorsque nous avons voulu créer cette classification, nous avons aussi rencontré des imprécisions dans les statistiques recueillies par les centres de jour. Par exemple, certaines données sont enregistrées sous le nom d'un groupe qui pouvait contenir plusieurs types d'activités (par exemple, groupe répit) et nous avons fait des inférences sur les présences à chaque catégories d'activités, à partir du pourcentage approximatif de ces activités à l'intérieur du groupe, selon le responsable du centre de jour. De plus, certains centres de jour n'enregistraient pas les activités individuelles, alors nous n'avons pas pu inclure ces activités dans les analyses. 
Les résultats de cette étude, avec leurs forces et leurs limites, permettent d'émettre diverses recommandations à l'intention des centres de jour et offrent plusieurs suggestions pour des recherches futures.

\section{Recommandations pour les centres de jour}

L'objectif général de ce projet de doctorat était d'améliorer la compréhension des facteurs qui influencent l'utilisation des centres de jour dans le but ultime de mieux cibler les interventions pour les personnes les plus susceptibles d'en bénéficier ou encore de revoir l'offre de services de façon à mieux l'adapter aux besoins de la clientèle cible. À la lumière des résultats de cette étude et de certaines données dégagées de la recension des écrits, il est possible d'émettre des recommandations qui seront utiles tant pour les gestionnaires et les intervenants des centres de jour que pour les personnes qui dirigent les clients vers ce service. Ces recommandations sont présentées au tableau $\mathrm{X}$ et expliquées cidessous. Elles portent sur les thèmes suivants :

1) Mieux rejoindre la clientèle le plus susceptible de bénéficier du centre de jour

2) Offrir une programmation d'activités de groupe mieux arrimée aux besoins individuels des utilisateurs

3) Viser une intensité d'exposition optimale

4) Prévoir une utilisation efficiente des ressources

\section{1) Mieux rejoindre la clientèle le plus susceptible de bénéficier du centre de jour}

Les gestionnaires de centres de jour peuvent se réjouir du fait que ces centres remplissent leur mission d'offrir des services aux personnes en perte d'autonomie, tout en intensifiant leurs efforts pour encore mieux cibler la clientèle le plus susceptible de bénéficier de leurs services.

Il sera pertinent de vérifier si les besoins d'une personne très âgée peuvent être réellement comblés par le centre de jour ou s'ils seraient mieux satisfaits par d'autres services. Ceci doit continuer d'être évalué au cas par cas, selon l'état de santé de la personne âgée, sa capacité à se déplacer et sa motivation à fréquenter un centre de jour, tout 
en réfléchissant aux possibilités de faciliter l'accès au centre de jour pour les personnes très âgées. Par exemple, des adaptations aux modes de transport et à la séquence des périodes d'activités et de repos pourraient être étudiées.

Il serait pertinent d'approfondir les raisons qui font que les diplômés universitaires utilisent moins le centre de jour. Est-ce réellement parce que leurs besoins sont moins grands ou est-ce parce que la programmation d'activités en centre de jour n'est pas adaptée aux besoins de ce type de clients? S'il s'avère que c'est la seconde option qui décrit le mieux la plus faible participation observée, il y aurait lieu de se questionner sur les façons d'adapter les activités des centres de jour aux besoins et aux intérêts des gens plus instruits.

Les hommes et les femmes n'utilisent pas le centre de jour de la même façon et ont probablement des attentes différentes envers ce type de service. Ainsi, en prenant connaissance des résultats de cette étude, les intervenants qui dirigent les personnes âgées vers les centres de jour pourront réaliser l'importance de discuter de cette possibilité avec les hommes qui ont fait un AVC et qui présentent des incapacités importantes puisque, parmi le clientèle masculine, c'est le groupe qui semble répondre le plus positivement à l'offre de fréquentation du centre de jour. Ils pourront aussi se questionner sur les façons de mieux rejoindre les autres hommes, en particulier ceux atteints de troubles cognitifs pour lesquels les personnes de soutien présentent un fort besoin de répit.

En effet, les intervenants, tant à l'intérieur des centres de jour que ceux qui y dirigent des clients, voudront vérifier s'ils prennent les bons moyens pour rejoindre les personnes atteintes de troubles cognitifs et les personnes dont l'aidant est épuisé. Répondre au besoin de répit de ceux qui prennent soin d'une personne atteinte de troubles cognitifs présente un défi et un effort additionnel peut être nécessaire pour bien les aider. Parmi les suggestions à cette fin, notons l'aide pour préparer la personne âgée avant sa journée au centre de jour, ainsi qu'une fréquence d'inscription et des durées suffisamment importantes pour compenser les efforts de préparation et de stimulation nécessaires pour amener la personne âgée à participer au centre de jour. Des activités de soutien et de résolution de 


\section{Tableau X : Recommandations pour les centres de jour}

\section{RECOMMANDATIONS}

\section{Mieux rejoindre la clientèle le plus susceptible de bénéficier du centre de jour}

- Orienter les personnes très âgées (95 ans et plus) avec grande fatigabilité vers d'autres services.

- Documenter les raisons qui font que les diplômés universitaires utilisent moins le centre de jour.

- Les hommes qui ont fait un AVC et qui présentent des incapacités importantes répondent généralement bien au programme de centre de jour et pourraient y être dirigés plus systématiquement.

- Pour rejoindre les personnes atteintes de troubles cognitifs dont l'aidant est épuisé, offrir du soutien pour la préparation de la personne âgée à sa venue au centre de jour.

- Offrir des activités attrayantes et non menaçantes pour les personnes qui présentent des atteintes cognitives.

- Offrir des périodes de répit suffisamment longues pour compenser les efforts de préparation.

- Limiter le coût des services (transport et repas) qui peut créer un frein à l'utilisation.

Offrir une programmation d'activités de groupe mieux arrimée aux besoins individuels des utilisateurs

- Mieux définir les programmes d'activités en centre de jour. En fonction des profils de participants identifiés dans la présente recherche, quatre sous-programmes sont proposés comme point de départ à cette réflexion :

- programme préventif pour personnes fragiles ou à risque, en raison de problèmes de santé physique;

- programme préventif et thérapeutique pour les personnes fragiles ou à risque, en lien avec la santé mentale;

- programme de stimulation et de répit pour les personnes qui ont des incapacités principalement d'origine cognitive;

- programme thérapeutique et de répit pour les personnes qui ont des incapacités principalement d'origine motrice.

- Lors des programmes de nature préventive, favoriser l'adoption d'activités dans lesquelles les participants sont actifs.

- Offrir des activités comportant différents niveaux de difficulté pour s'adapter au niveau fonctionnel des différents groupes.

- Offrir les activités de réminiscence plus régulièrement et de manière plus ciblées envers les clientèles le plus à même d'en bénéficier, par exemple, les personnes qui présentent des atteintes cognitives ou des symptômes dépressifs.

- Éviter d'intégrer dans des groupes pour les personnes qui présentent des atteintes cognitives, des personnes cognitivement intactes qui présentent d'autres besoins.

\section{Viser une intensité d'exposition minimale}

- Privilégier la participation en journées entières plutôt qu'en demi-journées.

- Pour le répit: privilégier l'inscription à raison de deux fois par semaine ou plus.

- S'assurer que les diverses catégories d'activités du centre de jour soient offertes avec des fréquences suffisantes pour engendrer les effets bénéfiques escomptés de ces activités.

\section{Prévoir une utilisation efficiente des ressources}

- Documenter le taux d'absentéisme, pour planifier un plus grand nombre d'inscriptions par jour en fonction de ce taux. 
problèmes pour les aidants pourraient aussi être utiles pour favoriser la participation de cette clientèle au centre de jour (Gitlin et coll., 2006).

De plus, des activités attrayantes et non menaçantes pour les personnes qui présentent des atteintes cognitives peuvent faciliter leur participation au centre de jour et diminuer l'effort des aidants pour stimuler cette participation. Dans notre échantillon, les personnes qui ont des atteintes cognitives tendent à participer plus régulièrement aux activités des centres de jour lorsqu'elles sont inscrites à des groupes qui leur sont destinés. Si cette observation était confirmée par d'autres données, il serait pertinent d'encourager ce type de groupe à l'intérieur des centres de jour. De plus, pour les hommes en particulier, on pourrait se demander si les activités actuelles sont trop associées aux loisirs féminins et s'il y aurait des types d'activités plus attrayantes pour ces derniers?

Les intervenants des centres de jour devraient aussi miser sur un bon arrimage avec le CLSC, puisque l'aide du CLSC peut favoriser la participation au centre de jour.

Les résultats de la présente étude suggèrent aussi de porter attention au coût des services qui, s'il est trop élevé, crée une contrainte qui peut réduire l'accès au centre de jour.

\section{2) Offrir une programmation d'activités mieux arrimée aux besoins des utilisateurs}

Bien qu'ils méritent d'être confirmés par des recherches plus poussées, les éléments mis en lumière dans cette étude soulèvent des questions sur l'adéquation entre la programmation d'activités, les besoins des individus et les objectifs visés. Nous aborderons d'abord les questions reliées au cadre de référence pour la programmation d'activités, puis celles reliées aux activités elles-mêmes.

\section{Le cadre de référence pour la programmation d'activités}

Le Cadre de référence pour les centres de jour de l'ACAQ (1991) suggéraient une programmation d'activités répartie en six sous-programmes portant sur les aspects suivants : incapacités fonctionnelles d'origine motrice, incapacités fonctionnelles d'origine 
sensorielle, incapacités fonctionnelles d'origine cognitive, facteurs socioenvironnementaux, santé physique, et santé mentale. La présente étude dénote peu de concordance entre les activités dont ont bénéficié les participants en centre de jour et les recommandations d'experts établies à partir des ces six sous-programmes. Ceci soulève la question à savoir si ces sous-programmes représentent bien la réalité des centres de jour et les besoins des participants. D'autres observations nous amènent aussi vers ce questionnement. Par exemple, dans les recommandations de notre groupe d'experts, les activités de deux sous-programmes (incapacités fonctionnelles d'origine motrice et santé physique) semblaient relativement similaires. Ensuite, les données empiriques ne démontrent aucune activité particulière pour les personnes qui présentent des difficultés sensorielles. Il y a donc lieu de remettre en question l'existence réelle de ce sousprogramme dans les centres de jour. Enfin, les divers sous-programmes de ce cadre de référence distinguent les individus davantage en fonction de l'origine de leurs difficultés que selon leur niveau fonctionnel. Cependant, lorsqu'on planifie des activités de groupe, il semble important de tenir compte du niveau fonctionnel pour prévoir des activités qui présentent le niveau de stimulation approprié aux capacités des individus qui participent au groupe.

Par ailleurs, les quatre groupes de participants identifiés par nos analyses en regroupement semblent suggérer des besoins différents à combler par le centre de jour. Les personnes fragiles et les utilisateurs précoces qui présentent moins d'incapacités que la moyenne des participants, semblent avoir besoin davantage des activités de prévention comme la surveillance de l'état de santé et, lorsqu'elle survient, l'identification rapide de la détérioration de la santé, pour permettre une intervention précoce par une référence à une ressource appropriée. Elles profiteront aussi d'activités de groupe pour prévenir les conséquences négatives de l'isolement qui peut survenir en raison de la fragilité. Une attention particulière devrait être apportée aux personnes qui présentent des symptômes dépressifs et qui profiteraient certainement d'activités démontrées efficaces pour réduire ces symptômes. Les personnes moins autonomes des deux derniers groupes semblent déjà présenter des incapacités fonctionnelles importantes, dont l'origine est surtout attribuable à des atteintes cognitives pour les personnes du troisième groupe et à des atteintes motrices 
pour celles du quatrième groupe. Ces personnes semblent avoir davantage besoin d'activités thérapeutiques qui favorisent l'utilisation des capacités résiduelles ainsi que l'apprentissage de moyens de compensation pour les pertes de capacités. Elles profiteront certainement aussi des activités qui visent le maintien ou l'amélioration de la qualité de vie, de l'estime de soi et des liens sociaux et préviennent les conséquences négatives de l'isolement qui peut survenir en raison des incapacités. De plus, le répit aux personnes de soutien semble indiqué pour une majorité de ces personnes. Les personnes dont l'autonomie est compromise surtout en raison de troubles cognitifs seront davantage valorisées dans des activités qui sont adaptées à leurs pertes cognitives et leur garantiront des chances de succès, tout en présentant certains défis au plan physique. Celles dont l'autonomie semble compromise surtout en raison d'incapacités locomotrices se sentiront sans doute plus valorisées dans des activités comportant un plus haut niveau de défi au plan cognitif.

À la réunion du Groupe d'intérêt des responsables de centres de jour de Montréal le 30 mai 2008, l'idée de revoir le cadre de référence pour la programmation d'activités en fonction des quatre groupes de participants identifiés par la présente recherche est apparue intéressante pour les personnes présentes. Elle mériterait d'être étudiée plus à fond.

Nous suggérons quatre sous-programmes qui pourraient servir de point de départ à cette réflexion :

- programme préventif pour personnes fragiles ou à risque, en raison de problèmes de santé physique;

- programme préventif et thérapeutique pour les personnes fragiles ou à risque, en lien avec la santé mentale;

- programme de stimulation et de répit pour les personnes qui ont des incapacités principalement d'origine cognitive;

- programme thérapeutique et de répit pour les personnes qui ont des incapacités principalement d'origine motrice. 
Les activités des centres de jour

Peu importe le type de regroupement de clientèle retenu, il sera pertinent de porter davantage attention aux types d'activités à offrir à l'intérieur des divers sous-programmes des centres de jour.

En premier lieu, on note que les activités de prévention et de promotion qui ont été observées dans cette étude sont plus souvent des activités de type éducatif au cours desquelles les individus sont passifs. Ces activités sont peut-être moins appréciées des participants puisqu'elles sont associées à une plus faible régularité de participation. On devrait sans doute utiliser les activités éducatives passives avec parcimonie et chercher à transmettre les messages de promotion de la santé dans des activités plus dynamiques. Des activités de nature préventive dans lesquels les participants sont actifs, par exemple des groupes de cuisine santé, sont sans doute plus stimulantes que des présentations formelles sur le même sujet. L'effet de ces deux types d'activités sur la régularité de la participation reste à évaluer.

En second lieu, on peut penser qu'il est pertinent de maintenir certaines activités comme les exercices physiques et les activités d'intégration sociale pour toutes les clientèles du centre de jour. Ces activités correspondent aux besoins d'un grand nombre de personnes âgées qui fréquentent les centres de jour. De plus, ces deux types d'activités sont facilement réalisables en groupe et probablement facilement acceptées par les participants des centres de jour. De même, en portant attention à choisir le niveau approprié à chaque clientèle, des activités de stimulation cognitive pourraient aussi être offertes à l'ensemble des clientèles, puisqu'il existe certaines données voulant que la stimulation cognitive puisse aider à prévenir l'apparition de déficits cognitifs (Wilson et coll., 2002).

On insistera cependant aussi sur le fait que certaines catégories d'activités devraient être davantage ciblées en fonction des diverses clientèles les plus à même d'en bénéficier.

Par exemple, diverses approches ayant démontré des résultats probants pour diminuer les symptômes dépressifs (réminiscence, thérapie cognitive behaviorale - voir Frazer et coll., 2005) pourraient être offertes davantage et de façon plus ciblée pour cette 
clientèle. Les centres de jour devraient s'assurer d'avoir suffisamment d'intervenants formés à l'intervention psychosociale pour animer ce type d'activités de groupe.

De même, les activités destinées aux personnes qui présentent des atteintes cognitives pourraient inclure davantage d'activités de réminiscence et de stimulation cognitive adaptée à leurs capacités, puisque des travaux ont démontré l'utilité de ces activités pour cette clientèle (Knapp et coll., 2006; Spector et coll., 2005; Spector et coll., 2003; Woods et coll., 2005)

Troisièmement, il y a lieu de s'interroger sur la participation de personnes sans atteintes cognitives selon le SPMSQ dans les groupes destinés aux personnes qui ont des troubles cognitifs puisque, même si la différence n'est pas statistiquement significative, la participation de ces personnes au centre de jour semble moins assidue. Une explication

possible est que le SPMSQ n'identifie peut-être pas tous les types d'atteintes cognitives. Il reflèterait mieux les difficultés de mémoire et d'orientation et moins bien les difficultés des fonctions exécutives comme la planification et le jugement. Cependant, d'après nos discussions avec des responsables de centre de jour, il est aussi possible que les lourdes incapacités physiques soient faussement associées à des pertes cognitives. Il est possible que des personnes avec de lourdes atteintes physiques soient inscrites dans ces groupes pour offrir plus de journées de répit à leur aidant ou parce qu'elles auraient de la difficulté à participer aux activités d'autres groupes en raison de leurs déficiences physiques. Bien que cela réponde à des impératifs pratiques, cette situation n'est peut-être pas idéale pour répondre aux besoins des individus sans atteintes cognitives et la question mériterait d'être étudiée plus à fond par les responsables de la programmation des activités en centre de jour.

\section{3) Viser une intensité d'exposition optimale}

Il existe peu de données sur l'intensité optimale d'exposition aux activités du centre de jour afin d'induire des résultats bénéfiques chez les participants.

Dans la présente étude, les personnes inscrites à des journées entières sont des utilisateurs plus réguliers du centre de jour que celles inscrites en demi-journée. Ceci 
suggère que la participation pendant une journée entière serait peut-être nécessaire pour compenser l'effort de préparation par la personne âgée elle-même ou par son aidant et sentir que les bénéfices de la participation au centre de jour excèdent les inconvénients.

Les quelques indications dont nous disposons portent aussi à croire que lorsque le but du centre de jour est de réduire le fardeau des aidants, une participation réelle d'une fois par semaine serait un minimum nécessaire (Baumgarten et coll., 2002). À la lumière de nos résultats faisant état d'un taux moyen de participation réelle de $68,1 \%$ des participations prévues et un taux médian de $72,8 \%$, il devient évident que, pour atteindre une participation réelle minimale d'une fois par semaine pour une majorité de participants, la fréquence d'inscription doit être plus élevée. Ainsi, lorsque le but de la participation au centre de jour est de réduire le fardeau des aidants, il semble pertinent de recommander une fréquence d'inscription d'au moins deux fois par semaine. Les connaissances actuelles ne permettent pas de porter un jugement sur la fréquence optimale de participation au centre de jour pour répondre à d'autres types de besoins.

Comme mentionné précédemment, il sera aussi pertinent de s'interroger sur la fréquence de la participation aux diverses activités du centre de jour pour s'assurer qu'elle soit suffisante pour engendrer les effets bénéfiques escomptés de chacune d'elles. Il y aurait peut-être lieu de distinguer entre les activités offertes dans un but de prévention et les activités offertes dans un but thérapeutique, pour chaque type de clientèle. Il est probable que les activités offertes dans un but thérapeutique nécessitent une fréquence de participation plus élevée. Il y aurait lieu de documenter les fréquences et les intensités recommandées dans les écrits scientifiques pour que divers types d'activités puissent induire des effets thérapeutiques chez différentes clientèles.

\section{4) Prévoir une utilisation efficiente des ressources}

Les programmes qui ont des listes d'attentes doivent porter une attention particulière aux taux de participation. Pour une utilisation efficiente de leurs ressources, les centres de jour qui ont des taux de participation similaires à ceux observés dans cette étude 
(68,1\% des présences possibles) peuvent inscrire de 25 à $30 \%$ plus de participants que leur capacité estimée, sans que la participation quotidienne n'excède la capacité souhaitée.

\section{Pistes de recherche pour le futur}

Il serait certainement intéressant de poursuivre l'analyse des déterminants de l'utilisation des centres de jour dans le contexte québécois et de vérifier si les résultats de cette étude peuvent être reproduits avec des échantillons aléatoires de personnes âgées qui présentent des incapacités, tirés d'une plus grande variété de régions du Québec. Pour éviter certaines des limites de la présente recherche, une telle étude devrait chercher à :

- Recueillir des données sur certaines variables incluses dans le cadre conceptuel et non disponibles pour la présente recherche, comme par exemple, les attitudes envers les activités de groupe, les caractéristiques des intervenants des CLSC qui dirigent les personnes âgées vers le centre de jour, etc.

- Lors du recueil de données sur la santé mentale, distinguer les symptômes reliés à la dépression de ceux reliés à l'anxiété, puisque ces facteurs peuvent avoir des influences divergentes sur l'utilisation du centre de jour.

- S'interroger sur les meilleures façons de rejoindre les personnes de soutien afin de pouvoir obtenir un meilleur taux de réponse et de pouvoir inclure leurs caractéristiques dans toutes les analyses.

- Recueillir des données auprès d'un nombre plus important de participants au centre de jour. En particulier, tenir compte de l'analyse des possibilités d'interaction de certaines variables dans le calcul de la taille échantillonnale afin de s'assurer d'une puissance statistique adéquate.

- En préalable à une telle étude, implanter une classification des activités en centre de jour qui permettrait un recueil de données plus fiable et subséquemment une meilleure comparaison entre les centres de jour. Cette classification devrait inclure autant les activités individuelles que les activités de groupe et les activités de soutien aux proches aidants. Ceci permettrait une étude plus approfondie des liens entre les activités offertes et la régularité de la participation au centre de jour. 
La réflexion sur l'utilisation des centres de jour entreprise dans cette thèse soulève aussi de nouvelles questions de recherche.

Des études qualitatives auprès de personnes auxquelles on a recommandé la participation au centre de jour pourraient apporter un éclairage intéressant quant à certaines observations de la présente étude. Par exemple, il serait intéressant de documenter comment l'expérience des hommes diffère de celle des femmes en ce qui concerne la recommandation de fréquentation et la participation à un centre de jour.

Il serait intéressant d'explorer, parmi les personnes âgées qui présentent des incapacités et n'utilisent pas un centre de jour, les différences entre les personnes qui sont moins dirigées vers ce service et celles qui y sont dirigées mais refusent d'y participer ou cessent plus rapidement leur participation. Ceci pourrait se faire par des recherches auprès des CLSC qui dirigent les clients vers les centres de jour et sont tenus de faire un suivi des résultats puisqu'ils demeurent responsables des plans de services de ces clients.

Les résultats de cette étude suggèrent que la collaboration des services de soutien à domicile des CLSC pour préparer la personne âgée pour le centre de jour peut améliorer la régularité de la participation. Il serait intéressant d'explorer si d'autres types de collaboration peuvent faciliter la participation au centre de jour.

Enfin, le but ultime de la recherche sur les centres de jour est de mieux comprendre comment ces derniers peuvent atteindre leurs objectifs. L'objectif général du centre de jour est de «favoriser le maintien à domicile de la clientèle âgée et donc de retarder, sinon éviter, le recours à un milieu d'hébergement institutionnel, et ce, dans le contexte d'une qualité de vie optimale » (Agence de la santé et des services sociaux de Montréal, 2005). L'amélioration de l'état de santé ou des capacités fonctionnelles des participants ainsi que l'amélioration du bien-être psychologique des personnes de soutien et la réduction de leur fardeau subjectif et objectif peuvent constituer des résultats intermédiaires. Les recherches futures sur l'efficacité des centres de jour à atteindre ces résultats devraient idéalement inclure comme variables de contrôle, des données sur les taux d'utilisation et sur les processus d'intervention. 
Des études sur l'intensité minimale d'exposition au centre de jour nécessaire pour induire des effets bénéfiques sont encore pertinentes. Nous disposons actuellement de données probantes seulement pour la fréquentation minimale pour réduire le fardeau des aidants.

Des recherches plus approfondies sur les liens entre l'atteinte des objectifs des centres de jour et les processus d'intervention seraient aussi indiquées. Les processus d'intervention étudiés devraient inclure le type d'activités offertes en centre de jour et leur fréquence, de même que les activités offertes par les CLSC en complémentarité des activités des centres de jour. On pense ici en particulier aux activités de soutien pour les proches aidants. En effet, ce sera souvent une combinaison d'interventions qui permettra de favoriser le maintien à domicile de la clientèle âgée. 


\section{Chapitre 7 : Conclusion}

Dans le but de favoriser le maintien à domicile de la clientèle âgée et donc de retarder, sinon éviter, le recours à l'hébergement en milieu institutionnel, les centres de jour offrent des services aux personnes âgées qui demeurent à domicile et présentent des incapacités, principalement au moyen d'activités de groupe, et ce, en complémentarité avec les autres services de soutien à domicile des CLSC.

Ce projet de doctorat avait pour but d'améliorer notre compréhension des centres de jour en étudiant leur utilisation. Cela nous a permis de constater que conformément à leur mission, les centres de jour rejoignent une bonne proportion des personnes âgées qui présentent des incapacités. Nous avons pu faire ressortir les caractéristiques des clients les plus susceptibles d'y participer, les types d'activités auxquelles les utilisateurs ont participé le plus fréquemment, de même que les caractéristiques des personnes qui ont une participation plus régulière en centre de jour. Nous avons aussi constaté que l'intensité d'exposition au centre de jour, et en particulier à certaines catégories d'activités thérapeutiques, semble faible. Ceci soulève la question des seuils d'exposition nécessaires pour induire des effets favorables sur le maintien à domicile et sur la qualité de vie de la clientèle cible, bien que les données disponibles sur la participation minimale pour induire divers types d'effets sont encore insuffisantes.

L'intégration des résultats de la présente étude aux connaissances obtenues de la recension des écrits a permis d'émettre certaines recommandations pour mieux rejoindre la clientèle cible et améliorer la régularité de participation de certaines catégories de clients, mieux cibler les activités qui sont offertes en centre de jour, de même que pour viser une fréquence d'inscription minimale pour assurer une intensité d'exposition adéquate lorsque le but du centre de jour est de réduire le fardeau des proches aidants.

Le maintien à domicile des personnes âgées et la diminution des taux d'hébergement en institution s'inscrivent dans les priorités du gouvernement du Québec pour faire face au vieillissement de la population et réduire les coûts qui y sont associés. 
Dans le but de s'assurer que les ressources consacrées aux centres de jour sont utilisées de façon efficace et responsable, il importe de poursuivre la recherche sur la façon dont ceuxci contribuent à ces grands objectifs. Cela ne peut se faire qu'en tenant compte de l'influence de divers processus d'intervention et des taux d'utilisation sur l'efficacité de ce service. 


\section{Références $^{3}$}

Aday, L. A., \& Andersen, R. M. (1974). A framework for the study of access to medical care. Health Services Research, 9, 208-220.

Agence de la santé et des services sociaux de Montréal (2005). Les services destinés aux personnes en perte d'autonomie liée au vieillissement : Enjeux - perspectives balises régionales. Montréal : Auteur.

Agence de la santé et des services sociaux de Montréal. (2004a). Portrait de la population :

Centre de santé Côte-des-Neiges, Métro et Parc Extension. En ligne [http://www. santemontreal.qc.ca/pdf/PDF_CSSS/pop_05.pdf]. Consulté le 28 juillet 2007.

Agence de la santé et des services sociaux de Montréal. (2004b). Portrait de la population :

Centre de santé Nord de l'île et Saint-Laurent. En ligne [http://www. santemontreal.qc.ca/pdf/PDF_CSSS/pop_06.pdf]. Consulté le 28 juillet 2007.

Andersen, R., \& Newman, J. F. (1973). Societal and individual determinants of medical care utilization in the United States. Milbank Memorial Fund Quarterly, 51, 95-124.

Andersen, R. M. (1995). Revisiting the behavioral model and access to medical care: Does it matter? Journal of Health and Social Behavior, 36, 1-10.

Anderson, A. J. (2002). Treatment of Depression in Older Adults [Electronic version]. International Journal of Psychosocial Rehabilitation, 6, 69-78.

Association des centres d'accueil du Québec (ACAQ). (1991). Le centre de jour: Un cadre de référence. Montréal : Auteur.

Barber, G. M., Paton, R. N., \& Wishnia, G. C. (1993). Public's perceived need for adult day care versus actual use. Home Health Care Services Quarterly, 14(2-3), 53-71.

\footnotetext{
${ }^{3}$ Cette liste de références inclut tous les documents cités dans la thèse, y compris ceux déjà inclus dans les trois articles qui présentent les résultats et font partie intégrante de la thèse. Elle n'inclut pas les références citées uniquement dans l'article en Annexe.
} 
Bass, D. M., Looman, W. J., \& Ehrlich, P. (1992). Predicting the volume of health and social services: Integrating cognitive impairment into the Modified Andersen Framework. The Gerontologist, 32(1), 33-43.

Bass, D. M., \& Noelker, L. S. (1987). The influence of family caregivers on elder's use of in-home services: An expanded conceptual framework. Journal of Health and Social Behavior, 28, 184-196.

Baumgarten, M., Lebel, P., Laprise, H., Leclerc, C., \& Quinn, C. (2002). Adult day care for the frail elderly: Outcomes, satisfaction, and cost. Journal of Aging and Health, 14(2), 237-259.

Bea van Beveren, A. J., \& Hetherington, R.W. (1998). The One Percent Solution: A Basis for Adult Day Program Development? Activities, Adaptation and Aging, 22(4), 4152.

Beisecker, A. E., Wright, L. J., Chrisman, S. K., \& Ashworth, J. (1996). Family caregiver perceptions of benefits and barriers to the use of adult day care for individuals with Alzheimer's disease. Research on Aging, 18(4), 430-450.

Béland, F., Bergman, H., \& Lebel, P. (2001). SIPA : Un Système de services intégrés pour personnes âgées en perte d'autonomie. Évaluation de la phase I, juin 1999 à mai 2000. Montréal : Groupe de recherche Université de Montréal et Université McGill sur les services intégrés aux personnes âgées.

Béland, F., Bergman, H., Lebel, P., Clarfield, A. M., Tousignant, P., Contandriopoulos, A. P., et al. (2006a). A system of integrated care for older persons with disabilities in Canada: Results from a randomized controlled trial. Journal of Gerontology Series A: Biological Sciences and Medical Sciences, 61(4), 367-373.

Béland, F., Bergman, H., Lebel, P., Dallaire, L., Fletcher, J., Contandriopoulos, A. P., et al. (2006b). Integrated services for frail elders (SIPA): A trial of a model for Canada. / Des services intégrés pour les personnes âgées fragiles (SIPA): Expérimentation d'un modèle pour le Canada. Canadian Journal on Aging / Revue canadienne du vieillissement, 25(1), 5-42. 
Béland, F., Haldemann, V., Martin, J.-C., Bourque, P., Ouellette, P., \& Lavoie, J.-P. (1998). Vieillir dans la communauté : Santé et autonomie (No. R98-05). Montréal : Groupe de recherche interdisciplinaire en santé, Université de Montréal.

Biegel, D. E., Bass, D. M., Schulz, R., \& Morycz, R. K. (1993). Predictors in home and out-of-home service use by family caregivers of alzheimer's disease patients. Journal of Aging and Health, 5(4), 419-438.

Bloom, M. (1996). Primary prevention practices. Thousand Oaks, CA: Sage Publications.

Bohlmeijer, E., Smit, F., \& Cuijpers, P. (2003). Effects of reminiscence and life review on late-life depression: A meta-analysis. International Journal of Geriatric Psychiatry, 18(12), 1088-1094.

Boucher, L., Renvall, M. J., \& Jackson, J. E. (1996). Cognitively impaired spouses as primary caregivers for demented elderly people. Journal of the American Geriatrics Society, 44(7), 828-831.

Bourque, P., Blanchard, L., \& Vézina, J. (1990). Étude psychométrique de l'Échelle de dépression gériatrique. Canadian Journal on Aging / Revue canadienne du vieillissement, 9(4), 348-355.

Brodaty, H., Thomson, C., Thomson, C., \& Fine, M. (2005). Why caregivers of people with dementia and memory loss don't use services. International Journal of Geriatric Psychiatry, 20, 537-546.

Calsyn, R. J., \& Winter, J. P. (2000). Predicting different types of service use by the elderly: The strength of the Behavioral Model and the value of interaction terms. Journal of Applied Gerontology, 19(3), 284-303.

Canadian Study of Health and Aging Working Group. (1994). Patterns of care for people with dementia in Canada. Canadian Journal on Aging / Revue canadienne du vieillissement, 13(1), 470-487.

Caserta, M. S., Lund, D. A., Wright, S. D., \& Redburn, D. E. (1987). Caregivers to dementia patients: The utilisation of community services. The Gerontologist, 27(2), 209-214. 
Cefalu, C. A., Ettinger, W. H., \& Espeland, M. (1996). A study of the characteristics of the dementia patients and caregivers in dementia-nonspecific adult day care programs. Journal of the American Geriatrics Society, 44(6), 654-659.

Centers for Disease Control and Prevention (CDC). (2007). Characteristics and health of caregivers and care recipients: North Carolina, 2005. Morbidity and Mortality Weekly Report, 56(21), 529-532.

Chappell, N. L. (1983). Who benefits from adult day care: Changes in functional ability and mental functioning during attendance. Canadian Journal on Aging / Revue canadienne du vieillissement, 2(1), 9-26.

Chenoweth, B., \& Spencer, B. (1986). Dementia: The experience of family caregivers. The gerontologist, 26(3), 267-272.

Cohen-Mansfield, J., Besansky, J., Watson, V., \& Bernhard, L. J. (1994). Underutilization of adult day care: An exploratory study. Journal of Gerontological Social Work, 22(1-2), 21-39.

Cohen-Mansfield, J., Lipson, S., Brenneman, K. S., \& Pawlson, L. G. (2001). Health status of participants of adult day care centers. Journal of Health and Social Policy, 14(2), 71-89.

Conrad, K. J., \& Guttman, R. (1991). Characteristics of Alzheimer's versus nonAlzheimer's adult day care centers. Research on Aging, 13(1), 96-116.

Conrad, K. J., Hanrahan, P., \& Hughes, S. L. (1990). Survey of adult day care in the United States: National and regional findings. Research on Aging, 12(1), 36-56.

Conrad, K. J., Hughes, S. L., Hanrahan, P., \& Wang, S. (1993). Classification of adult day care: A cluster analysis of services and activities. Journal of Gerontology, 48(3), S112-122.

Conrad, K. J., Hughes, S. L., \& Wang, S. (1992). Program factors that influence utilization of adult day care. Health Services Research, 27(4), 481-503

Contandriopoulos, A. P., Champagne, F., Potvin, L., Denis, J. L., \& Boyle, P. (1990). Savoir préparer une recherche : la définir, la structurer, la financer. Montréal : Les Presses de l'Université de Montréal. 
Cornoni-Huntley, J., Brook, D. B., Ostfeld, A., Taylor, J. O., \& Wallace, R. B. (1986). Established Populations for Epidemiological Studies of the Elderly, Resources Data Book. National Institutes of Health: NIH no. 86-2443.

Cotrell, V. (1996). Respite use by dementia caregivers: Preferences and reasons for initial use. Journal of Gerontological Social Work, 26(3/4), 35-55.

Cotrell, V., \& Engel, R. J. (1998). The role of secondary supports in mediating formal services to dementia caregivers. Journal of Gerontological Social Work, 30(3/4), 117-132.

Coulton, C., \& Frost, A. K. (1982). Use of social and health services by the elderly. Journal of Health and Social Behavior, 23(4), 330-339.

Cox, C. (1997). Findings from a statewide program of respite care: A comparison of service users, stoppers, and nonusers. The Gerontologist, 37(4), 511-517.

Cox, N. J., \& Reifler, B. V. (1994). Dementia care and respite services program. Alzheimer's Disease and Associated Disorders, 8(supp.3), 113-121.

Cox, N. J., Reifler, B. V., \& Yates, K. (1998). Building adult day center census by preventing premature terminations. American Journal of Alzheimer's Disease, 13(3), 124-130.

Crets, S. (1996). Determinants of the use of ambulant social care by the elderly. Social Science and Medicine, 43(12), 1709-1720.

Curran, J. S. M. (1996). The evolution of daycare services for people with dementia. In: R. Bland (Ed.), Developing Services for Older People and Their Families (Vol. 29). London: Jessica Kingsley Publishers.

Dabelko, H. I. (2004). Individual and environmental factors that influence length of stay in adult health care programs. Journal of Gerontological Social Work, 43(1), 83-105.

Dabelko, H. I. (2005). A comparative analysis of short stays versus long stays in adult day health care programs. Social Work in Health Care, 42(1), 57-71.

Dabelko, H. I., \& Balaswamy, S. (2000). Use of adult day services and home health care services by older adults: A comparative analysis. Home Health Care Services Quarterly, 18(3), 65-79 
Dabelko, H. I., \& Zimmerman, J.A. (2008). Outcomes of adult day services for participants: A conceptual model. Journal of Applied Gerontology, 27(1), 78-92.

Delbecq, A. L., Van De Ven, A. H., \& Gustavson, D. H. (1975). Group Techniques for Program Planning, A Guide to Nominal Group and Delphi Processes. Glenview, Illinois: Scott, Foresman and Company.

Desrosiers, J., Bravo, G., Hébert, R., \& Dubuc, N. (1995). Reliability of the revised functional autonomy measurement system (SMAF) for epidemiological research. Age and Ageing, 24(5), 402-406.

Développement des ressources humaines Canada. (2003). L'incapacité au Canada : Un profil en 2001. En ligne [http://www.dsc.gc.ca/fr/pip/bcph/documents/EPLA /EPLA.pdf]. Consulté le 28 juillet 2005.

Donabedian, A. (1973). Aspect of Medical Care Administration. Cambridge: Harvard University Press.

Douglass, C., \& Visconti, C. (1998). Factors influencing the use of adult day care by individuals with Alzheimer's Disease: A multivariate examination of the California Alzheimer's Disease Diagnostic and Treatment Center Program. Home Health Care Services Quarterly, 17(2), 53-69.

Ducharme, F., Lebel, P., \& Bergman, H. (2001). Vieillissement et soins, l'urgence d'offrir des services de santé intégrés aux familles du XXIe siècle. Ruptures, revue transdisciplinaire en santé, 8(2), 110-121.

Ducharme, F., Levesque, L., Zarit, S. H., Lachance, L., Giroux, F. (2007). Changes in health outcomes among older husband caregivers: A one-year longitudinal study. International Journal of Aging and Human Development, 65(1), 73-96.

Everitt, B. S., Landau, S., \& Leese, M. (2001). Cluster Analysis (4 ${ }^{\text {th }}$ ed.). London: Arnold.

Fillenbaum, G. G. (1988). Multidimensional Functional Assessment of Older Adults: The Duke Older American Resources and Services Procedures. Hillsdale, NJ: Lawrence Erlbaum Associates.

Fillenbaum, G. G., \& Smyer, M. A. (1981). The development, validity and reliability of the OARS multidimentional functional assessment questionnaire. Journal of Gerontology, 36, 428-434. 
Folstein, M. F., Folstein, S. E., \& McHugh, P. R. (1975). Mini-Mental State: A practical method for grading the cognitive state of patients for the clinician. Journal of Psychiatric Research, 12(3), 189-198.

Forbes, D. A., \& Janzen, B. L. (2004). Comparison of rural and urban users and non-users of home care in Canada. Canadian Journal of Rural Medicine, 9(4), 227-235.

Foreman, M. D. (1987). Reliability and validity of mental status questionnaires in elderly hospitalized patients. Nursing Research, 36(4), 216-220.

Fortinsky, R. H., \& Hathaway, T. J. (1990). Information and service needs among active and former family caregivers of persons with Alzheimer's disease. The Gerontologist, 30(5), 604-609.

Frazer, C. J., Christensen, H., \& Griffiths, K. M. (2005). Effectiveness of treatments for depression in older people. Medical Journal of Australia, 182(12), 627-632.

Gaugler, J. E., Jarrot, S. E., Zarit, S. H., Stephens, M. A. P., Townsend, A., \& Greene, R. (2003). Respite for dementia caregivers: The effects of adult day service use on caregiving hours and care demands. International Psychogeriatrics, 15(1), 37-58.

Gaugler, J. E., Kane, R. L., Kane, R. A., \& Newcomer, R. (2005). Early community- based service utilization and its effects on institutionalization in dementia caregiving. The Gerontologist, 45, 177-185.

Gaugler, J. E., \& Zarit, S. H. (2001). The effectiveness of adult day services for disabled older people. Journal of Aging and Social Policy, 12(2), 23-47.

Gauthier, H. (2004). Les personnes âgées et le vieillissement démographique. Dans: Institut de la statistique du Québec (Ed.), Vie des générations et personnes âgées : aujourd'hui et demain, vol. 1, p. 43-89. Québec: Institut de la statistique du Québec.

Germain, C.B., \& Bloom, M. (1999). Human behavior in the social environment: An ecological view. New York: Columbia University Press.

Gitlin, L. N., Reever, K., Dennis, M. P., Mathieu, E., \& Hauck, W. W. (2006 ). Enhancing quality of life of families who use adult day services: Short- and long-term effects of the adult day services plus program. The Gerontologist, 46(5), 630-639. 
Goldston, S. M. (1989). Adult day care: A basic guide. Owings Mills, Maryland: National Health Publishing (a division of Williams \& Wilkins).

Gottlieb, B.H., \& Johnson, J. (2000). Respite programs for caregivers of persons with dementia: A review with practice implications. Aging and Mental Health, 4(2), 119129.

Graham, R. W. (1989). Adult day care: How families of the dementia patient respond. Journal of Gerontological Nursing, 15(3), 27-31, 40-41.

Granger, C. V., Albrecht, G. L., \& Hamilton, B. B. (1979). Outcome of comprehensive medical rehabilitation: Measurement by PULSES profile and the Bartel Index. Archives of Physiology and Medical Rehabilitation, 60, 145-154.

Gratton, G. (1995). L'hôpital de jour: Concept, coûts et impacts sur le taux d'institutionnalisation et le taux d'hospitalisation en courte durée. Thèse de maîtrise : Université du Québec en Abitibi-Témiscamingue

Greene, V. L. (1983). Substitution between formally and informally provided care for the impaired elderly in the community. Medical Care, 21(6), 609-619.

Griffin, L. W. (1993). Adult day care centers and adult protective services. Journal of Gerontological Social Work, 20(1-2), 115-133.

Gutman, G. M., Milstein, S., Killam, J., Lewis, D., \& Hollander, M. J. (1993a). Adult day care centres in British Columbia: Client characteristics, reasons for referral and reasons for non-attendance / Les centres de soins de jour pour adultes en ColombieBritannique: Caractéristiques des clients, raisons de l'aiguillage et raisons de l'omission de se présenter. Health Reports / Rapports sur la santé, 5(3), 321-333.

Gutman, G. M., Milstein, S., Killam, J., Lewis, D., \& Hollander, M. J. (1993b). Adult Day Care centres in British Columbia: Models, characteristics and services / Les centres de soins de jour pour adultes en Colombie-Britannique : Modèles, caractéristiques et services. Health Reports / Rapports sur la santé, 5(2), 189-207.

Hébert, R., Bravo, G., \& Girouard, D. (1993). Fidélité de la traduction française de trois instruments d'évaluation des aidants naturels de malades déments. Canadian Journal on Aging / La revue canadienne du vieillissement, 12(3), 324-337. 
Hébert, R., Carrier, R., \& Bilodeau, A. (1988). The Functional Autonomy Measurement System (SMAF): Description and validation of an instrument for the measurement of handicaps. Age and Ageing, 17, 293-302.

Hébert, R., Carrier, R., \& Bilodeau, A. (1988). Le Système de mesure de l'autonomie fonctionnelle (SMAF). La revue de gériatrie, 13(4), 161-167.

Henry, M. E., \& Capitman, J. A. (1995). Finding satisfaction in adult day care: Analysis of a national demonstration of dementia care and respite services. Journal of Applied Gerontology, 14(3), 302-320.

Hosmer, D. W., \& Lemeshow, S. (2000). Applied Logistic Regression (2 $2^{\text {nd }}$ ed.). New-York: John Wiley and Sons, inc.

Institut de la statistique du Québec. (31 janvier 2007). Données démographiques régionales: Estimation de la population des régions métropolitaines de recensement selon le groupe d'âge et le sexe, Québec, au ler juillet des années 1996 à 2006. En ligne [http://www.stat.gouv.qc.ca/donstat/societe/demographie/dons_regnl/regional/index. htm]. Consulté le 3 juillet 2007.

Institut de la statistique du Québec. (2005-2006). Données générales sur la population du Québec: Population par année d'âge et par sexe, Québec, le ler juillet 2005-révisé et Population par année d'âge et par sexe, Québec le ler juillet 2006- provisoire. En ligne [http://www.stat.gouv.qc.ca/donstat/societe/demographie/struc_poplt/ index.htm]. Consulté le 3 juillet 2007.

Jackson, S. A., \& Mittelmark, M. B. (1997). Unmet needs for formal home and community services among african american and white older adults: The Forsyth County Aging Study. The Journal of Applied Gerontology, 16(3), 298-316.

Jarrott, S. E. , Zarit, S. H., Parris-Stephens, M. A., Townsend, A., \& Greene, R. (1999). Caregiver satisfaction with adult day service programs. American Journal of Alzheimer's Disease, 14(4), 233-244.

Jarrott, S. E., Zarit, S. H., Berg, S., \& Johansson, L. (1998). Adult day care for dementia: A comparison of programs in Sweden and the United States. Journal of CrossCultural Gerontology, 13(2), 99-108. 
Kadushin, G. (2004). Home health care utilization: A review of the research for social work. Health and Social Work, 29(3), 219-244.

Knapp, M., Thorgrimsen, L., Patel, A., Spector, A., Hallam, A., Woods, B., et al. (2006). Cognitive stimulation therapy for people with dementia: Cost-effectiveness analysis. British Journal of Psychiatry, 188, 574-580.

Kosloski, K., \& Montgomery, R. J. V. (1994). Investigating patterns of service use by families providing care for dependent elders. Journal of Aging and Health, 6(1), 17-37.

Kosloski, K., \& Montgomery, R. J. V. (1993). Perceptions of respite services as predictors of utilization. Research on Aging, 15(4), 393-413.

Kosloski, K., Montgomery, R. J. V., \& Karner, T. X. (1999). Differences in the perceived need for assistive services by culturally diverse caregivers of persons with dementia. Journal of Applied Gerontology, 18(2), 239-256.

Kosloski, K., Montgomery, R. J. V., \& Youngbauer, J. G. (2001). Utilization of respite services: A comparison of users, seekers, and nonseekers. Journal of Applied Gerontology, 20(1), 111-132.

Lawton, M. P., Brody, E. M., \& Saperstein, A. R. (1989). A controlled study of respite services for caregivers of Alzheimer's patients. The Gerontologist, 29(1), 8-16.

Lawton, M. P., Brody, E. M., \& Saperstein, A. R. (1991). Respite for caregivers of Alzheimer's patients: Research and practice. New-York: Springer Publishing Company.

Leitsch, S. A., Zarit, S. H., Townsend, A., \& Greene, R. (2001). Medical and social adult day service programs. Research on Aging, 23(4), 473-498.

Li, L. W. (2004). Caregiving network compositions and use of support services by community-dwelling dependent elders. Journal of Gerontological Social Work, 43(2-3), 147-164.

Lindeman, D. A., Corby, N. H., Downing, R., \& Sanborn, B. (1991). Alzheimer's Day Care: A Basic Guide. Washington, DC: Hemisphere Publishing Corp. 
Lynch, M., Harrington, C., \& Newcomer, R. (1999). Predictors of use of chronic services by impaired members in the Social Health Maintenance Organization demonstration. Journal of Applied Gerontology, 18(3), 283-304.

Mace, N., \& Rabins, P. (1984). Day care \& dementia. Generations, Winter, 41-45.

Mason, A., Weatherly, H., Spilsbury, K., Golder, S., Arksey, H., Adamson, J., \& Drummund, M. (2007). The effectiveness and cost-effectiveness of respite for caregivers of frail older people. Journal of the American Geriatrics Society, 55(2), 290-299.

Mavall, L., \& Thorslund, M. (2007). Does day care also provide care for the caregiver? Archives of Gerontology and Geriatrics, 45(2), 137-150.

Miller, B., \& McFall, S. (1991). The effect of caregiver's burden on change in frail older persons' use of formal helpers. Journal of Health and Social Behavior, 32(2), 165-179.

Ministère de la Santé et des Services sociaux. (1986). Le centre de jour : orientations et programmes (document de travail). Québec: Auteur.

Ministère de la Santé et des Services sociaux (1986). Hôpital de jour: orientations. Québec: Auteur.

Monahan, D. (1993). Utilization of dementia-specific respite day care for clients and their caregivers in a social model program. Journal of Gerontological Social Work, 20(3/4), 57-70.

Montgomery, R. J. V., \& Borgotta, E. F. (1989). The effects of alternative support strategies on family caregiving. The Gerontologist, 29(4), 457-464.

Montgomery, R. J. V., Kosloski, K., \& Borgotta, E. F. (1988-1989). The influence of cognitive impairment on service use and caregiver response. The Journal of Applied Social Sciences, 13(1), 142-169.

Montoro-Rodriguez, J., Kosloski, K., \& Montgomery, R. J. V. (2003). Evaluating a practice-oriented service model to increase the use of respite services among minorities and rural caregivers. The Gerontologist, 43(6), 916-924.

National Institute on Adult Day Care. (1990). Standards and Guidelines for Adult Day Care. Washington, DC: National Council on Aging. 
Neufeld, A., \& Strang, V. (1992). Issues in the evaluation of small-scale adult day care programs. International Journal of Nursing Studies, 29(3), 261-273.

Nissenboim, S., \& Vroman, C. (1998). The Positive Interactions Program of Activities for People with Alzheimer's Disease. Baltimore, MD: Health Professions Press.

Norman, J. L., \& Horton, E. R. (1996). Adult Day Care Therapeutic Activity Manual: A Continuous Quality Improvement Approach. Gaithersburg, MD: Aspen Publishers.

O’Brien, C. L. (1982). Program development. In: C.L. O’Brien (Ed.) Adult Day Care: A Practical Guide. Monterey, CA: Wadsworth Health Sciences Division.

Paquet, M. (1996). La réticence des personnes-soutien de personnes âgées dépendantes visà-vis l'utilisation des services de soutien formel. Canadian journal on Aging / Revue canadienne du vieillissement, 15(3), 442-462.

Pearlin, L. I., Mullan, J. T., Semple, S. J., \& Skaff, M. M. (1990). Caregiving and the stress process: An overview of concepts and their measures. The Gerontologist, 30(5), 583-594.

Pedlar, D. J., \& Biegel, D. E. (1999). The impact of family caregiver attitudes on the use of community services for dementia care. Journal of Applied Gerontology, 18(2), 201221.

Pfeiffer, E. (1975). A Short Portable Mental Status Questionnaire for the assessment of organic brain deficit in elderly patients. Journal of the American Geriatrics Society, 23(10), 433-441.

Philp, I., McKee, K. J., Meldrum, P., Ballinger, B. R., Gilhooly, M. L. M., Gordon, D. S., et al. (1995). Community care for demented and non-demented elderly people: A comparison study of financial burden, service use, and unmet needs in family supporters. British Medical Journal, 310, 1503-1506.

Pinquart, M., \& Sörensen, S. (2003). Differences between caregivers and noncaregivers in psychological health and physical health: A meta-analysis. Psychology and Aging, $18(2), 250-267$.

Pope, S. K., Shue, V. M., \& Beck, C. (2003). Will a healthy lifestyle help prevent Alzheimer's disease. Annual Review of Public Health, 24, 111-132. 
Pushkar-Gold, D., Feldman-Reis, M., Markiewicz, D., \& Andres, D. (1995). When home caregiving ends: A longitudinal study of outcomes for caregiver of relatives with dementia. Journal of the American Geriatrics Society, 43, 10-16.

Regroupement des centres d'hébergement et de soins de longue durée, Région de Montréal. (2001). Les centres de jour aujourd'hui... et demain! Document III: Synthèse des consultations et propositions d'orientations. Montréal : Auteur.

Regroupement des centres d'hébergement et de soins de longue durée, Région de Montréal. (2005). Cadre de référence régional pour le programme Centre de jour. Montréal : Auteur.

Reilly, S., Venables, D., Hughes, J., Challis D., \& Abendstern, M. (2006). Standards of care in day hospitals and day centres: A comparison of services for older people with dementia. International Journal of Geriatric Psychiatry, 21, 460-468

Robinson, K., \& Yates, K. (1994). Effects of two caregiver-training programs on burden and attitude toward help. Archives of Psychiatric Nursing, 8(5), 312-319

Ross-Kerr, J. C., Warren, S., \& Godkin, M. D. (1995). Evaluation of adult day programs in Alberta: Final report. Edmonton: Alberta Health: Long Term Care Branch.

Ross-Kerr, J.C., Warren, S., Schalm, C., Smith, D.L., Godkin, M.D. (2003). Adult Day Programs: Who Needs Them? Journal of Gerontological Nursing, 29(12), 11-17.

Savard, J., Leduc, N., Lebel, P., Béland, F., \& Bergman, H. (2007). L'utilisation des services de centres de jour par les personnes âgées qui présentent des incapacités. Canadian Journal on Aging / Revue canadienne du vieillissement, 26(3), 255-274.

Schacke, C., \& Zank, S. R. (2006). Measuring the effectiveness of adult day care as a facility to support family caregivers of dementia patients. Journal of Applied Gerontology, 25(1), 65-80.

Schulz, R., \& Beach, S. R. (1999). Caregiving as a risk factor for mortality: The caregiver health effects study. Journal of the American Medical Association, 282(23), 2215-2219.

Sheikh, J. I., \& Yesavage, J. A. (1986). Geriatric Depression Scale (GDS): Recent evidence and development of a shorter version. Clinical Gerontologist, 5, 165-173. 
Spector, A., Orrell, M., Davies, S., \& Woods, B. (2005). Reality orientation for dementia. Cochrane Database of Systematic Reviews.

Spector, A., Thorgrimsen, L., Woods, B., Royan, L., Davies, S., Butterworth, M., et al. (2003). Efficacy of an evidence-based cognitive stimulation therapy programme for people with dementia. British Journal of Psychiatry, 183, 248-254.

Starrett, R. A., Rogers, D., \& Walters, G. (1988). Home health care utilization: A causal model. Home Health Care Services Quarterly, 9(4), 125-140.

Statistique Canada. (02/01/2007). Profil des communautés en 2001 : Faits saillants pour la communauté urbaine de Montréal. En ligne [http:/www12.statcan.ca/english/ profi101/CP01/Details/Page.cfm?Lang $=$ F\&Geo1 $=$ CD\&Code1 $=2466 \& G e o 2=$ PR\&C ode2=24\&Data $=$ Count $\&$ SearchText $=$ montréal $\&$ SearchType $=$ Begins $\&$ SearchPR $=24$ $\&$ B1=Population]. Consutlé le 1 mars 2008.

Strain, L. A., \& Blandford, A. A. (2002). Community-based services for the taking but few takers: Reasons for nonuse. Journal of Applied Gerontology, 21, 220-235.

Strain, L. A., Chappell, N. L., \& Blandford, A. A. (1987). Changes in life satisfaction among participants of Adult Day Care and their informal caregivers. Journal of Gerontological Social Work, 11(3-4), 115-129.

Strang, V., Greschuk, J., McIlveen, K., Gadacz, R. R., \& Neufeld, A. (1992). Adult day care: An evaluation study. Edmonton: Victorian Order of Nurses, Edminton Brabch.

Szekais, B. (1985). Adult day-care centres: Geriatric day health services in the community. Journal of Family Practice, 20(2), 157-161.

Tate, L. A. (1988). Adult day care: A practical guidebook and manual. Activities, Adaptation and Aging, 11(2), 1-59.

Tennstedt, S. L., McKinlay, J. B., \& Sullivan, L. M. (1989). Informal care for frail elders: The role of secondary caregivers. The Gerontologist, 29(5), 677-683.

Theis, S., Moss, J., \& Pearson, M. (1994). Respite for caregivers: An evaluation study. Journal of Community Health Nursing, 11(1), 31-44. 
Tourigny, A., Côté, L., Laberge, A., Paradis, M., \& Joubert, P. (1993). Évaluation du programme québécois des centres de jour. Québec: Centre hospitalier de l'Université Laval: Unité de recherche en santé communautaire, Centre de santé publique de Québec.

Wallace, J. (1987). Factors affecting attendance in adult day care centers. Journal of Gerontological Social Work, 11(3/4), 155-165.

Warren, S., Ross-Kerr, J., Smith, D., \& Schalm, C. (2003). The impact of adult day programs on family caregivers of elderly relatives. Journal of Community Health Nursing, 20(4), 209-221.

Webb, L.C. (Eds.) (1989). Planning and Managing Adult Day Care: Pathways to Success. Owing Mills, Maryland: National Health Publishing (a division of Williams \& Wilkins).

Weissert, W. G. (1976). Two models of geriatric day care: findings from a comparative study. The Gerontologist, 16(5), 420-427.

Weissert, W. G., Elston, J. M., Bolda, E. J., Cready, C. M., Zelman, W. N., Sloane, P. D., et al. (1989). Models of adult day care: Findings from a national survey. The Gerontologist, 29(5), 640-649.

Weissert, W. G., Elston, J. M., Bolda, E. J., Zelman, W. N., Mutran, E., \& Magnum, A. B. (1990). Adult day care: Findings form a national survey. Baltimore, MD: John Hopkins University Press.

Wells, Y., Jorm, A., Jordan, F., \& Lefroy, R. (1990). Effects on care-givers of special day care programmes for dementia sufferers. Australian and New Zealand Journal of Psychiatry, 24(1), 82-90.

Wilson, R. S., McCann, J. J., Li, Y., Aggarwal, N. T., Gilley, D. W., \& Evans, D. A. (2007). Nursing home placement, day care use, and cognitive decline in Alzheimer's disease. American Journal of Psychiatry, 164(6), 910-915.

Wilson, R. S., Mendes de Leon, C. F., Barnes, L. L., Schneider, J. A., Bienias, J. L., et al. (2002). Participation in cognitively stimulating activities and risk of incident Alzheimer disease. Journal of the American Medical Association, 287, 742-748. 
Wimo, A., Mattsson, B., Adolfsson, R., Eriksson, T., \& Nelvig, A. (1993). Dementia day care and its effects on symptoms and institutionalization: A controlled Swedish study. Scandinavian Journal of Primary Health Care, 11, 117-123.

Wolinsky, F. D., \& Johnson, R. J. (1991). The use of health services by older adults. Journal of Gerontoloy: Social Sciences, 46(6), S345-257.

Woods, B., Spector, A., Jones, C., Orrell, M., \& Davies, S. (2005). Reminiscence therapy for dementia. [update of Cochrane Database Systematic Reviews 2000. Issue 4, Art. No.: CD001120. PMID: 11034700]. The Cochrane Database of Systematic Reviews 2005. Issue 2, Art. No.: CD001120.

Yeatts, D. E., Crow, T., \& Folts, E. (1992). Service use among low-income minority elderly: Strategies for overcoming barriers. The Gerontologist, 32(1), 24-32.

Yesavage, J. A., Brink, T. L., Rose, T. L., Lum, O., Huang, V., Adey, M., \& Leirer, V. O. (1983). Development and validation of a geriatric depression screening scale: A premiminary report. Journal of Psychiatric Research, 17(1), 37-49.

Zank S., \& Schacke, C. (2002). Evaluation of geriatric day care units: Effects on patients and caregivers. Journal of Gerontology, Series B: Psychological Sciences and Social Sciences, 52(4), 348-357.

Zarit, S. H., Orr, N. K., \& Zarit, J. M. (1985). The Hidden Victims of Alzheimer's Disease. New York: New York University Press.

Zarit, S. H., Stephens, M. A., Townsend, A., \& Greene, R. (1998). Stress reduction for family caregivers: Effects of adult day care use. Journals of Gerontology, Series B: Psychological Sciences and Social Sciences, 53, S267-277.

Zarit, S. H., Gaugler, J. E., \& Jarrott, S. E. (1999a). Useful services for families: Research findings and directions. International Journal of Geriatric Psychiatry, 14(3), 165177; discussion 178-181.

Zarit, S. H., Stephens, M. A. P., Townsend, A., Greene, R., \& Leitsch, S. A. (1999b). Patterns of adult day service use by family caregivers: A comparison of brief versus sustained use. Family Relations: Interdisciplinary Journal of Applied Family Studies, 48(4), 355-361. 


\title{
Annexe I \\ Article rédigé pour répondre aux exigences de l'examen de synthèse du programme d'études doctorales en Santé publique
}

\author{
Caregiver satisfaction with support services: \\ Influence of different types of services.
}

\author{
Jacinthe Savard ${ }^{1,2}$ \\ Nicole Leduc ${ }^{1,3}$ \\ Paule Lebel ${ }^{1,4}$ \\ François Béland ${ }^{1,3}$ \\ Howard Bergman 1,3,5
}

${ }^{1}$ Groupe de recherche SOLIDAGE, Faculté de Médecine, Université de Montréal et Université McGill

${ }^{2}$ Clinique universitaire interprofessionnelle de réadaptation, Université d'Ottawa

${ }^{3}$ Groupe de recherche interdisciplinaire en santé (GRIS), Faculté de Médecine, Université de Montréal

${ }^{4}$ Centre d'expertise sur la santé des personnes âgées et des aidants (CESPA), Institut universitaire de gériatrie de Montréal

${ }^{5}$ Division of Geriatric Medicine, Université McGill et Jewish General Hospital, Montréal

Soumis : 10/05/04, accepté pour publication : 08/04/05

Publié : Journal of Aging and Health, 18(1), 3-27 (2006) 


\section{AUTHOR'S NOTE}

The first author was supported during the present study through a doctoral award from the Alzheimer Society of Canada. The study used data collected during the System of Integrated Care for Older Persons (SIPA) project, funded by the Health Transition Funds of Health Canada, the Canadian Health Services Research Foundation and the Ministry of Health and Social Services of the Quebec Province. The authors wish to thank Luc Dallaire and John Fletcher, research coordinators with the SIPA project for their helpful cooperation during data analysis, Mary Egan for her helpful comments on the article, and Danielle Boisvert, for her help with reference management.

Reprints can be obtained from Jacinthe Savard, c/o Nicole Leduc, Groupe de recherche interdisciplinaire en santé, Faculté de Médecine, Université de Montréal, C.P. 6128, Succ. Centre-Ville, Montréal (Québec) H3C 3J7; e-mail: savard.gelinas@sympatico.ca or nicole.leduc@umontreal.ca. 


\section{ABSTRACT}

Objectives: To examine factors influencing satisfaction with support services of caregivers of frail older adults, and to determine what types of support services are associated with greater satisfaction, controlling for frail individual and caregiver characteristics. Methods: The study includes 291 frail older adults-caregiver dyads from Montreal in which caregivers had received support services. The CSQ-8 was used to measure caregiver satisfaction with these services. Results: Caregivers receiving information, advice or emotional support, and those caring for seniors receiving integrated care were more likely to be highly satisfied. Other factors increasing satisfaction were fewer number of health problems of frail individuals, caregiver being the spouse of the frail person, as well as greater caregiver perceived health, autonomy in IADL, and available social support. Discussion: The results support the importance of integrated care for frail seniors and informational services for their caregivers.

Keywords: caregivers, client satisfaction, community long-term care, frail seniors, integrated care 


\section{Introduction}

Frail older adults receive the vast majority of their support from family members and, in some cases, from friends or neighbors (Connidis, 2001). Many studies have pointed out the perceived burden resulting from caregiving as well as the risks for the health of the caregivers (Chenoweth \& Spencer, 1986; Schulz \& Beach, 1999). In light of these findings, it is imperative that health care providers for older clients also consider caregivers' needs for care and support services (Brotman \& Yaffe, 1994; Brown, Potter, \& Foster, 1990; Ducharme, Lebel, \& Bergman, 2001; Schulz \& Beach, 1999). In this context, research on caregivers' perceptions of services is also needed (Rosenthal \& Shannon, 1997). Satisfaction is an important outcome of care. Studies of satisfaction with medical care have demonstrated its positive link with higher use of care, greater compliance to treatment, and higher chances to obtain positive results from care (Attkisson \& Greenfield, 1995; Pascoe, 1983; Wellwood, Dennis, \& Warlow, 1995). In the area of geriatric care, Proctor, MorrowHowell, Albaz, and Weir (1992) report a study in which it was found that the perceptions of benefits obtained from a geriatric rehabilitation unit 1 week after discharge predicted continued community stay at 6 weeks post-discharge. The important role of caregivers in the use of services by frail seniors is also well documented (Bass \& Noelker, 1987).

Common support services for caregivers include information and advice on the illness of their older relative or friend and the resources available to help them with their care; emotional support either through a support group or individual counselling; respite offered at home, in day care centers or by temporary admission to an institution; and provision of home care services to assist the frail seniors to complete activities of daily living (ADL) or instrumental activities of daily living (IADL) tasks, relieving caregivers from some of these responsibilities.

Existing studies of caregiver satisfaction are often limited to one type of services. For example, caregivers report high levels of satisfaction with respite services (Henry \& Capitman, 1995; Jarrott, Zarit, Parris-Stephens, Townsend \& Greene, 1999; Weissert et al., 1990) and home care services (Chiu, 1997). Most of these studies did not examine factors 
leading to different levels of satisfaction. We found only four studies that examined factors influencing caregiver satisfaction with services designed to support them in the caregiving role. These studies measured satisfaction with home-based respite (Bear, Griffin, Sauer, \& Milspaugh, 1999), day care for older persons in general (Weissert et al., 1990) or for cognitively impaired seniors (Jarrott et al., 1999), and a variety of community long-term care services, including some support services for caregivers (Durand, Krueger, Chambers, Grek, \& Charles, 1995). In these studies, the characteristics of the frail seniors associated with lower caregiver satisfaction are ADL impairment, dementia or at least one disruptive behavior (Durand et al., 1995), and increasing age (Weissert et al., 1990). The caregiver variables associated with lower caregiver satisfaction are being a female, being of a different cultural heritage than the majority, not living with the frail senior or thinking about placement for the frail senior (Durand et al., 1995), and caregivers not working (Weissert et al., 1990).

Other researchers have studied caregiver satisfaction with medical care or discharge planning of their older relative. Areas that generally receive lower satisfaction scores in these studies are often related to information they received on diagnosis, course of illness or community services (Haley, Clair, \& Saulsberry, 1992; van Hout, Vernooij-Dassen, Hoefnagels, \& Grol, 2001; Venohr et al., 2001), or communication between hospital staff and the caregiver (Jones \& Lester, 1994; Wellwood et al., 1995). Other variables that have demonstrated association with satisfaction with medical care in general are also identified as important control variables to include in any studies of satisfaction. They are age, education, marital status and social status (Hall \& Dornan, 1990; Hall, Roter and Katz, 1988); life satisfaction; and health status (Cleary \& Mc Neil, 1988; Hall, Feldstein, Fretwell, Rowe, \& Epstein, 1990).

The objective of the present study is to examine which types of services (information and advice, emotional support, respite, help with ADL or IADL tasks, other) and what model of care delivery (regular or integrated care) are associated with greater satisfaction controlling for health and functional status of the frail senior as well as caregiver sociodemographic characteristics, relationship with the frail individual, health 
i-6

and functional status, feeling of burden and social support. From the literature review on caregiver satisfaction, it was expected that receiving information and advice would be associated with greater satisfaction. As respite is often identified as an area of great need in studies of caregivers' needs (Caserta, Lund, Wright, \& Redburn, 1987; Fortinsky \& Hathaway, 1990; Theis, Moss, \& Pearson, 1994), it was also expected to be related to greater caregiver satisfaction. Finally, it was expected that receiving integrated care would lead to greater caregiver satisfaction (Bergman et al., 1997).

\section{Design and Method}

This study uses data collected as part of a larger study, the Services Intégrés pour Personnes Agées (System of Integrated care for Older Persons; SIPA) project, which evaluated integrated services for the frail seniors, based on a model of care developed by a group of researchers and health care managers from McGill University and Université de Montréal (Bergman et al., 1997). The model was applied with some changes in a demonstration project implemented in two Centres locaux de services communautaires (CLSC) of Montreal.

In Quebec, regular home care services are provided by CLSCs. CLSCs are part of the Quebec Ministry of Health and Social Services network and exist throughout the province. Their home care services include home nursing, social work, rehabilitation services, help with personal care and IADL, home respite, referrals to community agencies, and evaluation of admissibility and referrals for admission to long term care institutions. These services are free of charge, and access is on the basis of evaluated need. As the amount of services available from the CLSC is often limited, some clients may buy additional services from other service providers. This happens mainly in the area of help with IADL, an area receiving less priority for CLSC publicly funded services. The government promotes the development of nonprofit organizations to provide house cleaning services or meal delivery at low cost for seniors. In the CLSCs, a team member designated as the primary respondent for the client is responsible for coordinating the services provided through this organization. Although CLSC care providers often refer 
clients to other health care organizations, they are rarely involved in the planning of care to be provided by these other organizations. The caseload of a primary respondent can be as high as 100 clients.

The SIPA project was administered by the two participating CLSCs as a very distinct program. As a demonstration project, it was offered to a limited number of clients. In contrast to the regular CLSC care, in the SIPA project, a case manager with a multidisciplinary team assumes the clinical responsibility as well as the responsibility for the optimal use of resources throughout the system of care for the client. The team is responsible for assessing and carrying out most of the community-based services including the same kind of services as those regularly offered by the CLSC. Other important characteristics of the demonstration project include availability of a telephone service 24 hours a day, 7 days a week, for quick and personalized response; the use of interdisciplinary protocols linking community and primary medical care; capacity to rapidly mobilize resources to respond to needs and avoid inappropriate hospital utilization; and in case of hospitalization, follow-up with the hospital team. To facilitate rapid mobilization of resources, the SIPA team had access to reserved places in residences for seniors and to a budget for increased temporary home care services. Also, the caseload of case managers was limited to 40 clients to insure adequate and personalized response to needs.

\section{Study Population}

From January to August 1999, the SIPA team recruited 1,230 senior participants from persons receiving home care services from the two participating CLSCs, who met the following criteria: presented with disabilities as measured by a score of -10 or less on the Functional Autonomy Measurement System (Hébert, Carrier \& Bilodeau, 1984; 1988), were aged 65 or over, and were not institutionalized. In addition, they needed to speak French or English or have a relative or close friend who could communicate with health care professionals and research personal in French or English. Of the 1,230 senior subjects recuited in 1999, 824 were followed-up in 2000, and 734 of these participants indicated that they had a caregiver. 
Participants for the present study are limited to frail older adults - caregiver dyads, in which the caregiver reported having received at least one support service during the study period and answered the questions on caregiver satisfaction with support services. Caregivers needed to understand French or English to be included in this analysis. There were 441 dyads meeting these criteria. From this number, 130 were excluded from the analysis because of missing data on important variables either from the caregiver or the frail senior questionnaire. Twenty dyads were also excluded because the frail person had been admitted to an institution before the administration of the satisfaction questionnaire. Comparison between included and excluded dyads on the sociodemographic variables listed in Table 1 revealed that they were similar except for the two following statistically significant differences $(\mathrm{p}<.05)$ : Excluded caregivers were less likely to be married, and excluded caregivers and frail seniors were more likely to speak a language other than French or English at home. Analyses are based on a final sample of 291 dyads. A little more than half of these dyads $(n=163)$ received the integrated care being evaluated in the larger study. The others $(n=128)$ received regular care from their CLSC.

\section{Measurements}

The dependent variable, caregiver satisfaction, was measured by the Client Satisfaction Questionnaire (CSQ-8; Larsen, Attkisson, Hargreaves, \& Nguyen, 1979). The CSQ-8 is a measure of general satisfaction that has been used in a wide variety of settings. It can be used to measure global satisfaction with various types of services. Questions are rated on four-level Likert-type scales. This instrument includes questions on the following eight items: the general quality of services, if the participant received the kind of services he or she wanted, the extent to which the services met the participant's needs, if the participant would recommand the same services to a friend, satisfaction with the amount of help received, if services helped the participant to deal more effectively with his or her problems, a general evaluation of the degree of satisfaction with the services received, and if the participant would use the same services again if needed. Possible scores range from 8 to 32, a higher score indicating a higher degree of satisfaction. Cronbach's alphas for internal consistency of the CSQ-8 range from .83 to .93 across studies (Attkisson \& 
Greenfield, 1995). It is one of the rare general satisfaction tools that has been validated in both French and English (Sabourin, Pérusse, \& Gendreau, 1989). In our study, questions were asked relating to the general satisfaction considering all services received by the caregivers during the last 12 months. Chronbach's alpha for our sample of caregivers was .93 .

There were two independent variables. The first independent variable was caregiver support services. Caregivers were asked which of the following six types of services they had received in the past 12 months: (a) information and advice, (b) emotional support, (c) respite, (d) help with personal care tasks previously completed by the caregiver, (e) help with domestic tasks previously completed by the caregiver, and (f) other services. These services could have been provided by the CLSC regular team or the SIPA team, other community agencies, or purchased privately. The second independent variable was the model of care delivery: integrated care or regular CLSC care.

The several control variables included sociodemographic information, health, and functional status variables for caregivers and frail seniors, as well as caregiver social support and perceived burden.

Sociodemographic information for caregivers. This information included caregiver's age, gender, marital status, country of birth, language spoken at home and with health professionals, religion, education, working status, type of relationship with the frail individual, coresidency status, and length of time of caregiving on a continuous basis. As type of relationship and coresidency status was expected to be highly correlated, a combined variable with three categories was created: spouse, other coresident caregiver, non coresident caregiver. For the length of time of caregiving, many caregivers did not give an exact number of years so the variable was coded on a four-point scale (fewer than 3 years, 3 to 5 years, 5 to 10 years and equal to or more than 10 years). Sociodemographic information for frail seniors included age, gender, and whether the individual was awaiting placement in an institution. Marital status, country of birth, language spoken, religion, and education were recorded mainly for descriptive purposes as they were expected to be too highly correlated to caregiver variables to be included in the analysis. 
Health and functional status of caregivers and frail seniors. Measurements used with both caregivers and frail seniors included number of health problems, presence of sensory or communication problems, and independence in ADL and IADL. To evaluate the number of health problems, respondents were asked if they had any of the 14 conditions included in a list of conditions taken from the Established Populations for Epidemiologic Studies of the Elderly (Cornoni-Huntley, Brook, Ostfeld, Taylor, \& Wallace, 1986). The conditions include high blood pressure, heart disease, circulation problems, stroke, diabetes, respiratory problems, rheumatism or arthritis (joint or bone pain), tumor or cancer, emotional problems (nervousness, depression, anxiety, or insomnia), Parkinson's disease, cataracts, glaucoma, stomach problems (gastritis, ulcer, hiatus hernia), or recent hip or leg fracture (during the past year). Memory problems or Alzheimer's disease, as well as bladder and kidneys problems, were excluded from that list to test the influence of cognitive impairment and incontinence separately. The total positive answers gave a single score for the number of health problems. Presence of sensory or communication problems was evaluated by asking respondents if they had no difficulty, some difficulty or a lot of difficulty recognizing people or reading, hearing, or being understood when they speak, even when using appropriate technical aids (glasses, hearing aid) if applicable. A dichotomous score was then created with those indicating a lot of difficulty in at least one of the four activities categorized as having sensory or communication problems. ADL independence was measured with a version of the Barthel Index described by Granger, Albrecht, and Hamilton (1979). Scores can range from 0 to 100, the higher score indicating total independence. IADL independence was measured using 7 items from the Older American Resources and Services questionnaire (Fillenbaum, 1988). Scores can range from 0 to 14, the higher score indicating total independence. As an additional measure of caregiver health, caregivers' perceived health was also recorded. It was assessed with a single question asking caregivers to rank their own health as compared to other persons of the same age group on a 6 -point scale $(1=$ excellent to $6=$ very poor $)$. Additional health measures of the frail seniors were presence of incontinence and cognitive impairment. Presence of incontinence, included in the Barthel Index, was treated as a distinct variable in some analyses. It was coded on a t3-point scale (no incontinence; occasional urinary or 
fecal incontinence; frequent urinary or fecal incontinence, and using catheters or having a colostomy). Cognitive impairment was assessed with the Short Portable Mental Status Questionnaire (Pfeiffer, 1975). Chronbach's alpha for our sample was .83. Pfeiffer (1975) suggests that a score of 3 or more errors on the 10 questions is indicative of possible cognitive impairment for persons with at least some high school education. He suggests allowing one additional error for persons with less than high school education and one less error for those with college or university education, as well as an additional error for African Americans. Pfeiffer's recommendations were used to classify participants as cognitively impaired or not, adapting the correction for ethnicity to the multiethnic context of the city of Montreal, allowing one additional error for subjects who were not born in Canada.

Quality of social support for the caregivers. This was assessed with 15 items taken from the 19-item Medical Outcomes Study Social Support Survey (MOS-SSS; Sherbourne $\&$ Stewart, 1991). The items used represent 3 of the 4 dimensions of support included in the MOS-SSS scale: emotional and informational support, positive social interaction, and affectionate support. The instrumental support scale was not used because this type of support was assessed in more detail with the questions on the type of services received. Possible scores for the 15-item scale range from 15 to 75 , with higher scores indicating more frequent availability of social support. Chronbach's alpha for our caregiver sample was .96 .

Subjective burden of the caregivers. This was measured with the 22-item Burden Interview (Hébert, Bravo, \& Girouard, 1993; Zarit, Orr, \& Zarit, 1985). Possible scores range from 0 to 88 , with higher scores indicating higher level of burden. Chronbach's alpha for our caregiver sample was .91 .

\section{Data collection}

Data were obtained from two questionnaires administered by interview from May to October 2000, one to the caregivers and the other to the frail seniors. Respondents were interviewed at home by trained interviewers. Each interview required an average of an hour 
to complete. In $25.8 \%$ of the sample, the frail seniors' interviews were completed by a proxy, as the individual was unable to answer because of frailty, cognitive impairment, or language barrier. In another $15.4 \%$ of the sample, the frail seniors were helped by a relative or a friend to answer the questionnaire. To ascertain if an individual was admitted to a longterm care institution at the time of the survey or awaiting placement, the study team accessed databases from the Montreal Regional Health Board, which records every request for admission and actual admission to publicly funded institutions in Montreal. ${ }^{1}$

\section{Analyses}

Descriptive statistics were computed first for each of the variables, followed by bivariate analysis of the relationship between each independent and control variable and satisfaction. To better understand the influence of the different variables on caregiver satisfaction, regression analyses were performed. Multiple linear regression was not appropriate, as the distribution of the satisfaction score was not normal and traditional methods of transformation did not improve the distribution. This is not unusual with satisfaction scores (see Hall et al., 1990). Instead, polytomous logistic regressions were performed with the satisfaction score categorized into a three-level outcome variable: lower satisfaction (8 to 20), average satisfaction (21 to 30), and higher satisfaction (31 and 32). The satisfaction cut-offs correspond approximately to one standard deviation below and above the mean for our sample. Identifying factors that distinguish extreme satisfaction levels seemed important, as it is possible that dissatisfaction of the caregiver could affect negatively the continuation of care in the community for the frail senior.

Correlations between independent and control variables were examined, and variables were selected to avoid multicolinearity problems. Continuous variables were tested for linearity. If their relationship with satisfaction was not linear, the variables were categorized. Variables were grouped into two categories: Caregiver and frail senior variables, and service variables. Caregiver and frail senior variables were first entered in the model. As suggested by Hosmer and Lemeshow (2000), variables that demonstrated relationship with satisfaction at the $p \leq .25$ level in the bivariate analysis were considered 
for entry in the regression analysis, as it is possible that variables that are weakly associated to the outcome at the bivariate level may become important predictors when taken together. A backward elimination procedure was then performed. When the model contained only variables significant at $p \leq .05$ for at least one of the comparisons, variables previously eliminated, were reentered one by one into the analysis to see if they would be significant in a model containing fewer variables. All service variables were then entered in the model and the process repeated to generate the final model. Pearson chi-square and deviance residuals statistics were used to assess the quality of the model. All analyses were conducted with SPSS software for Windows, version 11.0.

\section{Results}

\section{Description of the sample}

Sociodemographic characteristics of caregivers and frail seniors are reported in Table 1. The caregivers in our sample were between 22 and 92 years of age (mean 63.4 \pm 13.9 ), and somewhat more educated than the general population. ${ }^{2}$ Consistent with other caregiver studies, they were mostly women. Frail seniors were also mostly women. Their mean age was $83.2( \pm 6.9)$. Their education level was somewhat lower than the general population, which could be expected from their older age. Reflecting the higher multiethnic composition of the areas of the city where the study took place, ${ }^{2}$ only about half of both the caregivers and the frail seniors were born in Canada. Half of the sample is Francophone and about 20\% is Anglophone. The remaining 30\% had another maternal language and were, for the most part, multilingual. Only a small number of frail seniors were awaiting admission to an institution prior to the interview. In half of our sample, the caregiver lived with the frail individual. More than half of the caregivers have been providing care for more than 5 years.

\section{(Insert Table 1 around here)}

Health and functional status of the caregivers and the frail seniors are presented in Table 2 . The caregivers were in relatively good health, with $50.9 \%$ reporting excellent or 
very good health. Their mean number of health problems was $1.97( \pm 2.01)$. Their median scores on both the ADL and the IADL scales were the maximum possible, indicating total independence for the vast majority of the caregivers. In contrast, the frail seniors had on average $4.01( \pm 2.13)$ health problems. About one third (34.7\%) presented with important sensory problems, $21.3 \%$ had regular incontinence problems, and 36.8\% presented with cognitive impairments. Their ADL score (Barthel Index) indicated a variety of independence levels (range 0-100).

(Insert Table 2 around here)

\section{Caregiver support services}

Table 3 presents the percentage of caregivers who received each type of support services. The three services received most frequently were information and advice, help with personal care of the frail senior, and respite. Services mentioned under the "other services" category included medical and therapeutic services for the frail senior delivered at home $(n=30)$; help with personal care or domestic tasks of the frail individual that caregivers were not necessarily completing before $(n=9)$; arrangements for various services such as finding a doctor, a social worker or a residence; obtaining a handicapped parking sticker $(n=6)$; information and advice on various subjects, such as moving $(n=6)$; other therapeutic or evaluation services for the frail senior provided outside the home $(n=3)$; transportation $(n=2)$; and material provided for the care of the frail senior $(n=2)$. Most of the services were received from the CLSC regular team or SIPA team (over $83 \%$ for all services except help with domestic tasks [61\%] and other services [71\%]).

\section{(Insert Table 3 around here)}

Correlations among some services were high. Most of the caregivers who received emotional support also received information and advice (odds ratio $=3.47, p<.05$ ). Similarly, those receiving help with IADL tasks they previously assumed for the frail senior, were likely to be receiving help with the frail senior personal care (odds ratio $=$ $4.93, p<.05)$. Services highly correlated were grouped together to form four groups of 
services for further analyses: information, advice, and emotional support; respite; help with ADL or IADL; and other services.

\section{Caregiver satisfaction}

Mean caregiver satisfaction score was 26.08 out of $32( \pm 5.26)$. The distribution of the satisfaction scores was skewed toward the satisfied end with $27.1 \%$ of the caregivers $(n=79)$ reporting perfect or almost perfect satisfaction (CSQ-8 score: 31 or 32$)$. Another 38 caregivers (13\%) were markedly less satisfied with scores of 20 or less. Mean scores for each of the 8 questions were somewhat consistent with Item 3 (services met your needs) receiving the lowest mean score ( 3.07 out of $4 ; \pm 0.84$ ) and Item 4 (would recommend same services to a friend) receiving the highest mean score $(3.44$ out of $4 ; \pm 0.70)$.

\section{Factors influencing satisfaction}

In bivariate analysis of associations between various measures and satisfaction score, the following variables were associated with higher caregiver satisfaction $(p<.05)$ : higher caregiver IADL score; better perceived caregiver health; higher availability of social support; lower number of health problems for the frail senior; caregiver receiving information, advice or emotional support; caregiver receiving other services, and frail senior receiving integrated care.

The above sociodemographic and health variables were entered in the regression analysis along with the following: caregiver gender, education, working status, type of relationship with the frail person, number of health problems, burden score, and frail senior gender, sensory and communication problems, ADL score, and incontinence. Models were tested with and without caregiver working status and number of health problems, as well as frail senior gender, incontinence and ADL scores because of their high correlation with other variables. Inclusion of these variables did not change the results. All service variables were also entered in the model at the final step of the analysis. Results of the polytomous logistic regression comparing three groups of caregivers are presented in Table 4. Goodness-of-fit statistics for the polytomous logistic regression model were satisfactory. 
(Insert Table 4 around here)

Caregivers who report higher availability of social support and an intermediate level of burden, care for a person with a lower number of health problems, and receive information, advice or emotional support were more likely to be in the highly satisfied group than in the two other groups. Caregivers who were the spouse of the frail individual, had no difficulty in performing their IADL, had received other services, and who cared for a person receiving integrated care were also more likely to be in the highly satisfied group than in the lower satisfaction group. However, these later differences were not present when they were compared to those in the average satisfaction group.

More specifically, comparing the higher satisfaction group to the lower satisfaction group, the following significant differences were observed. Caregivers reporting no difficulty with their IADL were 4.5 times more likely to be highly satisfied. Caregivers reporting an intermediate level of burden were 8.2 times more likely to be highly satisfied than those reporting a low level of burden. For each increment of 1 on the Social Support Scale, odds of being in the higher satisfaction group were of 1.047. That means, for each increment of 10 on the Social Support Scale, the odds of being in the higher satisfaction group increase by $58 \%{ }^{3}$. Spouse caregivers were 3.5 times more likely to be in the higher satisfaction group. Caregivers of frail seniors with 5 or fewer health problems were 10.9 times more likely to be in the higher satisfaction group than those caring for persons with 6 or more health problems. Controlling for the previous variables, caregivers who had received information, advice or emotional support, and those who had received other services were respectively 6.2 times and 7.7 times more likely to be in the higher satisfaction group. Caregivers of seniors in the integrated care group were 2.9 times more likely to be in the highly satisfied group.

When the higher satisfaction group was compared to the average satisfaction group, four of the variables mentioned above remained significant: caregiver burden, caregiver social support, number of health problems of frail seniors and caregiver having received information, advice and/or emotional support. The differences were in the same direction, 
although of a lesser magnitude. Caregivers' perceived health also became significant. Caregivers who perceived themselves in excellent or very good health were 3.8 times more likely to be highly satisfied than those who perceived their health as good, fair or poor.

\section{Discussion}

The objective of the present study was to better understand caregiver satisfaction with support services by identifying factors associated with greater satisfaction. This study focused on the global experience of caregivers with a diversity of support services. Among the various factors identified, this discussion will differentiate between the individual characteristics of caregivers and frail seniors, and the characteristics of services. This is important because, as satisfaction with health care is usually high (Pascoe, 1983; Williams, 1994), interpretation of satisfaction surveys needs to take into account the relative satisfaction of different groups of users. Wellwood et al. (1995) state that if consumer satisfaction is to be used in program evaluation, it is important to differentiate those factors predicting satisfaction that are independent of the provider from those related to the services provided. However, associations between individual characteristics and the propensity to answer questions on satisfaction more positively may also reflect the fact that certain types of services are more appropriate for certain types of clients.

At the global level, our results are consistent with what has been found in the literature on satisfaction studies. Our mean satisfaction score was high, and the distribution was negatively skewed with more than one quarter of the sample reporting perfect or almost perfect satisfaction. Various factors were found to influence satisfaction, some related to the frail senior's or caregiver's characteristics and others related to the services provided. When examining these factors, our results reveal some similarities with previous findings, but also shed new light on other factors.

For the factors related to the services provided, this study took a unique orientation by trying to determine which type of services are associated with greater satisfaction. Our results showed that the reception of information, advice, and emotional support services increased the odds of being in the highly satisfied group. This is consistent with studies of 
caregiver satisfaction with medical care which have obtained lower satisfaction scores mainly in areas related to information exchange between health care professionals and caregivers (Haley et al., 1992; Jones \& Lester, 1994; van Hout et al., 2001; Venohr et al., 2001; Wellwood et al., 1995). As well, having received services other than those usually listed as caregiver support services decreased the odds of being in the lower satisfaction group. Although this finding is hard to interpret because of the wide variety of services included is this category, it may be indicative of a more personalized approach to responding to caregiver needs. The expected positive association between respite and caregiver satisfaction was not found. It is possible that among those receiving respite, some did not receive satisfactory amounts of this service. This research did not inquire about the amount of service received. Help with ADL or IADL was not related to caregiver satisfaction.

It is apparent that services associated with increased caregiver satisfaction were those that respond to caregivers' informational needs, rather than instrumental support needs. This observation relates to the findings of other research (Browers, 1987; Corcoran, 1994) that sheds light on the invisible work of the caregivers, such as planning for the future, protecting the frail senior self-image, and so forth. According to Lavoie (2000), adequate support to caregivers in these intangible tasks requires more intense and flexible services that include accompaniment, information, and advice.

As expected, caregivers of seniors receiving integrated care were also more likely to be highly satisfied. Part of the increased satisfaction could be due to the integrated care providing access to a wider variety of services or to more appropriate services. However, our data did not show associations between integrated care and caregivers receiving any particular type of service. It is also possible that the integrated care provided a greater amount of these services. Again, this research did not inquire about the amount of specific services received by the caregivers. However, we know that SIPA participants, in general, received more community care (possibly including more support services for caregivers) and less institutional care than non participants (Béland, Bergman, Lebel, et al., in press). As most seniors prefer to stay at home whenever possible, this transfer of resources from 
institutional care to community care may increase their quality of life as well as the satisfaction of their caregivers. In addition, in anecdotal qualitative data, caregivers from the integrated care group reported they felt secure, knowing that if something happened, they could easily get in touch with the case manager and there would be a rapid response. This may also explain the increased satisfaction.

For the factors related to the frail senior or caregiver characteristics, caregiver assessments of their perceived health, IADL scores, burden, available social support, and relationship with the frail individual, as well as number of health problems of the frail senior, were found to be predictors of high caregiver satisfaction.

The link between caregiver health variables and satisfaction is consistent with what has been reported in studies of satisfaction with medical care: Users in better health are usually more satisfied (Cleary \& Mc Neil, 1988; Hall et al., 1990).

Caregivers reporting greater availability of social support were more likely to be highly satisfied. Spouse caregivers were less likely to have lower satisfaction. Spouse caregivers also reported higher availability of social support (odds ratio: 2.79, $p<.05$ ). Caregiver informal support networks and formal support services can complement each other. Thus, it is possible that caregivers with greater social support may have been more satisfied with the support services they received because they expected and required less, having already had some of their needs met through their informal networks. Another possible explanation is that caregivers included formal support when answering some of the social support questions such as "Do you have someone who understands your problems?" If this had been the case, social support could have correlated with the amount or type of services received. In our data, social support did not correlate with the receipt of any particular type of formal services.

Caregivers with intermediate levels of burden had increased odds of being highly satisfied, compared to those reporting lower or higher burden. A tentative explanation may be that those with low burden may not be recognized as needing services from health care professionals, and for this reason, they may receive less services. Those with higher burden 
may be in need of more intensive services than what is available. Our data do not permit the validation of these assumptions as we do not know the amount of services received. We can simply observe that, in this sample, burden is not correlated to receiving any particular type of services.

The link between number of health problems of the frail seniors and caregiver satisfaction is also consistent with previous studies of caregiver satisfaction, although expressed differently. Other researchers (Durand et al., 1995; Weissert et al., 1990) have found age of the frail individual, presence of disruptive behaviors (because of cognitive impairment), or higher ADL impairments to be associated with lower satisfaction. These three frail senior characteristics were somewhat associated with their number of health problems. In our study, frail senior age and cognitive impairment were not associated with caregiver satisfaction. Frail senior ADL impairment and incontinence were not associated with caregiver satisfaction when their number of health problems was controlled. Our findings suggest that the latter would be the most important factor influencing caregiver satisfaction. Furthermore, the data showed that the presence of specific types of impairments seemed to be compensated at least in part by an increased proportion of caregivers receiving some type of services. For example, in our sample, a greater proportion of caregivers of cognitively impaired older adults received information, advice, and emotional support as well as respite (odds ratio 2.45 and 2.00 respectively, $p<.05$ ) than caregivers of cognitively intact seniors did, and a greater proportion of caregivers of incontinent older adults received respite (odds ratio $=2.20, p<.05$ ) than caregivers of continent individuals did. In contrast, an increased number of health problems of the frail seniors was not significantly associated with an increased proportion of caregivers receiving any particular type of services.

Contrary to what is often reported in studies of satisfaction with medical care (Hall \& Dornan, 1990), we did not find any association between caregivers' age and their satisfaction with support services. Contrary to Durand et al. (1995), caregivers' cultural background was not associated with caregivers' satisfaction. 
The study contained a number of strengths and limitations. In terms of strengths, contrary to most of the existing caregiver satisfaction studies, this research used a validated instrument (the CSQ-8) to measure caregiver satisfaction. This is viewed as positive, as it will allow comparison with future studies using the same instrument. As well, study participants were from a population-based sample of caregivers and older adult care recipients.

In terms of limitations, the present study recorded only the type of service and not the amount of service received by the caregivers. The amount of services used may be an important factor associated with satisfaction. Another limitation comes from the conceptual distinction between services directed to caregivers and services directed to the frail seniors. This service orientation was not always clear for our respondents. When asked about other services they had received as caregivers, many respondents mentioned services that, as researchers, we would have considered to be directed to the frail seniors. This limits the interpretation of our findings regarding the effects of the category "other services" on caregiver satisfaction. Another point that deserves attention is the fact that, in this study, cost of services was not included in the variables under investigation, as most of the services studied were provided free of charge by the Quebec public Health and Social Services system. This is a limitation to the generalization of the findings to other contexts.

In conclusion, one important function of a consumer satisfaction survey is to provide useful information to help improve service delivery in the future (Wellwood et al., 1995; Jarrott et al., 1999). It is our hope that, despite the study's limitations, the findings presented here can serve such purpose. These findings indicate frail senior and caregiver characteristics which are important to control for when analyzing results of caregiver satisfaction surveys. They also demonstrate the importance of information, advice, and emotional support services to caregiver satisfaction. This should encourage every health care professional involved with frail older adults to communicate with primary caregivers whenever possible, as information appears to be very important for them. Finally, the results of this study confirmed the positive effects of integrated care on caregiver satisfaction. 
Notes

${ }^{1}$ In Quebec, the vast majority of nursing homes and chronic care institutions are publicly funded. However, although health care is provided for free in these institutions, a resident's contribution is expected to cover the housing costs.

2 According to the 1996 census, 43\% of the Montreal population have post secondary education. Immigrants make up $30 \%$ of the population of Montreal (Statistique Canada, 2002).

${ }^{3}$ This results comes from: $\mathrm{B}=0.046, \mathrm{e}^{\mathrm{B}}=1.047$ and $\mathrm{e}^{10 \mathrm{~B}}=1.584$ 
Table 1: Sociodemographic characteristics of and relationships in caregiver-elder dyads

\begin{tabular}{|c|c|c|c|c|}
\hline \multirow[b]{2}{*}{ Variables } & \multicolumn{2}{|l|}{ Caregivers } & \multicolumn{2}{|l|}{ Frail seniors } \\
\hline & $\%$ or mean & $\mathrm{n}$ & $\%$ or mean & $\mathrm{n}$ \\
\hline Age: mean ( \pm s.d.) & $63.4( \pm 13.9)$ & 291 & $83.2( \pm 6.9)$ & 291 \\
\hline Gender & & & & \\
\hline $\begin{array}{l}\text { - men } \\
\text { - women }\end{array}$ & $\begin{array}{l}32.0 \% \\
68.0 \%\end{array}$ & 291 & $\begin{array}{l}31.3 \% \\
68.7 \%\end{array}$ & 291 \\
\hline $\begin{array}{l}\text { Marital status } \\
\text { - married or marital relationship } \\
\text { - other }\end{array}$ & $\begin{array}{l}71.5 \% \\
28.5 \%\end{array}$ & 291 & $\begin{array}{l}38.3 \% \\
61.7 \%\end{array}$ & 290 \\
\hline $\begin{array}{c}\text { Country of birth } \\
\text { - Canada } \\
\text { - other }\end{array}$ & $\begin{array}{l}57.2 \% \\
42.8 \%\end{array}$ & 290 & $\begin{array}{l}49.0 \% \\
51.0 \%\end{array}$ & 290 \\
\hline $\begin{array}{l}\text { Religion } \\
\text { - Catholic } \\
\text { - Jewish } \\
\text { - other (including none) }\end{array}$ & $\begin{array}{l}59.9 \% \\
22.1 \% \\
18.0 \%\end{array}$ & 289 & $\begin{array}{l}58.3 \% \\
22.6 \% \\
19.1 \%\end{array}$ & 288 \\
\hline $\begin{array}{l}\text { Language } \\
\text { - French at home and with health professionals } \\
\text { - English at home and with health professionals } \\
\text { - French or English or both with health prof. } \\
\text { - other than French or English with health prof. }\end{array}$ & $\begin{array}{l}50.9 \% \\
22.0 \% \\
26.8 \% \\
0.3 \%\end{array}$ & 291 & $\begin{array}{l}52.2 \% \\
20.7 \% \\
23.5 \% \\
3.6 \%\end{array}$ & 276 \\
\hline $\begin{array}{l}\text { Education } \\
\text { - none } \\
\text { - elementary school (grade school) } \\
\text { - high school } \\
\text { - technical or trade school } \\
\text { - university }\end{array}$ & $\begin{array}{l}0.0 \% \\
12.7 \% \\
26.1 \% \\
17.2 \% \\
44.0 \%\end{array}$ & 291 & $\begin{array}{l}3.2 \% \\
31.4 \% \\
35.0 \% \\
10.5 \% \\
19.9 \%\end{array}$ & 277 \\
\hline $\begin{array}{c}\text { Working } \\
\text { - yes } \\
\text { - no }\end{array}$ & $\begin{array}{l}33.3 \% \\
66.7 \%\end{array}$ & 291 & & \\
\hline $\begin{array}{l}\text { Type of relationship and co-residency } \\
\text { - spouse } \\
\text { - other co-resident caregiver } \\
\text { - other non co-resident caregiver }\end{array}$ & $\begin{array}{l}33.7 \% \\
18.9 \% \\
47.4 \%\end{array}$ & 291 & & \\
\hline $\begin{array}{l}\text { Length of time caregiving on a continuous basis } \\
\text { - less than } 3 \text { years } \\
-3 \text { to } 5 \text { years } \\
-5 \text { to } 10 \text { years } \\
-10 \text { years or more }\end{array}$ & $\begin{array}{l}22.0 \% \\
21.6 \% \\
24.7 \% \\
31.7 \%\end{array}$ & 287 & & \\
\hline Awaiting placement in an institution & & & $4.1 \%$ & 291 \\
\hline
\end{tabular}


Table 2: Health and functional status of caregivers and elders

\begin{tabular}{|c|c|c|c|c|}
\hline \multirow[b]{2}{*}{ Variables } & \multicolumn{2}{|c|}{ Caregivers } & \multicolumn{2}{|c|}{ Frail seniors } \\
\hline & $\%$ or mean & $\mathrm{n}$ & $\%$ or mean & $\mathrm{n}$ \\
\hline Number of health problems: mean ( \pm s.d.) & $1.97( \pm 2.0)$ & 291 & $4.01( \pm 2.13)$ & 291 \\
\hline Presence of important sensory problems & $3.4 \%$ & 291 & $34.7 \%$ & 291 \\
\hline ADL score: mean ( \pm s.d.) & $99.2( \pm 2.4)$ & 290 & $75.6( \pm 24.9)$ & 291 \\
\hline IADL score: mean ( \pm s.d.) & $13.5( \pm 1.0)$ & 291 & $7.0( \pm 4.0)$ & 289 \\
\hline $\begin{array}{l}\text { Perceived health } \\
\text { - excellent } \\
\text { - very good } \\
\text { - good } \\
\text { - fair } \\
\text { - poor } \\
\text { - very poor }\end{array}$ & $\begin{array}{c}27.2 \% \\
23.7 \% \\
33.3 \% \\
11.3 \% \\
3.1 \% \\
1.4 \%\end{array}$ & 291 & & \\
\hline Availability of social support: mean ( \pm s.d.) & $57.9( \pm 15.0)$ & 291 & & \\
\hline Burden: mean ( \pm s.d.) & $28.6( \pm 15.8)$ & 291 & & \\
\hline $\begin{array}{l}\text { Burden categorized } \\
\quad \text { - low (0 to } 26) \\
\quad \text { - intermediate (26.1 to } 35) \\
\text { - high ( } 35.1 \text { to } 72)\end{array}$ & $\begin{array}{l}49.5 \% \\
19.5 \% \\
31.0 \%\end{array}$ & & & \\
\hline $\begin{array}{l}\text { Presence of incontinence } \\
\text { - none } \\
\text { - occasionally } \\
\text { - regularly (including using a catheter or colostomy) }\end{array}$ & & & $\begin{array}{l}53.3 \% \\
25.4 \% \\
21.3 \%\end{array}$ & 291 \\
\hline $\begin{array}{l}\text { Presence of cognitive impairment } \\
\text { - yes } \\
\text { - no }\end{array}$ & & & $\begin{array}{l}36.8 \% \\
63.2 \%\end{array}$ & 291 \\
\hline
\end{tabular}

Note: $A D L=$ activities of daily living; IADL = instrumental activities of daily living. 
Table 3: Support services received by the caregivers $(n=291)$

$\begin{array}{cc}\text { Type of services } & \begin{array}{c}\text { Percentage who received } \\ \text { this type of services }\end{array}\end{array}$

\begin{tabular}{lc}
\hline Information and advice & 70.1 \\
$\begin{array}{l}\text { Help with personal care tasks previously } \\
\text { completed by the caregiver }\end{array}$ & 66.0 \\
Respite & 44.3 \\
$\begin{array}{l}\text { Help with domestic tasks previously } \\
\text { completed by the caregiver }\end{array}$ & 34.4 \\
Emotional support & 21.0 \\
Other services & 20.3 \\
\hline
\end{tabular}


Table 4: Polytomous logistic regression of caregiver satisfaction on caregiver and frail senior variables, and type of services received (n=291)

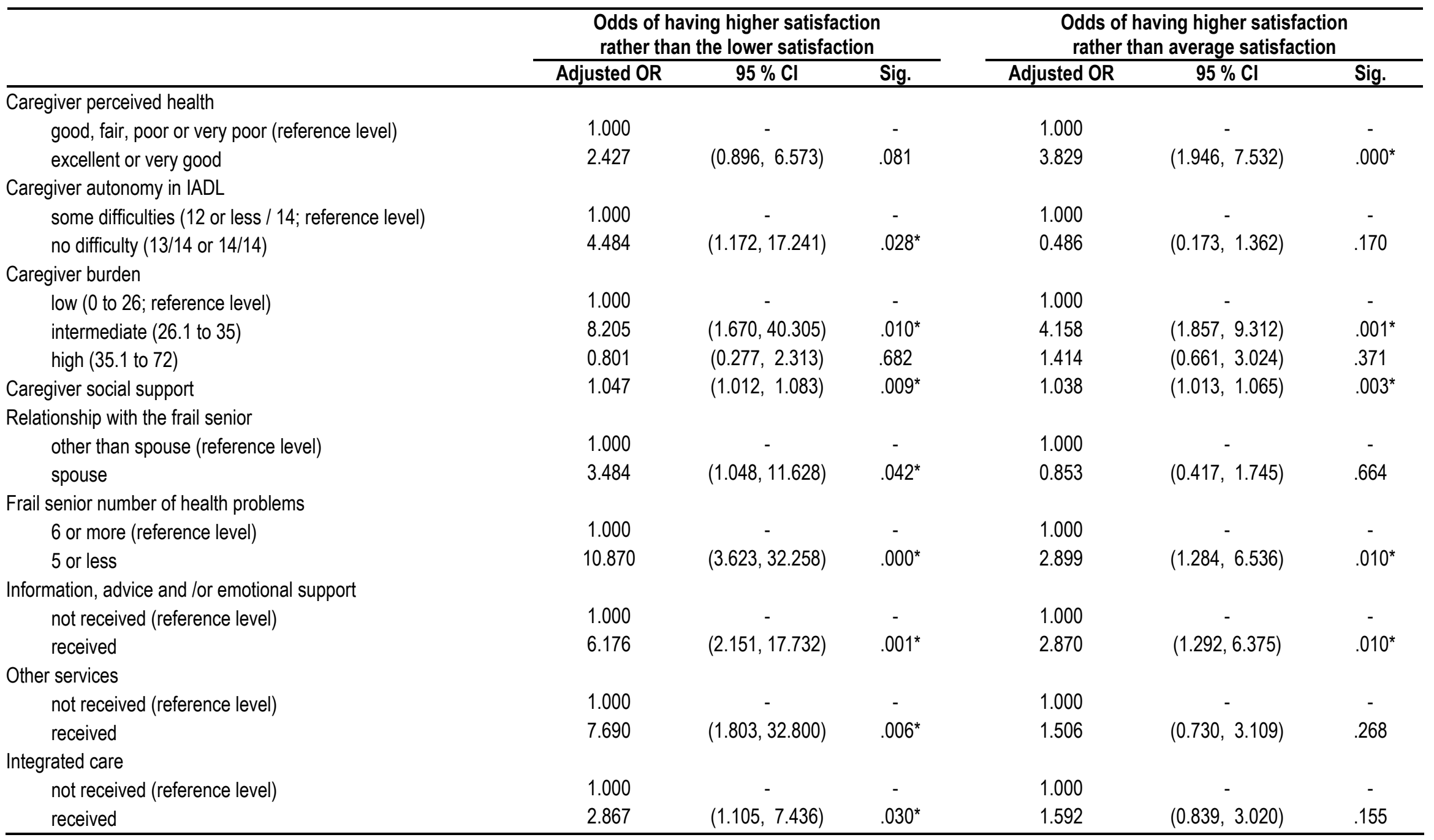

Note: $\mathrm{OR}=$ Odds ratio, $\mathrm{Cl}=$ Confidence interval. Goodness-of-fit statistics: Pearson: 531.279, $p=0.193$; Deviance: $393.226, p=1.000$

${ }^{*} p \leq .05$ 


\section{References}

Attkisson, C. C., \& Greenfield, T. K. (1995). The client satisfaction questionnaire (CSQ) scales: A history of scale development and a guide for users. San Francisco: University of California, Department of Psychiatry.

Bass, D. M., \& Noelker, L. S. (1987). The influence of family caregivers on elder's use of in-home services: An expanded conceptual framework. Journal of Health and Social Behavior, 28, 184-196.

Bear, M., Griffin, R., Sauer, M., \& Milspaugh, C. (1999). Measuring client satisfaction with home-based respite services. American Journal of Alzheimer's Disease, 14(6), 357-363.

Béland, F., Bergman, H., Lebel, P. (2005, in press). A system of integrated care for frail older persons (SIPA) in Canada: Results from a randomized controlled trial. Journal of Gerontology: Biological Sciences and Medical Sciences.

Bergman, H., Béland, F., Lebel, P., Contandriopoulos, A. P., Tousignant, P., Brunelle, Y., et al. (1997). Care for Canada's frail elderly population: Fragmentation or integration? Canadian Medical Association Journal, 157, 1116-1121.

Brotman, S. L., \& Yaffe, M. J. (1994). Are physicians meeting the needs of family caregivers of the frail elderly? Canadian Family Physician, 40, 679-685.

Browers, B. J. (1987). Intergenerational caregiving: Adult caregivers and their aging parents. Advanced Nursing Science, 9, 20-31.

Brown, L. J., Potter, J. F., \& Foster, B. G. (1990). Caregiver burden should be evaluated during geriatric assessment. Journal of the American Geriatrics Society, 38, 455460.

Caserta, M. S., Lund, D. A., Wright, S. D., \& Redburn, D. E. (1987). Caregivers to dementia patients: The utilisation of community services. The Gerontologist, 27, 209-214.

Chenoweth, B., \& Spencer, B. (1986). Dementia: The experience of family caregivers. The Gerontologist, 26, 267-272.

Chiu, L. (1997). Family caregiver's satisfaction with home care in the Taipei metropolitan area. Public Health Nursing, 14, 42-50. 
Cleary, P. D., \& Mc Neil, B. J. (1988). Patient satisfaction as an indicator of quality care. Inquiry, 25, 25-36.

Corcoran, M. A. (1994). Management decisions made by caregiver spouses of persons with Alzheimer's disease. American Journal of Occupational Therapy, 48, 38-45

Coroni-Huntley, J., Brook, D. B., Ostfeld, A., Taylor, J. O., \& Wallace, R. B. (1986). Established Populations for Epidemiological Studies of the Elderly, Resources Data Book: National Institutes of Health.

Connidis, I. A. (2001). Family Ties and Aging. Thousand Oaks, CA: Sage Publications, Inc. Ducharme, F., Lebel, P., \& Bergman, H. (2001). Vieillissement et soins, l'urgence d'offrir des services de santé intégrés aux familles du XXIe siècle. [Aging and care: Urgency to offer integrated health services to families of 21 st century]. Ruptures, revue transdisciplinaire en santé, 8(2), 110-121.

Durand, P. J., Krueger, P. D., Chambers, L. W., Grek, A., \& Charles, C. (1995). Predictors of caregivers' dissatisfaction with community long-term care services for seniors: Results from the Canadian Study of Health and Aging. Canadian Journal of Public Health, 86, 325-332.

Fillenbaum, G. G. (1988). Multidimensional functional assessment of older adults: The Duke Older American Resources and Services procedures. Hillsdale, NJ: Lawrence Erlbaum Associates.

Fortinsky, R. H., \& Hathaway, T. J. (1990). Information and service needs among active and former family caregivers of persons with Alzheimer's disease. The Gerontologist, 30, 604-609.

Granger, C. V., Albrecht, G. L., \& Hamilton, B. B. (1979). Outcome of comprehensive medical rehabilitation: Measurement by PULSES profile and the Bartel Index. Archives of Physiology \& Medical Rehabilitation, 60, 145-154.

Haley, W. E., Clair, J. M., \& Saulsberry, K. (1992). Family caregiver satisfaction with medical care of their demented relatives. The Gerontologist, 32, 219-226.

Hall, J., \& Dornan, M. (1990). Patient sociodemographic characteristics predictors of satisfaction with medical care: A meta-analysis. Social Science and Medicine, 30, 811. 
Hall, J.A., Feldstein, M., Fretwell, M. D., Rowe, J. W., \& Epstein, A. M. (1990). Older patient's health status and satisfaction with medical care in an HMO population. Medical Care, 28, 261-270.

Hall, J. A., Roter, D. L., \& Katz, N. R. (1988). Meta-analysis of correlates of provider behavior in medical encounters. Medical Care, 26, 657-675.

Hébert, R., Bravo, G., \& Girouard, D. (1993). Fidélité de la traduction française de trois instruments d'évaluation des aidants naturels de malades déments. [Reliability of the French translation of three evaluation instruments for family caregivers and dementia patients]. Canadian Journal on Aging, 12, 324-337.

Hébert, R., Carrier, R., \& Bilodeau, A. (1984). Système de mesure de l'autonomie fonctionnelle. [Functional Autonomy Measurement System]. Lévis, Québec, Canada: Centre hospitalier Hôtel-Dieu de Lévis.

Hébert, R., Carrier, R., \& Bilodeau, A. (1988). The Functional Autonomy Measurement System (SMAF). Description and validation of an instrument for the measurement of handicaps. Age \& Ageing, 17, 293-302.

Henry, M. E., \& Capitman, J. A. (1995). Finding satisfaction in adult day care: Analysis of a national demonstration of dementia care and respite services. Journal of Applied Gerontology, 14, 302-320.

Hosmer, D.W., \& Lemeshow, S. (2000). Applied Logistic Regression (2 ${ }^{\text {nd }}$ ed.). New-York: John Wiley \& sons, inc.

Jarrott, S. E., Zarit, S. H., Parris-Stephens, M. A., Townsend, A., \& Greene, R. (1999). Caregiver satisfaction with adult day service programs. American Journal of Alzheimer's Disease, 14, 233-244.

Jones, D., \& Lester, C. (1994). Hospital care and discharge: Patients' and carers' opinions. Age \& Ageing, 23, 91-96.

Larsen, D. L., Attkisson, C. C., Hargreaves, W. A., \& Nguyen, T. D. (1979). Assessment of client/patient satisfaction: Development of a general scale. Evaluation and Program Planning, 2, 197-207.

Lavoie, J.-P. (2000). Les aidantes familiales au Québec: De la redécouverte à la prise en compte de leur réalité. [Family caregivers in Québec: Taking into account their reality]. Le gérontophile, 22(1), 15-19. 
Pascoe, G. C. (1983). Patient satisfaction in primary care: A literature review and analysis. Evaluation and Program Planning, 6, 185-210.

Pfeiffer, E. (1975). A Short Portable Mental Status Questionnaire for the assessment of organic brain deficit in elderly patients. Journal of the American Geriatrics Society, $23,433-441$.

Proctor, E., Morrow-Howell, N., Albaz, R., \& Weir, C. (1992). Patient and family satisfaction with discharge plans. Medical Care, 30(3), 262-275.

Rosenthal, G. E., \& Shannon, S. E. (1997). The use of patient perceptions in the evaluation of health-care delivery systems. Medical Care, 35(11 Suppl), NS58-NS68.

Sabourin, S., Pérusse, D., \& Gendreau, P. (1989). Les qualités psychométriques de la version canadienne française du Questionnaire de satisfaction du consommateur de services thérapeutiques. [The psychometric qualities of the French Canadian version of the Client Satisfaction Questionnaire]. Canadian Journal of Behavioural Science, $21,149-159$.

Schulz, R., \& Beach, S. R. (1999). Caregiving as a risk factor for mortality: The caregiver health effects study. Journal of the American Medical Association, 282, 2215-2219.

Sherbourne, C. D., \& Stewart, A. L. (1991). The MOS Social Support Survey. Social Science and Medicine, 32, 705-714.

Statistiques Canada. (2002-12-27). Profil des communautés de 1996- Montréal (ville), Québec [Profileof communities from 1996-Montreal, Quebec]. Retrieved December 30, 2002, from http://www12.statcan.ca/francais/Profil/PlaceSearch Form1_F.cfm?LANG $=\mathrm{F}$

Theis, S., Moss, J., \& Pearson, M. (1994). Respite for caregivers: An evaluation study. Journal of Community Health Nursing, 11(1), 31-44.

van Hout, H. P., Vernooij-Dassen, M. J., Hoefnagels, W. H., \& Grol, R. P. (2001). Measuring the opinions of memory clinic users: Patients, relatives and general practitioners. International Journal of Geriatric Psychiatry, 16, 846-851.

Venohr, I., Fine, R., Saunders, V., Tenney, E., Vahan, V., \& Williams, M. (2001). Improving dementia care through community linkages: A multi-site demonstration project. Home Health Care Services Quarterly, 19(4), 51-63. 
Weissert, W.G., Elston, J.M., Bolda, E.J., Zelman, W.N., Mutran, E., \& Magnum, A.B. (1990). Adult day care: Findings form a national survey. Baltimore, MD: John Hopkins Univeersity Press.

Wellwood, I., Dennis, M., \& Warlow, C. (1995). Patients' and carers' satisfaction with acute stroke management. Age \& Ageing, 24, 519-524.

Williams, B. (1994). Patient satisfaction: A valid concept? Social Science and Medicine, $38,509-516$.

Zarit, S. H., Orr, N. K., \& Zarit, J. M. (1985). The Hidden Victims of Alzheimer's Disease. New York: New York University Press. 



\section{Annexe II \\ Variables étudiées dans les principales recherches présentées à la recension des écrits.}

Les tableaux qui suivent présentent la liste des variables étudiées dans les études recensées selon trois catégories:

a) études auprès des centres de jour dont l'échantillon est composé d'une clientèle âgée générale (avec ou sans démence) et de leur personne de soutien

b) études auprès des centres de jour dont l'échantillon est constitué de personnes de soutien de personnes atteintes de démence

c) étude auprès de services connexes

Les abréviations utilisées dans ces tableaux sont les suivantes :

\begin{tabular}{|c|c|c|}
\hline \multirow[t]{11}{*}{ Légende : } & $\#$ : nombre de... & HTA : haute tension artérielle \\
\hline & ADDTC : Alzheimer Disease Diagnostic and & MMSE : Mini- Mental State Evaluation \\
\hline & Treatment Center Program & PA : personne âgée \\
\hline & AVC : Accident vasculaire cérébral & PS : personne de soutien \\
\hline & AVQ : Activité de vie quotidienne & Prob. : problèmes \\
\hline & AVD : Activité de vie domestique & $\mathrm{p} / \mathrm{r}:$ par rapport \\
\hline & BRDRS : Blessed-Roth Dementia Rating Scale & SCO : Syndrome cérébral organique \\
\hline & $\mathrm{CJ}:$ centre de jour & Sympt. : symptômes \\
\hline & HMO : Health Maintenance Organization & \\
\hline & $*$ : facteurs qui ont démontré une relation significa & $<, 05)$ \\
\hline & $\ddagger$ : facteurs pour lesquels une tendance est observ & statistiquement significatif car « $\mathrm{n} »$ petit) \\
\hline
\end{tabular}


a) : études auprès des centres de jour dont l'échantillon est composé d'une clientèle âgée générale et de leur personne de soutien

\begin{tabular}{|c|c|c|c|c|c|c|c|}
\hline Variables & Chappell, 1983 & Wallace, 1987 & $\begin{array}{l}\text { Kosloski et coll., } \\
1994\end{array}$ & $\begin{array}{l}\text { Henry \& Capitman, } \\
1995\end{array}$ & Lynch et coll., 1999 & $\begin{array}{l}\text { Baumgarten et } \\
\text { coll., } 2002\end{array}$ & $\begin{array}{l}\text { Dabelko } \\
2004 \text { (1) et } 2005 \text { (2) }\end{array}$ \\
\hline \multicolumn{8}{|c|}{ Facteurs prédisposants } \\
\hline \multicolumn{8}{|c|}{ Personnes âgées } \\
\hline $\begin{array}{l}\text { Socio- } \\
\text { démographique }\end{array}$ & $\begin{array}{l}\text { - âge } \\
\text { - sexe } \\
\text { - éducation* } \\
\text { - profession* } \\
\text { - présence d'un } \\
\text { conjoint }\end{array}$ & $\begin{array}{l}\text { - } \text { sexe }^{*} \\
\text { - } \text { vit seule } \\
\text { - } \text { a des enfants* } \\
\text { - } \text { a un conjoint* } \\
\text { - } \text { a des frères et sœurs }\end{array}$ & - âge & - âge* & \begin{tabular}{|l} 
- âge \\
- sexe \\
- vit seule \\
- vit avec conjoint*
\end{tabular} & $\begin{array}{l}\text { - âge* } \\
\text { - } \text { sexe }^{*} \\
\text { - éducation } \\
\text { - présence d'une PS* } \\
\text { - vit seul }\end{array}$ & $\begin{array}{l}\text { - } \text { âge }^{\star 1} \\
\text { - sexe } \\
\text { - pratique religion ou pas } \\
\text { - éducation }{ }^{\star 2} \\
\text { - nombre d'individus dans } \\
\text { la maisonnée }{ }^{* 2} \\
\text { - } \text { a un conjoint*1 }\end{array}$ \\
\hline \multicolumn{8}{|c|}{ Personnes de soutien } \\
\hline $\begin{array}{l}\text { Socio- } \\
\text { démographique }\end{array}$ & & & & $\begin{array}{l}\text { - âge* } \\
\text { - sexe } \\
\text { - éducation* } \\
\text { - réseau soutien informel }\end{array}$ & & $\begin{array}{l}\text { - } \text { âge }^{*} \\
\text { - } \text { sexe }^{*} \\
\text { - éducation } \\
\text { - vit seule } \\
\end{array}$ & \\
\hline \multicolumn{8}{|c|}{ Personnes âgées et personnes de soutien } \\
\hline $\begin{array}{l}\text { Appartenance } \\
\text { culturelle } \\
\text { Lien PS / PA } \\
\text { Croyances et } \\
\text { attitudes }\end{array}$ & \begin{tabular}{|l} 
- langue parlée \\
- lieu de naissance \\
(Canada ou autre) ${ }^{*}$ \\
- religion
\end{tabular} & & - lien PS / PA * & $\begin{array}{l}\text { - } \text { origine ethnique* }^{*} \\
\text { - lien PS / PA * } \\
\text { - cohabitation PS / PA } \\
\text { - durée d'utilisation du } \\
\text { CJ, avant l'étude* }\end{array}$ & & $\begin{array}{l}\text { - lien PS / PA * } \\
\text { - cohabitation PS / } \\
\text { PA }\end{array}$ & $\begin{array}{l}\text { - } \text { origine ethnique }^{* 1,2} \\
\text { - lien PS / PA }\end{array}$ \\
\hline \multicolumn{8}{|c|}{ Facteurs de capacité personnels ou communautaires } \\
\hline \multicolumn{8}{|c|}{ Personnes âgées ou personnes de soutien, souvent sans distinction } \\
\hline $\begin{array}{l}\text { Revenus } \\
\text { Source de } \\
\text { financement } \\
\text { Milieu }\end{array}$ & & $\begin{array}{l}\text { - financement externe } \\
\text { pour } \mathrm{CJ} \text { * }\end{array}$ & - revenus de la PA & $\begin{array}{l}\text { - } \text { revenus de la PS } \\
\text { - coûts partagés PA / PS }\end{array}$ & $\begin{array}{l}\text { - revenu de la PA } \\
\text { - service disponible } \\
\text { dans le HMO* }\end{array}$ & & $\begin{array}{l}\text { - revenus de la PA } \\
\text { - financement externe pour } \\
\text { CJ *1,2 }\end{array}$ \\
\hline \multicolumn{8}{|c|}{ Facteurs de besoin } \\
\hline \multicolumn{8}{|c|}{ Personnes âgées } \\
\hline Santé physique & & $\begin{array}{l}\text { - HTA } \\
\text { - ACV } \\
\text { - problèmes visuels } \\
\text { - diabètes } \\
\text { - maladie chronique } \\
\text { - SCO } \\
\text { - problèmes auditifs } \\
\text { - immobilité }\end{array}$ & $\begin{array}{l}\text { - } \text { santé perçue } \mathrm{p} / \mathrm{r} \\
\text { aux autres du } \\
\text { même âge }\end{array}$ & $\begin{array}{l}\text { - présence de plus d'un } \\
\text { prob. de santé } \\
\text { - hospitalisation ou } \\
\text { hébergement } \\
\text { temporaire dans les } 2 \\
\text { mois précédant le } \\
\text { questionnaire }\end{array}$ & 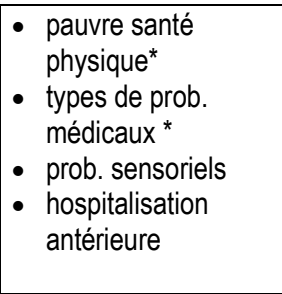 & & $\begin{array}{l}\text { - nombre d'hospitalisations } \\
\text { dans les } 3 \text { années } \\
\text { précédentes } \\
\text { - } \text { risque nutritionnel }{ }^{\star 1} \\
\text { - multiples diagnostics } \\
\text { (plus de 4) }\end{array}$ \\
\hline
\end{tabular}




\begin{tabular}{|c|c|c|c|c|c|c|c|}
\hline Variables & Chappell, 1983 & Wallace, 1987 & $\begin{array}{l}\text { Kosloski et coll., } \\
1994\end{array}$ & $\begin{array}{l}\text { Henry \& Capitman, } \\
1995\end{array}$ & Lynch et coll., 1999 & $\begin{array}{l}\text { Baumgarten et } \\
\text { coll., } 2002\end{array}$ & $\begin{array}{l}\text { Dabelko } \\
2004 \text { (1) et } 2005 \text { (2) }\end{array}$ \\
\hline $\begin{array}{l}\text { Santé } \\
\text { psychologique }\end{array}$ & $\begin{array}{l}\text { - échelle d'AVQ et } \\
\text { AVD* }^{*}\end{array}$ & $\begin{array}{l}\text { - dépression } \\
\text { - } \text { alcoolisme } \\
\text { - } \text { instabilité émotionnelle } \\
\text { - } \text { confusion } \\
\text { Raison de référence : } \\
\text { - } \text { socialisation } \\
\text { - diminuer stress PS * } \\
\text { - surveillance état santé } \\
\text { - besoin de thérapie } \\
\text { - maintenir autonomie } \\
\text { - prévenir placement* }\end{array}$ & $\begin{array}{l}\text { - } \text { maladie d'Alzheimer } \\
\text { - } \text { sentiment de } \\
\text { dépendre des } \\
\text { autres } \\
\text { - échelle d'AVQ } \\
\text { - échelle d'AVD }\end{array}$ & $\begin{array}{l}\text { - échelle d'AVQ } \\
\text { - incontinence } \\
\text { - durée de la } \\
\text { dépendance due à la } \\
\text { maladie* }^{*}\end{array}$ & 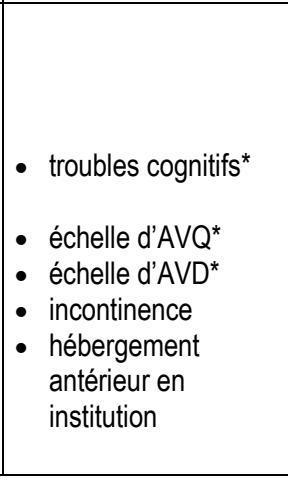 & $\begin{array}{l}\text { - dépression* } \\
\text { - anxiété* } \\
\text { - troubles cognitifs : } \\
\text { MMSE<22* } \\
\text { - échelle d'AVQ et } \\
\text { AVD* }\end{array}$ & $\begin{array}{l}\text { - } \text { présence de dépression } \\
\text { ou anxiétét1,2 } \\
\text { - } \text { diagnostic de démence } \\
\text { - } \text { comportements } \\
\text { suggérant la démence } \\
\text { - échelle d'AVQ et d'AVD } \\
\text { - incontinence } \\
\text { - hébergement antérieur en } \\
\text { institution }\end{array}$ \\
\hline \multicolumn{8}{|c|}{ Personnes de soutien } \\
\hline $\begin{array}{l}\text { Santé physique } \\
\text { Santé } \\
\text { psychologique } \\
\text { Facteurs reliés } \\
\text { au rôle de PS }\end{array}$ & & & $\begin{array}{l}\text { - évaluation globale } \\
\text { de sa santé } \\
\text { - heures consacrées } \\
\text { aux soins } \\
\text { personnels } \\
\text { - heures consacrées } \\
\text { à aider à la gestion }\end{array}$ & $\begin{array}{l}\text { - } \text { durée de l'aide* } \\
\text { - a choisi de devenir PS* } \\
\text { - attitude vs rôle de PS } \\
\text { - impact des prob. de } \\
\text { comportement de la PA } \\
\text { sur la PS } \\
\text { - PS croit que CJ est } \\
\text { arrivé trop tard } \\
\text { - PS a un emploi } \\
\text { - impact sur l'emploi }\end{array}$ & & - Fardeau* & \\
\hline \multicolumn{8}{|c|}{ Caractéristiques des services } \\
\hline $\begin{array}{l}\text { Caractéristiques } \\
\text { de services } \\
\text { connexes }\end{array}$ & & $\begin{array}{l}\text { - fréquence de } \\
\text { participation prévue* }\end{array}$ & & $\begin{array}{l}\text { - } \text { coût du CJ r* } \\
\text { - } \text { coûts services }^{*} \text { auxiliaires* } \\
\text { - } \text { insatisfait vs coût }^{*} \\
\text { - } \text { programme axé sur : } \\
\text { - santé } \\
\text { - activités de } \\
\text { stimulation } \\
\text { - répit + contacts } \\
\text { avec les PS* } \\
\text { - difficulté à obtenir } \\
\text { d'autres services de } \\
\text { soutien }\end{array}$ & & & $\begin{array}{l}\text { - } \text { coût pour le client*2 }^{*} \\
\text { - fréquence de participation } \\
\text { prévue } \\
\text { - transport fourni par CJ*2 } \\
\text { - ratio clients/ personnel } \\
\text { - diversité du personnel } \\
\text { - capacité du CJ (\# clients) } \\
\text { - pourcentage d'utilisation } \\
\text { - espace en pieds carrés } \\
\text { - programme accepte ou } \\
\text { non les personnes avec } \\
\text { - } \text { atteinte cognitive } \\
\text { programme localisé dans } \\
\text { une institution de soins } \\
\text { de longue durée ou pas }\end{array}$ \\
\hline
\end{tabular}




\section{b) : études auprès des centres de jour dont l'échantillon est constitué de personnes de soutien de personnes atteintes de démence}

\begin{tabular}{|c|c|c|c|c|c|c|}
\hline Variables & Wells et coll., 1990 & Beisecker et coll., 1996 & \begin{tabular}{|l|} 
Zarit et coll., 1999 \\
1= analyse bivariée \\
2= analyse multivariée
\end{tabular} & Douglass \& Visconti. 1998 & $\begin{array}{l}\text { Montoro-Rodriguez et coll., } \\
2003\end{array}$ & Mävall et Thorslund, 2006 \\
\hline \multicolumn{7}{|c|}{ Facteurs prédisposants } \\
\hline \multicolumn{7}{|c|}{ Personnes âgées } \\
\hline $\begin{array}{l}\text { Socio- } \\
\text { démographique }\end{array}$ & & & $\begin{array}{l}\text { - âge } \\
\text { - } \text { sexe }^{\star 1,2}\end{array}$ & $\begin{array}{l}\text { - âge } \\
\text { - sexe } \\
\text { - éducation } \\
\text { - emploi antérieur } \\
\text { - présence d'une PS } \\
\text { - vit seule ou avec d'autres } \\
\text { - a un conjoint }\end{array}$ & & \\
\hline \multicolumn{7}{|c|}{ Personnes de soutien } \\
\hline $\begin{array}{l}\text { Socio- } \\
\text { démographique }\end{array}$ & & & $\begin{array}{l}\text { - âge } \\
\text { - sexe } \\
\text { - éducation* } 1,2 \\
\text { - a un conjoint }\end{array}$ & & $\begin{array}{l}\text { - âge } \\
\text { - sexe } \\
\text { - éducation }\end{array}$ & \\
\hline \multicolumn{7}{|c|}{ Personnes âgées et personnes de soutien } \\
\hline $\begin{array}{l}\text { Appartenance } \\
\text { culturelle }\end{array}$ & & 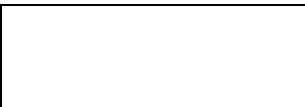 & - origine ethnique ${ }^{* 1}$ & - origine ethnique & $\begin{array}{l}\text { - ethnicité (blanc, noir ou } \\
\text { latino) }\end{array}$ & \\
\hline Lien PS / PA & & & - lien PA/PS *1,2 & - lien PA/PS & - lien PA/PS & - cohabitation PS / PA \\
\hline $\begin{array}{l}\text { Croyances et } \\
\text { attitudes }\end{array}$ & & $\begin{array}{l}\text { - utilisation d'autres } \\
\text { services de soutien* }\end{array}$ & & $\begin{array}{l}\text { - utilisation du CJ déjà } \\
\text { présente au début de la } \\
\text { recherche* }^{*}\end{array}$ & $\begin{array}{l}\text { - PS préfère aide formelle ou } \\
\text { de la famille } \\
\text { - perception de l'utilité des } \\
\text { services de répit' } \\
\text { - attitude négative envers } \\
\text { services gouvernementaux } \\
\text { - attitude négative, stigma } \\
\text { associé à la démence }\end{array}$ & \\
\hline \multicolumn{7}{|c|}{ Facteurs de capacité personnels ou communautaires } \\
\hline \multicolumn{7}{|c|}{ Personnes de soutien } \\
\hline $\begin{array}{l}\text { Connaissance des } \\
\text { services }\end{array}$ & & & 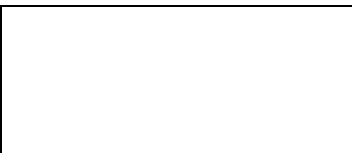 & $\begin{array}{l}\text { - PS a déjà aidé un autre } \\
\text { parent atteint de démence }\end{array}$ & $\begin{array}{l}\text { - PS connaît l'existence des } \\
\text { services d'aide formelle* } \\
\text { - PS sait comment obtenir } \\
\text { services d'aide }\end{array}$ & \\
\hline & & - revenu de PS & & $\begin{array}{l}\text { - présence de prob. } \\
\text { financiers (PS) }\end{array}$ & - revenu PS* & \\
\hline $\begin{array}{l}\text { Source de } \\
\text { financement }\end{array}$ & & & $\begin{array}{l}\text { - financement externe pour } \\
\text { CJ*1 }\end{array}$ & - type d'assurance & 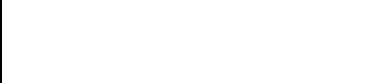 & \\
\hline Milieu & & & & & - milieu urbain ou rural & \\
\hline
\end{tabular}




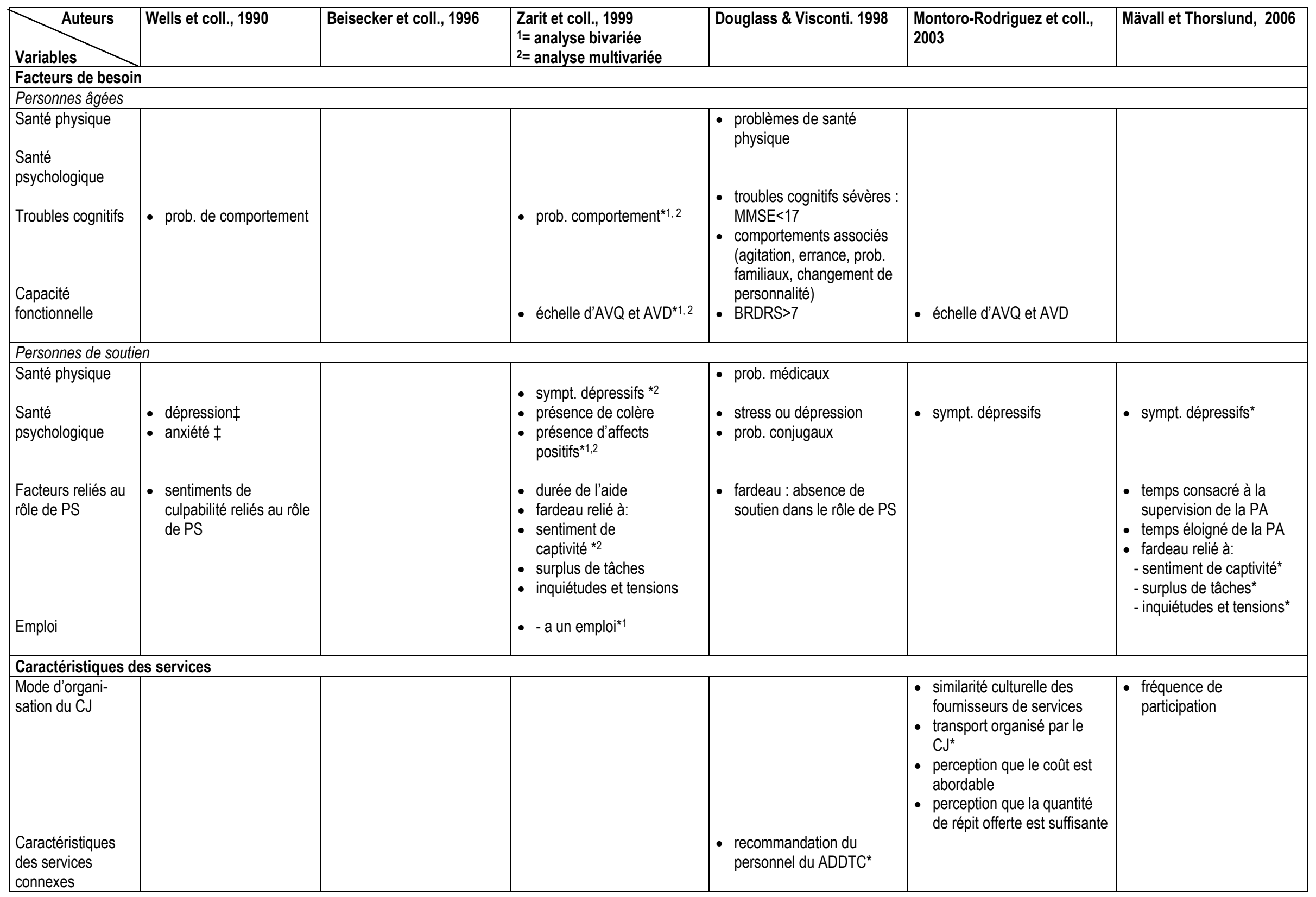




\section{c) : étude auprès de services connexes}

\begin{tabular}{|c|c|c|c|c|c|c|}
\hline Variables & Casserta et coll., 1987 & Montgomery et coll., 1988 & Lawton et coll., 1989; 1991 & Biegel et coll., 1993 & Kosloski et coll., 1993 & Cox, 1997 \\
\hline \multicolumn{7}{|c|}{ Facteurs prédisposants } \\
\hline \multicolumn{7}{|c|}{ Personnes âgées } \\
\hline $\begin{array}{l}\text { Socio- } \\
\text { démographiques }\end{array}$ & $\begin{array}{l}\text { - âge* } \\
\text { - sexe } \\
\text { - éducation }\end{array}$ & & - âge $^{*}$ & & & \\
\hline \multicolumn{7}{|c|}{ Personnes de soutien } \\
\hline $\begin{array}{l}\text { Socio- } \\
\text { démographiques }\end{array}$ & $\begin{array}{l}\text { - âge } \\
\text { - sexe } \\
\text { - éducation } \\
\text { - nombre d'individus dans } \\
\text { la maisonnée } \\
\text { - nombre d'aidants } \\
\text { secondaires } \\
\text { - satisfaction envers ce } \\
\text { réseau* }\end{array}$ & & $\begin{array}{l}\text { - } \text { âge }^{*} \\
\text { - heures de soutien informel } \\
\text { reçues dans l'année } \\
\text { précédant l'étude }\end{array}$ & $\begin{array}{l}\text { - sexe } \\
\text { - présence de soutien } \\
\text { secondaire } \\
\text { - PS trouve son réseau de } \\
\text { soutien adéquat* }^{*}\end{array}$ & & $\begin{array}{l}\text { - sexe } \\
\text { - présence de soutien } \\
\text { informel } \\
\text { - qualité des relations dans } \\
\text { la famille* }\end{array}$ \\
\hline \multicolumn{7}{|c|}{ Personnes âgées ou personnes de soutien } \\
\hline $\begin{array}{l}\text { Appartenance } \\
\text { culturelle } \\
\text { Lien PS / PA } \\
\text { Croyances et } \\
\text { attitudes }\end{array}$ & $\begin{array}{l}\text { - origine ethnique } \\
\text { - lien PS / PA* }\end{array}$ & & $\begin{array}{l}\text { - Lien PS / PA } \\
\text { - Cohabitation PS / PA } \\
\text { - heures de soutien formel } \\
\text { reçues dans l'année } \\
\text { précédant l'étude }\end{array}$ & - lien PS / PA & $\begin{array}{l}\text { - } \text { perception de la difficulté à } \\
\text { obtenir et utiliser services* } \\
\text { - perception de la qualité } \\
\text { des services de répit } \\
\text { offerts par cette agence* } \\
\text { - perception de l'utilité de } \\
\text { ces services* } \\
\text { - utilisation d'autres services } \\
\text { de répit } \\
\text { - utilisation d'autres services } \\
\text { de soutien }\end{array}$ & $\begin{array}{l}\text { - } \text { origine ethnique }{ }^{*} \\
\text { - lien PS / PA } \\
\text { - utilisation CJ } \\
\text { - utilisation soins à dom. }\end{array}$ \\
\hline \multicolumn{7}{|c|}{ Facteurs de capacité personnels ou communautaires } \\
\hline \multicolumn{7}{|c|}{ Personnes âgées ou personnes de soutien } \\
\hline $\begin{array}{l}\text { Connaissance des } \\
\text { services } \\
\text { Revenus / Source } \\
\text { de financement } \\
\text { Milieu }\end{array}$ & $\begin{array}{l}\text { - population de la zone de } \\
\text { résidence }\end{array}$ & & & $\begin{array}{l}\text { - expérience antérieure } \\
\text { dans le rôle de PS } \\
\text { - revenus de la PS }\end{array}$ & & \\
\hline
\end{tabular}




\begin{tabular}{|c|c|c|c|c|c|c|}
\hline Variables & Casserta et coll., 1987 & Montgomery et coll., 1988 & Lawton et coll., 1989; 1991 & Biegel et coll., 1993 & Kosloski et coll., 1993 & Cox, 1997 \\
\hline \multicolumn{7}{|l|}{ Facteurs de besoin } \\
\hline \multicolumn{7}{|l|}{ Personnes âgées } \\
\hline Santé physique & & & $\begin{array}{l}\text { - sévérité des problèmes } \\
\text { autres que cognitifs }\end{array}$ & & & \\
\hline $\begin{array}{l}\text { Santé } \\
\text { psychologique } \\
\text { Troubles cognitifs }\end{array}$ & $\begin{array}{l}\text { - résultat à la Functional } \\
\text { Dementia Scale* }\end{array}$ & $\begin{array}{l}\text { - } \begin{array}{l}\text { maladie d'Alzheimer } \\
\text { probable* } \\
\text { - troubles cognitifs d'autre } \\
\text { origine }\end{array} \\
\end{array}$ & $\begin{array}{l}\text { - sévérité des troubles } \\
\text { cognitifs } \\
\text { - présence de troubles de } \\
\text { comportement* }\end{array}$ & $\begin{array}{l}\text { - importance des troubles } \\
\text { cognitifs }\end{array}$ & & $\begin{array}{l}\text { - importance des troubles } \\
\text { cognitifs* } \\
\text { - problèmes de } \\
\text { comportement }\end{array}$ \\
\hline $\begin{array}{l}\text { Capacité } \\
\text { fonctionnelle ou } \\
\text { Besoin perçu }\end{array}$ & & & & - échelle d'AVQ* & $\begin{array}{l}\text { - échelle d'AVQ* } \\
\text { - échelle d'AVD }\end{array}$ & - échelle d'AVQ \\
\hline \multicolumn{7}{|l|}{ Personnes de soutien } \\
\hline Santé physique & & & $\begin{array}{l}\text { - un indicateur de santé } \\
\text { physique }\end{array}$ & & - santé perçue & \\
\hline $\begin{array}{l}\text { Santé } \\
\text { psychologique }\end{array}$ & & & - symptômes dépressifs * & & & $\begin{array}{l}\text { - anxiété } \\
\text { - dépression* } \\
\end{array}$ \\
\hline $\begin{array}{l}\text { Facteurs reliés au } \\
\text { rôle de PS }\end{array}$ & $\begin{array}{l}\text { - fardeau* } \\
\text { - durée de l'aide } \\
\text { - temps depuis le } \\
\text { diagnostic }\end{array}$ & & $\begin{array}{l}\text { - } \text { fardeau ressenti }^{*} \\
\text { - \# d'heures consacrées aux } \\
\text { soins* } \\
\text { - impacts négatifs de ce rôle } \\
\text { - sentiments de compétence } \\
\text { vs ce rôle } \\
\text { - sentiments positifs vs ce } \\
\text { rôle }\end{array}$ & $\begin{array}{l}\text { - temps depuis le } \\
\text { diagnostic } \\
\text { - fardeau } \\
\text { - émotionnel* } \\
\text { - tensions dans la } \\
\text { relation avec la PA }\end{array}$ & & $\begin{array}{l}\text { - } \text { fardeau* } \\
\text { - gains ressentis du rôle de } \\
\text { PS }^{*}\end{array}$ \\
\hline Emploi & & & & - PS a un emploi & & - PS a un emploi \\
\hline \multicolumn{7}{|c|}{ Caractéristiques des services } \\
\hline $\begin{array}{l}\text { Caractéristiques des } \\
\text { services de répit }\end{array}$ & & & & & & \\
\hline $\begin{array}{l}\text { Caractéristiques des } \\
\text { services connexes }\end{array}$ & $\begin{array}{l}\text { - participe à un groupe de } \\
\text { soutien }\end{array}$ & & & & & \\
\hline
\end{tabular}


c) : étude auprès de services connexes (suite)

\begin{tabular}{|c|c|c|c|c|c|}
\hline Variables & Cotrell \& Engel, 1998 & Pedlar et coll., 1999 & Kosloski et coll., 1999 & $\begin{array}{l}\text { Kosloski et coll., } 2001 \\
1=\text { analyse bivariée } \\
2=\text { analyse multivariée }\end{array}$ & Li, 2004 \\
\hline \multicolumn{6}{|c|}{ Facteurs prédisposants } \\
\hline \multicolumn{6}{|c|}{ Personnes âgées } \\
\hline $\begin{array}{l}\text { Socio- } \\
\text { démographiques }\end{array}$ & & & - âge & - âge & \\
\hline \multicolumn{6}{|l|}{ Personnes de soutien } \\
\hline $\begin{array}{l}\text { Socio- } \\
\text { démographiques }\end{array}$ & $\begin{array}{l}\text { - } \text { âge } \\
\text { - sexe } \\
\text { - } \text { présence d'un médiateur qui } \\
\text { encourage utilisation dans le } \\
\text { réseau formel ou informel * } \\
\text { - soutien reçu } \\
\text { - soutien perçu }\end{array}$ & $\begin{array}{l}\text { - âge } \\
\text { - éducation } \\
\text { - nombre de personnes de } \\
\text { soutien secondaires }\end{array}$ & - sexe & $\begin{array}{l}\text { - âge } \\
\text { - sexe } \\
\text { - présence de soutien } \\
\text { secondaire }{ }^{* 1,2} \\
\text { - nombre de personnes ds } \\
\text { famille } \\
\text { - enfants à moins de } 1 \text { heure } \\
\text { route }\end{array}$ & $\begin{array}{l}\text { - âge } \\
\text { - présence d'aidants } \\
\text { secondaires } \\
\text { - type d'aidants secondaires } \\
\text { (membre de la famille ou } \\
\text { amis)* }\end{array}$ \\
\hline \multicolumn{6}{|c|}{ Personnes âgées ou personnes de soutien } \\
\hline $\begin{array}{l}\text { Appartenance } \\
\text { culturelle } \\
\text { Lien PS / PA } \\
\text { Croyance et attitudes }\end{array}$ & - lien PS / PA * & $\begin{array}{l}\text { - PS préfère aide de la famille } \\
\text { à aide formelle } \\
\text { - s'inquiète de l'opinion des } \\
\text { autres membres de famille } \\
\text { - confiance envers les services } \\
\text { d'aide* } \\
\text { - expérience de services de } \\
\text { mauvaise qualité }\end{array}$ & - lien PS / PA & $\begin{array}{l}\text { - } \text { appartenance culturelle }{ }^{* 1} \\
\text { - lien PS / PA } \\
\text { - sentiment que la } \\
\text { responsabilité des soins est un } \\
\text { devoir *1 } \\
\text { - utilisation d'autres services de } \\
\text { répit }\end{array}$ & - lien PS / PA* \\
\hline \multicolumn{6}{|c|}{ Facteurs de capacité personnels ou communautaires } \\
\hline \multicolumn{6}{|c|}{ Personnes âgées ou personnes de soutien } \\
\hline $\begin{array}{l}\text { Connaissance des } \\
\text { services } \\
\text { Revenus } \\
\text { Source de } \\
\text { financement } \\
\text { Milieu }\end{array}$ & $\begin{array}{l}\text { - connaissance des services* } \\
\text { - revenus de la PS }\end{array}$ & $\begin{array}{l}\text { - revenu familial (PS + PA) } \\
\text { - vétéran }\end{array}$ & & & \\
\hline
\end{tabular}




\begin{tabular}{|c|c|c|c|c|c|}
\hline Variables & Cotrell \& Engel, 1998 & Pedlar et coll., 1999 & Kosloski et coll., 1999 & $\begin{array}{l}\text { Kosloski et coll., } 2001 \\
\text { 1= analyse bivariée } \\
\text { 2= analyse multivariée }\end{array}$ & Li, 2004 \\
\hline \multicolumn{6}{|l|}{ Facteurs de besoin } \\
\hline \multicolumn{6}{|l|}{ Personnes âgées } \\
\hline Santé physique & & - échelle de Nagi* & & & \\
\hline Santé psychologique & & & & & \\
\hline Troubles cognitifs & $\begin{array}{l}\text { - } \text { troubles cognitifs } \\
\text { (orientation) }^{*}\end{array}$ & $\begin{array}{l}\text { - } \text { troubles cognitifs } \\
\text { - } \text { problèmes de comportement* }\end{array}$ & $\begin{array}{l}\text { - diagnostic de maladie } \\
\text { d'Alzheimer ou non }\end{array}$ & $\begin{array}{l}\text { - } \text { atteintes cognitives } \\
\text { - prob de comportement }\end{array}$ & $\begin{array}{l}\text { - prob. de mémoire } \\
\text { - prob. de comportement* }\end{array}$ \\
\hline Capacité fonctionnelle & - échelle d'AVQ* & $\begin{array}{l}\text { - échelle d'AVQ } \\
\text { - échelle d'AVD }\end{array}$ & $\begin{array}{l}\text { - échelle d'AVQ } \\
\text { - échelle d'AVD }\end{array}$ & - échelles d'AVQ *1,2 & - échelle d'AVQ et AVD* \\
\hline \multicolumn{6}{|l|}{ Personnes de soutien } \\
\hline Santé physique & & $\begin{array}{l}\text { - échelle de Nagi } \\
\text { - santé perçue }\end{array}$ & & - santé perçue & - auto-évaluation de sa santé \\
\hline Santé psychologique & & & & - santé mentale & \\
\hline $\begin{array}{l}\text { Facteurs reliés au rôle } \\
\text { de PS }\end{array}$ & $\begin{array}{l}\text { - fardeau: } \\
\text { - limitation de ses } \\
\text { activités } \\
\text { - inquiétudes } \\
\end{array}$ & $\begin{array}{l}\text { - fardeau } \\
\text { - durée de l'aide }\end{array}$ & & - fardeau & \\
\hline Emploi & - PS a un emploi* & & $\begin{array}{ll}\text { - } & \text { statut d'emploi de la PS } \\
\text { (temps plein, temps partiel, } \\
\text { aucun emploi) }\end{array}$ & - PS a un emploi & - PS a un emploi \\
\hline \multicolumn{6}{|c|}{ Caractéristiques des services } \\
\hline $\begin{array}{l}\text { Caractéristiques des } \\
\text { services de répit } \\
\text { Caractéristiques des } \\
\text { services connexes }\end{array}$ & & $\begin{array}{l}\text { - difficulté à obtenir et utiliser } \\
\text { ce type de services* }\end{array}$ & & $\begin{array}{l}\text { - } \text { transport organisé par le CJ } \\
\text { - fiabilité de l'horaire de répit*1,2 } \\
\text { - possibilité de choisir les temps } \\
\text { de répit } \\
\text { - coût du répit }\end{array}$ & \\
\hline
\end{tabular}





\section{Annexe III}

\section{Guide d'entrevue}

Entrevue individuelle avec les coordonnateurs des cinq centres de jour à l'étude

Les informations dont nous avons besoin concernent l'année financière 2000-2001 (1 avril 2000 au 31 mars 2001). Si des changements sont survenus depuis cette période, se reporter toujours à cette période.

\section{INFORMATION GENERALE}

Quels étaient les objectifs de votre centre de jour (si des dépliants ou autres documents répondent à cette question, pouvons-nous avoir des copies des documents) ?

Quels étaient les critères d'admission ou d'exclusion?

À cette époque, votre centre acceptait-il les personnes qui :

- présentaient des troubles cognitifs?

- présentaient des problèmes de comportement?

- présentaient de l'incontinence ?

- avaient besoin d'assistance pour se rendre à la toilette ?

- avaient besoin d'assistance pour manger ?

Quel était le processus de référence et prise en charge?

Qui pouvaient diriger des clients vers le centre de jour?

Comment se faisait l'évaluation de l'admission par le centre de jour? 


\section{INFORMATION GENERALE}

Est-ce que votre centre de jour avait une liste d'attente?

Si oui, comment se faisait la gestion de la liste d'attente?

Quelle était la durée moyenne d'attente pour être admis à votre centre de jour en avril 2000 ?

Est-ce que des plans d'intervention individualisés étaient établis pour chacun des clients du centre de jour? Si oui, à quelle fréquence étaient-ils révisés?

\section{STRUCTURE DE PERSONNEL}

Nous aimerions connaître le nombre de personnes qui travaillaient dans votre centre de jour en 2000-2001. Pour chacune des fonctions les plus couramment rencontrés en centre de jour, nous voulons savoir si votre centre emploi ce type d'intervenant; si oui, en quelle quantité et pour quelles tâches.

Personnel total du centre de jour (nombre d'équivalent temps complet) :

\section{Coordonnateur/trice :}

Le coordonnateur a-t-il la responsabilité d'autres services en plus du centre de jour?

Le coordonnateur assume-t-il des tâches cliniques en plus des tâches de coordonnateur?

Si oui, quel type d'interventions cliniques? Quelle est sa formation?

Proportion de son temps accordé aux différentes tâches :

- gestion relié au centre de jour :

$\mathrm{h} / \mathrm{sem}$.

- tâches cliniques au centre de jour :

- autre tâches (à l'extérieur du CJ) : $\mathrm{h} / \mathrm{sem}$. $\mathrm{h} / \mathrm{sem}$.

\section{Secrétaire / adjointe administrative :}

Heures de présences au centre de jour : $\mathrm{h} / \mathrm{sem}$. Cette personne assume-t-elle des tâches cliniques en plus des tâches de secrétariat? Si oui, quel type d'interventions cliniques? Quelle est sa formation?

Proportion de son temps accordé aux différentes tâches :

- soutien administratif au centre de jour : $\mathrm{h} / \mathrm{sem}$.

- tâches cliniques au centre de jour : $\mathrm{h} / \mathrm{sem}$. 


\section{STRUCTURE DE PERSONNEL}

\section{Infirmière :}

Présence d'infirmière(s) au centre de jour : $\ldots \mathrm{h} / \mathrm{sem}$.

Présence d'aide-infirmière(s) au centre de jour : __ _ $\mathrm{h} / \mathrm{sem}$.

Leurs principales tâches (interventions individuelles, groupes, etc.) :

\section{Ergothérapie}

Présence d'ergothérapeute(s) au centre de jour : ___

Principales tâches (interventions individuelles, groupes, etc.) :

\section{Physiothérapie :}

Présence de physiothérapeute(s) au centre de jour : __ $\mathrm{h} / \mathrm{sem}$.

Présence de thérapeute(s) en réadaptation physique au $\mathrm{CJ}$ : $\mathrm{h} / \mathrm{sem}$.

Principales tâches (interventions individuelles, groupes, etc.) :

\section{Éducation spécialisée :}

Présence d'éducateur(s) spécialisé(s) au centre de jour : $\mathrm{h} / \mathrm{sem}$ au CJ

Principales tâches (interventions individuelles, groupes, etc.) :

\section{Loisirs : (récréologue, technicien en loisirs, animateur)}

Présence de (préciser la formation) $\mathrm{h} / \mathrm{sem}$.

Présence de (préciser la formation) $\mathrm{h} / \mathrm{sem}$.

Principales tâches (interventions individuelles, groupes, etc.) : 


\section{STRUCTURE DE PERSONNEL}

\section{Intervention psychosociale :}

Présence de travailleur(s) social(aux) au centre de jour :

Présence de psychologue(s) au centre de jour : h/sem.

Autre intervenant psycho-social (formation $\mathrm{h} / \mathrm{sem}$.

Autre intervenant psycho-social (formation ) $h / s e m$. ) $\mathrm{h} / \mathrm{sem}$.

Principales tâches (interventions individuelles, groupes, etc.) :

\section{Assistance aux participants :}

Présence de préposé(s) ou auxiliaire(s) familial(aux) au CJ : $\mathrm{h} / \mathrm{sem}$.

Autres : $\mathrm{h} / \mathrm{sem}$.

Principales tâches (interventions individuelles, groupes, etc.) :

\section{Hôtellerie :}

Le personnel du centre de jour comprend-il des personnes affectées aux tâches associées à l'hôtellerie (préparation des repas, services aux tables, entretien ménager, etc.). Ne pas inclure l'assistance un à un pour faire manger qui doit être plutôt rapportée à la section précédente (assistance aux participants) $\mathrm{h} / \mathrm{sem}$.

\section{Transport :}

Présence d'un chauffeur au centre de jour : $\mathrm{h} / \mathrm{sem}$.

Cette personne assume-t-elle des tâches cliniques en plus du transport de la clientèle? Si oui, quel type d'interventions cliniques? Quelle est sa formation?

Proportion de son temps accordé aux différentes tâches :

- Transport de la clientèle :

- Tâches cliniques au centre de jour :

$\mathrm{h} / \mathrm{sem}$. $\mathrm{h} / \mathrm{sem}$.

\section{Autres :}

Y a-t-il d'autres catégories de personnel dont nous aurions oublié de praler?

Autre (préciser : $\mathrm{h} / \mathrm{sem}$.

Autre (préciser : $\mathrm{h} / \mathrm{sem}$.

Principales tâches (interventions individuelles, groupes, etc.) : 
CAPACITE DU CENTRE DE JOUR

Inscriptions : participants / jour ou participants / semaine

Participation réelle (moyenne): semaine

Pour l'année financière 2000/2001 :

Quel a été le nombre de jours-présences?

Quel était le budget total de votre centre?

Quel était le per diem?

\section{LES ACTIVITES DU CENTRE DE JOUR}

Est-il possible d'avoir une copie de la grille des activités de votre centre de jour en vigueur pour 2000-2001?

La durée de la participation est-elle identique pour toute la clientèle? Durée

$\mathrm{Si}$ elle variait en fonction de type de groupe auquel la personne était inscrite, (inscrire la durée sur la grille des activités.

Comment déterminez-vous à quelle activité un client va participer (place disponible, profil santé ou fonctionnel, intérêt, contraintes de transport, etc.)?

Quel était la fréquence maximale de participation pour un client?

Nous aimerions connaître les objectifs de chacune des activités auxquelles des participants de notre étude ont été inscrits (voir annexe propre à chacun des centres de jour), de même que les types de clients ciblés pour ces activités. Quels étaient les buts de ces activités? Quel type de participation exigent-elles? etc.

En 2000-2001, est-ce que vous avez offert des activités pour les aidants?

Est-ce qu'elles apparaîtraient dans les données du logiciel JEDI des participants, si leurs aidants en avaient bénéficié ?

Quels types d'assistance individuelle étaient fournis aux participants?

Est-ce que l'assistance individuelle étaient compilée dans les données du logiciel JEDI? 
POUR LA PERIODE ALLANT DU 1 AVRIL 2000 AU 31 MARS 2001

Le Centre de jour était-il ouvert normalement du lundi au vendredi ?

$\square$ Oui

Non : $\quad$ autre horaire :

Quels étaient les heures normales d'ouverture :

Pour le personnel :

Pour les clients :

Était-ce le même horaire pour tous ? Ou l'horaire variait-il selon les groupes?

Si c'est le cas, joindre l'horaire des groupes pour la période de référence.

Pouvez-vous nous fournir la liste des jours où le CJ a été fermé pour les clients (jours fériés, vacances, tempêtes, journées de planification, etc.) pendant cette période?

Quel était le coût normalement demandé aux usagers qui fréquentaient votre centre de jour pendant cette période?

Repas_ Transport___ Matériel ___ Autre

Était-il possible à votre centre de jour de réduire les coûts de repas et de transport pour ceux dont les moyens financiers sont limités ? Oui Non

Quelle était la durée moyenne d'un trajet en autobus ou taxi pour se rendre au CJ ?

Comment le transport est-il organisé?

Qu'est-ce que vous faites pour favoriser l'assuidité de la participation au centre de jour?

Est-ce que le CJ appelle les clients chaque semaine pour confirmer leur présence ? le matin de la présence prévue? La veille? Non 
Annexe : Exemple de tableau pour recueillir l'information sur les activités inscrites dans les statistiques d'activités de chaque centre de jour

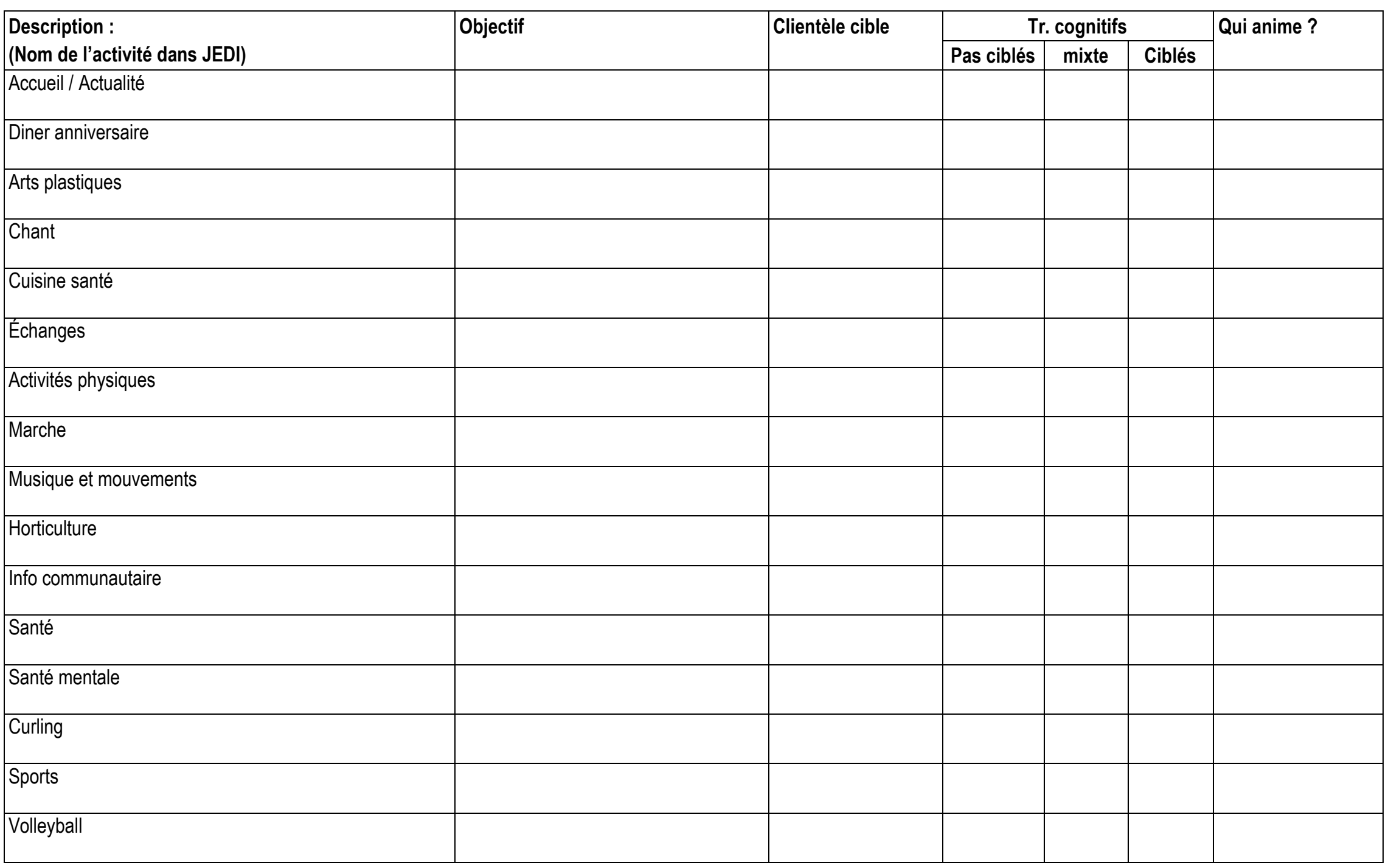





\section{Annexe IV \\ Curriculum vitae \\ Jacinthe SAVARD}

\section{FORMATION :}

Ph. D. en Santé publique, Université de Montréal. (Décembre 2008, date de soutenance prévue)

Sujet de la thèse : L'utilisation des centres de jour par les personnes âgées en perte d'autonomie

M. Sc. en Administration des services de santé, Université de Montréal, 1992.

B. Sc. Ergothérapie, Université Laval, Québec, 1982.

\section{DISTINCTIONS :}

2006 Bourse de rédaction, Faculté des études supérieures, Université de Montréal

2006 Bourse d'études doctorales de la Fondation canadienne d'ergothérapie

2004-2005 Bourse d'études doctorales du groupe de recherche SOLIDAGE

2003-2004 Bourse d'études doctorales de la Société Alzheimer du Canada (renouvellement)

2001-2003 Bourse d'études doctorales de la Société Alzheimer du Canada

2001 Bourse d'études supérieures en gérontologie et gériatrie Marthe-Demers, de la Faculté de médecine de l'Université de Montréal

2001 Bourse d'études doctorales Goldwin Howland, de la Fondation canadienne d'ergothérapie

1982 Prix de l'Association canadienne des ergothérapeutes pour les meilleurs résultats scolaires au cours de la formation universitaire en ergothérapie.

\section{EXPÉRIENCES PROFESSIONNELLES:}

Sept 2006 - ...Coordonnatrice de la formation et des services, Clinique universitaire interprofessionnelle de réadaptation en soins de santé primaire, Univiversité d'Ottawa.

2000 - 2006 Chargé de cours (temps partiel), Programme d'ergothérapie, Université d'Ottawa

$1997-2000$ Directrice du programme de premier cycle, Programme d'ergothérapie, Université d'Ottawa.

1996 - 1997 Professeur remplaçante (temps complet), Programme d'ergothérapie, Université d'Ottawa.

1992 - 1996 Coordonnatrice du Centre de jour et des Services de réadaptation et d'animation, Centre d'accueil et CLSC de Rosemont (Résidence Robert-Cliche), Montréal.

1987 - 1991 Chef du service d'ergothérapie, Hôpital Notre-Dame-de-Lourdes, Montréal. (Centre d'hébergement et de soins de longue durée)

1985 -1987 Chef de service, Centre de jour de l'Accueil de Ville St-Georges, St-Georges de Beauce.

1983 - 1985 Ergothérapeute, Unité de réadaptation et Hôpital de jour, Hôpital D'Youville, Sherbrooke 


\section{PUBLICATIONS (sept dernières années):}

\section{Direction de livre :}

Tremblay, L.E., Savard, J., Casimiro, L., \& Tremblay, M. (Dir.). Répertoire des outils d'évaluation en français pour la réadaptation. Ottawa: Centre franco-ontarien de ressources pédagogiques. (2004) $585 \mathrm{p}$.

\section{Articles dans des revues arbitrées :}

Savard, J., Leduc, N., Lebel, P., Béland, F., \& Bergman, H. L'utilisation des centres de jour par les personnes âgées qui présentent des incapacités. Canadian Journal on Aging / Revue canadienne $d u$ vieillissement. (2007) 26(3):255-274.

Savard, J., Leduc, N., Lebel, P., Béland, F., \& Bergman, H. Caregiver satisfaction with support services: Influence of different types of services. Journal of Aging and Health. (2006) 18(1):3-27.

\section{Résumé de communications et présentations arbitrées :}

Lagacé, J., Bélanger-Schaadt, M.A., Cormier, I., Savard, J., Dubouloz C-J. Interprofessional approach to Auditory Processing Disorders. School 2008 conference of the American Speech and Audiology Association, Florida. (Juillet 2008). (affiche)

Savard, J., Metthé, L., Dubouloz, C.-J. Guitard, P., Brasset-Latulippe, A. An Interprofessional University Clinic: Interprofessional learning at its best! Congrès de l'association canadienne des ergothérapeutes. Whitehorse, Yukon. (Juin 2008). (affiche)

Savard, J., Leduc, N., Lebel, P., Béland, F., \& Bergman, H. L'utilisation des services de centre de jour par les personnes âgées. Congrès de l'association canadienne des ergothérapeutes. St-Jean, TerreNeuve. (Juillet 2007). (affiche)

Gauthier, V., St-Jean, J., Dubé, K., Pineault, L.P., Savard, J. Evaluation practices of Canadian Francophone and Anglophone occupational therapists. Congrès de l'association canadienne des ergothérapeutes. St-Jean, Terre-Neuve. (Juillet 2007).

Dubouloz, C.J., Savard, J., Metthé, L., Guitard, P., Casimiro, L. Enhancing interprofessional education through a rehabilitation university clinic. Congrès de l'association canadienne des ergothérapeutes. St-Jean, Terre-Neuve. (Juillet 2007).

Savard, J., Dubouloz, C.J., Metthé, L., Guitard, P. Améliorer l'accessibilité aux services de réadaptation. Congrès de l'association canadienne des ergothérapeutes. St-Jean, Terre-Neuve. (Juillet 2007). (affiche)

Metthé, L., Dubouloz, C.J., Savard, J., Guitard, P., Thelland, M.J. Implementation strategies for an interprofessional rehabilitation university clinic in primary health care. Congrès de l'Association canadienne des orthophonistes et des audiologistes. Moncton, N.B. (avril 2007). (affiche)

Savard, J., Leduc, N., Lebel, P., Béland, F., \& Bergman, H. L'utilisation des services de centre de jour par les personnes âgées qui ont des incapacités fonctionnelles. VIIe Congrès international francophone de gérontologie et gériatrie. Québec, QC. (octobre 2006). (affiche)

Guitard, P., Egan, M., Tremblay, M., Dubouloz, C.J., Coutu-Wakulczyk, G., Etchevery, E., Restall, G., Leclair, L., Savard, J. Étude pilote : Fidélité test-retest de la Mesure canadienne du rendement occupationnel. Congrès de l'Association canadienne des ergothérapeutes, Montréal, QC. (2006). (affiche) 
Tremblay, M., Savard, J., Tremblay, L., \& Casimiro, L. Répertoire des outils d'évaluation en français pour la réadaptation. Congrès de l'Association canadienne des ergothérapeutes, Charlottetown, I.P.E. (2004). (affiche)

Dubouloz, C.J., Savard, J., Egan, M., Beaudoin, J.P., Haddad, L., Martin, H., Massicotte, D., Macintosh, D., \& Vallée, C. Integrating science with the human touch in a new Occupational Therapy master's program. Congrès de l'Association canadienne des ergothérapeutes, St-John, N.B. (2002). (affiche)

\section{Autres publications :}

Savard, J. \& Guitard, P. Promotion de l'ergothérapie : Défis et opportunités. Publié en trois parties dans Les Nouvelles du REFO : (2006) 9(2):4-6; (2007) 10(1):2-5 et (2008) 11(1).

Savard, J. \& Egan, M. L'ergothérapeute : un gestionnaire de cas idéal à domicile et dans la communauté. Sommaire des études récentes. Actualités ergothérapiques. (2003) 5(5):29-32.

Savard, J., Dubouloz, C.J., \& Burnett, D. Une ère de changement pour la formation en réadaptation. Reflets : Revue ontaroise d'intervention sociale et communautaire. (2002) 8(1):95-105.

\section{Présentations sans comité de lecture :}

\section{Présentations sur invitation :}

Donnelly, C., Krupa, T., Lee, K., Lee, M., Paterson, M., Savard, J., Stewart, D., Van Benthem, K. Interprofessional Education - ACOTUP panel. Congrès de l'association canadienne des ergothérapeutes. Whitehorse, Yukon. (Juin 2008).

Savard J. Les centres de jour pour les personnes âgées: réflexions éclairées par les données de recherche. Présentation au Groupe d'intérêt des centres de jour de la région de Montréal. (Mai 08).

Bélanger-Schaadt, M.A., Lagacé, J., Cormier, I., Savard, J., Dubouloz C-J. L'approche interprofessionnelle auprès de la clientèle pédiatrique à la Clinique universitaire interprofessionnelle de réadaptation en soins de santé primaire. 3e Journées orthophoniques du Regroupement des audiologistes et orthophonistes francophones de l'Ontario. Ottawa, ON. (Avril 2008).

Savard, J. Utilisation de centres de jour: Recension des écrits et premiers résultats. Présentation aux gestionnaires de centres de jour, à l'Agence de santé de la Mauricie et du Centre du Québec, Trois-Rivières. (Novembre 2006).

Savard, J., \& Guitard, P. Promotion de l'ergothérapie : Défis et opportunités. Présentation à l'assemblée générale annuelle du Regroupement des ergothérapeutes francophones de l'Ontario. (Mars 2006).

Savard, J. Études sur les centres de jour : Recension des écrits. Présentation à la table de concertation des centres de jour de Montréal-centre, Montréal. (Février 2002).

\section{Autres présentations :}

Savard, J. L'utilisation des centres de jour par les personnes âgées en perte d'autonomie. Présentation au Colloque du Réseau québécois de recherche sur le vieillissement. Montréal. (Mai 2005).

Savard, J. Facteurs qui influencent la satisfaction des personnes de soutien envers les services d'aide formelle qu'elles ont reçus. Présentation par affiche au Colloque La recherche sur le vieillissement: accomplissements et enjeux de la prochaine décennie, Réseau québécois de recherche sur le vieillissement. Sherbrooke. (Mai 2003). 